\title{
EROSION AND DEPOSITION OF SEDIMENT AT CHANNEL CROSS SECTIONS ON POWDER RIVER BETWEEN MOORHEAD AND BROADUS, MONTANA, 1980-98
}
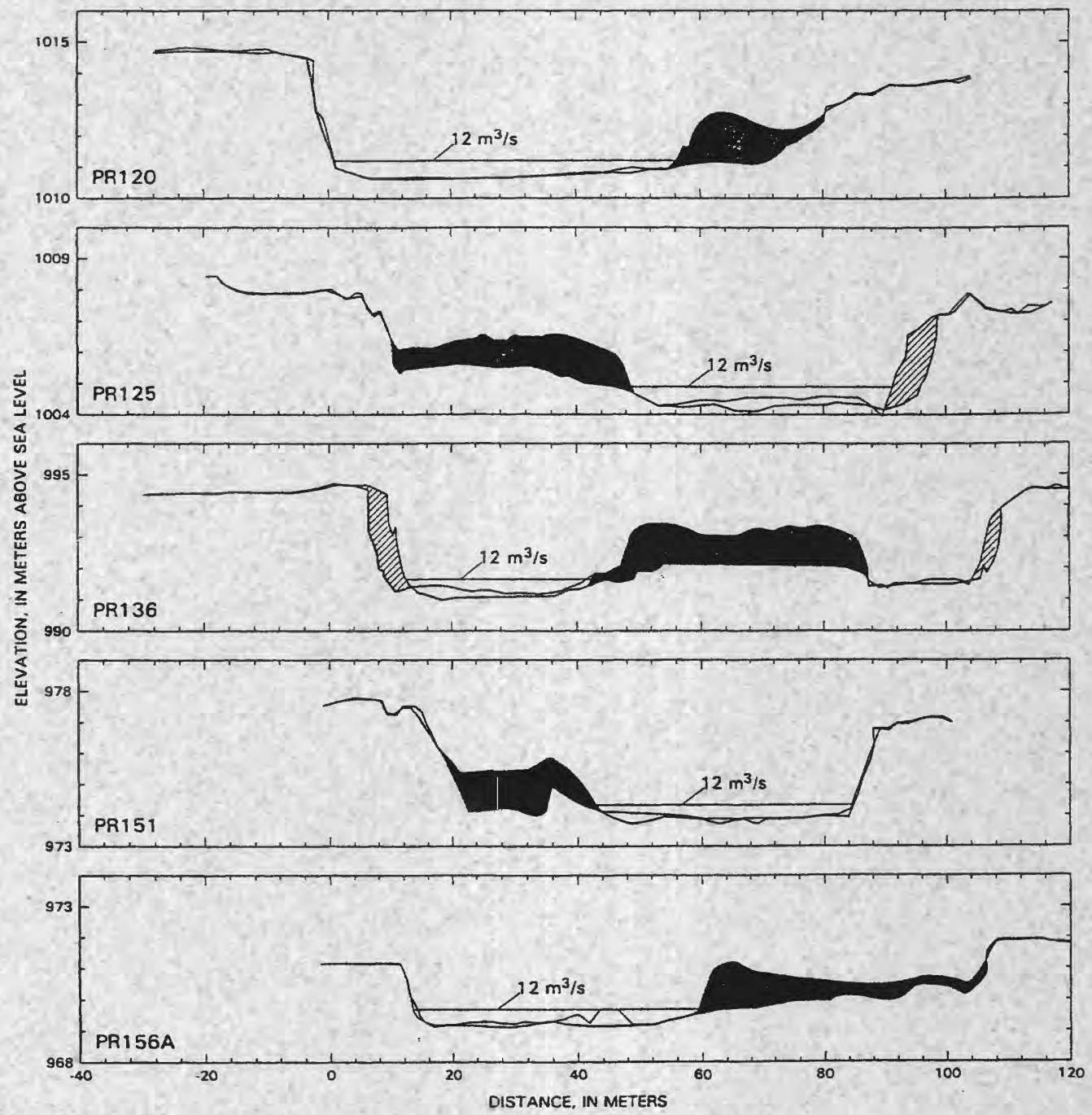

U.S. Geological Survey

Water-Resources Investigations Report 02-4219 



\section{EROSION AND DEPOSITION OF SEDIMENT AT CHANNEL CROSS}

SECTIONS ON POWDER RIVER BETWEEN MOORHEAD AND BROADUS, MONTANA, 1980-98

by John A. Moody, Robert H. Meade, and Holly A. Martinson

U.S. Geological Survey

Water-Resources Investigations Report 02-4219 


\title{
U. S. DEPARTMENT OF THE INTERIOR
}

\author{
GALE A. NORTON, Secretary
}

U.S. GEOLOGICAL SURVEY

CHARLES G. GROAT, Director

The use of trade, product, industry, or firm names is for descriptive purposes only and does not imply endorsement by the U. S. Government

For addition information write to:

Chief, Branch of Regional Research

U.S. Geological Survey

Box 25046, MS 418

Denver Federal Center

Lakewood, CO 80225
Copies of this report can be purchased from:

U. S. Geological Survey

Earth Science Information Center

Reports Section

Box 25286, MS 517

Denver Federal Center

Lakewood, CO 80225 


\section{CONTENTS}

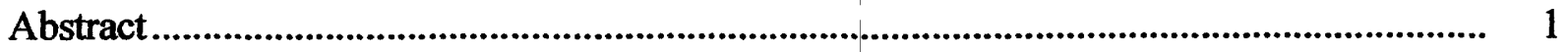

Introduction............................................................................................................................ 2

Purpose and scope

Acknowledgments ............................................................................................... 2

Suspended sediment ..................................................................................................................... 6

Deposited sediment .................................................................................................................... 9

Cross sections .......................................................................................................... 14

Survey method .......................................................................................................... 18

Channel change ................................................................................................ 18

Minimum riverbed elevation ................................................................................ 18

Area of Erosion and Deposition................................................................................. 19

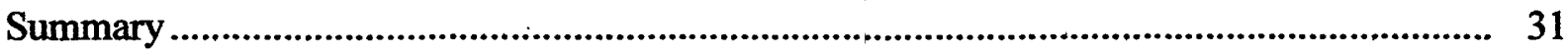

References cited .................................................................................................................... 32

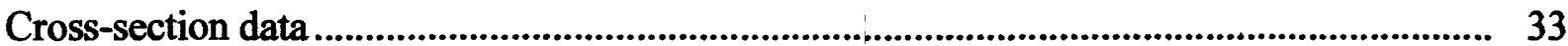

Description of cross section PR113 ….............................................................. 34

Description of cross section PR116 _................................................................ 44

Description of cross section PR120 …..................................................................... 53

Description of cross section PR122 …................................................................ 63

Description of cross section PR122A ............................................................. 67

Description of cross section PR125 …............................................................... 78

Description of cross section PR130 ….................................................................... 88

Description of cross section PR136 ....................................................................... 97

Description of cross section PR141 ........................................................................ 107

Description of cross section PR141A ....................................................................... 111

Description of cross section PR141.7 ..................................................................... 122

Description of cross section PR147 ............................................................................. 129

Description of cross section PR151 ................................................................ 139

Description of cross section PR156A ...................................................................... 148

Description of cross section PR156 ................................................................. 158

Description of cross section PR163 ….............................................................. 168

Description of cross section PR164.8 ........................................................... 178

Description of cross section PR165.6 ........................................................................ 183

Description of cross section PR166.0 .............................................................. 188

Description of cross section PR166.6 ...................................................................... 193

Description of cross section PR167 .............................................................. 198

Description of cross section PR167.5 …............................................................. 208

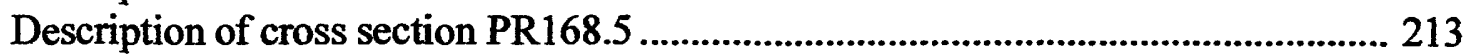

Description of cross section PR169.2 …............................................................... 218

Description of cross section PR169.8 …............................................................ 223

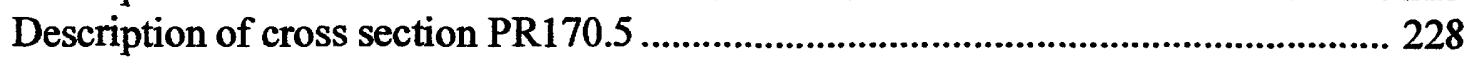

Description of cross section PR175 …............................................................. 233

Description of cross section PR180 …....................................................................... 244

Description of cross section PR183 ….................................................................. 254 
Description of cross section PR191 ......................................................................... 263

Description of cross section PR194 …....................................................................... 273

Description of cross section PR200A …………........................................................ 282

Description of cross section PR206 ........................................................................ 296

\section{FIGURES}

1. Map showing location of Powder River drainage basin and the study reach .

2. Graph showing daily mean discharge for Powder River at Moorhead, Montana, for each water year 1989, 1990, 1991, 1992, and 1993.

3. Graph showing daily mean discharge for Powder River at Moorhead, Montana, for each water year 1994, 1995, 1996, 1997, and 1998.

4. Graph showing relations between suspended-sediment concentration and water discharge for single floods.

5. Graph showing relations between suspended-sediment concentration and water discharge for multiple floods

6. Graph showing percentage of sand and silt \& clay in eroded and deposited sediment.... 11

7. Map showing location of 20 channel cross sections and tributaries of

Powder River in the study reach

8. Map showing location of 10 channel cross sections between PR163 and PR175 ........... 17

9. Map showing the location of cross section PR113 in the Bradshaw Creek quadrangle... 35

10. Profiles of cross section PR113 from 1988 to 1992 ....................................................... 36

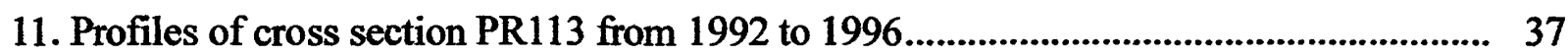

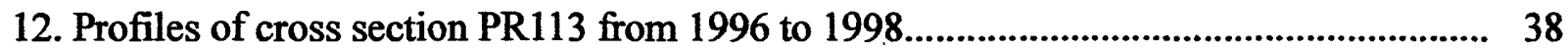

13. Map showing the location of cross section PR116 in the Moorhead quadrangle ............ 45

14. Profiles of cross section PR116 from 1988 to 1992 .......................................................... 46

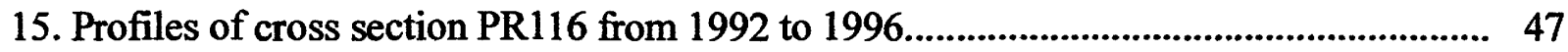

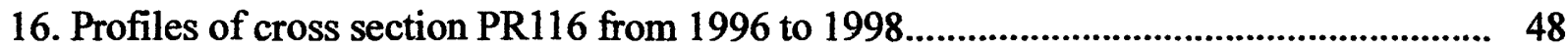

17. Map showing the location of cross section PR120 in the Moorhead quadrangle............ 54

18. Profiles of cross section PR120 from 1988 to 1992 ...................................................... 55

19. Profiles of cross section PR120 from 1992 to 1996 ......................................................... 56

20. Profiles of cross section PR120 from 1996 to 1998 ................................................. 57

22. Map showing the location of cross section PR122 in the Moorhead quadrangle............ 64

22. Profiles of cross section PR122 from 1987 to 1995 ...................................................... 65

23. Map showing the location of cross section PR122A in the Moorhead quadrangle ........ 68

24. Profiles of cross section PR122A from 1988 to 1992 .................................................. 69

25. Profiles of cross section PR122A from 1992 to 1996 .................................................. 70

26. Profiles of cross section PR122A from 1996 to 1998 ................................................. 71

27. Map showing the location of cross section PR125 in the Moorhead quadrangle............ 79

28. Profiles of cross section PR125 from 1988 to 1992 ..................................................... 80

29. Profiles of cross section PR125 from 1992 to 1996 ......................................................... 81

30. Profiles of cross section PR125 from 1996 to 1998 .................................................... 82

31. Map showing the location of cross section PR130 in the Bloom Creek quadrangle...... 89

32. Profiles of cross section PR130 from 1988 to 1992 ..................................................... 90 
33. Profiles of cross section PR130 from 1992 to 1996 ..................................................... 91

34. Profiles of cross section PR130 from 1996 to 1998 .................................................... 92

35. Map showing the location of cross section PR136 in the Bloom Creek quadrangle....... 98

36. Profiles of cross section PR136 from 1988 to 1992 ..................................................... 99

37. Profiles of cross section PR136 from 1992 to 1996 ........................................................ 100

38. Profiles of cross section PR136 from 1996 to 1998 ....................................................... 101

39. Map showing the location of cross section PR141 in the Bloom Creek quadrangle...... 108

40. Map showing the location of cross section PR141A in the Huckins School quadrangle 112

41. Profiles of cross section PR141A from 1988 to 1992 .................................................... 113

42. Profiles of cross section PR141A from 1992 to 1996 ....................................................... 114

43. Profiles of cross section PR141A from 1996 to 1998 ................................................. 115

44. Map showing the location of cross section PR141.7 in the Huckins School quadrangle 123

45. Profiles of cross section PR141.7 from 1994 to 1998 .................................................... 124

46. Map showing the location of cross section PR147 in the Huckins School quadrangle... 130

47. Profiles of cross section PR147 from 1988 to 1992 ................................................... 131

48. Profiles of cross section PR147 from 1992 to 1996 .................................................. 132

49. Profiles of cross section PR147 from 1996 to 1998 .................................................... 133

50. Map showing the location of cross section PR151 in the Huckins School quadrangle... 140

51. Profiles of cross section PR151 from 1988 to 1992 .................................................... 141

52. Profiles of cross section PR151 from 1992 to 1996 ................................................... 142

53. Profiles of cross section PR151 from 1996 to 1998 .................................................... 143

54. Map showing the location of cross section PR156A in the Huckins School quadrangle 149

55. Profiles of cross section PR156A from 1988 to 1992 ..................................................... 150

56. Profiles of cross section PR156Afrom 1992 to 1996 ........................................................ 151

57. Profiles of cross section PR156A from 1996 to 1998 ................................................... 152

58. Map showing the location of cross section PR156 in the Huckins School quadrangle... 159

59. Profiles of cross section PR156 from 1988 to 1992 ...................................................... 160

60. Profiles of cross section PR156 from 1992 to 1996 .................................................... 161

61. Profiles of cross section PR156 from 1996 to 1998 ...................................................... 162

62. Map showing the location of cross section PR163 in the Yarger Butte quadrangle........ 169

63. Profiles of cross section PR163 from 1988 to 1992 ........................................................ 170

64. Profiles of cross section PR163 from 1992 to 1996 ....................................................... 171

65. Profiles of cross section PR163 from 1996 to 1998 ................................................... 172

66. Map showing the location of cross section PR164.8 in the Lonesome Peak quadrangle 179

67. Profiles of cross section PR164.8 from 1986 to 1998 ................................................... 180

68. Map showing the location of cross section PR165.6 in the Lonesome Peak quadrangle 184

69. Profiles of cross section PR165.6 from 1980 to 1998 ................................................... 185

70. Map showing the location of cross section PR166.0 in the Lonesome Peak quadrangle 189

71. Profiles of cross section PR166.0 from 1980 to 1998 ................................................. 190

72. Map showing the location of cross section PR166.6 in the Lonesome Peak quadrangle 194

73. Profiles of cross section PR166.6 from 1980 to 1998 ..................................................... 195

74. Map showing the location of cross section PR167 in the Lonesome Peak quadrangle... 199

75. Profiles of cross section PR167 from 1988 to 1992 .................................................... 200

76. Profiles of cross section PR167 from 1992 to 1996 ..................................................... 201 
77. Profiles of cross section PR167 from 1996 to 1998 ...................................................... 202

78. Map showing the location of cross section PR167.5 in the Lonesome Peak quadrangle 209

79. Profiles of cross section PR167.5 from 1980 to 1992 ................................................... 210

80. Map showing the location of cross section PR168.5 in the Lonesome Peak quadrangle 214

81. Profiles of cross section PR168.5 from 1980 to 1998 ................................................... 215

82. Map showing the location of cross section PR169.2 in the Lonesome Peak quadrangle 219

83. Profiles of cross section PR169.2 from 1980 to 1998 ................................................... 220

84. Map showing the location of cross section PR169.8 in the Lonesome Peak quadrangle 224

85. Profiles of cross section PR169.8 from 1980 to 1998 .................................................... 225

86. Map showing the location of cross section PR170.5 in the Lonesome Peak quadrangle 229

87. Profiles of cross section PR170.5 from 1980 to 1998 .................................................. 230

88. Map showing the location of cross section PR175 in the Lonesome Peak quadrangle... 234

89. Profiles of cross section PR175 from 1988 to 1992 ......................................................... 235

90. Profiles of cross section PR175 from 1992 to 1996 .................................................... 236

91. Profiles of cross section PR175 from 1996 to 1998 .................................................. 237

92. Map showing the location of cross section PR180 in the Lonesome Peak quadrangle... 245

93. Profiles of cross section PR180 from 1988 to 1992 ....................................................... 246

94. Profiles of cross section PR180 from 1992 to 1996 ..................................................... 247

95. Profiles of cross section PR180 from 1996 to 1998 ...................................................... 248

96. Map showing the location of cross section PR183 in the Lonesome Peak quadrangle... 255

97. Profiles of cross section PR183 from 1988 to 1992 ..................................................... 256

98. Profiles of cross section PR183 from 1992 to 1996 ...................................................... 257

99. Profiles of cross section PR183 from 1996 to 1998 ................................................... 258

100. Map showing the location of cross section PR191 in the Eldon Mountain quadrangle 264

101. Profiles of cross section PR191 from 1988 to 1992 ...................................................... 265

102. Profiles of cross section PR191 from 1992 to 1996 ....................................................... 266

103. Profiles of cross section PR191 from 1996 to 1998 .................................................... 267

104. Map showing the location of cross section PR194 in the Eldon Mountain quadrangle 274

105. Profiles of cross section PR194 from 1988 to 1992 ..................................................... 275

106. Profiles of cross section PR194 from 1992 to 1996 .................................................. 276

107. Profiles of cross section PR194 from 1996 to 1998 .................................................. 277

108. Map showing the location of cross section PR200A in the Broadus quadrangle .......... 283

109. Profiles of cross section PR200A from 1988 to 1991 ................................................ 284

110. Profiles of cross section PR200A from 1991 to 1994 ..................................................... 285

111. Profiles of cross section PR200A from 1994 to 1997.................................................. 286

112. Profiles of cross section PR200A from 1997 to 1998................................................ 287

113. Map showing the location of cross section PR206 in the Broadus quadrangle ............. 297

114. Profiles of cross section PR206 from 1988 to 1992..................................................... 298

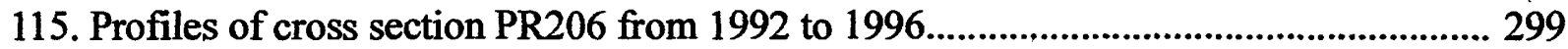

116. Profiles of cross section PR206 from 1996 to 1998 ...................................................... 300 


\section{TABLES}

1. Suspended-sediment characteristics of samples collected during floods on

Powder River at Moorhead and Broadus, Montana.

2. Particle-size analysis of sediment deposited on the flood plain and on the bed

of Powder River...................................................................................... cross sections of Powder River between Moorhead and Broadus, Montana.................. 16

4. Minimum riverbed elevation at the end of the water year for 1989-1998 ........................ 20

5. Changes in the minimum riverbed elevation for 1978-1998 ........................................... 21

6. Erosion and deposition of sediment at channel cross sections on the Powder River ....... 22

7-39. Listings of horizontal stations and elevations for cross sections

7. PR113

8. PR116.

9. PR120

10. PR122

11. PR122A

12. PR125

13. PR130

14. PR136

15. PR141

16. PR141A

17. PR141.7

18. PR147

19. PR151

20. PR156A

21. PR156

22. PR163

23. PR164.8

24. PR165.6

25. PR166.0

26. PR166.6

27. PR167

28. PR167.5

29. PR168.5

30. PR169.2

31. PR169.8

32. PR170.5

33. PR175

34. PR180

35. PR183

36. PR191

37. PR194

38. PR200A

39. PR206

Appendix Sand-Silt and Clay analysis of suspended-sediment samples collected at Moorhead and Broadus, Montana, on the Powder River. 


\section{CONVERSION FACTORS}

Metric units (International System) in this report may be converted to inch-pound units by using the following conversion factors:

\section{Multiply}

meter (m)

kilometer $(\mathrm{km})$

square meter $\left(\mathrm{m}^{2}\right)$

square kilometer $(\mathrm{km} 2)$

cubic meter $\left(\mathrm{m}^{3}\right)$

meter per second $(\mathrm{m} / \mathrm{s})$

cubic meter per second $\left(\mathrm{m}^{3} / \mathrm{s}\right)$

cubic meter per year $\left(\mathrm{m}^{3} / \mathrm{yr}\right)$

kilogram (kg)

metric tons per day

metric tons per year

metric tons per square kilometer per year (metric tons $/ \mathrm{km}^{2} / \mathrm{yr}$ )
By

To obtain

\section{Length}

3.281

0.6214

Area

10.76

0.3861

Volume

1.308

cubic yard $\left(\mathrm{yd}^{3}\right)$

\section{Velcity}

3.281

foot per second (ft/s)

Flow

35.31

cubic foot per second (cfs)

35.31

cubic foot per year $\left(\mathrm{ft}^{3} / \mathrm{yr}\right)$

Mass

2.205

pounds avoirdupois (lb)

Mass Flow

1.102

1.102

tons per day (tons/d)

tons per year (tons/yr)

\section{Mass Yield}

2.854 tons per square mile

per year (tons $/ \mathrm{mi}^{2} / \mathrm{yr}$ )

Sea level: In this report "sea level' refers to the National Geodetic Vertical Datum of 1929 (NGVD of 1929)--a geodetic datum derived from a general adjustment of the first-order level nets of both the United State and Canada, formerly called Sea Level Datum of 1929. 


\section{Errata}

These are corrections for the first Open-File Report 89-407 titled:

Channel Changes at cross sections of the Powder River between Moorhead and Broadus, Montana, 1975-88 by John A. Moody and Robert H. Meade.

p. 6 and 7. Elevation for cross section PR163 is 965.53 meters above sea level.

p. 13. The labels in figure 4 should read Moorhead not Moorehead.

p. 143. Location of bench mark BM-PR156 is shown (not BM-PR151).

p. 244. Bench mark for PR206 is on the right bank (not on the left bank). 


\title{
SECTIONS ON POWDER RIVER BETWEEN
}

\section{MOORHEAD AND BROADUS, MONTANA, 1980-98}

\author{
John A. Moody, Robert H. Meade, and Holly A. Martinson
}

\begin{abstract}
Erosion and deposition of sediment and some characteristics of these sediments were measured along a 95-kilometer study reach of Powder River between Moorhead and Broadus, Montana from 1988 to 1998 . Suspended-sediment samples were collected at two gaging stations (Moorhead and Broadus, Montana). Sediment samples were also collected from the flood plain and channel within the study reach. Bed elevations were measured each year after the peak runoff at 21 cross sections and for different time intervals at an additional 12 cross sections.

The proportions of sand (particle diameters greater than or equal to 0.063 millimeters) and silt and clay (less than 0.063 millimeters) in the suspended sediment depended upon the type of flood. Ice break-up floods in February and March carried 28 percent sand, snowmelt floods in May and June carried 23 percent sand, and flash floods during the summer carried 7 percent sand. The proportion of sand in the deposited sediment depended upon the distance of the sample from the channel. Samples from the channel contained about 99 percent sand, samples from the flood plain crest adjacent to the channel contained about 70 percent sand, and samples from the flood plain trough farthest from the channel contained about 10 percent sand.

Changes in erosion and deposition of sediment were measured by changes in the minimum riverbed elevation and changes in the cross-sectional area. In general, the minimum riverbed elevation fluctuated from year to year and showed no strong trend toward either increase or decrease. Standard deviations of the minimum riverbed elevation ranged from 0.01 to 0.30 meter. Areas of erosion and deposition were calculated for successive years at each cross section and subjectively assigned to four features where erosional and depositional processes were active: 1) bank, 2) flood plain, 3) channel, and 4) point bar. Erosion exceeded deposition by 47 percent at one cross section and deposition exceeded erosion by 41 percent at another cross section. However, the average of all the cross sections indicated, in general, that the erosion of sediment was balanced by the deposition of sediment.
\end{abstract}




\section{INTRODUCTION}

Powder River flows east and northeast from the Bighorn Mountains in north-central Wyoming through northeastern Wyoming and southeastern Montana (fig. 1). At its confluence with the Yellowstone River, Powder River drains an area of 34, $706 \mathrm{~km}^{2}$ and has an average discharge of about 500 million $\mathrm{m}^{3} / \mathrm{yr}$ or $16 \mathrm{~m}^{3} / \mathrm{s}$. Near the Wyoming-Montana State line (at the gaging station at Moorhead, Montana), Powder River discharges an average of 2 to 3 million metric tons of suspended sediment per year, which corresponds to a sediment yield of about $\mathbf{4 0 0}$ metric tons/ $\mathrm{km}^{2} / \mathrm{yr}$. The Powder River drainage basin includes areas of conventional production of oil and coal (Teapot Dome oil fields and part of the Gillette coal field) and areas of coal-bed methane production. At the present time, the primary use of river water in the basin is for irrigation. No dams or other large engineering structures obstruct the flow of Powder River or its principal tributaries.

Three types of floods occur on Powder River: ice break-up floods, snowmelt floods, and flash floods (figs. 2 and 3). Ice break-up floods are caused by snowmelt or rain at low elevation in February or March. They occur along most of the mainstem and last a few days to a week, often breaking up the ice cover and creating ice dams, which amplify the flooding in localized areas. Snowmelt floods are caused by snowmelt at higher elevations, primarily in the Bighorn Mountains. Snowmelt floods usually are larger than the ice break-up floods, typically occur in May or June along the entire mainstem, and may last several weeks. Flash floods are caused by localized summer (June through September) convective rain storms. They affect only a few tributaries and often only part of the mainstem Powder River.

In 1975, a program of measurements was begun to define long-term sediment budgets and channel changes along a study reach of Powder River. This river was selected for several reasons. First, Powder River carries a large load of suspended sediment (Hembree and others, 1952), which is an indication of active sedimentary processes in the river and in its drainage basin. Second, Powder River is one of the few remaining rivers of its size in the region that is not controlled by a dam (in contrast with the neighboring Bighorn, Tongue, and Belle Fourche Rivers). Third, a major flood occurred on the river in 1978, and these post-flood measurements continue a longterm record (Moody and Meade, 1990) of how an active river-floodplain system adjusts to a major disturbance.

\section{Purpose and Scope}

The purpose of this report is to present measurements of the erosion and deposition of sediment at channel cross sections following a major flood in a river that carries a large sediment load. The reach selected for detailed study was the $95-\mathrm{km}$ reach of Powder River between Moorhead and Broadus, Montana (fig. 1). Samples of suspended sediment (eroded sediment) were collected at the gaging stations at Moorhead and Broadus, and samples of sediment deposited on the flood plain were collected at various locations. Data are presented that describe the characteristics of this eroded and deposited sediment. The amounts of erosion and deposition of sediment at channel cross sections were computed from the distances and elevations listed in this report and subjectively assigned to four features where erosional and depositional processes were active: 1) bank, 2) flood plain, 3) channel, and 4) point bar.

\section{Acknowledgments}

We thank the ranchers and landowners along Powder River for their hospitality and their continuing courtesy in allowing us repeated access to the river. They are, in downstream order: Hugh and Cheri Fulton, George and Evelene Fulton, Larry and Linda Thomas, Jim and Ellie Bowers, Tom and Carla Bowers, Jim and Sherry Bowers, Bill and Dinah Gay, Glenn Gay, Hubert and Mary Gay, Bunk Huckins, Gary Huckins, Floyd and Dora Huckins, Butch Samuelson, the late 


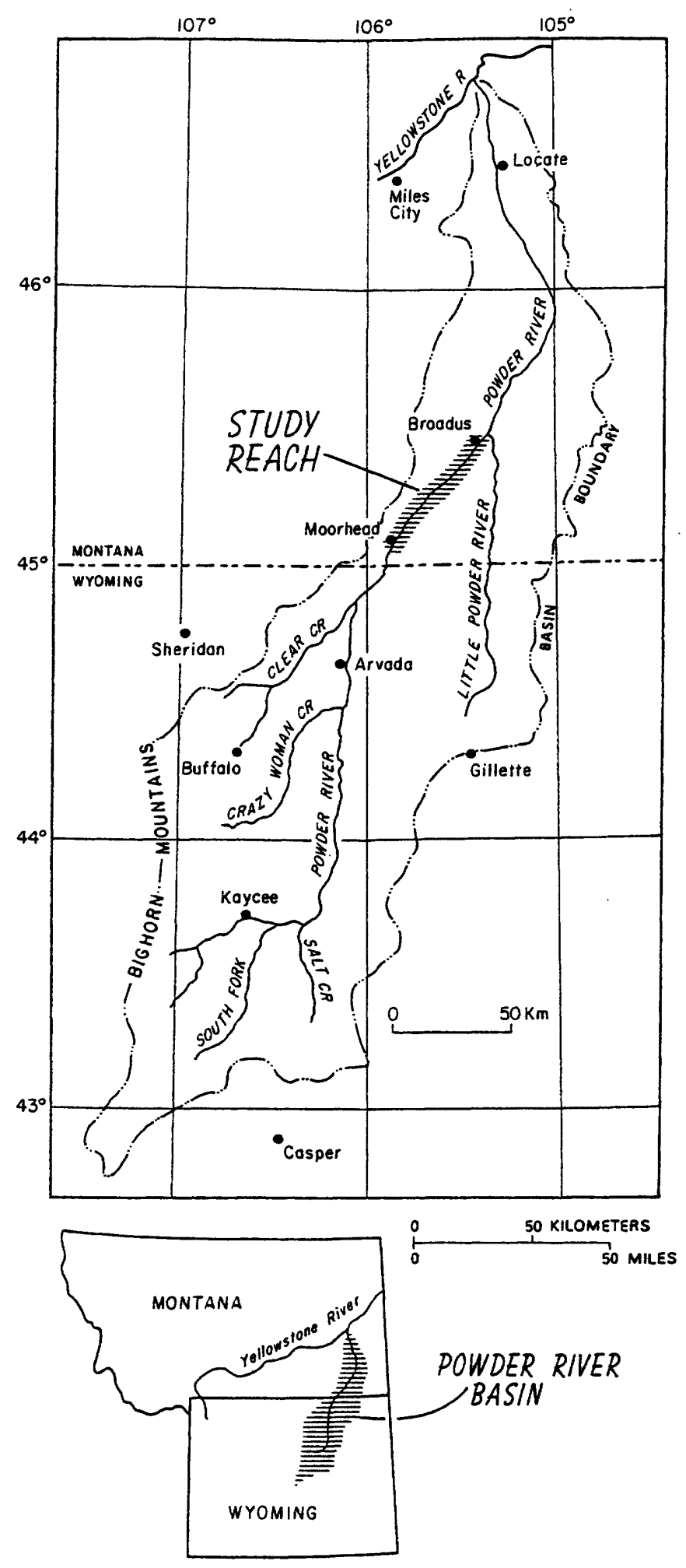

Figure 1.--Location of Powder River drainage basin and the study reach. 

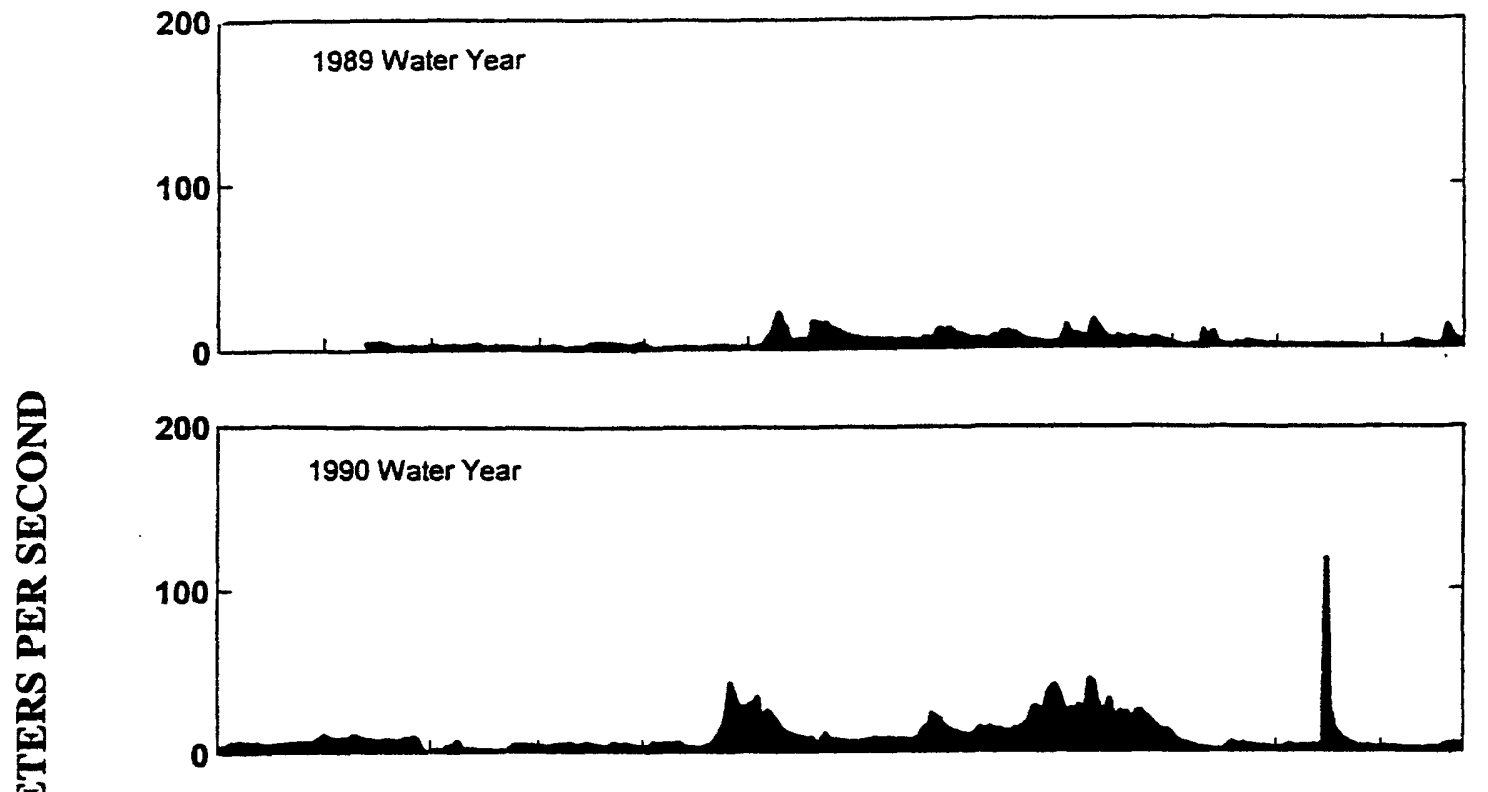

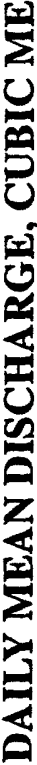
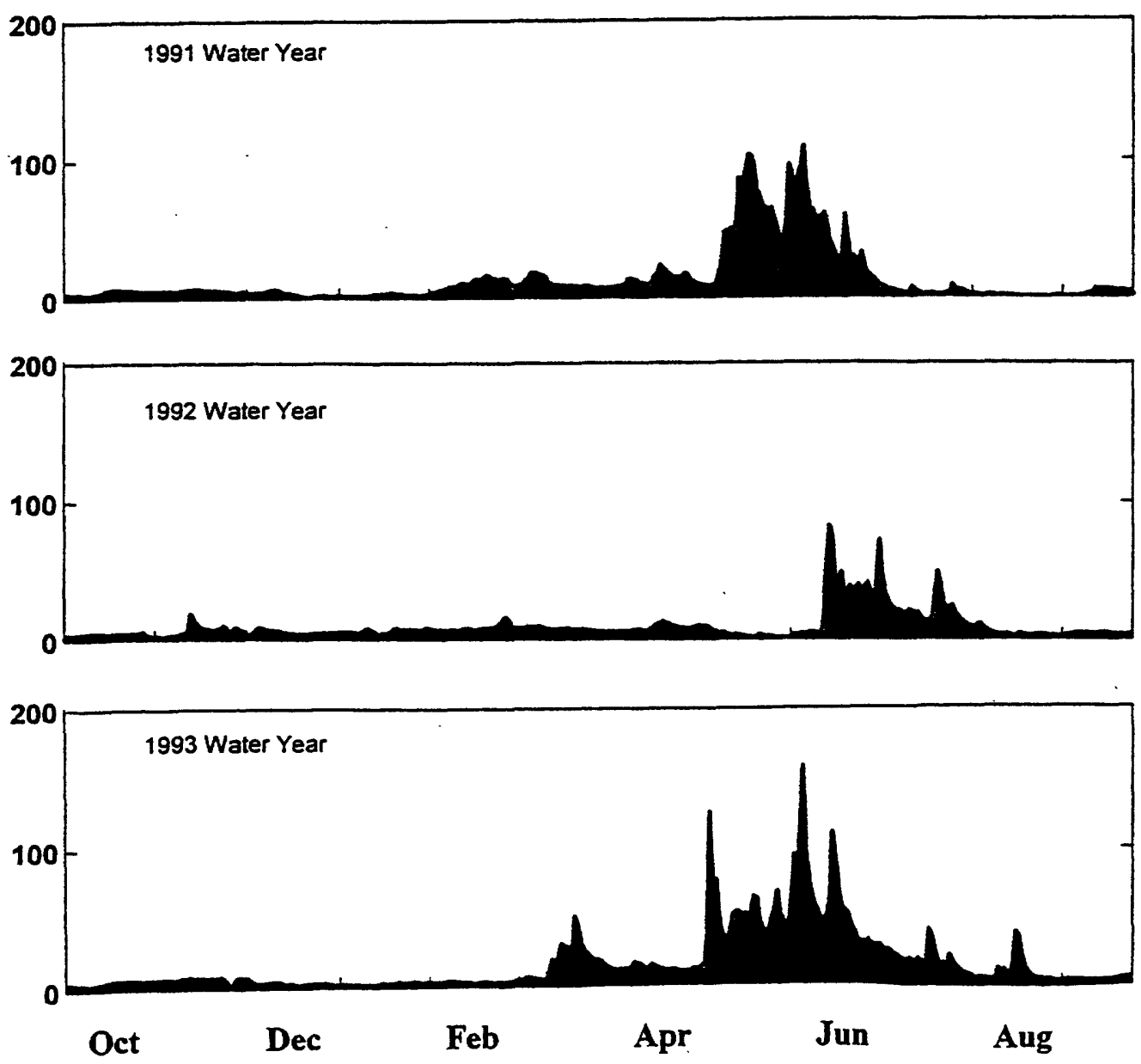

Figure 2. Daily mean water discharge for Powder River at Moorhead, Montana, for each water year 1989, 1990, 1991, 1992, and 1993. 


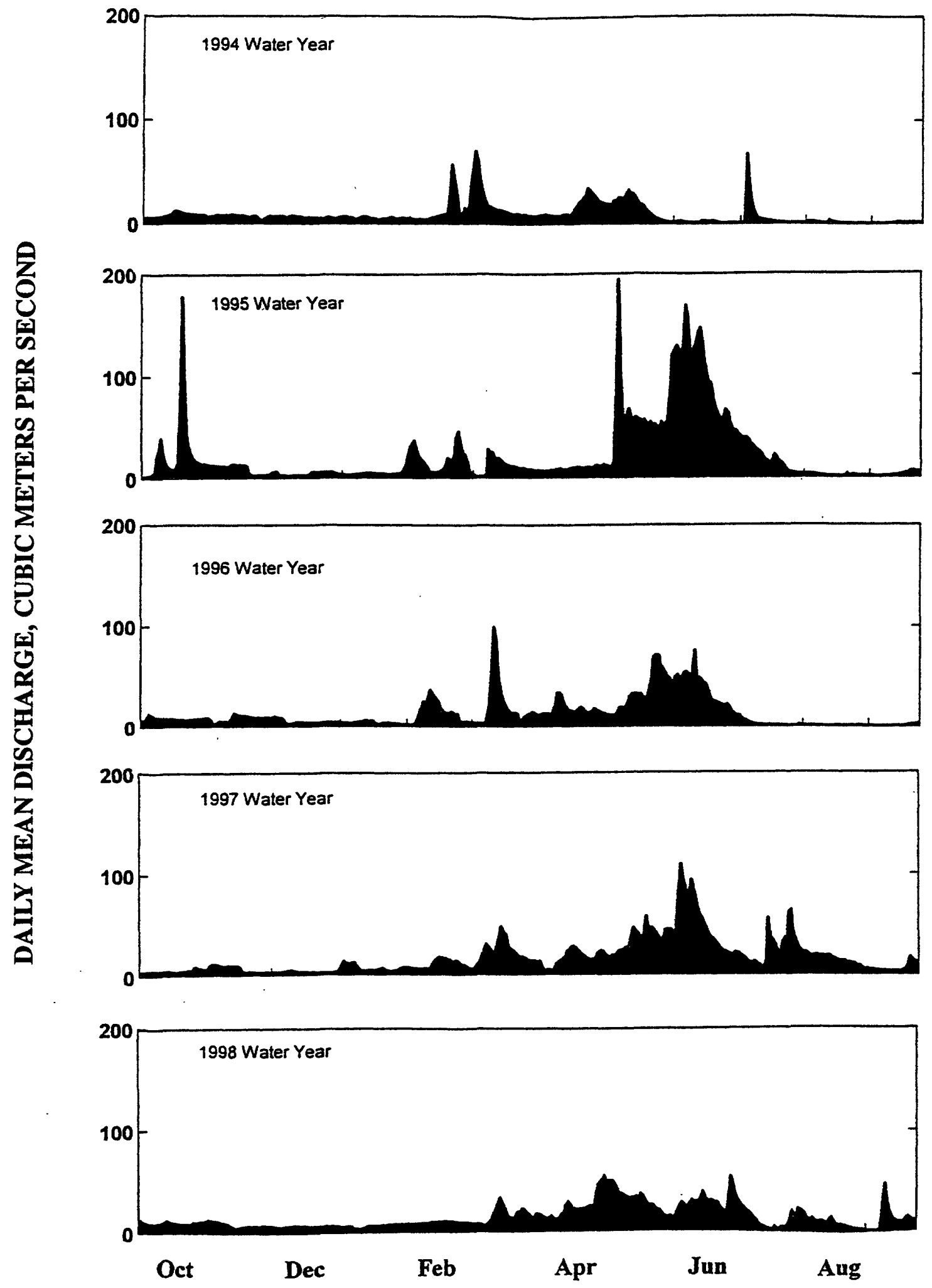

Figure 3. Daily mean water discharge for Powder River at Moorhead, Montana, for each water year 1994, 1995, 1996, 1997, and 1998. 
John Daily, James Cossitt, the late J.L. Wilson and his wife Phyllis, Dick and Connie Wilson, Craig and Jackee Randall, Doug and Lucille Randall, the late Shirley Stuver, John and Diane Stuver, Charlie and Shirley Russell, Ron and Twila Talcott, and Jean Hough. We also acknowledge the stimulating conversations and encouragement, along the banks of the Powder River, with Jim Pizzuto, Nick Allmendinger, Koichi Fujita, Nicole Surian, and J. Dungan Smith. Deborah Martin was instrumental in helping with the GPS measurements in 1998, which provided accurate locations of the cross sections on Powder River for geomorphological research in the future. Jim Pizzuto provided some sediment data for this report. Deborah Martin and Jim Pizzuto provided useful suggestions to improve the original report.

\section{SUSPENDED-SEDIMENT}

Depth-integrated samples of suspended sediment were collected from bridges near the gaging stations at Moorhead, Montana, from 1975 through 1997 and at Broadus, Montana, from 1975 through 1995. These samples represent typical sediment eroded from the banks and bed of Powder River. Particle-size analyses of 305 of these samples included either a determination of sand fraction and silt and clay fraction by sieve analyses at whole phi intervals [phi $=-\log _{2}$ (particle diameter in millimeters)], or a determination of sand fraction, silt fraction, and clay fraction by settling analyses at whole phi intervals (U.S. Geological Survey, 1975-97). These analyses were sorted first by the type of flood and then by whether or not the mean daily discharge was increasing (rising stage), at a maximum, decreasing (falling stage), or at a minimum. From the 305 samples, 51 samples were collected during ice break-up floods, 47 samples were collected during snowmelt floods, 17 were collected during flash floods, and 190 samples were collected during other times of the year. Because all analyses (115 samples, see Appendix) obtained data for the percent sand $(>0.063 \mathrm{~mm})$ and for the percent silt and clay $(<0.063 \mathrm{~mm})$, the sediment characteristics of the three types of floods are summarized by these two size classes (table 1 ).

The maximum total suspended-sediment concentration and the relative proportions of sand versus silt and clay were different for the three types of floods. Snowmelt floods carried the greatest maximum concentration $(52,200 \mathrm{mg} / \mathrm{L})$, followed by flash floods $(49,700 \mathrm{mg} / \mathrm{L})$, and ice break-up floods $(26,000 \mathrm{mg} / \mathrm{L})$. Ice break-up floods carried a greater percentage of sand ( 28 percent) than either the snowmelt floods ( 23 percent) or the flash floods ( 7 percent). In ice break-up floods, the greatest mean concentration of sand $(3,820 \mathrm{mg} / \mathrm{L})$ occurred during falling stages, whereas in snowmelt and flash floods, the greatest mean concentrations $(3,160$ and $2,880 \mathrm{mg} / \mathrm{L}$, respectively) occurred during maximum stage. Flash floods during maximum and falling stages (no samples were collected during rising stages) carried a greater percentage of silt and clay (9096 percent) than ice break-up floods (66-81 percent) and snowmelt floods (75-79 percent). Mean concentrations of silt and clay $(15,300-24,300 \mathrm{mg} / \mathrm{L})$ during the maximum and falling stages of flash floods were also greater than those measured for ice break-up floods $(5,110-5,120 \mathrm{mg} / \mathrm{L})$ and for snowmelt floods $(6,130-16,600 \mathrm{mg} / \mathrm{L})$.

Many samples were collected during a snowmelt flood (13 May 1981 to 6 July 1981) and during an ice break-up flood (19-21 March 1997). Both data sets were collected at the gage at Moorhead, Montana. For the snowmelt flood (fig. 4A), the silt and clay concentration indicated a clockwise loop relation with discharge. The maximum concentration of silt and clay $(18,200 \mathrm{mg} /$ L) occurred during the rising stage $\left(33.1 \mathrm{~m}^{3} / \mathrm{s}\right)$ before the maximum discharge $\left(56.9 \mathrm{~m}^{3} / \mathrm{s}\right)$. The sand concentration approximated a linear relation with discharge and reached a maximum at maximum discharge $\left(3,580 \mathrm{mg} / \mathrm{L}\right.$ at $\left.56.9 \mathrm{~m}^{3} / \mathrm{s}\right)$. During the ice break-up flood (fig. 4B), in contrast, the silt and clay concentration showed a counterclockwise hysteresis loop and reached a maximum concentration $(7,680 \mathrm{mg} / \mathrm{L})$ on the falling stage $\left(63.1 \mathrm{~m}^{3} / \mathrm{s}\right)$ after the maximum discharge $\left(129 \mathrm{~m}^{3} / \mathrm{s}\right)$. The sand concentrations showed a similar relation with discharge but a greater con- 
Table 1. Suspended-sediment characteristics of samples collected during floods on Powder River at Moorhead and Broadus, Montana

[Data source is annual publications (U.S. Geological Survey, 1975-97); mm, millimeter; \%, percent of total concentration; $\mathrm{mg} / \mathrm{L}$, milligram per liter; Slope, slope of linear regression of concentration and water discharge in cubic meters per second $\left(\mathrm{m}^{3} / \mathrm{s}\right)$; Intercept, intercept of linear regression of concentration and water discharge in cubic meters per second; $\mathrm{r}^{2}$, coefficient of determination; $\mathrm{nr}$, insufficient data or coefficient of determination is less than 0.1]

\begin{tabular}{|c|c|c|c|c|c|c|c|c|c|c|}
\hline \multirow{2}{*}{ Characteristic } & \multicolumn{5}{|c|}{ Silt and Clay $(<0.063 \mathrm{~mm})$} & \multicolumn{5}{|c|}{ Sand $(>0.063 \mathrm{~mm})$} \\
\hline & Rising & $\begin{array}{l}\text { Maxi- } \\
\text { mum }\end{array}$ & Falling & $\begin{array}{l}\text { Mini- } \\
\text { mum }\end{array}$ & Total & Rising & $\begin{array}{l}\text { Maxi- } \\
\text { mum }\end{array}$ & Falling & $\begin{array}{l}\text { Mini- } \\
\text { mum }\end{array}$ & Total \\
\hline & \multicolumn{10}{|c|}{ Ice break-up floods } \\
\hline Number samples & 14 & 5 & 32 & 2 & 53 & 14 & 5 & 32 & 2 & 53 \\
\hline Mean (\%) & 82 & 81 & 66 & 78 & 72 & 18 & 19 & 34 & 22 & 28 \\
\hline $\begin{array}{l}\text { Mean concentration } \\
(\mathrm{mg} / \mathrm{L})\end{array}$ & 2,610 & . 5,110 & 5,120 & 2,390 & 4,350 & 570 & 1,240 & 3,820 & 1,010 & 2,610 \\
\hline $\begin{array}{l}\text { Slope }\left(\mathrm{mg} / \mathrm{L} \text { per } \mathrm{m}^{3} / \mathrm{s}\right) \\
\text { Slope for intercept }=0\end{array}$ & $\begin{array}{l}24 \\
41\end{array}$ & $\begin{array}{r}-25 \\
\mathrm{nr}\end{array}$ & $\begin{array}{l}\mathrm{nr} \\
\mathrm{nr}\end{array}$ & $\begin{array}{l}\mathrm{nr} \\
\mathrm{nr}\end{array}$ & $\begin{array}{l}\mathrm{nr} \\
\mathrm{nr}\end{array}$ & $\begin{array}{c}11 \\
9.7\end{array}$ & $\begin{array}{l}\mathrm{nr} \\
\mathrm{nr}\end{array}$ & $\begin{array}{r}100 \\
80\end{array}$ & $\begin{array}{l}\mathrm{nr} \\
\mathrm{nr}\end{array}$ & 551 \\
\hline Intercept (mg/L) & 1,200 & 6,300 & $\mathrm{nr}$ & $\mathrm{nr}$ & nr & -110 & nr & $-1,500$ & nr & -200 \\
\hline Intercept set $=0$ & 0 & $\mathrm{nr}$ & $\mathrm{nr}$ & $\mathrm{nr}$ & $\mathrm{nr}$ & 0 & $\mathrm{nr}$ & 0 & $\mathrm{nr}$ & 0 \\
\hline \multirow[t]{2}{*}{$r^{2}$ for intercept $=0$} & $\begin{array}{l}0.56 \\
0.24\end{array}$ & $\begin{array}{c}0.68 \\
\mathrm{nr}\end{array}$ & $\mathrm{nr}$ & $\mathrm{nr}$ & $\begin{array}{l}\mathrm{nr} \\
\mathrm{nr}\end{array}$ & $\begin{array}{l}0.54 \\
0.53\end{array}$ & $\begin{array}{l}\mathrm{nr} \\
\mathrm{nr}\end{array}$ & $\begin{array}{l}0.48 \\
0.44\end{array}$ & $\begin{array}{l}\mathrm{nr} \\
\mathrm{nr}\end{array}$ & $\begin{array}{l}0.19 \\
0.19\end{array}$ \\
\hline & \multicolumn{10}{|c|}{ Snowmelt floods } \\
\hline Number samples & 13 & 14 & 17 & 4 & 48 & 13 & 14 & 17 & 4 & 48 \\
\hline Mean (\%) & 79 & 79 & 75 & 68 & 77 & 21 & 21 & 25 & 32 & 23 \\
\hline $\begin{array}{c}\text { Mean concentration } \\
(\mathbf{m g} / \mathbf{L})\end{array}$ & 8,940 & 16,600 & 6,130 & 1,180 & 9,590 & 1,740 & 3,160 & 1,570 & 488 & 2,000 \\
\hline Slope $\left(\mathrm{mg} / \mathrm{L}\right.$ per $\left.\mathrm{m}^{3} / \mathrm{s}\right)$ & 92 & 240 & 63 & 52 & 120 & 14 & 34 & 9.6 & 37 & 19 \\
\hline Slope for intercept $=0$ & 133 & 210 & 79 & 62 & 140 & 25 & 37 & $n r$ & 33 & 27 \\
\hline Intercept (mg/L) & 3,700 & $-3,000$ & 2,000 & 320 & 1,700 & 940 & 360 & 930 & -130 & 750 \\
\hline Intercept set $=0$ & 0 & 0 & 0 & 0 & 0 & 0 & 0 & $\mathrm{nr}$ & $\mathbf{0}$ & 0 \\
\hline$r^{2}$ & 0.30 & 0.67 & 0.20 & 0.92 & 0.38 & 0.34 & 0.70 & 0.28 & 0.98 & 0.43 \\
\hline \multirow[t]{2}{*}{$r^{2}$ for intercept $=0$} & 0.20 & 0.66 & 0.21 & 0.85 & 0.37 & 0.05 & 0.69 & $\mathrm{nr}$ & 0.96 & 0.34 \\
\hline & \multicolumn{10}{|c|}{ Flash floods } \\
\hline Number samples & $\mathbf{0}$ & 4 & 13 & 1 & 18 & 0 & 4 & 13 & 1 & 18 \\
\hline Mean (\%) & - & 90 & 96 & 68 & 93 & -- & 10 & 4 & 32 & 7 \\
\hline $\begin{array}{c}\text { Mean concentration } \\
(\mathbf{m g} / \mathbf{L})\end{array}$ & - & 24,300 & 15,300 & 2,260 & 16,600 & $\cdots$ & 2,880 & 583 & 1,070 & 1,120 \\
\hline Slope $\left(\mathrm{mg} / \mathrm{L}\right.$ per $\left.\mathrm{m}^{3} / \mathrm{s}\right)$ & nr & 130 & 700 & $\mathrm{nr}$ & 150 & $\mathrm{nr}$ & 20 & 51 & $\mathrm{nr}$ & 22 \\
\hline Slope for intercept $=0$ & $\mathrm{nr}$ & 190 & 850 & $\mathrm{nr}$ & 210 & $\mathrm{nr}$ & 24 & 44 & $\mathrm{nr}$ & 25 \\
\hline Intercept (mg/L) & $\mathrm{nr}$ & 11,000 & 3,600 & $\mathrm{nr}$ & 10,000 & $\mathrm{nr}$ & 940 & -150 & $\mathrm{nr}$ & 410 \\
\hline Intercept set $=0$ & $\mathrm{nr}$ & 0 & 0 & nr & 0 & $\mathrm{nr}$ & 0 & 0 & $\mathrm{nr}$ & 0 \\
\hline $\mathbf{r}^{2}$ & $\mathrm{nr}$ & 0.60 & 0.42 & $\mathrm{nr}$ & 0.43 & $\mathrm{nr}$ & 0.82 & 0.86 & $\mathrm{nr}$ & 0.84 \\
\hline $\mathbf{r}^{2}$ for intercept $=0$ & $\mathrm{nr}$ & 0.40 & 0.39 & nr & 0.10 & $\mathrm{nr}$ & 0.73 & 0.84 & $\mathrm{nr}$ & 0.80 \\
\hline
\end{tabular}




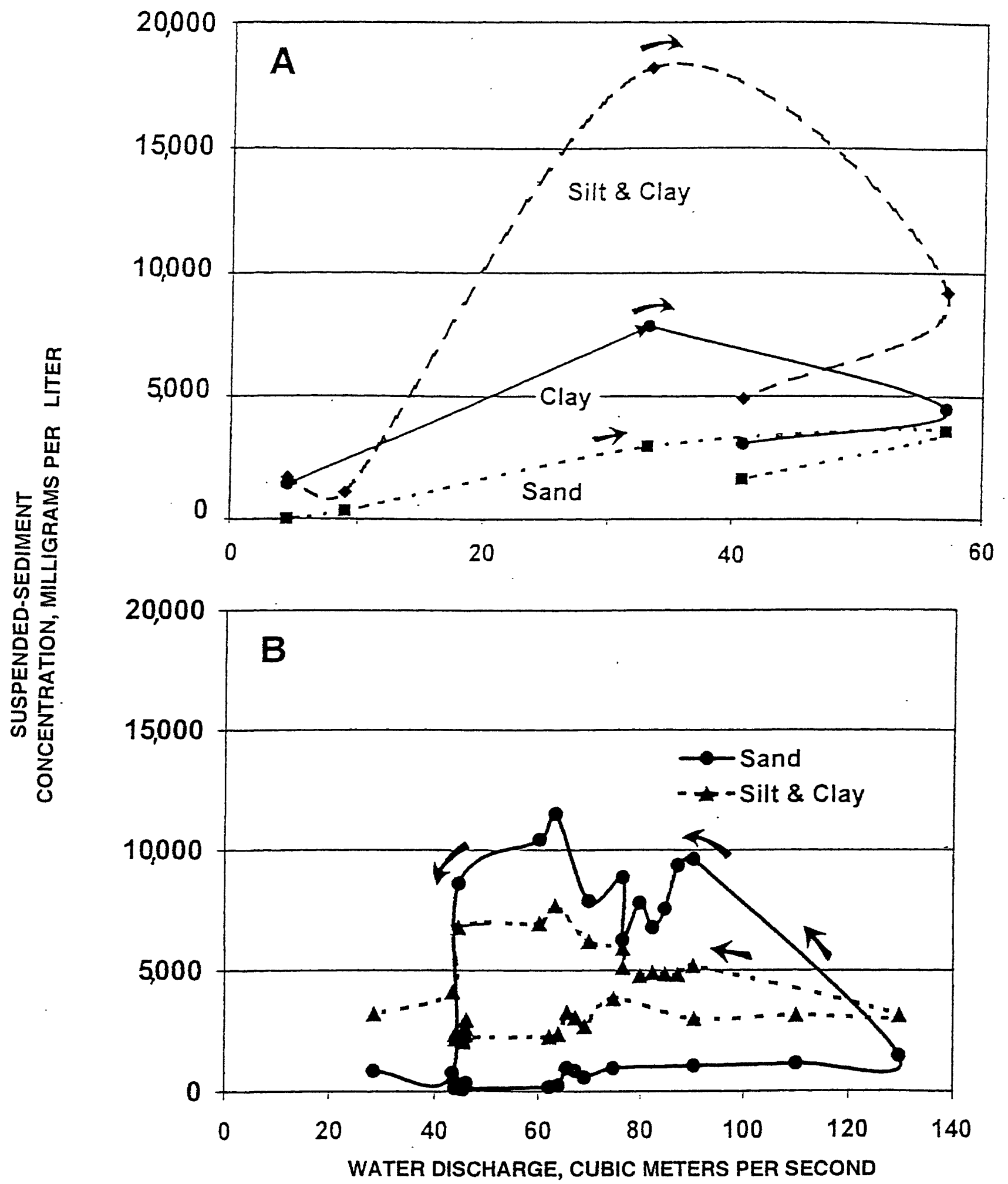

Figure 4. Relations between suspended-sediment concentration and water discharge for single floods. A. Snowmelt flood samples collected between 13 May 1981 and 6 July 1981 . The clay value was not determined for the second sample on 19 May 1981 but was certainly less than the silt and clay value. B. Ice break-up flood samples collected between 19 and 21 March 1997. 
centration $(11,500 \mathrm{mg} / \mathrm{L})$ during the falling stage $\left(63.1 \mathrm{~m}^{3} / \mathrm{s}\right)$.

The relation between suspended-sediment concentration and discharge was variable for the three types of floods. Visual inspection of the patterns of the data indicated that linear regressions would approximate the relations as well as more complex equations. Thus, two straight lines were fit to the data for each flood and for each stage category (rising, maximum, falling, and minimum). One line allowed the concentration at zero discharge to differ from zero and the other line set the concentration at zero discharge equal to zero (table 1). Most of the linear relations had coefficients of determination $\left(r^{2}\right)$ less than 0.50 . However, the relations between the concentration of silt and clay and maximum discharge and the relations between the concentration of sand and the maximum discharge had coefficients of determination greater than 0.50 (see lines in figure 5). The one exception was for ice break-up floods where the concentration of silt and clay decreased with increase in the maximum discharge. During falling discharge in ice break-up floods, sand concentrations were the largest (fig. $5 \mathrm{~A}$, right panel), but the relation with discharge had a correlation coefficient of only 0.48 and 0.44 for the two linear regressions.

\section{DEPOSITED SEDIMENT}

Samples of the sediment deposited on the flood plains and in the channels were collected over several years at different cross sections. Some samples were sediment cores, some were composite samples collected from the sides of holes dug in the flood plain, some were surficial samples, and one sample was collected underwater from newly deposited sediment adjacent to the river bank. All samples were processed by either wet sieving or dry sieving on a Ro-Tap for 20 minutes to determine the sand fractions $(>0.063 \mathrm{~mm})$ and the silt and clay fraction $(<0.063 \mathrm{~mm})$. The silt and clay fraction, in some cases, was further analyzed by settling in a solution of sodium hexametaphosphate and using standard pipette methods $(\mathrm{Guy}, 1969)$ to separate the silt fraction and the clay fraction.

Sample collection was concentrated on examples of the channel-expansion flood plains (PR120, PR125, PR136, PR151, and PR156A), which developed slowly in annual increments following the 1978 flood (Moody and others, 1999). The percentage of sand in sediment deposited on the flood plain crest at PR120 (table 2) decreased in the downstream direction. At $400 \mathrm{~m}$ upstream from the cross section, sand comprised 87.3 percent and decreased to 68.4 percent at $200 \mathrm{~m}$ downstream. In general, after many floods, the sediments on the flood-plain crest adjacent to the channel at other cross sections were mostly sand (38.5-87.3 percent) and the average of 12 samples in table 2 was 72.2 percent. Sediments in the flood-plain trough were mostly clay (36.752.0 percent) with an average sand content of 13.2 percent for 6 samples in table 2. Sediment deposited after a single flood in October 1994 was coarser ( 80 percent sand) at the flood-plain crest of PR156A and finer (24.1 percent sand) in the flood-plain trough (table 2).

The channel banks and beds are sources of sediment that may be eroded or resuspended and deposited on the flood plain. The bank sediment was mostly sand ( 60.8 percent) in the top layer (0-0.30 m) and mostly silt and clay (59.6-89.9 percent) in the remaining layers, down to a basal gravel layer (table 2). The bed of Powder River is mostly sand and gravel ( 99.2 percent sand). Patches of sand move over the top of the gravel and change location along the cross sections from year to year. The sand is mostly fine and medium sand (77.3-95.8 percent). However, newly deposited sediment on the bed can be finer. For example, sediment deposited adjacent to the bank of cross section PR125 during a snowmelt flood in 1996 contained 99 percent silt and clay and 1 percent sand. The particle-size distribution of these deposited sediments is compared with the suspended or eroded sediments in figure 6. 

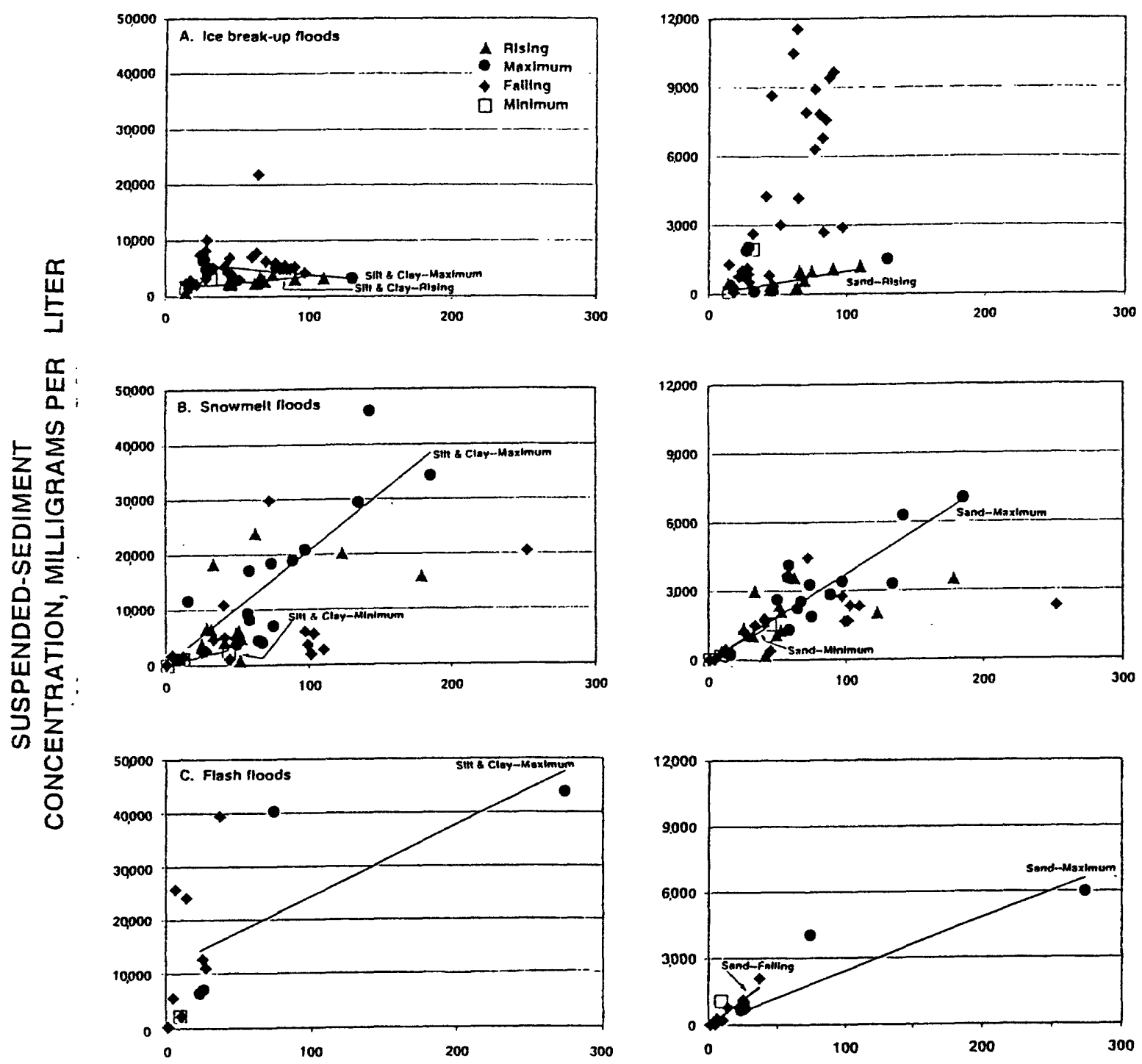

WATER DISCHARGE, CUBIC METERS PER SECOND

Figure 5. Relations between suspended-sediment concentration and water discharge for multiple floods. A. Ice break-up floods B. Snowmelt floods and C. Flash floods. 


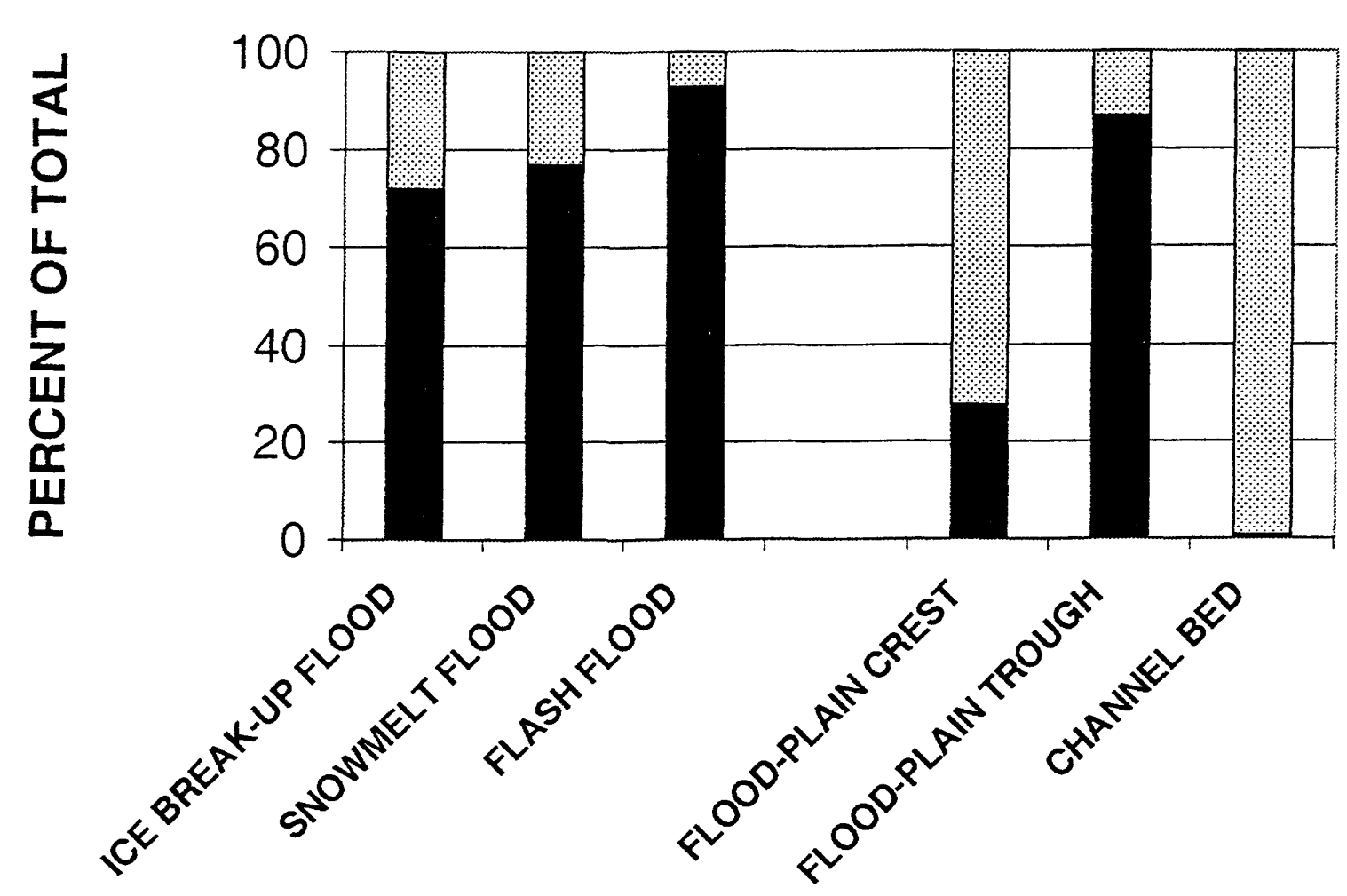

Figure 6. Percentages of sand and silt and clay in eroded and deposited sediments. Sand is shown by the stippled bar and silt and clay by the black bar. 
Table 2. Particle-size analysis of sediment deposited on the flood plain and on the bed of Powder River

[mm, millimeter; $\mathrm{m}$, meter; <, less than; >, greater than; Location is given by the cross section number fol-

lowed by the distance in meters upstream (negative) or downstream (positive); sta., station)]

\begin{tabular}{|c|c|c|c|c|c|c|c|c|c|c|c|c|c|c|c|c|}
\hline \multirow[b]{3}{*}{ Date } & \multirow[b]{3}{*}{ Location } & \multirow[b]{3}{*}{$\begin{array}{l}\text { Sta. } \\
\text { (m) }\end{array}$} & \multirow[b]{3}{*}{$\begin{array}{c}\text { Depth } \\
\text { (m) }\end{array}$} & \multicolumn{10}{|c|}{ Percent of total sample } & \multirow{2}{*}{\multicolumn{3}{|c|}{ Total percentage }} \\
\hline & & & & \multirow{2}{*}{$\begin{array}{c}\text { Clay } \\
\begin{array}{l}\text { C.004 } \\
\text { (mm) }\end{array}\end{array}$} & \multicolumn{4}{|c|}{ Silt } & \multicolumn{5}{|c|}{ Sand } & & & \\
\hline & & & & & $\begin{array}{l}0.004- \\
0.008 \\
(\mathrm{~mm})\end{array}$ & $\begin{array}{l}0.008- \\
0.016 \\
(\mathrm{~mm})\end{array}$ & $\begin{array}{l}0.016- \\
0.032 \\
(\mathrm{~mm})\end{array}$ & $\begin{array}{l}0.032- \\
0.063 \\
(\mathrm{~mm})\end{array}$ & $\begin{array}{l}0.063- \\
0.125 \\
(\mathrm{~mm})\end{array}$ & $\begin{array}{l}0.125- \\
0.250 \\
(\mathrm{~mm})\end{array}$ & $\begin{array}{l}0.250- \\
0.500 \\
(\mathrm{~mm})\end{array}$ & $\begin{array}{c}0.500- \\
1.00 \\
(\mathrm{~mm})\end{array}$ & $\begin{array}{l}>1.00 \\
(\mathrm{~mm})\end{array}$ & Clay & silt & Sand \\
\hline \multicolumn{17}{|c|}{$1992^{\mathrm{a}}$} \\
\hline \multirow[t]{11}{*}{10 Aug. } & PR120-400 & crest & $0-0.25$ & 2.7 & & 10. & & & 36.1 & 48.4 & 2.4 & 0.4 & 0.0 & 2.7 & 10.0 & 87.3 \\
\hline & PR120-330 & crest & $0-0.25$ & 3.0 & & 11. & & & 39.5 & 43.0 & 2.3 & 0.3 & 0.0 & 3.0 & 11.8 & 85.1 \\
\hline & PR120-220 & crest & $0-0.25$ & 6.2 & & 20. & & & 44.2 & 27.9 & 1.2 & 0.4 & 0.0 & 6.2 & 20.2 & 73.7 \\
\hline & PR120-110 & crest & $0-0.25$ & 4.9 & & 23. & & & 40.2 & 30.4 & 0.9 & 0.3 & 0.0 & 4.9 & 23.3 & 71.8 \\
\hline & PR120+70 & crest & $0-0.25$ & 3.9 & & 18. & & & 43.9 & 32.2 & 0.9 & 0.2 & 0.0 & 3.9 & 18.9 & 77.2 \\
\hline & PR120+200 & crest & $0-0.25$ & 5.1 & & 26. & & & 44.1 & 23.5 & 0.7 & 0.1 & 0.0 & 5.1 & 26.7 & 68.4 \\
\hline & PR120+8 & 56 & $0-0.25$ & 8.1 & & 57. & & & 25.8 & 7.3 & 1.0 & 0.3 & 0.0 & 8.1 & 57.4 & 34.4 \\
\hline & PR120+8 & 58 & $0-0.25$ & 1.5 & & 4.3 & & & 24.1 & 67.3 & 2.3 & 0.4 & 0.0 & 1.5 & 4.3 & 94.1 \\
\hline & PR120+8 & 65 & $0-0.25$ & 5.2 & & 30. & & & 44.2 & 19.3 & 0.7 & 0.1 & 0.0 & 5.2 & 30.4 & 64.3 \\
\hline & PR120+8 & 72 & $0-0.25$ & 41.0 & & 46. & & & 7.2 & 4.8 & 0.6 & 0.0 & 0.0 & 41.0 & 46.3 & 12.6 \\
\hline & & & & & & & 1994 & & & & & & & & & \\
\hline \multirow[t]{5}{*}{24 Sept. } & PR120-220 & bed & $0-0.25$ & & & 0.2 & & & 0.9 & 22.5 & 58.5 & 13.4 & 4.5 & 0.2 & & 99.8 \\
\hline & PR120-170 & bed & $0-0.25$ & & & 0.4 & & & 0.7 & 29.2 & 55.9 & 9.3 & 4.5 & 0.4 & & 99.6 \\
\hline & PR120-110 & bed & $0-0.25$ & & & 0.8 & & & 2.2 & 48.1 & 47.7 & 0.9 & 0.2 & 0.8 & & 99.1 \\
\hline & PR120 & bed & $0-0.25$ & & & 0.3 & & & 4.9 & 77.7 & 15.6 & 0.9 & 0.5 & 0.3 & & 99.6 \\
\hline & PR120+35 & bed & $0-0.25$ & & & 2.2 & & & 19.2 & 68.0 & 9.3 & 0.6 & 0.6 & 2.2 & & 97.7 \\
\hline \multirow[t]{5}{*}{5 Nov. } & PR120+2 & 60.0 & $0-1.15$ & 7.4 & 1.2 & 1.9 & 5.5 & 14.4 & 31.0 & 37.2 & 1.2 & 0.1 & 0.0 & 7.4 & 23.0 & 69.5 \\
\hline & & 62.0 & $0-1.00$ & 4.6 & 0.7 & 2.1 & 5.2 & 8.5 & 35.4 & 43.0 & 0.3 & 0.0 & 0.0 & 4.6 & 16.5 & 78.7 \\
\hline & & 65.0 & $0-1.00$ & 12.0 & 1.1 & 5.4 & 10.7 & 21.6 & 23.8 & 23.5 & 1.9 & 0.0 & 0.0 & 12.0 & 38.8 & 49.2 \\
\hline & & 69.0 & $0-1.00$ & 25.0 & 5.8 & 14.2 & 24.0 & 15.7 & 10.9 & 4.4 & 0.0 & 0.0 & 0.0 & 25.0 & 59.7 & 15.3 \\
\hline & & 75.0 & $0-0.40$ & 36.7 & 6.4 & 15.2 & 15.9 & 13.2 & 7.2 & 5.0 & 0.4 & 0.0 & 0.0 & 36.7 & 50.7 & 12.6 \\
\hline \multirow[t]{4}{*}{5 Nov. } & PR125+2 & 12.0 & $0-0.40$ & 38.9 & 3.1 & 13.5 & 20.4 & 16.5 & 5.5 & 1.8 & 0.2 & 0.0 & 0.0 & 38.9 & 53.5 & 7.5 \\
\hline & & 30.0 & $0-0.95$ & 13.9 & 2.2 & 4.7 & 11.4 & 18.5 & 29.9 & 18.2 & 1.1 & 0.1 & 0.0 & 13.9 & 36.8 & 49.3 \\
\hline & & 36.0 & $0-1.00$ & 13.6 & 1.9 & 4.7 & 11.8 & 23.2 & 23.9 & 18.0 & 2.3 & 0.6 & 0.0 & 13.6 & 41.6 & 44.8 \\
\hline & & 42.0 & $0-1.00$ & 10.3 & 1.6 & 3.9 & 9.9 & 19.5 & 30.2 & 23.8 & 0.7 & 0.0 & 0.0 & 10.3 & 34.9 & 54.7 \\
\hline \multirow[t]{3}{*}{5 Nov. } & PR136+2 & 51.0 & $0-0.95$ & 7.7 & 0.7 & 2.8 & 5.1 & 10.0 & 18.0 & 44.1 & 10.8 & 0.7 & 0.0 & 7.7 & 18.6 & 73.6 \\
\hline & & 57.0 & $0-1.00$ & 15.6 & 1.4 & 6.7 & 13.3 & 22.7 & 22.4 & 14.6 & 2.7 & 0.6 & 0.0 & 15.6 & 44.1 & 40.3 \\
\hline & & 65 & $0-0.85$ & 14.0 & 1.6 & 5.6 & 9.4 & 15.1 & 21.3 & 28.3 & 3.2 & 1.3 & 0.2 & 14.0 & 31.7 & 54.3 \\
\hline \multirow[t]{3}{*}{6 Nov. } & PR151+2 & 22.0 & $0-0.60$ & 37.1 & 3.4 & 14.8 & 16.8 & 16.1 & 10.6 & 1.1 & 0.0 & 0.0 & 0.0 & 37.1 & 51.1 & 11.7 \\
\hline & & 30.0 & $0-1.00$ & 19.5 & 2.9 & 5.9 & 15.1 & 25.6 & 20.7 & 9.3 & 0.9 & 0.0 & 0.0 & 19.5 & 49.5 & 30.9 \\
\hline & & 37.0 & $0-0.80$ & 15.7 & 2.0 & 6.5 & 12.7 & 24.6 & 25.7 & 12.2 & 0.6 & 0.0 & 0.0 & 15.7 & 45.8 & 38.5 \\
\hline
\end{tabular}


Table 2. (Continued) Particle-size analysis of sediment deposited on the flood plain and on the bed of Powder River

\begin{tabular}{|c|c|c|c|c|c|c|c|c|c|c|c|c|c|c|c|c|}
\hline \multirow[b]{3}{*}{ Date } & \multirow[b]{3}{*}{ Location } & \multirow[b]{3}{*}{$\begin{array}{l}\text { Sta. } \\
\text { (m) }\end{array}$} & \multirow[b]{3}{*}{$\begin{array}{c}\text { Depth } \\
\text { (m) }\end{array}$} & \multicolumn{10}{|c|}{ Percent of total sample } & \multirow{2}{*}{\multicolumn{3}{|c|}{ Total percentage }} \\
\hline & & & & \multirow{2}{*}{$\begin{array}{c}\text { Clay } \\
\begin{array}{c}<.004 \\
(\mathrm{~mm})\end{array}\end{array}$} & \multicolumn{4}{|c|}{ Silt } & \multicolumn{5}{|c|}{ Sand } & & & \\
\hline & & & & & $\begin{array}{l}0.004- \\
0.008 \\
(\mathrm{~mm})\end{array}$ & $\begin{array}{l}0.008 \\
0.016 \\
(\mathrm{~mm})\end{array}$ & $\begin{array}{l}0.016- \\
0.032 \\
(\mathrm{~mm})\end{array}$ & $\begin{array}{l}0.032- \\
0.063 \\
(\mathrm{~mm})\end{array}$ & $\begin{array}{l}0.063- \\
0.125 \\
(\mathrm{~mm})\end{array}$ & $\begin{array}{l}0.125- \\
0.250 \\
(\mathrm{~mm})\end{array}$ & $\begin{array}{l}0.250- \\
0.500 \\
(\mathrm{~mm})\end{array}$ & $\begin{array}{c}0.500- \\
1.00 \\
(\mathrm{~mm})\end{array}$ & $\begin{array}{l}>1.00 \\
(\mathrm{~mm})\end{array}$ & Clay & Slit & Sand \\
\hline \multirow[t]{10}{*}{6 Nov. } & PR156A+2 & $61.0^{b}$ & $0-0.02$ & 3.4 & 0.7 & 1.7 & 4.1 & 10.1 & 31.4 & 48.1 & 0.5 & 0.0 & 0.0 & 3.4 & 16.6 & 80.0 \\
\hline & & 61.5 & $0-0.95$ & 12.9 & 1.8 & 6.3 & 12.9 & 20.4 & 26.0 & 19.3 & 0.5 & 0.0 & 0.0 & 12.9 & 41.4 & 45.8 \\
\hline & & 64.0 & $0-1.00$ & 13.8 & 1.8 & 4.3 & 11.0 & 21.7 & 32.8 & 14.1 & 0.5 & 0.0 & 0.0 & 13.8 & 38.8 & 47.4 \\
\hline & & $64.5^{b}$ & $0-0.02$ & 10.8 & 2.5 & 5.6 & 10.8 & 18.1 & 42.7 & 9.3 & 0.1 & 0.0 & 0.0 & 10.8 & 37.0 & 52.1 \\
\hline & & $67.0^{b}$ & $0-0.05$ & 19.1 & 1.7 & 7.1 & 13.1 & 19.1 & 31.3 & 8.5 & 0.0 & 0.0 & 0.0 & 19.1 & 41.0 & 39.8 \\
\hline & & 67.0 & $0-0.98$ & 16.3 & 1.6 & 6.4 & 14.9 & 28.1 & 22.8 & 9.3 & 0.6 & 0.0 & 0.0 & 16.3 & 51.0 & 32.7 \\
\hline & & 80.0 & $0-0.45$ & 42.0 & 7.2 & 16.2 & 17.5 & 11.4 & 4.4 & 1.2 & 0.0 & 0.0 & 0.0 & 42.0 & 52.3 & 5.6 \\
\hline & & $90.0^{b}$ & $0-0.05$ & 42.2 & 1.4 & 12.0 & 12.2 & 8.0 & 15.1 & 8.8 & 0.2 & 0.0 & 0.0 & 42.2 & 33.6 & 24.1 \\
\hline & & 90.0 & $0-0.45$ & 52.0 & 5.9 & 13.3 & 11.3 & 6.7 & 8.3 & 2.1 & 0.4 & 0.0 & 0.0 & 52.0 & 37.2 & 10.8 \\
\hline & \multicolumn{16}{|c|}{1996} \\
\hline $\begin{array}{c}28 \text { May } \\
2001\end{array}$ & PR 125 & $28.0^{c}$ & $0-0.10$ & & & 99 & & & & & 1 & & & 99 & & 1 \\
\hline 26 Sept. & PR125+2 & \multicolumn{2}{|c|}{$101.0^{d} 0.00-0.30$} & \multicolumn{5}{|c|}{39.2} & 37.1 & 19.9 & 3.6 & 0.2 & 0.0 & \multicolumn{2}{|c|}{39.2} & 60.8 \\
\hline & PR125+2 & \multicolumn{2}{|c|}{$101.0^{d} 0.30-0.65$} & \multicolumn{5}{|c|}{65.2} & 34.7 & 0.0 & 0.0 & 0.0 & 0.0 & \multicolumn{2}{|c|}{65.2} & 34.7 \\
\hline & PR125+2 & \multicolumn{2}{|c|}{$101.0^{d} 0.65-0.76$} & \multicolumn{5}{|c|}{79.9} & 20.1 & 0.0 & 0.0 & 0.0 & 0.0 & \multicolumn{2}{|c|}{79.9} & 20.1 \\
\hline & PR $125+2$ & \multicolumn{2}{|c|}{$101.0^{d} 0.76-0.81$} & \multicolumn{5}{|c|}{72.2} & 27.8 & 0.0 & 0.0 & 0.0 & 0.0 & \multicolumn{2}{|c|}{72.2} & 27.8 \\
\hline & PR125+2 & \multicolumn{2}{|c|}{$101.0^{\mathrm{d}} 0.81-0.91$} & \multicolumn{5}{|c|}{87.4} & 12.6 & 0.1 & 0.0 & 0.0 & 0.0 & \multicolumn{2}{|c|}{87.4} & 12.7 \\
\hline & PR 125+2 & \multicolumn{2}{|c|}{$101.0^{d} 0.91-0.94$} & \multicolumn{5}{|c|}{89.9} & 10.0 & 0.1 & 0.0 & 0.0 & 0.0 & \multicolumn{2}{|c|}{89.9} & 10.1 \\
\hline & PR125+2 & \multicolumn{2}{|c|}{$101.0^{d} 0.94-0.99$} & & & 74.4 & & & 23.1 & 2.4 & 0.0 & 0.0 & 0.0 & 74.4 & & 25.5 \\
\hline & PR 125+2 & $101.0^{d}$ & $0.99-1.14$ & & & 59.6 & & & 40.1 & 0.2 & 0.1 & 0.0 & 0.0 & 59.6 & & 40.4 \\
\hline
\end{tabular}

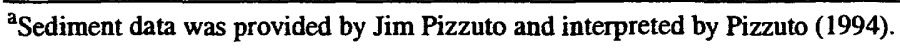

${ }^{b}$ Deposited during a flood in October 1994 according to Tori Franklin.

${ }^{\mathrm{c}}$ Newly deposited sediment collected during a snowmelt flood adjacent to the bank.

${ }^{\mathrm{d}}$ Sediment was collected from a cut bank. 


\section{CROSS SECTIONS}

Ten cross sections (identified as PR113 to PR151) were established during 1975 to monitor the erosion and deposition of sediment and channel changes (fig. 7). The section number, for example 113 or 151 (table 3), is the river distance in kilometers downstream from the mouth of Crazy Woman Creek near Arvada, Wyoming, as shown in the U.S. Geological Survey river-survey maps of 1946 (Martinson and Meade, 1983). The first cross section was located about $3 \mathrm{~km}$ upstream from the Moorhead gaging station, and the remaining sections were located 2 to $6 \mathrm{~km}$ apart for $39 \mathrm{~km}$ (river distance) downstream from the Moorhead gaging station (table 3). Ten more cross sections (PR156 to PR206) were established during 1977 from $43 \mathrm{~km}$ to about $95 \mathrm{~km}$ (river distance) downstream from the Moorhead gaging station. These sections were spaced farther apart ( 3 to $8 \mathrm{~km}$ ), and the last section was about $1 \mathrm{~km}$ downstream from the U.S. Highway 212 bridge, where the Broadus gaging station was located.

After intense rainfall, a major flood occurred on Powder River between 17 May and 23 May 1978 (Parrett and others, 1984) bringing peak discharges of water and suspended sediment at Moorhead of $800 \mathrm{~m}^{3} / \mathrm{s}$ and 2 million metric tons per day, respectively. Two cross sections (PR122 and PR141) located on bends of the river were cut off from the main channel during the flood, and new measurement sections (PR122A and PR141A) were established in 1979 on the new cutoffs. These cutoffs shortened the total river distance of the study reacli by about $2.7 \mathrm{~km}$. All reference marks for section PR200 were completely obliterated in the flood, and a new measurement section (PR200A) was established in approximately the same location as the old section in the fall of 1978. One section (PR156) was believed to have been lost during the flood, and a new section (PR156A) was established about $40 \mathrm{~m}$ upstream for the location of the original section. Later, the original metal reference monuments were found using a metal detector, and both sections have been resurveyed regularly since 1978. Cross-sectional measurements, from 1975 to 1988 , for these 23 cross sections are listed in U.S. Geological Survey Open-File Report 89-407 (Moody and Meade, 1990).

Additional cross sections have been added after the 1978 flood. Nine "cut-off" cross sections were established in 1980 and 1986 on a meander bend that was nearly cut off by the 1978 flood. The "cut-off" cross sections were spaced about $0.5-1.0 \mathrm{~km}$ apart to monitor the potential channel changes upstream from, along, and downstream from the meander bend between PR163 and PR175 (figs. 7 and 8). These cross sections were not resurveyed annually but resurveyed about every 5-7 years. One "ice-deposit" section was established in 1994 on a bend where an icejam flood had deposited sediment. The single "ice-deposit" cross section (PR141.7) has been resurveyed at varying time intervals to monitor the fate of these ice-deposited sediments.

There are now 33 cross sections along Powder River spanning a total river distance of 92.4 $\mathrm{km}$. In the "CROSS-SECTION DATA" section at the back of this report, the township location, U.S. Geological Survey quadrangle map, access, and landowners are listed for eacli cross section. The horizontal location and average elevation (above sea level) for the lorizontal reference monuments (1/2-inch- or 12-mm-diameter, pieces of 4-foot long rebar) also are listed. The location of the original 23 cross sections is shown on part of a U.S. Geological Survey 7.5-minute topographical map $(1: 24,000)$ and the locations of the 10 additional cross sections are shown in figure 8. The bench mark location at eacli cross section is shown on a more detailed map, which includes surrounding local landmarks. All azimuths are reported in degrees magnetic. Profiles of each cross section liave been plotted in the "CROSS-SECTION DATA" section in pairs of successive surveys to provide a general indication of the areas of erosion and deposition and are not meant to provide actual data. Following these section profiles is a listing of the actual stations and elevations for each cross-section profile. 


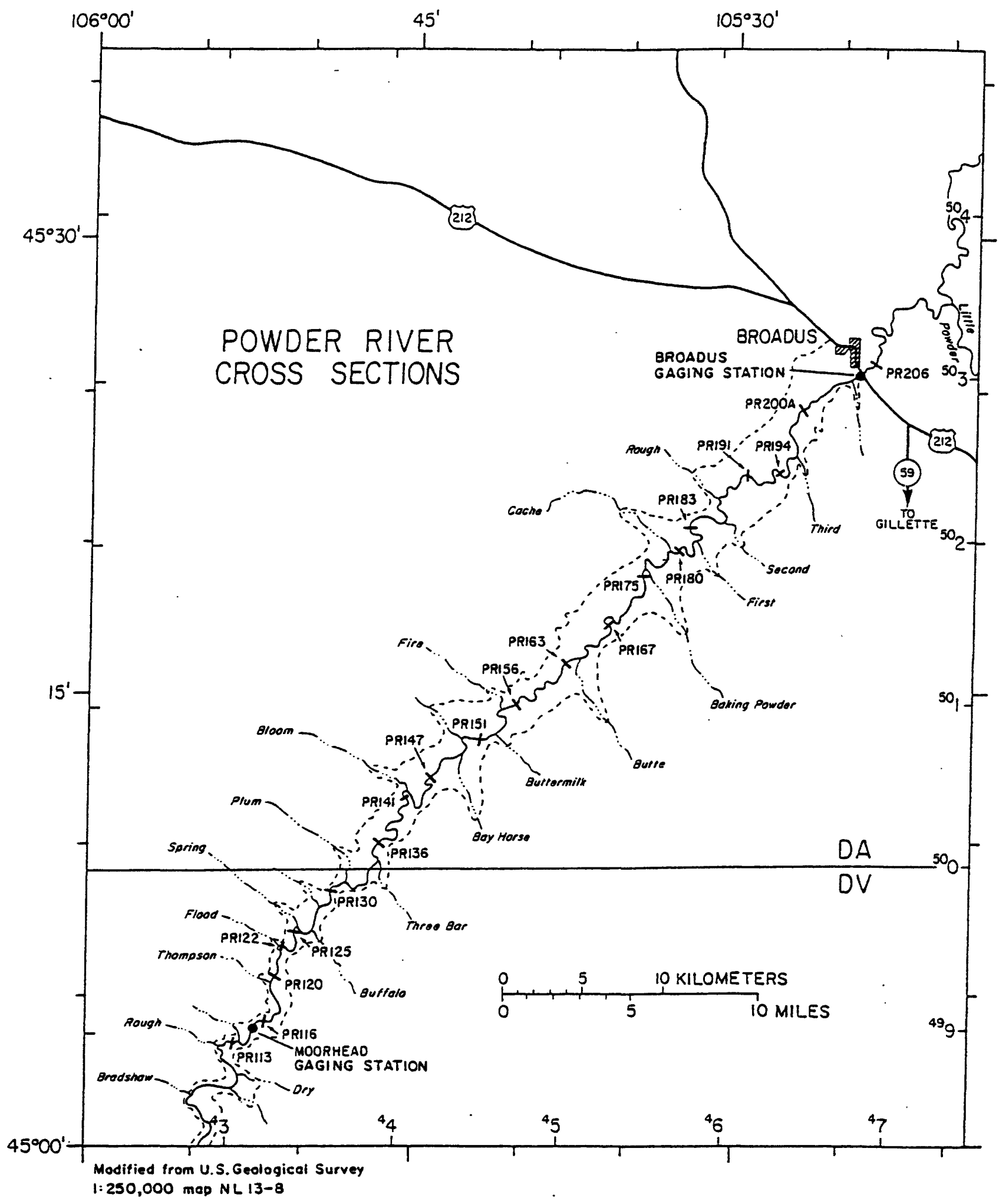

Figure 7. Locations of 20 channel cross sections and the tributaries of Powder River in the study reach. The dashed line indicates the approximate boundary of the visually-obvious valley. Longitude and latitude tick marks are on the outside of the border, and tick marks for the universal transverse mercator (UTM) grid are on the inside of the border. DA and DV are the identification letters for the 100,000 -meter-square grid. PR113 is the cross section identification. 
Table 3. Vertical and geographical locations of bench marks or reference monuments for cross sections of Powder River between Moorhead and Broadus, Montana

[The bench mark is a brass circular plate embedded in concrete unless other wise noted. Distance is measured from the Moorhead gaging station and based on aerial photographs taken after 1978 (from Martinson, 1983); km, kilometer; Elevation is meters above sea level; m, meter; Azimuth is in degrees magnetic]

\begin{tabular}{|c|c|c|c|c|c|c|c|}
\hline \multirow[b]{2}{*}{$\begin{array}{l}\text { Cross } \\
\text { section }\end{array}$} & \multirow[b]{2}{*}{$\begin{array}{c}\text { Distance } \\
(\mathbf{k m})\end{array}$} & \multirow[b]{2}{*}{$\begin{array}{l}\text { Elevation } \\
\text { (m) }\end{array}$} & \multirow[b]{2}{*}{$\begin{array}{c}\text { Aximuth } \\
\text { (\%) }\end{array}$} & \multicolumn{4}{|c|}{ Bench mark location } \\
\hline & & & & Latitude & Longitude & $\begin{array}{l}\text { Standard } \\
\text { deviation } \\
\text { (m) }\end{array}$ & $\begin{array}{l}\text { Horizontal } \\
\text { precision } \\
\text { (m) }\end{array}$ \\
\hline$\overline{\text { PR113 }}$ & -2.23 & 1023.34 & 171.5 & $45^{\circ} 03^{\prime} 21.49^{\prime \prime}$ & $105^{\circ} 53^{\prime} 03.31^{\prime \prime}$ & 0.412 & 0.432 \\
\hline PR116 & 0.65 & 1020.00 & 187.5 & $45^{\circ} 04^{\prime} 07.22^{\prime \prime}$ & $105^{\circ} 51^{\prime} 46.75^{\prime \prime}$ & 0.548 & 0.554 \\
\hline PR120 & 4.68 & 1014.70 & 114.5 & $45^{\circ} 05^{\prime} 32.49^{\prime \prime}$ & $105^{\circ} 51^{\prime} 14.17^{\prime \prime}$ & 0.619 & 0.624 \\
\hline PR122A ${ }^{a}$ & 6.60 & 1010.37 & 133.5 & $45^{\circ} 06^{\prime} 29.84^{\prime \prime}$ & $105^{\circ} 50^{\prime} 47.05^{\prime \prime}$ & 0.356 & 0.551 \\
\hline PR122 ${ }^{b}$ & 7.07 & 1014.56 & 135.5 & $45^{\circ} 06^{\prime} 37.88^{\prime \prime}$ & $105^{\circ} 51^{\prime} 00.00^{\prime \prime}$ & 0.280 & 0.500 \\
\hline PR125 & 8.61 & 1008.45 & 084.5 & $45^{\circ} 07^{\prime} 06.40^{\prime \prime}$ & $105^{\circ} 50^{\prime} 17.91^{\prime \prime}$ & 0.193 & 0.608 \\
\hline PR130 & 13.92 & 1002.67 & 075 & $45^{\circ} 08^{\prime} 23.46^{\prime \prime}$ & $105^{\circ} 48^{\prime} 39.01^{\prime \prime}$ & 0.554 & 0.922 \\
\hline PR136 & 20.19 & 994.30 & 129 & $45^{\circ} 10^{\prime} 05.91^{\prime \prime}$ & $105^{\circ} 46^{\prime} 26.89^{\prime \prime}$ & 0.283 & 0.433 \\
\hline PR141 & 26.10 & 988.93 & 021 & $45^{\circ} 11^{\prime} 35.80^{\prime \prime}$ & $105^{\circ} 45^{\prime} 09.56^{\prime \prime}$ & 0.336 & 0.486 \\
\hline PR141A ${ }^{a}$ & 26.22 & 986.99 & 181.5 & $45^{\circ} 11^{\prime} 38.08^{\prime \prime}$ & $105^{\circ} 44^{\prime} 47.90^{\prime \prime}$ & 0.443 & 0.425 \\
\hline PR141.7 & 26.9 & 987.34 & 240.5 & $45^{\circ} 11^{\prime} 20.20^{\prime \prime}$ & $105^{\circ} 44^{\prime} 44.86^{\prime \prime}$ & 0.527 & 0.759 \\
\hline PR147 & 30.24 & 983.92 & 130 & $45^{\circ} 12^{\prime} 19.56^{\prime \prime}$ & $105^{\circ} 44^{\prime} 06.61^{\prime \prime}$ & 0.557 & 0.460 \\
\hline PR151 & 36.27 & 977.46 & 176.5 & $45^{\circ} 13^{\prime} 32.83^{\prime \prime}$ & $105^{\circ} 41^{\prime} 47.36^{\prime \prime}$ & 0.251 & 0.531 \\
\hline PR156A & 40.24 & 972.17 & 124.5 & $45^{\circ} 14^{\prime} 44.90^{\prime \prime}$ & $105^{\circ} 39^{\prime} 56.56^{\prime \prime}$ & 1.241 & 0.637 \\
\hline PR156 & 40.28 & 972.17 & 109.5 & $45^{\circ} 14^{\prime} 44.90^{\prime \prime}$ & $105^{\circ} 39^{\prime} 56.56^{\prime \prime}$ & 1.241 & 0.637 \\
\hline PR163 & 48.30 & 965.53 & 151 & $45^{\circ} 16^{\prime} 12.70^{\prime \prime}$ & $105^{\circ} 37^{\prime} 52.08^{\prime \prime}$ & 0.191 & 0.532 \\
\hline PR164. $8^{\mathrm{a}}$ & 49.6 & 962.75 & 185 & $45^{\circ} 16^{\prime} 39.98^{\prime \prime}$ & $105^{\circ} 36^{\prime} 35.00^{\prime \prime}$ & 0.413 & 0.475 \\
\hline PR $165.6^{\mathrm{a}}$ & 50.4 & 961.60 & 105 & $45^{\circ} 16^{\prime} 49.90^{\prime \prime}$ & $105^{\circ} 36^{\prime} 13.39^{\prime \prime}$ & 0.334 & 0.513 \\
\hline PR166.0 & 50.8 & 960.47 & 150 & $45^{\circ} 17^{\prime} 00.10^{\prime \prime}$ & $105^{\circ} 35^{\prime} 59.67^{\prime \prime}$ & 0.290 & 0.622 \\
\hline PR166. $6^{\mathrm{a}}$ & 51.2 & 960.62 & 078 & $45^{\circ} 17^{\prime} 07.49^{\prime \prime}$ & $105^{\circ} 35^{\prime} 45.17^{\prime \prime}$ & 1.313 & 0.701 \\
\hline PR167 & 51.77 & 959.79 & 006 & $45^{\circ} 17^{\prime} 21.96^{\prime \prime}$ & $105^{\circ} 35^{\circ} 49.97^{\prime \prime}$ & 0.527 & 0.490 \\
\hline PR167.5 & 52.3 & 963.34 & 057 & \multicolumn{4}{|c|}{ No measurements were made in 1998.} \\
\hline PR $168.5^{\mathrm{a}}$ & 53.3 & 958.61 & 221 & $45^{\circ} 17^{\prime} 34.14^{\prime \prime}$ & $105^{\circ} 35^{\prime} 34.22^{\prime \prime}$ & 0.257 & 0.772 \\
\hline PR $169.2^{\mathrm{a}}$ & 54.0 & 957.69 & 114 & $45^{\circ} 17^{\prime} 24.67^{\prime \prime}$ & $105^{\circ} 35^{\prime} 23.82^{\prime \prime}$ & 0.466 & 0.495 \\
\hline PR169.8 ${ }^{\mathrm{a}}$ & 54.6 & 958.45 & 127 & $45^{\circ} 17^{\prime} 41.85^{\prime \prime}$ & $105^{\circ} 35^{\prime} 16.46^{\prime \prime}$ & 0.268 & 0.779 \\
\hline PR170.5 & 55.3 & 957.07 & 101 & $45^{\circ} 17^{\prime} 57.44^{\prime \prime}$ & $105^{\circ} 35^{\prime} 00.38^{\prime \prime}$ & 0.523 & 0.711 \\
\hline PR175 & 58.32 & 953.58 & 058 & $45^{\circ} 18^{\prime} 59.74^{\prime \prime}$ & $105^{\circ} 34^{\prime} 37.31^{\prime \prime}$ & 0.468 & 0.546 \\
\hline PR $180^{\mathrm{a}}$ & 63.10 & 950.15 & 103 & $45^{\circ} 19^{\prime} 51.46^{\prime \prime}$ & $105^{\circ} 32^{\prime} 38.16^{\prime \prime}$ & 0.615 . & 0.711 \\
\hline PR183 & 67.85 & 945.89 & 059 & $45^{\circ} 20^{\prime} 33.33^{\prime \prime}$ & $105^{\circ} 32^{\prime} 10.76^{\prime \prime}$ & 0.531 & 0.504 \\
\hline PR191 & 76.53 & 938.00 & 177 & $45^{\circ} 22^{\prime} 18.87^{\prime \prime}$ & $105^{\circ} 29^{\prime} 27.95^{\prime \prime}$ & 0.166 & 0.521 \\
\hline PR194 & 80.08 & 934.41 & 225 & $45^{\circ} 22^{\prime} 17.71^{\prime \prime}$ & $105^{\circ} 27^{\prime} 51.05^{\prime \prime}$ & 0.409 & 0.765 \\
\hline PR200A & 86.28 & 927.80 & 140 & $45^{\circ} 24^{\prime} 32.43^{\prime \prime}$ & $105^{\circ} 27^{\prime} 02.73^{\prime \prime}$ & 0.223 & 0.652 \\
\hline PR206 ${ }^{\mathrm{a}}$ & 92.19 & 921.00 & 266 & $45^{\circ} 26^{\prime} 03.23^{\prime \prime}$ & $105^{\circ} 23^{\prime} 33.50^{\prime \prime}$ & 0.404 & 0.434 \\
\hline
\end{tabular}

${ }^{\mathrm{a}}$ This is a 12 -mm-diameter steel rod or $1 / 2$-inch rebar.

${ }^{\mathrm{b}}$ This is the monument at station $-1.2 \mathrm{~m}$, the bench mark is a 12 -mm-diameter steel rod or $1 / 2$-inch rebar located at station 0.0 . 


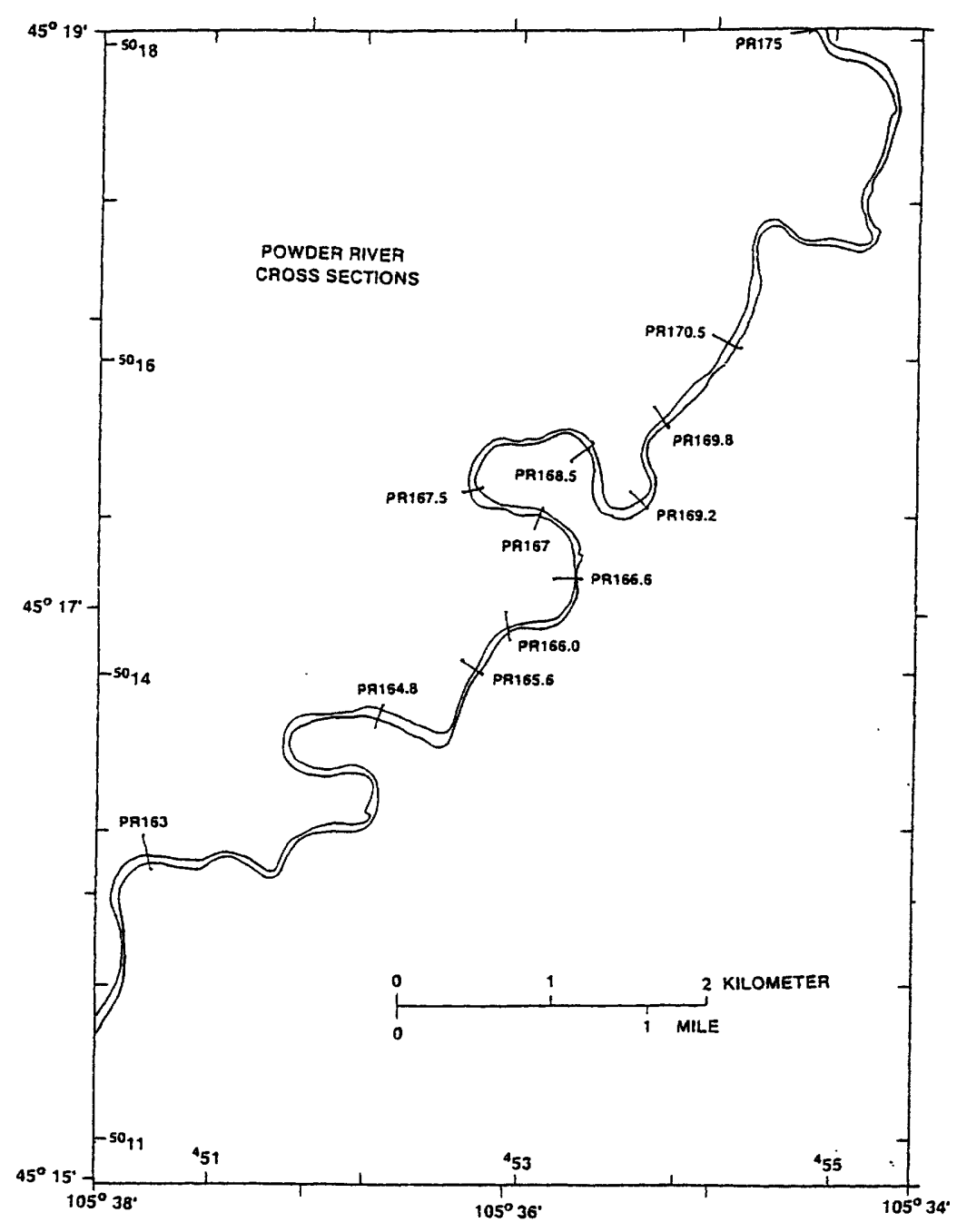

Figure 8. Location of 10 channel cross sections between PR163 and PR175. These sections were established to monitor the evolution of the channel upstream from, along, and downstream from a large river bend that may cut off in the future. 


\section{SURVEY METHOD}

Initially, at each cross section a steel reference monument (1/2-inch rebar, $12 \mathrm{~mm}$ in diameter and about $1.2 \mathrm{~m}$ long) was driven almost flush with the ground to mark the horizontal zero point (station 0.0) on the left bank (left when facing downstream), and a second monument was driven in on the right bank to mark a convenient horizontal reference distance. Since the cross sections were established in the 1970 s, the river has changed and some of the zero reference monuments have been lost to the river. Therefore, new reference monuments were established with negative values for the station. The reference monuments were resurveyed in 1998 using a GPS (Global Positioning System, Trimble Pro XRS). The coordinates [NAD83 (1992)] and measurement accuracy and precision are listed in the CROSS-SECTION DATA section of this report. The horizontal precision of the GPS measurements ranged from 0.3 to $1.1 \mathrm{~m}$ and averaged $0.6 \pm 0.2 \mathrm{~m}$. Two additional steel pins were driven into the ground on the line of section but behind the reference monuments to hold the cable clamps used to stretch a steel cable or tagline $(150 \mathrm{~m}$ long and $3 \mathrm{~mm}$ in diameter) across the river. The cable was marked every meter by a brass bead, and horizontal distances were measured to $0.1 \mathrm{~m}$ by estimating between beads. Distances between reference monuments ( 108 to $321 \mathrm{~m}$ ) were measured using both the GPS and the tagline and the difference between these two methods was $\pm 0.7 \mathrm{~m}$ or about 0.2 to 0.6 percent. Elevations were not measured by the GPS method.

A local vertical bench mark was installed at each cross section, and the elevation above sea level was determined by leveling from U.S. Coast and Geodetic Survey bench marks shown on the U.S. Geological Survey topographic maps. The local bencli mark consisted of a brass circular plate embedded in reinforced concrete, which was flush with the ground. The cross-section identification number (for example, PR113) is stamped into the plate, and an arrow in the center of the brass plate points to station 0.0 . The location of each bench mark is shown in a figure in the CROSS-SECTION DATA section. Typical errors in the measurement of elevation $(1.4-1.9 \mathrm{~cm})$ and change in cross-sectional area (0.6-1.2 $\left.\mathrm{m}^{2}\right)$ are discussed by Moody and Meade (1990) and applied to this report because the same methods were used.

\section{CHANNEL CHANGE}

Two measurements of the changes in erosion and deposition have been used for this report: (1) changes in the minimum riverbed elevation, and (2) changes in the cross-sectional area. These are net annual changes between the time of two successive cross-section surveys and do not represent the total amount of erosion or deposition. For example, during an ice break-up flood, sediment may be deposited on the banks and adjacent flood plain. A later snowmelt flood may erode these sediment deposits and perhaps some of the bank. A late summer flash flood may deposit some sediment in the area where the bank existed before the snowmelt flood. The net result, when measured in the fall, may therefore indicate only erosion near the bank while in reality there may have been substantially more of both deposition and erosion.

\section{Minimum Riverbed Elevation}

The minimum riverbed elevations for each cross section are listed in table 4. These data were combined with similar data for 1975-1988 (Moody and Meade, 1990) and linear trends were calculated for 20 years (table 5). The minimum riverbed elevation decreased with time for 8 cross sections and increased with time for 15 cross sections. However, only 5 of the linear trends had coefficients of determination greater than or equal to 0.50 . Two sections, PR122 $\left(r^{2}=0.92\right)$ and PR141 $\left(r^{2}=0.50\right)$, were cut off and abandoned during the 1978 flood. Of the remaining 3 cross 
sections, two (PR120, $\mathrm{r}^{2}=0.53$ and $\mathrm{PR} 151, \mathrm{r}^{2}=0.68$ ) showed a decrease in the minimum riverbed elevation and one $\left(\mathrm{PR} 122 \mathrm{~A}, \mathrm{r}^{2}=0.70\right)$ showed an increase in the minimum riverbed elevation. In general, the minimum riverbed elevation was stable and simply fluctuated from year to year as indicated by the standard deviation of the elevation, which ranged from 0.01 to $0.30 \mathrm{~m}$ (table 5).

\section{Area of Erosion and Deposition}

The cross-sectional area of erosion and deposition was computed for successive surveys by linearly interpolating the elevations between horizontal measurements at every $0.1 \mathrm{~m}$. Then the positive (deposition) and negative (erosion) differences were summed separately between a starting station and an ending station. The starting and ending stations corresponded to four features where erosional and depositional processes were active: 1) bank, 2) flood plain, 3) channel, and 4) point bar. Sometimes distinguishing between features (for example, where the channel ended and the point bar started, or whether deposition was on a point bar or flood plain) was sufficiently difficult that the identification of these features must be considered subjective. This method of calculation approximated the cross-sectional area as a sum of narrow $(0.1 \mathrm{~m}$ wide) rectangular strips. The error in this approximation was estimated by calculating the exact areas as sums of triangles and by subtracting the areas computed using the rectangular approximation. The difference, for one cross section, was $0.01 \mathrm{~m}^{2}$ for the area of deposition and $0.11 \mathrm{~m}^{2}$ for the area of erosion. This was a 1.4-percent difference for the total area of channel change. The fieldleveling error was determined by computing the area of erosion and deposition for duplicate surveys of cross sections PR141A, PR163, and PR206 (Moody and Meade, 1990). The average error (for the six values of erosion and deposition) was $0.88 \mathrm{~m}^{2}$ per 100 horizontal meters of surveyed cross section.

During the 10 years from 1988 to 1998 , the erosion of sediment balanced the deposition of sediment in the study reach of Powder River (table 6). The average ratio of the net erosion divided by the net deposition for the 21 cross sections surveyed annually was 1.02 . The 2 percent difference is less than the error of the measurements. Erosion was greater than deposition at 10 cross sections. Cross section PR122A, on a new channel formed during the 1978 flood, had the largest ratio (1.47), but for PR141A, the other cross section on a new channel, the ratio (1.08) was less. PR200A had the second largest ratio (1.39) but this section was influenced by human activity. Cross sections PR163 and PR180 with evolving point bars also had large ratios (1.38 and 1.32 , respectively). Deposition was greater than erosion at 11 cross sections (ratios less than 1 ). The greatest deposition (smallest ratio) was at PR175 (0.59) followed by PR130 (0.61) and PR120 (0.66). PR120 is an example of a channel-expansion flood plain (Moody and others, 1999), which is still evolving by vertical accretion 20 years after the 1978 flood. The other examples of channel-expansion flood plains (PR125, PR136, PR151, and PR156A) had ratios of 1.04, $0.92,0.90$, and 1.11 .

The greatest amounts of bank erosion during the 10 years occurred at cross sections with point bars. This excludes PR200A, which had the greatest erosion $\left(253.9 \mathrm{~m}^{2}\right)$, but may have been affected by human changes made in the channel upstream from the cross section. However, the sections with the next greatest bank erosion, PR141A $\left(94.9 \mathrm{~m}^{2}\right)$, PR113 $\left(92.1 \mathrm{~m}^{2}\right)$, and PR180 $\left(89.8 \mathrm{~m}^{2}\right)$, all contained active point bars. At these three cross sections most of the bank erosion between 1988 and 1998 occurred during two years, 1991 and 1995.

The greatest amount of flood plain deposition during the 10 years occurred where the channel had shifted and the old channel was being filled in to create a flood plain. At PR200A $\left(235.9 \mathrm{~m}^{2}\right)$, the main channel shifted from the left bank to a secondary channel on the right bank and deposition may have been influenced by human activity. At PR206 $\left(135.6 \mathrm{~m}^{2}\right)$, the main 
Table 4. Minimum riverbed elevation at the end of the water year for 1989-1998

[--, cross section was not surveyed]

\begin{tabular}{|c|c|c|c|c|c|c|c|c|c|c|}
\hline \multirow{2}{*}{$\begin{array}{l}\text { Cross } \\
\text { sections }\end{array}$} & \multicolumn{10}{|c|}{$\begin{array}{l}\text { Minimum riverbed elevation } \\
\text { (meters above sea level) }\end{array}$} \\
\hline & 1989 & 1990 & 1991 & 1992 & 1993 & 1994 & 1995 & 1996 & 1997 & 1998 \\
\hline PR113 & 1019.64 & 1019.51 & 1019.59 & 1019.63 & 1019.63 & 1019.64 & 1019.14 & 1019.56 & 1019.48 & 1019.60 \\
\hline PR116 & 1015.56 & 1015.57 & 1015.48 & 1015.45 & 1015.58 & 1015.40 & 1015.21 & 1015.38 & 1015.31 & 1015.43 \\
\hline PR120 & 1010.65 & 1010.65 & 1010.62 & 1010.63 & 1010.60 & 1010.59 & 1010.63 & 1010.62 & 1010.62 & 1010.60 \\
\hline PR122 & -- & -- & - & -- & 1008.28 & -- & 1008.39 & -- & -- & -- \\
\hline PR122A & 1006.58 & 1006.55 & 1006.58 & 1006.59 & 1006.62 & 1006.62 & 1006.81 & 1006.83 & 1006.86 & 1006.86 \\
\hline PR125 & 1004.20 & 1004.18 & 1004.33 & 1004.07 & 1004.15 & 1004.22 & 1004.19 & 1004.12 & 1004.14 & 1004.22 \\
\hline PR130 & 998.39 & 998.45 & 998.31 & 998.40 & 998.32 & 998.33 & 998.41 & 998.30 & 998.32 & 998.17 \\
\hline PR136 & 991.15 & 991.09 & 991.03 & 991.16 & 991.25 & 991.15 & 990.69 & 991.14 & 991.14 & 991.09 \\
\hline PR141 & - & -- & - & - & -- & -- & -- & -- & -- & 987.08 \\
\hline PR141A & 984.83 & 984.61 & 984.72 & 984.72 & 984.55 & 984.69 & 984.61 & 984.58 & 984.58 & 984.62 \\
\hline PR147 & 980.19 & 980.20 & 980.15 & 980.19 & 980.11 & 980.19 & 980.12 & 979.89 & 979.89 & 979.90 \\
\hline PR151 & 973.72 & 973.73 & 973.76 & 973.73 & 973.70 & 973.68 & 973.64 & 973.70 & 973.49 & 973.62 \\
\hline PR156A & 969.17 & 969.00 & 969.06 & 969.20 & 969.14 & 969.11 & 969.08 & 969.09 & 969.13 & 969.09 \\
\hline PR156 & 969.16 & 968.98 & 969.16 & 969.15 & 969.12 & 969.16 & 969.18 & 969.06 & 969.18 & 968.89 \\
\hline PR163 & 961.64 & 961.65 & 961.83 & 961.94 & 961.72 & 961.92 & 961.82 & 961.92 & 961.96 & 961.65 \\
\hline PR167 & 956.97 & 956.70 & 956.96 & 956.75 & 956.72 & 956.93 & 956.68 & 956.77 & 957.02 & 956.67 \\
\hline PR175 & 950.65 & 950.79 & 950.68 & 950.80 & 950.83 & 950.49 & 950.81 & 950.98 & 950.68 & 950.70 \\
\hline PR180 & 946.35 & 946.33 & 946.37 & 946.39 & 946.20 & 946.24 & 946.48 & 946.46 & 946.42 & 946.22 \\
\hline PR183 & 942.82 & 942.82 & 942.91 & 942.71 & 942.77 & 942.87 & 942.66 & 942.83 & 942.72 & 942.60 \\
\hline PR191 & 934.54 & 934.46 & 934.50 & 934.44 & 934.61 & 934.50 & 934.59 & 934.61 & 934.69 & 934.51 \\
\hline PR194 & 931.40 & 931.26 & 931.28 & 931.27 & 931.28 & 931.24 & 931.01 & 931.08 & 931.18 & 931.22 \\
\hline PR200A & 924.39 & 923.90 & 924.24 & 924.29 & 924.56 & 924.49 & 923.59 & 924.21 & 924.18 & 924.17 \\
\hline PR206 & 917.42 & 917.29 & 916.64 & 916.95 & 916.33 & 916.88 & 917.19 & 917.22 & 917.38 & 917.23 \\
\hline
\end{tabular}


Table 5. Changes in the minimum riverbed elevation for 1978-1998

[ $\mathrm{m}$, meter; $\mathrm{m} / \mathrm{yr}$, meter per year; $\mathrm{r}^{2}$, coefficient of determination]

\begin{tabular}{|c|c|c|c|c|c|}
\hline \multirow[b]{2}{*}{$\begin{array}{c}\text { Cross } \\
\text { sections }\end{array}$} & \multirow[b]{2}{*}{$\begin{array}{c}\text { Number of } \\
\text { measure- } \\
\text { ments }\end{array}$} & \multirow[b]{2}{*}{$\begin{array}{l}\text { Standard } \\
\text { deviation } \\
\text { (m) }\end{array}$} & \multicolumn{3}{|c|}{ Linear trend } \\
\hline & & & $\begin{array}{l}\text { Slope of trend } \\
(\mathrm{m} / \mathrm{yr})\end{array}$ & $\begin{array}{l}\text { Intercept } \\
\text { (m) }\end{array}$ & $r^{2}$ \\
\hline PR113 & 19 & 0.12 & -0.001 & 1019.54 & 0.00 \\
\hline PR116 & 18 & 0.12 & -0.011 & 1015.61 & 0.28 \\
\hline PR120 & 19 & 0.02 & -0.003 & 1010.66 & 0.53 \\
\hline PR122 & 8 & 0.10 & 0.016 & 1008.08 & 0.92 \\
\hline PR122A & 18 & 0.17 & 0.025 & 1006.33 & 0.70 \\
\hline PR125 & 19 & 0.09 & 0.004 & 1004.12 & 0.07 \\
\hline PR130 & 19 & 0.08 & 0.001 & 998.33 & 0.01 \\
\hline PR136 & 19 & 0.13 & 0.007 & 990.99 & 0.11 \\
\hline PR141 & 6 & 0.01 & 0.001 & 987.06 & 0.50 \\
\hline PR141A & 18 & 0.15 & 0.009 & 984.52 & 0.11 \\
\hline PR147 & 18 & 0.12 & 0.005 & 979.99 & 0.05 \\
\hline PR151 & 19 & 0.09 & -0.012 & 973.85 & 0.68 \\
\hline PR156A & 19 & 0.07 & 0.000 & 969.12 & 0.00 \\
\hline PR156 & 19 & 0.08 & 0.001 & 969.113 & 0.00 \\
\hline PR163 & 19 & 0.12 & 0.011 & 961.63 & 0.31 \\
\hline PR167 & 19 & 0.17 & -0.010 & 956.98 & 0.13 \\
\hline PR175 & 19 & 0.17 & 0.018 & 950.47 & 0.43 \\
\hline PR180 & 19 & 0.14 & 0.015 & 946.12 & 0.42 \\
\hline PR183 & 19 & 0.10 & -0.003 & 942.82 & 0.05 \\
\hline PR191 & 19 & 0.10 & 0.007 & 934.45 & 0.18 \\
\hline PR194 & 19 & 0.16 & -0.008 & 931.37 & 0.09 \\
\hline PR200A & 19 & 0.21 & -0.004 & 924.27 & 0.01 \\
\hline PR206 & 19 & 0.30 & 0.000 & 917.08 & 0.00 \\
\hline
\end{tabular}


Table 6. Erosion and deposition of sediment at channel cross sections on the Powder River

[Bank includes both left and right banks and width is variable; flood plain, represents vertical erosion or deposition on the flood plain within the channel widened by the 1978 flood and older flood plains adjacent to the river; Eros.,erosion; Depo.,deposition; $\mathrm{m}$, meter; $\mathrm{m}^{2}$, square meters]

\begin{tabular}{|c|c|c|c|c|c|c|c|c|c|c|c|c|c|c|c|}
\hline \multirow{2}{*}{$\begin{array}{l}\text { Water } \\
\text { Year }\end{array}$} & \multicolumn{3}{|c|}{ Bank } & \multicolumn{3}{|c|}{ Flood plain } & \multicolumn{3}{|c|}{ Channel } & \multicolumn{3}{|c|}{ Point bar } & \multicolumn{3}{|c|}{ Total } \\
\hline & $\begin{array}{l}\text { Width } \\
\text { (m) }\end{array}$ & $\begin{array}{c}\text { Eros. } \\
\left(\mathrm{m}^{2}\right)\end{array}$ & $\begin{array}{c}\text { Depo. } \\
\left(\mathrm{m}^{2}\right)\end{array}$ & $\begin{array}{l}\text { Width } \\
\text { (m) }\end{array}$ & $\begin{array}{c}\text { Eros. } \\
\left(\mathrm{m}^{2}\right)\end{array}$ & $\begin{array}{c}\text { Depo. } \\
\left(\mathrm{m}^{2}\right)\end{array}$ & $\begin{array}{l}\text { Width } \\
\text { (m) }\end{array}$ & $\begin{array}{l}\text { Eros. } \\
\left(\mathrm{m}^{2}\right)\end{array}$ & $\begin{array}{c}\text { Depo. } \\
\left(\mathrm{m}^{2}\right)\end{array}$ & $\begin{array}{l}\text { Width } \\
\text { (m) }\end{array}$ & $\begin{array}{l}\text { Eros. } \\
\left(\mathrm{m}^{2}\right)\end{array}$ & $\begin{array}{c}\text { Depo. } \\
\left(\mathrm{m}^{2}\right)\end{array}$ & $\begin{array}{l}\text { Width } \\
\text { (m) }\end{array}$ & $\begin{array}{l}\text { Eros. } \\
\left(m^{2}\right)\end{array}$ & $\begin{array}{r}\text { Depc } \\
\left(\mathrm{m}^{2}\right)\end{array}$ \\
\hline \multicolumn{16}{|c|}{ PR113 } \\
\hline 1989 & 5.5 & 0.3 & 0.0 & 46.5 & 1.1 & 0.8 & 33.5 & 0.5 & 3.1 & 24.5 & 4.8 & 0.0 & 110.0 & 6.7 & 3.9 \\
\hline 1990 & 6.0 & 6.9 & 0.0 & 53.0 & 0.0 & 2.5 & 28.0 & 2.3 & 0.1 & 23.0 & 0.0 & 11.1 & 110.0 & 9.2 & 13.7 \\
\hline 1991 & 15.0 & 27.4 & 0.0 & 57.0 & 0.1 & 2.6 & 30.5 & 0.0 & 2.9 & 19.5 & 8.1 & 0.0 & 122.0 & 35.6 & 5.5 \\
\hline 1992 & 11.0 & 4.0 & 0.0 & 48.0 & 0.3 & 0.5 & 39.5 & 0.9 & 1.8 & 26.5 & 0.4 & 0.6 & 125.0 & 5.6 & 2.9 \\
\hline 1993 & 14.0 & 19.6 & 0.0 & 48.0 & 0.1 & 2.6 & 26.0 & 0.1 & 1.4 & 44.0 & 0.0 & 23.2 & 132.0 & 19.8 & 27.2 \\
\hline 1994 & 4.0 & 2.8 & 0.0 & 68.5 & 1.3 & 2.6 & 34.0 & 0.9 & 1.1 & 25.5 & 0.1 & 4.9 & 132.0 & 5.1 & 8.6 \\
\hline 1995 & 11.5 & 19.5 & 0.0 & 58.0 & 0.0 & 14.0 & 25.5 & 0.9 & 1.2 & 45.0 & 2.7 & 16.5 & 140.0 & 23.1 & 31.7 \\
\hline 1996 & 4.5 & 4.7 & 0.0 & 90.0 & 0.7 & 1.6 & 27.5 & 2.1 & 1.6 & 18.0 & 0.3 & 4.2 & 140.0 & 7.8 & 7.4 \\
\hline 1997 & 10.0 & 5.2 & 0.0 & 90.0 & 1.4 & 2.8 & 19.0 & 0.1 & 0.3 & 23.0 & 0.8 & 1.3 & 142.0 & 7.5 & 4.4 \\
\hline 1998 & 5.0 & 1.7 & 0.1 & 108.0 & 0.7 & 1.8 & 30.0 & 0.1 & 4.0 & 0.0 & 0.0 & 0.0 & 143.0 & 2.5 & 5.9 \\
\hline Total & -- & 92.1 & 0.1 & -- & 5.7 & 31.8 & -- & 7.9 & 17.5 & - & 17.2 & 61.8 & - & 122.9 & 111.2 \\
\hline
\end{tabular}

Bank erosion is approximately balanced by point-bar and flood-plain deposition

\begin{tabular}{|c|c|c|c|c|c|c|c|c|c|c|c|c|c|c|c|}
\hline 1989 & 16.5 & 0.8 & 0.1 & 0.0 & 0.0 & 0.0 & 66.5 & 4.6 & 2.5 & 0.0 & 0.0 & 0.0 & 83.0 & 5.4 & 2.6 \\
\hline 1990 & 17.5 & 0.1 & 1.4 & 0.0 & 0.0 & 0.0 & 65.5 & 6.2 & 6.6 & 0.0 & 0.0 & 0.0 & 83.0 & 6.3 & 8.0 \\
\hline 1991 & 17.5 & 0.8 & 0.3 & 0.0 & 0.0 & 0.0 & 65.5 & 4.6 & 3.4 & 0.0 & 0.0 & 0.0 & 83.0 & 5.4 & 3.7 \\
\hline 1992 & 17.0 & 0.2 & 0.3 & 0.0 & 0.0 & 0.0 & 66.0 & 4.6 & 4.4 & 0.0 & 0.0 & 0.0 & 83.0 & 4.8 & 4.7 \\
\hline 1993 & 17.0 & 0.7 & 0.5 & 0.0 & 0.0 & 0.0 & 66.0 & 5.2 & 3.2 & 0.0 & 0.0 & 0.0 & 83.0 & 5.9 & 3.7 \\
\hline 1994 & 16.5 & 0.5 & 0.8 & 0.0 & 0.0 & 0.0 & 66.5 & 2.9 & 5.6 & 0.0 & 0.0 & 0.0 & 83.0 & 3.4 & 6.4 \\
\hline 1995 & 16.5 & 0.7 & 0.2 & 0.0 & 0.0 & 0.0 & 66.5 & 15.5 & 5.3 & 0.0 & 0.0 & 0.0 & 83.0 & 16.2 & 5.5 \\
\hline 1996 & 16.5 & 1.1 & 0.4 & 0.0 & 0.0 & 0.0 & 66.5 & 1.9 & 6.7 & 0.0 & 0.0 & 0.0 & 83.0 & 3.0 & 7.1 \\
\hline 1997 & 17.0 & 0.9 & 0.7 & 0.0 & 0.0 & 0.0 & 66.0 & 6.4 & 4.1 & 0.0 & 0.0 & 0.0 & 83.0 & 7.3 & 4.8 \\
\hline 1998 & 17.0 & 1.8 & 0.0 & 0.0 & 0.0 & 0.0 & 66.0 & 2.6 & 6.4 & 0.0 & 0.0 & 0.0 & 83.0 & 4.4 & 6.4 \\
\hline Total & -- & 7.6 & 4.7 & -- & 0.0 & 0.0 & -- & 54.5 & 48.2 & 0.0 & 0.0 & 0.0 & - & 62.1 & 52.9 \\
\hline \multicolumn{16}{|c|}{ Cross section was stable. It is in a bedrock reach. } \\
\hline 1989 & 6.0 & 0.3 & 0.0 & 32.0 & 0.1 & 0.4 & 57.0 & 1.2 & 1.0 & 0.0 & 0.0 & 0.0 & 95.0 & 1.6 & 1.4 \\
\hline 1990 & 6.0 & 0.1 & 0.4 & 34.0 & 0.1 & 2.7 & 55.0 & 0.6 & 2.7 & 0.0 & 0.0 & 0.0 & 95.0 & 0.8 & 5.8 \\
\hline 1991 & 6.0 & 0.2 & 0.1 & 34.0 & 0.6 & 0.2 & 55.0 & 2.9 & 0.1 & 0.0 & 0.0 & 0.0 & 95.0 & 3.7 & 0.4 \\
\hline 1992 & 6.0 & 0.1 & 0.4 & 34.0 & 0.2 & 0.7 & 55.0 & 0.5 & 0.4 & 0.0 & 0.0 & 0.0 & 95.0 & 0.8 & 1.5 \\
\hline 1993 & 11.5 & 0.0 & 0.9 & 34.0 & 0.0 & 2.6 & 49.5 & 0.4 & 2.1 & 0.0 & 0.0 & 0.0 & 95.0 & 0.4 & 5.6 \\
\hline 1994 & 10.0 & 0.7 & 0.0 & 34.0 & 0.1 & 0.7 & 51.0 & 1.3 & 2.4 & 0.0 & 0.0 & 0.0 & 95.0 & 2.1 & 3.1 \\
\hline 1995 & 6.5 & 0.3 & 0.3 & 33.5 & 0.4 & 3.4 & 55.0 & 4.5 & 0.2 & 0.0 & 0.0 & 0.0 & 95.0 & 5.2 & 3.9 \\
\hline 1996 & 6.0 & 0.3 & 0.2 & 33.5 & 0.2 & 0.9 & 55.5 & 0.4 & 0.6 & 0.0 & 0.0 & 0.0 & 95.0 & 0.9 & 1.7 \\
\hline 1997 & 6.5 & 0.1 & 0.0 & 34.0 & 0.4 & 0.1 & 54.5 & 0.5 & 0.4 & 0.0 & 0.0 & 0.0 & 95.0 & 1.0 & 0.5 \\
\hline 1998 & 6.5 & 0.0 & 0.2 & 34.0 & 0.1 & 0.4 & 54.5 & 0.2 & 0.8 & 0.0 & 0.0 & 0.0 & 95.0 & 0.3 & 1.4 \\
\hline Total & -. & 2.1 & 2.5 & -- & 2.2 & 12.1 & -- & 12.5 & 10.7 & 0.0 & 0.0 & 0.0 & -- & 16.8 & 25.3 \\
\hline
\end{tabular}

Channel and bank erosion and deposition are balanced. The net increase at the section is a result of new flood-plain deposition PR122

\begin{tabular}{|c|c|c|c|c|c|c|c|c|c|c|c|c|c|c|c|}
\hline $\begin{array}{c}1988- \\
1993\end{array}$ & 8.5 & 0.4 & 0.2 & 0.0 & 0.0 & 0.0 & 82.0 & 0.0 & 2.7 & 28.0 & 0.4 & 0.2 & 118.5 & 0.8 & 3.1 \\
\hline $\begin{array}{c}1994- \\
1995\end{array}$ & 8.5 & 1.2 & 0.3 & 0.0 & 0.0 & 0.0 & 82.0 & 0.1 & 4.4 & 28.0 & 0.4 & 0.4 & 118.5 & 1.7 & 5.1 \\
\hline Total & -- & 1.6 & 0.5 & 0.0 & 0.0 & 0.0 & -- & 0.1 & 7.1 & - & 0.8 & 0.6 & - & 2.5 & 8.2 \\
\hline
\end{tabular}


Table 6. (Continued) Erosion and deposition of sediment at channel cross sections on the Powder River

\begin{tabular}{|c|c|c|c|c|c|c|c|c|c|c|c|c|c|c|c|}
\hline \multirow{2}{*}{$\begin{array}{l}\text { Water } \\
\text { Year }\end{array}$} & \multicolumn{3}{|c|}{ Bank } & \multicolumn{3}{|c|}{ Flood plain } & \multicolumn{3}{|c|}{ Channel } & \multicolumn{3}{|c|}{ Point bar } & \multicolumn{3}{|c|}{ Total } \\
\hline & $\begin{array}{c}\text { Width } \\
\text { (m) }\end{array}$ & $\begin{array}{c}\text { Eros. } \\
\left(\mathrm{m}^{2}\right)\end{array}$ & $\begin{array}{c}\text { Depo. } \\
\left(\mathrm{m}^{2}\right)\end{array}$ & $\begin{array}{l}\text { Width } \\
\text { (m) }\end{array}$ & $\begin{array}{l}\text { Eros. } \\
\left(m^{2}\right)\end{array}$ & $\begin{array}{c}\text { Depo. } \\
\left(m^{2}\right)\end{array}$ & $\begin{array}{c}\text { Width } \\
\text { (m) }\end{array}$ & $\begin{array}{c}\text { Eros. } \\
\left(\mathrm{m}^{2}\right)\end{array}$ & $\begin{array}{c}\text { Depo. } \\
\left(\mathrm{m}^{2}\right)\end{array}$ & $\begin{array}{c}\text { Width } \\
\text { (m) }\end{array}$ & $\begin{array}{c}\text { Eros. } \\
\left(\mathrm{m}^{2}\right)\end{array}$ & $\begin{array}{c}\text { Depo. } \\
\left(m^{2}\right)\end{array}$ & $\begin{array}{l}\text { Width } \\
\text { (m) }\end{array}$ & $\begin{array}{c}\text { Eros. } \\
\left(\mathrm{m}^{2}\right)\end{array}$ & $\begin{array}{c}\text { Depo. } \\
\left(m^{2}\right)\end{array}$ \\
\hline \multicolumn{16}{|c|}{ PR122A } \\
\hline 1989 & 8.5 & 0.8 & 0.2 & 21.5 & 1.0 & 0.7 & 36.0 & 0.2 & 0.4 & 4.0 & 0.0 & 0.5 & 70.0 & 2.0 & 1.8 \\
\hline 1990 & 11.5 & 0.8 & 0.1 & 20.5 & 0.0 & 3.4 & 30.0 & 1.9 & 0.2 & 8.0 & 0.1 & 0.8 & 70.0 & 2.8 & 4.5 \\
\hline 1991 & 11.5 & 1.4 & 0.3 & 12.5 & 0.0 & 0.8 & 28.5 & 0.1 & 0.6 & 17.5 & 3.3 & 0.0 & 70.0 & 4.8 & 1.7 \\
\hline 1992 & 7.5 & 0.2 & 0.2 & 12.0 & 0.0 & 0.2 & 32.5 & 0.6 & 0.5 & 18.0 & 0.2 & 0.2 & 70.0 & 1.0 & 1.1 \\
\hline 1993 & 14.0 & 10.8 & 0.0 & 13.0 & 0.0 & 2.2 & 23.0 & 1.6 & 0.1 & 24.0 & 0.5 & 2.8 & 74.0 & 12.9 & 5.1 \\
\hline 1994 & 10.5 & 1.6 & 0.1 & 37.0 & 0.0 & 2.3 & 27.5 & 0.1 & 1.2 & 0.0 & 0.0 & 0.0 & 75.0 & 1.7 & 3.5 \\
\hline 1995 & 18.5 & 21.2 & 0.2 & 11.0 & 0.0 & 3.2 & 16.5 & 0.6 & 0.0 & 37.0 & 0.3 & 14.0 & 83.0 & 22.1 & 17.3 \\
\hline 1996 & 14.5 & 5.4 & 0.0 & 10.0 & 0.0 & 0.1 & 27.5 & 0.3 & 1.4 & 35.0 & 0.5 & 1.1 & 87.0 & 6.2 & 2.6 \\
\hline 1997 & 12.0 & 3.3 & 0.1 & 15.0 & 0.1 & 0.1 & 30.0 & 0.4 & 0.4 & 30.0 & 0.4 & 0.5 & 87.0 & 4.2 & 1.1 \\
\hline 1998 & 7.5 & 0.5 & 0.1 & 45.0 & 0.2 & 0.7 & 34.5 & 0.2 & 0.4 & 0.0 & 0.0 & 0.0 & 87.0 & 0.9 & 1.2 \\
\hline Total & -- & 46.0 & 1.1 & -- & 1.3 & 13.7 & - & 6.0 & 5.2 & - & 5.3 & 19.9 & & 58.6 & 39.9 \\
\hline
\end{tabular}

Erosion on the left bank was partly balanced by deposition on the point bar and on the new flood plain.

$\begin{array}{rrrrrrrrrrrrrrrr}1989 & 4.0 & 0.4 & 0.0 & 40.0 & 0.4 & 0.4 & 42.0 & 3.1 & 0.2 & 0.0 & 0.0 & 0.0 & 86.0 & 3.9 & 0.6 \\ 1990 & 4.0 & 0.5 & 0.0 & 40.0 & 0.0 & 2.4 & 42.0 & 3.9 & 0.8 & 0.0 & 0.0 & 0.0 & 86.0 & 4.4 & 3.2 \\ 1991 & 4.0 & 1.1 & 0.0 & 40.0 & 0.2 & 1.5 & 42.0 & 0.8 & 6.3 & 0.0 & 0.0 & 0.0 & 86.0 & 2.1 & 7.8 \\ 1992 & 4.0 & 0.1 & 0.0 & 40.0 & 0.2 & 0.5 & 42.0 & 4.4 & 0.3 & 0.0 & 0.0 & 0.0 & 86.0 & 4.7 & 0.8 \\ 1993 & 5.0 & 2.5 & 0.0 & 40.0 & 0.0 & 5.0 & 42.0 & 1.0 & 2.1 & 0.0 & 0.0 & 0.0 & 87.0 & 3.5 & 7.1 \\ 1994 & 5.0 & 0.2 & 0.2 & 40.0 & 0.2 & 1.0 & 42.0 & 4.5 & 1.2 & 0.0 & 0.0 & 0.0 & 87.0 & 4.9 & 2.4 \\ 1995 & 5.0 & 2.1 & 0.0 & 38.0 & 0.2 & 6.2 & 46.0 & 1.1 & 6.7 & 0.0 & 0.0 & 0.0 & 89.0 & 3.4 & 12.9 \\ 1996 & 10.0 & 4.5 & 0.0 & 38.0 & 0.3 & 0.9 & 41.0 & 3.7 & 0.1 & 0.0 & 0.0 & 0.0 & 89.0 & 8.5 & 1.0 \\ 1997 & 4.5 & 0.8 & 0.0 & 38.0 & 0.5 & 0.4 & 47.0 & 2.4 & 0.5 & 0.0 & 0.0 & 0.0 & 89.5 & 3.7 & 0.9 \\ 1998 & 4.0 & 0.5 & 0.0 & 38.0 & 0.4 & 0.4 & 47.0 & 1.2 & 2.6 & 0.0 & 0.0 & 0.0 & 89.0 & 2.1 & 3.0 \\ \text { Total } & -- & 12.7 & 0.2 & -- & 2.4 & 18.7 & - & 26.1 & 20.8 & -- & 0.0 & 0.0 & - & 41.2 & 39.7\end{array}$

Bank erosion was balanced by flood-plain deposition. Erosion approximately balances deposition.

$\begin{array}{rrrrrrrrrrrrrrrrr}1989 & 6.0 & 0.1 & 0.1 & 45.0 & 0.1 & 1.1 & 46.5 & 1.9 & 1.1 & 0.0 & 0.0 & 0.0 & 97.5 & 2.1 & 2.3 \\ 1990 & 6.0 & 0.0 & 0.8 & 37.0 & 0.0 & 1.3 & 50.5 & 1.0 & 1.8 & 4.0 & 0.0 & 1.3 & 97.5 & 1.0 & 5.2 \\ 1991 & 6.0 & 0.7 & 0.0 & 39.0 & 0.3 & 1.4 & 51.5 & 1.8 & 0.5 & 1.0 & 0.2 & 0.0 & 97.5 & 3.0 & 1.9 \\ 1992 & 6.0 & 0.0 & 0.6 & 39.0 & 0.1 & 0.7 & 49.5 & 0.6 & 1.2 & 3.0 & 0.0 & 0.6 & 97.5 & 0.7 & 3.1 \\ 1993 & 5.5 & 0.2 & 0.3 & 42.0 & 0.1 & 3.4 & 50.0 & 1.7 & 1.5 & 0.0 & 0.0 & 0.0 & 97.5 & 2.0 & 5.2 \\ 1994 & 5.0 & 0.6 & 0.1 & 42.0 & 0.2 & 0.9 & 50.5 & 1.8 & 1.0 & 0.0 & 0.0 & 0.0 & 97.5 & 2.6 & 2.0 \\ 1995 & 5.5 & 0.4 & 0.5 & 42.0 & 0.1 & 8.2 & 50.0 & 1.2 & 1.5 & 0.0 & 0.0 & 0.0 & 97.5 & 1.7 & 10.2 \\ 1996 & 5.0 & 0.4 & 0.1 & 42.0 & 0.4 & 0.6 & 50.5 & 1.4 & 0.3 & 0.0 & 0.0 & 0.0 & 97.5 & 2.2 & 1.0 \\ 1997 & 5.0 & 0.4 & 0.1 & 42.0 & 0.4 & 0.2 & 50.5 & 3.4 & 0.4 & 0.0 & 0.0 & 0.0 & 97.5 & 4.2 & 0.7 \\ 1998 & 5.0 & 0.4 & 0.1 & 42.0 & 0.1 & 0.4 & 50.5 & 0.8 & 2.0 & 0.0 & 0.0 & 0.0 & 97.5 & 1.3 & 2.5 \\ \text { Total } & -- & 3.2 & 2.7 & -- & 1.8 & 18.2 & -- & 15.6 & 11.3 & -- & 0.2 & 1.9 & -- & 20.8 & 31.1\end{array}$

Bank erosion was balanced by deposition on flood plain at bottom of bank. Channel was approximately in equilibrium. 
Table 6. (Continued) Erosion and deposition of sediment at channel cross sections on the Powder River

\begin{tabular}{|c|c|c|c|c|c|c|c|c|c|c|c|c|c|c|c|}
\hline \multirow{2}{*}{$\begin{array}{l}\text { Water } \\
\text { Year }\end{array}$} & \multicolumn{3}{|c|}{ Bank } & \multicolumn{3}{|c|}{ Flood plain } & \multicolumn{3}{|c|}{ Channel } & \multicolumn{3}{|c|}{ Point bar } & \multicolumn{3}{|c|}{ Total } \\
\hline & $\begin{array}{l}\text { Width } \\
\text { (m) }\end{array}$ & $\begin{array}{c}\text { Eros. } \\
\left(\mathrm{m}^{2}\right)\end{array}$ & $\begin{array}{c}\text { Depo. } \\
\left(\mathrm{m}^{2}\right)\end{array}$ & $\begin{array}{c}\text { Width } \\
\text { (m) }\end{array}$ & $\begin{array}{l}\text { Eros. } \\
\left(\mathrm{m}^{2}\right)\end{array}$ & $\begin{array}{c}\text { Depo. } \\
\left(\mathrm{m}^{2}\right)\end{array}$ & $\begin{array}{c}\text { Width } \\
(\mathrm{m})\end{array}$ & $\begin{array}{c}\text { Eros. } \\
\left(\mathrm{m}^{2}\right)\end{array}$ & $\begin{array}{c}\text { Depo. } \\
\left(\mathrm{m}^{2}\right)\end{array}$ & $\begin{array}{c}\text { Width } \\
\text { (m) }\end{array}$ & $\begin{array}{c}\text { Eros. } \\
\left(\mathrm{m}^{2}\right)\end{array}$ & $\begin{array}{c}\text { Depo. } \\
\left(\mathrm{m}^{2}\right)\end{array}$ & $\begin{array}{c}\text { Width } \\
(\mathrm{m})\end{array}$ & $\begin{array}{c}\text { Eros. } \\
\left(\mathrm{m}^{2}\right)\end{array}$ & $\begin{array}{c}\text { Depo. } \\
\left(\mathrm{m}^{2}\right)\end{array}$ \\
\hline \multicolumn{16}{|c|}{ PR136 } \\
\hline 1989 & 17.5 & 0.7 & 0.3 & 40.5 & 0.3 & 0.4 & 52.0 & 3.6 & 0.7 & 0.0 & 0.0 & 0.0 & 110.0 & 4.6 & 1.4 \\
\hline 1990 & 17.0 & 0.1 & 0.0 & 41.5 & 0.2 & 2.1 & 51.5 & 2.4 & 3.3 & 0.0 & 0.0 & 0.0 & 110.0 & 2.7 & 5.4 \\
\hline 1991 & 17.5 & 0.5 & 0.3 & 41.0 & 0.6 & 0.7 & 51.5 & 3.9 & 2.5 & 0.0 & 0.0 & 0.0 & 110.0 & 5.0 & 3.5 \\
\hline 1992 & 15.5 & 0.4 & 0.3 & 39.5 & 0.1 & 0.9 & 52.0 & 0.3 & 6.4 & 3.0 & 0.2 & 0.0 & 110.0 & 1.0 & 7.6 \\
\hline 1993 & 16.0 & 1.2 & 0.1 & 40.0 & 0.1 & 5.2 & 45.0 & 4.6 & 0.7 & 9.0 & 0.0 & 2.4 & 110.0 & 5.9 & 8.4 \\
\hline 1994 & 15.5 & 1.6 & 0.2 & 45.0 & 0.2 & 2.0 & 49.5 & 2.0 & 3.9 & 0.0 & 0.0 & 0.0 & 110.0 & 3.8 & 6.1 \\
\hline 1995 & 18.0 & 8.2 & 0.0 & 45.0 & 0.2 & 11.9 & 48.0 & 6.3 & 1.6 & 0.0 & 0.0 & 0.0 & 111.0 & 14.7 & 13.5 \\
\hline 1996 & 15.0 & 6.4 & 0.0 & 37.0 & 0.2 & 0.2 & 50.0 & 1.9 & 5.1 & 8.0 & 0.4 & 1.9 & 110.0 & 8.9 & 7.2 \\
\hline 1997 & 11.5 & 5.3 & 0.1 & 41.0 & 0.3 & 0.7 & 44.0 & 0.5 & 5.7 & 3.5 & 0.6 & 0.0 & 110.0 & 6.7 & 6.5 \\
\hline 1998 & 10.0 & 1.3 & 0.3 & 39.0 & 0.2 & 0.4 & 57.0 & 5.4 & 6.8 & 4.0 & 1.8 & 0.0 & 110.0 & 8.7 & 7.5 \\
\hline Total & - & 25.7 & 1.6 & -- & 2.4 & 24.5 & - & 30.9 & 36.7 & - & 3.0 & 4.3 & -- & 62.0 & 67.1 \\
\hline
\end{tabular}

Bank erosion was balanced by deposition on flood plain in the middle of the channel. Erosion approximately balanced deposition

PR141

\begin{tabular}{|c|c|c|c|c|c|c|c|c|c|c|c|c|c|c|c|}
\hline $\begin{array}{l}1984- \\
1998\end{array}$ & 18.9 & 0.4 & 0.4 & 32.1 & 0.2 & 0.2 & 49.0 & 0.2 & 1.1 & 0.0 & 0.0 & 0.0 & 100.0 & 0.8 & 1.7 \\
\hline \multicolumn{16}{|c|}{ This section was cut off by the 1978 flood and these changes have occurred over 14 years. } \\
\hline \multicolumn{16}{|c|}{ PR141A } \\
\hline 1989 & 11.0 & 0.4 & 0.2 & 82.0 & 0.4 & 4.6 & 37.0 & 3.8 & 3.0 & 0.0 & 0.0 & 0.0 & 130.0 & 4.6 & 7.8 \\
\hline 1990 & 11.0 & 0.2 & 0.2 & 82.0 & 0.5 & 7.0 & 26.5 & 3.5 & 0.2 & 10.5 & 0.0 & 4.0 & 130.0 & 4.2 & 11.4 \\
\hline 1991 & 11.0 & 4.7 & 0.0 & 91.0 & 7.2 & 4.4 & 28.0 & 0.1 & 3.4 & 0.0 & 0.0 & 0.0 & 130.0 & 12.0 & 7.8 \\
\hline 1992 & 5.0 & 0.2 & 0.1 & 61.5 & 0.2 & 1.2 & 33.5 & 2.3 & 0.7 & 30.0 & 1.1 & 0.5 & 130.0 & 3.8 & 2.5 \\
\hline 1993 & 4.3 & 4.2 & 0.0 & 60.0 & 0.0 & 4.8 & 33.7 & 1.3 & 4.7 & 32.0 & 2.1 & 2.1 & 130.0 & 7.6 & 11.6 \\
\hline 1994 & 4.5 & 2.0 & 0.0 & 78.5 & 0.0 & 7.1 & 56.5 & 4.5 & 6.7 & 10.5 & 0.0 & 2.9 & 150.0 & 6.5 & 16.7 \\
\hline 1995 & 23.5 & 39.6 & 0.1 & 71.0 & 0.2 & 10.5 & 9.5 & 0.0 & 3.5 & 46.0 & 0.0 & 26.9 & 150.0 & 39.8 & 41.0 \\
\hline 1996 & 13.5 & 22.4 & 0.0 & 87.5 & 1.5 & 7.5 & 19.0 & 0.2 & 2.4 & 15.0 & 1.7 & 2.4 & 135.0 & 25.8 & 12.3 \\
\hline 1997 & 10.5 & 14.6 & 0.0 & 77.5 & 0.2 & 5.5 & 22.5 & 1.0 & 1.0 & 29.5 & 4.5 & 1.1 & 140.0 & 20.3 & 7.6 \\
\hline 1998 & 8.0 & 6.6 & 0.0 & 101.0 & 1.7 & 2.6 & 29.0 & 0.4 & 3.0 & 5.0 & 1.3 & 0.0 & 143.0 & 10.0 & 5.6 \\
\hline Total & - & 94.9 & 0.6 & - & 11.9 & 55.2 & -- & 17.1 & 28.6 & - & 10.7 & 39.9 & -- & 134.6 & 124.3 \\
\hline \multicolumn{16}{|c|}{$\begin{array}{l}\text { Left bank retreated and the point bar on the right bank advanced. } \\
\text { PR141.7 }\end{array}$} \\
\hline 1995 & 17.5 & 21.7 & 0.4 & 48.0 & 0.1 & 7.9 & 64.0 & 0.4 & 14.6 & 0.0 & 0.0 & 0.0 & 129.5 & 22.2 & 22.9 \\
\hline 1996 & 10.4 & 7.9 & 0.2 & 48.0 & 0.2 & 0.6 & 72.0 & 2.9 & 3.6 & 0.0 & 0.0 & 0.0 & 130.4 & 11.0 & 4.4 \\
\hline 1997 & 3.0 & 0.0 & 0.1 & 47.0 & 0.5 & 0.4 & \multicolumn{3}{|c|}{ partial survey } & 0.0 & 0.0 & 0.0 & 50.0 & 0.5 & 0.5 \\
\hline 1998 & 3.0 & 0.2 & 0.0 & 47.0 & 0.5 & 0.3 & \multicolumn{3}{|c|}{ partial survey } & 0.0 & 0.0 & 0.0 & 50.0 & 0.7 & 0.3 \\
\hline
\end{tabular}

This section was established in 1994 after an ice jam deposited sediment on the left bank. The surveys are focused on the left bank 
Table 6. (Continued) Erosion and deposition of sediment at channel cross sections on the Powder River

\begin{tabular}{|c|c|c|c|c|c|c|c|c|c|c|c|c|c|c|c|}
\hline \multirow{2}{*}{$\begin{array}{l}\text { Water } \\
\text { Year }\end{array}$} & \multicolumn{3}{|c|}{ Bank } & \multicolumn{3}{|c|}{ Flood plain } & \multicolumn{3}{|c|}{ Channel } & \multicolumn{3}{|c|}{ Point bar } & \multicolumn{3}{|c|}{ Total } \\
\hline & $\begin{array}{l}\text { Width } \\
\text { (m) }\end{array}$ & $\begin{array}{l}\text { Eros. } \\
\left(\mathrm{m}^{2}\right)\end{array}$ & $\begin{array}{c}\text { Depo. } \\
\left(\mathrm{m}^{2}\right)\end{array}$ & $\begin{array}{c}\text { Width } \\
\text { (m) }\end{array}$ & $\begin{array}{l}\text { Eros. } \\
\left(\mathrm{m}^{2}\right)\end{array}$ & $\begin{array}{c}\text { Depo. } \\
\left(\mathrm{m}^{2}\right)\end{array}$ & $\begin{array}{l}\text { Width } \\
\text { (m) }\end{array}$ & $\begin{array}{l}\text { Eros. } \\
\left(\mathrm{m}^{2}\right)\end{array}$ & $\begin{array}{c}\text { Depo. } \\
\left(\mathrm{m}^{2}\right)\end{array}$ & $\begin{array}{l}\text { Width } \\
(\mathrm{m})\end{array}$ & $\begin{array}{l}\text { Eros. } \\
\left(\mathrm{m}^{2}\right)\end{array}$ & $\begin{array}{c}\text { Depo. } \\
\left(\mathrm{m}^{2}\right)\end{array}$ & $\begin{array}{c}\text { Width } \\
\text { (m) }\end{array}$ & $\begin{array}{l}\text { Eros. } \\
\left(\mathrm{m}^{2}\right)\end{array}$ & $\begin{array}{c}\text { Depo. } \\
\left(\mathrm{m}^{2}\right)\end{array}$ \\
\hline \multicolumn{16}{|c|}{ PR147 } \\
\hline 1989 & 11.0 & 0.3 & 0.2 & 15.0 & 0.1 & 0.8 & 59.0 & 1.1 & 1.0 & 0.0 & 0.0 & 0.0 & 85.0 & 1.5 & 2.0 \\
\hline 1990 & 11.0 & 0.3 & 0.2 & 17.5 & 0.2 & 2.3 & 56.5 & 1.4 & 1.5 & 0.0 & 0.0 & 0.0 & 85.0 & 1.9 & 4.0 \\
\hline 1991 & 11.5 & 1.4 & 0.2 & 19.0 & 0.0 & 7.0 & 54.5 & 4.5 & 0.2 & 0.0 & 0.0 & 0.0 & 85.0 & 5.9 & 7.4 \\
\hline 1992 & 11.0 & 0.2 & 0.4 & 18.0 & 0.3 & 0.2 & 56.0 & 0.6 & 1.4 & 0.0 & 0.0 & 0.0 & 85.0 & 1.1 & 2.0 \\
\hline 1993 & 4.0 & 0.1 & 0.0 & 14.5 & 0.0 & 3.6 & 61.0 & 3.0 & 1.2 & 0.0 & 0.0 & 0.0 & 79.5 & 3.1 & 4.8 \\
\hline 1994 & 14.5 & 0.1 & 0.2 & 14.0 & 0.1 & 0.2 & 61.5 & 1.2 & 1.7 & 0.0 & 0.0 & 0.0 & 90.0 & 1.4 & 2.1 \\
\hline 1995 & 15.0 & 7.7 & 0.1 & 11.0 & 0.0 & 2.0 & 64.0 & 5.5 & 4.1 & 0.0 & 0.0 & 0.0 & 90.0 & 13.2 & 6.2 \\
\hline 1996 & 10.0 & 1.0 & 0.1 & 11.0 & 0.1 & 0.1 & 69.0 & 4.3 & 4.3 & 0.0 & 0.0 & 0.0 & 90.0 & 5.4 & 4.5 \\
\hline 1997 & 10.5 & 0.2 & 0.4 & 9.0 & 0.0 & 0.1 & 70.5 & 2.1 & 2.4 & 0.0 & 0.0 & 0.0 & 90.0 & 2.3 & 2.9 \\
\hline 1998 & 9.5 & 0.8 & 0.2 & 10.0 & 0.1 & 0.5 & 70.5 & 1.2 & 2.5 & 0.0 & 0.0 & 0.0 & 90.0 & 2.1 & 3.2 \\
\hline Total & -- & 12.1 & 2.0 & - & 0.9 & 16.8 & - & 24.9 & 20.3 & -- & 0.0 & 0.0 & -- & 37.9 & 39.1 \\
\hline \multicolumn{16}{|c|}{$\begin{array}{l}\text { Some bank erosion occurred on the right bank and a floodplain evolved on the left bank. } \\
\text { PR151 }\end{array}$} \\
\hline 1989 & 24.5 & 0.4 & 0.2 & 24.0 & 0.2 & 0.2 & 41.0 & 0.7 & 0.9 & 0.0 & 0.0 & 0.0 & 89.5 & 1.3 & 1.3 \\
\hline 1990 & 27.5 & 0.3 & 0.9 & 24.0 & 0.0 & 1.6 & 38.0 & 1.8 & 0.1 & 0.0 & 0.0 & 0.0 & 89.5 & 2.1 & 2.6 \\
\hline 1991 & 28.5 & 0.5 & 0.4 & 24.0 & 0.2 & 0.4 & 37.0 & 0.3 & 0.7 & 0.0 & 0.0 & 0.0 & 89.5 & 1.0 & 1.5 \\
\hline 1992 & 29.0 & 0.5 & 0.7 & 22.0 & 0.0 & 0.6 & 38.5 & 1.0 & 0.1 & 0.0 & 0.0 & 0.0 & 89.5 & 1.5 & 1.4 \\
\hline 1993 & 27.0 & 0.6 & 0.3 & 22.5 & 0.1 & 2.1 & 41.0 & 0.6 & 0.4 & 0.0 & 0.0 & 0.0 & 89.5 & 1.3 & 2.8 \\
\hline 1994 & 27.5 & 0.5 & 0.5 & 22.0 & 0.3 & 0.2 & 40.0 & 1.2 & 0.4 & 0.0 & 0.0 & 0.0 & 89.5 & 2.0 & 1.1 \\
\hline 1995 & 27.5 & 1.2 & 0.2 & 21.0 & 0.2 & 3.7 & 41.0 & 1.3 & 1.2 & 0.0 & 0.0 & 0.0 & 89.5 & 2.7 & 5.1 \\
\hline 1996 & 24.0 & 0.1 & 0.6 & 22.5 & 0.8 & 0.6 & 41.0 & 1.4 & 0.8 & 0.0 & 0.0 & 0.0 & 87.5 & 2.3 & 2.0 \\
\hline 1997 & 24.5 & 0.3 & 0.5 & 21.5 & 0.4 & 0.1 & 41.5 & 0.9 & 0.4 & 0.0 & 0.0 & 0.0 & 87.5 & 1.6 & 1.0 \\
\hline 1998 & 24.5 & 0.4 & 0.2 & 21.0 & 0.4 & 0.2 & 42.0 & 0.8 & 0.2 & 0.0 & 0.0 & 0.0 & 87.5 & 1.6 & 0.6 \\
\hline Total & -- & 4.8 & 4.5 & -- & 2.6 & 9.7 & -- & 10.0 & 5.2 & -- & 0.0 & 0.0 & - & 17.4 & 19.4 \\
\hline
\end{tabular}




\begin{tabular}{|c|c|c|c|c|c|c|c|c|c|c|c|c|c|c|c|}
\hline \multirow{2}{*}{$\begin{array}{l}\text { Water } \\
\text { Year }\end{array}$} & \multicolumn{3}{|c|}{ Bank } & \multicolumn{3}{|c|}{ Flood plain } & \multicolumn{3}{|c|}{ Channel } & \multicolumn{3}{|c|}{ Point bar } & \multicolumn{3}{|c|}{ Total } \\
\hline & $\begin{array}{l}\text { Width } \\
\text { (m) }\end{array}$ & $\begin{array}{l}\text { Eros. } \\
\left(\mathrm{m}^{2}\right)\end{array}$ & $\begin{array}{c}\text { Depo. } \\
\left(\mathrm{m}^{2}\right)\end{array}$ & $\begin{array}{c}\text { Width } \\
\text { (m) }\end{array}$ & $\begin{array}{l}\text { Eros. } \\
\left(\mathrm{m}^{2}\right)\end{array}$ & $\begin{array}{c}\text { Depo. } \\
\left(\mathrm{m}^{2}\right)\end{array}$ & $\begin{array}{l}\text { Width } \\
\text { (m) }\end{array}$ & $\begin{array}{l}\text { Eros. } \\
\left(\mathrm{m}^{2}\right)\end{array}$ & $\begin{array}{c}\text { Depo. } \\
\left(\mathrm{m}^{2}\right)\end{array}$ & $\begin{array}{l}\text { Width } \\
\text { (m) }\end{array}$ & $\begin{array}{l}\text { Eros. } \\
\left(\mathrm{m}^{2}\right)\end{array}$ & $\begin{array}{c}\text { Depo. } \\
\left(\mathrm{m}^{2}\right)\end{array}$ & $\begin{array}{l}\text { Width } \\
\text { (m) }\end{array}$ & $\begin{array}{l}\text { Eros. } \\
\left(m^{2}\right)\end{array}$ & $\begin{array}{l}\text { Depo. } \\
\left(m^{2}\right)\end{array}$ \\
\hline \multicolumn{16}{|c|}{ PR156A } \\
\hline 1989 & 5.0 & 0.2 & 0.1 & 60.0 & 0.5 & 1.0 & 45.0 & 0.7 & 1.9 & 0.0 & 0.0 & 0.0 & 110.0 & 1.4 & 3.0 \\
\hline 1990 & 5.0 & 0.1 & 0.1 & 60.0 & 0.2 & 1.9 & 45.0 & 10.6 & 0.5 & 0.0 & 0.0 & 0.0 & 110.0 & 10.9 & 2.5 \\
\hline 1991 & 5.0 & 0.0 & 0.2 & 60.0 & 0.4 & 0.6 & 45.0 & 0.3 & 9.0 & 0.0 & 0.0 & 0.0 & 110.0 & 0.7 & 9.8 \\
\hline 1992 & 5.0 & 0.1 & 0.2 & 60.0 & 0.6 & 0.4 & 45.0 & 4.4 & 1.3 & 0.0 & 0.0 & 0.0 & 110.0 & 5.1 & 1.9 \\
\hline 1993 & 5.0 & 0.1 & 0.1 & 60.0 & 0.3 & 4.7 & 45.0 & 1.8 & 2.0 & 0.0 & 0.0 & 0.0 & 110.0 & 2.2 & 6.8 \\
\hline 1994 & 5.0 & 0.1 & 0.1 & 60.0 & 0.5 & 0.8 & 45.0 & 2.9 & 4.0 & 0.0 & 0.0 & 0.0 & 110.0 & 3.5 & 4.9 \\
\hline 1995 & 5.0 & 0.5 & 0.0 & 60.0 & 0.2 & 6.1 & 45.0 & 4.1 & 2.8 & 0.0 & 0.0 & 0.0 & 110.0 & 4.8 & 8.9 \\
\hline 1996 & 5.0 & 0.9 & 0.0 & 60.0 & 1.0 & 0.3 & 45.0 & 3.8 & 1.1 & 0.0 & 0.0 & 0.0 & 110.0 & 5.7 & 1.4 \\
\hline 1997 & 5.0 & 1.0 & 0.2 & 60.0 & 0.3 & 0.8 & 45.0 & 0.1 & 2.9 & 0.0 & 0.0 & 0.0 & 110.0 & 1.4 & 3.9 \\
\hline 1998 & 5.0 & 0.8 & 0.0 & 60.0 & 0.5 & 0.7 & 45.0 & 3.7 & 0.1 & 0.0 & 0.0 & 0.0 & 110.0 & 5.0 & 0.8 \\
\hline Total & - & 3.8 & 1.0 & -- & 4.5 & 17.3 & -- & 32.4 & 25.6 & -- & 0.0 & 0.0 & -. & 40.7 & 43.9 \\
\hline
\end{tabular}

Vertical accretion on the flood plain adjacent to the right bank. Channel is approximately in equilibrium every two years.

$\begin{array}{rrrrrrrrrrrrrrrr}1989 & 30.0 & 0.7 & 0.2 & 14.5 & 0.1 & 0.4 & 75.5 & 1.6 & 2.5 & 0.0 & 0.0 & 0.0 & 120.0 & 2.4 & 3.1 \\ 1990 & 30.0 & 0.2 & 0.4 & 14.5 & 0.1 & 0.4 & 75.5 & 8.8 & 0.4 & 0.0 & 0.0 & 0.0 & 120.0 & 9.1 & 1.2 \\ 1991 & 30.0 & 0.3 & 0.5 & 18.5 & 0.1 & 2.2 & 71.5 & 0.1 & 6.3 & 0.0 & 0.0 & 0.0 & 120.0 & 0.5 & 9.0 \\ 1992 & 29.0 & 0.4 & 0.8 & 18.5 & 1.3 & 0.3 & 72.5 & 2.3 & 0.2 & 0.0 & 0.0 & 0.0 & 120.0 & 4.0 & 1.3 \\ 1993 & 29.0 & 0.6 & 0.5 & 18.5 & 0.2 & 1.1 & 72.5 & 0.8 & 1.6 & 0.0 & 0.0 & 0.0 & 120.0 & 1.6 & 3.2 \\ 1994 & 29.0 & 0.3 & 0.5 & 15.0 & 0.3 & 0.3 & 76.0 & 2.9 & 3.2 & 0.0 & 0.0 & 0.0 & 120.0 & 3.5 & 4.0 \\ 1995 & 29.0 & 0.6 & 0.5 & 15.0 & 0.3 & 2.7 & 76.0 & 3.4 & 3.3 & 0.0 & 0.0 & 0.0 & 120.0 & 4.3 & 6.5 \\ 1996 & 29.5 & 1.0 & 0.8 & 14.5 & 0.3 & 0.2 & 76.0 & 3.2 & 1.1 & 0.0 & 0.0 & 0.0 & 120.0 & 4.5 & 2.1 \\ 1997 & 30.5 & 0.6 & 0.3 & 14.5 & 0.4 & 0.3 & 75.0 & 1.6 & 3.1 & 0.0 & 0.0 & 0.0 & 120.0 & 2.6 & 3.7 \\ 1998 & 30.5 & 0.8 & 0.4 & 14.5 & 0.4 & 0.3 & 75.0 & 5.4 & 0.4 & 0.0 & 0.0 & 0.0 & 120.0 & 6.6 & 1.1 \\ \text { Total } & -- & 5.5 & 4.9 & -- & 3.5 & 8.2 & -- & 30.1 & 22.1 & -- & -- & -- & -- & 39.1 & 35.2\end{array}$

Vertical accretion of the flood plain along the right bank. In 1994 and 1995 a stump was on section in the channel. After 10 years net erosion is approximately equal to net deposition.

\begin{tabular}{|c|c|c|c|c|c|c|c|c|c|c|c|c|c|c|c|}
\hline 1989 & 12.0 & 0.8 & 0.0 & 52.0 & 0.2 & 1.4 & 36.0 & 2.5 & 0.5 & 0.0 & 0.0 & 0.0 & 100.0 & 3.5 & 1.9 \\
\hline 1990 & 12.0 & 0.5 & 0.1 & 58.0 & 0.2 & 6.3 & 30.0 & 1.6 & 1.2 & 0.0 & 0.0 & 0.0 & 100.0 & 2.3 & 7.6 \\
\hline 1991 & 13.5 & 7.3 & 0.0 & 44.0 & 0.4 & 0.5 & 31.0 & 0.0 & 6.2 & 11.5 & 3.8 & 0.0 & 100.0 & 11.5 & 6.7 \\
\hline 1992 & 9.0 & 0.6 & 0.0 & 47.5 & 0.2 & 0.7 & 43.5 & 5.9 & 1.5 & 0.0 & 0.0 & 0.0 & 100.0 & 6.7 & 2.2 \\
\hline 1993 & 9.0 & 6.9 & 0.1 & 45.5 & 0.0 & 2.0 & 24.5 & 1.8 & 1.6 & 21.0 & 0.1 & 5.5 & 100.0 & 8.8 & 9.2 \\
\hline 1994 & 6.5 & 1.2 & 0.2 & 45.5 & 0.1 & 0.8 & 27.0 & 2.4 & 2.4 & 21.0 & 0.0 & 2.8 & 100.0 & 3.7 & 6.2 \\
\hline 1995 & 9.0 & 12.1 & 0.1 & 45.0 & 0.0 & 7.5 & 27.5 & 0.4 & 2.8 & 21.0 & 0.2 & 4.2 & 102.5 & 12.7 & 14.6 \\
\hline 1996 & 12.0 & 17.9 & 0.0 & 45.0 & 0.1 & 1.7 & 33.0 & 1.1 & 3.6 & 20.0 & 2.2 & 0.1 & 110.0 & 21.3 & 5.4 \\
\hline 1997 & 7.0 & 4.7 & 0.0 & 45.5 & 0.1 & 1.1 & 38.0 & 2.5 & 2.7 & 19.5 & 1.5 & 0.2 & 110.0 & 8.8 & 4.0 \\
\hline 1998 & 4.5 & 3.3 & 0.0 & 44.5 & 0.5 & 0.3 & 40.5 & 2.7 & 2.5 & 20.5 & 0.0 & 1.6 & 110.0 & 6.5 & 4.4 \\
\hline Total & -- & 55.3 & 0.5 & - & 1.8 & 22.3 & -- & 20.9 & 25.0 & -. & 7.8 & 14.4 & -- & 85.8 & 62.2 \\
\hline
\end{tabular}

Left bank retreated about $15 \mathrm{~m}$. On right bank, the point bar accreted laterally and the flood plain farther to the right accreted vertically.

\section{PR164.8}

\begin{tabular}{|c|c|c|c|c|c|c|c|c|c|c|c|c|c|c|c|}
\hline $\begin{array}{c}1986- \\
1992\end{array}$ & 9.0 & 2.7 & 0.1 & 45.0 & 0.2 & 6.6 & 40.0 & 1.6 & 1.3 & 0.0 & 0.0 & 0.0 & 94.0 & 4.5 & 8.0 \\
\hline $\begin{array}{l}1992- \\
1998\end{array}$ & 8.0 & 11.6 & 0.0 & 43.5 & 1.1 & 16.9 & 42.5 & 7.6 & 3.6 & 0.0 & 0.0 & 0.0 & 94.0 & 20.3 & 20.5 \\
\hline Total & -- & 14.3 & 0.1 & -- & 1.3 & 23.5 & -- & 9.2 & 4.9 & 0.0 & 0.0 & 0.0 & -- & 24.8 & 28.5 \\
\hline
\end{tabular}


Table 6. (Continued) Erosion and deposition of sediment at channel cross sections on the Powder River

\begin{tabular}{|c|c|c|c|c|c|c|c|c|c|c|c|c|c|c|c|}
\hline \multirow{2}{*}{$\begin{array}{l}\text { Water } \\
\text { Year }\end{array}$} & \multicolumn{3}{|c|}{ Bank } & \multicolumn{3}{|c|}{ Flood plain } & \multicolumn{3}{|c|}{ Channel } & \multicolumn{3}{|c|}{ Point bar } & \multicolumn{3}{|c|}{ Total } \\
\hline & $\begin{array}{l}\text { Width } \\
\text { (m) }\end{array}$ & $\begin{array}{c}\text { Eros. } \\
\left(\mathrm{m}^{2}\right)\end{array}$ & $\begin{array}{c}\text { Depo. } \\
\left(\mathrm{m}^{2}\right)\end{array}$ & $\begin{array}{l}\text { Width } \\
\text { (m) }\end{array}$ & $\begin{array}{c}\text { Eros. } \\
\left(\mathrm{m}^{2}\right)\end{array}$ & $\begin{array}{c}\text { Depo. } \\
\left(\mathrm{m}^{2}\right)\end{array}$ & $\begin{array}{l}\text { Width } \\
\text { (m) }\end{array}$ & $\begin{array}{l}\text { Eros. } \\
\left(\mathrm{m}^{2}\right)\end{array}$ & $\begin{array}{c}\text { Depo. } \\
\left(\mathrm{m}^{2}\right)\end{array}$ & $\begin{array}{l}\text { Width } \\
\text { (m) }\end{array}$ & $\begin{array}{c}\text { Eros. } \\
\left(\mathbf{m}^{2}\right)\end{array}$ & $\begin{array}{c}\text { Depo. } \\
\left(\mathrm{m}^{2}\right)\end{array}$ & $\begin{array}{l}\text { Width } \\
\text { (m) }\end{array}$ & $\begin{array}{l}\text { Eros. } \\
\left(\mathrm{m}^{2}\right)\end{array}$ & $\begin{array}{c}\text { Depo. } \\
\left(\mathrm{m}^{2}\right)\end{array}$ \\
\hline \multicolumn{16}{|c|}{ PR165.6 } \\
\hline $\begin{array}{l}1980- \\
1985\end{array}$ & 5.5 & 0.3 & 0.1 & 14.0 & 0.1 & 3.3 & 65.5 & 3.9 & 14.6 & 0.0 & 0.0 & 0.0 & 85.0 & 4.3 & 18.0 \\
\hline $\begin{array}{l}1985- \\
1992\end{array}$ & 7.0 & 0.1 & 0.6 & 14.0 & 0.7 & 0.9 & 64.0 & 7.8 & 8.9 & 0.0 & 0.0 & 0.0 & 85.0 & 8.6 & 10.4 \\
\hline $\begin{array}{c}1992- \\
1998\end{array}$ & 7.0 & 0.5 & 0.5 & 14.0 & 1.5 & 1.5 & 64.0 & 5.8 & 3.8 & 0.0 & 0.0 & 0.0 & 85.0 & 7.8 & 5.8 \\
\hline Total & -- & 0.9 & 1.2 & - & 2.3 & 5.7 & - & 17.5 & 27.3 & -- & 0.0 & 0.0 & -- & 20.7 & 34.2 \\
\hline \multicolumn{16}{|c|}{$\begin{array}{l}\text { The channel aggraded. } \\
\text { PR166.0 }\end{array}$} \\
\hline $\begin{array}{l}1980- \\
1985\end{array}$ & 5.5 & 7.9 & 0.0 & 92.5 & 1.2 & 7.6 & 47.0 & 3.8 & 4.8 & 0.0 & 0.0 & 0.0 & 145.0 & 12.9 & 12.4 \\
\hline $\begin{array}{l}1985- \\
1992\end{array}$ & 6.0 & 11.9 & 0.0 & 86.5 & 0.4 & 7.4 & 52.5 & 2.6 & 2.2 & 0.0 & 0.0 & 0.0 & 145.0 & 14.9 & 9.6 \\
\hline $\begin{array}{l}1992- \\
1998\end{array}$ & 11.5 & 17.5 & 0.1 & 78.0 & 0.8 & 20.0 & 55.5 & 2.1 & 6.0 & 0.0 & 0.0 & 0.0 & 145.0 & 20.4 & 26.1 \\
\hline Total & - & 37.3 & 0.1 & - & 2.4 & 35.0 & -- & 8.5 & 13.0 & -- & 0.0 & 0.0 & -- & 48.2 & 48.1 \\
\hline
\end{tabular}

Sediment lost by $15 \mathrm{~m}$ of erosion of the left bank was balanced by deposition of the right bank flood plain and aggradation of the channel.

\begin{tabular}{|c|c|c|c|c|c|c|c|c|c|c|c|c|c|c|c|}
\hline $\begin{array}{l}1980- \\
1985\end{array}$ & 3.0 & 1.9 & 0.0 & 6.0 & 0.1 & 0.0 & 55.0 & 0.6 & 3.8 & 29.0 & 0.1 & 5.1 & 93.0 & 2.7 & 8.9 \\
\hline $\begin{array}{l}1985- \\
1992\end{array}$ & 1.5 & 0.9 & 0.0 & \multicolumn{3}{|c|}{$\begin{array}{l}\text { dirt pile from ramp } \\
\text { excavation }\end{array}$} & 353.5 & 4.3 & 8.5 & 31.5 & 2.0 & 4.0 & 86.5 & 7.2 & 12.5 \\
\hline $\begin{array}{l}1992- \\
1998\end{array}$ & 1.5 & 0.8 & 0.0 & \multicolumn{3}{|c|}{$\begin{array}{l}\text { dirt pile from ramp } \\
\text { excavating }\end{array}$} & 54.0 & 9.5 & 6.9 & 31.0 & 0.8 & 6.0 & 86.5 & 11.1 & 12.9 \\
\hline Total & - & 3.6 & 0.0 & - & 0.1 & 0.0 & -- & 14.4 & 19.2 & -- & 2.9 & 15.1 & -- & 21.0 & 34.3 \\
\hline \multicolumn{16}{|c|}{$\begin{array}{l}\text { Right bank eroded and the point bar aggraded } \\
\text { PR167 }\end{array}$} \\
\hline 1989 & 4.0 & 0.1 & 0.0 & 56.5 & 0.6 & 0.5 & 54.5 & 3.7 & 8.2 & 0.0 & 0.0 & 0.0 & 115.0 & 4.4 & 8.7 \\
\hline 1990 & 4.0 & 0.2 & 0.0 & 55.5 & 0.4 & 0.5 & 13.5 & 3.6 & 0.0 & 42.0 & 0.0 & 20.8 & 115.0 & 4.2 & 21.3 \\
\hline 1991 & 4.0 & 0.4 & 0.1 & 12.5 & 0.1 & 0.1 & 33.5 & 5.2 & 1.6 & 65.0 & 9.9 & 1.0 & 115.0 & 15.6 & 2.8 \\
\hline 1992 & 4.5 & 0.4 & 0.1 & 58.5 & 0.5 & 0.5 & 51.0 & 5.2 & 3.2 & 1.0 & 0.1 & 0.0 & 115.0 & 6.2 & 3.8 \\
\hline 1993 & 4.5 & 0.1 & 0.1 & 61.0 & 0.5 & 3.6 & 49.5 & 3.2 & 7.4 & 0.0 & 0.0 & 0.0 & 115.0 & 3.8 & 11.1 \\
\hline 1994 & 3.0 & 3.1 & 0.0 & 54.5 & 0.7 & 0.7 & 57.5 & 2.8 & 8.6 & 0.0 & 0.0 & 0.0 & 115.0 & 6.6 & 9.3 \\
\hline 1995 & 8.5 & 10.5 & 0.0 & 62.0 & 0.2 & 14.6 & 44.5 & 6.6 & 4.3 & 0.0 & 0.0 & 0.0 & 115.0 & 17.3 & 18.9 \\
\hline 1996 & 6.5 & 3.2 & 0.0 & 65.0 & 0.3 & 4.0 & 43.5 & 4.7 & 2.5 & 0.0 & 0.0 & 0.0 & 115.0 & 8.2 & 6.5 \\
\hline 1997 & 4.5 & 0.4 & 0.1 & 66.0 & 1.7 & 0.5 & 44.5 & 3.2 & 1.9 & 0.0 & 0.0 & 0.0 & 115.0 & 5.3 & 2.5 \\
\hline 1998 & 5.5 & 0.2 & 0.4 & 64.5 & 0.9 & 0.8 & 45.0 & 6.5 & 3.2 & 0.0 & 0.0 & 0.0 & 115.0 & 7.6 & 4.4 \\
\hline Total & -- & 18.6 & 0.8 & - & 5.9 & 25.8 & -- & 44.7 & 40.9 & -- & 10.0 & 21.8 & - & 79.2 & 89.3 \\
\hline \multicolumn{16}{|c|}{ Point bar appeared but became one of those point-bar flood plains with vertical rather than sloping bank } \\
\hline $\begin{array}{l}1980- \\
1985\end{array}$ & 12.0 & 0.8 & 0.8 & 0.0 & 0.0 & 0.0 & 218.0 & 9.0 & 11.6 & 0.0 & 0.0 & 0.0 & 230.0 & 9.8 & 12.4 \\
\hline $\begin{array}{l}1985- \\
1992\end{array}$ & 13.0 & 0.7 & 1.3 & 0.0 & 0.0 & 0.0 & 48.0 & 1.9 & 3.4 & 0.0 & 0.0 & 0.0 & 61.0 & -- & 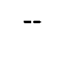 \\
\hline
\end{tabular}

Island section had mostly deposition in a secondary channel. Did not survey from station 149 to 259 in 1992 . Did not survey in 1998. 
Table 6. (Continued) Erosion and deposition of sediment at channel cross sections on the Powder River

\begin{tabular}{|c|c|c|c|c|c|c|c|c|c|c|c|c|c|c|c|}
\hline \multirow{2}{*}{$\begin{array}{l}\text { Water } \\
\text { Year }\end{array}$} & \multicolumn{3}{|c|}{ Bank } & \multicolumn{3}{|c|}{ Flood plain } & \multicolumn{3}{|c|}{ Channel } & \multicolumn{3}{|c|}{ Point bar } & \multicolumn{3}{|c|}{ Total } \\
\hline & $\begin{array}{c}\text { Width } \\
\text { (m) }\end{array}$ & $\begin{array}{l}\text { Eros. } \\
\left(\mathrm{m}^{2}\right)\end{array}$ & $\begin{array}{c}\text { Depo. } \\
\left(\mathrm{m}^{2}\right)\end{array}$ & $\begin{array}{l}\text { Width } \\
\text { (m) }\end{array}$ & $\begin{array}{l}\text { Eros. } \\
\left(\mathrm{m}^{2}\right)\end{array}$ & $\begin{array}{c}\text { Depo. } \\
\left(\mathrm{m}^{2}\right)\end{array}$ & $\begin{array}{c}\text { Width } \\
(\mathbf{m})\end{array}$ & $\begin{array}{l}\text { Eros. } \\
\left(\mathrm{m}^{2}\right)\end{array}$ & $\begin{array}{c}\text { Depo. } \\
\left(\mathrm{m}^{2}\right)\end{array}$ & $\begin{array}{c}\text { Width } \\
\text { (m) }\end{array}$ & $\begin{array}{l}\text { Eros. } \\
\left(\mathrm{m}^{2}\right)\end{array}$ & $\begin{array}{c}\text { Depo. } \\
\left(\mathrm{m}^{2}\right)\end{array}$ & $\begin{array}{c}\text { Width } \\
(\mathbf{m})\end{array}$ & $\begin{array}{c}\text { Eros. } \\
\left(\mathrm{m}^{2}\right)\end{array}$ & $\begin{array}{c}\text { Depo. } \\
\left(\mathrm{m}^{2}\right)\end{array}$ \\
\hline \multicolumn{16}{|c|}{ PR168.5 } \\
\hline $\begin{array}{c}1980- \\
1985\end{array}$ & 5.0 & 4.1 & 0.0 & 0.0 & 0.0 & 0.0 & 28.0 & 0.6 & 4.4 & 61.0 & 0.9 & 5.3 & 94.0 & 5.6 & 9.7 \\
\hline $\begin{array}{l}1985- \\
1992\end{array}$ & 8.5 & 9.9 & 0.0 & 0.0 & 0.0 & 0.0 & 23.5 & 1.7 & 2.9 & 66.5 & 0.1 & 17.5 & 98.5 & 11.7 & 20.4 \\
\hline $\begin{array}{l}1992- \\
1998\end{array}$ & 17.0 & 33.8 & 0.0 & 0.0 & 0.0 & 0.0 & 15.0 & 0.0 & 5.9 & 77.0 & 0.1 & 37.5 & 109.0 & 33.9 & 43.4 \\
\hline Total & -- & 47.8 & 0.0 & 0.0 & 0.0 & 0.0 & -- & 2.3 & 13.2 & -- & 1.1 & 60.3 & - & 51.2 & 73.5 \\
\hline \multicolumn{16}{|c|}{ Left bank retreated about $15 \mathrm{~m}$ and right bank point bar advanced and aggraded. } \\
\hline $\begin{array}{l}1980- \\
1985\end{array}$ & 4.5 & 2.9 & 0.0 & 0.0 & 0.0 & 0.0 & 59.0 & 1.7 & 6.7 & 32.5 & 0.3 & 1.6 & 96.0 & 4.9 & 8.3 \\
\hline $\begin{array}{l}1985- \\
1992\end{array}$ & 4.5 & 4.0 & 0.0 & 40.5 & 0.1 & 5.2 & 52.0 & 2.6 & 2.1 & 0.0 & 0.0 & 0.0 & 97.0 & 6.7 & 7.3 \\
\hline $\begin{array}{l}1992- \\
1998\end{array}$ & 7.5 & 5.7 & 0.0 & 42.0 & 0.6 & 5.8 & 49.0 & 4.2 & 1.9 & 0.0 & 0.0 & 0.0 & 98.5 & 10.5 & 7.7 \\
\hline Total & - & 12.6 & 0.0 & -- & 0.7 & 11.0 & -- & 8.5 & 10.7 & -- & 0.3 & 1.6 & -- & 22.1 & 23.3 \\
\hline \multicolumn{16}{|c|}{ The right bank eroded about $4 \mathrm{~m}$ and sediment was deposited on the point bar in the form of a scroll bar or bench } \\
\hline \multicolumn{16}{|c|}{ PR169.8 } \\
\hline $\begin{array}{r}1980- \\
1985\end{array}$ & 6.0 & 1.5 & 0.0 & $(51.0)$ & 0.0 & 4.4 & 51.0 & 4.6 & 0.0 & 33.0 & 0.4 & 0.6 & 90.0 & 6.5 & 5.0 \\
\hline $\begin{array}{l}1985- \\
1992\end{array}$ & 7.0 & 5.1 & 0.0 & $(48.5)$ & 0.0 & 2.8 & 48.5 & 3.1 & 0.0 & 35.5 & 0.0 & 2.2 & 91.0 & 8.2 & 5.0 \\
\hline $\begin{array}{l}1992- \\
1998\end{array}$ & 9.0 & 13.0 & 0.0 & $(51.5)$ & 0.0 & 1.9 & 51.5 & 9.6 & 0.0 & 35.5 & 1.2 & 1.1 & 96.0 & 23.8 & 3.0 \\
\hline Total & -- & 19.6 & 0.0 & - & 0.0 & 9.1 & -- & 17.3 & 0.0 & -- & 1.6 & 3.9 & - & 38.5 & 13.0 \\
\hline
\end{tabular}

Widths in the () represent the same location as widths in the channel but sediment was deposited on a low floodplain rather than in the channel. PR170.5

\begin{tabular}{|c|c|c|c|c|c|c|c|c|c|c|c|c|c|c|c|}
\hline $\begin{array}{c}1980 \\
1985\end{array}$ & 5.5 & 3.2 & 0.0 & 9.5 & 0.0 & 2.0 & 68.0 & 4.9 & 8.8 & 0.0 & 0.0 & 0.0 & 83.0 & 8.1 & 10.8 \\
\hline $\begin{array}{l}1985- \\
1992\end{array}$ & 5.0 & 2.2 & 0.0 & 9.5 & 0.0 & 1.9 & 69.5 & 9.1 & 0.9 & 0.0 & 0.0 & 0.0 & 84.0 & 11.3 & 2.8 \\
\hline $\begin{array}{c}1992- \\
1998\end{array}$ & 5.0 & 4.1 & 0.0 & 9.5 & 0.0 & 1.6 & 71.0 & 13.2 & 0.4 & 0.0 & 0.0 & 0.0 & 85.5 & 17.3 & 2.0 \\
\hline Total & - & 9.5 & 0.0 & -- & 0.0 & 5.5 & -- & 27.2 & 10.1 & -- & 0.0 & 0.0 & -. & 36.7 & 15.6 \\
\hline \multicolumn{16}{|c|}{ Right bank eroded while a small bench was deposited on the left bank } \\
\hline \multicolumn{16}{|c|}{ PR175 } \\
\hline 1989 & 21.5 & 0.3 & 0.3 & 0.0 & 0.0 & 0.0 & 55.0 & 9.7 & 0.8 & 43.5 & 0.5 & 2.5 & 120.0 & 10.5 & 3.6 \\
\hline 1990 & 21.5 & 0.1 & 0.5 & 0.0 & 0.0 & 0.0 & 57.0 & 1.9 & 6.3 & 41.5 & 0.1 & 6.5 & 120.0 & 2.1 & 13.3 \\
\hline 1991 & 22.0 & 0.2 & 0.7 & 0.0 & 0.0 & 0.0 & 55.0 & 1.3 & 7.2 & 43.0 & 11.5 & 2.1 & 120.0 & 13.0 & 10.0 \\
\hline 1992 & 21.0 & 0.1 & 0.5 & 0.0 & 0.0 & 0.0 & 70.0 & 3.1 & 5.6 & 29.0 & 0.5 & 1.0 & 120.0 & 3.7 & 7.1 \\
\hline 1993 & 28.0 & 0.2 & 4.0 & 0.0 & 0.0 & 0.0 & 54.5 & 5.1 & 2.3 & 37.5 & 0.0 & 8.4 & 120.0 & 5.3 & 14.7 \\
\hline 1994 & 20.0 & 0.2 & 0.3 & 0.0 & 0.0 & 0.0 & 62.5 & 8.8 & 3.8 & 37.5 & 0.0 & 3.8 & 120.0 & 9.0 & 7.9 \\
\hline 1995 & 20.0 & 0.0 & 2.4 & 0.0 & 0.0 & 0.0 & 73.0 & 6.5 & 19.6 & 27.0 & 0.0 & 7.9 & 120.0 & 6.5 & 29.9 \\
\hline 1996 & 20.0 & 0.2 & 0.7 & 0.0 & 0.0 & 0.0 & 73.0 & 2.3 & 9.0 & 27.0 & 0.7 & 1.3 & 120.0 & 3.2 & 11.0 \\
\hline 1997 & 20.0 & 0.3 & 0.2 & 0.0 & 0.0 & 0.0 & 73.0 & 14.2 & 18.1 & 27.0 & 0.3 & 1.4 & 120.0 & 14.8 & 19.7 \\
\hline 1998 & 22.0 & 2.3 & 0.1 & 0.0 & 0.0 & 0.0 & 60.5 & 2.0 & 3.5 & 37.5 & 0.4 & 1.6 & 120.0 & 4.7 & 5.2 \\
\hline Total & -- & 3.9 & 9.7 & -- & 0.0 & 0.0 & -. & 54.9 & 76.2 & -- & 14.0 & 36.5 & -- & 72.8 & 122.4 \\
\hline
\end{tabular}

Left bank retreated a little and the point bar grew episodically by vertical accretion in different locations at different times. 
Table 6. (Continued) Erosion and deposition of sediment at channel cross sections on the Powder River

\begin{tabular}{|c|c|c|c|c|c|c|c|c|c|c|c|c|c|c|c|}
\hline \multirow{2}{*}{$\begin{array}{l}\text { Water } \\
\text { Year }\end{array}$} & \multicolumn{3}{|c|}{ Bank } & \multicolumn{3}{|c|}{ Flood plain } & \multicolumn{3}{|c|}{ Channel } & \multicolumn{3}{|c|}{ Point bar } & \multicolumn{3}{|c|}{ Total } \\
\hline & $\begin{array}{c}\text { Width } \\
(\mathrm{m})\end{array}$ & $\begin{array}{l}\text { Eros. } \\
\left(\mathrm{m}^{2}\right)\end{array}$ & $\begin{array}{c}\text { Depo. } \\
\left(\mathrm{m}^{2}\right)\end{array}$ & $\begin{array}{c}\text { Width } \\
\text { (m) }\end{array}$ & $\begin{array}{l}\text { Eros. } \\
\left(\mathrm{m}^{2}\right)\end{array}$ & $\begin{array}{c}\text { Depo. } \\
\left(\mathrm{m}^{2}\right)\end{array}$ & $\begin{array}{c}\text { Width } \\
\text { (m) }\end{array}$ & $\begin{array}{l}\text { Eros. } \\
\left(\mathrm{m}^{2}\right)\end{array}$ & $\begin{array}{c}\text { Depo. } \\
\left(\mathrm{m}^{2}\right)\end{array}$ & $\begin{array}{c}\text { Width } \\
(\mathrm{m})\end{array}$ & $\begin{array}{l}\text { Eros. } \\
\left(\mathrm{m}^{2}\right)\end{array}$ & $\begin{array}{c}\text { Depo. } \\
\left(\mathrm{m}^{2}\right)\end{array}$ & $\begin{array}{c}\text { Width } \\
(\mathrm{m})\end{array}$ & $\begin{array}{c}\text { Eros. } \\
\left(\mathrm{m}^{2}\right)\end{array}$ & $\begin{array}{c}\text { Depo. } \\
\left(\mathrm{m}^{2}\right)\end{array}$ \\
\hline \multicolumn{16}{|c|}{ PR180 } \\
\hline 1989 & 8.0 & 2.2 & 0.0 & 0.0 & 0.0 & 0.0 & 22.0 & 1.4 & 1.1 & 58.0 & 0.7 & 3.6 & 88.0 & 4.3 & 4.7 \\
\hline 1990 & 13.0 & 6.8 & 0.0 & 0.0 & 0.0 & 0.0 & 17.5 & 0.6 & 1.4 & 57.5 & 0.6 & 4.4 & 88.0 & 8.0 & 5.8 \\
\hline 1991 & 15.0 & 29.0 & 3.8 & 0.0 & 0.0 & 0.0 & 33.0 & 0.0 & 7.8 & 50.0 & 0.5 & 4.1 & 98.0 & 29.5 & 15.7 \\
\hline 1992 & 9.0 & 2.3 & 0.1 & 0.0 & 0.0 & 0.0 & 39.0 & 0.5 & 4.8 & 50.0 & 0.1 & 2.5 & 98.0 & 2.9 & 7.4 \\
\hline 1993 & 14.0 & 15.5 & 0.0 & 0.0 & 0.0 & 0.0 & 24.0 & 0.3 & 3.1 & 65.0 & 0.7 & 7.6 & 103.0 & 16.5 & 10.7 \\
\hline 1994 & 6.5 & 0.8 & 0.1 & 0.0 & 0.0 & 0.0 & 31.5 & 2.7 & 0.8 & 65.0 & 1.3 & 2.1 & 103.0 & 4.8 & 3.0 \\
\hline 1995 & 11.0 & 14.1 & 0.0 & 0.0 & 0.0 & 0.0 & 29.0 & 0.9 & 5.7 & 68.0 & 0.0 & 16.9 & 108.0 & 15.0 & 22.6 \\
\hline 1996 & 10.0 & 8.9 & 0.0 & 0.0 & 0.0 & 0.0 & 33.0 & 3.9 & 3.7 & 70.0 & 1.8 & 1.9 & 113.0 & 14.6 & 5.6 \\
\hline 1997 & 7.0 & 7.9 & 0.0 & 0.0 & 0.0 & 0.0 & 36.0 & 3.6 & 3.2 & 70.0 & 1.0 & 1.7 & 113.0 & 12.5 & 4.9 \\
\hline 1998 & 6.5 & 2.3 & 0.0 & 0.0 & 0.0 & 0.0 & 33.5 & 2.0 & 1.2 & 73.0 & 0.1 & 3.2 & 113.0 & 4.4 & 4.4 \\
\hline Total & -- & 89.8 & 4.0 & -- & 0.0 & 0.0 & - & 15.9 & 32.8 & - & 6.8 & 48.0 & - & 112.5 & 84.8 \\
\hline \multicolumn{16}{|c|}{ Left bank retreated about $30 \mathrm{~m}$ and point bar advanced } \\
\hline \multicolumn{16}{|c|}{ PR183 } \\
\hline 1989 & 11.0 & 0.4 & 0.1 & 30.0 & 0.4 & 0.6 & 39.0 & 0.5 & 4.0 & 0.0 & 0.0 & 0.0 & 80.0 & 1.3 & 4.7 \\
\hline 1990 & 11.0 & 0.1 & 0.4 & 30.0 & 0.1 & 2.0 & 39.0 & 4.0 & 0.2 & 0.0 & 0.0 & 0.0 & 80.0 & 4.2 & 2.6 \\
\hline 1991 & 11.5 & 0.4 & 0.1 & 30.0 & 0.5 & 0.9 & 38.5 & 0.7 & 1.8 & 0.0 & 0.0 & 0.0 & 80.0 & 1.6 & 2.8 \\
\hline 1992 & 11.0 & 0.1 & 0.2 & 30.0 & 0.1 & 0.5 & 39.0 & 1.7 & 1.9 & 0.0 & 0.0 & 0.0 & 80.0 & 1.9 & 2.6 \\
\hline 1993 & 11.0 & 0.7 & 0.1 & 30.0 & 0.2 & 2.5 & 39.0 & 4.4 & 1.8 & 0.0 & 0.0 & 0.0 & 80.0 & 5.3 & 4.4 \\
\hline 1994 & 9.5 & 0.7 & 0.1 & 30.0 & 0.2 & 0.4 & 40.5 & 0.2 & 3.1 & 0.0 & 0.0 & 0.0 & 80.0 & 1.1 & 3.6 \\
\hline 1995 & 9.5 & 1.3 & 0.4 & 30.0 & 0.4 & 4.6 & 40.5 & 4.3 & 2.7 & 0.0 & 0.0 & 0.0 & 80.0 & 6.0 & 7.7 \\
\hline 1996 & 8.0 & 0.4 & 0.0 & 30.0 & 0.4 & 0.2 & 42.0 & 2.5 & 4.2 & 0.0 & 0.0 & 0.0 & 80.0 & 3.3 & 4.4 \\
\hline 1997 & 9.5 & 0.3 & 0.1 & 30.0 & 0.4 & 0.2 & 40.5 & 4.2 & 2.8 & 0.0 & 0.0 & 0.0 & 80.0 & 4.9 & 3.1 \\
\hline 1998 & 8.0 & 0.2 & 0.1 & 30.0 & 0.3 & 0.8 & 42.0 & 5.0 & 1.3 & 0.0 & 0.0 & 0.0 & 80.0 & 5.5 & 2.2 \\
\hline Total & -- & 4.6 & 1.6 & - & 3.0 & 12.7 & -- & 27.5 & 23.8 & - & 0.0 & 0.0 & -- & 35.1 & 38.1 \\
\hline \multicolumn{16}{|c|}{ Vertical accretion on the left bank flood plain. } \\
\hline 1989 & 13.0 & 0.2 & 0.3 & 79.0 & 0.5 & 0.3 & 33.0 & 0.1 & 2.4 & 0.0 & 0.0 & 0.0 & 125.0 & 0.8 & 3.0 \\
\hline 1990 & 10.5 & 0.6 & 0.2 & 79.0 & 0.0 & 1.2 & 35.5 & 7.9 & 0.0 & 0.0 & 0.0 & 0.0 & 125.0 & 8.5 & 1.4 \\
\hline 1991 & 11.5 & 0.7 & 0.4 & 89.0 & 0.6 & 0.3 & 34.5 & 0.1 & 7.2 & 0.0 & 0.0 & 0.0 & 135.0 & 1.4 & 7.9 \\
\hline 1992 & 12.0 & 0.2 & 0.2 & 88.5 & 0.6 & 0.6 & 34.5 & 0.6 & 0.5 & 0.0 & 0.0 & 0.0 & 135.0 & 1.4 & 1.3 \\
\hline 1993 & 12.0 & 2.0 & 0.4 & 88.5 & 0.1 & 8.9 & 34.5 & 1.5 & 2.4 & 0.0 & 0.0 & 0.0 & 135.0 & 3.6 & 11.7 \\
\hline 1994 & 9.0 & 0.2 & 0.3 & 89.0 & 1.7 & 0.7 & 37.0 & 5.6 & 0.2 & 0.0 & 0.0 & 0.0 & 135.0 & 7.5 & 1.2 \\
\hline 1995 & 7.5 & 2.5 & 0.2 & 90.5 & 0.1 & 15.5 & 37.0 & 0.1 & 8.9 & 0.0 & 0.0 & 0.0 & 135.0 & 2.7 & 24.6 \\
\hline 1996 & 12.0 & 2.2 & 0.1 & 83.5 & 0.6 & 1.4 & 39.5 & 1.7 & 1.3 & 0.0 & 0.0 & 0.0 & 135.0 & 4.5 & 2.8 \\
\hline 1997 & 10.0 & 1.0 & 0.3 & 85.5 & 0.2 & 1.0 & 39.5 & 5.0 & 2.6 & 0.0 & 0.0 & 0.0 & 135.0 & 6.2 & 3.9 \\
\hline 1998 & 10.0 & 0.9 & 0.1 & 85.0 & 0.9 & 0.6 & 40.0 & 4.4 & 0.1 & 0.0 & 0.0 & 0.0 & 135.0 & 6.2 & 0.8 \\
\hline Total & -- & 10.5 & 2.5 & -- & 5.3 & 30.5 & -- & 27.0 & 25.6 & -- & 0.0 & 0.0 & & 42.8 & 58.6 \\
\hline
\end{tabular}

Vertical accretion on both the left and right bank flood plains. 
Table 6. (Continued) Erosion and deposition of sediment at channel cross sections on the Powder River

\begin{tabular}{|c|c|c|c|c|c|c|c|c|c|c|c|c|c|c|c|}
\hline \multirow{2}{*}{$\begin{array}{l}\text { Water } \\
\text { Year }\end{array}$} & \multicolumn{3}{|c|}{ Bank } & \multicolumn{3}{|c|}{ Flood plain } & \multicolumn{3}{|c|}{ Channel } & \multicolumn{3}{|c|}{ Point bar } & \multicolumn{3}{|c|}{ Total } \\
\hline & $\begin{array}{l}\text { Width } \\
\text { (m) }\end{array}$ & $\begin{array}{l}\text { Eros. } \\
\left(\mathrm{m}^{2}\right)\end{array}$ & $\begin{array}{l}\text { Depo. } \\
\left(m^{2}\right)\end{array}$ & $\begin{array}{l}\text { Width } \\
\text { (m) }\end{array}$ & $\begin{array}{l}\text { Eros. } \\
\left(\mathrm{m}^{2}\right)\end{array}$ & $\begin{array}{l}\text { Depo. } \\
\left(\mathrm{m}^{2}\right)\end{array}$ & $\begin{array}{l}\text { Width } \\
\text { (m) }\end{array}$ & $\begin{array}{l}\text { Eros. } \\
\left(m^{2}\right)\end{array}$ & $\begin{array}{l}\text { Depo. } \\
\left(\mathrm{m}^{2}\right)\end{array}$ & $\begin{array}{l}\text { Width } \\
\text { (m) }\end{array}$ & $\begin{array}{l}\text { Eros. } \\
\left(\mathrm{m}^{2}\right)\end{array}$ & $\begin{array}{l}\text { Depo. } \\
\left(\mathrm{m}^{2}\right)\end{array}$ & $\begin{array}{l}\text { Width } \\
\text { (m) }\end{array}$ & $\begin{array}{l}\text { Eros. } \\
\left(\mathrm{m}^{2}\right)\end{array}$ & $\begin{array}{l}\text { Depo. } \\
\left(\mathrm{m}^{2}\right)\end{array}$ \\
\hline \multicolumn{16}{|c|}{ PR194 } \\
\hline 1989 & 14.0 & 0.1 & 1.1 & 38.5 & 0.1 & 0.2 & 52.5 & 3.7 & 3.7 & 0.0 & 0.0 & 0.0 & 105.0 & 3.9 & 5.0 \\
\hline 1990 & 14.0 & 0.7 & 1.5 & 38.5 & 0.1 & 0.4 & 52.5 & 9.0 & 1.6 & 0.0 & 0.0 & 0.0 & 105.0 & 9.8 & 3.5 \\
\hline 1991 & 14.5 & 1.4 & 2.7 & 38.0 & 0.5 & 0.1 & 52.5 & 0.3 & 1.9 & 0.0 & 0.0 & 0.0 & 105.0 & 2.2 & 4.7 \\
\hline 1992 & 15.5 & 0.0 & 1.9 & 37.5 & 0.2 & 0.6 & 52.0 & 2.3 & 2.9 & 0.0 & 0.0 & 0.0 & 105.0 & 2.5 & 5.4 \\
\hline 1993 & 13.5 & 0.9 & 0.7 & 39.5 & 0.1 & 1.2 & 52.0 & 3.3 & 1.2 & 0.0 & 0.0 & 0.0 & 105.0 & 4.3 & 3.1 \\
\hline 1994 & 14.5 & 0.7 & 1.0 & 39.0 & 0.2 & 0.6 & 51.5 & 1.8 & 1.4 & 0.0 & 0.0 & 0.0 & 105.0 & 2.7 & 3.0 \\
\hline 1995 & 16.5 & 4.4 & 0.3 & 37.0 & 0.3 & 1.8 & 51.5 & 3.6 & 4.2 & 0.0 & 0.0 & 0.0 & 105.0 & 8.3 & 6.3 \\
\hline 1996 & 16.5 & 2.1 & 0.2 & 36.0 & 0.2 & 0.3 & 52.5 & 3.2 & 3.6 & 0.0 & 0.0 & 0.0 & 105.0 & 5.5 & 4.1 \\
\hline 1997 & 16.0 & 1.5 & 0.5 & 35.5 & 0.0 & 0.4 & 53.5 & 5.6 & 0.8 & 0.0 & 0.0 & 0.0 & 105.0 & 7.1 & 1.7 \\
\hline 1998 & 16.5 & 0.9 & 0.3 & 35.5 & 0.3 & 0.0 & 53.0 & 2.8 & 1.5 & 0.0 & 0.0 & 0.0 & 105.0 & 4.0 & 1.8 \\
\hline Total & -- & 12.7 & 10.2 & - & 2.0 & 5.6 & -- & 35.6 & 22.8 & -- & 0.0 & 0.0 & - & 50.3 & 38.6 \\
\hline \multicolumn{16}{|c|}{$\begin{array}{l}\text { Flood plain accretion on the left bank. } \\
\text { PR200A }\end{array}$} \\
\hline 1989 & 19.0 & 0.9 & 1.0 & 170.5 & 1.3 & 4.2 & 38.0 & 1.4 & 0.7 & 0.0 & 0.0 & 0.0 & 227.5 & 3.6 & 5.9 \\
\hline 1990 & 16.5 & 7.6 & 0.5 & 184.5 & 1.2 & 12.7 & 27.0 & 6.4 & 0.8 & 0.0 & 0.0 & 0.0 & 228.0 & 15.2 & 14.0 \\
\hline 1991 & 26.0 & 24.3 & 0.3 & 192.0 & 2.4 & 26.8 & 11.0 & 8.4 & 0.0 & 0.0 & 0.0 & 0.0 & 229.0 & 35.1 & 27.1 \\
\hline 1992 & 33.5 & 52.9 & 0.2 & 195.5 & 1.2 & 35.7 & 0.0 & 0.0 & 0.0 & 0.0 & 0.0 & 0.0 & 229.0 & 54.1 & 35.9 \\
\hline 1993 & 39.0 & 64.5 & 0.0 & 195.0 & 3.5 & 61.6 & 0.0 & 0.0 & 0.0 & 0.0 & 0.0 & 0.0 & 234.0 & 68.0 & 61.6 \\
\hline 1994 & 24.0 & 28.3 & 0.0 & 216.0 & 1.8 & 23.8 & 0.0 & 0.0 & 0.0 & 0.0 & 0.0 & 0.0 & 240.0 & 30.1 & 23.8 \\
\hline 1995 & 43.5 & 66.6 & 0.0 & 159.0 & 0.5 & 40.3 & 50.0 & 10.2 & 1.9 & 0.0 & 0.0 & 0.0 & 252.5 & 77.3 & 42.2 \\
\hline 1996 & 7.0 & 5.3 & 0.0 & 170.0 & 2.4 & 11.6 & 77.0 & 11.2 & 6.7 & 0.0 & 0.0 & 0.0 & 254.0 & 18.9 & 18.3 \\
\hline 1997 & 3.5 & 0.5 & 0.3 & 190.0 & 2.0 & 17.5 & 61.5 & 38.0 & 0.0 & 0.0 & 0.0 & 0.0 & 255.0 & 40.5 & 17.8 \\
\hline 1998 & 23.0 & 3.0 & 0.3 & 179.5 & 2.7 & 1.7 & 52.5 & 0.9 & 2.1 & 0.0 & 0.0 & 0.0 & 255.0 & 6.6 & 4.1 \\
\hline Total & -- & 253.9 & 2.6 & -- & 19.0 & 235.9 & - & 76.5 & 12.2 & -- & 0.0 & 0.0 & - & 349.4 & 250.7 \\
\hline
\end{tabular}

Left bank retreated. The channel along the left bank filled. The secondary channel shifted toward the left bank (this appears as bank erosion and no apparent channel activity, see 1992-1994 above) and evolved into the main channel with some human assistance.

\begin{tabular}{|c|c|c|c|c|c|c|c|c|c|c|c|c|c|c|c|}
\hline 1989 & 10.0 & 0.5 & 0.0 & 23.5 & 0.0 & 0.6 & 77.5 & 0.6 & 3.1 & 0.0 & 0.0 & 0.0 & 111.0 & 1.1 & 3.7 \\
\hline 1990 & 15.5 & 21.2 & 0.0 & 23.5 & 0.1 & 0.4 & 77.5 & 2.9 & 8.1 & 0.0 & 0.0 & 0.0 & 116.5 & 24.2 & 8.5 \\
\hline 1991 & 13.5 & 21.4 & 0.4 & 45.5 & 0.1 & 22.1 & 65.0 & 24.2 & 5.1 & 0.0 & 0.0 & 0.0 & 124.0 & 45.7 & 27.6 \\
\hline 1992 & 7.5 & 0.5 & 0.0 & 63.5 & 0.6 & 3.2 & 54.0 & 5.1 & 4.4 & 0.0 & 0.0 & 0.0 & 125.0 & 6.2 & 7.6 \\
\hline 1993 & 6.5 & 1.3 & 0.6 & 53.5 & 0.0 & 13.8 & 65.0 & 10.1 & 0.5 & 0.0 & 0.0 & 0.0 & 125.0 & 11.4 & 14.9 \\
\hline 1994 & 6.5 & 0.0 & 0.3 & 54.5 & 0.1 & 2.3 & 65.0 & 0.6 & 6.4 & 0.0 & 0.0 & 0.0 & 126.0 & 0.7 & 9.0 \\
\hline 1995 & 6.5 & 0.4 & 0.0 & 93.5 & 0.0 & 74.9 & 27.0 & 26.9 & 0.1 & 0.0 & 0.0 & 0.0 & 127.0 & 27.3 & 75.0 \\
\hline 1996 & 13.5 & 10.1 & 0.1 & 98.5 & 4.1 & 4.9 & 15.0 & 0.2 & 3.6 & 0.0 & 0.0 & 0.0 & 127.0 & 14.4 & 8.6 \\
\hline 1997 & 15.5 & 16.4 & 0.2 & 92.0 & 3.5 & 9.0 & 19.5 & 0.0 & 5.2 & 0.0 & 0.0 & 0.0 & 127.0 & 19.9 & 14.4 \\
\hline 1998 & 9.5 & 3.3 & 0.3 & 93.5 & 1.8 & 4.4 & 24.0 & 4.7 & 0.0 & 0.0 & 0.0 & 0.0 & 127.0 & 9.8 & 4.7 \\
\hline Total & -- & 75.1 & .1 .9 & -- & 10.3 & 135.6 & -- & 75.3 & 36.5 & -- & 0.0 & 0.0 & - & 160.7 & 174.0 \\
\hline
\end{tabular}

Left bank retreated about $15 \mathrm{~m}$. A flood plain developed adjacent to the left bank and was larger than the flood plain that developed on the right bank. 
channel also shifted from the left bank to the right bank. The shift at PR206 primarily occurred in just two years (1991 and 1995) when 71.5 percent of the sediment was deposited. This contrasts with PR200A where the shift was more gradual and never exceeded 30 percent in any year.

\section{SUMMARY}

This report represents the second decade (1988-1998) of measurements of erosion and deposition at cross sections on Powder River following the major flood in 1978. Snowmelt floods carried the greatest maximum sediment concentration $(52,200 \mathrm{mg} / \mathrm{L})$, followed by flash floods $(49,700 \mathrm{mg} / \mathrm{L})$ and ice break-up floods $(26,000 \mathrm{mg} / \mathrm{L})$. The proportion of sand in the suspended sediment or eroded sediment also depended upon the type of flood (ice break-up, 28-percent; snowmelt, 23-percent; and flash, 7-percent). Sediment deposited on the flood-plain trough averaged 13 percent sand, while sediment deposited on the flood-plain crest averaged 72 percent sand. The finer-grained sediment sampled from the bed of the channel averaged 99 percent sand.

The minimum riverbed elevation decreased with time at 8 cross sections and increased with time at 15 cross sections. However, there was no consistent trend. The minimum riverbed elevation fluctuated from year to year as indicated by the standard deviation of the elevation, which ranged from 0.01 to $0.30 \mathrm{~m}$.

Likewise, the areas of erosion of sediment and areas of deposition of sediment fluctuated from year to year. At some specific cross sections, erosion of sediment exceed deposition of sediment by 47 percent and at the other sections, deposition exceeded erosion by 41 percent. However, in general in this study reach of Powder River, the sedimentary processes were in equilibrium such that erosion balanced deposition during the second decade after the 1978 flood. 


\section{REFERENCES CITED}

Guy, H. P., 1969, Laboratory theory and methods for sediment analysis: U.S. Geological Survey Techniques of Water-Resources Investigations, book 5, chap. C1, 58 p.

Hembree, C. H., Colby, B. R., Swenson, H. A., and Davis, J. R., 1952, Sedimentation and chemical quality of water in the Powder River drainage basin, Wyoming and Montana: U.S. Geological Survey Circular 170, 92 p.

Martinson, H. A., 1983, Channel changes of Powder River between Moorhead and Broadus, Montana, 1939 to 1978: U.S. Geological Survey Water-Resources Investigations Report 83-4128, 62 p.

Martinson, H. A. and Meade, R. H., 1983, Channel changes of Powder River, 1938-78, Powder River County, Montana: U.S. Geological Survey Hydrologic Investigations Atlas HA-661, scale 1:24,000, 3 sheets.

Moody, J. A. and Meade, R. H., 1990, Channel changes at cross sections of the Powder River between Moorhead and Broadus, Montana, 1975-88: U.S. Geological Survey Open-File Report 89-407, 252 p.

Moody, J. A., Pizzuto, J. E., and Meade, R. H., 1999, Ontogeny of a flood plain: Geological Society of America Bulletin, 111(2), 291-303.

Parrett, Charles, Carlson, D. D., Craig, G. S., Jr., and Chin, E. H., 1984, Floods of May 1978 in southeastern Montana and northeastern Wyoming: U.S. Geological Survey Professional Paper 1244, 74 p.

Pizzuto, J. E., 1994, Channel adjustments to changing discharges, Powder River, Montana: Geological Society of America Bulletin, 106, 1494-1501.

U.S. Geological Survey, 1975-1998, Water resources data for Montana, water years 197598--volume 1; U.S. geological Survey Water-Data Reports, MT-75-1 to MT-98-1 (published annually) 


\section{CROSS-SECTION DATA}




\section{Description of Cross Section PR113}

Location: Township 9 South/Range 48 East--section 18

U. S. Geological Survey quadrangle (1:24,000): Bradshaw Creek

Landowners--left bank: Lloyd Sams (Moorhead Cattle Company)

--right bank: U. S. Government

Access: Right bank

Permission from: None necessary

Distance from Moorhead Gaging Station: -2.23 kilometers

Azimuth of Section (degrees magnetic): 171.5

\section{Reference Monuments}

[Monuments at stations $100.0,100.4$, and 114.7 were closest to the leveling instrument]

\begin{tabular}{|c|c|c|c|c|c|c|}
\hline \multirow[b]{2}{*}{ Description } & \multirow[b]{2}{*}{$\begin{array}{l}\text { Station } \\
\text { (m) }\end{array}$} & \multicolumn{2}{|c|}{ GPS-NAD83(1992) } & \multicolumn{2}{|c|}{ Measurement } & \multirow{2}{*}{$\begin{array}{l}\text { Elevation } \\
\text { (NGVD1929 } \\
\text { (m) }\end{array}$} \\
\hline & & Latitude & Longitude & $\begin{array}{l}\text { Standard } \\
\text { deviation } \\
\text { (m) }\end{array}$ & $\begin{array}{l}\text { Horizontal } \\
\text { precision } \\
(\mathrm{m})\end{array}$ & \\
\hline $\begin{array}{l}\text { 1/2-inch-rebar; } 0.14 \text { meter above } 1998 \\
\text { ground level; under 3-strand barbed- } \\
\text { wire fence with all wooden posts }\end{array}$ & -83.9 & $45^{\circ} 03^{\prime} 28.78^{\prime \prime}$ & $105^{\circ} 53^{\prime} 02.22^{\prime \prime}$ & 0.225 & 0.496 & 1024.02 \\
\hline $\begin{array}{l}\text { 1/2-inch-rebar; } 0.16 \mathrm{~m} \text { above } 1998 \text { ground } \\
\text { level }\end{array}$ & -60.0 & & & & & 1023.05 \\
\hline $\begin{array}{l}\text { 1/2-inch-rebar; } 0.17 \text { meter above } 1998 \\
\text { ground level }\end{array}$ & 70.0 & & & & & 1022.08 \\
\hline $\begin{array}{l}\text { 1/2-inch-rebar; } 0.04 \text { meter below } 1998 \\
\text { ground level }\end{array}$ & 100.0 & & & & & 1023.02 \\
\hline $\begin{array}{l}\text { 1/2-inch-rebar; } 0.06 \text { meter above } 1998 \\
\text { ground level }\end{array}$ & 100.4 & & & & & 1023.18 \\
\hline $\begin{array}{l}\text { 1/2-inch-rebar; } 0.12 \text { meter above } 1998 \\
\text { ground level; under 3-strand, barbed- } \\
\text { wire fence with all metal posts }\end{array}$ & 114.7 & & & & & 1023.57 \\
\hline Benchmark--brass circular plate & 142.0 & $45^{\circ} 03^{\prime} 21.49^{\prime \prime}$ & $105^{\circ} 53^{\prime} 03.31^{\prime \prime}$ & 0.412 & 0.432 & 1023.34 \\
\hline
\end{tabular}




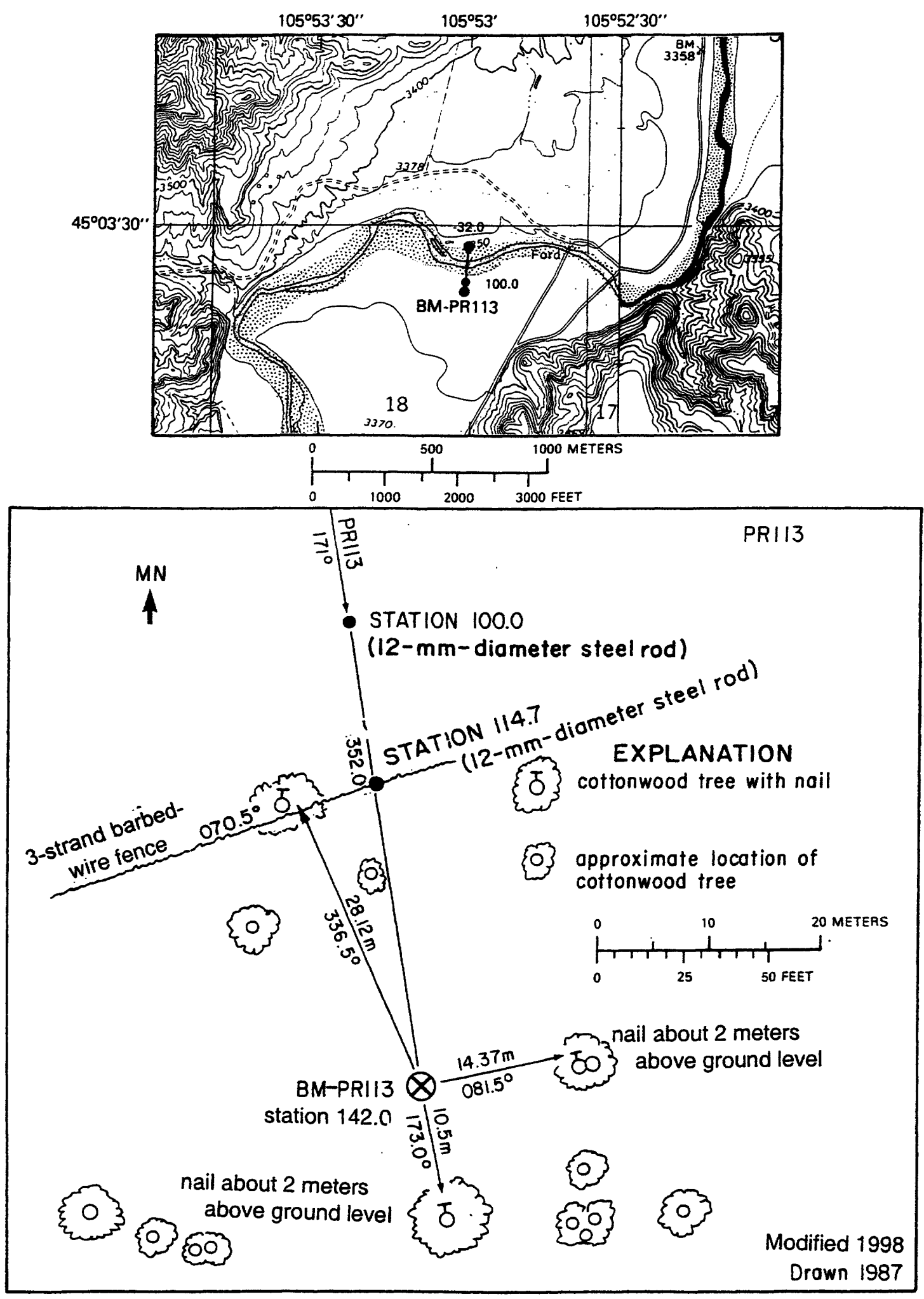

Figure 9. Upper: Location of cross section PR113, bench mark BM-PR113, and left and right bank reference monuments in the Bradshaw Creek quadrangle. Lower: Location of the bench mark on the right bank. MN is magnetic north. 

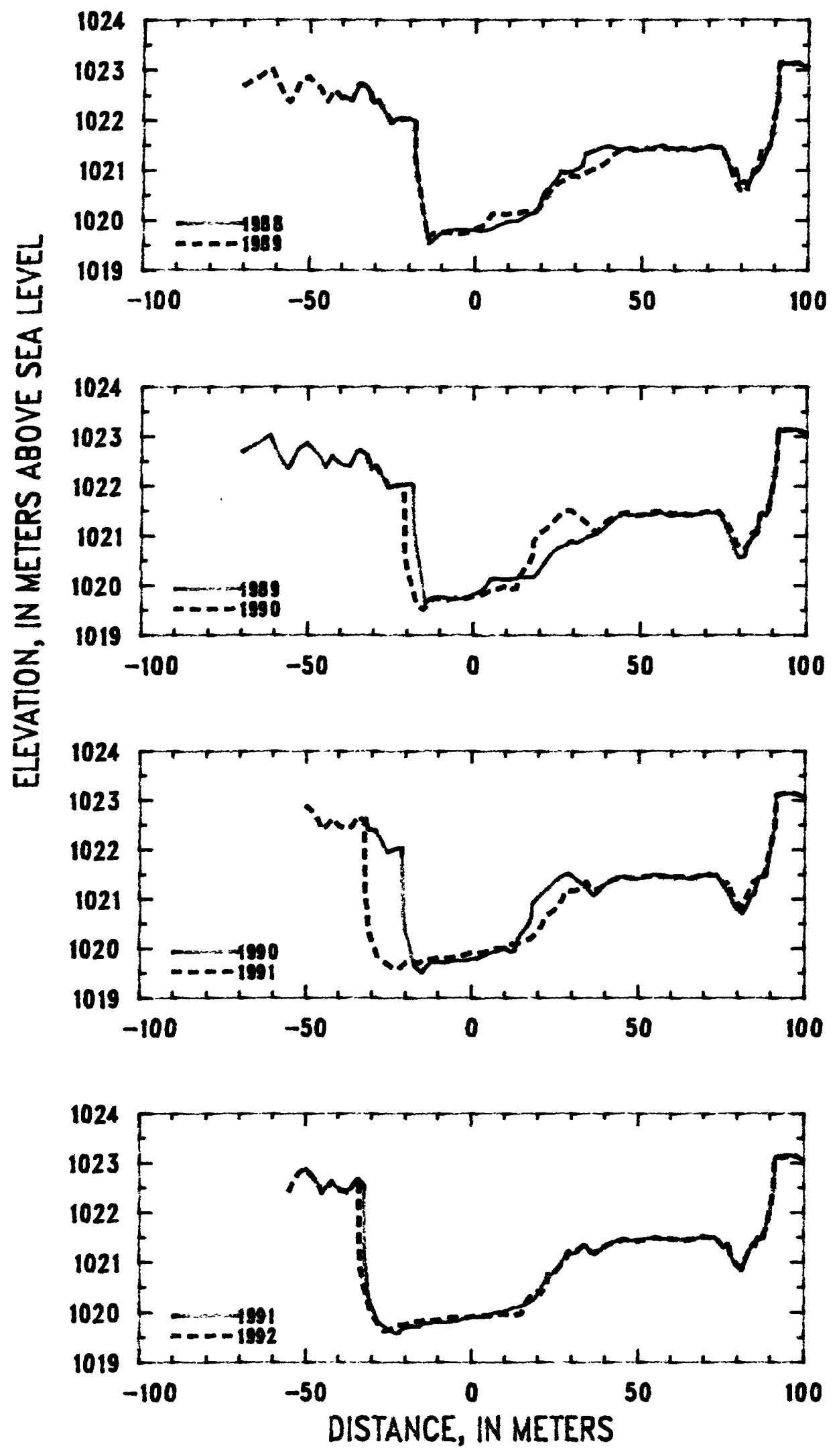

Figure 10. Profiles of cross section PR113 from 1988 to 1992. 

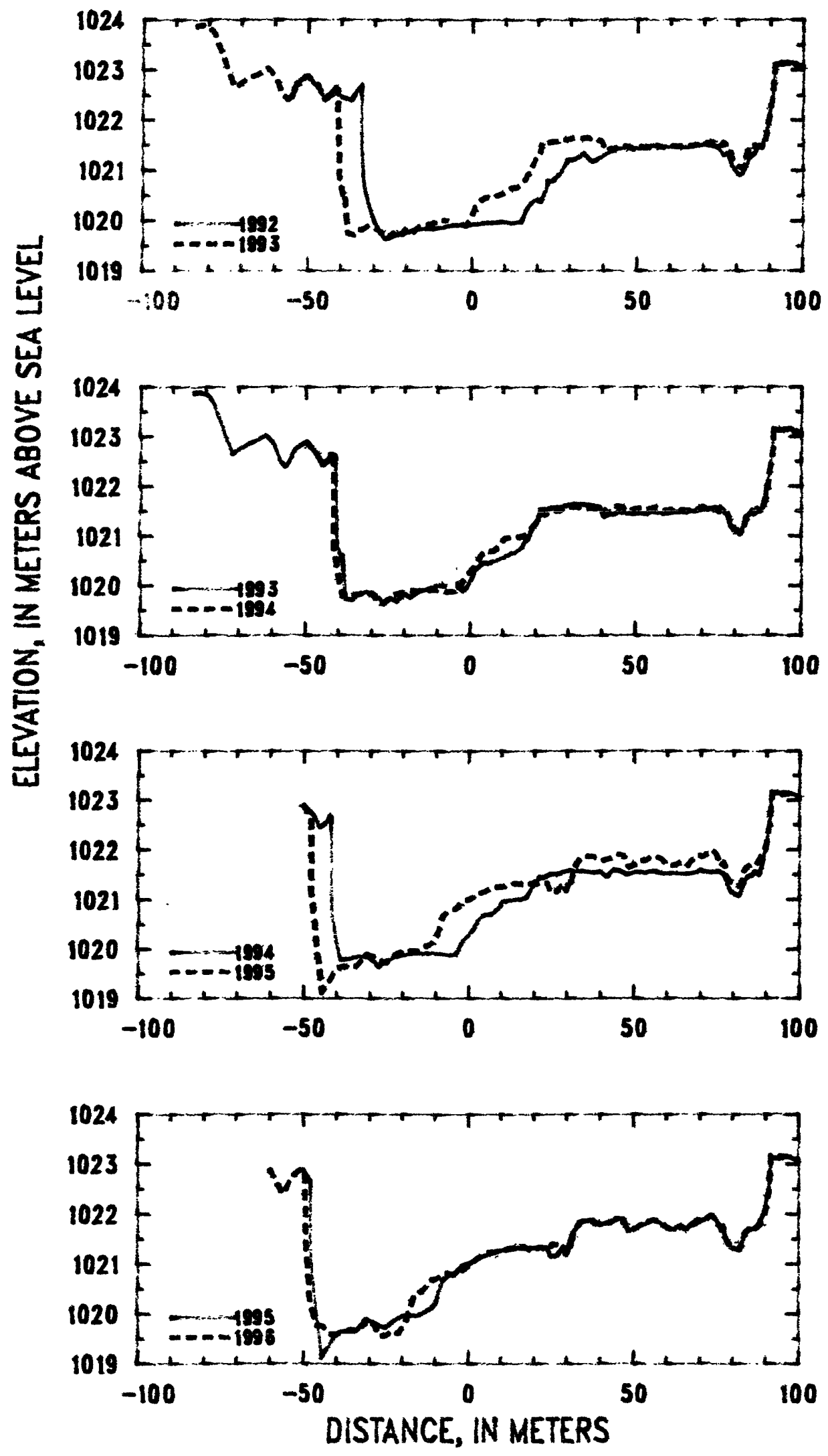

Figure 11. Profiles of cross section PR113 from 1992 to 1996. 


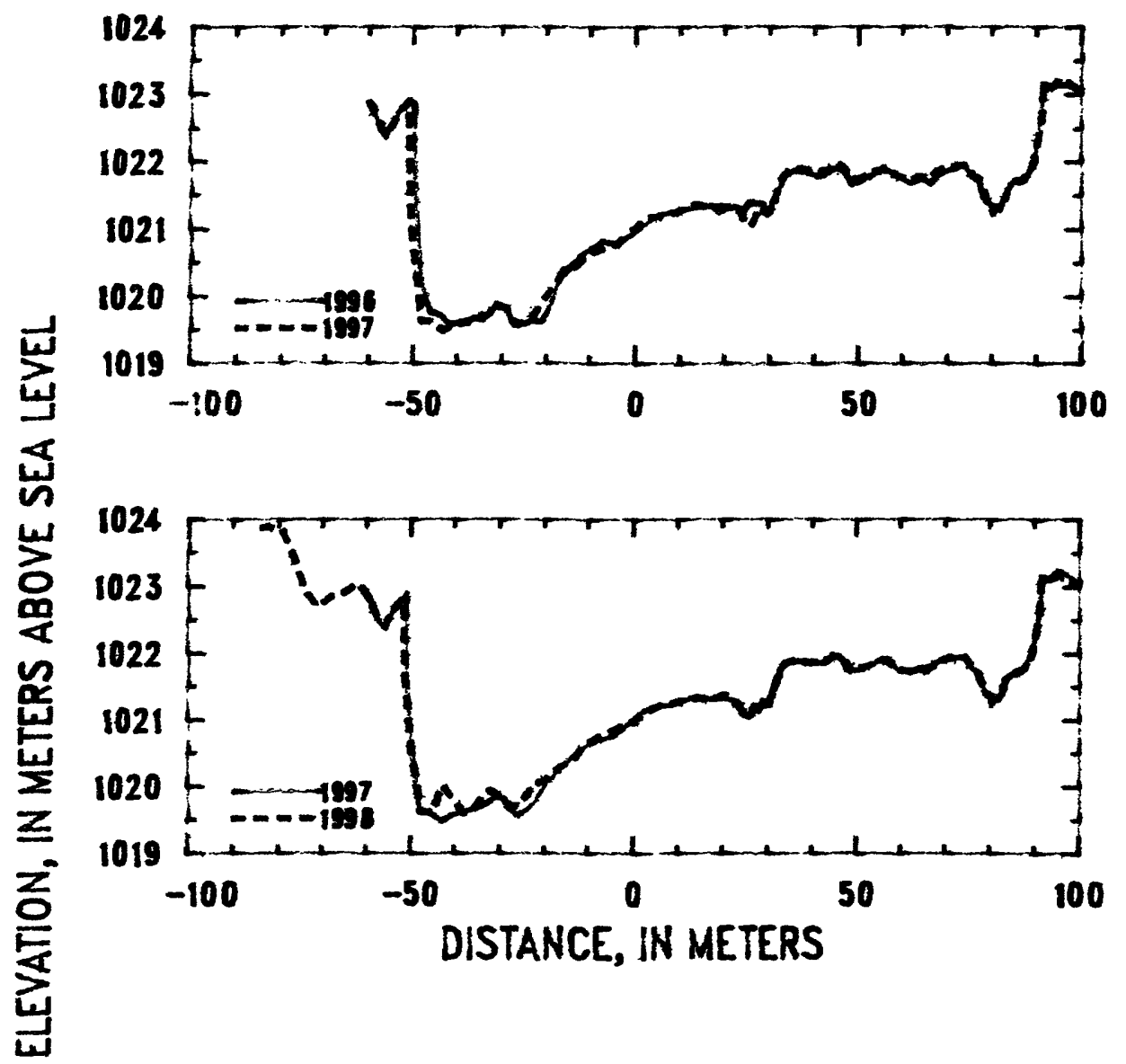

Figure 12. Profiles of cross section PR113 from 1996 to 1998. 
Table 7. Listing of horizontal stations and elevations for cross section PR113

[Sta., station, distance in meters from a reference pin on the left bank; Elev., elevation, in meters above sea level]

\begin{tabular}{|c|c|c|c|c|c|c|c|c|c|}
\hline \multirow{2}{*}{\multicolumn{2}{|c|}{$\begin{array}{c}1989 \\
16 \text { September }\end{array}$}} & \multicolumn{2}{|c|}{1989} & \multicolumn{2}{|c|}{1989} & \multicolumn{2}{|c|}{1990} & \multicolumn{2}{|c|}{1990} \\
\hline & & $16 S$ & tember & $16 \mathrm{~S}$ & tember & $13 \mathrm{~S}$ & tember & $13 \mathrm{Se}$ & tember \\
\hline Sta. & Elev. & Sta. & Elev. & Sta. & Elev. & Sta. & Elev. & Sta. & Elev. \\
\hline-70.0 & 1022.70 & 19.3 & 1020.24 & 97.0 & 1023.15 & -32.0 & 1022.63 & 44.0 & 1021.44 \\
\hline-65.0 & 1022.89 & 21.0 & 1020.45 & 99.0 & 1023.07 & -31.0 & 1022.41 & 46.0 & 1021.48 \\
\hline-61.4 & 1023.04 & 23.0 & 1020.62 & 100.0 & 1023.05 & -29.0 & 1022.39 & 48.0 & 1021.41 \\
\hline-57.5 & 1022.48 & 25.0 & 1020.76 & 100.4 & 1023.08 & -27.0 & 1022.18 & 50.0 & 1021.41 \\
\hline-55.9 & 1022.37 & 28.0 & 1020.82 & 102.8 & 1023.07 & -25.3 & 1021.95 & 52.0 & 1021.45 \\
\hline-54.9 & 1022.45 & 29.0 & 1020.89 & 105.0 & 1023.31 & -23.0 & 1022.02 & 54.0 & 1021.49 \\
\hline-53.0 & 1022.75 & 31.0 & 1020.87 & 107.0 & 1023.21 & -21.0 & 1022.04 & 56.0 & 1021.49 \\
\hline-50.2 & 1022.88 & 33.0 & 1020.94 & 110.0 & 1023.42 & -20.6 & 1020.58 & 58.0 & 1021.45 \\
\hline-46.7 & 1022.65 & 35.0 & 1021.01 & 114.7 & 1023.40 & -19.5 & 1020.22 & 60.0 & 1021.45 \\
\hline-44.5 & 1022.39 & 37.0 & 1021.05 & & & -16.9 & 1019.62 & 62.0 & 1021.49 \\
\hline-42.5 & 1022.61 & 39.0 & 1021.14 & & & -15.0 & 1019.51 & 64.0 & 1021.44 \\
\hline-40.0 & 1022.45 & 41.0 & 1021.30 & & & -14.0 & 1019.59 & 66.0 & 1021.41 \\
\hline-37.0 & 1022.40 & 43.0 & 1021.38 & & & -12.0 & 1019.74 & 68.0 & 1021.45 \\
\hline-35.0 & 1022.71 & 45.0 & 1021.45 & & & -10.0 & 1019.73 & 70.0 & 1021.49 \\
\hline-34.0 & 1022.72 & 48.0 & 1021.40 & & & -8.0 & 1019.71 & 72.0 & 1021.50 \\
\hline-32.0 & 1022.64 & 51.0 & 1021.44 & & & -5.0 & 1019.76 & 74.0 & 1021.48 \\
\hline-30.5 & 1022.35 & 53.0 & 1021.41 & & & -2.0 & 1019.75 & 76.0 & 1021.28 \\
\hline-29.4 & 1022.41 & 56.0 & 1021.48 & & & 0.0 & 1019.78 & 77.8 & 1021.05 \\
\hline-28.0 & 1022.26 & 59.0 & 1021.40 & & & 2.0 & 1019.81 & 78.7 & 1020.93 \\
\hline-26.0 & 1022.05 & 62.0 & 1021.45 & & & 5.0 & 1019.90 & 79.0 & 1020.85 \\
\hline-25.0 & 1021.98 & 65.0 & 1021.40 & & & 8.0 & 1019.95 & 81.5 & 1020.71 \\
\hline-23.0 & 1022.03 & 67.0 & 1021.44 & & & 10.0 & 1020.01 & 83.0 & 1020.87 \\
\hline-21.0 & 1022.03 & 70.0 & 1021.48 & & & 12.0 & 1019.94 & 84.0 & 1021.06 \\
\hline-19.0 & 1022.05 & 72.0 & 1021.47 & & & 13.0 & 1019.95 & 85.2 & 1021.10 \\
\hline-18.1 & 1022.02 & 74.0 & 1021.45 & & & 14.0 & 1020.20 & 86.2 & 1021.44 \\
\hline-18.0 & 1021.24 & 76.0 & 1021.21 & & & 16.0 & 1020.39 & 88.0 & 1021.46 \\
\hline-17.0 & 1020.77 & 78.0 & 1020.82 & & & 17.0 & 1020.49 & 88.6 & 1021.48 \\
\hline-15.8 & 1020.26 & 80.0 & 1020.56 & & & 17.9 & 1020.63 & 90.0 & 1022.03 \\
\hline-14.2 & 1019.64 & 81.8 & 1020.59 & & & 18.0 & 1020.91 & 91.0 & 1022.43 \\
\hline-13.0 & 1019.72 & 82.3 & 1020.76 & & & 20.0 & 1021.05 & 91.5 & 1023.10 \\
\hline-11.0 & 1019.76 & 84.0 & 1020.98 & & & 22.0 & 1021.17 & 93.0 & 1023.13 \\
\hline-9.0 & 1019.76 & 85.4 & 1021.05 & & & 24.0 & 1021.30 & 95.0 & 1023.14 \\
\hline-6.0 & 1019.73 & 86.1 & 1021.43 & & & 26.0 & 1021.43 & 97.0 & 1023.14 \\
\hline-3.0 & 1019.73 & 87.0 & 1021.46 & & & 28.0 & 1021.51 & 100.0 & 1023.05 \\
\hline 0.0 & 1019.82 & 88.0 & 1021.41 & & & 29.0 & 1021.52 & 100.4 & 1023.09 \\
\hline 3.0 & 1019.92 & 88.6 & 1021.47 & & & 31.0 & 1021.42 & 114.7 & 1023.41 \\
\hline 5.0 & 1020.13 & 90.0 & 1022.05 & & & 33.0 & 1021.31 & & \\
\hline 7.0 & 1020.15 & 91.1 & 1022.42 & & & 35.0 & 1021.20 & . & \\
\hline 9.0 & 1020.12 & 91.4 & 1023.10 & & & 36.5 & 1021.07 & & \\
\hline 12.0 & 1020.14 & 91.6 & 1023.15 & & & 38.0 & 1021.14 & & \\
\hline 15.0 & 1020.17 & 93.0 & 1023.12 & & & 40.0 & 1021.31 & & \\
\hline 18.0 & 1020.16 & 95.0 & 1023.13 & & & 42.0 & 1021.39 & & \\
\hline
\end{tabular}


Table 7. (Continued) Listing of horizontal stations and elevations for cross section PR113

[Sta., station, distance in meters from a reference pin on the left bank; Elev., elevation, in meters above sea level]

\begin{tabular}{|c|c|c|c|c|c|c|c|c|c|}
\hline \multirow{2}{*}{\multicolumn{2}{|c|}{$\begin{array}{c}1991 \\
28 \text { August }\end{array}$}} & \multirow{2}{*}{\multicolumn{2}{|c|}{$\begin{array}{c}1991 \\
28 \text { August }\end{array}$}} & \multirow{2}{*}{\multicolumn{2}{|c|}{$\begin{array}{c}1992 \\
25 \text { August }\end{array}$}} & \multirow{2}{*}{\multicolumn{2}{|c|}{$\begin{array}{c}1992 \\
25 \text { August }\end{array}$}} & \multirow{2}{*}{\multicolumn{2}{|c|}{$\begin{array}{c}1992 \\
25 \text { August }\end{array}$}} \\
\hline & & & & & & & & & \\
\hline Sta. & Elev. & Sta. & Elev. & Sta. & Elev. & Sta. & Elev. & Sta. & Elev. \\
\hline-50.0 & 1022.88 & 30.3 & 1021.16 & -55.0 & 1022.44 & 18.0 & 1020.35 & 97.0 & 1023.15 \\
\hline-47.0 & 1022.69 & 31.5 & 1021.19 & -52.0 & 1022.81 & 20.0 & 1020.42 & 100.0 & 1023.06 \\
\hline-45.0 & 1022.40 & 34.4 & 1021.35 & -50.0 & 1022.88 & 20.9 & 1020.38 & 100.5 & 1023.10 \\
\hline-42.0 & 1022.63 & 35.9 & 1021.19 & -48.0 & 1022.73 & 23.0 & 1020.67 & & \\
\hline-40.0 & 1022.48 & 38.0 & 1021.20 & -46.0 & 1022.55 & 23.0 & 1020.79 & & \\
\hline-38.0 & 1022.43 & 40.0 & 1021.31 & -45.0 & 1022.40 & 24.8 & 1020.79 & & \\
\hline-36.0 & 1022.47 & 42.0 & 1021.39 & -43.0 & 1022.53 & 27.0 & 1020.95 & & \\
\hline-34.0 & 1022.68 & 45.0 & 1021.47 & -42.0 & 1022.63 & 29.0 & 1021.14 & & \\
\hline-32.2 & 1022.56 & 48.0 & 1021.42 & -40.0 & 1022.47 & 29.1 & 1021.20 & & \\
\hline-32.0 & 1021.11 & 51.0 & 1021.45 & -37.0 & 1022.39 & 31.0 & 1021.21 & & \\
\hline-31.3 & 1020.73 & 54.0 & 1021.48 & -35.0 & 1022.61 & 34.0 & 1021.35 & & \\
\hline-31.2 & 1020.40 & 57.0 & 1021.48 & -33.8 & 1022.69 & 36.0 & 1021.20 & & \\
\hline-29.7 & 1020.08 & 60.0 & 1021.44 & -33.7 & 1021.48 & 37.0 & 1021.17 & & \\
\hline-28.2 & 1019.83 & 63.0 & 1021.47 & -33.3 & 1020.88 & 39.0 & 1021.26 & & \\
\hline-26.0 & 1019.71 & 66.0 & 1021.43 & -32.0 & 1020.42 & 41.0 & 1021.35 & & \\
\hline-24.0 & 1019.62 & 69.0 & 1021.50 & -31.0 & 1020.24 & 43.5 & 1021.41 & & \\
\hline-22.0 & 1019.58 & 71.0 & 1021.51 & -30.5 & 1020.07 & 46.0 & 1021.45 & & \\
\hline-20.0 & 1019.71 & 72.0 & 1021.49 & -29.0 & 1019.87 & 49.0 & 1021.42 & & \\
\hline-18.0 & 1019.72 & 73.5 & 1021.49 & -27.0 & 1019.67 & 52.0 & 1021.45 & & \\
\hline-16.0 & 1019.71 & 75.0 & 1021.39 & -26.0 & 1019.63 & 55.0 & 1021.49 & & \\
\hline-14.0 & 1019.79 & 76.0 & 1021.29 & -24.0 & 1019.68 & 58.0 & 1021.45 & & \\
\hline-12.0 & 1019.78 & 77.4 & 1021.36 & -22.0 & 1019.75 & 61.0 & 1021.47 & & \\
\hline-10.0 & 1019.82 & 78.0 & 1021.16 & -20.0 & 1019.75 & 64.0 & 1021.44 & & \\
\hline-8.0 & 1019.80 & 80.0 & 1020.90 & -18.0 & 1019.81 & 67.0 & 1021.46 & & \\
\hline-6.0 & 1019.81 & 81.5 & 1020.83 & -16.0 & 1019.83 & 70.0 & 1021.51 & & \\
\hline-4.0 & 1019.83 & 84.0 & 1021.26 & -14.0 & 1019.85 & 72.0 & 1021.49 & & \\
\hline-2.0 & 1019.87 & 85.0 & 1021.36 & -12.0 & 1019.83 & 74.0 & 1021.46 & & \\
\hline 0.0 & 1019.91 & 85.7 & 1021.32 & -10.0 & 1019.85 & 76.0 & 1021.30 & & \\
\hline 2.0 & 1019.92 & 86.2 & 1021.46 & -8.0 & 1019.89 & 77.5 & 1021.36 & & \\
\hline 4.0 & 1019.94 & 88.2 & 1021.47 & -6.0 & 1019.91 & 78.2 & 1021.12 & & \\
\hline 6.0 & 1019.98 & 89.6 & 1021.96 & -4.0 & 1019.91 & 80.0 & 1020.94 & & \\
\hline 8.0 & 1020.01 & 91.1 & 1022.45 & -2.0 & 1019.90 & 81.0 & 1020.90 & & \\
\hline 10.0 & 1020.01 & 91.5 & 1023.11 & 0.0 & 1019.91 & 82.0 & 1020.97 & & \\
\hline 12.0 & 1020.09 & 93.0 & 1023.13 & 2.0 & 1019.94 & 84.0 & 1021.28 & & \\
\hline 15.0 & 1020.12 & 95.0 & 1023.15 & 4.0 & 1019.94 & 85.1 & 1021.32 & & \\
\hline 18.0 & 1020.21 & 97.0 & 1023.14 & 6.0 & 1019.95 & 86.5 & 1021.49 & & \\
\hline 20.0 & 1020.35 & 100.0 & 1023.06 & 8.0 & 1019.96 & 87.5 & 1021.45 & & \\
\hline 21.5 & 1020.57 & 100.4 & 1023.10 & 10.0 & 1019.98 & 89.0 & 1021.72 & & \\
\hline 23.0 & 1020.69 & & & -55.0 & 1022.44 & 91.0 & 1022.48 & & \\
\hline 25.0 & 1020.80 & & & -52.0 & 1022.81 & 91.4 & 1023.10 & & \\
\hline 26.0 & 1020.87 & & & -50.0 & 1022.88 & 92.0 & 1023.14 & & ' \\
\hline 28.5 & 1021.18 & & & -48.0 & 1022.73 & 94.0 & 1023.10 & & \\
\hline
\end{tabular}


Table 7. (Continued) Listing of horizontal stations and elevations for cross section PR113

[Sta., station, distance in meters from a reference pin on the left bank; Elev., elevation, in meters above sea level]

\begin{tabular}{|c|c|c|c|c|c|c|c|c|c|}
\hline \multirow{2}{*}{\multicolumn{2}{|c|}{$\begin{array}{c}1993 \\
25 \text { August }\end{array}$}} & \multirow{2}{*}{\multicolumn{2}{|c|}{$\begin{array}{c}1993 \\
25 \text { August }\end{array}$}} & \multirow{2}{*}{\multicolumn{2}{|c|}{$\begin{array}{c}1993 \\
25 \text { August }\end{array}$}} & \multirow{2}{*}{\multicolumn{2}{|c|}{$\begin{array}{c}1994 \\
24 \text { September }\end{array}$}} & \multirow{2}{*}{\multicolumn{2}{|c|}{$\begin{array}{c}1994 \\
4 \text { September }\end{array}$}} \\
\hline & & & & & & & & & \\
\hline Sta. & Elev. & Sta. & Elev. & Sta. & Elev. & Sta. & Elev. & Sta. & Elev. \\
\hline-83.9 & 1023.87 & -10.0 & 1020.02 & $\overline{61.0}$ & 1021.48 & -50.0 & 1022.89 & 40.0 & 1021.57 \\
\hline-82.0 & 1023.89 & -8.0 & 1020.00 & 63.0 & 1021.48 & -48.0 & 1022.77 & 42.0 & 1021.45 \\
\hline-80.0 & 1023.86 & -6.0 & 1020.92 & 65.0 & 1021.47 & -47.0 & 1022.71 & 44.0 & 1021.59 \\
\hline-78.0 & 1023.73 & -4.0 & 1019.98 & 67.0 & 1021.50 & -45.0 & 1022.44 & 46.0 & 1021.59 \\
\hline-76.0 & 1023.37 & -2.0 & 1019.88 & 69.0 & 1021.51 & -43.0 & 1022.55 & 48.0 & 1021.50 \\
\hline-74.0 & 1022.99 & 0.0 & 1020.08 & 70.0 & 1021.55 & -42.0 & 1022.69 & 51.0 & 1021.56 \\
\hline-71.9 & 1022.65 & 1.0 & 1020.29 & 71.0 & 1021.53 & -41.5 & 1022.64 & 53.0 & 1021.52 \\
\hline-70.0 & 1022.74 & 2.4 & 1020.38 & 73.0 & 1021.56 & -41.4 & 1020.73 & 56.0 & 1021.59 \\
\hline-67.5 & 1022.85 & 4.0 & 1020.46 & 75.0 & 1021.48 & -40.0 & 1020.17 & 59.0 & 1021.52 \\
\hline-65.0 & 1022.91 & 6.0 & 1020.45 & 76.0 & 1021.56 & -38.7 & 1019.78 & 62.0 & 1021.53 \\
\hline-62.0 & 1023.03 & 8.0 & 1020.50 & 77.0 & 1021.47 & -36.0 & 1019.81 & 65.0 & 1021.51 \\
\hline-59.5 & 1022.85 & 10.0 & 1020.56 & 77.9 & 1021.42 & -33.0 & 1019.85 & 68.0 & 1021.52 \\
\hline-57.5 & 1022.50 & 12.0 & 1020.65 & 78.7 & 1021.19 & -30.0 & 1019.85 & 70.0 & 1021.60 \\
\hline-56.0 & 1022.39 & 14.0 & 1020.67 & 80.0 & 1021.09 & -27.0 & 1019.64 & 72.0 & 1021.55 \\
\hline-55.0 & 1022.46 & 16.0 & 1020.79 & 81.6 & 1021.03 & -24.0 & 1019.79 & 74.0 & 1021.56 \\
\hline-53.2 & 1022.75 & 17.0 & 1020.93 & 82.1 & 1021.08 & -21.0 & 1019.86 & 76.0 & 1021.57 \\
\hline-51.5 & 1022.84 & 17.5 & 1020.93 & 83.1 & 1021.39 & -18.0 & 1019.91 & 77.5 & 1021.50 \\
\hline-50.0 & 1022.88 & 18.5 & 1021.10 & 84.2 & 1021.42 & -15.0 & 1019.91 & 78.8 & 1021.21 \\
\hline-48.0 & 1022.74 & 19.1 & 1021.22 & 84.8 & 1021.50 & -12.0 & 1019.92 & 80.0 & 1021.14 \\
\hline-47.0 & 1022.70 & 20.0 & 1021.24 & 85.2 & 1021.52 & -9.0 & 1019.91 & 82.0 & 1021.06 \\
\hline-45.0 & 1022.41 & 21.0 & 1021.41 & 85.5 & 1021.44 & -6.0 & 1019.87 & 83.5 & 1021.38 \\
\hline-43.2 & 1022.49 & 21.3 & 1021.55 & 87.0 & 1021.49 & -4.0 & 1019.87 & 84.5 & 1021.46 \\
\hline-42.2 & 1022.65 & 23.0 & 1021.55 & 87.7 & 1021.50 & -1.8 & 1020.17 & 85.8 & 1021.48 \\
\hline-41.0 & 1022.62 & 25.0 & 1021.57 & 88.6 & 1021.63 & 0.0 & 1020.29 & 86.6 & 1021.56 \\
\hline-40.5 & 1020.92 & 27.0 & 1021.58 & 89.1 & 1021.78 & 2.0 & 1020.45 & 87.8 & 1021.51 \\
\hline-39.5 & 1020.53 & 29.0 & 1021.61 & 90.8 & 1022.49 & 3.0 & 1020.60 & 89.0 & 1021.70 \\
\hline-39.0 & 1020.59 & 31.0 & 1021.64 & 91.4 & 1023.16 & 5.0 & 1020.70 & 91.3 & 1022.66 \\
\hline-38.8 & 1020.41 & 33.0 & 1021.64 & 93.0 & 1023.13 & 7.0 & 1020.69 & 91.5 & 1023.16 \\
\hline-38.0 & 1019.73 & 35.0 & 1021.65 & 95.0 & 1023.14 & 9.0 & 1020.80 & 93.0 & 1023.13 \\
\hline-36.0 & 1019.71 & 37.0 & 1021.63 & 97.0 & 1023.16 & 10.5 & 1020.95 & 95.0 & 1023.15 \\
\hline-34.0 & 1019.81 & 39.3 & 1021.58 & 98.0 & 1023.06 & 12.0 & 1020.98 & 97.0 & 1023.15 \\
\hline-32.0 & 1019.88 & 40.6 & 1021.38 & 100.0 & 1023.06 & 15.0 & 1020.99 & 100.0 & 1023.05 \\
\hline-30.0 & 1019.86 & 42.0 & 1021.43 & 100.4 & 1023.10 & 17.5 & 1021.04 & 100.4 & 1023.09 \\
\hline-28.0 & 1019.78 & 43.5 & 1021.43 & & & 20.0 & 1021.29 & 114.7 & 1023.43 \\
\hline-26.0 & 1019.63 & 45.0 & 1021.50 & & & 21.5 & 1021.45 & & \\
\hline-24.0 & 1019.75 & 47.0 & 1021.47 & & & 23.0 & 1021.44 & & \\
\hline-22.0 & 1019.69 & 49.0 & 1021.43 & & & 25.0 & 1021.52 & & \\
\hline-20.0 & 1019.85 & 51.0 & 1021.47 & & & 27.0 & 1021.50 & & \\
\hline-18.0 & 1019.76 & 53.0 & 1021.44 & & & 30.0 & 1021.59 & & \\
\hline-16.0 & 1019.88 & 55.0 & 1021.51 & & & 33.0 & 1021.59 & & \\
\hline-14.0 & 1019.92 & 57.0 & 1021.47 & & & 36.0 & 1021.57 & & \\
\hline-12.0 & 1019.93 & 59.0 & 1021.44 & & & 38.5 & 1021.53 & & \\
\hline
\end{tabular}


Table 7. (Continued) Listing of horizontal stations and elevations for cross section PR113

[Sta., station, distance in meters from a reference pin on the left bank; Elev., elevation, in meters above sea level]

\begin{tabular}{|c|c|c|c|c|c|c|c|c|c|}
\hline \multirow{2}{*}{\multicolumn{2}{|c|}{$\begin{array}{c}1995 \\
23 \text { September }\end{array}$}} & \multicolumn{2}{|c|}{1995} & \multicolumn{2}{|c|}{1995} & \multicolumn{2}{|c|}{1996} & \multicolumn{2}{|c|}{1996} \\
\hline & & $23 \mathrm{Se}$ & tember & $23 \mathrm{Se}$ & tember & $15 \mathrm{C}$ & tober & $15 C$ & tober \\
\hline Sta. & Elev. & Sta. & Elev. & Sta. & Elev. & Sta. & Elev. & Sta. & Elev. \\
\hline-50.0 & 1022.89 & 29.8 & 1021.19 & 91.5 & 1023.15 & -60.0 & 1022.89 & 24.5 & 1021.28 \\
\hline-47.7 & 1022.67 & 30.2 & 1021.25 & 94.0 & 1023.09 & -56.2 & 1022.38 & 25.5 & 1021.40 \\
\hline-47.6 & 1021.15 & 30.9 & 1021.42 & 97.0 & 1023.15 & -53.2 & 1022.76 & 28.5 & 1021.38 \\
\hline-46.2 & 1020.27 & 31.3 & 1021.36 & 100.0 & 1023.06 & -51.5 & 1022.86 & 30.0 & 1021.23 \\
\hline-44.3 & 1019.14 & 32.6 & 1021.69 & 100.4 & 1023.08 & -50.0 & 1022.89 & 32.0 & 1021.60 \\
\hline-40.0 & 1019.60 & 34.0 & 1021.83 & 114.7 & 1023.42 & -49.6 & 1022.87 & 34.0 & 1021.83 \\
\hline-38.0 & 1019.65 & 36.0 & 1021.87 & & & -49.1 & 1021.29 & 36.0 & 1021.88 \\
\hline-36.0 & 1019.64 & 38.0 & 1021.88 & & & -47.9 & 1020.20 & 39.0 & 1021.82 \\
\hline-34.0 & 1019.66 & 40.0 & 1021.78 & & & -46.0 & 1019.78 & 41.0 & 1021.78 \\
\hline-32.0 & 1019.81 & 42.0 & 1021.81 & & & -44.0 & 1019.74 & 43.5 & 1021.84 \\
\hline-31.0 & 1019.91 & 44.0 & 1021.85 & & & -42.0 & 1019.60 & 45.0 & 1021.91 \\
\hline-29.0 & 1019.85 & 45.0 & 1021.91 & & & -40.0 & 1019.59 & 47.0 & 1021.86 \\
\hline-27.0 & 1019.77 & 47.0 & 1021.89 & & & -38.0 & 1019.61 & 48.5 & 1021.66 \\
\hline-25.0 & 1019.72 & 48.4 & 1021.65 & & & -36.0 & 1019.69 & 50.0 & 1021.68 \\
\hline-23.0 & 1019.84 & 50.0 & 1021.68 & & & -34.0 & 1019.66 & 53.0 & 1021.79 \\
\hline-21.0 & 1019.93 & 52.0 & 1021.76 & & & -32.0 & 1019.79 & 56.0 & 1021.89 \\
\hline-19.0 & 1019.97 & 54.0 & 1021.80 & & & -31.0 & 1019.90 & 58.0 & 1021.84 \\
\hline-17.0 & 1019.97 & 56.0 & 1021.88 & & & -29.0 & 1019.83 & 60.0 & 1021.73 \\
\hline-15.0 & 1019.96 & 58.0 & 1021.83 & & & -27.0 & 1019.56 & 62.0 & 1021.68 \\
\hline-13.0 & 1020.05 & 60.0 & 1021.71 & & & -25.0 & 1019.57 & 64.3 & 1021.76 \\
\hline-11.0 & 1020.12 & 62.0 & 1021.68 & & & -23.0 & 1019.66 & 66.2 & 1021.67 \\
\hline-9.9 & 1020.23 & 64.0 & 1021.75 & & & -21.0 & 1019.62 & 68.0 & 1021.79 \\
\hline-8.0 & 1020.68 & 66.0 & 1021.67 & & & -19.0 & 1019.83 & 70.0 & 1021.86 \\
\hline-6.0 & 1020.78 & 67.0 & 1021.76 & & & -17.0 & 1020.23 & 72.0 & 1021.91 \\
\hline-4.0 & 1020.81 & 69.0 & 1021.88 & & & -16.0 & 1020.41 & 74.0 & 1021.97 \\
\hline-2.0 & 1020.98 & 71.0 & 1021.86 & & & -15.0 & 1020.41 & 76.0 & 1021.76 \\
\hline 0.0 & 1021.00 & 73.0 & 1021.96 & & & -13.0 & 1020.53 & 77.2 & 1021.73 \\
\hline 2.0 & 1021.07 & 74.0 & 1021.97 & & & -11.0 & 1020.68 & 78.2 & 1021.45 \\
\hline 4.0 & 1021.14 & 75.0 & 1021.90 & & & -9.0 & 1020.73 & 80.0 & 1021.34 \\
\hline 6.0 & 1021.18 & 75.8 & 1021.76 & & & -7.0 & 1020.83 & 82.0 & 1021.28 \\
\hline 8.0 & 1021.25 & 76.5 & 1021.67 & & & -4.0 & 1020.78 & 84.0 & 1021.63 \\
\hline 10.0 & 1021.25 & 77.2 & 1021.75 & & & -2.0 & 1020.87 & 85.4 & 1021.73 \\
\hline 12.0 & 1021.29 & 78.2 & 1021.43 & & & 0.0 & 1020.96 & 86.5 & 1021.70 \\
\hline 14.0 & 1021.33 & 79.5 & 1021.33 & & & 3.0 & 1021.12 & 88.0 & 1021.76 \\
\hline 16.0 & 1021.31 & 82.0 & 1021.27 & & & 6.0 & 1021.22 & 89.5 & 1021.97 \\
\hline 18.0 & 1021.32 & 83.0 & 1021.43 & & & 9.0 & 1021.25 & 91.0 & 1022.52 \\
\hline 20.0 & 1021.33 & 83.5 & 1021.62 & & & 12.0 & 1021.30 & 91.6 & 1023.16 \\
\hline 22.0 & 1021.30 & 85.0 & 1021.70 & & & 15.0 & 1021.33 & 93.0 & 1023.13 \\
\hline 24.0 & 1021.34 & 87.0 & 1021.70 & & & 17.0 & 1021.35 & 95.0 & 1023.15 \\
\hline 25.0 & 1021.14 & 88.5 & 1021.89 & & & 19.0 & 1021.25 & 97.0 & 1023.15 \\
\hline 27.0 & 1021.16 & 89.5 & 1021.97 & & & 20.0 & 1021.29 & 100.0 & 1023.06 \\
\hline 29.0 & 1021.33 & 91.0 & 1022.51 & & & 22.0 & 1021.34 & 100.4 & 1023.11 \\
\hline
\end{tabular}


Table 7. (Continued) Listing of horizontal stations and elevations for cross section PR113

[Sta., station, distance in meters from a reference pin on the left bank; Elev., elevation, in meters above sea level]

\begin{tabular}{|c|c|c|c|c|c|c|c|c|c|}
\hline \multirow{2}{*}{\multicolumn{2}{|c|}{$\begin{array}{c}1997 \\
18 \text { September }\end{array}$}} & \multicolumn{2}{|c|}{1997} & \multicolumn{2}{|c|}{1998} & \multicolumn{2}{|c|}{1998} & \multicolumn{2}{|c|}{1998} \\
\hline & & $18 \mathrm{~S}$ & tember & $25 \mathrm{~S}$ & tember & $25 \mathrm{~S}$ & tember & $25 \mathrm{~S}$ & tember \\
\hline Sta. & Elev. & Sta. & Elev. & Sta. & Elev. & Sta. & Elev. & Sta. & Elev. \\
\hline-60.0 & 1022.88 & 28.0 & 1021.15 & -83.9 & 1023.88 & 9.0 & 1021.26 & 100.0 & 1023.05 \\
\hline-59.0 & 1022.79 & 29.0 & 1021.29 & -82.0 & 1023.90 & 12.0 & 1021.33 & 100.5 & 1023.11 \\
\hline-57.0 & 1022.46 & 29.5 & 1021.22 & -79.0 & 1023.83 & 15.0 & 1021.34 & 103.0 & 1023.11 \\
\hline-56.0 & 1022.41 & 30.5 & 1021.30 & -76.5 & 1023.44 & 18.0 & 1021.32 & 105.0 & 1023.34 \\
\hline-54.5 & 1022.58 & 32.5 & 1021.73 & -74.0 & 1022.94 & 20.0 & 1021.39 & 106.5 & 1023.21 \\
\hline-52.5 & 1022.78 & 34.0 & 1021.86 & -71.0 & 1022.71 & 22.0 & 1021.31 & 110.0 & 1023.38 \\
\hline-50.9 & 1022.90 & 35.0 & 1021.88 & -68.0 & 1022.86 & 25.0 & 1021.08 & 114.7 & 1023.45 \\
\hline-50.4 & 1021.12 & 37.0 & 1021.90 & -65.0 & 1022.93 & 26.2 & 1021.06 & 120.0 & 1023.28 \\
\hline-49.0 & 1020.27 & 40.0 & 1021.85 & -62.0 & 1023.06 & 26.9 & 1021.22 & 125.0 & 1023.24 \\
\hline-48.0 & 1019.63 & 43.0 & 1021.87 & -60.0 & 1022.88 & 29.2 & 1021.27 & 130.0 & 1023.28 \\
\hline-46.0 & 1019.63 & 45.0 & 1021.99 & -58.0 & 1022.60 & 30.2 & 1021.24 & 135.0 & 1023.27 \\
\hline-43.0 & 1019.48 & 47.0 & 1021.91 & -56.0 & 1022.41 & 32.0 & 1021.60 & 140.0 & 1023.26 \\
\hline-41.0 & 1019.57 & 48.0 & 1021.75 & -54.0 & 1022.67 & 34.0 & 1021.88 & 142.5 & 1023.30 \\
\hline-38.0 & 1019.62 & 50.0 & 1021.73 & -51.7 & 1022.84 & 36.0 & 1021.91 & 145.0 & 1023.07 \\
\hline-35.0 & 1019.69 & 53.0 & 1021.83 & -51.5 & 1021.83 & 39.0 & 1021.88 & 150.0 & 1022.86 \\
\hline-33.0 & 1019.73 & 56.0 & 1021.92 & -51.1 & 1021.47 & 42.0 & 1021.87 & 152.0 & 1022.84 \\
\hline-31.0 & 1019.87 & 59.0 & 1021.78 & -49.9 & 1020.52 & 45.0 & 1021.97 & 155.0 & 1022.97 \\
\hline-29.0 & 1019.83 & 62.0 & 1021.71 & -47.5 & 1019.62 & 47.0 & 1021.93 & 160.0 & 1022.72 \\
\hline-28.0 & 1019.67 & 64.0 & 1021.78 & -45.0 & 1019.62 & 49.0 & 1021.72 & 165.0 & 1022.67 \\
\hline-26.0 & 1019.57 & 66.0 & 1021.73 & -43.0 & 1020.02 & 51.0 & 1021.78 & 170.0 & 1022.74 \\
\hline-23.0 & 1019.70 & 68.0 & 1021.88 & -40.0 & 1019.79 & 54.0 & 1021.86 & 175.0 & 1023.09 \\
\hline-20.0 & 1020.00 & 70.0 & 1021.92 & -38.0 & 1019.60 & 57.0 & 1021.92 & 180.0 & 1023.57 \\
\hline-17.0 & 1020.25 & 72.0 & 1021.94 & -36.0 & 1019.63 & 60.0 & 1021.75 & 185.0 & 1023.74 \\
\hline-16.0 & 1020.34 & 74.0 & 1021.96 & -34.0 & 1019.84 & 63.0 & 1021.73 & & \\
\hline-13.0 & 1020.47 & 76.0 & 1021.80 & -32.0 & 1019.95 & 65.0 & 1021.78 & & \\
\hline-10.0 & 1020.65 & 77.5 & 1021.72 & -30.0 & 1019.88 & 67.0 & 1021.81 & & \\
\hline-7.0 & 1020.72 & 79.0 & 1021.41 & -28.0 & 1019.71 & 70.0 & 1021.92 & & \\
\hline-4.0 & 1020.78 & 80.0 & 1021.39 & -26.0 & 1019.71 & 73.0 & 1021.98 & & \\
\hline-1.0 & 1020.96 & 80.5 & 1021.24 & -24.0 & 1019.87 & 74.0 & 1021.99 & & \\
\hline 1.0 & 1021.08 & 81.0 & 1021.33 & -22.0 & 1020.06 & 77.0 & 1021.76 & & \\
\hline 3.0 & 1021.17 & 82.0 & 1021.32 & -20.0 & 1020.10 & 79.0 & 1021.42 & & \\
\hline 6.0 & 1021.21 & 83.0 & 1021.41 & -18.0 & 1020.21 & 81.0 & 1021.32 & & \\
\hline 9.0 & 1021.24 & 84.0 & 1021.65 & -16.0 & 1020.32 & 82.4 & 1021.36 & & \\
\hline 11.0 & 1021.30 & 86.0 & 1021.72 & -14.0 & 1020.38 & 83.7 & 1021.63 & & \\
\hline 14.0 & 1021.37 & 87.0 & 1021.73 & -12.7 & 1020.46 & 86.0 & 1021.73 & & \\
\hline 17.0 & 1021.32 & 89.0 & 1021.90 & -11.0 & 1020.62 & 88.0 & 1021.79 & & \\
\hline 19.0 & 1021.34 & 90.0 & 1022.15 & -8.0 & 1020.75 & 89.7 & 1022.05 & & \\
\hline 21.0 & 1021.33 & 91.2 & 1022.61 & -5.0 & 1020.84 & 91.1 & 1022.61 & & \\
\hline 23.5 & 1021.26 & 91.7 & 1023.16 & -2.5 & 1020.93 & 91.5 & 1023.13 & & \\
\hline 24.5 & 1021.09 & 93.5 & 1023.11 & 0.0 & 1020.98 & 93.0 & 1023.12 & & \\
\hline 26.0 & 1021.05 & 95.4 & 1023.25 & 3.0 & 1021.14 & 95.6 & 1023.18 & & \\
\hline 27.0 & 1021.20 & 100.0 & 1023.05 & 6.0 & 1021.23 & 98.0 & 1023.09 & & \\
\hline & & 100.4 & 1023.11 & & & & & & \\
\hline
\end{tabular}




\section{Description of Cross Section PR116}

Location: Township 9 South/Range 48 East--section 8

U. S. Geological Survey quadrangle (1:24,000): Moorhead

Landowners--left bank: County road

--right bank: 3 Bar Ranch

Access: Left bank

Permission from: None necessary

Distance from Moorhead Gaging Station: 0.65 kilometer

Azimuth of Section (degrees magnetic): 187.5

\section{Reference Monuments}

[ Monuments on the left bank were closest to the leveling instrument ]

\begin{tabular}{|c|c|c|c|c|c|c|}
\hline \multirow[b]{2}{*}{ Description } & \multirow[b]{2}{*}{$\begin{array}{c}\text { Station } \\
\text { (m) }\end{array}$} & \multicolumn{2}{|c|}{ GPS-NAD83 (1992) } & \multicolumn{2}{|c|}{ Measurement } & \multirow{2}{*}{$\begin{array}{c}\text { Elevation } \\
\text { (NGVD1929) } \\
(\mathrm{m}) \\
\end{array}$} \\
\hline & & Latitude & Longitude & $\begin{array}{l}\text { Standard } \\
\text { deviation } \\
\text { (m) }\end{array}$ & $\begin{array}{l}\text { Horizontal } \\
\text { precision } \\
\text { (m) }\end{array}$ & \\
\hline $\begin{array}{l}\text { 1/2-inch-rebar; } 0.20 \text { meter above } 1998 \text { ground } \\
\text { level }\end{array}$ & & & & & & 1020.36 \\
\hline $\begin{array}{l}\text { Benchmark, bolt in downstream concrete foot- } \\
\text { ing; about } 0.9 \text { meter downstream }\end{array}$ & -1.3 & $45^{\circ} 04^{\prime} 07.22^{\prime \prime}$ & $105^{\circ} 51^{\prime} 46.75^{\prime \prime}$ & 0.548 & 0.554 & 1020.00 \\
\hline $\begin{array}{l}\text { Bolt in upstream concrete footing; about } 0.9 \\
\text { meter upstream }\end{array}$ & -1.1 & & & & & 1019.99 \\
\hline $\begin{array}{l}\text { 1/2-inch-rebar; } 0.02 \text { meter above } 1998 \text { ground } \\
\text { level }\end{array}$ & 0.0 & $45^{\circ} 04^{\prime} 07.19^{\prime \prime}$ & $105^{\circ} 51^{\prime} 46.81^{\prime \prime}$ & 0.441 & 0.596 & 1019.69 \\
\hline Metal hoop in concrete deadman & 109.1 & $45^{\circ} 04^{\prime} 03.92$ & $105^{\circ} 51^{\prime} 09.40^{\prime \prime}$ & 0.135 & 0.445 & $-\cdots$ \\
\hline
\end{tabular}



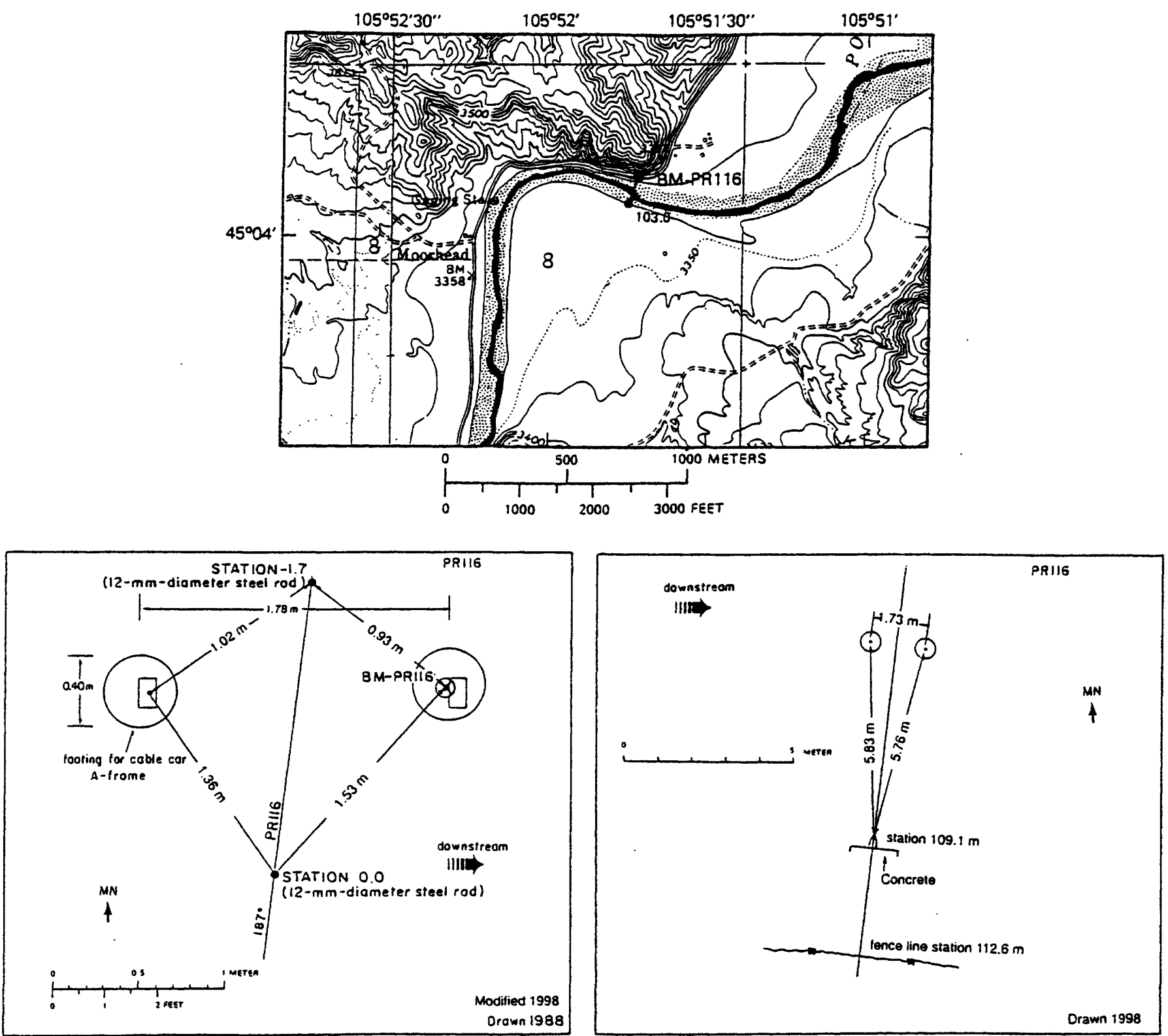

Figure 13. Upper: Location of cross section PR116, bench mark BM-PR113, and left and right bank reference monuments in the Moorhead quadrangle. Lower left: Location of the bench mark on the left bank. Lower right: Location of station 109.1 at the end of a Ushaped steel rod anchored in concrete on the right bank. MN is magnetic north. 

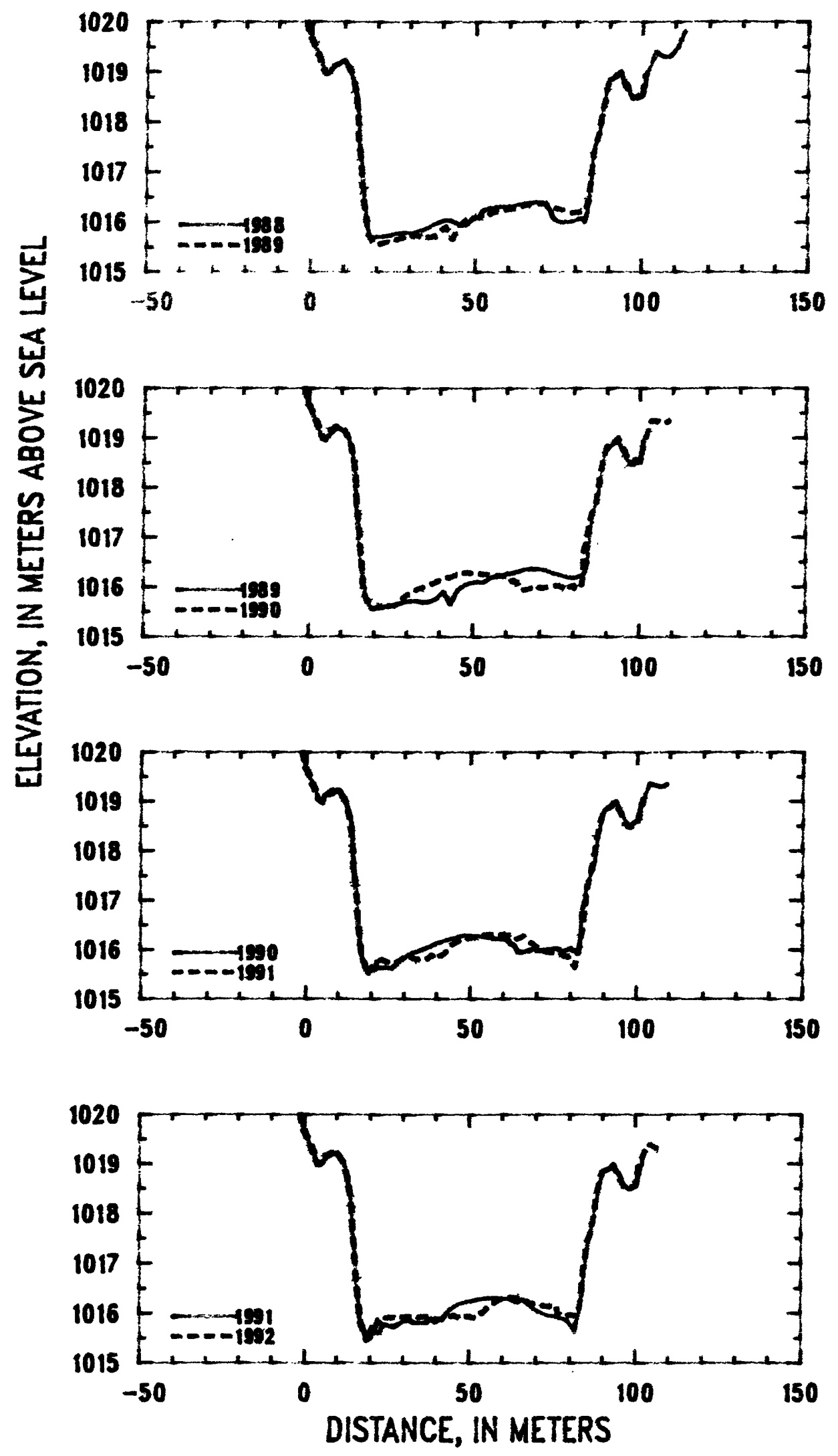

Figure 14. Profiles of cross section PR116 from 1988 to 1992. 

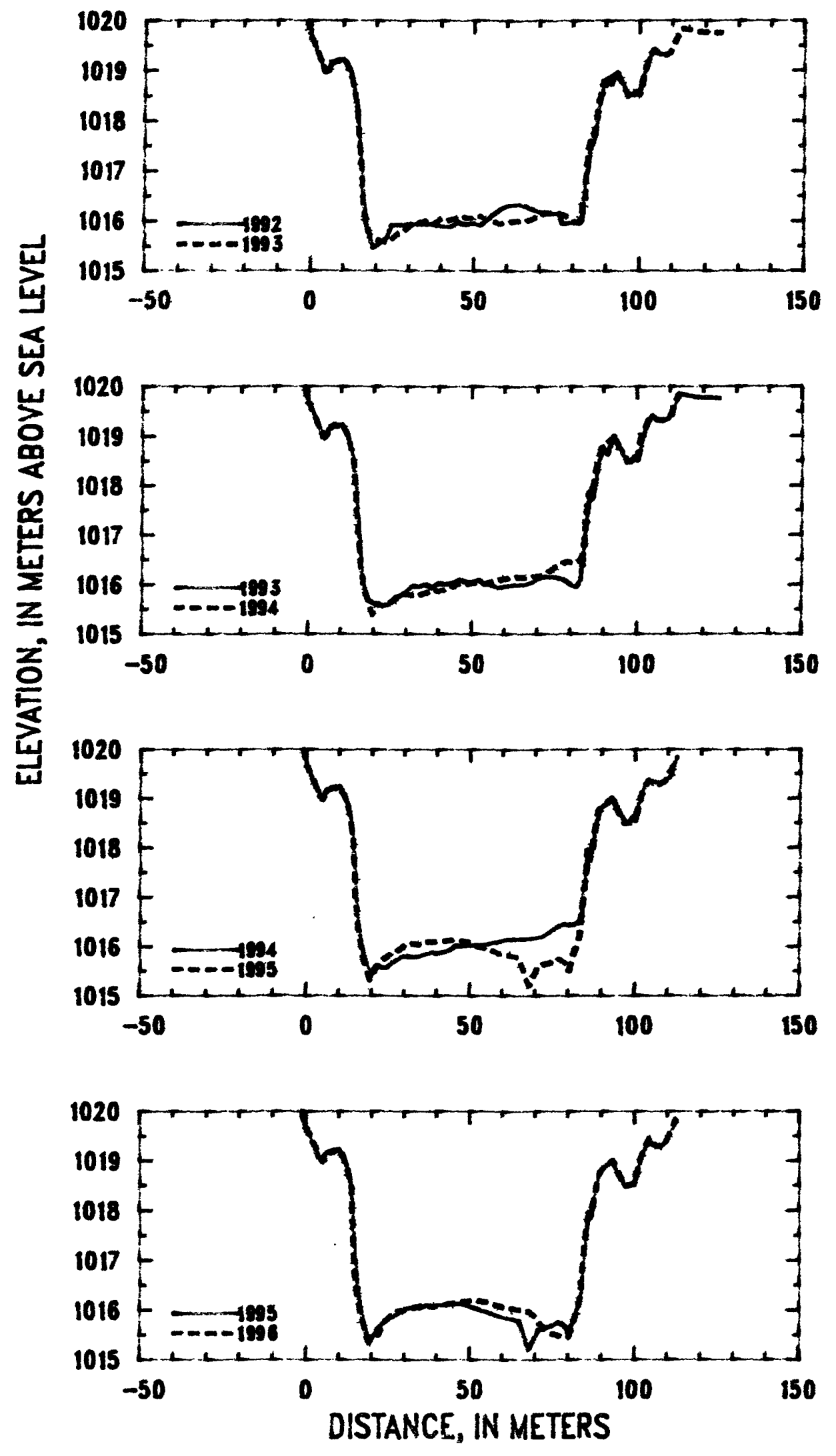

Figure 15. Profiles of cross section PR116 from 1992 to 1996. 


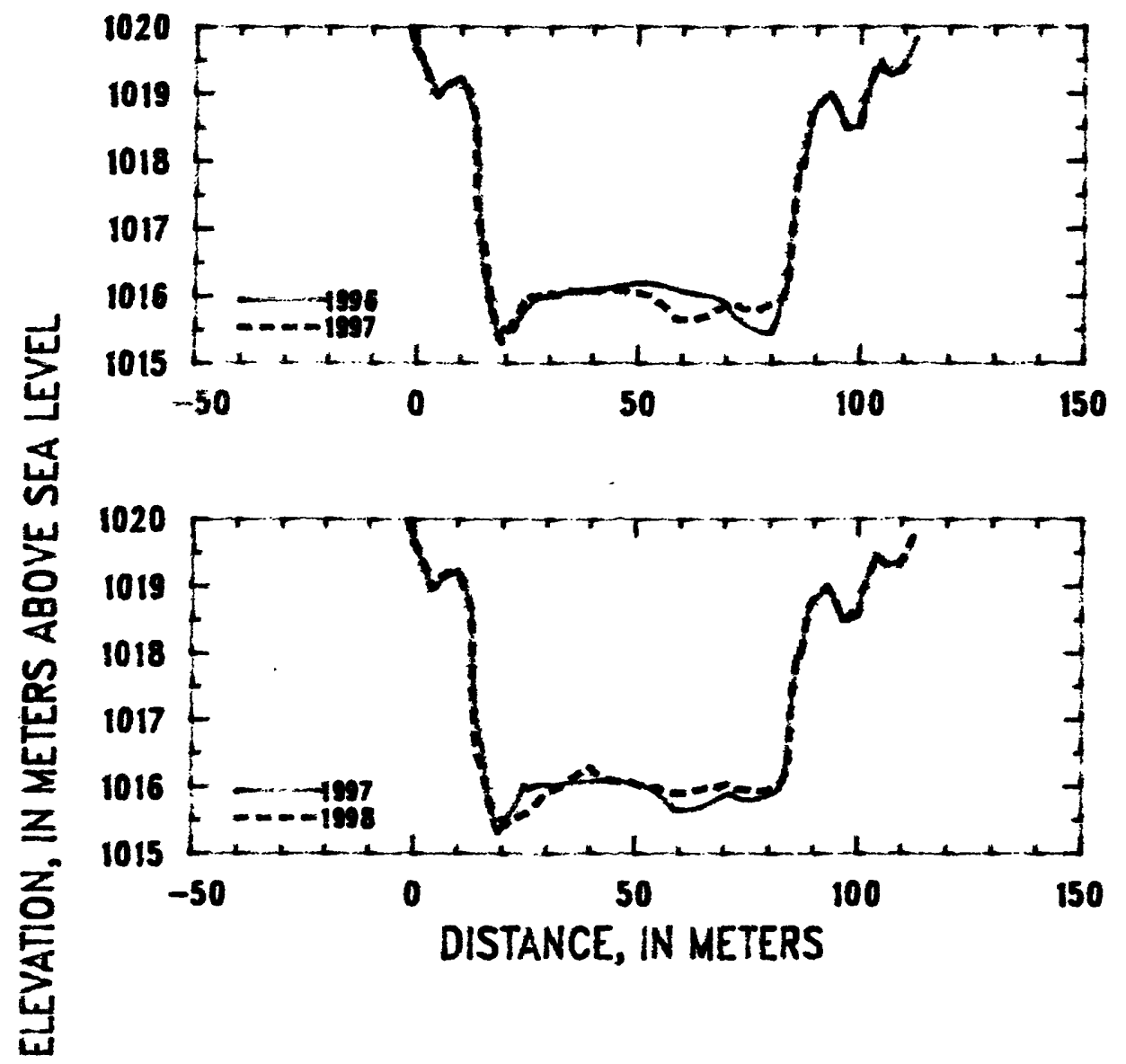

Figure 16. Profiles of cross section PR116 from 1996 to 1998. 
Table 8. Listing of horizontal stations and elevations for cross section PR116

[Sta., station, distance in meters from a reference pin on the left bank; Elev., elevation, in meters above sea level]

\begin{tabular}{|c|c|c|c|c|c|c|c|c|c|}
\hline \multirow{2}{*}{\multicolumn{2}{|c|}{$\begin{array}{c}1989 \\
16 \text { September }\end{array}$}} & \multicolumn{2}{|c|}{1989} & \multicolumn{2}{|c|}{1990} & \multicolumn{2}{|c|}{1990} & \multicolumn{2}{|c|}{1991} \\
\hline & & $16 \mathrm{~S}$ & tember & $13 \mathrm{Se}$ & tember & $13 \mathrm{Se}$ & tember & 28 & ugust \\
\hline Sta. & Elev. & Sta. & Elev. & Sta. & Elev. & Sta. & Elev. & Sta. & Elev. \\
\hline-1.7 & 1020.18 & 69.0 & 1016.37 & -1.7 & 1020.17 & 70.0 & 1015.98 & -1.7 & 1020.18 \\
\hline-1.2 & 1019.89 & 71.0 & 1016.35 & 0.0 & 1019.65 & 73.0 & 1016.00 & 0.0 & 1019.65 \\
\hline 0.0 & 1019.65 & 73.0 & 1016.29 & 2.0 & 1019.39 & 75.0 & 1016.04 & 2.0 & 1019.35 \\
\hline 2.0 & 1019.38 & 75.0 & 1016.26 & 4.0 & 1019.03 & 76.5 & 1016.01 & 4.0 & 1019.02 \\
\hline 4.0 & 1019.00 & 77.0 & 1016.23 & 5.0 & 1018.96 & 77.0 & 1015.95 & 5.0 & 1018.96 \\
\hline 5.0 & 1018.96 & 79.0 & 1016.18 & 6.0 & 1019.15 & 80.0 & 1016.04 & 7.0 & 1019.13 \\
\hline 7.0 & 1019.13 & 81.0 & 1016.19 & 8.0 & 1019.23 & 80.2 & 1016.00 & 9.0 & 1019.19 \\
\hline 9.0 & 1019.20 & 83.0 & 1016.26 & 9.0 & 1019.20 & 82.0 & 1015.96 & 10.0 & 1019.22 \\
\hline 11.0 & 1019.13 & 84.0 & 1016.48 & 10.0 & 1019.22 & 82.5 & 1016.05 & 12.0 & 1019.00 \\
\hline 12.0 & 1019.01 & 85.2 & 1017.33 & 12.0 & 1019.01 & 83.0 & 1016.39 & 13.0 & 1018.74 \\
\hline 13.0 & 1018.75 & 87.0 & 1017.80 & 13.0 & 1018.76 & 83.2 & 1016.74 & 14.2 & 1018.17 \\
\hline 14.1 & 1018.22 & 89.5 & 1018.75 & 13.6 & 1018.56 & 83.7 & 1016.85 & 14.3 & 1017.72 \\
\hline 14.2 & 1017.75 & 91.0 & 1018.86 & 14.2 & 1018.21 & 84.6 & 1017.25 & 14.9 & 1017.49 \\
\hline 15.0 & 1017.20 & 93.0 & 1018.99 & 14.4 & 1017.67 & 86.0 & 1017.57 & 16.0 & 1016.51 \\
\hline 16.0 & 1016.40 & 94.5 & 1018.76 & 14.8 & 1017.51 & 86.8 & 1017.71 & 17.0 & 1015.87 \\
\hline 16.7 & 1016.09 & 96.0 & 1018.57 & 16.6 & 1016.05 & 88.0 & 1018.30 & 18.0 & 1015.69 \\
\hline 17.5 & 1015.73 & 97.0 & 1018.47 & 17.4 & 1015.76 & 89.0 & 1018.62 & 18.7 & 1015.48 \\
\hline 19.0 & 1015.58 & 98.0 & 1018.47 & 18.0 & 1015.74 & 90.0 & 1018.84 & 20.4 & 1015.71 \\
\hline 21.0 & 1015.56 & 99.0 & 1018.56 & 18.7 & 1015.57 & 92.0 & 1018.90 & 21.2 & 1015.61 \\
\hline 23.0 & 1015.60 & 100.0 & 1018.51 & 20.0 & 1015.57 & 93.4 & 1019.01 & 22.3 & 1015.87 \\
\hline 25.0 & 1015.63 & 101.0 & 1018.95 & 23.0 & 1015.64 & 95.0 & 1018.75 & 23.2 & 1015.78 \\
\hline 27.0 & 1015.66 & & & 26.0 & 1015.62 & 97.0 & 1018.47 & 25.0 & 1015.75 \\
\hline 29.0 & 1015.71 & & & 29.0 & 1015.80 & 98.0 & 1018.52 & 27.0 & 1015.73 \\
\hline 31.0 & 1015.73 & & & 32.0 & 1015.92 & 100.6 & 1018.66 & 29.6 & 1015.83 \\
\hline 33.0 & 1015.72 & & & 35.0 & 1016.00 & 101.0 & 1018.93 & 32.0 & 1015.87 \\
\hline 35.0 & 1015.68 & & & 37.2 & 1016.06 & 102.0 & 1019.06 & 33.0 & 1015.82 \\
\hline 37.0 & 1015.70 & & & 39.0 & 1016.11 & 103.5 & 1019.36 & 35.0 & 1015.80 \\
\hline 39.0 & 1015.77 & & & 42.0 & 1016.19 & 105.0 & 1019.35 & 37.0 & 1015.81 \\
\hline 41.0 & 1015.91 & & & 45.0 & 1016.25 & 107.0 & 1019.28 & 38.3 & 1015.90 \\
\hline 43.0 & 1015.66 & & & 48.0 & 1016.30 & 109.0 & 1019.35 & 39.7 & 1015.82 \\
\hline 45.0 & 1015.92 & & & 51.0 & 1016.27 & & & 41.0 & 1015.88 \\
\hline 47.0 & 1016.04 & & & 54.0 & 1016.25 & & & 42.0 & 1015.92 \\
\hline 49.5 & 1016.09 & & & 57.0 & 1016.22 & & & 44.2 & 1016.11 \\
\hline 51.0 & 1016.10 & & & 59.0 & 1016.22 & & & 47.0 & 1016.20 \\
\hline 53.0 & 1016.10 & & & 61.0 & 1016.21 & & & 50.0 & 1016.25 \\
\hline 55.0 & 1016.19 & & & 62.0 & 1016.11 & & & 53.0 & 1016.28 \\
\hline 57.0 & 1016.22 & & & 63.0 & 1016.11 & & & 56.0 & 1016.31 \\
\hline 59.0 & 1016.25 & & & 63.5 & 1016.04 & & & 59.0 & 1016.31 \\
\hline 61.0 & 1016.27 & & & 64.3 & 1015.94 & & & 62.0 & 1016.28 \\
\hline 63.0 & 1016.31 & & & 66.5 & 1015.95 & & & 64.0 & 1016.22 \\
\hline 65.0 & 1016.35 & & & 68.3 & 1016.02 & & & 66.0 & 1016.28 \\
\hline 67.0 & 1016.37 & & & 69.0 & 1016.04 & & & 68.0 & 1016.15 \\
\hline
\end{tabular}


Table 8. (Continued) Listing of horizontal stations and elevations for cross section PR116

[Sta., station, distance in meters from a reference pin on the left bank; Elev., elevation, in meters above sea level]

\begin{tabular}{|c|c|c|c|c|c|c|c|c|c|}
\hline \multirow{2}{*}{\multicolumn{2}{|c|}{$\begin{array}{c}1991 \\
28 \text { August }\end{array}$}} & \multicolumn{2}{|c|}{1992} & \multicolumn{2}{|c|}{1992} & \multicolumn{2}{|c|}{1993} & \multicolumn{2}{|c|}{1993} \\
\hline & & \multicolumn{2}{|c|}{25 August } & \multicolumn{2}{|c|}{25 August } & \multicolumn{2}{|c|}{25 August } & \multicolumn{2}{|c|}{25 August } \\
\hline Sta. & Elev. & Sta. & Elev. & Sta. & Elev. & Sta. & Elev. & Sta. & Elev. \\
\hline 70.0 & 1016.04 & -1.7 & 1020.15 & 70.0 & 1016.17 & -1.7 & 1020.16 & 70.0 & 1016.11 \\
\hline 73.0 & 1015.95 & 0.0 & 1019.65 & 73.0 & 1016.14 & 0.0 & 1019.66 & 72.0 & 1016.16 \\
\hline 76.0 & 1015.94 & 2.0 & 1019.37 & 75.0 & 1016.14 & 2.0 & 1019.39 & 74.0 & 1016.16 \\
\hline 79.0 & 1015.86 & 4.3 & 1018.96 & 76.1 & 1016.16 & 4.3 & 1018.97 & 76.0 & 1016.15 \\
\hline 81.4 & 1015.64 & 5.4 & 1019.01 & 76.8 & 1015.95 & 5.4 & 1018.99 & 78.0 & 1016.10 \\
\hline 83.0 & 1016.17 & 6.0 & 1019.14 & 78.0 & 1015.93 & 6.1 & 1019.15 & 80.0 & 1015.99 \\
\hline 83.4 & 1016.35 & 8.0 & 1019.23 & 80.0 & 1015.97 & 8.0 & 1019.20 & 82.0 & 1015.95 \\
\hline 83.6 & 1016.39 & 10.0 & 1019.22 & 82.0 & 1015.96 & 10.0 & 1019.24 & 83.1 & 1016.11 \\
\hline 84.0 & 1016.67 & 11.0 & 1019.10 & 82.4 & 1015.96 & 12.0 & 1018.99 & 83.3 & 1016.31 \\
\hline 85.0 & 1017.26 & 12.2 & 1018.96 & 83.0 & 1016.08 & 13.0 & 1018.71 & 83.5 & 1016.45 \\
\hline 86.0 & 1017.57 & 13.0 & 1018.73 & 84.3 & 1016.97 & 14.3 & 1018.18 & 84.0 & 1016.42 \\
\hline 88.0 & 1018.29 & 14.0 & 1018.30 & 84.7 & 1017.04 & 15.0 & 1017.39 & 84.4 & 1016.96 \\
\hline 89.0 & 1018.62 & 14.3 & 1018.12 & 85.3 & 1017.40 & 15.7 & 1016.93 & 84.9 & 1017.34 \\
\hline 90.0 & 1018.84 & 15.0 & 1017.36 & 86.6 & 1017.65 & 16.1 & 1016.31 & 85.7 & 1017.59 \\
\hline 92.0 & 1018.90 & 16.0 & 1016.51 & 88.0 & 1018.29 & 17.0 & 1015.95 & 85.8 & 1017.75 \\
\hline 93.0 & 1018.99 & 16.7 & 1016.09 & 89.4 & 1018.76 & 18.2 & 1015.68 & 86.7 & 1017.73 \\
\hline 95.0 & 1018.76 & 17.3 & 1015.76 & 90.0 & 1018.83 & 19.3 & 1015.65 & 87.4 & 1017.99 \\
\hline 96.2 & 1018.55 & 19.0 & 1015.45 & 92.0 & 1018.90 & 21.0 & 1015.59 & 88.1 & 1018.36 \\
\hline 98.0 & 1018.47 & 21.0 & 1015.56 & 93.0 & 1018.98 & 23.0 & 1015.58 & 89.0 & 1018.62 \\
\hline 100.0 & 1018.55 & 23.0 & 1015.63 & 95.0 & 1018.73 & 24.5 & 1015.58 & 89.6 & 1018.78 \\
\hline 100.6 & 1018.72 & 24.6 & 1015.91 & 96.3 & 1018.53 & 26.0 & 1015.72 & 91.0 & 1018.64 \\
\hline 102.0 & 1019.07 & 27.0 & 1015.93 & 98.0 & 1018.51 & 28.0 & 1015.78 & 93.0 & 1018.99 \\
\hline & & 29.0 & 1015.92 & 100.0 & 1018.54 & 30.0 & 1015.86 & 95.0 & 1018.73 \\
\hline & & 31.0 & 1015.94 & 101.0 & 1018.94 & 32.0 & 1015.98 & 96.7 & 1018.47 \\
\hline & & 33.0 & 1015.94 & 103.0 & 1019.29 & 34.0 & 1015.96 & 98.2 & 1018.48 \\
\hline & & 35.0 & 1015.93 & 104.0 & 1019.41 & 36.0 & 1016.01 & 99.0 & 1018.58 \\
\hline & & 37.0 & 1015.94 & 106.0 & 1019.33 & 38.0 & 1015.93 & 100.0 & 1018.52 \\
\hline & & 40.0 & 1015.89 & 108.0 & 1019.31 & 40.0 & 1016.04 & 100.8 & 1018.72 \\
\hline & & 43.0 & 1015.87 & & & 42.0 & 1016.00 & 102.0 & 1019.07 \\
\hline & & 45.0 & 1015.97 & & & 44.0 & 1016.03 & 103.0 & 1019.27 \\
\hline & & 47.0 & 1015.92 & & & 46.0 & 1016.11 & 104.3 & 1019.42 \\
\hline & & 50.0 & 1015.95 & & & 48.0 & 1016.05 & 106.0 & 1019.33 \\
\hline & & 52.0 & 1015.90 & & & 50.0 & 1016.05 & 108.0 & 1019.31 \\
\hline & & 54.0 & 1016.02 & & & 52.0 & 1016.10 & 109.4 & 1019.36 \\
\hline & & 54.8 & 1016.05 & & & 54.0 & 1016.00 & 112.6 & 1019.84 \\
\hline & & 55.2 & 1016.10 & & & 56.0 & 1015.99 & 114.0 & 1019.84 \\
\hline & & 56.0 & 1016.14 & & & 58.0 & 1015.94 & 116.0 & 1019.80 \\
\hline & & 58.0 & 1016.23 & & & 60.0 & 1015.96 & 118.0 & 1019.77 \\
\hline & & 60.0 & 1016.30 & & & 62.0 & 1015.99 & 120.0 & 1019.76 \\
\hline & & 63.0 & 1016.33 & & & 64.0 & 1016.00 & 125.0 & 1019.76 \\
\hline & & 65.0 & 1016.32 & & & 66.0 & 1016.00 & & \\
\hline & & 68.0 & 1016.21 & & & 68.0 & 1016.04 & & \\
\hline
\end{tabular}


Table 8. (Continued) Listing of horizontal stations and elevations for cross section PR116

[Sta., station, distance in meters from a reference pin on the left bank; Elev., elevation, in meters above sea level]

\begin{tabular}{|c|c|c|c|c|c|c|c|c|c|}
\hline \multirow{2}{*}{\multicolumn{2}{|c|}{$\begin{array}{c}1994 \\
\text { 24 September }\end{array}$}} & \multicolumn{2}{|c|}{1994} & \multicolumn{2}{|c|}{1995} & \multicolumn{2}{|c|}{1995} & \multicolumn{2}{|c|}{1996} \\
\hline & & $24 \mathrm{~S}$ & tember & $23 \mathrm{~S}$ & tember & $23 \mathrm{~S}$ & tember & 15 & tober \\
\hline Sta. & Elev. & Sta. & Elev. & Sta. & Elev. & Sta. & Elev. & Sta. & Elev. \\
\hline-1.7 & 1020.12 & 86.6 & 1017.76 & -1.7 & 1020.15 & 82.0 & 1015.91 & -1.7 & 1020.15 \\
\hline 0.0 & 1019.67 & 88.0 & 1018.37 & 0.0 & 1019.67 & 83.0 & 1016.08 & 0.0 & 1019.66 \\
\hline 3.0 & 1019.22 & 89.0 & 1018.65 & 2.0 & 1019.39 & 83.5 & 1016.22 & 2.0 & 1019.40 \\
\hline 5.0 & 1018.98 & 90.0 & 1018.83 & 4.0 & 1019.03 & 85.8 & 1017.61 & 5.0 & 1018.97 \\
\hline 6.0 & 1019.15 & 92.0 & 1018.91 & 5.0 & 1018.97 & 86.1 & 1017.93 & 7.0 & 1019.14 \\
\hline 8.0 & 1019.23 & 93.2 & 1019.01 & 6.0 & 1019.15 & 87.0 & 1017.85 & 9.0 & 1019.19 \\
\hline 9.0 & 1019.19 & 94.5 & 1018.79 & 8.0 & 1019.21 & 89.2 & 1018.77 & 10.0 & 1019.24 \\
\hline 10.0 & 1019.25 & 95.5 & 1018.67 & 10.0 & 1019.23 & 91.0 & 1018.88 & 11.0 & 1019.12 \\
\hline 12.0 & 1019.00 & 97.0 & 1018.50 & 12.0 & 1018.99 & 93.0 & 1019.01 & 12.5 & 1018.85 \\
\hline 13.0 & 1018.74 & 98.0 & 1018.49 & 13.0 & 1018.73 & 94.5 & 1018.78 & 13.9 & 1018.41 \\
\hline 13.8 & 1018.44 & 99.0 & 1018.61 & 14.1 & 1018.27 & 96.8 & 1018.49 & 14.5 & 1016.96 \\
\hline 15.9 & 1016.36 & 100.0 & 1018.54 & 14.8 & 1017.23 & 100.0 & 1018.55 & 15.3 & 1016.36 \\
\hline 17.0 & 1015.95 & 101.0 & 1018.95 & 15.8 & 1016.42 & 102.0 & 1019.08 & 16.2 & 1016.14 \\
\hline 19.5 & 1015.40 & 103.5 & 1019.35 & 16.7 & 1016.03 & 104.0 & 1019.37 & 16.5 & 1015.99 \\
\hline 21.0 & 1015.61 & 106.0 & 1019.33 & 17.4 & 1015.75 & 107.0 & 1019.28 & 17.2 & 1015.77 \\
\hline 24.0 & 1015.55 & 108.0 & 1019.31 & 19.2 & 1015.32 & 109.0 & 1019.33 & 18.8 & 1015.38 \\
\hline 27.0 & 1015.73 & 110.7 & 1019.45 & 22.0 & 1015.65 & 112.6 & 1019.79 & 20.0 & 1015.53 \\
\hline 30.0 & 1015.82 & 111.0 & 1019.57 & 24.0 & 1015.77 & & & 22.0 & 1015.50 \\
\hline 33.0 & 1015.77 & 112.6 & 1019.84 & 26.0 & 1015.86 & & & 24.0 & 1015.77 \\
\hline 36.0 & 1015.83 & & & 28.0 & 1015.93 & & & 26.0 & 1015.90 \\
\hline 39.0 & 1015.89 & & & 30.0 & 1016.03 & & & 28.0 & 1015.99 \\
\hline 40.0 & 1015.86 & & & 33.0 & 1016.06 & & & 30.0 & 1015.99 \\
\hline 43.0 & 1015.90 & & & 35.0 & 1016.04 & & & 32.6 & 1016.04 \\
\hline 44.6 & 1015.97 & & & 36.7 & 1016.09 & & & 35.0 & 1016.08 \\
\hline 45.4 & 1016.02 & & & 39.0 & 1016.09 & & & 37.5 & 1016.09 \\
\hline 48.0 & 1016.02 & & & 41.0 & 1016.10 & & & 38.6 & 1016.07 \\
\hline 51.0 & 1016.03 & & & 43.0 & 1016.12 & & & 41.0 & 1016.09 \\
\hline 54.0 & 1016.02 & & & 45.0 & 1016.13 & & & 44.0 & 1016.12 \\
\hline 57.0 & 1016.08 & & & 47.0 & 1016.12 & & & 47.0 & 1016.16 \\
\hline 60.0 & 1016.12 & & & 49.0 & 1016.09 & & & 50.0 & 1016.19 \\
\hline 63.0 & 1016.15 & & & 50.4 & 1016.07 & & & 52.0 & 1016.19 \\
\hline 66.0 & 1016.15 & & & 53.0 & 1015.99 & & & 55.0 & 1016.16 \\
\hline 69.0 & 1016.16 & & & 56.0 & 1015.94 & & & 58.0 & 1016.11 \\
\hline 72.0 & 1016.19 & & & 59.0 & 1015.87 & & & 60.5 & 1016.05 \\
\hline 75.0 & 1016.34 & & & 62.0 & 1015.83 & & & 62.0 & 1016.04 \\
\hline 77.0 & 1016.42 & & & 65.0 & 1015.78 & & & 65.0 & 1016.00 \\
\hline 79.0 & 1016.47 & & & 68.0 & 1015.21 & & & 68.0 & 1015.97 \\
\hline 81.0 & 1016.43 & & & 71.0 & 1015.61 & & & 70.0 & 1015.86 \\
\hline 83.0 & 1016.48 & & & 74.0 & 1015.66 & & & 72.0 & 1015.66 \\
\hline 84.1 & 1016.65 & & & 77.0 & 1015.76 & & & 75.0 & 1015.53 \\
\hline 85.5 & 1017.66 & & & 79.0 & 1015.68 & & & 78.0 & 1015.45 \\
\hline 86.0 & 1017.84 & & & 80.2 & 1015.50 & & & 80.0 & 1015.46 \\
\hline
\end{tabular}


Table 8. (Continued) Listing of horizontal stations and elevations for cross section PR116

[Sta, station, distance in meters from a reference pin on the left bank; Elev., elevation, in meters above sea level]

\begin{tabular}{|c|c|c|c|c|c|c|c|c|c|}
\hline \multirow{2}{*}{\multicolumn{2}{|c|}{$\begin{array}{c}1996 \\
15 \text { October }\end{array}$}} & \multicolumn{2}{|c|}{1997} & \multicolumn{2}{|c|}{1997} & \multicolumn{2}{|c|}{1998} & \multicolumn{2}{|c|}{1998} \\
\hline & & \multicolumn{2}{|c|}{18 September } & \multicolumn{2}{|c|}{18 September } & \multicolumn{2}{|c|}{22 September } & \multicolumn{2}{|c|}{22 September } \\
\hline Sta. & Elev. & Sta. & Elev. & Sta. & Elev. & Sta. & Elev. & Sta. & Elev. \\
\hline 81.0 & 1015.61 & -1.7 & 1020.12 & 85.0 & 1017.24 & -1.7 & 1020.16 & 69.0 & 1016.01 \\
\hline 83.0 & 1016.06 & 0.0 & 1019.66 & 86.2 & 1017.95 & 0.0 & 1019.67 & 71.0 & 1016.06 \\
\hline 83.6 & 1016.23 & 2.0 & 1019.39 & 87.0 & 1017.96 & 2.0 & 1019.38 & 73.0 & 1015.97 \\
\hline 84.4 & 1016.95 & 4.0 & 1019.04 & 89.0 & 1018.73 & 4.6 & 1018.95 & 75.0 & 1015.95 \\
\hline 86.2 & 1017.94 & 5.0 & 1018.97 & 90.0 & 1018.81 & 5.6 & 1019.01 & 77.0 & 1015.94 \\
\hline 87.1 & 1017.94 & 7.0 & 1019.14 & 93.0 & 1019.00 & 6.2 & 1019.14 & 79.0 & 1015.94 \\
\hline 89.0 & 1018.67 & 9.0 & 1019.20 & 95.0 & 1018.77 & 8.0 & 1019.23 & 81.0 & 1015.98 \\
\hline 90.0 & 1018.82 & 10.0 & 1019.24 & 96.7 & 1018.48 & 9.2 & 1019.17 & 83.0 & 1016.08 \\
\hline 93.2 & 1019.01 & 12.0 & 1019.00 & 98.0 & 1018.49 & 11.0 & 1019.11 & 84.0 & 1016.24 \\
\hline 95.0 & 1018.77 & 13.5 & 1018.59 & 100.0 & 1018.57 & 12.9 & 1018.73 & 84.3 & 1016.42 \\
\hline 96.8 & 1018.48 & 13.7 & 1017.46 & 101.0 & 1018.94 & 13.3 & 1018.63 & 85.2 & 1017.39 \\
\hline 99.9 & 1018.51 & 14.2 & 1017.08 & 103.0 & 1019.31 & 13.5 & 1017.47 & 86.2 & 1017.94 \\
\hline 101.0 & 1018.92 & 15.5 & 1016.65 & 104.0 & 1019.48 & 14.0 & 1016.84 & 87.2 & 1018.00 \\
\hline 103.0 & 1019.28 & 16.6 & 1016.01 & 106.0 & 1019.32 & 14.8 & 1016.42 & 89.0 & 1018.69 \\
\hline 104.5 & 1019.48 & 18.5 & 1015.43 & 109.0 & 1019.33 & 15.8 & 1016.22 & 90.0 & 1018.81 \\
\hline 104.9 & 1019.35 & 19.3 & 1015.31 & 110.6 & 1019.46 & 17.5 & 1015.70 & 92.0 & 1018.91 \\
\hline 107.0 & 1019.28 & 21.0 & 1015.52 & & & 19.0 & 1015.61 & 93.1 & 1019.01 \\
\hline 109.0 & 1019.33 & 23.5 & 1015.78 & & & 20.6 & 1015.43 & 95.0 & 1018.75 \\
\hline 112.6 & 1019.84 & 25.0 & 1016.03 & & & 23.0 & 1015.53 & 96.7 & 1018.49 \\
\hline & & 26.0 & 1015.97 & & & 25.0 & 1015.60 & 98.7 & 1018.62 \\
\hline & & 28.0 & 1016.02 & & & 27.0 & 1015.69 & 99.8 & 1018.52 \\
\hline & & 30.0 & 1016.04 & & & 29.0 & 1015.85 & 101.2 & 1018.98 \\
\hline & & 32.0 & 1016.00 & & & 31.0 & 1015.93 & 102.2 & 1019.11 \\
\hline & & 35.0 & 1016.07 & & & 33.0 & 1015.98 & 104.2 & 1019.47 \\
\hline & & 38.0 & 1016.06 & & & 35.0 & 1016.08 & 106.0 & 1019.32 \\
\hline & & 41.0 & 1016.08 & & & 37.0 & 1016.16 & 108.0 & 1019.30 \\
\hline & & 44.0 & 1016.10 & & & 38.0 & 1016.22 & 109.3 & 1019.30 \\
\hline & & 47.0 & 1016.07 & & & 39.9 & 1016.31 & 110.7 & 1019.44 \\
\hline & & 50.0 & 1016.05 & & & 41.3 & 1016.20 & 112.6 & 1019.81 \\
\hline & & 53.0 & 1015.99 & & & 43.0 & 1016.14 & & \\
\hline & & 56.0 & 1015.85 & & & 45.0 & 1016.09 & & \\
\hline & & 59.0 & 1015.64 & & & 47.0 & 1016.07 & & \\
\hline & & 62.0 & 1015.64 & & & 49.0 & 1016.07 & & \\
\hline & & 65.0 & 1015.69 & & & 51.0 & 1016.02 & & \\
\hline & & 68.0 & 1015.80 & & & 53.0 & 1015.99 & & \\
\hline & & 71.0 & 1015.90 & & & 55.0 & 1015.99 & & \\
\hline & & 74.0 & 1015.80 & & & 57.0 & 1015.90 & & \\
\hline & & 77.0 & 1015.80 & & & 59.0 & 1015.90 & & \\
\hline & & 80.0 & 1015.88 & & & 61.0 & 1015.90 & & \\
\hline & & 82.0 & 1015.93 & & & 63.0 & 1015.93 & & \\
\hline & & 83.1 & 1016.07 & & & 65.0 & 1015.96 & & \\
\hline & & 83.6 & 1016.13 & & & 67.0 & 1016.03 & & \\
\hline
\end{tabular}




\section{Description of Cross Section PR120}

Location: Township 8 South/Range 48 East--section 33

U. S. Geological Survey quadrangle (1:24,000): Moorhead

Landowners--left bank: Larry and Linda Thomas

--right bank: 3 Bar Ranch

Access: Left bank

Permission from: Larry and Linda Thomas

Distance from Moorhead Gaging Station: 4.68 kilometers

Azimuth of Section (degrees magnetic): 114.5

\section{Reference Monuments}

[ Monuments at stations -82.0 and -30.0 were closest to leveling instrument]

\begin{tabular}{|c|c|c|c|c|c|c|}
\hline \multirow[b]{2}{*}{ Description } & \multirow[b]{2}{*}{$\begin{array}{c}\text { Station } \\
\text { (m) }\end{array}$} & \multicolumn{2}{|c|}{ GPS-NAD83 (1992) } & \multicolumn{2}{|c|}{ Measurement } & \multirow{2}{*}{$\begin{array}{c}\text { Elevation } \\
\text { (NGVD1929) } \\
\text { (m) }\end{array}$} \\
\hline & & Latitude & Longitude & $\begin{array}{l}\text { Standard } \\
\text { deviation } \\
\text { (m) }\end{array}$ & $\begin{array}{l}\text { Horizontal } \\
\text { precision } \\
\text { (m) }\end{array}$ & \\
\hline $\begin{array}{l}\text { 1/2-inch-rebar; } 0.06 \text { meter below } 1998 \text { ground } \\
\text { level }\end{array}$ & -82.0 & & & & & \\
\hline Benchmark--brass circular plate & -30.0 & $45^{\circ} 05^{\prime} 32.49^{\prime \prime}$ & $105^{\circ} 51^{\prime} 14.17^{\prime \prime}$ & 0.619 & 0.624 & 1014.70 \\
\hline $\begin{array}{l}\text { 1/2-inch-rebar; } 0.16 \text { meter above } 1998 \text { ground } \\
\text { level }\end{array}$ & 100.9 & & & & & 1013.93 \\
\hline $\begin{array}{l}\text { 1/2-inch-rebar; } 0.20 \text { meter above } 1998 \text { ground } \\
\text { level }\end{array}$ & 104.0 & $45^{\circ} 05^{\prime} 29.73^{\prime \prime}$ & $105^{\circ} 51^{\prime} 09.40^{\prime \prime}$ & 0.243 & 0.481 & 1014.05 \\
\hline
\end{tabular}




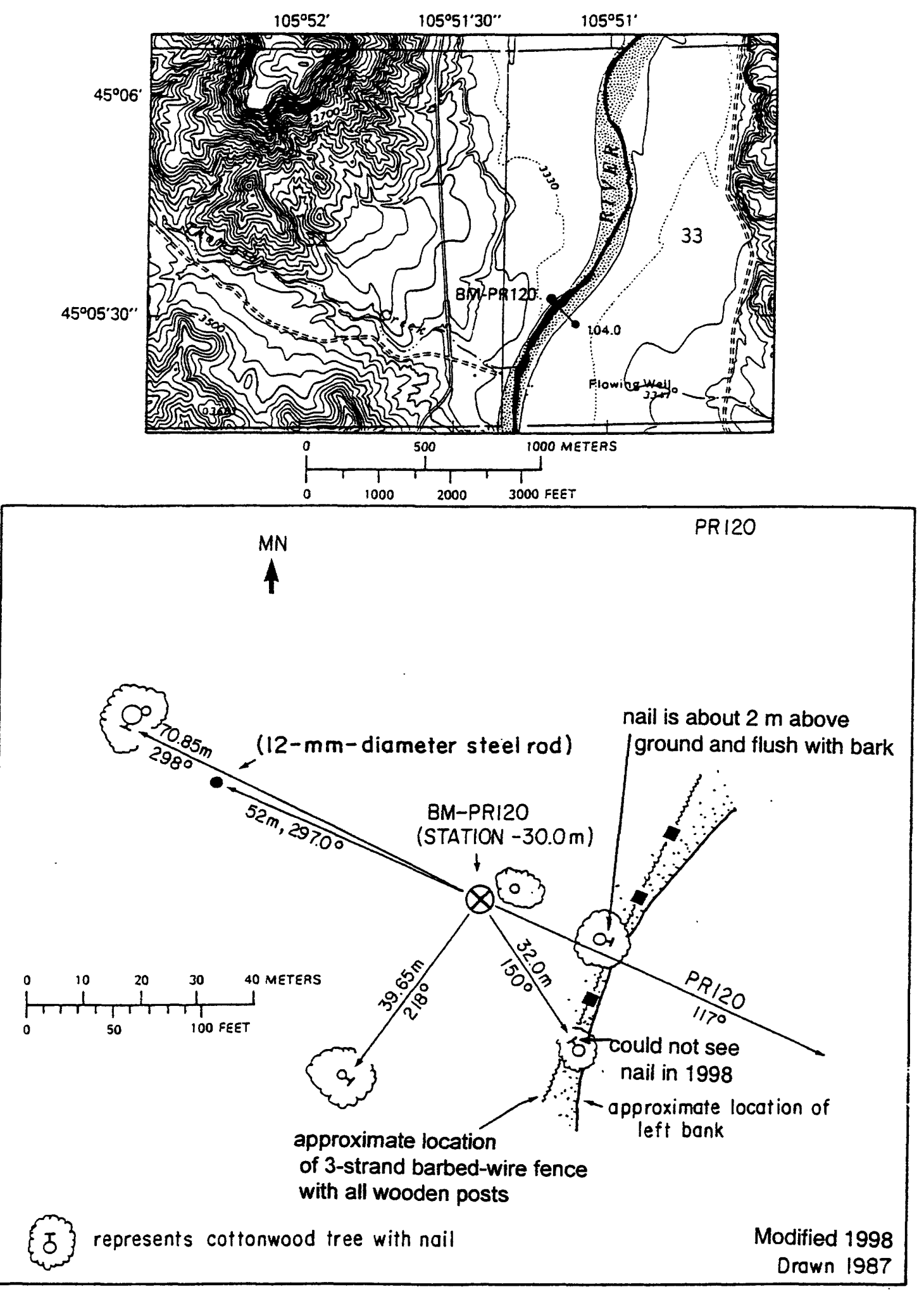

Figure 17. Upper: Location of cross section PR120, bench mark BM-PR120, and the right bank reference monument in the Moorhead quadrangle. Lower: Location of the bench mark on the left bank. MN is magnetic north. 

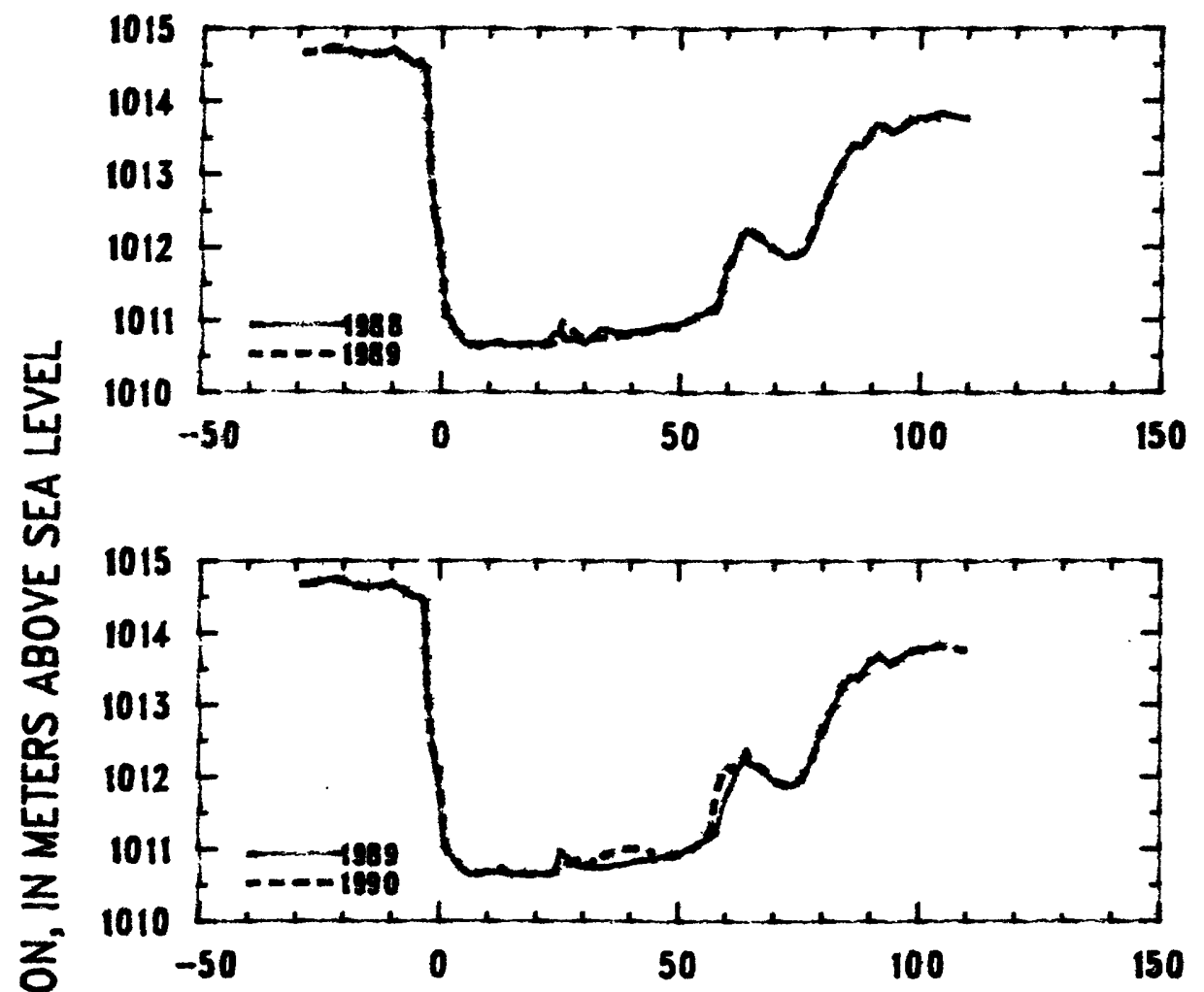

톤
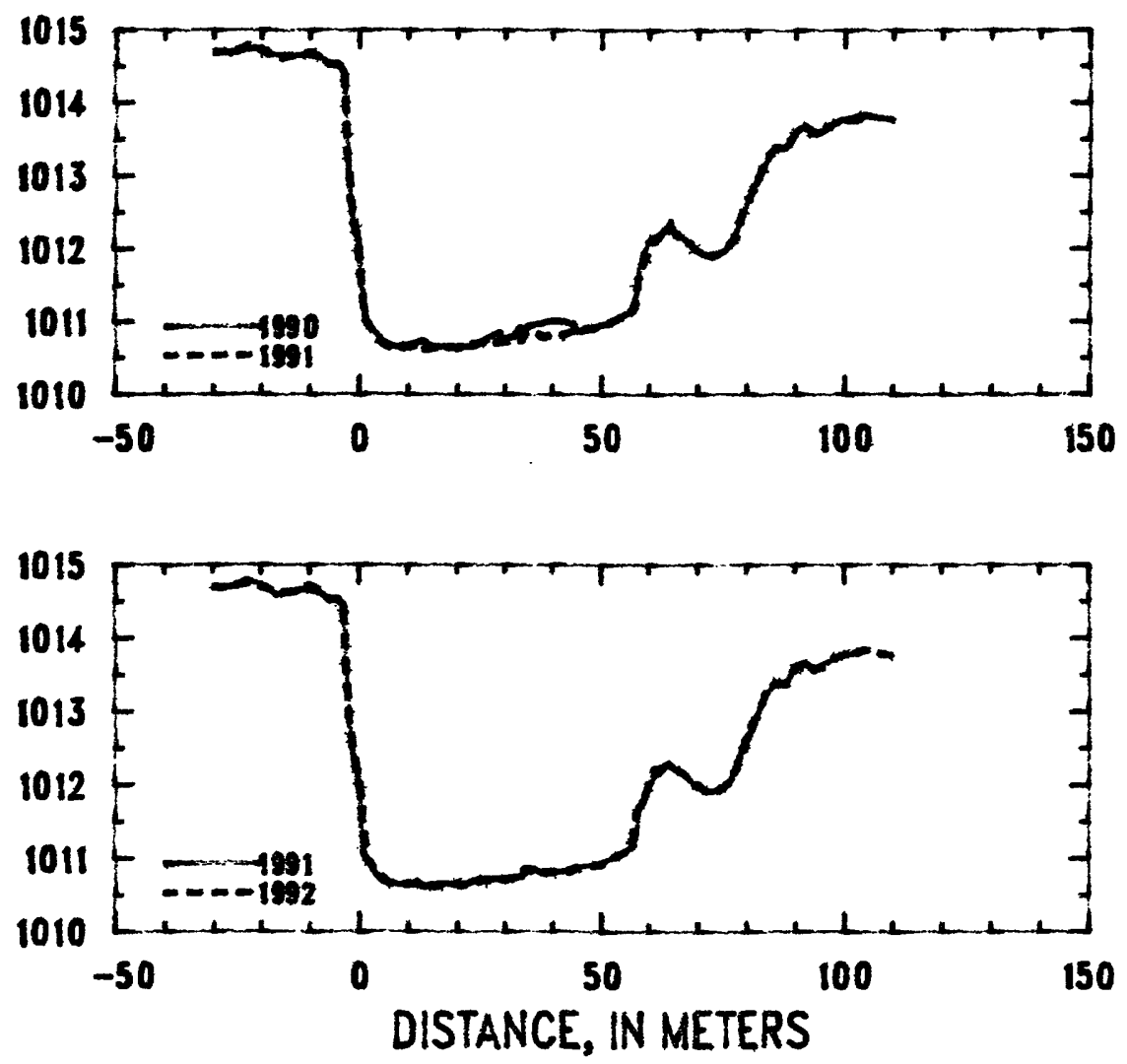

Figure 18. Profiles of cross section PR120 from 1988 to 1992. 

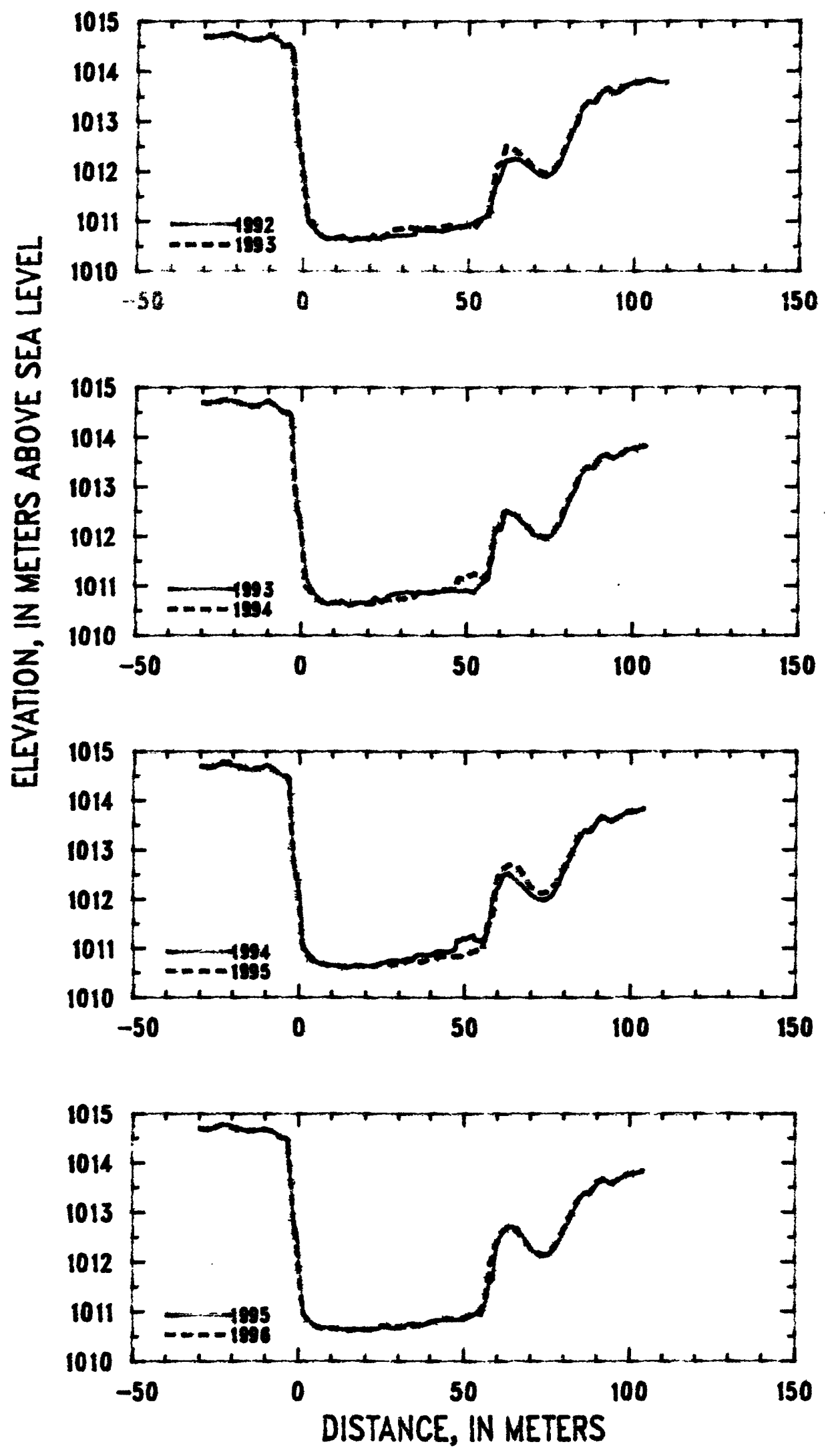

Figure 19. Profiles of cross section PR120 from 1992 to 1996. 


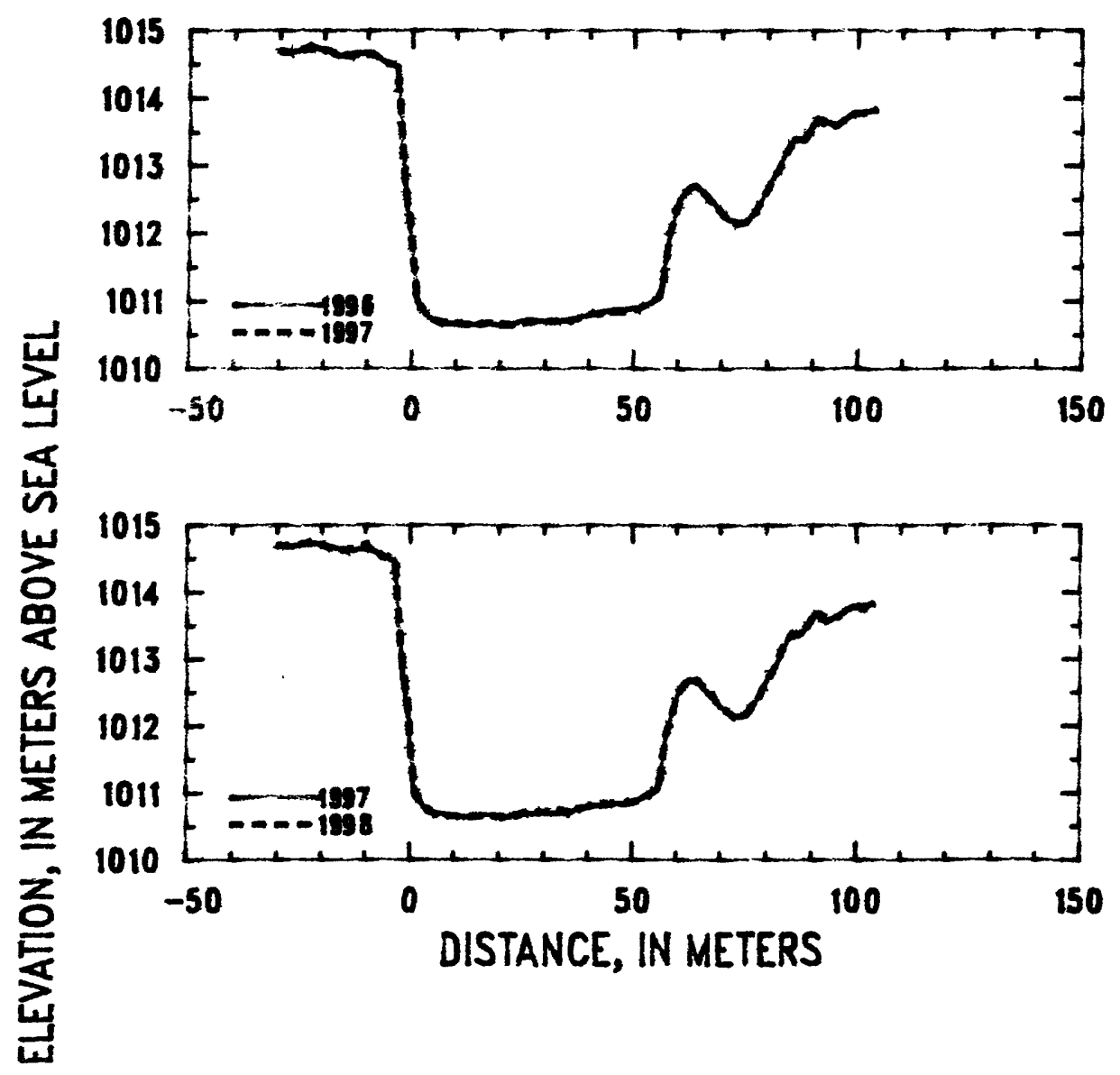

Figure 20. Profiles of cross section PR120 from 1996 to 1998. 
Table 9. Listing of horizontal stations and elevations for cross section PR120

[Sta., station, distance in meters from a reference pin on the left bank; Elev., elevation, in meters above sea level]

\begin{tabular}{|c|c|c|c|c|c|c|c|c|c|}
\hline \multirow{2}{*}{\multicolumn{2}{|c|}{$\begin{array}{r}1989 \\
\text { 16 September } \\
\end{array}$}} & \multicolumn{2}{|c|}{1989} & \multicolumn{2}{|c|}{1990} & \multicolumn{2}{|c|}{1990} & \multicolumn{2}{|c|}{1991} \\
\hline & & $16 \mathrm{Se}$ & tember & $14 \mathrm{Se}$ & tember & $14 \mathrm{Se}$ & tember & 28 & ugust \\
\hline Sta. & Elev. & Sta. & Elev. & Sta. & Elev. & Sta. & Elev. & Sta. & Elev. \\
\hline-29.0 & 1014.68 & 45.0 & 1010.87 & -27.0 & 1014.68 & 44.4 & 1010.97 & -30.0 & 1014.70 \\
\hline-26.0 & 1014.68 & 47.0 & 1010.91 & -24.0 & 1014.74 & 44.8 & 1010.86 & -29.0 & 1014.69 \\
\hline-23.0 & 1014.75 & 49.0 & 1010.88 & -21.0 & 1014.76 & 47.0 & 1010.90 & -26.0 & 1014.69 \\
\hline-20.0 & 1014.72 & 50.6 & 1010.95 & -18.0 & 1014.66 & 49.0 & 1010.94 & -23.0 & 1014.79 \\
\hline-17.0 & 1014.64 & 52.0 & 1011.00 & -15.0 & 1014.65 & 51.0 & 1010.96 & -20.0 & 1014.73 \\
\hline-14.0 & 1014.66 & 54.0 & 1011.08 & -12.0 & 1014.64 & 53.0 & 1011.02 & -17.0 & 1014.59 \\
\hline-11.0 & 1014.68 & 56.0 & 1011.14 & -10.0 & 1014.71 & 55.0 & 1011.11 & -14.0 & 1014.64 \\
\hline-9.0 & 1014.66 & 58.2 & 1011.22 & -8.0 & 1014.62 & 56.0 & 1011.12 & -11.0 & 1014.67 \\
\hline-7.0 & 1014.56 & 60.0 & 1011.74 & -6.0 & 1014.52 & 57.2 & 1011.34 & -8.0 & 1014.63 \\
\hline-5.0 & 1014.50 & 61.6 & 1011.92 & -4.6 & 1014.52 & 58.0 & 1011.76 & -6.0 & 1014.52 \\
\hline-4.0 & 1014.50 & 63.0 & 1012.17 & -4.0 & 1014.51 & 59.0 & 1011.96 & -4.7 & 1014.53 \\
\hline-3.2 & 1014.45 & 64.0 & 1012.24 & -3.0 & 1014.41 & 60.0 & 1012.11 & -3.1 & 1014.46 \\
\hline-3.0 & 1014.25 & 66.0 & 1012.17 & -2.4 & 1013.17 & 60.7 & 1012.15 & -2.4 & 1013.20 \\
\hline-2.6 & 1013.71 & 68.0 & 1012.08 & -1.6 & 1012.56 & 61.4 & 1012.10 & -1.2 & 1012.42 \\
\hline-2.5 & 1013.28 & 70.0 & 1011.95 & -0.9 & 1012.28 & 63.0 & 1012.25 & -0.3 & 1012.17 \\
\hline-1.2 & 1012.38 & 72.0 & 1011.87 & -0.2 & 1012.20 & 63.9 & 1012.28 & 1.1 & 1011.04 \\
\hline-0.3 & 1012.07 & 74.0 & 1011.89 & 1.3 & 1011.03 & 64.4 & 1012.36 & 3.7 & 1010.78 \\
\hline 0.9 & 1011.17 & 76.0 & 1011.96 & 2.2 & 1010.92 & 65.0 & 1012.24 & 5.0 & 1010.68 \\
\hline 1.2 & 1011.02 & 77.2 & 1012.17 & 3.0 & 1010.87 & 66.0 & 1012.18 & 7.0 & 1010.66 \\
\hline 2.0 & 1010.95 & 78.4 & 1012.30 & 5.0 & 1010.71 & 68.0 & 1012.12 & 8.7 & 1010.64 \\
\hline 4.0 & 1010.78 & 79.5 & 1012.62 & 7.0 & 1010.67 & 70.0 & 1011.96 & 10.6 & 1010.66 \\
\hline 6.0 & 1010.67 & 80.3 & 1012.63 & 9.0 & 1010.66 & 72.0 & 1011.91 & 11.9 & 1010.69 \\
\hline 8.0 & 1010.65 & 82.0 & 1012.92 & 11.0 & 1010.70 & 74.0 & 1011.92 & 13.0 & 1010.62 \\
\hline 10.0 & 1010.68 & 84.0 & 1013.19 & 13.0 & 1010.74 & 76.0 & 1012.00 & 15.0 & 1010.63 \\
\hline 12.0 & 1010.69 & 86.0 & 1013.40 & 15.0 & 1010.65 & 78.0 & 1012.26 & 17.0 & 1010.67 \\
\hline 14.0 & 1010.65 & 88.0 & 1013.38 & 17.0 & 1010.67 & 80.0 & 1012.64 & 19.0 & 1010.65 \\
\hline 16.0 & 1010.65 & 90.0 & 1013.61 & 19.0 & 1010.65 & 81.0 & 1012.85 & 21.0 & 1010.62 \\
\hline 18.0 & 1010.67 & 92.0 & 1013.68 & 21.0 & 1010.67 & 82.0 & 1012.90 & 23.0 & 1010.68 \\
\hline 20.0 & 1010.66 & 94.0 & 1013.57 & 23.0 & 1010.66 & 84.0 & 1013.20 & 25.0 & 1010.72 \\
\hline 22.0 & 1010.65 & 96.0 & 1013.64 & 25.0 & 1010.71 & 85.0 & 1013.32 & 27.0 & 1010.72 \\
\hline 23.0 & 1010.66 & 98.0 & 1013.73 & 27.0 & 1010.80 & 86.0 & 1013.40 & 30.0 & 1010.73 \\
\hline 24.3 & 1010.70 & 100.0 & 1013.78 & 29.0 & 1010.86 & 88.0 & 1013.38 & 31.6 & 1010.74 \\
\hline 25.2 & 1010.97 & 101.0 & 1013.76 & 29.5 & 1010.74 & 90.0 & 1013.60 & 33.6 & 1010.75 \\
\hline 27.0 & 1010.87 & 104.0 & 1013.82 & 32.0 & 1010.79 & 92.0 & 1013.68 & 34.0 & 1010.85 \\
\hline 29.0 & 1010.76 & & & 33.0 & 1010.91 & 93.5 & 1013.57 & 36.0 & 1010.86 \\
\hline 31.0 & 1010.75 & & & 33.3 & 1010.91 & 95.0 & 1013.58 & 38.0 & 1010.79 \\
\hline 33.0 & 1010.75 & & & 34.0 & 1010.88 & 96.0 & 1013.64 & 40.0 & 1010.82 \\
\hline 35.0 & 1010.74 & & & 35.0 & 1010.94 & 99.0 & 1013.76 & 42.0 & 1010.82 \\
\hline 37.0 & 1010.78 & & & 37.0 & 1010.96 & 101.0 & 1013.77 & 45.0 & 1010.90 \\
\hline 39.0 & 1010.78 & & & 39.0 & 1011.01 & 102.5 & 1013.75 & 48.0 & 1010.91 \\
\hline 41.0 & 1010.82 & & & 41.0 & 1011.01 & 104.0 & 1013.83 & 51.0 & 1010.96 \\
\hline 43.0 & 1010.84 & & & 43.0 & 1010.99 & 107.0 & 1013.78 & 54.0 & 1011.07 \\
\hline & & & & & & 110.0 & 1013.76 & & \\
\hline
\end{tabular}


Table 9. (Continued) Listing of horizontal stations and elevations for cross section PR120

[Sta., station, distance in meters from a reference pin on the left bank; Elev., elevation, in meters above sea level]

\begin{tabular}{|c|c|c|c|c|c|c|c|c|c|}
\hline \multirow{2}{*}{\multicolumn{2}{|c|}{$\begin{array}{c}1991 \\
28 \text { August }\end{array}$}} & \multicolumn{2}{|c|}{1992} & \multicolumn{2}{|c|}{1992} & \multicolumn{2}{|c|}{1993} & \multicolumn{2}{|c|}{1993} \\
\hline & & \multicolumn{2}{|c|}{25 August } & \multicolumn{2}{|c|}{25 August } & \multicolumn{2}{|c|}{25 August } & \multicolumn{2}{|c|}{25 August } \\
\hline Sta. & Elev. & Sta. & Elev. & Sta. & Elev. & Sta. & Elev. & Sta. & Elev. \\
\hline 56.8 & 1011.17 & -30.0 & 1014.70 & 50.0 & 1010.92 & -30.0 & 1014.70 & 56.2 & 1011.14 \\
\hline 58.1 & 1011.73 & -28.0 & 1014.69 & 52.0 & 1011.00 & -28.0 & 1014.70 & 57.1 & 1011.52 \\
\hline 58.6 & 1011.75 & -25.0 & 1014.73 & 54.0 & 1011.06 & -25.0 & 1014.73 & 57.3 & 1011.53 \\
\hline 61.0 & 1012.14 & -22.0 & 1014.77 & 56.0 & 1011.13 & -22.0 & 1014.75 & 57.6 & 1011.64 \\
\hline 62.0 & 1012.17 & -19.0 & 1014.68 & 56.3 & 1011.18 & -19.0 & 1014.70 & 58.3 & 1012.08 \\
\hline 64.0 & 1012.30 & -16.0 & 1014.63 & 57.4 & 1011.59 & -16.0 & 1014.63 & 59.0 & 1012.14 \\
\hline 66.0 & 1012.18 & -13.0 & 1014.64 & 58.3 & 1011.77 & -13.0 & 1014.65 & 59.5 & 1012.15 \\
\hline 68.0 & 1012.11 & -10.0 & 1014.72 & 59.0 & 1011.83 & -10.0 & 1014.74 & 60.0 & 1012.15 \\
\hline 70.0 & 1011.95 & -8.0 & 1014.64 & 60.0 & 1012.01 & -8.0 & 1014.65 & 60.5 & 1012.22 \\
\hline 72.0 & 1011.90 & -6.0 & 1014.52 & 61.0 & 1012.20 & -6.0 & 1014.51 & 61.2 & 1012.50 \\
\hline 74.0 & 1011.92 & -4.5 & 1014.54 & 63.0 & 1012.25 & -4.0 & 1014.51 & 62.0 & 1012.50 \\
\hline 76.0 & 1012.00 & -3.2 & 1014.45 & 65.0 & 1012.25 & -3.0 & 1014.41 & 63.5 & 1012.45 \\
\hline 78.0 & 1012.25 & -2.5 & 1013.40 & 68.0 & 1012.10 & -2.4 & 1013.43 & 65.0 & 1012.39 \\
\hline 79.5 & 1012.61 & -1.8 & 1012.75 & 71.0 & 1011.93 & -1.0 & 1012.45 & 66.4 & 1012.29 \\
\hline 80.0 & 1012.63 & -0.6 & 1012.28 & 73.0 & 1011.88 & -0.6 & 1012.41 & 68.0 & 1012.21 \\
\hline 82.0 & 1012.90 & 0.5 & 1011.80 & 74.0 & 1011.93 & 1.6 & 1011.13 & 70.0 & 1012.03 \\
\hline 83.0 & 1013.09 & 1.5 & 1011.00 & 76.0 & 1012.02 & 4.0 & 1010.84 & 72.0 & 1011.99 \\
\hline 85.0 & 1013.32 & 2.4 & 1010.92 & 78.0 & 1012.26 & 6.0 & 1010.69 & 73.5 & 1011.95 \\
\hline 86.0 & 1013.39 & 4.3 & 1010.77 & 80.0 & 1012.63 & 8.0 & 1010.63 & 75.0 & 1012.01 \\
\hline 88.0 & 1013.37 & 6.0 & 1010.67 & 81.5 & 1012.88 & 10.0 & 1010.66 & 76.5 & 1012.11 \\
\hline 90.0 & 1013.60 & 8.0 & 1010.65 & 82.5 & 1012.96 & 12.0 & 1010.69 & 78.0 & 1012.27 \\
\hline 92.0 & 1013.67 & 10.0 & 1010.66 & 84.0 & 1013.26 & 14.0 & 1010.61 & 79.0 & 1012.46 \\
\hline 94.0 & 1013.57 & 12.0 & 1010.66 & 86.0 & 1013.39 & 16.0 & 1010.66 & 80.0 & 1012.62 \\
\hline 97.0 & 1013.70 & 14.0 & 1010.63 & 88.0 & 1013.38 & 18.0 & 1010.66 & 81.5 & 1012.88 \\
\hline 100.0 & 1013.78 & 16.0 & 1010.63 & 90.0 & 1013.60 & 20.0 & 1010.64 & 82.5 & 1012.95 \\
\hline 101.0 & 1013.77 & 18.0 & 1010.66 & 92.0 & 1013.67 & 22.0 & 1010.76 & 84.0 & 1013.26 \\
\hline 104.0 & 1013.84 & 20.0 & 1010.66 & 94.0 & 1013.57 & 24.0 & 1010.67 & 86.0 & 1013.40 \\
\hline & & 22.0 & 1010.64 & 96.0 & 1013.64 & 26.0 & 1010.81 & 88.0 & 1013.38 \\
\hline & & 24.0 & 1010.69 & 98.0 & 1013.74 & 28.0 & 1010.85 & 90.0 & 1013.61 \\
\hline & & 26.0 & 1010.70 & 100.0 & 1013.79 & 30.0 & 1010.85 & 92.0 & 1013.67 \\
\hline & & 28.0 & 1010.72 & 101.0 & 1013.76 & 32.0 & 1010.89 & 94.0 & 1013.58 \\
\hline & & 30.0 & 1010.72 & 104.0 & 1013.84 & 34.0 & 1010.85 & 97.0 & 1013.70 \\
\hline & & 32.0 & 1010.72 & 107.0 & 1013.79 & 36.0 & 1010.88 & 100.0 & 1013.79 \\
\hline & & 33.8 & 1010.75 & 110.0 & 1013.77 & 38.0 & 1010.86 & 101.0 & 1013.77 \\
\hline & & 34.3 & 1010.86 & & & 40.0 & 1010.87 & 104.0 & 1013.83 \\
\hline & & 36.0 & 1010.83 & & & 42.0 & 1010.88 & & \\
\hline & & 38.0 & 1010.81 & & & 44.0 & 1010.92 & & \\
\hline & & 40.0 & 1010.82 & & & 46.0 & 1010.91 & & \\
\hline & & 42.0 & 1010.80 & & & 48.0 & 1010.88 & & \\
\hline & & 44.0 & 1010.83 & & & 50.0 & 1010.92 & & \\
\hline & & 46.0 & 1010.89 & & & 52.0 & 1010.86 & & \\
\hline & & 48.0 & 1010.90 & & & 54.0 & 1011.04 & & \\
\hline
\end{tabular}


Table 9. (Continued) Listing of horizontal stations and elevations for cross section PR120

[Sta., station, distance in meters from a reference pin on the left bank; Elev., elevation, in meters above sea level]

\begin{tabular}{|c|c|c|c|c|c|c|c|c|c|}
\hline \multirow{2}{*}{\multicolumn{2}{|c|}{$\begin{array}{c}1994 \\
24 \text { September } \\
\end{array}$}} & \multicolumn{2}{|c|}{1994} & \multicolumn{2}{|c|}{1995} & \multicolumn{2}{|c|}{1995} & \multicolumn{2}{|c|}{1996} \\
\hline & & $24 \mathrm{Se}$ & tember & $23 \mathrm{Se}$ & tember & $23 \mathrm{Se}$ & tember & 150 & tober \\
\hline Sta. & Elev. & Sta. & Elev. & Sta. & Elev. & Sta. & Elev. & Sta. & Elev. \\
\hline-30.0 & 1014.70 & 50.0 & 1011.19 & -28.0 & 1014.68 & 66.0 & 1012.67 & -30.0 & 1014.70 \\
\hline-28.0 & 1014.68 & 52.5 & 1011.26 & -26.0 & 1014.69 & 68.0 & 1012.47 & -29.0 & 1014.68 \\
\hline-26.0 & 1014.69 & 53.0 & 1011.18 & -23.0 & 1014.78 & 70.0 & 1012.25 & -26.0 & 1014.69 \\
\hline-24.0 & 1014.76 & 54.5 & 1011.15 & -20.0 & 1014.75 & 72.0 & 1012.15 & -23.0 & 1014.78 \\
\hline-21.0 & 1014.75 & 56.0 & 1011.20 & -17.0 & 1014.67 & 73.0 & 1012.12 & -20.0 & 1014.72 \\
\hline-18.0 & 1014.67 & 56.5 & 1011.29 & -14.0 & 1014.64 & 75.0 & 1012.15 & -17.0 & 1014.63 \\
\hline-16.0 & 1014.62 & 57.2 & 1011.52 & -11.0 & 1014.68 & 76.0 & 1012.20 & -14.0 & 1014.66 \\
\hline-13.0 & 1014.63 & 58.3 & 1012.02 & -8.0 & 1014.64 & 78.0 & 1012.37 & -11.0 & 1014.68 \\
\hline-10.0 & 1014.73 & 58.7 & 1012.13 & -6.0 & 1014.53 & 80.0 & 1012.66 & -9.0 & 1014.67 \\
\hline-8.0 & 1014.64 & 60.3 & 1012.27 & -4.0 & 1014.50 & 82.0 & 1012.91 & -6.0 & 1014.52 \\
\hline-6.0 & 1014.53 & 61.3 & 1012.49 & -3.3 & 1014.46 & 84.0 & 1013.24 & -3.3 & 1014.47 \\
\hline-3.1 & 1014.44 & 61.9 & 1012.51 & -2.7 & 1013.75 & 86.0 & 1013.39 & -1.5 & 1012.74 \\
\hline-2.5 & 1013.57 & 63.0 & 1012.53 & -1.5 & 1012.69 & 88.0 & 1013.38 & -0.8 & 1012.42 \\
\hline-1.7 & 1012.74 & 65.0 & 1012.41 & -1.0 & 1012.63 & 90.0 & 1013.60 & 1.3 & 1010.98 \\
\hline-1.0 & 1012.45 & 67.0 & 1012.28 & -0.4 & 1012.41 & 92.0 & 1013.67 & 1.8 & 1010.93 \\
\hline-0.3 & 1012.29 & 69.0 & 1012.17 & 1.2 & 1010.96 & 94.0 & 1013.58 & 3.0 & 1010.79 \\
\hline 0.5 & 1011.81 & 70.0 & 1012.08 & 4.0 & 1010.75 & 96.0 & 1013.64 & 6.0 & 1010.66 \\
\hline 1.2 & 1011.00 & 72.0 & 1012.00 & 7.0 & 1010.68 & 98.5 & 1013.76 & 9.0 & 1010.66 \\
\hline 3.0 & 1010.87 & 74.0 & 1011.99 & 10.0 & 1010.65 & 101.0 & 1013.78 & 12.0 & 1010.66 \\
\hline 5.5 & 1010.70 & 75.0 & 1012.02 & 13.0 & 1010.63 & 104.0 & 1013.83 & 15.0 & 1010.62 \\
\hline 8.0 & 1010.64 & 76.0 & 1012.07 & 16.0 & 1010.65 & & & 18.0 & 1010.66 \\
\hline 11.0 & 1010.67 & 77.5 & 1012.25 & 19.0 & 1010.64 & & & 21.0 & 1010.63 \\
\hline 14.0 & 1010.59 & 78.6 & 1012.37 & 22.0 & 1010.63 & & & 24.0 & 1010.70 \\
\hline 17.0 & 1010.66 & 79.0 & 1012.51 & 25.0 & 1010.70 & & & 27.0 & 1010.72 \\
\hline 20.0 & 1010.63 & 80.0 & 1012.64 & 28.0 & 1010.66 & & & 30.0 & 1010.69 \\
\hline 23.0 & 1010.67 & 82.0 & 1012.90 & 31.0 & 1010.71 & & & 33.0 & 1010.70 \\
\hline 26.0 & 1010.74 & 83.4 & 1013.14 & 34.0 & 1010.77 & & & 36.0 & 1010.72 \\
\hline 29.0 & 1010.75 & 85.0 & 1013.33 & 37.0 & 1010.73 & & & 39.0 & 1010.78 \\
\hline 31.0 & 1010.74 & 86.0 & 1013.40 & 40.0 & 1010.79 & & & 42.0 & 1010.83 \\
\hline 32.0 & 1010.78 & 88.0 & 1013.37 & 43.0 & 1010.84 & & & 45.0 & 1010.86 \\
\hline 33.0 & 1010.77 & 90.0 & 1013.60 & 46.0 & 1010.85 & & $\cdot$ & 48.0 & 1010.87 \\
\hline 34.5 & 1010.80 & 91.0 & 1013.68 & 49.0 & 1010.84 & & & 51.0 & 1010.89 \\
\hline 35.2 & 1010.86 & 93.0 & 1013.62 & 51.0 & 1010.89 & & & 53.0 & 1010.94 \\
\hline 36.2 & 1010.88 & 94.0 & 1013.57 & 53.5 & 1010.97 & & & 55.0 & 1011.02 \\
\hline 38.0 & 1010.87 & 96.0 & 1013.65 & 55.0 & 1010.94 & & & 56.2 & 1011.16 \\
\hline 40.0 & 1010.87 & 99.0 & 1013.78 & 56.3 & 1011.20 & & & 57.1 & 1011.58 \\
\hline 42.0 & 1010.94 & 101.0 & 1013.77 & 57.4 & 1011.61 & & & 57.9 & 1011.96 \\
\hline 45.0 & 1010.93 & 104.0 & 1013.84 & 58.4 & 1011.66 & & & 60.0 & 1012.43 \\
\hline 47.0 & 1010.98 & & & 59.2 & 1012.14 & & & 61.0 & 1012.56 \\
\hline 47.5 & 1011.16 & & & 60.1 & 1012.46 & & & 63.0 & 1012.70 \\
\hline 48.5 & 1011.15 & & & 62.0 & 1012.65 & & & 64.0 & 1012.71 \\
\hline 49.5 & 1011.21 & & & 64.0 & 1012.71 & & & 66.0 & 1012.60 \\
\hline
\end{tabular}


Table 9. (Continued) Listing of horizontal stations and elevations for cross section PR120

[Sta., station, distance in meters from a reference pin on the left bank; Elev., elevation, in meters above sea level]

\begin{tabular}{|c|c|c|c|c|c|c|c|c|c|}
\hline \multirow{2}{*}{\multicolumn{2}{|c|}{$\begin{array}{c}1996 \\
15 \text { October }\end{array}$}} & \multicolumn{2}{|c|}{1997} & \multicolumn{2}{|c|}{1997} & \multicolumn{2}{|c|}{1998} & \multicolumn{2}{|c|}{1998} \\
\hline & & \multicolumn{2}{|c|}{18 September } & \multicolumn{2}{|c|}{18 September } & \multicolumn{2}{|c|}{25 September } & \multicolumn{2}{|c|}{25 September } \\
\hline Sta. & Elev. & Sta. & Elev. & Sta. & Elev. & Sta. & Elev. & Sta. & Elev. \\
\hline 68.0 & 1012.47 & -30.0 & 1014.70 & 69.0 & 1012.37 & -30.0 & 1014.70 & 54.5 & 1011.00 \\
\hline 70.0 & 1012.26 & -27.0 & 1014.67 & 71.0 & 1012.23 & -28.0 & 1014.70 & 56.0 & 1011.09 \\
\hline 72.0 & 1012.18 & -24.0 & 1014.73 & 73.0 & 1012.13 & -26.0 & 1014.70 & 56.5 & 1011.27 \\
\hline 74.0 & 1012.15 & -21.0 & 1014.74 & 75.0 & 1012.16 & -24.0 & 1014.73 & 57.0 & 1011.55 \\
\hline 75.0 & 1012.15 & -18.0 & 1014.68 & 77.0 & 1012.30 & -22.3 & 1014.77 & 57.5 & 1011.79 \\
\hline 77.0 & 1012.31 & -15.0 & 1014.63 & 79.0 & 1012.53 & -20.0 & 1014.71 & 57.8 & 1011.94 \\
\hline 78.0 & 1012.39 & -12.0 & 1014.64 & 81.0 & 1012.79 & -18.0 & 1014.66 & 58.0 & 1011.98 \\
\hline 80.0 & 1012.67 & -9.0 & 1014.66 & 82.5 & 1012.97 & -16.8 & 1014.62 & 58.4 & 1012.06 \\
\hline 82.0 & 1012.93 & -6.0 & 1014.53 & 85.0 & 1013.32 & -15.0 & 1014.63 & 59.0 & 1012.20 \\
\hline 84.0 & 1013.21 & -3.3 & 1014.47 & 88.0 & 1013.38 & -13.0 & 1014.65 & 60.0 & 1012.41 \\
\hline 86.0 & 1013.39 & -1.6 & 1012.79 & 91.0 & 1013.70 & -11.0 & 1014.68 & 60.5 & 1012.49 \\
\hline 88.0 & 1013.38 & -1.2 & 1012.63 & 93.5 & 1013.56 & -9.8 & 1014.73 & 61.0 & 1012.57 \\
\hline 90.0 & 1013.61 & -0.5 & 1012.25 & 96.0 & 1013.64 & -9.2 & 1014.65 & 62.0 & 1012.66 \\
\hline 92.0 & 1013.68 & 1.3 & 1010.97 & 99.0 & 1013.77 & -8.0 & 1014.66 & 63.0 & 1012.71 \\
\hline 95.0 & 1013.58 & 1.6 & 1010.95 & 101.0 & 1013.77 & -6.0 & 1014.52 & 64.0 & 1012.71 \\
\hline 98.0 & 1013.74 & 4.0 & 1010.73 & 104.0 & 1013.84 & -5.0 & 1014.52 & 65.0 & 1012.68 \\
\hline 100.9 & 1013.78 & 7.0 & 1010.68 & & & -3.2 & 1014.47 & 66.0 & 1012.61 \\
\hline 104.0 & 1013.84 & 10.0 & 1010.67 & & & -2.9 & 1014.11 & 67.0 & 1012.53 \\
\hline & & 13.0 & 1010.62 & & & -1.7 & 1012.85 & 67.5 & 1012.48 \\
\hline & & 16.0 & 1010.66 & & & -0.5 & 1012.37 & 68.0 & 1012.50 \\
\hline & & 19.0 & 1010.69 & & & 0.0 & 1011.88 & 69.0 & 1012.37 \\
\hline & & 22.0 & 1010.64 & & & 0.8 & 1011.33 & 69.5 & 1012.34 \\
\hline & & 25.0 & 1010.67 & & & 1.2 & 1011.08 & 70.0 & 1012.30 \\
\hline & & 28.0 & 1010.70 & & & 1.5 & 1010.96 & 71.0 & 1012.25 \\
\hline & & 31.0 & 1010.70 & & & 3.0 & 1010.83 & 72.0 & 1012.19 \\
\hline & & 34.0 & 1010.71 & & & 6.0 & 1010.67 & 73.0 & 1012.13 \\
\hline & & 37.0 & 1010.73 & & & 9.0 & 1010.66 & 74.0 & 1012.14 \\
\hline & & 40.0 & 1010.80 & & & 12.0 & 1010.66 & 75.0 & 1012.15 \\
\hline & & 43.0 & 1010.82 & & & 15.0 & 1010.65 & 75.4 & 1012.17 \\
\hline & & 46.0 & 1010.84 & & & 18.0 & 1010.67 & 76.0 & 1012.20 \\
\hline & & 49.0 & 1010.84 & & & 21.0 & 1010.63 & 77.0 & 1012.33 \\
\hline & & 51.5 & 1010.89 & & & 24.0 & 1010.69 & 78.0 & 1012.38 \\
\hline & & 52.6 & 1010.95 & & & 27.0 & 1010.74 & 78.5 & 1012.43 \\
\hline & & 55.0 & 1011.04 & & & 30.0 & 1010.72 & 79.0 & 1012.53 \\
\hline & & 55.8 & 1011.06 & & & 33.0 & 1010.77 & 80.0 & 1012.65 \\
\hline & & 56.5 & 1011.23 & & & 36.0 & 1010.72 & 80.8 & 1012.74 \\
\hline & & 58.0 & 1011.98 & & & 39.0 & 1010.79 & 81.0 & 1012.78 \\
\hline & & 59.5 & 1012.32 & & & 42.0 & 1010.83 & 82.0 & 1012.91 \\
\hline & & 61.0 & 1012.57 & & & 45.0 & 1010.85 & 82.7 & 1013.00 \\
\hline & & 63.0 & 1012.70 & & & 48.0 & 1010.85 & 83.0 & 1013.07 \\
\hline & & 65.0 & 1012.67 & & & 51.0 & 1010.88 & 83.7 & 1013.16 \\
\hline & & 67.0 & 1012.52 & & & 53.0 & 1010.95 & 84.0 & 1013.22 \\
\hline
\end{tabular}


Table 9. (Continued) Listing of horizontal stations and elevations for cross section PR120

[Sta., station, distance in meters from a reference pin on the left bank; Elev., elevation, in meters above sea level] 1998

\begin{tabular}{cc}
\multicolumn{3}{c}{ 25 September } \\
\hline Sta. & Elev. \\
\hline 85.0 & 1013.33 \\
86.0 & 1013.39 \\
87.0 & 1013.38 \\
87.7 & 1013.35 \\
88.0 & 1013.38 \\
88.5 & 1013.43 \\
89.0 & 1013.49 \\
90.0 & 1013.58 \\
91.0 & 1013.71 \\
92.0 & 1013.68 \\
93.3 & 1013.57 \\
96.0 & 1013.63 \\
98.0 & 1013.74 \\
100.0 & 1013.80 \\
101.0 & 1013.78 \\
102.0 & 1013.73
\end{tabular}




\section{Description of Cross Section PR122}

Location: Township 8 South/Range 48 East--section 28

U. S. Geological Survey quadrangle (1:24,000): Moorhead

Landowners--left bank: Glenn and William Gay

--right bank: U. S. Government

Access: Left bank

Permission from: Gay Ranch

Distance from Moorhead Gaging Station: 7.07 kilometers

Azimuth of Section (degrees magnetic): 135.5

\section{Reference Monuments}

[ Monuments at stations -1.2 and 0.0 were closest to leveling instrument; benchmark (brass circular plate in concrete) was removed between 1988 and 1998 ]

\begin{tabular}{|c|c|c|c|c|c|c|}
\hline \multirow[b]{2}{*}{ Description } & \multirow[b]{2}{*}{$\begin{array}{c}\text { Station } \\
\text { (m) }\end{array}$} & \multicolumn{2}{|c|}{ GPS-NAD83 (1992) } & \multicolumn{2}{|c|}{ Measurement } & \multirow[b]{2}{*}{$\begin{array}{l}\text { Elevation } \\
\text { (NGVD1929) } \\
\text { (m) }\end{array}$} \\
\hline & & Latitude & Longitude & $\begin{array}{l}\text { Standard } \\
\text { deviation } \\
\text { (m) }\end{array}$ & $\begin{array}{l}\text { Horizontal } \\
\text { precision } \\
(\mathrm{m})\end{array}$ & \\
\hline $\begin{array}{l}\text { 1/2-inch-rebar; bent, } 0.18 \text { meter above } 1998 \\
\text { ground level }\end{array}$ & -1.2 & $45^{\circ} 06^{\prime} 37.88^{\prime \prime}$ & $105^{\circ} 51^{\prime} 00.00^{\prime \prime} 0$ & 0.280 & 0.500 & 1014.76 \\
\hline Benchmark--1/2-inch-rebar & 0.0 & & & & & 1014.56 \\
\hline $\begin{array}{l}\text { 1/2-inch-rebar; bent, about } 0.08 \text { meter below } \\
1978 \text { ground level; could not see it in } 1998\end{array}$ & 101.8 & & & & & 1009.29 \\
\hline $\begin{array}{l}\text { 1/2-inch-rebar; bent, } 0.13 \text { meter above } 1998 \\
\text { ground level }\end{array}$ & 136.2 & & & & & 1010.36 \\
\hline $\begin{array}{l}\text { 1/2-inch-rebar; in sand ridge above surround- } \\
\text { ing floodplain; } 0.29 \text { meter above } 1998 \\
\text { ground level }\end{array}$ & 141.0 & $45^{\circ} 06^{\prime} 33.88^{\prime \prime}$ & $105^{\circ} 51^{\prime} 56.78^{\prime \prime}$ & 0.475 & 0.472 & 1010.93 \\
\hline
\end{tabular}




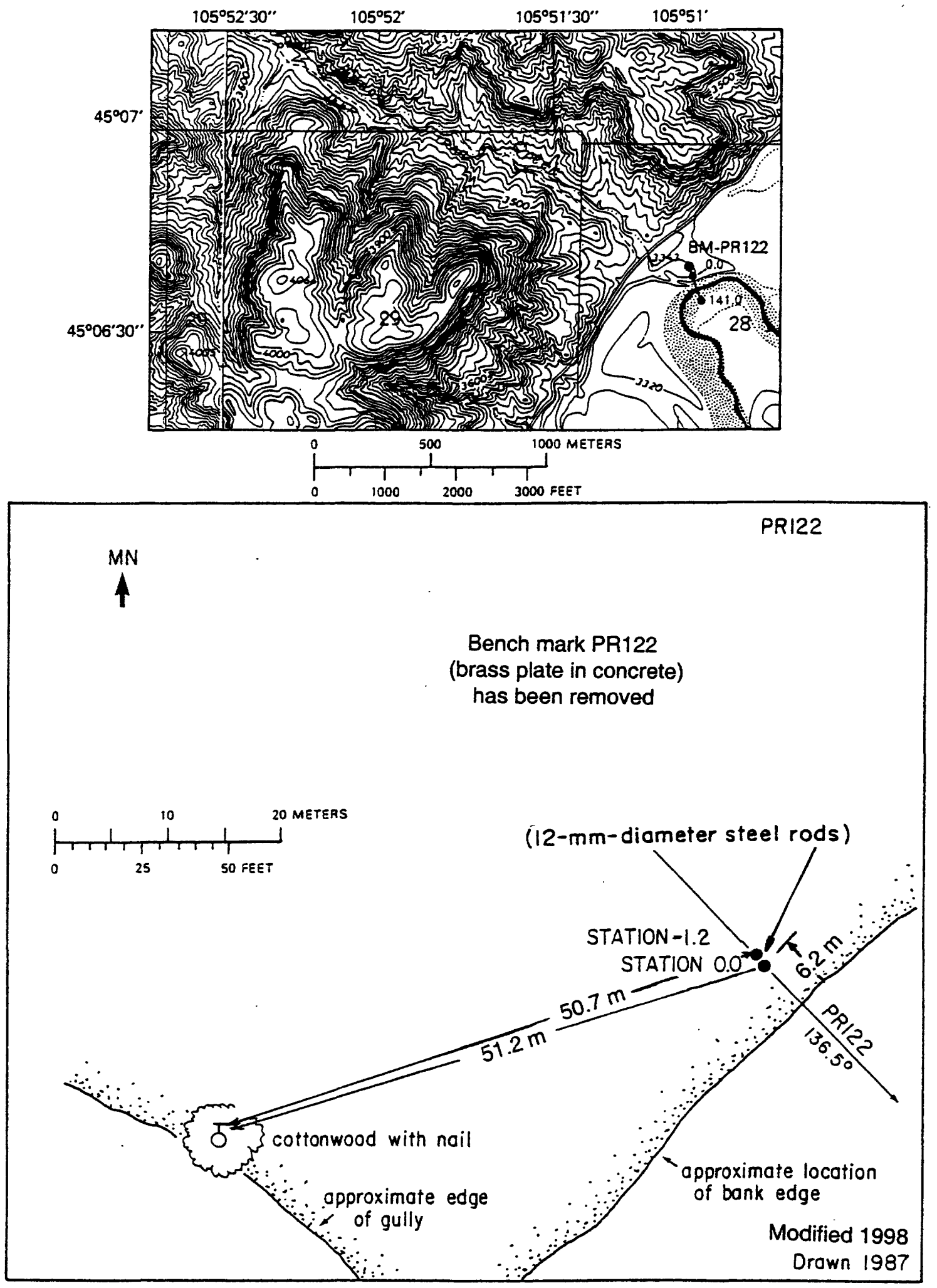

Figure 21. Upper: Location of cross section PR122 and the left and right bank reference monuments in the Moorhead quadrangle. Lower: Location of stations 0.0 and -1.2 on the left bank. MN is magnetic north. 
Table 10. Listing of horizontal stations and elevations for cross section PR122

[Sta., station, distance in meters from a reference pin on the left bank; Elev., elevation, in meters above sea level]

\begin{tabular}{|c|c|c|c|c|c|c|c|}
\hline \multirow{2}{*}{\multicolumn{2}{|c|}{$\begin{array}{c}1993 \\
26 \text { August }\end{array}$}} & \multicolumn{2}{|c|}{1993} & \multicolumn{2}{|c|}{1995} & \multicolumn{2}{|c|}{1995} \\
\hline & & 26 & ugust & $26 \mathrm{Se}$ & tember & $26 \mathrm{Se}$ & tember \\
\hline Sta. & Elev. & Sta. & Elev. & Sta. & Elev. & Sta. & Elev. \\
\hline 0.0 & 1014.50 & 86.0 & 1008.46 & -1.2 & 1014.57 & 84.0 & 1008.55 \\
\hline 1.0 & 1014.46 & 89.0 & 1008.52 & 0.0 & 1014.48 & 86.0 & 1008.55 \\
\hline 3.0 & 1014.55 & 91.0 & 1008.64 & 2.0 & 1014.53 & 88.0 & 1008.57 \\
\hline 5.1 & 1014.59 & 93.0 & 1008.80 & 4.0 & 1014.61 & 90.0 & 1008.61 \\
\hline 5.7 & 1013.84 & 95.0 & 1008.94 & 4.9 & 1014.62 & 92.0 & 1008.71 \\
\hline 6.0 & 1013.26 & 97.0 & 1008.99 & 6.1 & 1011.54 & 93.0 & 1008.79 \\
\hline 6.2 & 1011.47 & 98.5 & 1009.05 & 9.0 & 1009.82 & 95.0 & 1008.92 \\
\hline 6.6 & 1011.24 & 100.0 & 1009.16 & 11.0 & 1009.12 & 97.0 & 1009.00 \\
\hline 8.5 & 1009.98 & 102.0 & 1009.42 & 14.0 & 1008.58 & 99.0 & 1009.12 \\
\hline 10.0 & 1009.42 & 104.0 & 1009.60 & 16.0 & 1008.39 & 101.0 & 1009.29 \\
\hline 11.0 & 1009.22 & 106.0 & 1009.46 & 18.0 & 1008.41 & 103.0 & 1009.52 \\
\hline 13.0 & 1008.66 & 107.0 & 1009.52 & 20.0 & 1008.45 & 105.0 & 1009.59 \\
\hline 15.0 & 1008.39 & 108.5 & 1009.39 & 22.0 & 1008.50 & 107.0 & 1009.52 \\
\hline 16.0 & 1008.29 & 110.0 & 1009.47 & 24.0 & 1008.56 & 109.0 & 1009.40 \\
\hline 17.0 & 1008.28 & 112.0 & 1009.59 & 26.0 & 1008.56 & 111.0 & 1009.54 \\
\hline 19.0 & 1008.38 & 115.0 & 1009.63 & 28.0 & 1008.53 & 113.0 & 1009.61 \\
\hline 21.0 & 1008.36 & 117.0 & 1009.62 & 30.0 & 1008.52 & 115.0 & 1009.63 \\
\hline 23.0 & 1008.46 & 119.0 & 1009.46 & 32.0 & 1008.61 & 117.0 & 1009.57 \\
\hline 25.0 & 1008.52 & 121.0 & 1009.32 & 34.0 & 1008.69 & 119.0 & 1009.37 \\
\hline 27.0 & 1008.46 & 123.0 & 1009.05 & 36.0 & 1008.63 & 121.0 & 1009.33 \\
\hline 29.0 & 1008.41 & 124.0 & 1009.10 & 38.0 & 1008.62 & 123.0 & 1009.12 \\
\hline 31.0 & 1008.44 & & & 40.0 & 1008.66 & 125.0 & 1009.19 \\
\hline 32.5 & 1008.70 & & & 42.0 & 1008.69 & & \\
\hline 34.0 & 1008.67 & & & 44.0 & 1008.70 & & \\
\hline 35.0 & 1008.58 & & & 47.0 & 1008.71 & & \\
\hline 37.0 & 1008.55 & & & 49.0 & 1008.71 & & \\
\hline 39.0 & 1008.58 & & & 51.0 & 1008.69 & & \\
\hline 41.0 & 1008.64 & & & 53.0 & 1008.70 & & \\
\hline 44.0 & 1008.65 & & & 55.0 & 1008.69 & & \\
\hline 47.0 & 1008.69 & & & 57.0 & 1008.72 & & \\
\hline 50.0 & 1008.66 & & & 60.0 & 1008.72 & & \\
\hline 53.0 & 1008.68 & & & 62.0 & 1008.69 & & \\
\hline 56.0 & 1008.66 & & & 64.0 & 1008.68 & & \\
\hline 59.0 & 1008.69 & & & 66.0 & 1008.67 & & \\
\hline 62.0 & 1008.65 & & & 68.0 & 1008.65 & & \\
\hline 65.0 & 1008.60 & & & 70.0 & 1008.65 & & \\
\hline 68.0 & 1008.61 & & & 72.0 & 1008.64 & & \\
\hline 71.0 & 1008.61 & & & 74.0 & 1008.61 & & \\
\hline 74.0 & 1008.57 & & & 76.0 & 1008.61 & & \\
\hline 77.0 & 1008.56 & & & 78.0 & 1008.61 & & \\
\hline 80.0 & 1008.56 & & & 80.0 & 1008.61 & & \\
\hline 83.0 & 1008.47 & & & 82.0 & 1008.59 & & \\
\hline
\end{tabular}




\section{Description of Cross Section PR122A}

Location: Township 8 South/Range 48 East--section 28

U. S. Geological Survey quadrangle (1:24,000): Moorhead

Landowners--left bank: U.S. Government

--right bank: U.S. Government

Access: Left bank

Permission from: Gay Ranch

Distance from Moorhead Gaging Station: 6.60 kilometers

Azimuth of Section (degrees magnetic): 133.5

\section{Reference Monuments}

[ Monuments on left bank were closest to leveling instrument]

\begin{tabular}{|c|c|c|c|c|c|c|}
\hline \multirow[b]{2}{*}{ Description } & \multirow[b]{2}{*}{$\begin{array}{l}\text { Station } \\
(\mathrm{m})\end{array}$} & \multicolumn{2}{|c|}{ GPS-NAD83 (1992) } & \multicolumn{2}{|c|}{ Measurement } & \multirow{2}{*}{$\begin{array}{c}\text { Elevation } \\
\text { (NGVD1929) } \\
\text { (m) }\end{array}$} \\
\hline & & Latitude & Longitude & $\begin{array}{l}\text { Standard } \\
\text { deviation } \\
\text { (m) }\end{array}$ & $\begin{array}{l}\text { Horizontal } \\
\text { precision } \\
\text { (m) }\end{array}$ & \\
\hline $\begin{array}{l}\text { 1/2-inch-rebar; } 0.17 \text { meter above } 1998 \text { ground } \\
\text { level }\end{array}$ & -50.0 & $45^{\circ} 06^{\prime} 29.84^{\prime \prime}$ & $105^{\circ} 50^{\prime} 47.05^{\prime \prime}$ & 0.356 & 0.551 & 1010.37 \\
\hline $\begin{array}{l}\text { Benchmark--1/2-inch-rebar; } 0.06 \text { meter above } \\
1998 \text { ground level }\end{array}$ & -20.0 & & & & & 1010.33 \\
\hline $\begin{array}{l}\text { 1/2-inch-rebar; } 0.05 \text { meter above } 1998 \text { ground } \\
\text { level }\end{array}$ & 100.0 & & & & & 1010.00 \\
\hline $\begin{array}{l}\text { 1/2-inch-rebar; bent, } 0.13 \text { meter above } 1998 \\
\text { ground level }\end{array}$ & 101.8 & & & & & 1010.12 \\
\hline $\begin{array}{l}\text { 1/2-inch-rebar; bent flat with } 1998 \text { ground } \\
\text { level }\end{array}$ & 145.0 & $45^{\circ} 06^{\prime} 24.50^{\prime \prime}$ & $105^{\circ} 50^{\prime} 42.28^{\prime \prime}$ & 0.453 & 0.781 & 1009.98 \\
\hline
\end{tabular}



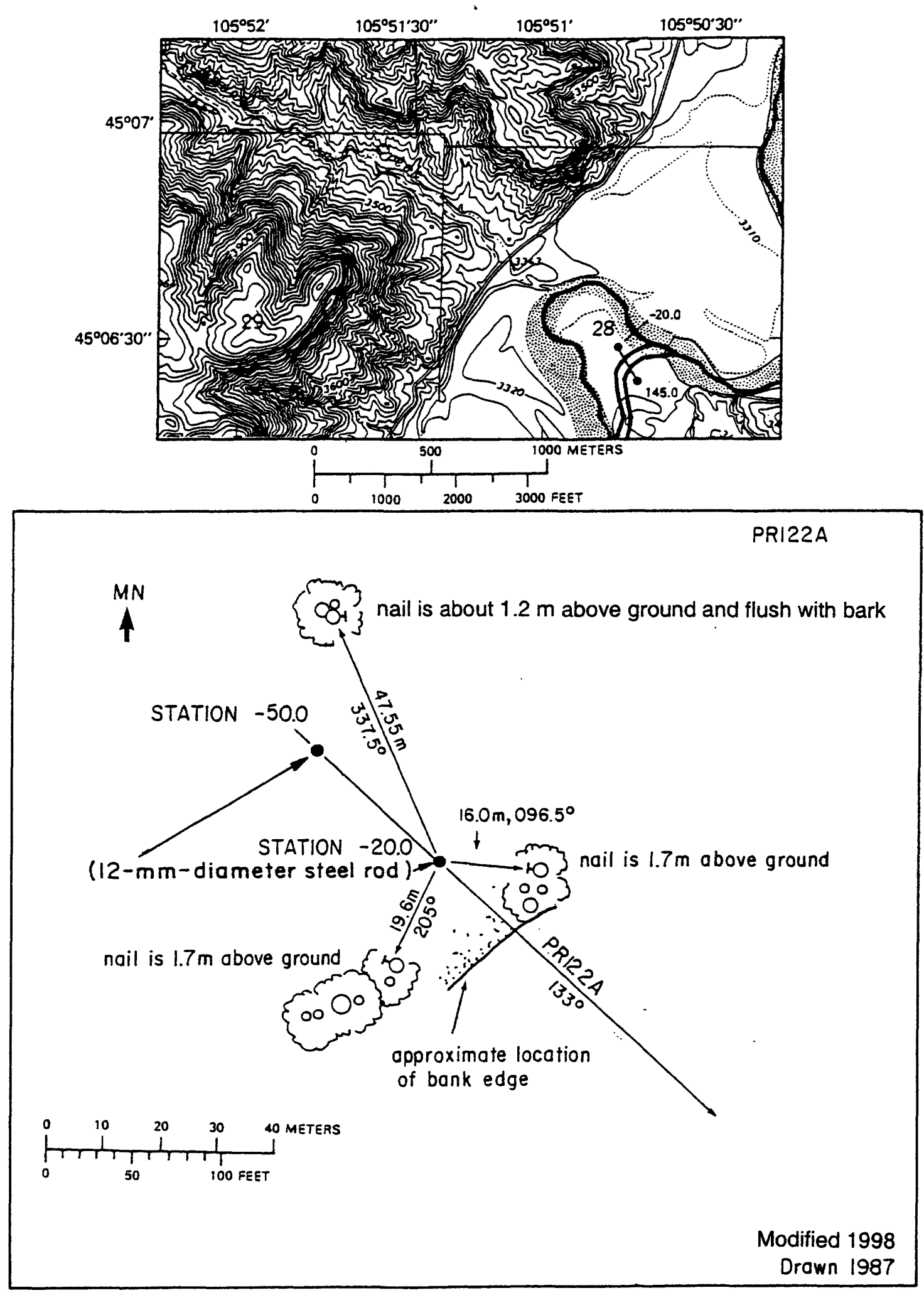

Figure 23. Upper: Location of cross section PR122A and the left and right bank reference monuments in the Moorhead quadrangle. New channel created during the 1978 flood is shown in its approximate location. Lower: Location of the bench marks (12-mm-diameter steel rods) on the left bank. $\mathrm{MN}$ is magnetic north. 

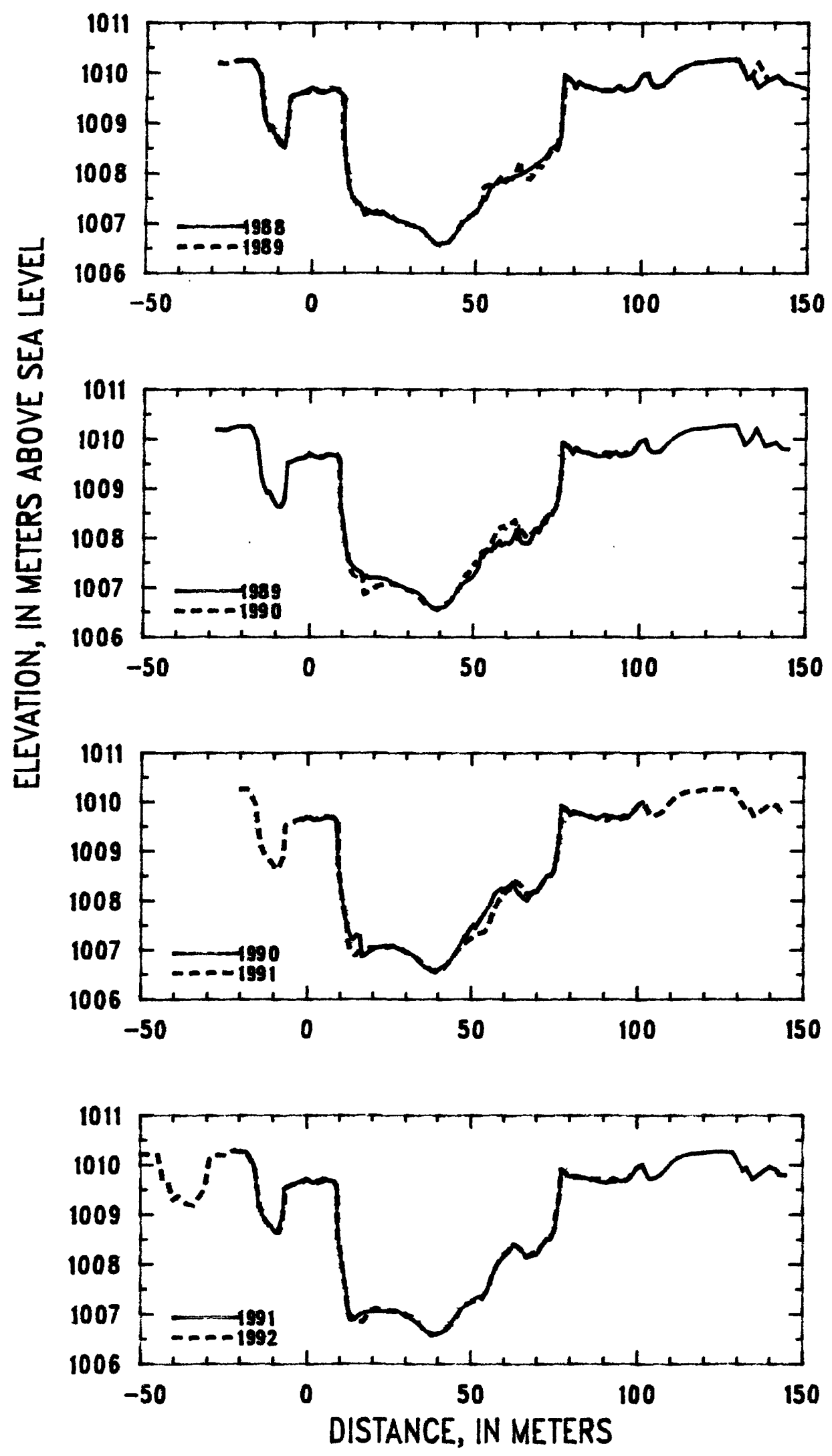

Figure 24. Profiles of cross section PR122A from 1988 to 1992. 

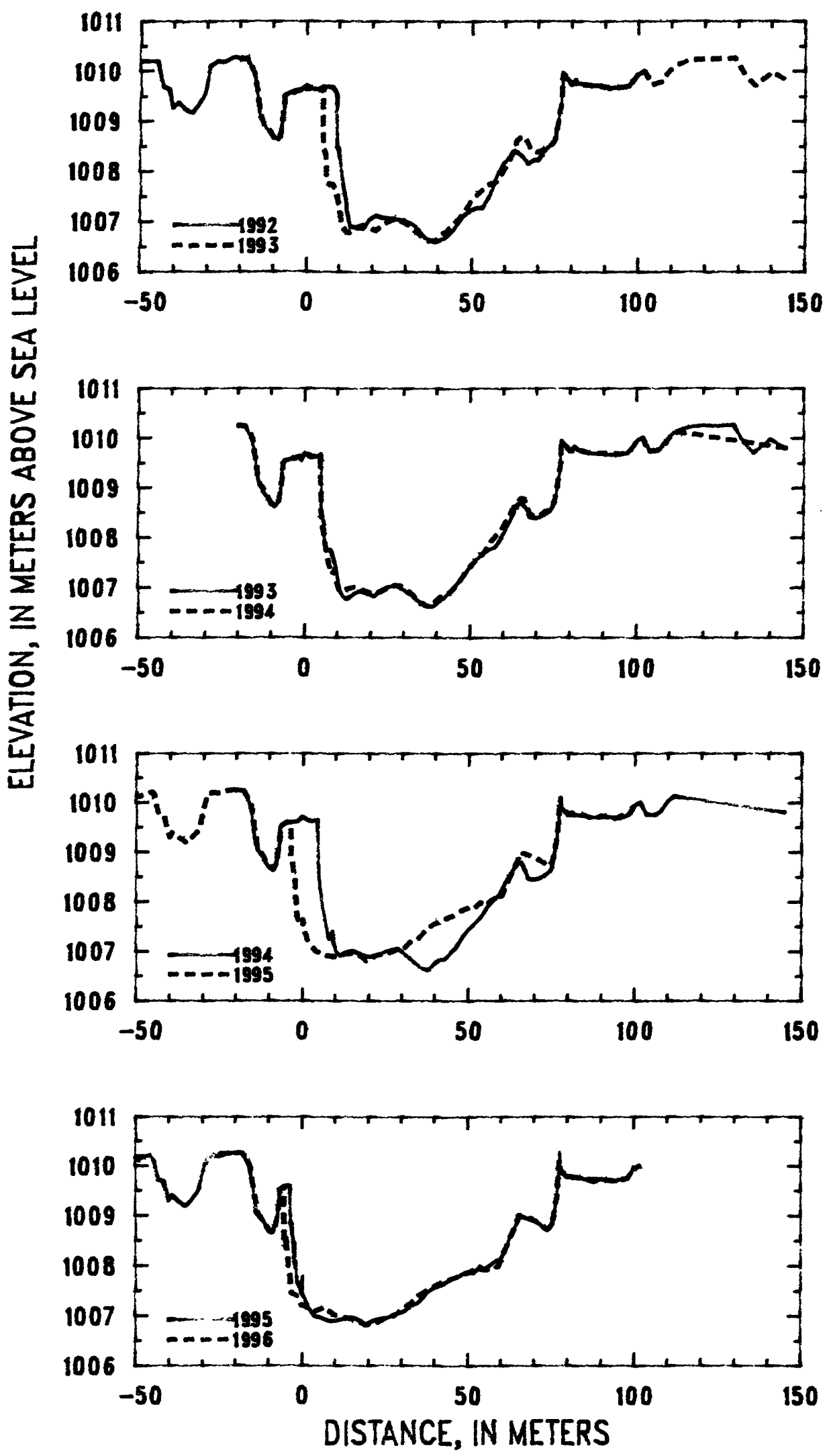

Figure 25. Profiles of cross section PR122A from 1992 to 1996. 


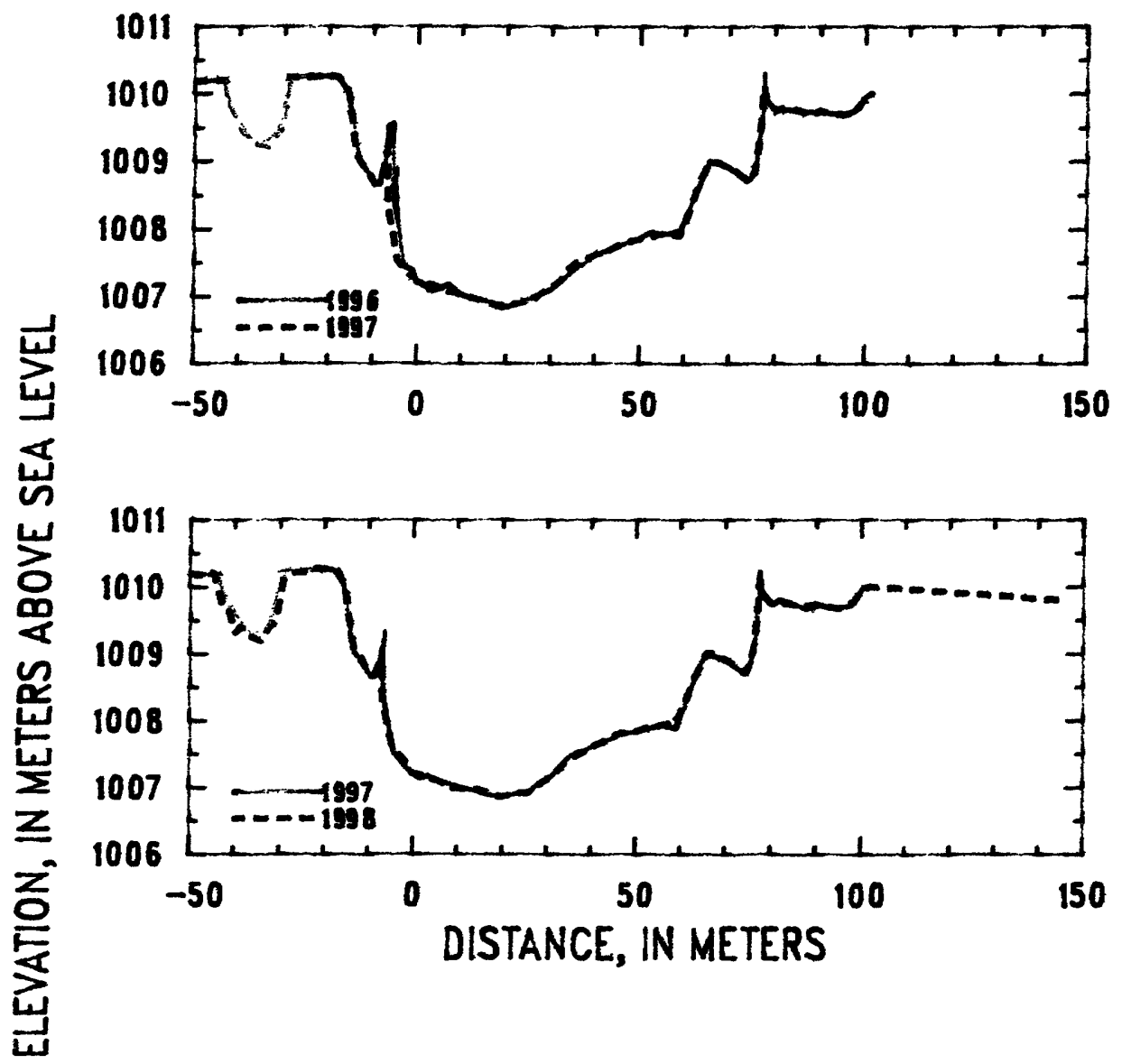

Figure 26. Profiles of cross section PR122A from 1996 to 1998. 
Table 11. Listing of horizontal stations and elevations for cross section PR122A

[Sta., station, distance in meters from a reference pin on the left bank; Elev., elevation, in meters above sea level]

\begin{tabular}{|c|c|c|c|c|c|c|c|c|c|}
\hline \multirow{2}{*}{\multicolumn{2}{|c|}{$\begin{array}{c}1989 \\
17 \text { September }\end{array}$}} & \multicolumn{2}{|c|}{1989} & \multicolumn{2}{|c|}{1989} & \multicolumn{2}{|c|}{1989} & \multicolumn{2}{|c|}{1990} \\
\hline & & $17 \mathrm{Sc}$ & tember & $17 \mathrm{~S}$ & tember & $17 \mathrm{Se}$ & tember & $15 \mathrm{Se}$ & tember \\
\hline Sta. & Elev. & Sta. & Elev. & Sta. & Elev. & Sta. & Elev. & Sta. & Elev. \\
\hline-28.0 & 1010.21 & 13.0 & 1007.45 & 66.8 & 1007.89 & 135.0 & 1010.20 & -1.5 & 1009.63 \\
\hline-25.0 & 1010.18 & 15.0 & 1007.34 & 68.0 & 1008.01 & 138.0 & 1009.86 & 0.0 & 1009.70 \\
\hline-22.5 & 1010.25 & 15.8 & 1007.31 & 68.4 & 1008.11 & 141.0 & 1009.93 & 2.0 & 1009.64 \\
\hline-20.0 & 1010.26 & 16.8 & 1007.19 & 69.0 & 1008.18 & 143.0 & 1009.80 & 4.0 & 1009.64 \\
\hline-18.0 & 1010.26 & 17.5 & 1007.24 & 70.1 & 1008.13 & 145.0 & 1009.79 & 6.0 & 1009.70 \\
\hline-17.0 & 1010.21 & 18.1 & 1007.20 & 71.0 & 1008.30 & & & 8.0 & 1009.68 \\
\hline-16.0 & 1010.03 & 19.0 & .1007 .19 & 72.0 & 1008.32 & & & 8.8 & 1009.63 \\
\hline-15.3 & 1009.95 & 20.1 & 1007.21 & 73.0 & 1008.48 & & & 9.1 & 1009.55 \\
\hline-14.9 & 1009.64 & 22.0 & 1007.19 & 74.0 & 1008.49 & & & 9.3 & 1008.67 \\
\hline-13.9 & 1009.10 & 24.0 & 1007.16 & 75.0 & 1008.61 & & & 11.0 & 1007.80 \\
\hline-12.7 & 1008.92 & 26.0 & 1007.05 & 76.0 & 1008.85 & & & 12.0 & 1007.56 \\
\hline-12.0 & 1008.94 & 28.0 & 1007.04 & 76.7 & 1009.27 & & & 13.0 & 1007.30 \\
\hline-11.0 & 1008.81 & 30.0 & 1006.96 & 77.1 & 1009.65 & & & 14.0 & 1007.23 \\
\hline-9.6 & 1008.65 & 32.0 & 1006.95 & 77.2 & 1009.93 & & & 15.0 & 1007.31 \\
\hline-9.5 & 1008.64 & 34.0 & 1006.85 & 78.0 & 1009.90 & & & 16.1 & 1007.27 \\
\hline-8.6 & 1008.64 & 36.0 & 1006.70 & 79.0 & 1009.86 & & & 16.3 & 1007.13 \\
\hline-8.3 & 1008.67 & 38.0 & 1006.58 & 80.0 & 1009.75 & & & 16.8 & 1006.86 \\
\hline-7.6 & 1008.77 & 40.0 & 1006.59 & 80.3 & 1009.71 & & & 18.0 & 1006.90 \\
\hline-7.4 & 1008.87 & 42.0 & 1006.63 & 81.3 & 1009.83 & & & 19.0 & 1006.97 \\
\hline-6.8 & 1009.25 & 44.0 & 1006.81 & 83.0 & 1009.74 & & & 21.0 & 1007.04 \\
\hline-6.6 & 1009.53 & 46.0 & 1007.03 & 84.5 & 1009.73 & & & 23.0 & 1007.07 \\
\hline-6.0 & 1009.55 & 48.0 & 1007.15 & 86.0 & 1009.67 & & & 25.0 & 1007.09 \\
\hline-5.0 & 1009.56 & 49.7 & 1007.21 & 88.0 & 1009.65 & & & 26.0 & 1007.08 \\
\hline-4.0 & 1009.60 & 51.0 & 1007.32 & 89.5 & 1009.68 & & & 28.0 & 1007.03 \\
\hline-3.0 & 1009.62 & 52.1 & 1007.51 & 91.0 & 1009.64 & & & 30.0 & 1006.96 \\
\hline-1.5 & 1009.62 & 52.3 & 1007.71 & 93.0 & 1009.74 & & & 32.0 & 1006.91 \\
\hline 0.0 & 1009.72 & 53.0 & 1007.75 & 94.0 & 1009.67 & & & 33.0 & 1006.85 \\
\hline 1.0 & 1009.67 & 54.0 & 1007.77 & 95.0 & 1009.67 & & & 35.0 & 1006.69 \\
\hline 2.0 & 1009.63 & 55.5 & 1007.74 & 97.0 & 1009.70 & & & 37.0 & 1006.63 \\
\hline 3.0 & 1009.63 & 56.0 & 1007.83 & 98.0 & 1009.75 & & & 39.0 & 1006.55 \\
\hline 4.0 & 1009.63 & 56.5 & 1007.81 & 100.0 & 1009.95 & & & 41.0 & 1006.65 \\
\hline 5.0 & 1009.69 & 57.0 & 1007.88 & 101.7 & 1009.99 & & & 43.0 & 1006.73 \\
\hline 6.0 & 1009.69 & 58.0 & 1007.95 & 103.0 & 1009.78 & & & 45.0 & 1006.92 \\
\hline 7.0 & 1009.68 & 58.5 & 1007.85 & 104.0 & 1009.74 & & & 47.0 & 1007.14 \\
\hline 8.0 & 1009.66 & 60.0 & 1007.92 & 106.0 & 1009.75 & & & 47.9 & 1007.28 \\
\hline 9.0 & 1009.62 & 61.0 & 1007.90 & 110.0 & 1010.03 & & & 48.3 & 1007.26 \\
\hline 9.3 & 1009.53 & 62.0 & 1007.98 & 115.0 & 1010.20 & & & 49.5 & 1007.43 \\
\hline 9.4 & 1008.63 & 63.0 & 1008.15 & 120.0 & 1010.22 & & & 50.8 & 1007.52 \\
\hline 10.0 & 1008.46 & 63.4 & 1008.04 & 125.0 & 1010.26 & & & 51.3 & 1007.46 \\
\hline 11.0 & 1007.93 & 63.9 & 1008.17 & 129.0 & 1010.27 & & & 52.0 & 1007.53 \\
\hline 11.3 & 1007.70 & 64.2 & 1007.98 & 131.7 & 1009.88 & & & 53.0 & 1007.67 \\
\hline 12.1 & 1007.56 & 65.0 & 1007.91 & 133.0 & 1009.95 & & & 55.0 & 1007.85 \\
\hline
\end{tabular}


Table 11. (Continued) Listing of horizontal stations and elevations for cross section PR122A

[Sta., station, distance in meters from a reference pin on the left bank; Elev., elevation, in meters above sea level]

\begin{tabular}{|c|c|c|c|c|c|c|c|c|c|}
\hline \multirow{2}{*}{\multicolumn{2}{|c|}{$\begin{array}{c}1990 \\
\text { 15 September }\end{array}$}} & \multirow{2}{*}{\multicolumn{2}{|c|}{$\begin{array}{c}1991 \\
29 \text { August }\end{array}$}} & \multirow{2}{*}{\multicolumn{2}{|c|}{$\begin{array}{c}1991 \\
29 \text { August }\end{array}$}} & \multirow{2}{*}{\multicolumn{2}{|c|}{$\begin{array}{c}1991 \\
29 \text { August }\end{array}$}} & \multirow{2}{*}{\multicolumn{2}{|c|}{$\begin{array}{c}1992 \\
25 \text { August }\end{array}$}} \\
\hline & & & & & & & & & \\
\hline Sta. & Elev. & Sta. & Elev. & Sta. & Elev. & Sta. & Elev. & Sta. & Elev. \\
\hline 57.0 & 1008.13 & -20.0 & 1010.27 & 42.0 & 1006.66 & 120.0 & 1010.24 & -50.0 & 1010.22 \\
\hline 58.0 & 1008.21 & -18.0 & 1010.26 & 44.0 & 1006.82 & 123.0 & 1010.26 & -44.7 & 1010.21 \\
\hline 59.2 & 1008.25 & -17.0 & 1010.20 & 45.2 & 1006.93 & 126.0 & 1010.26 & -43.0 & 1009.69 \\
\hline 59.6 & 1008.19 & -15.3 & 1009.96 & 47.0 & 1007.08 & 129.0 & 1010.25 & -41.4 & 1009.64 \\
\hline 60.0 & 1008.25 & -14.0 & 1009.15 & 50.0 & 1007.25 & 132.0 & 1009.89 & -40.2 & 1009.29 \\
\hline 61.0 & 1008.28 & -12.7 & 1008.94 & 51.7 & 1007.33 & 133.0 & 1009.95 & -38.6 & 1009.38 \\
\hline 62.6 & 1008.37 & -11.2 & 1008.83 & 54.0 & 1007.37 & 135.0 & 1009.71 & -35.8 & 1009.21 \\
\hline 64.0 & 1008.19 & -9.5 & 1008.67 & 55.6 & 1007.56 & 138.0 & 1009.86 & -34.2 & 1009.18 \\
\hline 65.0 & 1008.14 & -8.8 & 1008.63 & 56.6 & 1007.79 & 140.0 & 1009.96 & -31.3 & 1009.46 \\
\hline 66.0 & 1008.05 & -7.4 & 1008.86 & 58.0 & 1007.98 & 142.0 & 1009.92 & -30.2 & 1009.68 \\
\hline 67.0 & 1008.01 & -6.8 & 1009.22 & 60.0 & 1008.16 & 143.2 & 1009.79 & -29.2 & 1010.11 \\
\hline 68.0 & 1008.11 & -6.6 & 1009.52 & 61.5 & 1008.24 & 145.0 & 1009.79 & -27.0 & 1010.21 \\
\hline 69.0 & 1008.20 & -5.0 & 1009.59 & 63.0 & 1008.41 & & & -25.0 & 1010.19 \\
\hline 70.0 & 1008.19 & -3.0 & 1009.63 & 65.0 & 1008.33 & & & -22.0 & 1010.29 \\
\hline 71.0 & 1008.32 & -1.5 & 1009.66 & 67.0 & 1008.13 & & & -20.0 & 1010.27 \\
\hline 72.0 & 1008.40 & 0.0 & 1009.72 & 69.0 & 1008.18 & & & -18.0 & 1010.26 \\
\hline 73.0 & 1008.52 & 2.0 & 1009.63 & 70.0 & 1008.20 & & & -15.4 & 1009.96 \\
\hline 74.0 & 1008.52 & 5.0 & 1009.72 & 72.0 & 1008.40 & & & -14.0 & 1009.20 \\
\hline 75.0 & 1008.62 & 7.0 & 1009.68 & 73.0 & 1008.51 & & & -12.8 & 1008.93 \\
\hline 75.7 & 1008.80 & 8.5 & 1009.66 & 74.0 & 1008.51 & & & -10.0 & 1008.68 \\
\hline 76.8 & 1009.34 & 9.3 & 1009.55 & 74.8 & 1008.57 & & & -8.4 & 1008.64 \\
\hline 77.0 & 1009.53 & 9.3 & 1008.68 & 75.8 & 1008.80 & & & -7.4 & 1008.87 \\
\hline 77.3 & 1009.92 & 10.0 & 1008.33 & 77.1 & 1009.47 & & & -6.7 & 1009.23 \\
\hline 78.0 & 1009.90 & 10.7 & 1008.00 & 77.5 & 1009.90 & & & -6.5 & 1009.53 \\
\hline 79.0 & 1009.85 & 12.1 & 1007.54 & 80.0 & 1009.76 & & & -4.0 & 1009.61 \\
\hline 80.0 & 1009.74 & 12.6 & 1007.11 & 82.0 & 1009.79 & & & -1.5 & 1009.64 \\
\hline 81.0 & 1009.82 & 13.0 & 1007.04 & 85.0 & 1009.73 & & & 0.0 & 1009.71 \\
\hline 82.0 & 1009.78 & 14.6 & 1006.90 & 88.0 & 1009.66 & & & 2.0 & 1009.64 \\
\hline 84.0 & 1009.75 & 16.0 & 1006.99 & 91.0 & 1009.63 & & & 4.0 & 1009.64 \\
\hline 86.0 & 1009.69 & 18.0 & 1007.04 & 93.0 & 1009.73 & & & 6.0 & 1009.70 \\
\hline 88.0 & 1009.66 & 20.0 & 1007.05 & 95.0 & 1009.67 & & & 8.0 & 1009.68 \\
\hline 90.0 & 1009.74 & 22.0 & 1007.06 & 97.0 & 1009.70 & & & 9.2 & 1009.58 \\
\hline 92.0 & 1009.71 & 24.0 & 1007.06 & 98.0 & 1009.74 & & & 9.3 & 1009.48 \\
\hline 94.0 & 1009.67 & 26.0 & 1007.09 & 100.0 & 1009.95 & & & 9.3 & 1008.50 \\
\hline 96.0 & 1009.74 & 28.0 & 1007.05 & 101.7 & 1010.00 & & & 9.8 & 1008.46 \\
\hline 97.0 & 1009.70 & 30.0 & 1006.96 & 103.7 & 1009.72 & & & 11.4 & 1007.69 \\
\hline 98.0 & 1009.75 & 31.1 & 1006.95 & 105.0 & 1009.72 & & & 12.1 & 1007.45 \\
\hline 100.0 & 1009.95 & 32.0 & 1006.92 & 107.0 & 1009.78 & & & 12.3 & 1007.30 \\
\hline \multirow[t]{4}{*}{101.7} & 1010.00 & 34.0 & 1006.80 & 109.0 & 1009.94 & & & 12.5 & 1007.15 \\
\hline & & 36.0 & 1006.69 & 111.0 & 1010.10 & & & 13.3 & 1006.89 \\
\hline & & 38.0 & 1006.58 & 114.0 & 1010.19 & & & 15.0 & 1006.88 \\
\hline & & 40.0 & 1006.61 & 117.0 & 1010.23 & & & 17.2 & 1006.85 \\
\hline
\end{tabular}


Table 11. (Continued) Listing of horizontal stations and elevations for cross section PR122A

[Sta., station, distance in meters from a reference pin on the left bank; Elev., elevation, in meters above sea level]

\begin{tabular}{|c|c|c|c|c|c|c|c|c|c|}
\hline \multirow{2}{*}{\multicolumn{2}{|c|}{$\begin{array}{c}1992 \\
25 \text { August }\end{array}$}} & \multirow{2}{*}{\multicolumn{2}{|c|}{$\begin{array}{c}1992 \\
25 \text { August }\end{array}$}} & \multirow{2}{*}{\multicolumn{2}{|c|}{$\begin{array}{c}1993 \\
26 \text { August }\end{array}$}} & \multirow{2}{*}{\multicolumn{2}{|c|}{$\begin{array}{c}1993 \\
26 \text { August }\end{array}$}} & \multirow{2}{*}{\multicolumn{2}{|c|}{$\begin{array}{c}1993 \\
26 \text { August }\end{array}$}} \\
\hline & & & & & & & & & \\
\hline Sta. & Elev. & Sta. & Elev. & Sta. & Elev. & Sta. & Elev. & Sta. & Elev. \\
\hline 19.0 & 1007.04 & 99.0 & 1009.87 & -20.0 & 1010.26 & 35.0 & 1006.71 & 100.0 & 1009.95 \\
\hline 21.0 & 1007.12 & 100.0 & 1009.95 & -17.7 & 1010.27 & 37.0 & 1006.63 & 101.7 & 1010.01 \\
\hline 23.0 & 1007.08 & 101.7 & 1010.01 & -15.4 & 1009.96 & 39.0 & 1006.62 & 104.0 & 1009.74 \\
\hline 25.0 & 1007.07 & & & -13.8 & 1009.14 & 41.0 & 1006.74 & 107.0 & 1009.78 \\
\hline 27.0 & 1007.05 & & & -12.6 & 1008.94 & 43.0 & 1006.86 & 109.0 & 1009.99 \\
\hline 29.0 & 1007.03 & & & -11.0 & 1008.83 & 45.0 & 1006.99 & 112.0 & 1010.15 \\
\hline 31.0 & 1006.98 & & & -9.6 & 1008.66 & 46.5 & 1007.12 & 115.0 & 1010.21 \\
\hline 33.0 & 1006.93 & & & -8.8 & 1008.63 & 48.0 & 1007.23 & 120.0 & 1010.25 \\
\hline 35.0 & 1006.75 & & & -8.0 & 1008.69 & 50.0 & 1007.44 & 125.0 & 1010.25 \\
\hline 37.0 & 1006.64 & & & -7.5 & 1008.82 & 52.0 & 1007.57 & 129.0 & 1010.27 \\
\hline 39.0 & 1006.59 & & & -6.7 & 1009.27 & 54.0 & 1007.68 & 132.0 & 1009.92 \\
\hline 41.0 & 1006.63 & & & -6.6 & 1009.52 & 55.0 & 1007.74 & 135.0 & 1009.71 \\
\hline 43.0 & 1006.73 & & & -6.0 & 1009.56 & 57.2 & 1007.79 & 140.0 & 1009.98 \\
\hline 45.0 & 1006.89 & & & -3.0 & 1009.61 & 58.4 & 1007.87 & 145.0 & 1009.80 \\
\hline 47.0 & 1007.09 & & & -1.5 & 1009.64 & 60.0 & 1008.06 & & \\
\hline 48.0 & 1007.17 & & & -1.0 & 1009.58 & 62.0 & 1008.34 & & \\
\hline 50.0 & 1007.24 & & & 0.0 & 1009.71 & 63.3 & 1008.53 & & \\
\hline 52.0 & 1007.28 & & & 2.0 & 1009.63 & 63.4 & 1008.61 & & \\
\hline 53.0 & 1007.26 & & & 4.0 & 1009.64 & 64.0 & 1008.65 & & \\
\hline 55.3 & 1007.51 & & & 5.0 & 1009.69 & 65.0 & 1008.71 & & \\
\hline 56.6 & 1007.79 & & & 5.1 & 1008.51 & 66.0 & 1008.68 & & \\
\hline 58.0 & 1007.98 & & & 6.0 & 1008.34 & 66.7 & 1008.60 & & \\
\hline 60.0 & 1008.20 & & & 6.2 & 1007.90 & 68.0 & 1008.41 & & \\
\hline 62.0 & 1008.31 & & & 6.7 & 1007.77 & 69.0 & 1008.40 & & \\
\hline 63.0 & 1008.42 & & & 7.2 & 1007.75 & 70.0 & 1008.38 & & \\
\hline 65.0 & 1008.33 & & & 8.2 & 1007.75 & 72.0 & 1008.45 & & \\
\hline 67.0 & 1008.15 & & & 8.8 & 1007.66 & 74.0 & 1008.53 & & \\
\hline 69.0 & 1008.23 & & & 9.5 & 1007.48 & 75.0 & 1008.63 & & \\
\hline 70.0 & 1008.23 & & & 10.1 & 1007.26 & 76.0 & 1008.97 & & \\
\hline 72.0 & 1008.42 & & & 10.7 & 1006.96 & 77.0 & 1009.39 & & \\
\hline 74.0 & 1008.55 & & & 12.0 & 1006.81 & 77.3 & 1009.86 & & \\
\hline 75.0 & 1008.64 & & & 13.0 & 1006.78 & 77.5 & 1009.95 & & \\
\hline 77.0 & 1009.42 & & & 15.0 & 1006.88 & 79.0 & 1009.84 & & \\
\hline 77.5 & 1009.92 & & & 17.0 & 1006.94 & 80.0 & 1009.74 & & \\
\hline 78.3 & 1009.88 & & & 19.0 & 1006.90 & 81.5 & 1009.83 & & \\
\hline 80.0 & 1009.76 & & & 21.0 & 1006.82 & 83.0 & 1009.75 & & \\
\hline 83.0 & 1009.75 & & & 23.0 & 1006.95 & 85.0 & 1009.73 & & \\
\hline 86.0 & 1009.70 & & & 25.0 & 1007.01 & 87.0 & 1009.70 & & \\
\hline 89.0 & 1009.72 & & & 27.0 & 1007.06 & 89.0 & 1009.69 & & \\
\hline 91.0 & 1009.65 & & & 29.0 & 1007.01 & 91.0 & 1009.67 & & \\
\hline 94.0 & 1009.68 & & & 31.0 & 1006.92 & 94.0 & 1009.67 & & \\
\hline 97.0 & 1009.70 & & & 33.0 & 1006.82 & 97.0 & 1009.70 & & \\
\hline
\end{tabular}


Table 11. (Continued) Listing of horizontal stations and elevations for cross section PR122A

[Sta., station, distance in meters from a reference pin on the left bank; Elev., elevation, in meters above sea level]

\begin{tabular}{|c|c|c|c|c|c|c|c|c|c|}
\hline \multirow{2}{*}{\multicolumn{2}{|c|}{$\begin{array}{c}1994 \\
24 \text { September }\end{array}$}} & \multicolumn{2}{|c|}{1994} & \multicolumn{2}{|c|}{1995} & \multicolumn{2}{|c|}{1995} & \multicolumn{2}{|c|}{1995} \\
\hline & & $24 \mathrm{Se}$ & tember & $26 \mathrm{~S}$ & tember & $26 \mathrm{~S}$ & tember & $26 \mathrm{Se}$ & tember \\
\hline Sta. & Elev. & Sta. & Elev. & Sta. & Elev. & Sta. & Elev. & Sta. & Elev. \\
\hline-20.0 & 1010.26 & 50.0 & 1007.44 & -50.0 & 1010.19 & 1.0 & 1007.34 & 75.0 & 1008.78 \\
\hline-18.0 & 1010.25 & 52.0 & 1007.56 & -49.0 & 1010.12 & 2.0 & 1007.17 & 75.3 & 1008.81 \\
\hline-17.0 & 1010.21 & 54.0 & 1007.69 & -47.0 & 1010.20 & 4.0 & 1007.00 & 76.0 & 1009.00 \\
\hline-15.4 & 1009.97 & 56.0 & 1007.91 & -45.0 & 1010.22 & 6.0 & 1006.95 & 77.0 & 1009.46 \\
\hline-14.2 & 1009.31 & 57.7 & 1008.03 & -44.5 & 1010.17 & 8.0 & 1006.91 & 77.7 & 1010.10 \\
\hline-13.3 & 1009.05 & 59.6 & 1008.15 & -43.0 & 1009.72 & 10.0 & 1006.90 & 78.0 & 1009.90 \\
\hline-12.0 & 1008.94 & 60.6 & 1008.32 & -41.5 & 1009.70 & 12.0 & 1006.94 & 80.0 & 1009.77 \\
\hline-10.0 & 1008.70 & 62.0 & 1008.47 & -40.0 & 1009.31 & 14.0 & 1006.97 & 82.0 & 1009.79 \\
\hline-8.8 & 1008.64 & 64.0 & 1008.70 & -39.0 & 1009.40 & 16.0 & 1006.96 & 84.0 & 1009.77 \\
\hline-7.8 & 1008.74 & 65.0 & 1008.79 & -37.0 & 1009.29 & 18.0 & 1006.90 & 86.0 & 1009.72 \\
\hline-6.6 & 1009.28 & 66.0 & 1008.79 & -36.0 & 1009.24 & 19.0 & 1006.81 & 88.0 & 1009.68 \\
\hline-6.5 & 1009.51 & 67.0 & 1008.66 & -35.0 & 1009.18 & 21.0 & 1006.89 & 90.0 & 1009.74 \\
\hline-6.0 & 1009.57 & 68.0 & 1008.48 & -33.0 & 1009.32 & 23.0 & 1006.94 & 92.0 & 1009.72 \\
\hline-4.0 & 1009.60 & 69.0 & 1008.44 & -31.2 & 1009.49 & 25.0 & 1006.95 & 94.0 & 1009.68 \\
\hline-1.5 & 1009.62 & 71.0 & 1008.46 & -30.0 & 1009.83 & 27.0 & 1007.00 & 96.0 & 1009.75 \\
\hline 0.0 & 1009.72 & 73.0 & 1008.53 & -28.7 & 1010.17 & 29.0 & 1007.05 & 98.0 & 1009.75 \\
\hline 2.0 & 1009.63 & 75.0 & 1008.64 & -27.0 & 1010.21 & 31.0 & 1007.08 & 100.0 & 1009.95 \\
\hline 4.0 & 1009.65 & 76.0 & 1008.99 & -25.0 & 1010.19 & 33.0 & 1007.20 & 101.7 & 1010.00 \\
\hline 4.6 & 1009.65 & 77.2 & 1009.49 & -23.0 & 1010.25 & 35.0 & 1007.26 & & \\
\hline 4.9 & 1008.69 & 77.5 & 1009.88 & -21.0 & 1010.26 & 36.6 & 1007.37 & & \\
\hline 5.2 & 1008.36 & 78.0 & 1009.91 & -20.0 & 1010.27 & 38.0 & 1007.47 & & \\
\hline 6.7 & 1007.76 & 80.0 & 1009.75 & -18.0 & 1010.26 & 40.0 & 1007.57 & & \\
\hline 8.0 & 1007.34 & 83.0 & 1009.76 & -15.4 & 1009.99 & 42.0 & 1007.61 & & \\
\hline 8.5 & 1007.27 & 86.0 & 1009.70 & -13.5 & 1009.06 & 44.0 & 1007.71 & & \\
\hline 9.2 & 1007.37 & 89.0 & 1009.71 & -12.0 & 1008.94 & 46.0 & 1007.74 & & \\
\hline 10.3 & 1006.99 & 92.0 & 1009.72 & -10.0 & 1008.72 & 48.0 & 1007.83 & & \\
\hline 11.5 & 1006.92 & 95.0 & 1009.68 & -8.7 & 1008.65 & 50.0 & 1007.88 & & \\
\hline 13.0 & 1006.98 & 98.0 & 1009.75 & -7.6 & 1008.88 & 51.7 & 1007.87 & & \\
\hline 15.0 & 1007.02 & 100.0 & 1009.95 & -6.7 & 1009.32 & 53.7 & 1008.00 & & \\
\hline 17.0 & 1006.97 & 101.7 & 1010.00 & -6.6 & 1009.52 & 55.2 & 1007.92 & & \\
\hline 20.0 & 1006.89 & 103.5 & 1009.75 & -5.0 & 1009.60 & 57.0 & 1008.03 & & \\
\hline 23.0 & 1006.93 & 106.0 & 1009.75 & -3.5 & 1009.59 & 59.0 & 1008.11 & & \\
\hline 26.0 & 1007.04 & 108.0 & 1009.84 & -3.5 & 1008.86 & 59.7 & 1008.07 & & \\
\hline 29.0 & 1007.05 & 109.5 & 1009.98 & -3.0 & 1008.82 & 61.0 & 1008.27 & & \\
\hline 32.0 & 1006.91 & 110.5 & 1010.06 & -2.5 & 1008.47 & 63.0 & 1008.57 & & \\
\hline 35.0 & 1006.71 & 112.0 & 1010.12 & -2.2 & 1008.44 & 65.0 & 1008.87 & & \\
\hline 38.0 & 1006.62 & 145.0 & 1009.79 & -2.2 & 1008.08 & 65.3 & 1008.99 & & \\
\hline 41.0 & 1006.82 & & & -1.9 & 1008.07 & 67.0 & 1008.97 & & \\
\hline 43.0 & 1006.84 & & & -1.4 & 1007.64 & 69.0 & 1008.91 & & \\
\hline 45.5 & 1007.02 & & & -0.4 & 1007.54 & 71.0 & 1008.86 & & \\
\hline 46.5 & 1007.10 & & & 0.0 & 1007.66 & 73.0 & 1008.77 & & , \\
\hline 48.0 & 1007.28 & & & 0.0 & 1007.45 & 74.0 & 1008.71 & & \\
\hline
\end{tabular}


Table 11. (Continued) Listing of horizontal stations and elevations for cross section PR122A

[Sta., station, distance in meters from a reference pin on the left bank; Elev., elevation, in meters above sea level]

\begin{tabular}{|c|c|c|c|c|c|c|c|c|c|}
\hline \multirow{2}{*}{\multicolumn{2}{|c|}{$\begin{array}{c}1996 \\
17 \text { October }\end{array}$}} & \multicolumn{2}{|c|}{1996} & \multicolumn{2}{|c|}{1997} & \multicolumn{2}{|c|}{1997} & \multicolumn{2}{|c|}{1998} \\
\hline & & 17 & ctober & $19 \mathrm{Se}$ & tember & $19 \mathrm{Se}$ & tember & $25 \mathrm{Se}$ & tember \\
\hline Sta. & Elev. & Sta. & Elev. & Sta. & Elev. & Sta. & Elev. & Sta. & Elev. \\
\hline-50.0 & 1010.19 & 59.3 & 1007.99 & -50.0 & 1010.19 & 60.0 & 1008.06 & -50.0 & 1010.19 \\
\hline-20.0 & 1010.27 & 61.5 & 1008.36 & -20.0 & 1010.27 & 62.0 & 1008.43 & -48.0 & 1010.18 \\
\hline-18.0 & 1010.26 & 64.0 & 1008.72 & -18.0 & 1010.26 & 63.0 & 1008.62 & -46.0 & 1010.24 \\
\hline-16.0 & 1010.07 & 65.4 & 1009.00 & -16.5 & 1010.15 & 65.4 & 1009.00 & -44.5 & 1010.19 \\
\hline-15.3 & 1009.99 & 67.0 & 1008.98 & -15.3 & 1009.99 & 67.0 & 1008.99 & -42.8 & 1009.72 \\
\hline-13.2 & 1009.03 & 69.0 & 1008.94 & -14.1 & 1009.28 & 69.0 & 1008.94 & -41.5 & 1009.70 \\
\hline-11.3 & 1008.85 & 70.0 & 1008.91 & -13.2 & 1009.02 & 71.0 & 1008.87 & -39.8 & 1009.32 \\
\hline-9.4 & 1008.67 & 72.0 & 1008.81 & -11.5 & 1008.88 & 73.0 & 1008.77 & -38.9 & 1009.40 \\
\hline-8.4 & 1008.67 & 74.0 & 1008.72 & -9.5 & 1008.67 & 74.4 & 1008.72 & -37.8 & 1009.38 \\
\hline-7.3 & 1008.98 & 75.4 & 1008.80 & -8.4 & 1008.67 & 75.5 & 1008.85 & -36.0 & 1009.24 \\
\hline-6.6 & 1009.30 & 76.9 & 1009.37 & -6.8 & 1009.19 & 76.5 & 1009.24 & -34.3 & 1009.19 \\
\hline-6.5 & 1009.52 & 77.7 & 1010.12 & -6.7 & 1008.38 & 77.7 & 1010.11 & -32.5 & 1009.38 \\
\hline-5.4 & 1009.56 & 78.0 & 1009.92 & -6.3 & 1008.21 & 78.1 & 1009.93 & -31.1 & 1009.48 \\
\hline-5.3 & 1008.50 & 80.0 & 1009.77 & -5.5 & 1007.96 & 80.3 & 1009.74 & -30.0 & 1009.79 \\
\hline-4.9 & 1008.36 & 83.0 & 1009.77 & -4.4 & 1007.52 & 82.0 & 1009.80 & -29.0 & 1010.12 \\
\hline-4.7 & 1008.60 & 86.0 & 1009.72 & -2.0 & 1007.34 & 85.0 & 1009.76 & -27.0 & 1010.21 \\
\hline-4.4 & 1008.23 & 89.0 & 1009.73 & 0.2 & 1007.21 & 88.0 & 1009.67 & -24.0 & 1010.21 \\
\hline-3.0 & 1007.45 & 92.0 & 1009.72 & 1.5 & 1007.14 & 90.0 & 1009.75 & -22.0 & 1010.28 \\
\hline-1.0 & 1007.38 & 95.0 & 1009.68 & 3.5 & 1007.17 & 92.0 & 1009.71 & -20.0 & 1010.26 \\
\hline 0.0 & 1007.21 & 98.0 & 1009.76 & 6.0 & 1007.09 & 95.0 & 1009.69 & -18.0 & 1010.25 \\
\hline 1.0 & 1007.19 & 100.0 & 1009.95 & 9.0 & 1007.04 & 97.5 & 1009.72 & -17.0 & 1010.23 \\
\hline 3.0 & 1007.08 & 101.8 & 1010.00 & 12.0 & 1006.98 & 100.0 & 1009.95 & -15.4 & 1010.00 \\
\hline 5.0 & 1007.12 & & & 15.0 & 1006.93 & 100.8 & 1010.00 & -13.4 & 1009.07 \\
\hline 7.0 & 1007.17 & & & 18.0 & 1006.86 & & & -12.0 & 1008.96 \\
\hline 10.0 & 1007.02 & & & 21.0 & 1006.86 & & & -10.0 & 1008.75 \\
\hline 13.0 & 1006.97 & & & 24.0 & 1006.92 & & & -8.7 & 1008.70 \\
\hline 16.0 & 1006.93 & & & 26.0 & 1006.92 & & & -7.4 & 1008.91 \\
\hline 19.0 & 1006.83 & & & 28.0 & 1007.04 & & & -7.1 & 1008.87 \\
\hline 22.0 & 1006.86 & & & 30.0 & 1007.12 & & & -7.0 & 1008.35 \\
\hline 25.0 & 1006.96 & & & 32.4 & 1007.25 & & & -6.0 & 1007.99 \\
\hline 28.0 & 1007.02 & & & 33.4 & 1007.37 & & & -4.5 & 1007.57 \\
\hline 30.0 & 1007.10 & & & 35.7 & 1007.51 & & & -2.3 & 1007.43 \\
\hline 32.6 & 1007.24 & & & 38.0 & 1007.55 & & & 0.0 & 1007.21 \\
\hline 35.0 & 1007.39 & & & 40.0 & 1007.62 & & & 3.0 & 1007.17 \\
\hline 38.0 & 1007.53 & & & 43.0 & 1007.68 & & & 6.0 & 1007.09 \\
\hline 41.0 & 1007.63 & & & 46.0 & 1007.79 & & & 9.0 & 1006.99 \\
\hline 44.0 & 1007.72 & & & 49.0 & 1007.84 & & & 12.0 & 1006.98 \\
\hline 47.0 & 1007.79 & & & 52.0 & 1007.87 & & & 15.0 & 1006.97 \\
\hline 50.0 & 1007.87 & & & 54.0 & 1007.91 & & & 18.0 & 1006.89 \\
\hline 53.0 & 1007.95 & & & 55.5 & 1007.93 & & & 20.0 & 1006.86 \\
\hline 55.0 & 1007.91 & & & 57.2 & 1007.93 & & & 23.0 & 1006.91 \\
\hline 57.0 & 1007.94 & & & 59.0 & 1007.89 & & & & \\
\hline
\end{tabular}


Table 11. (Continued) Listing of horizontal stations and elevations for cross section PR122A

[Sta., station, distance in meters from a reference pin on the left bank; Elev., elevation, in meters above sea level]

1998

\begin{tabular}{cc}
25 September \\
\hline Sta. & Elev. \\
\hline 29.0 & 1007.07 \\
32.0 & 1007.23 \\
35.1 & 1007.44 \\
38.0 & 1007.53 \\
41.0 & 1007.64 \\
44.0 & 1007.74 \\
46.5 & 1007.83 \\
49.0 & 1007.83 \\
52.0 & 1007.89 \\
55.0 & 1007.93 \\
57.0 & 1007.95 \\
57.8 & 1008.02 \\
58.7 & 1007.98 \\
60.0 & 1008.12 \\
61.5 & 1008.37 \\
63.0 & 1008.63 \\
65.0 & 1008.91 \\
65.9 & 1009.03 \\
68.0 & 1009.00 \\
70.0 & 1008.92 \\
72.0 & 1008.83 \\
74.3 & 1008.72 \\
75.6 & 1008.90 \\
76.7 & 1009.39 \\
77.6 & 1010.09 \\
78.3 & 1009.88 \\
80.0 & 1009.76 \\
83.0 & 1009.77 \\
86.0 & 1009.72 \\
89.0 & 1009.73 \\
92.0 & 1009.71 \\
95.0 & 1009.67 \\
98.0 & 1009.77 \\
100.0 & 1009.95 \\
101.7 & 1010.01 \\
145.0 & 1009.80
\end{tabular}




\section{Description of Cross Section PR125}

Location: Township 8 South/Range 48 East--section 21

U. S. Geological Survey quadrangle (1:24,000): Moorhead

Landowners--left bank: William and Glenn Gay

--right bank: 3 Bar Ranch

Access: Left bank

Permission from: Gay Ranch

Distance from Moorhead Gaging Station: 8.61 kilometers

Azimuth of Section (degrees magnetic): 84.5

\section{Reference Monuments}

[ Monuments $-14.3,0.0$ and 5.0 were closest to leveling instrument]

\begin{tabular}{|c|c|c|c|c|c|c|}
\hline \multirow[b]{2}{*}{ Description } & \multirow[b]{2}{*}{$\begin{array}{c}\text { Station } \\
(\mathrm{m})\end{array}$} & \multicolumn{2}{|c|}{ GPS-NAD83 (1992) } & \multicolumn{2}{|c|}{ Measurement } & \multirow{2}{*}{$\begin{array}{l}\text { Elevation } \\
\text { (NGVD1929) } \\
\text { (m) }\end{array}$} \\
\hline & & Latitude & Longitude & $\begin{array}{l}\text { Standard } \\
\text { deviation } \\
\text { (m) }\end{array}$ & $\begin{array}{l}\text { Horizontal } \\
\text { precision } \\
\text { (m) }\end{array}$ & \\
\hline Benchmark--brass circular plate & -20.0 & $45^{\circ} 07^{\prime} 06.40^{\prime \prime}$ & $105^{\circ} 50^{\prime} 17.91^{\prime \prime}$ & 0.193 & 0.608 & 1008.45 \\
\hline $\begin{array}{l}\text { 1/2-inch-rebar; on fence line, } 0.18 \text { meter } \\
\text { above } 1998 \text { ground level }\end{array}$ & -14.3 & & & & & 1008.06 \\
\hline $\begin{array}{l}\text { 1/2-inch-rebar; about } 0.05 \text { meter riverward of } \\
\text { wooden fence post; } 0.16 \text { meter above } 1998 \\
\text { ground level }\end{array}$ & 0.0 & & & & & 1008.16 \\
\hline $\begin{array}{l}\text { 1/2-inch-rebar; } 0.12 \text { meter above } 1998 \text { ground } \\
\text { level }\end{array}$ & 5.0 & & . & & & 1008.01 \\
\hline $\begin{array}{l}\text { 1/2-inch-rebar; } 0.07 \text { meter above } 1998 \text { ground } \\
\text { level }\end{array}$ & 100.8 & & & & & 1007.41 \\
\hline $\begin{array}{l}\text { 1/2-inch-rebar; probably bent and possibly } \\
\text { covered by sand in 1978; elevation was } \\
\text { measured in } 1977\end{array}$ & 105.4 & & & & & 1007.57 \\
\hline $\begin{array}{l}\text { 1/2-inch-rebar; } 0.23 \text { meter above } 1998 \text { ground } \\
\text { level }\end{array}$ & 117.1 & $45^{0} 07$ '05.73”' & $105^{\circ} 50^{\prime} 11.70^{\prime \prime}$ & 0.363 & 0.521 & 1007.84 \\
\hline
\end{tabular}




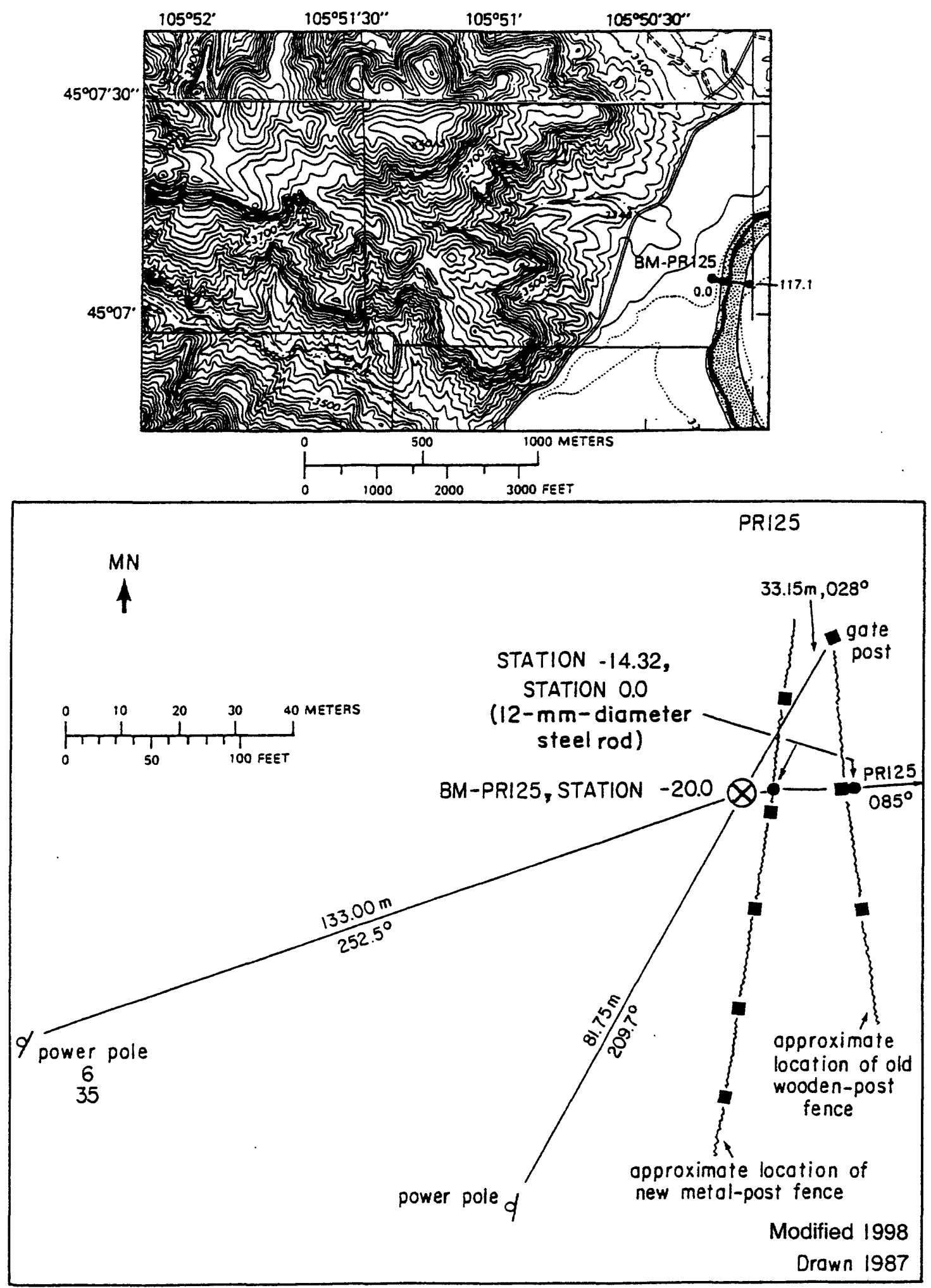

Figure 27. Upper: Location of cross section PR125, bench mark BM-PR125, and the left and right bank reference monuments in the Moorhead quadrangle. Lower: Location of the bench mark on the left bank. MN is magnetic north. 

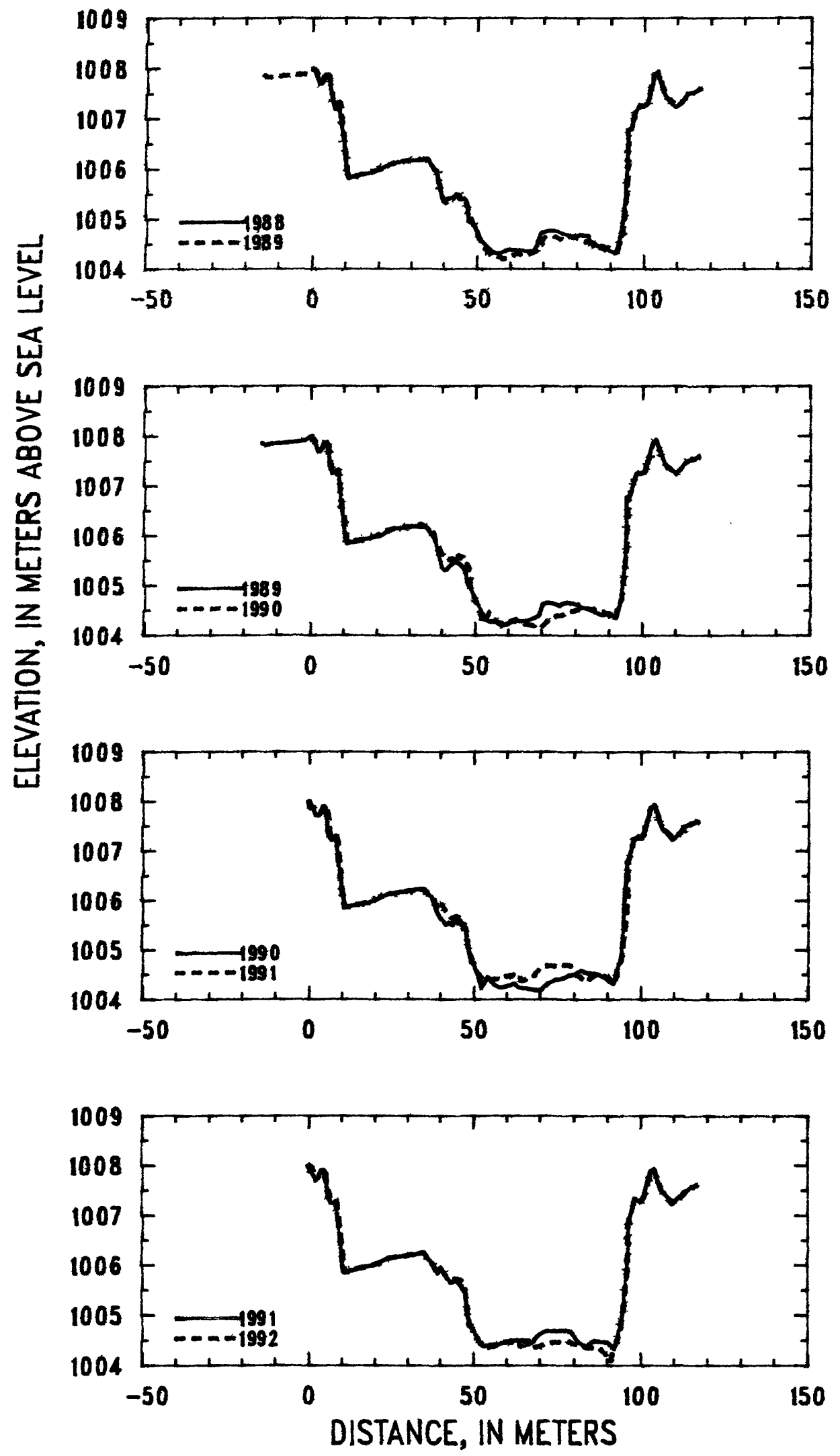

Figure 28. Profiles of cross section PR125 from 1988 to 1992. 

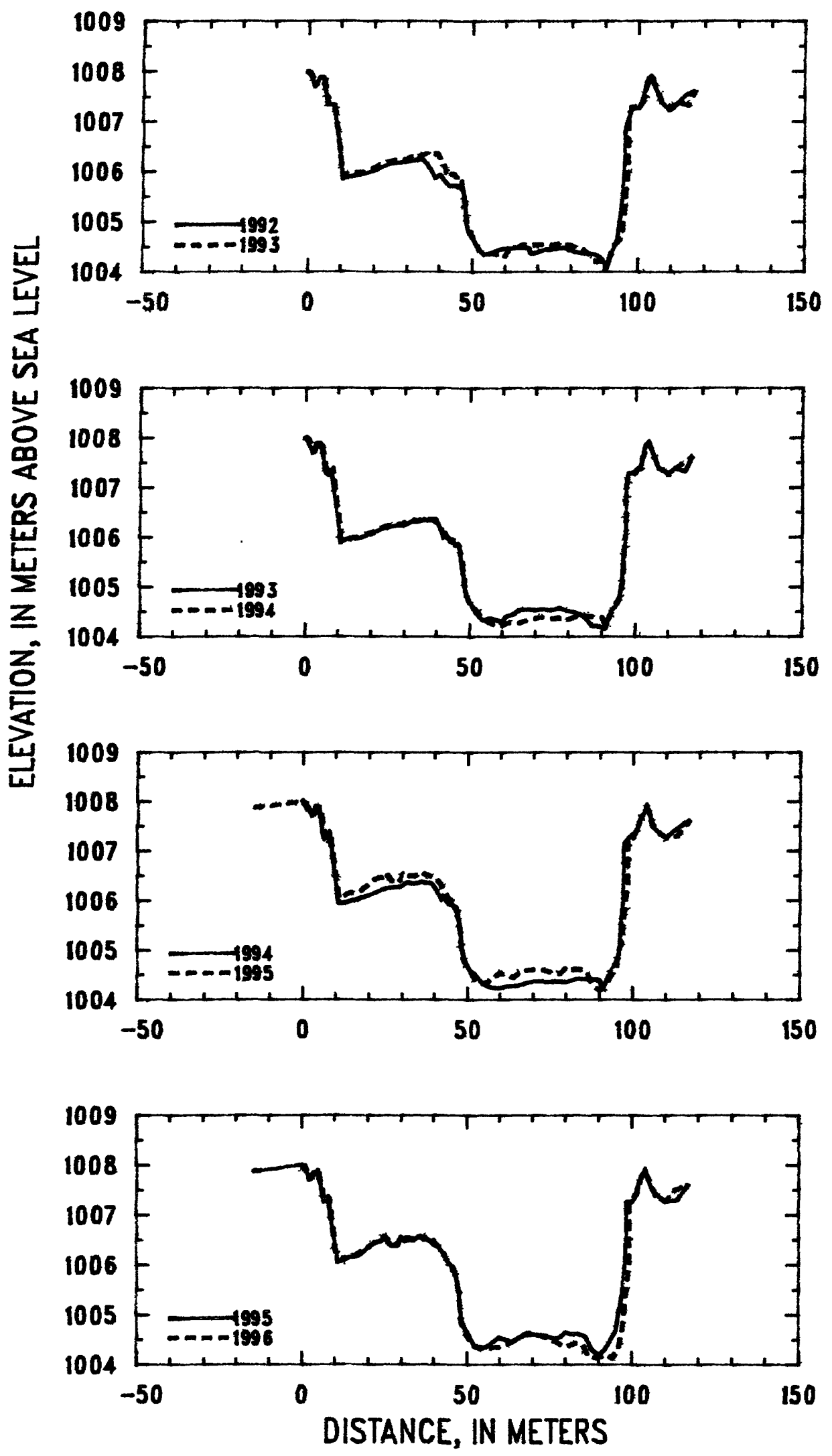

Figure 29. Profiles of cross section PR125 from 1992 to 1996. 


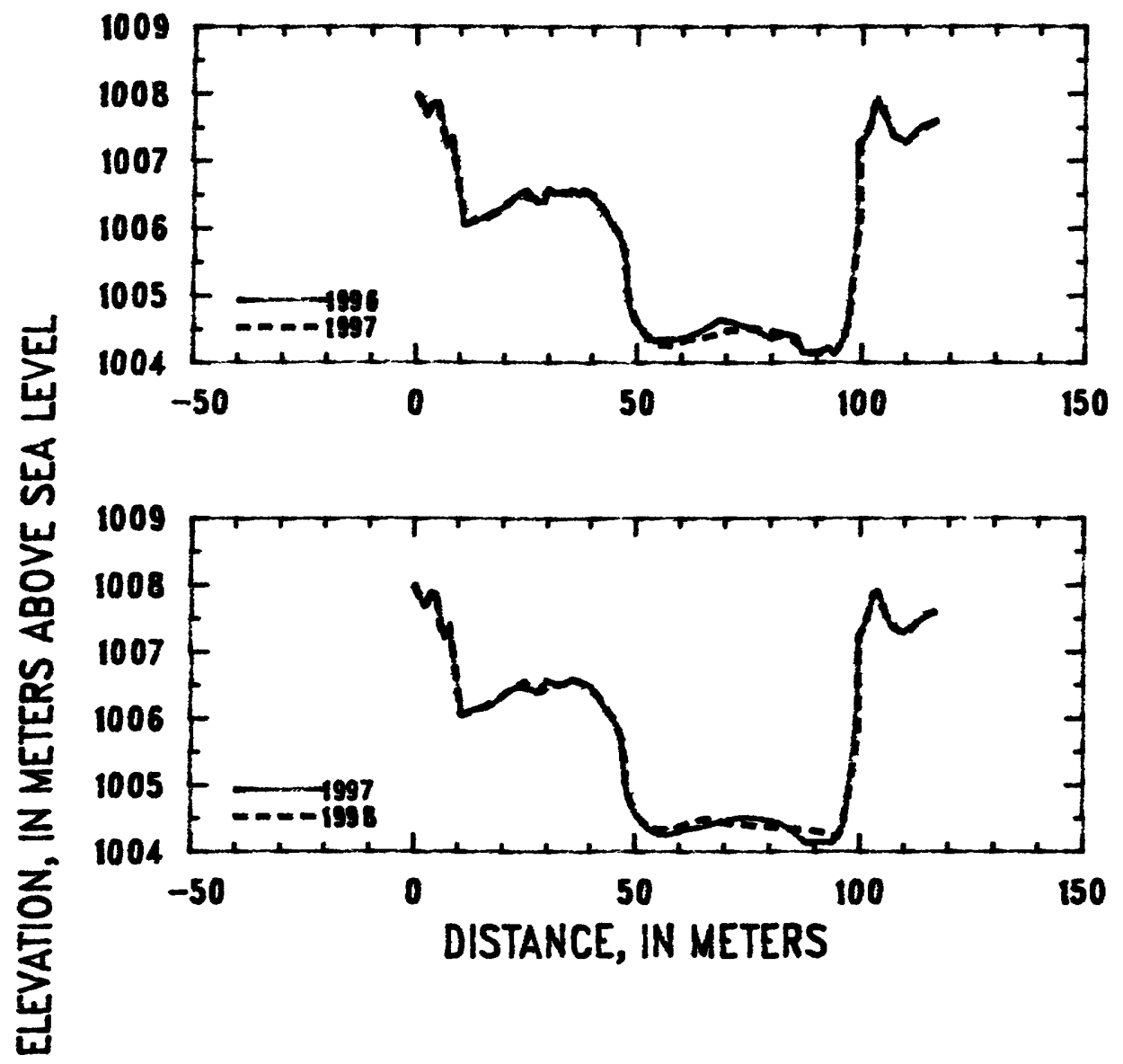

Figure 30. Profiles of cross section PR125 from 1996 to 1998. 
Table 12. Listing of horizontal stations and elevations for cross section PR125

[Sta., station, distance in meters from a reference pin on the left bank; Elev., elevation, in meters above sea level]

\begin{tabular}{|c|c|c|c|c|c|c|c|c|c|}
\hline \multirow{2}{*}{\multicolumn{2}{|c|}{$\begin{array}{c}1989 \\
17 \text { September }\end{array}$}} & \multicolumn{2}{|c|}{1989} & \multicolumn{2}{|c|}{1989} & \multicolumn{2}{|c|}{1990} & \multicolumn{2}{|c|}{1990} \\
\hline & & $17 \mathrm{~S}$ & tember & $17 \mathrm{~S}$ & tember & $14 \mathrm{Se}$ & tember & $14 \mathrm{~S}$ & tember \\
\hline Sta. & Elev. & Sta. & Elev. & Sta. & Elev. & Sta. & Elev. & Sta. & Elev. \\
\hline-14.5 & 1007.86 & 43.0 & 1005.44 & 104.0 & 1007.91 & 0.0 & 1008.01 & 54.0 & 1004.45 \\
\hline-13.0 & 1007.82 & 44.2 & 1005.48 & 105.0 & 1007.73 & 1.5 & 1007.87 & 56.0 & 1004.30 \\
\hline-12.0 & 1007.84 & 45.5 & 1005.42 & 107.0 & 1007.38 & 2.3 & 1007.72 & 58.0 & 1004.23 \\
\hline-10.0 & 1007.85 & 46.8 & 1005.31 & 109.0 & 1007.30 & 3.0 & 1007.71 & 60.0 & 1004.26 \\
\hline-8.0 & 1007.86 & 47.6 & 1005.04 & 110.0 & 1007.26 & 5.0 & 1007.89 & 62.0 & 1004.33 \\
\hline-6.0 & 1007.88 & 48.0 & 1005.02 & 111.0 & 1007.30 & 5.3 & 1007.86 & 64.0 & 1004.23 \\
\hline-4.0 & 1007.88 & 50.2 & .1004 .68 & 113.0 & 1007.49 & 6.0 & 1007.35 & 66.0 & 1004.22 \\
\hline-2.0 & 1007.90 & 52.5 & 1004.39 & 115.0 & 1007.53 & 7.0 & 1007.21 & 68.0 & 1004.19 \\
\hline 0.0 & 1007.99 & 54.0 & 1004.28 & 117.1 & 1007.61 & 8.3 & 1007.30 & 70.0 & 1004.18 \\
\hline 1.0 & 1007.97 & 56.0 & 1004.28 & & & 9.0 & 1006.77 & 72.0 & 1004.32 \\
\hline 2.0 & 1007.78 & 58.0 & 1004.20 & & & 10.0 & 1006.45 & 74.0 & 1004.39 \\
\hline 2.5 & 1007.69 & 60.0 & 1004.24 & & & 11.0 & 1005.86 & 76.0 & 1004.42 \\
\hline 3.0 & 1007.71 & 62.0 & 1004.31 & & & 12.0 & 1005.88 & 78.0 & 1004.48 \\
\hline 4.0 & 1007.87 & 64.0 & 1004.27 & & & 14.0 & 1005.91 & 80.0 & 1004.50 \\
\hline 5.0 & 1007.88 & 66.0 & 1004.30 & & & 16.0 & 1005.93 & 82.0 & 1004.59 \\
\hline 6.0 & 1007.38 & 68.0 & 1004.37 & & & 18.0 & 1005.96 & 84.0 & 1004.54 \\
\hline 6.6 & 1007.25 & 69.0 & 1004.40 & & & 20.0 & 1006.01 & 86.0 & 1004.52 \\
\hline 8.0 & 1007.32 & 70.1 & 1004.62 & & & 22.0 & 1006.09 & 88.0 & 1004.49 \\
\hline 8.4 & 1007.32 & 71.0 & 1004.65 & & & 24.0 & 1006.13 & 90.0 & 1004.41 \\
\hline 9.0 & 1006.92 & 73.0 & 1004.66 & & & 26.0 & 1006.15 & 91.7 & 1004.32 \\
\hline 10.0 & 1006.35 & 75.0 & 1004.59 & & & 28.0 & 1006.18 & 92.8 & 1004.59 \\
\hline 10.6 & 1005.95 & 76.0 & 1004.65 & & & 30.0 & 1006.19 & 93.9 & 1004.81 \\
\hline 11.0 & 1005.84 & 78.0 & 1004.66 & & & 32.0 & 1006.22 & 94.9 & 1005.54 \\
\hline 12.0 & 1005.87 & 80.0 & 1004.63 & & & 34.0 & 1006.24 & 95.0 & 1005.97 \\
\hline 13.0 & 1005.86 & 82.0 & 1004.56 & & & 36.0 & 1006.15 & 95.4 & 1006.27 \\
\hline 15.0 & 1005.90 & 84.0 & 1004.53 & & & 37.6 & 1006.06 & 95.8 & 1006.78 \\
\hline 17.0 & 1005.94 & 86.0 & 1004.46 & & & 38.0 & 1005.94 & 96.3 & 1006.87 \\
\hline 19.0 & 1005.97 & 88.0 & 1004.41 & & & 39.0 & 1005.76 & 97.0 & 1007.04 \\
\hline 21.0 & 1005.99 & 90.0 & 1004.46 & & & 40.0 & 1005.64 & 98.0 & 1007.26 \\
\hline 23.0 & 1006.09 & 92.0 & 1004.36 & & & 41.8 & 1005.51 & 99.0 & 1007.26 \\
\hline 25.0 & 1006.15 & 93.3 & 1004.67 & & & 42.7 & 1005.55 & 100.0 & 1007.26 \\
\hline 27.0 & 1006.15 & 94.0 & 1005.01 & & & 43.6 & 1005.50 & 100.7 & 1007.34 \\
\hline 29.0 & 1006.17 & 94.6 & 1005.46 & & & 44.4 & 1005.65 & 100.8 & 1007.41 \\
\hline 31.0 & 1006.19 & 95.0 & 1005.73 & & & 45.3 & 1005.54 & 101.0 & 1007.36 \\
\hline 33.0 & 1006.18 & 95.1 & 1006.77 & & & 46.0 & 1005.59 & 102.0 & 1007.66 \\
\hline 35.0 & 1006.19 & 96.0 & 1006.81 & & & 47.0 & 1005.42 & 103.0 & 1007.86 \\
\hline 36.0 & 1006.11 & 97.0 & 1007.07 & & & 47.6 & 1005.43 & 104.0 & 1007.93 \\
\hline 37.0 & 1006.00 & 98.0 & 1007.25 & & & 48.5 & 1004.96 & 105.0 & 1007.73 \\
\hline 37.7 & 1005.98 & 100.0 & 1007.26 & & & 49.0 & 1004.85 & 106.3 & 1007.45 \\
\hline 39.0 & 1005.62 & 100.8 & 1007.30 & & & 50.6 & 1004.59 & 108.0 & 1007.36 \\
\hline 40.0 & 1005.35 & 102.0 & 1007.65 & & & 51.4 & 1004.47 & 109.4 & 1007.22 \\
\hline 41.0 & 1005.31 & 103.0 & 1007.87 & & & 52.3 & 1004.24 & 111.0 & 1007.31 \\
\hline
\end{tabular}


Table 12. (Continued) Listing of horizontal stations and elevations for cross section PR125

[Sta., station, distance in meters from a reference pin on the left bank; Elev., elevation, in meters above sea level]

\begin{tabular}{|c|c|c|c|c|c|c|c|c|c|}
\hline \multirow{2}{*}{\multicolumn{2}{|c|}{$\begin{array}{c}1990 \\
14 \text { September }\end{array}$}} & \multirow{2}{*}{\multicolumn{2}{|c|}{$\begin{array}{l}1991 \\
29 \text { August }\end{array}$}} & \multirow{2}{*}{\multicolumn{2}{|c|}{$\begin{array}{c}1991 \\
29 \text { August }\end{array}$}} & \multicolumn{2}{|c|}{1992} & \multicolumn{2}{|c|}{1992} \\
\hline & & & & & & 26 & ugust & 26 & ugust \\
\hline Sta. & Elev. & Sta. & Elev. & Sta. & Elev. & Sta. & Elev. & Sta. & Elev. \\
\hline 113.0 & 1007.50 & 0.0 & 1007.99 & 63.8 & 1004.49 & 0.0 & 1008.00 & 76.0 & 1004.47 \\
\hline 115.0 & 1007.53 & 2.2 & 1007.74 & 64.2 & 1004.37 & 1.0 & 1007.96 & 78.0 & 1004.49 \\
\hline 117.1 & 1007.60 & 3.2 & 1007.74 & 66.0 & 1004.41 & 2.5 & 1007.70 & 80.0 & 1004.42 \\
\hline & & 4.0 & 1007.89 & 68.0 & 1004.46 & 4.0 & 1007.88 & 82.0 & 1004.39 \\
\hline & & 5.0 & 1007.90 & 68.6 & 1004.55 & 5.0 & 1007.88 & 84.0 & 1004.36 \\
\hline & & 5.3 & 1007.86 & 70.0 & 1004.63 & 6.3 & 1007.33 & 86.0 & 1004.36 \\
\hline & & 6.3 & 1007.33 & 72.0 & 1004.69 & 8.2 & 1007.33 & 88.0 & 1004.33 \\
\hline & & 7.0 & 1007.23 & 74.0 & 1004.67 & 9.5 & 1006.67 & 90.0 & 1004.19 \\
\hline & & 8.4 & 1007.27 & 76.0 & 1004.69 & 11.0 & 1005.85 & 90.8 & 1004.07 \\
\hline & & 9.6 & 1006.50 & 78.0 & 1004.70 & 13.0 & 1005.89 & 92.0 & 1004.35 \\
\hline & & 10.6 & 1005.93 & 79.2 & 1004.66 & 15.0 & 1005.92 & 93.7 & 1004.64 \\
\hline & & 11.0 & 1005.86 & 81.0 & 1004.47 & 18.0 & 1005.96 & 94.0 & 1004.75 \\
\hline & & 13.0 & 1005.88 & 83.8 & 1004.35 & 21.0 & 1006.04 & 94.7 & 1005.08 \\
\hline & & 15.0 & 1005.92 & 84.5 & 1004.42 & 24.0 & 1006.14 & 96.0 & 1006.03 \\
\hline & & 17.0 & 1005.96 & 86.0 & 1004.49 & 27.0 & 1006.18 & 96.0 & 1006.84 \\
\hline & & 20.0 & 1006.01 & 88.0 & 1004.48 & 30.0 & 1006.19 & 97.0 & 1007.04 \\
\hline & & 22.0 & 1006.08 & 90.0 & 1004.46 & 33.0 & 1006.23 & 98.0 & 1007.25 \\
\hline & & 24.0 & 1006.13 & 92.0 & 1004.33 & 35.0 & 1006.24 & 100.0 & 1007.27 \\
\hline & & 26.0 & 1006.15 & 92.7 & 1004.50 & 37.0 & 1006.10 & 100.8 & 1007.35 \\
\hline & & 28.0 & 1006.18 & 94.4 & 1004.92 & 39.0 & 1005.86 & 102.0 & 1007.63 \\
\hline & & 30.0 & 1006.20 & 96.0 & 1005.99 & 40.2 & 1005.94 & 103.0 & 1007.87 \\
\hline & & 32.0 & 1006.21 & 96.1 & 1006.81 & 41.5 & 1005.82 & 104.0 & 1007.92 \\
\hline & & 34.0 & 1006.23 & 97.0 & 1007.04 & 43.0 & 1005.71 & 105.0 & 1007.72 \\
\hline & & 35.0 & 1006.23 & 98.0 & 1007.34 & 45.0 & 1005.72 & 107.0 & 1007.39 \\
\hline & & 36.0 & 1006.17 & 100.0 & 1007.26 & 47.0 & 1005.65 & 109.4 & 1007.23 \\
\hline & & 37.0 & 1006.09 & 100.8 & 1007.39 & 47.6 & 1005.49 & 111.4 & 1007.31 \\
\hline & & 38.0 & 1006.03 & 103.0 & 1007.88 & 48.1 & 1005.17 & 114.0 & 1007.52 \\
\hline & & 39.3 & 1005.84 & 104.0 & 1007.91 & 48.4 & 1004.90 & 117.1 & 1007.61 \\
\hline & & 40.3 & 1005.95 & 105.0 & 1007.72 & 49.0 & 1004.77 & & \\
\hline & & 41.5 & 1005.79 & 106.0 & 1007.50 & 50.2 & 1004.66 & & \\
\hline & & 43.5 & 1005.64 & 108.0 & 1007.37 & 52.0 & 1004.44 & & \\
\hline & & 44.8 & 1005.69 & 110.0 & 1007.27 & 53.8 & 1004.35 & & \\
\hline & & 46.0 & 1005.61 & 112.0 & 1007.38 & 56.0 & 1004.37 & & \\
\hline & & 47.6 & 1005.45 & 115.0 & 1007.56 & 58.0 & 1004.44 & & \\
\hline & & 48.3 & 1004.94 & 117.1 & 1007.62 & 60.0 & 1004.46 & & \\
\hline & & 50.0 & 1004.71 & & & 62.0 & 1004.46 & & \\
\hline & & 51.2 & 1004.52 & & & 64.0 & 1004.49 & & \\
\hline & & 53.0 & 1004.38 & & & 67.2 & 1004.48 & & \\
\hline & & 55.0 & 1004.40 & & & 68.0 & 1004.36 & & \\
\hline & & 58.0 & 1004.42 & & & 70.0 & 1004.38 & & \\
\hline & & 60.0 & 1004.47 & & & 72.0 & 1004.44 & & \\
\hline & & 62.0 & 1004.50 & & & 74.0 & 1004.45 & & \\
\hline
\end{tabular}


Table 12. (Continued) Listing of horizontal stations and elevations for cross section PR125

[Sta., station, distance in meters from a reference pin on the left bank; Elev., elevation, in meters above sea level]

\begin{tabular}{|c|c|c|c|c|c|c|c|c|c|}
\hline \multirow{2}{*}{\multicolumn{2}{|c|}{$\begin{array}{c}1993 \\
26 \text { August }\end{array}$}} & \multicolumn{2}{|c|}{1993} & \multicolumn{2}{|c|}{1994} & \multicolumn{2}{|c|}{1994} & \multicolumn{2}{|c|}{1995} \\
\hline & & 26 & ugust & $25 \mathrm{Se}$ & tember & $25 \mathrm{Sc}$ & tember & $24 \mathrm{Se}$ & tember \\
\hline Sta. & Elev. & Sta. & Elev. & Sta. & Elev. & Sta. & Elev. & Sta. & Elev. \\
\hline 0.0 & 1008.00 & 56.0 & 1004.37 & 0.0 & 1008.02 & 65.0 & 1004.27 & -14.5 & 1007.87 \\
\hline 1.0 & 1007.96 & 58.0 & 1004.34 & 1.0 & 1007.97 & 68.0 & 1004.34 & 0.0 & 1008.00 \\
\hline 2.5 & 1007.71 & 60.0 & 1004.30 & 3.0 & 1007.71 & 71.0 & 1004.39 & 3.0 & 1007.71 \\
\hline 4.0 & 1007.89 & 61.0 & 1004.41 & 4.0 & 1007.90 & 74.0 & 1004.35 & 5.0 & 1007.88 \\
\hline 5.0 & 1007.89 & 63.0 & 1004.44 & 5.0 & 1007.88 & 77.0 & 1004.37 & 7.0 & 1007.26 \\
\hline 6.5 & 1007.29 & 65.0 & 1004.54 & 6.7 & 1007.25 & 80.0 & 1004.35 & 8.3 & 1007.33 \\
\hline 7.3 & 1007.24 & 67.0 & 1004.54 & 7.1 & 1007.24 & 83.0 & 1004.43 & 11.0 & 1006.05 \\
\hline 8.3 & 1007.28 & 69.0 & 1004.54 & 8.4 & 1007.37 & 86.0 & 1004.41 & 12.0 & 1006.07 \\
\hline 10.9 & 1005.89 & 71.0 & 1004.54 & 11.0 & 1005.95 & 89.0 & 1004.41 & 14.0 & 1006.15 \\
\hline 12.0 & 1005.94 & 73.0 & 1004.55 & 12.0 & 1005.94 & 90.0 & 1004.40 & 16.0 & 1006.15 \\
\hline 14.0 & 1005.99 & 75.0 & 1004.53 & 13.0 & 1005.95 & 91.0 & 1004.23 & 18.0 & 1006.22 \\
\hline 16.0 & 1005.97 & 77.0 & 1004.58 & 15.0 & 1005.99 & 93.0 & 1004.53 & 20.0 & 1006.34 \\
\hline 18.0 & 1006.03 & 79.0 & 1004.54 & 18.0 & 1006.06 & 95.6 & 1004.85 & 22.0 & 1006.45 \\
\hline 20.0 & 1006.07 & 81.0 & 1004.50 & 21.0 & 1006.13 & 96.3 & 1005.25 & 24.0 & 1006.47 \\
\hline 22.0 & 1006.14 & 83.0 & 1004.47 & 24.0 & 1006.23 & 96.5 & 1005.68 & 25.0 & 1006.56 \\
\hline 24.0 & 1006.19 & 85.0 & 1004.38 & 27.0 & 1006.26 & 97.0 & 1006.07 & 26.0 & 1006.45 \\
\hline 26.0 & 1006.23 & 87.0 & 1004.22 & 29.0 & 1006.27 & 97.1 & 1006.99 & 27.0 & 1006.38 \\
\hline 28.0 & 1006.24 & 89.0 & 1004.22 & 30.0 & 1006.30 & 97.4 & 1007.17 & 29.0 & 1006.38 \\
\hline 30.0 & 1006.25 & 91.0 & 1004.15 & 31.0 & 1006.35 & 99.0 & 1007.28 & 30.0 & 1006.55 \\
\hline 32.0 & 1006.32 & 92.0 & 1004.40 & 32.0 & 1006.36 & 100.8 & 1007.37 & 31.0 & 1006.47 \\
\hline 34.0 & 1006.31 & 93.0 & 1004.54 & 34.0 & 1006.33 & 102.0 & 1007.60 & 32.0 & 1006.50 \\
\hline 36.0 & 1006.36 & 94.7 & 1004.65 & 36.0 & 1006.38 & 104.0 & 1007.91 & 34.0 & 1006.49 \\
\hline 37.0 & 1006.34 & 95.4 & 1004.82 & 37.0 & 1006.36 & 105.0 & 1007.74 & 35.0 & 1006.55 \\
\hline 38.0 & 1006.36 & 96.2 & 1005.32 & 39.0 & 1006.35 & 106.5 & 1007.46 & 36.0 & 1006.57 \\
\hline 39.5 & 1006.36 & 96.3 & 1005.56 & 41.0 & 1006.18 & 108.0 & 1007.37 & 37.0 & 1006.53 \\
\hline 40.3 & 1006.26 & 97.0 & 1005.93 & 42.0 & 1006.02 & 110.0 & 1007.27 & 38.0 & 1006.52 \\
\hline 41.4 & 1006.14 & 97.1 & 1007.05 & 42.6 & 1006.09 & 112.0 & 1007.38 & 39.0 & 1006.48 \\
\hline 42.0 & 1006.00 & 98.0 & 1007.29 & 43.7 & 1005.96 & 115.0 & 1007.53 & 40.4 & 1006.39 \\
\hline 42.5 & 1006.07 & 100.0 & 1007.28 & 44.4 & 1005.93 & 117.1 & 1007.62 & 41.1 & 1006.40 \\
\hline 43.6 & 1005.96 & 100.8 & 1007.37 & 44.7 & 1005.96 & & & 43.0 & 1006.20 \\
\hline 44.0 & 1005.93 & 101.4 & 1007.40 & 46.0 & 1005.86 & & & 44.5 & 1006.04 \\
\hline 44.6 & 1005.95 & 103.0 & 1007.86 & 46.4 & 1005.87 & & & 46.0 & 1005.95 \\
\hline 45.5 & 1005.84 & 104.0 & 1007.92 & 47.0 & 1005.72 & & & 47.4 & 1005.56 \\
\hline 46.3 & 1005.87 & 105.0 & 1007.74 & 47.7 & 1005.51 & & & 48.5 & 1004.86 \\
\hline 46.8 & 1005.79 & 107.0 & 1007.39 & 48.4 & 1005.00 & & & 49.0 & 1004.78 \\
\hline 47.1 & 1005.58 & 110.0 & 1007.28 & 49.4 & 1004.73 & & & 52.5 & 1004.36 \\
\hline 47.8 & 1005.36 & 112.0 & 1007.39 & 50.8 & 1004.58 & & & 54.0 & 1004.33 \\
\hline 48.3 & 1005.00 & 115.0 & 1007.32 & 52.0 & 1004.54 & & & 56.0 & 1004.35 \\
\hline 49.0 & 1004.82 & 117.1 & 1007.61 & 54.0 & 1004.36 & & & 58.0 & 1004.45 \\
\hline 50.0 & 1004.70 & & & 57.0 & 1004.25 & & & 60.0 & 1004.55 \\
\hline 52.0 & 1004.45 & & & $60.0^{\circ}$ & 1004.23 & & & 62.0 & 1004.50 \\
\hline 54.0 & 1004.33 & & & 62.0 & 1004.27 & & & 64.0 & 1004.45 \\
\hline
\end{tabular}


Table 12. (Continued) Listing of horizontal stations and elevations for cross section PR125

[Sta., station, distance in meters from a reference pin on the left bank; Elev., elevation, in meters above sea level]

\begin{tabular}{|c|c|c|c|c|c|c|c|c|c|}
\hline \multirow{2}{*}{\multicolumn{2}{|c|}{$\begin{array}{c}1995 \\
24 \text { September }\end{array}$}} & \multirow{2}{*}{\multicolumn{2}{|c|}{$\begin{array}{c}1996 \\
17 \text { October }\end{array}$}} & \multirow{2}{*}{\multicolumn{2}{|c|}{$\begin{array}{c}1996 \\
17 \text { October }\end{array}$}} & \multicolumn{2}{|c|}{1997} & \multicolumn{2}{|c|}{1997} \\
\hline & & & & & & $18 \mathrm{~S}$ & tember & $18 \mathrm{Se}$ & tember \\
\hline Sta. & Elev. & Sta. & Elev. & Sta. & Elev. & Sta. & Elev. & Sta. & Elev. \\
\hline 66.0 & 1004.55 & 0.0 & 1008.00 & 66.0 & 1004.53 & 0.0 & 1008.00 & 94.0 & 1004.14 \\
\hline 68.0 & 1004.60 & 1.0 & 1007.97 & 68.5 & 1004.64 & 2.5 & 1007.71 & 96.0 & 1004.36 \\
\hline 70.0 & 1004.61 & 2.5 & 1007.71 & 71.0 & 1004.60 & 4.0 & 1007.90 & 97.0 & 1004.66 \\
\hline 72.0 & 1004.60 & 4.0 & 1007.89 & 74.0 & 1004.54 & 5.0 & 1007.88 & 99.5 & 1006.14 \\
\hline 74.0 & 1004.59 & 5.0 & 1007.88 & 77.0 & 1004.46 & 7.0 & 1007.24 & 99.5 & 1007.24 \\
\hline 76.0 & 1004.53 & 7.0 & 1007.25 & 80.0 & 1004.35 & 8.2 & 1007.34 & 100.6 & 1007.35 \\
\hline 78.0 & 1004.53 & 8.0 & 1007.35 & 83.0 & 1004.43 & 11.0 & 1006.07 & 101.5 & 1007.49 \\
\hline 80.0 & 1004.65 & 8.3 & 1007.31 & 85.5 & 1004.38 & 12.0 & 1006.08 & 103.0 & 1007.87 \\
\hline 82.0 & 1004.61 & 10.8 & 1006.07 & 87.0 & 1004.15 & 14.0 & 1006.16 & 104.0 & 1007.91 \\
\hline 84.0 & 1004.62 & 12.0 & 1006.08 & 90.0 & 1004.14 & 16.0 & 1006.16 & 107.0 & 1007.38 \\
\hline 86.0 & 1004.57 & 15.0 & 1006.16 & 92.5 & 1004.21 & 18.0 & 1006.23 & 110.0 & 1007.29 \\
\hline 88.0 & 1004.29 & 17.0 & 1006.23 & 94.0 & 1004.12 & 20.0 & 1006.34 & 112.0 & 1007.39 \\
\hline 90.0 & 1004.19 & 19.0 & 1006.29 & 96.0 & 1004.35 & 22.0 & 1006.46 & 115.0 & 1007.53 \\
\hline 92.0 & 1004.31 & 21.0 & 1006.40 & 97.0 & 1004.66 & 24.0 & 1006.49 & 117.1 & 1007.60 \\
\hline 94.0 & 1004.54 & 23.0 & 1006.50 & 99.0 & 1005.98 & 26.0 & 1006.45 & & \\
\hline 95.0 & 1004.64 & 25.0 & 1006.58 & 99.2 & 1007.27 & 28.0 & 1006.41 & & \\
\hline 95.3 & 1004.77 & 27.0 & 1006.41 & 100.8 & 1007.36 & 30.0 & 1006.58 & & \\
\hline 96.0 & 1005.08 & 29.0 & 1006.41 & 102.0 & 1007.61 & 32.0 & 1006.54 & & \\
\hline 96.5 & 1005.13 & 30.0 & 1006.59 & 103.5 & 1007.91 & 34.0 & 1006.52 & & \\
\hline 98.3 & 1006.32 & 31.0 & 1006.54 & 105.0 & 1007.71 & 36.0 & 1006.59 & & \\
\hline 98.4 & 1007.26 & 32.0 & 1006.54 & 107.0 & 1007.39 & 38.0 & 1006.55 & & \\
\hline 100.0 & 1007.26 & 34.0 & 1006.51 & 110.0 & 1007.27 & 40.0 & 1006.50 & & \\
\hline 100.8 & 1007.37 & 36.0 & 1006.56 & 113.0 & 1007.50 & 42.0 & 1006.31 & & \\
\hline 102.0 & 1007.62 & 37.0 & 1006.51 & 117.1 & 1007.61 & 44.0 & 1006.09 & & \\
\hline 104.0 & 1007.92 & 38.0 & 1006.58 & & & 46.0 & 1005.90 & & \\
\hline 106.0 & 1007.51 & 39.0 & 1006.55 & & & 47.1 & 1005.66 & & \\
\hline 108.0 & 1007.37 & 40.0 & 1006.47 & & & 48.0 & 1005.01 & & \\
\hline 110.0 & 1007.27 & 42.0 & 1006.31 & & & 49.4 & 1004.66 & & \\
\hline 113.5 & 1007.30 & 43.0 & 1006.23 & & & 52.5 & 1004.36 & & \\
\hline 117.1 & 1007.61 & 44.0 & 1006.07 & & & 55.0 & 1004.26 & & \\
\hline & & 45.5 & 1005.95 & & & 58.0 & 1004.25 & & \\
\hline & & 46.8 & 1005.76 & & & 61.0 & 1004.34 & & \\
\hline & & 47.8 & 1005.28 & & & 64.0 & 1004.36 & & \\
\hline & & 48.0 & 1005.03 & & & 67.0 & 1004.41 & & \\
\hline & & 48.3 & 1004.84 & & & 70.0 & 1004.48 & & \\
\hline & & 49.2 & 1004.67 & & & 73.0 & 1004.51 & & \\
\hline & & 50.0 & 1004.57 & & & 76.0 & 1004.52 & & \\
\hline & & 52.0 & 1004.42 & & & 79.0 & 1004.48 & & \\
\hline & & 54.0 & 1004.32 & & & 82.0 & 1004.44 & & \\
\hline & & 57.0 & 1004.34 & & & 85.0 & 1004.32 & & \\
\hline & & 60.0 & 1004.35 & & & 88.0 & 1004.14 & & \\
\hline & & 63.0 & 1004.42 & & & 91.0 & 1004.15 & & \\
\hline
\end{tabular}


Table 12. (Continued) Listing of horizontal stations and elevations for cross section PR125

[Sta., station, distance in meters from a reference pin on the left bank; Elev., elevation, in meters above sea level]

\begin{tabular}{|c|c|c|c|}
\hline \multirow{2}{*}{\multicolumn{2}{|c|}{$\begin{array}{c}1998 \\
22 \text { September }\end{array}$}} & \multicolumn{2}{|c|}{1998} \\
\hline & & $22 \mathrm{Se}$ & tember \\
\hline Sta. & Elev. & Sta. & Elev. \\
\hline 0.0 & 1008.00 & 69.0 & 1004.46 \\
\hline 2.7 & 1007.71 & 71.0 & 1004.43 \\
\hline 4.0 & 1007.89 & 73.0 & 1004.41 \\
\hline 5.0 & 1007.88 & 75.0 & 1004.41 \\
\hline 6.2 & 1007.36 & 77.0 & 1004.39 \\
\hline 7.3 & 1007.24 & 79.0 & 1004.39 \\
\hline 8.1 & 1007.37 & 81.0 & 1004.38 \\
\hline 10.8 & 1006.07 & 83.0 & 1004.36 \\
\hline 11.6 & 1006.07 & 85.0 & 1004.34 \\
\hline 13.0 & 1006.12 & 87.0 & 1004.34 \\
\hline 15.0 & 1006.16 & 89.0 & 1004.33 \\
\hline 17.0 & 1006.22 & 91.0 & 1004.30 \\
\hline 19.0 & 1006.29 & 93.0 & 1004.25 \\
\hline 21.0 & 1006.41 & 95.0 & 1004.22 \\
\hline 23.0 & 1006.47 & 96.0 & 1004.39 \\
\hline 25.0 & 1006.56 & 97.3 & 1004.81 \\
\hline 27.0 & 1006.40 & 99.4 & 1005.77 \\
\hline 29.0 & 1006.42 & 99.6 & 1006.04 \\
\hline 31.0 & 1006.53 & 99.6 & 1007.23 \\
\hline 33.0 & 1006.51 & 100.7 & 1007.34 \\
\hline 35.0 & 1006.53 & 103.0 & 1007.87 \\
\hline 37.0 & 1006.58 & 104.0 & 1007.90 \\
\hline 38.0 & 1006.55 & 106.3 & 1007.46 \\
\hline 40.0 & 1006.50 & 108.0 & 1007.35 \\
\hline 41.0 & 1006.38 & 110.0 & 1007.27 \\
\hline 43.0 & 1006.25 & 112.0 & 1007.36 \\
\hline 44.0 & 1006.10 & 114.0 & 1007.52 \\
\hline 45.0 & 1006.04 & 116.0 & 1007.58 \\
\hline 47.0 & 1005.70 & 117.1 & 1007.61 \\
\hline 47.4 & 1005.48 & & \\
\hline 47.8 & 1005.38 & & \\
\hline 48.0 & 1004.96 & & \\
\hline 48.8 & 1004.81 & & \\
\hline 50.5 & 1004.55 & & \\
\hline 53.0 & 1004.37 & & \\
\hline 55.0 & 1004.33 & & \\
\hline 57.0 & 1004.34 & & \\
\hline 59.0 & 1004.35 & & \\
\hline 61.0 & 1004.41 & & \\
\hline 63.0 & 1004.45 & & \\
\hline 65.0 & 1004.49 & & \\
\hline 67.0 & 1004.50 & & \\
\hline
\end{tabular}




\section{Description of Cross Section PR130}

Location: Township 8 South/Range 48 East--section 14

U. S. Geological Survey quadrangle (1:24,000): Bloom Creek

Landowners--left bank: Gay Ranch

--right bank: Bowers Ranch

Access: Left bank

Permission from: Gay Ranch

Distance from Moorhead Gaging Station: 13.92 kilometers

Azimuth of Section (degrees magnetic): 075

\section{Reference Monuments}

[Monuments at stations -1.1 and 0.0 were closest to leveling instrument]

\begin{tabular}{|c|c|c|c|c|c|c|}
\hline \multirow[b]{2}{*}{ Description } & \multirow[b]{2}{*}{$\begin{array}{l}\text { Station } \\
\text { (m) }\end{array}$} & \multicolumn{2}{|c|}{ GPS-NAD83 (1992) } & \multicolumn{2}{|c|}{ Measurement } & \multirow[b]{2}{*}{$\begin{array}{c}\text { Elevation } \\
\text { (NGVD1929) } \\
\text { (m) }\end{array}$} \\
\hline & & Latitude & Longitude & $\begin{array}{l}\text { Standard } \\
\text { deviation } \\
\text { (m) }\end{array}$ & $\begin{array}{l}\text { Horizontal } \\
\text { precision } \\
\text { (m) }\end{array}$ & \\
\hline Benchmark--brass circular plate & -53.0 & $45^{\circ} 08^{\prime} 23.46^{\prime \prime}$ & $105^{\circ} 48^{\prime} 39.01^{\prime \prime}$ & 0.554 & 0.922 & 1002.67 \\
\hline 1/2-inch-rebar; bent flat at 1998 ground level & -1.1 & $45^{\circ} 08^{\prime} 23.52^{\prime \prime}$ & $105^{\circ} 48^{\prime} 36.61^{\prime \prime}$ & 0.384 & 0.690 & 1002.69 \\
\hline 1/2-inch-rebar; bent flat at 1998 ground level & 0.0 & & & & & 1002.67 \\
\hline $\begin{array}{l}\text { 1/2-inch-rebar; } 0.02 \text { meter above } 1998 \text { ground } \\
\text { level }\end{array}$ & 101.9 & & & & & 1000.64 \\
\hline $\begin{array}{l}\text { 1/2-inch-rebar; } 0.01 \text { meter above } 1987 \text { ground } \\
\text { level; could not find in } 1998\end{array}$ & 108.4 & & & & & 1000.92 \\
\hline $\begin{array}{l}\text { 1/2-inch-rebar; } 0.20 \text { meter above } 1998 \text { ground } \\
\text { level }\end{array}$ & 110.6 & $45^{\circ} 08^{\prime} 23.65^{\prime \prime}$ & $105^{\circ} 48^{\prime} 31.52^{\prime \prime}$ & 0.364 & 1.064 & 1001.92 \\
\hline
\end{tabular}




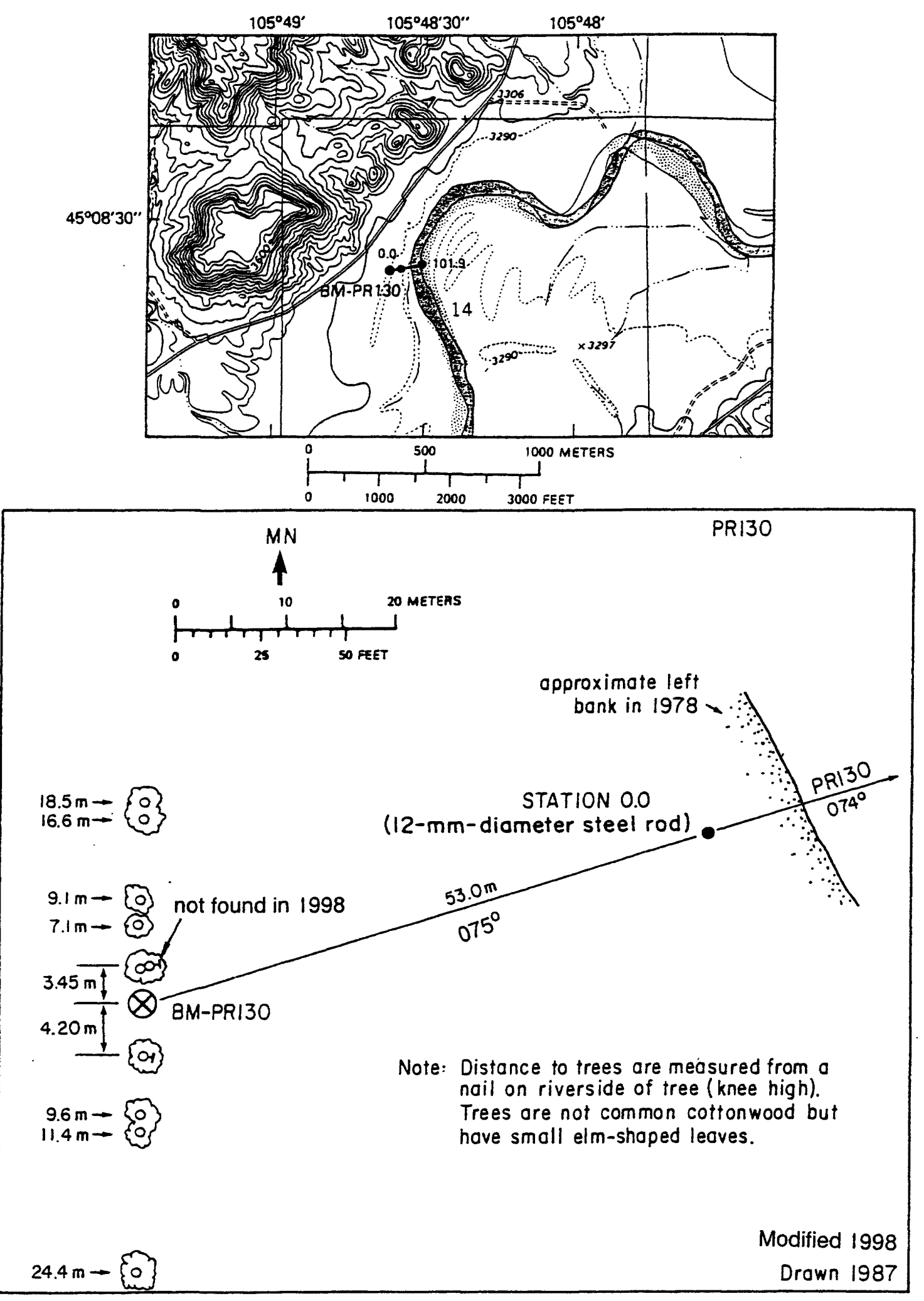

Figure 31. Upper: Location of cross section PR130, bench mark BM-PR130, and the left and right bank reference monuments in the Bloom Creek quadrangle. Lower: Location of the bench mark on the left bank. MN is magnetic north. 

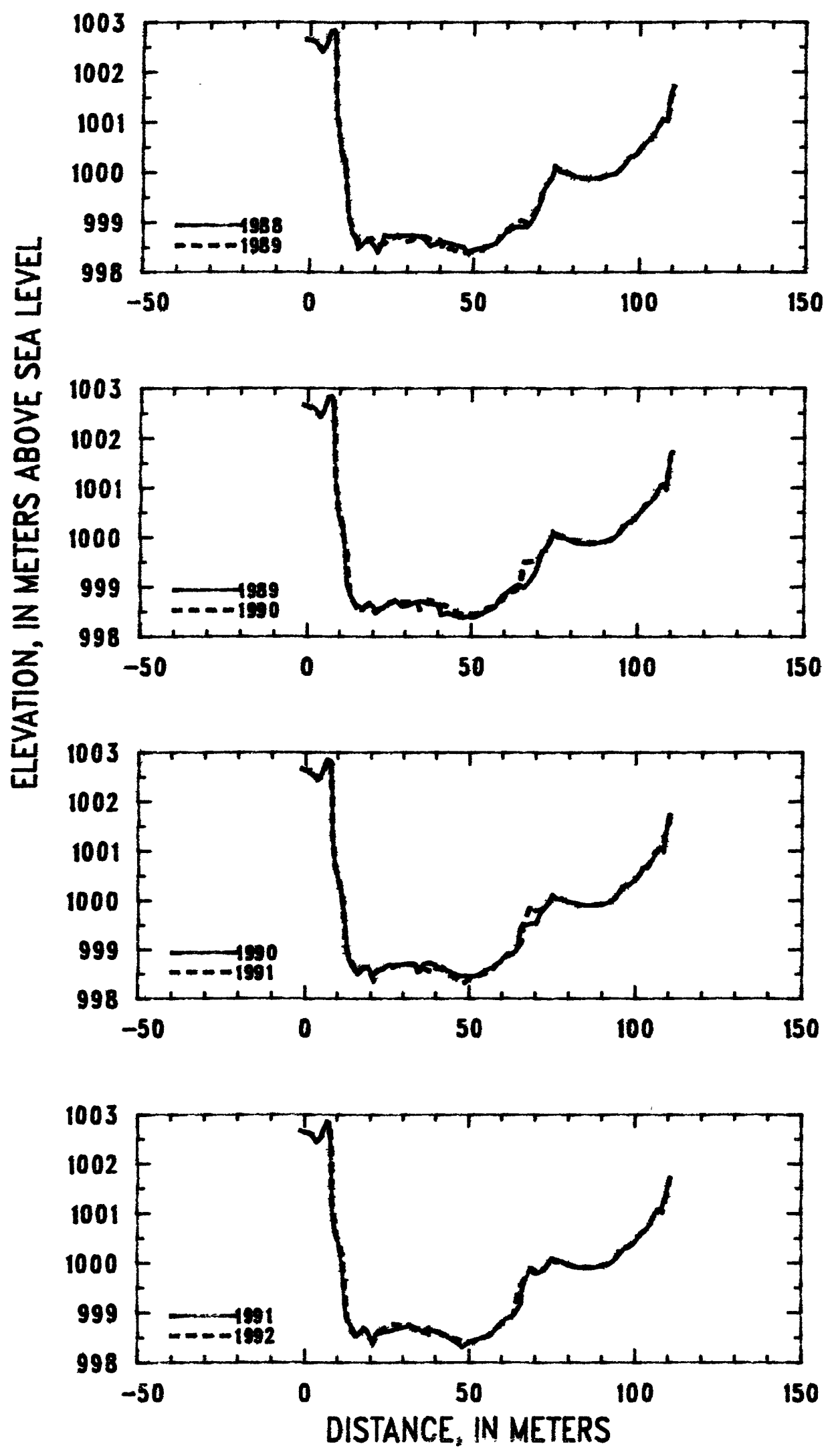

Figure 32. Profiles of cross section PR130 from 1988 to 1992. 

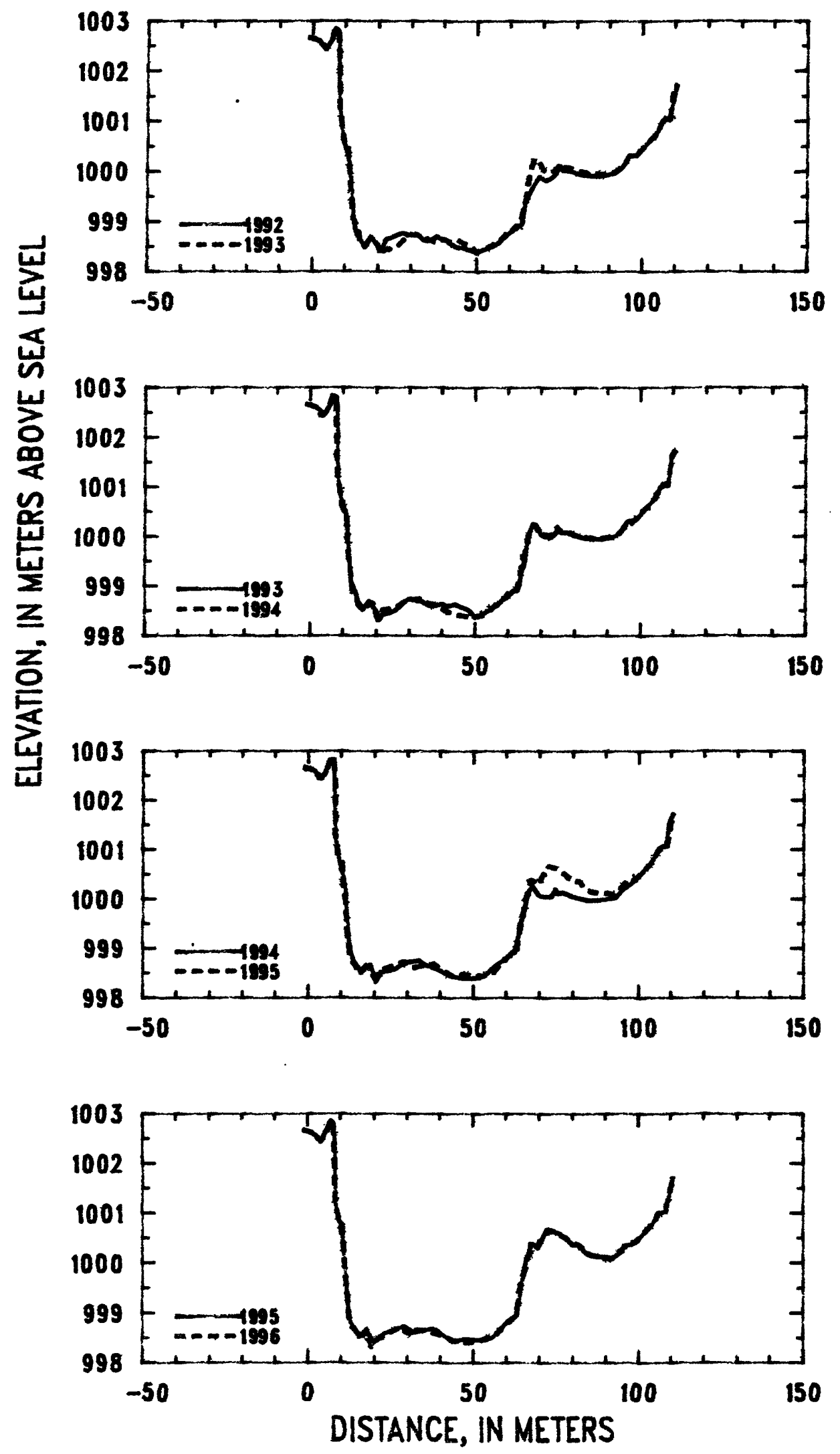

Figure 33. Profiles of cross section PR130 from 1992 to 1996. 


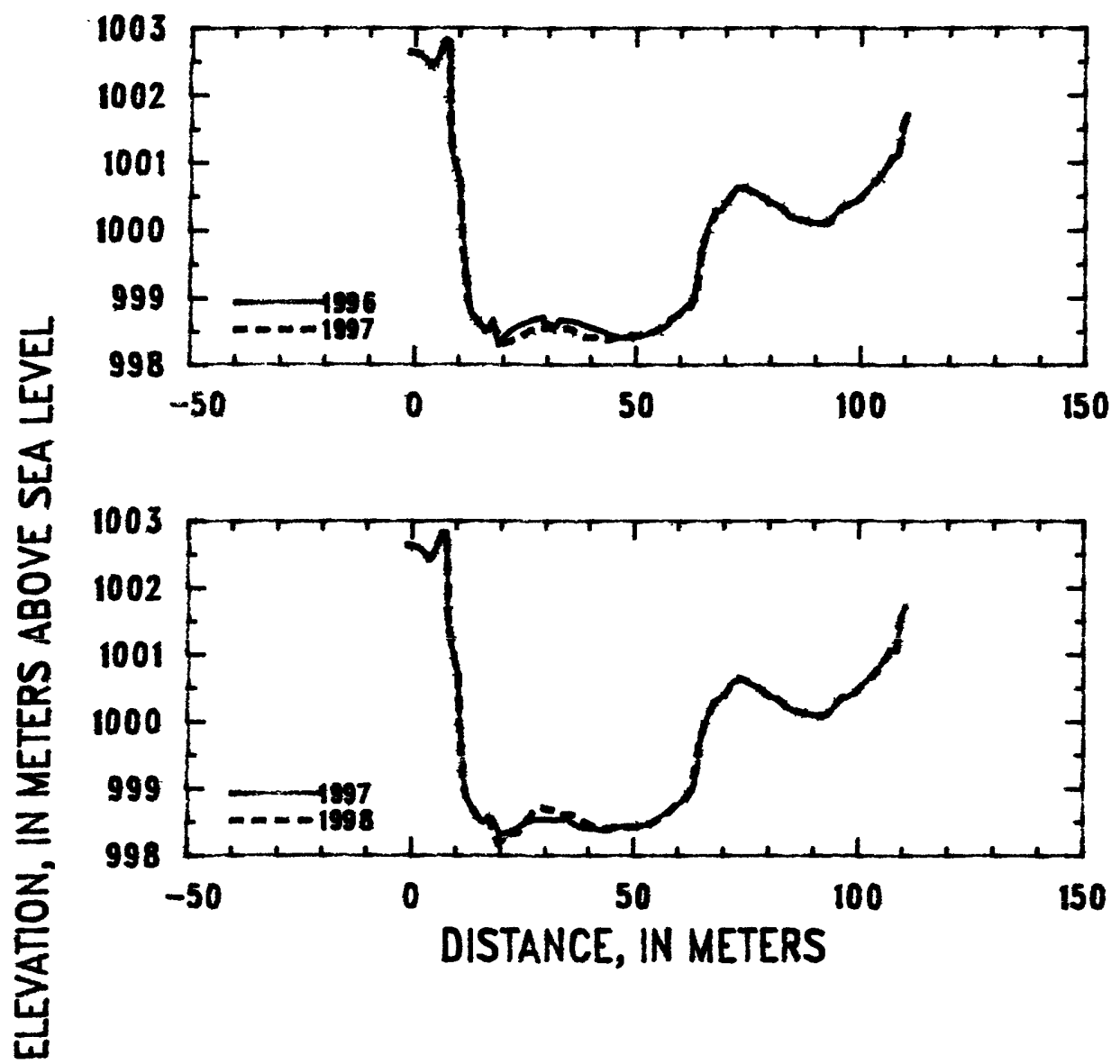

Figure 34. Profiles of cross section PR130 from 1996 to 1998. 
Table 13. Listing of horizontal stations and elevations for cross section PR130

[Sta., station, distance in meters from a reference pin on the left bank; Elev., elevation, in meters above sea level]

\begin{tabular}{|c|c|c|c|c|c|c|c|c|c|}
\hline \multirow{2}{*}{\multicolumn{2}{|c|}{$\begin{array}{c}1989 \\
17 \text { September }\end{array}$}} & \multicolumn{2}{|c|}{1989} & \multicolumn{2}{|c|}{1990} & \multicolumn{2}{|c|}{1990} & \multicolumn{2}{|c|}{1991} \\
\hline & & $17 \mathrm{Se}$ & tember & $15 \mathrm{Se}$ & tember & $15 \mathrm{Se}$ & tember & 28 & ugust \\
\hline Sta. & Elev. & Sta. & Elev. & Sta. & Elev. & Sta. & Elev. & Sta. & Elev. \\
\hline-1.1 & 1002.67 & 63.2 & 999.02 & -1.1 & 1002.67 & 51.0 & 998.45 & -1.1 & 1002.67 \\
\hline 0.0 & 1002.65 & 64.4 & 999.04 & 0.0 & 1002.65 & 53.0 & 998.48 & 0.0 & 1002.65 \\
\hline 2.0 & 1002.61 & 66.0 & 998.99 & 1.0 & 1002.61 & 55.0 & 998.57 & 2.0 & 1002.61 \\
\hline 4.0 & 1002.43 & 68.0 & 999.14 & 3.0 & 1002.51 & 57.0 & 998.62 & 4.0 & 1002.44 \\
\hline 5.0 & 1002.53 & 69.0 & 999.26 & 4.0 & 1002.44 & 57.7 & 998.68 & 5.0 & 1002.51 \\
\hline 6.4 & 1002.82 & 70.0 & 999.41 & 5.0 & 1002.53 & 59.5 & 998.74 & 6.0 & 1002.69 \\
\hline 7.6 & 1002.84 & 71.0 & 999.66 & 6.4 & 1002.80 & 60.3 & 998.83 & 7.0 & 1002.85 \\
\hline 8.1 & 1002.76 & 72.0 & 999.76 & 7.0 & 1002.85 & 61.7 & 998.92 & 7.8 & 1002.83 \\
\hline 8.3 & 1002.21 & 73.0 & 999.83 & 8.0 & 1002.81 & 62.5 & 998.90 & 8.3 & 1002.19 \\
\hline 8.4 & 1001.30 & 74.4 & 1000.02 & 8.5 & 1002.16 & 64.2 & 998.98 & 8.4 & 1001.22 \\
\hline 10.0 & 1000.38 & 74.5 & 1000.11 & 8.6 & 1001.18 & 65.0 & 999.16 & 9.4 & 1000.63 \\
\hline 11.0 & 1000.20 & 76.0 & 1000.01 & 9.5 & 1000.64 & 65.3 & 999.42 & 10.2 & 1000.46 \\
\hline 12.0 & 999.24 & 78.0 & 1000.01 & 10.6 & 1000.30 & 66.1 & 999.51 & 11.5 & 999.97 \\
\hline 12.4 & 999.03 & 80.0 & 999.93 & 11.0 & 1000.24 & 67.0 & 999.50 & 12.5 & 999.06 \\
\hline 13.3 & 998.89 & 82.0 & 999.88 & 11.4 & 999.94 & 69.0 & 999.53 & 13.4 & 998.83 \\
\hline 13.5 & 998.78 & 84.0 & 999.87 & 12.2 & 999.68 & 70.0 & 999.53 & 15.2 & 998.62 \\
\hline 15.0 & 998.64 & 86.0 & 999.87 & 12.3 & 999.51 & 71.4 & 999.75 & 16.0 & 998.53 \\
\hline 17.0 & 998.58 & 88.0 & 999.89 & 13.1 & 998.87 & 73.0 & 999.86 & 17.5 & 998.65 \\
\hline 19.0 & 998.68 & 90.0 & 999.92 & 13.8 & 998.75 & 75.0 & 1000.11 & 19.0 & 998.66 \\
\hline 21.0 & 998.54 & 92.0 & 999.94 & 16.0 & 998.49 & 76.0 & 1000.03 & 20.9 & 998.37 \\
\hline 23.0 & 998.57 & 94.0 & 1000.06 & 17.8 & 998.61 & 78.0 & 1000.02 & 21.8 & 998.57 \\
\hline 25.0 & 998.68 & 95.0 & 1000.13 & 19.2 & 998.64 & 80.0 & 999.96 & 23.0 & 998.59 \\
\hline 27.0 & 998.74 & 97.0 & 1000.30 & 20.8 & 998.48 & 82.0 & 999.95 & 26.0 & 998.64 \\
\hline 29.0 & 998.61 & 99.0 & 1000.35 & 22.0 & 998.55 & 84.0 & 999.90 & 29.0 & 998.69 \\
\hline 31.0 & 998.62 & 100.0 & 1000.46 & 24.3 & 998.62 & 86.0 & 999.89 & 32.0 & 998.72 \\
\hline 33.0 & 998.70 & 101.9 & 1000.59 & 24.6 & 998.66 & 88.0 & 999.91 & 33.0 & 998.73 \\
\hline 35.0 & 998.71 & 104.0 & 1000.72 & 26.0 & 998.70 & 90.0 & 999.91 & 34.8 & 998.67 \\
\hline 37.0 & 998.67 & 105.0 & 1000.83 & 28.0 & 998.69 & 92.0 & 999.96 & 38.0 & 998.55 \\
\hline 39.0 & 998.65 & 106.0 & 1000.96 & 30.0 & 998.71 & 94.0 & 1000.06 & 38.2 & 998.65 \\
\hline 40.0 & 998.64 & 107.0 & 1001.05 & 32.0 & 998.71 & 96.0 & 1000.28 & 40.8 & 998.62 \\
\hline 41.0 & 998.44 & 108.5 & 1001.00 & 34.0 & 998.67 & 98.0 & 1000.31 & 42.4 & 998.58 \\
\hline 43.0 & 998.48 & 109.9 & 1001.66 & 34.5 & 998.54 & 100.0 & 1000.44 & 44.0 & 998.49 \\
\hline 45.0 & 998.42 & 110.6 & 1001.74 & 35.4 & 998.57 & 101.8 & 1000.60 & 46.0 & 998.42 \\
\hline 47.0 & 998.39 & & & 36.2 & 998.70 & 101.9 & 1000.64 & 48.0 & 998.31 \\
\hline 49.0 & 998.40 & & & 38.0 & 998.73 & 104.0 & 1000.73 & 50.0 & 998.41 \\
\hline 51.0 & 998.39 & & & 40.0 & 998.68 & 105.0 & 1000.82 & 52.0 & 998.45 \\
\hline 53.0 & 998.46 & & & 41.0 & 998.61 & 107.0 & 1001.05 & 54.0 & 998.49 \\
\hline 55.0 & 998.53 & & & 42.0 & 998.64 & 108.0 & 1001.07 & 56.0 & 998.53 \\
\hline 57.0 & 998.58 & & & 43.0 & 998.59 & 108.5 & 1001.00 & 57.0 & 998.58 \\
\hline 58.9 & 998.78 & & & 45.0 & 998.51 & 110.0 & 1001.68 & 59.0 & 998.74 \\
\hline 60.0 & 998.83 & & & 47.0 & 998.46 & 110.6 & 1001.75 & 61.0 & 998.87 \\
\hline 62.0 & 998.95 & & & 49.0 & 998.46 & & & 63.0 & 998.91 \\
\hline
\end{tabular}


Table 13. (Continued) Listing of horizontal stations and elevations for cross section PR130

[Sta., station, distance in meters from a reference pin on the left bank; Elev., elevation, in meters above sea level]

\begin{tabular}{|c|c|c|c|c|c|c|c|c|c|}
\hline \multirow{2}{*}{\multicolumn{2}{|c|}{$\begin{array}{c}1991 \\
28 \text { August }\end{array}$}} & \multirow{2}{*}{\multicolumn{2}{|c|}{$\begin{array}{c}1992 \\
26 \text { August }\end{array}$}} & \multirow{2}{*}{\multicolumn{2}{|c|}{$\begin{array}{c}1992 \\
26 \text { August }\end{array}$}} & \multirow{2}{*}{\multicolumn{2}{|c|}{$\begin{array}{c}1993 \\
27 \text { August }\end{array}$}} & \multirow{2}{*}{\multicolumn{2}{|c|}{$\begin{array}{c}1993 \\
27 \text { August }\end{array}$}} \\
\hline & & & & & & & & & \\
\hline Sta. & Elev. & Sta. & Elev. & Sta. & Elev. & Sta. & Elev. & Sta. & Elev. \\
\hline 65.6 & 999.15 & 0.0 & 1002.65 & 65.5 & 999.50 & -1.2 & 1002.68 & 60.6 & 998.84 \\
\hline 66.1 & 999.52 & 2.0 & 1002.61 & 65.7 & 999.56 & 0.0 & 1002.65 & 61.4 & 998.88 \\
\hline 68.6 & 999.89 & 3.0 & 1002.51 & 68.0 & 999.81 & 2.0 & 1002.61 & 61.8 & 998.91 \\
\hline 69.8 & 999.79 & 4.0 & 1002.44 & 69.0 & 999.89 & 3.0 & 1002.52 & 62.8 & 998.91 \\
\hline 71.0 & 999.79 & 5.5 & 1002.57 & 71.0 & 999.82 & 4.0 & 1002.44 & 63.3 & 998.99 \\
\hline 73.0 & 999.88 & 7.0 & 1002.85 & 73.0 & 999.90 & 5.4 & 1002.55 & 64.3 & 999.34 \\
\hline 74.0 & 999.98 & 7.9 & 1002.80 & 74.0 & 999.99 & 6.6 & 1002.83 & 64.8 & 999.40 \\
\hline 75.0 & 1000.10 & 8.3 & 1002.28 & 75.0 & 1000.12 & 7.9 & 1002.81 & 65.0 & 999.49 \\
\hline 77.0 & 1000.05 & 8.4 & 1001.29 & 76.0 & 1000.02 & 8.4 & 1002.23 & 66.0 & 999.99 \\
\hline 78.0 & 1000.02 & 9.7 & 1000.59 & 78.0 & 1000.02 & 8.5 & 1001.24 & 66.5 & 1000.12 \\
\hline 80.0 & 999.96 & 10.4 & 1000.42 & 80.0 & 999.96 & 9.7 & 1000.60 & 67.4 & 1000.25 \\
\hline 82.0 & 999.92 & 11.5 & 1000.15 & 82.0 & 999.93 & 10.3 & 1000.51 & 69.0 & 1000.17 \\
\hline 84.0 & 999.91 & 13.0 & 998.90 & 84.0 & 999.92 & 10.8 & 1000.52 & 70.0 & 1000.03 \\
\hline 86.0 & 999.90 & 13.5 & 998.79 & 86.0 & 999.90 & 11.3 & 1000.20 & 71.0 & 1000.01 \\
\hline 88.0 & 999.92 & 14.5 & 998.64 & 88.0 & 999.91 & 12.0 & 999.60 & 73.0 & 1000.00 \\
\hline 90.0 & 999.94 & 16.0 & 998.48 & 90.0 & 999.95 & 12.5 & 999.06 & 74.0 & 1000.06 \\
\hline 92.0 & 999.97 & 18.0 & 998.69 & 92.0 & 999.97 & 14.0 & 998.87 & 75.0 & 1000.16 \\
\hline 93.5 & 1000.03 & 20.0 & 998.50 & 94.0 & 1000.08 & 14.6 & 998.62 & 76.0 & 1000.08 \\
\hline 95.0 & 1000.16 & 21.0 & 998.41 & 96.0 & 1000.32 & 16.0 & 998.52 & 78.0 & 1000.08 \\
\hline 97.0 & 1000.31 & 22.4 & 998.63 & 98.0 & 1000.32 & 18.0 & 998.69 & 80.0 & 1000.02 \\
\hline 99.0 & 1000.35 & 25.0 & 998.71 & 100.0 & 1000.48 & 20.0 & 998.50 & 82.0 & 1000.00 \\
\hline 101.0 & 1000.52 & 27.0 & 998.77 & 101.9 & 1000.60 & 21.0 & 998.32 & 84.0 & 999.96 \\
\hline 102.0 & 1000.60 & 28.8 & 998.75 & 104.0 & 1000.72 & 22.0 & 998.43 & 86.0 & 999.96 \\
\hline 104.0 & 1000.71 & 29.0 & 998.72 & 106.0 & 1001.01 & 24.0 & 998.46 & 88.0 & 999.94 \\
\hline 106.0 & 1000.99 & 32.0 & 998.75 & 107.0 & 1001.07 & 26.0 & 998.52 & 90.0 & 999.98 \\
\hline 108.0 & 1001.07 & 34.0 & 998.62 & 108.4 & 1001.02 & 28.0 & 998.64 & 92.0 & 1000.00 \\
\hline 110.6 & 1001.72 & 36.0 & 998.61 & 110.6 & 1001.73 & 30.0 & 998.74 & 94.0 & 1000.10 \\
\hline & & 38.0 & 998.69 & & & 32.0 & 998.72 & 95.0 & 1000.18 \\
\hline & & 40.0 & 998.63 & & & 34.0 & 998.69 & 95.4 & 1000.24 \\
\hline & & 42.0 & 998.56 & & & 36.0 & 998.61 & 96.0 & 1000.31 \\
\hline & & 44.0 & 998.47 & & & 38.0 & 998.60 & 98.0 & 1000.32 \\
\hline & & 46.0 & 998.47 & & & 40.0 & 998.64 & 100.0 & 1000.46 \\
\hline & & 48.0 & 998.42 & & & 42.0 & 998.60 & 101.9 & 1000.60 \\
\hline & & 50.0 & 998.40 & & & 44.0 & 998.64 & 103.0 & 1000.68 \\
\hline & & 52.0 & 998.43 & & & 46.0 & 998.56 & 104.6 & 1000.78 \\
\hline & & 54.0 & 998.48 & & & 48.0 & 998.52 & 106.0 & 1001.00 \\
\hline & & 56.0 & 998.54 & & & 50.0 & 998.38 & 108.0 & 1001.05 \\
\hline & & 58.0 & 998.66 & & & 52.0 & 998.42 & 108.5 & 1001.04 \\
\hline & & 60.0 & 998.78 & & & 54.0 & 998.51 & 109.6 & 1001.64 \\
\hline & & 62.0 & 998.88 & & & 56.0 & 998.57 & 110.6 & 1001.73 \\
\hline & & 63.7 & 998.96 & & & 58.0 & 998.68 & & \\
\hline & & 64.8 & 999.41 & & & 60.0 & 998.85 & & \\
\hline
\end{tabular}


Table 13. (Continued) Listing of horizontal stations and elevations for cross section PR130

[Sta., station, distance in meters from a reference pin on the left bank; Elev., elevation, in meters above sea level]

\begin{tabular}{|c|c|c|c|c|c|c|c|c|c|}
\hline \multirow{2}{*}{\multicolumn{2}{|c|}{$\begin{array}{c}1994 \\
\text { 25 September }\end{array}$}} & \multicolumn{2}{|c|}{1994} & \multicolumn{2}{|c|}{1995} & \multicolumn{2}{|c|}{1995} & \multicolumn{2}{|c|}{1996} \\
\hline & & $25 \mathrm{~S}$ & tember & $24 \mathrm{~S}$ & tember & $24 \mathrm{Se}$ & tember & 17 & ctober \\
\hline Sta. & Elev. & Sta. & Elev. & Sta. & Elev. & Sta. & Elev. & Sta. & Elev. \\
\hline-1.1 & 1002.67 & 66.0 & 999.99 & -1.2 & 1002.65 & 80.0 & 1000.36 & -1.2 & 1002.66 \\
\hline 0.0 & 1002.64 & 67.0 & 1000.19 & 0.0 & 1002.65 & 82.0 & 1000.36 & 0.0 & 1002.65 \\
\hline 2.0 & 1002.59 & 68.0 & 1000.25 & 2.0 & 1002.59 & 84.0 & 1000.20 & 2.0 & 1002.58 \\
\hline 3.2 & 1002.44 & 70.0 & 1000.06 & 4.0 & 1002.44 & 86.0 & 1000.14 & 4.0 & 1002.44 \\
\hline 5.0 & 1002.51 & 72.0 & 1000.03 & 6.0 & 1002.70 & 88.0 & 1000.12 & 5.5 & 1002.58 \\
\hline 6.5 & 1002.81 & 73.5 & 1000.05 & 7.0 & 1002.85 & 90.0 & 1000.12 & 7.0 & 1002.85 \\
\hline 7.8 & 1002.81 & 74.7 & 1000.20 & 7.8 & 1002.80 & 92.0 & 1000.09 & 7.8 & 1002.81 \\
\hline 8.1 & 1001.82 & 75.4 & 1000.11 & 8.3 & 1002.28 & 94.0 & 1000.18 & 8.0 & 1002.46 \\
\hline 8.4 & 1001.16 & 77.0 & 1000.14 & 8.3 & 1001.27 & 96.0 & 1000.34 & 8.3 & 1001.23 \\
\hline 10.0 & 1000.64 & 78.0 & 1000.10 & 9.3 & 1000.85 & 98.0 & 1000.37 & 9.5 & 1000.88 \\
\hline 11.2 & 1000.31 & 80.0 & 1000.04 & 10.3 & 1000.76 & 100.0 & 1000.48 & 10.3 & 1000.77 \\
\hline 11.7 & 999.67 & 82.5 & 1000.00 & 12.1 & 999.25 & 101.9 & 1000.61 & 11.8 & 999.38 \\
\hline 12.9 & 998.94 & 85.0 & 999.97 & 12.7 & 998.85 & 104.0 & 1000.74 & 12.1 & 999.29 \\
\hline 13.7 & 998.76 & 88.0 & 999.97 & 15.5 & 998.52 & 106.0 & 1000.99 & 12.7 & 998.85 \\
\hline 14.8 & 998.65 & 91.0 & 1000.01 & 17.7 & 998.66 & 108.5 & 1001.03 & 13.0 & 998.76 \\
\hline 16.0 & 998.51 & 93.0 & 1000.03 & 20.0 & 998.41 & 110.6 & 1001.72 & 15.0 & 998.60 \\
\hline 18.0 & 998.65 & 95.0 & 1000.19 & 23.0 & 998.59 & & & 16.0 & 998.49 \\
\hline 19.0 & 998.66 & 98.0 & 1000.32 & 26.0 & 998.67 & & & 17.7 & 998.65 \\
\hline 20.5 & 998.33 & 100.0 & 1000.47 & 29.0 & 998.74 & & & 19.0 & 998.30 \\
\hline 22.0 & 998.52 & 101.9 & 1000.60 & 32.0 & 998.61 & & & 21.0 & 998.47 \\
\hline 24.0 & 998.54 & 104.0 & 1000.73 & 35.0 & 998.66 & & & 24.0 & 998.59 \\
\hline 26.0 & 998.57 & 106.0 & 1001.00 & 38.0 & 998.68 & & & 27.0 & 998.68 \\
\hline 28.0 & 998.66 & 107.0 & 1001.06 & 41.0 & 998.55 & & & 29.0 & 998.71 \\
\hline 30.0 & 998.72 & 108.5 & 1001.07 & 44.0 & 998.43 & & & 29.5 & 998.62 \\
\hline 32.0 & 998.73 & 109.5 & 1001.58 & 47.0 & 998.48 & & & 32.0 & 998.56 \\
\hline 33.7 & 998.75 & 110.6 & 1001.73 & 50.0 & 998.46 & & & 33.0 & 998.66 \\
\hline 35.2 & 998.66 & & & 53.0 & 998.44 & & & 36.0 & 998.65 \\
\hline 38.0 & 998.62 & & & 56.0 & 998.50 & & & 39.0 & 998.57 \\
\hline 41.0 & 998.51 & & & 59.0 & 998.72 & & & 42.0 & 998.50 \\
\hline 44.0 & 998.43 & & & 60.7 & 998.85 & & & 45.0 & 998.42 \\
\hline 47.0 & 998.39 & & & 63.2 & 998.95 & & & 48.0 & 998.40 \\
\hline 50.0 & 998.39 & & & 64.5 & 999.51 & & & 51.0 & 998.41 \\
\hline 53.0 & 998.44 & & & 66.0 & 1000.05 & & & 54.0 & 998.50 \\
\hline 55.0 & 998.54 & & & 67.0 & 1000.22 & & & 57.0 & 998.57 \\
\hline 57.0 & 998.67 & & & 67.3 & 1000.38 & & & 59.5 & 998.76 \\
\hline 60.0 & 998.80 & & & 68.2 & 1000.37 & & & 61.0 & 998.84 \\
\hline 62.0 & 998.93 & & & 69.5 & 1000.30 & & & 63.0 & 998.94 \\
\hline 62.5 & 998.91 & & & 71.0 & 1000.48 & & & 63.5 & 999.14 \\
\hline 64.0 & 999.32 & & & 72.2 & 1000.67 & & & 64.5 & 999.68 \\
\hline 64.1 & 999.46 & & & 74.0 & 1000.65 & & & 65.2 & 999.78 \\
\hline 64.3 & 999.48 & & & 76.0 & 1000.58 & & & 66.2 & 1000.07 \\
\hline 65.2 & 999.70 & & & 78.0 & 1000.48 & & & 68.0 & 1000.29 \\
\hline
\end{tabular}


Table 13. (Continued) Listing of horizontal stations and elevations for cross section PR130

[Sta., station, distance in meters from a reference pin on the left bank; Elev., elevation, in meters above sea level]

\begin{tabular}{|c|c|c|c|c|c|c|c|c|c|}
\hline \multirow{2}{*}{\multicolumn{2}{|c|}{$\begin{array}{c}1996 \\
17 \text { October }\end{array}$}} & \multicolumn{2}{|c|}{1997} & \multicolumn{2}{|c|}{1997} & \multicolumn{2}{|c|}{1998} & \multicolumn{2}{|c|}{1998} \\
\hline & & \multicolumn{2}{|c|}{19 September } & \multicolumn{2}{|c|}{19 September } & \multicolumn{2}{|c|}{22 September } & \multicolumn{2}{|c|}{22 September } \\
\hline Sta. & Elev. & Sta. & Elev. & Sta. & Elev. & Sta. & Elev. & Sta. & Elev. \\
\hline 69.0 & 1000.29 & -1.2 & 1002.66 & 80.0 & 1000.38 & -1.1 & 1002.65 & 70.0 & 1000.38 \\
\hline 70.0 & 1000.41 & 0.0 & 1002.65 & 82.0 & 1000.35 & 0.0 & 1002.64 & 72.0 & 1000.59 \\
\hline 71.0 & 1000.49 & 2.0 & 1002.59 & 84.0 & 1000.20 & 2.0 & 1002.59 & 73.0 & 1000.65 \\
\hline 72.5 & 1000.63 & 4.0 & 1002.44 & 86.0 & 1000.16 & 4.0 & 1002.43 & 74.0 & 1000.64 \\
\hline 74.0 & 1000.63 & 5.5 & 1002.60 & 88.0 & 1000.12 & 6.0 & 1002.70 & 76.0 & 1000.58 \\
\hline 76.0 & 1000.57 & 6.7 & 1002.83 & 90.0 & 1000.09 & 7.7 & 1002.83 & 78.0 & 1000.49 \\
\hline 79.0 & 1000.44 & 7.7 & 1002.83 & 93.0 & 1000.11 & 8.0 & 1002.13 & 80.0 & 1000.37 \\
\hline 82.0 & 1000.34 & 7.9 & 1002.41 & 96.0 & 1000.35 & 8.1 & 1001.46 & 82.0 & 1000.34 \\
\hline 83.0 & 1000.31 & 8.1 & 1001.59 & 98.0 & 1000.38 & 9.9 & 1000.82 & 84.0 & 1000.21 \\
\hline 84.0 & 1000.20 & 8.4 & 1001.28 & 100.0 & 1000.49 & 10.4 & 1000.74 & 86.0 & 1000.16 \\
\hline 86.0 & 1000.15 & 9.7 & 1000.87 & 101.9 & 1000.62 & 10.9 & 999.76 & 88.0 & 1000.13 \\
\hline 88.0 & 1000.12 & 10.4 & 1000.76 & 105.0 & 1000.85 & 11.8 & 999.08 & 90.0 & 1000.09 \\
\hline 90.0 & 1000.10 & 11.1 & 999.77 & 107.0 & 1001.08 & 12.5 & 998.87 & 92.0 & 1000.08 \\
\hline 92.0 & 1000.09 & 12.3 & 998.94 & 108.5 & 1001.06 & 15.5 & 998.52 & 94.0 & 1000.18 \\
\hline 94.0 & 1000.19 & 13.3 & 998.75 & 109.5 & 1001.57 & 17.3 & 998.57 & 96.0 & 1000.36 \\
\hline 96.0 & 1000.35 & 16.0 & 998.51 & 110.6 & 1001.73 & 19.5 & 998.17 & 98.0 & 1000.37 \\
\hline 97.0 & 1000.38 & 18.0 & 998.56 & 80.0 & 1000.38 & 22.0 & 998.34 & 100.0 & 1000.50 \\
\hline 99.0 & 1000.42 & 20.0 & 998.32 & 82.0 & 1000.35 & 24.0 & 998.34 & 101.9 & 1000.61 \\
\hline 101.9 & 1000.61 & 23.0 & 998.38 & 84.0 & 1000.20 & 26.0 & 998.54 & 103.3 & 1000.69 \\
\hline 104.0 & 1000.78 & 26.0 & 998.52 & 86.0 & 1000.16 & 28.0 & 998.72 & 105.0 & 1000.85 \\
\hline 105.0 & 1000.84 & 29.0 & 998.56 & 88.0 & 1000.12 & 30.0 & 998.71 & 107.0 & 1001.07 \\
\hline 107.0 & 1001.08 & 32.0 & 998.53 & 90.0 & 1000.09 & 32.0 & 998.68 & 108.4 & 1001.01 \\
\hline 108.6 & 1001.12 & 35.0 & 998.55 & 93.0 & 1000.11 & 34.0 & 998.61 & 109.4 & 1001.56 \\
\hline 110.6 & 1001.73 & 38.0 & 998.42 & 96.0 & 1000.35 & 36.0 & 998.62 & 110.6 & 1001.73 \\
\hline & & 41.0 & 998.41 & 98.0 & 1000.38 & 38.0 & 998.55 & & \\
\hline & & 44.0 & 998.37 & 100.0 & 1000.49 & 40.0 & 998.47 & & \\
\hline & & 47.0 & 998.44 & 101.9 & 1000.62 & 42.0 & 998.41 & & \\
\hline & & 50.0 & 998.44 & 105.0 & 1000.85 & 44.0 & 998.40 & & \\
\hline & & 53.0 & 998.47 & 107.0 & 1001.08 & 46.0 & 998.44 & & \\
\hline & & 56.0 & 998.56 & 108.5 & 1001.06 & 48.0 & 998.42 & & \\
\hline & & 59.2 & 998.74 & 109.5 & 1001.57 & 50.0 & 998.43 & & \\
\hline & & 62.2 & 998.87 & 110.6 & 1001.73 & 52.0 & 998.43 & & \\
\hline & & 63.2 & 998.98 & & & 54.0 & 998.48 & & \\
\hline & & 63.8 & 999.15 & & & 56.0 & 998.56 & & \\
\hline & & 65.0 & 999.83 & & & 58.0 & 998.65 & & \\
\hline & & 66.0 & 999.99 & & & 60.0 & 998.79 & & \\
\hline & & 68.0 & 1000.30 & & & 61.6 & 998.85 & & \\
\hline & & 70.0 & 1000.39 & & & 62.8 & 998.95 & & \\
\hline & & 72.0 & 1000.59 & & & 63.8 & 999.26 & & \\
\hline & & 74.0 & 1000.64 & & & 64.4 & 999.63 & & \\
\hline & & 76.0 & 1000.57 & & & 65.5 & 999.92 & & \\
\hline & & 78.0 & 1000.50 & & & 68.0 & 1000.32 & & \\
\hline
\end{tabular}




\section{Description of Cross Section PR136}

Location: Township 8 South/Range 49 East--section 6

\section{U. S. Geological Survey quadrangle (1:24,000): Bloom Creek}

\section{Landowners--left bank: Gay Ranch}

--right bank: Bowers Ranch

Access: Left bank

Permission from: Gay Ranch

Distance from Moorhead Gaging Station: 20.19 kilometers

Azimuth of Section (degrees magnetic): 129

\section{Reference Monuments}

[Monuments at stations $-20.0,-1.5$, and 0.0 were closest to leveling instrument]

\begin{tabular}{|c|c|c|c|c|c|c|}
\hline \multirow[b]{2}{*}{ Description } & \multirow[b]{2}{*}{$\begin{array}{l}\text { Station } \\
\text { (m) }\end{array}$} & \multicolumn{2}{|c|}{ GPS-NAD83 (1992) } & \multicolumn{2}{|c|}{ Measurement } & \multirow{2}{*}{$\begin{array}{l}\text { Elevation } \\
\text { (NGVD1929) } \\
(\mathrm{m})\end{array}$} \\
\hline & & Latitude & Longitude & $\begin{array}{l}\text { Standard } \\
\text { deviation } \\
\text { (m) }\end{array}$ & $\begin{array}{l}\text { Horizontal } \\
\text { precision } \\
\text { (m) }\end{array}$ & \\
\hline $\begin{array}{l}\text { 1/2-inch-rebar; bent nearly flat with } 1998 \\
\text { ground level, } 0.13 \text { meter long }\end{array}$ & -82.5 & & & & & 994.18 \\
\hline Benchmark--brass circular plate & -32.6 & $45^{\circ} 10^{\prime} 05.91 "$ & $105^{\circ} 46^{\prime} 26.89^{\prime \prime}$ & 0.283 & 0.433 & 994.30 \\
\hline $\begin{array}{l}\text { 1/2-inch-rebar; } 0.19 \text { meter above } 1998 \text { ground } \\
\text { level }\end{array}$ & -20.0 & & & & & 994.50 \\
\hline $\begin{array}{l}\text { 1/2-inch-rebar; } 0.18 \text { meter above } 1998 \text { ground } \\
\text { level }\end{array}$ & -1.5 & & & & & 994.72 \\
\hline $\begin{array}{l}\text { 1/2-inch-rebar; } 0.01 \text { meter below } 1998 \text { ground } \\
\text { level; about } 0.02 \text { meter upstream and land- } \\
\text { ward from the other monument at }-1.5\end{array}$ & -1.5 & & & & & 994.53 \\
\hline 1/2-inch-rebar; at 1998 ground level & 0.0 & & & & & 994.59 \\
\hline $\begin{array}{l}\text { 1/2-inch-rebar; } 0.03 \text { meter above } 1998 \text { ground } \\
\text { level; about } 35 \text { meters upstream from a } \\
\text { large solitary cottonwood tree at the edge } \\
\text { of the right bank }\end{array}$ & 112.0 & & & & & 994.45 \\
\hline $\begin{array}{l}\text { 1/2-inch-rebar; } 0.16 \text { meter above } 1998 \text { ground } \\
\text { level; about } 35 \text { meters upstream from a } \\
\text { large solitary cottonwood tree at the edge } \\
\text { of the right bank }\end{array}$ & 115.0 & $45^{\circ} 10^{\prime} 02.15^{\prime \prime}$ & $105^{\circ} 46^{\prime} 22.71^{\prime \prime}$ & 0.255 & 0.645 & 994.80 \\
\hline
\end{tabular}




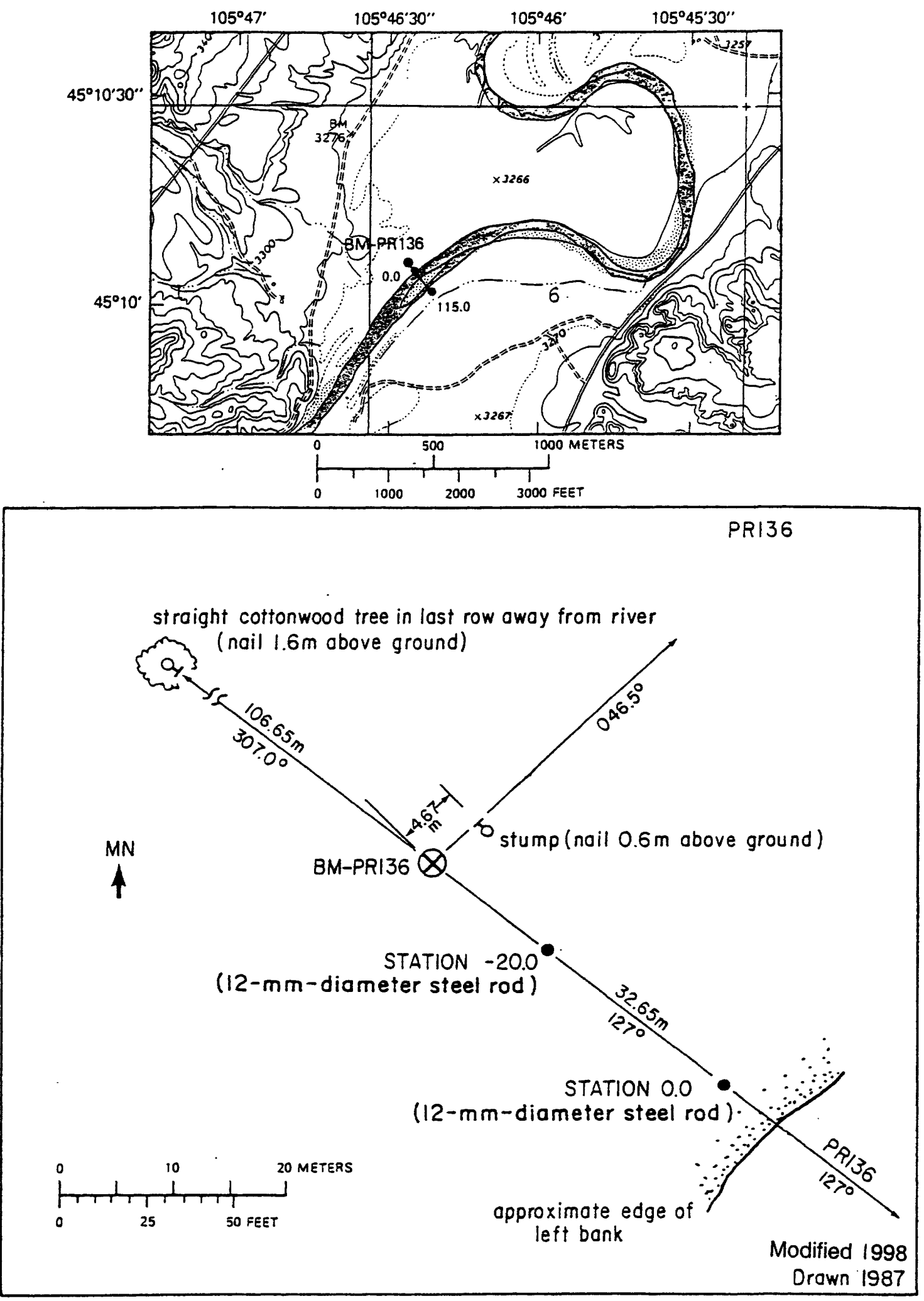

Figure 35. Upper: Location of cross section PR136, bench mark BM-PR136, and the left and right bank reference monuments in the Bloom Creek quadrangle. Lower: Location of the bench mark on the left bank. MN is magnetic north. 

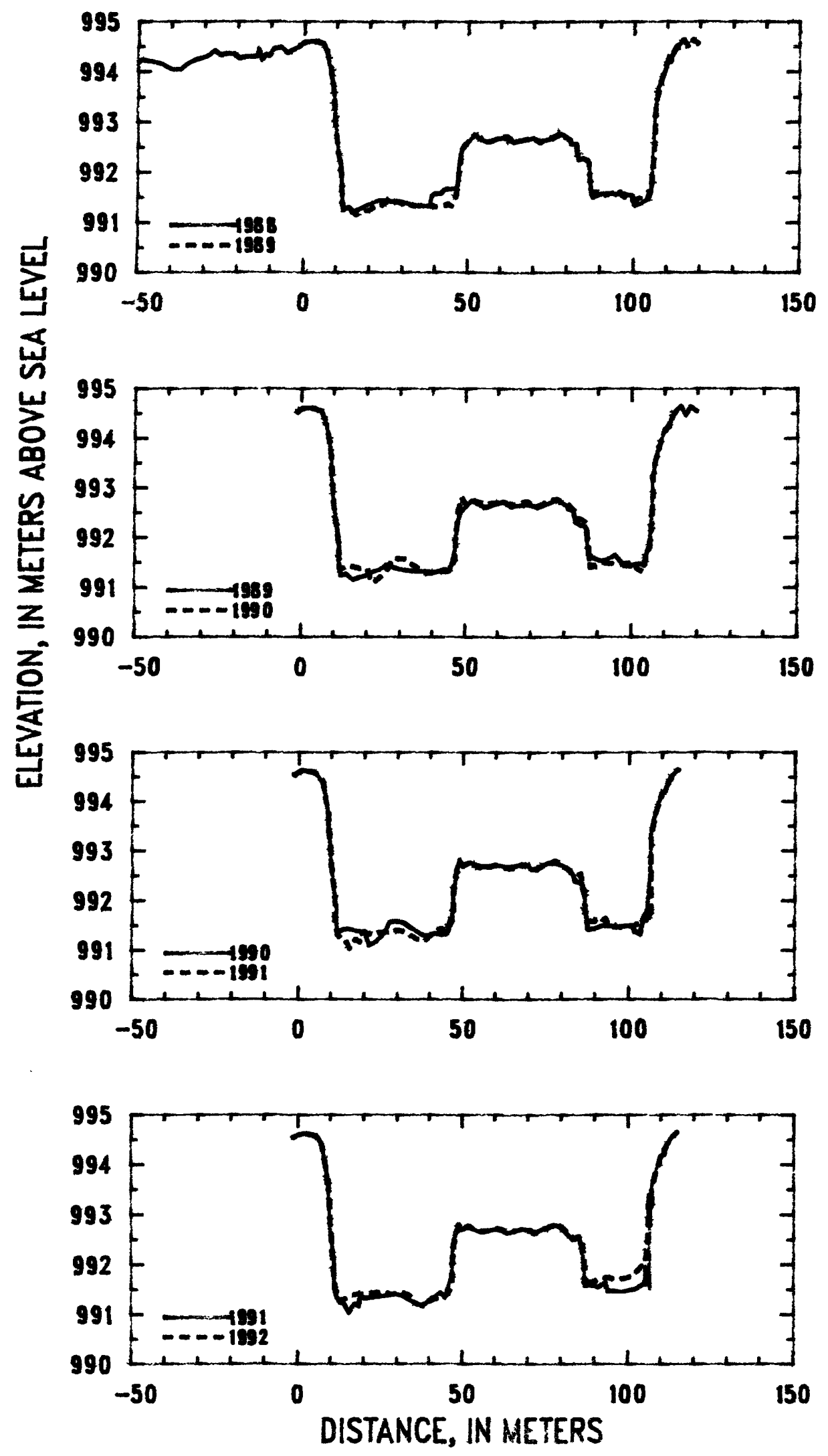

Figure 36. Profiles of cross section PR136 from 1988 to 1992. 

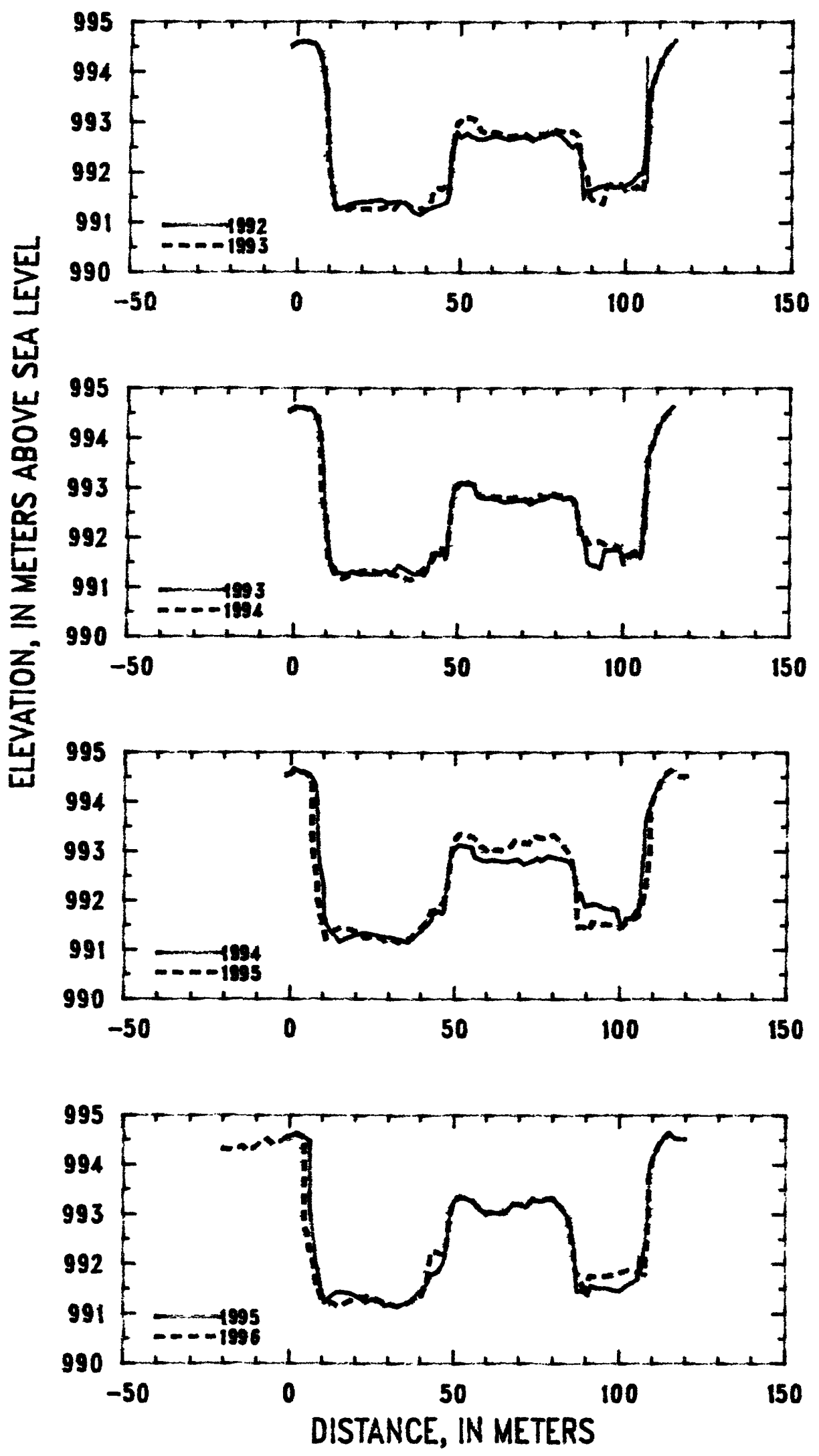

Figure 37. Profiles of cross section PR136 from 1992 to 1996. 


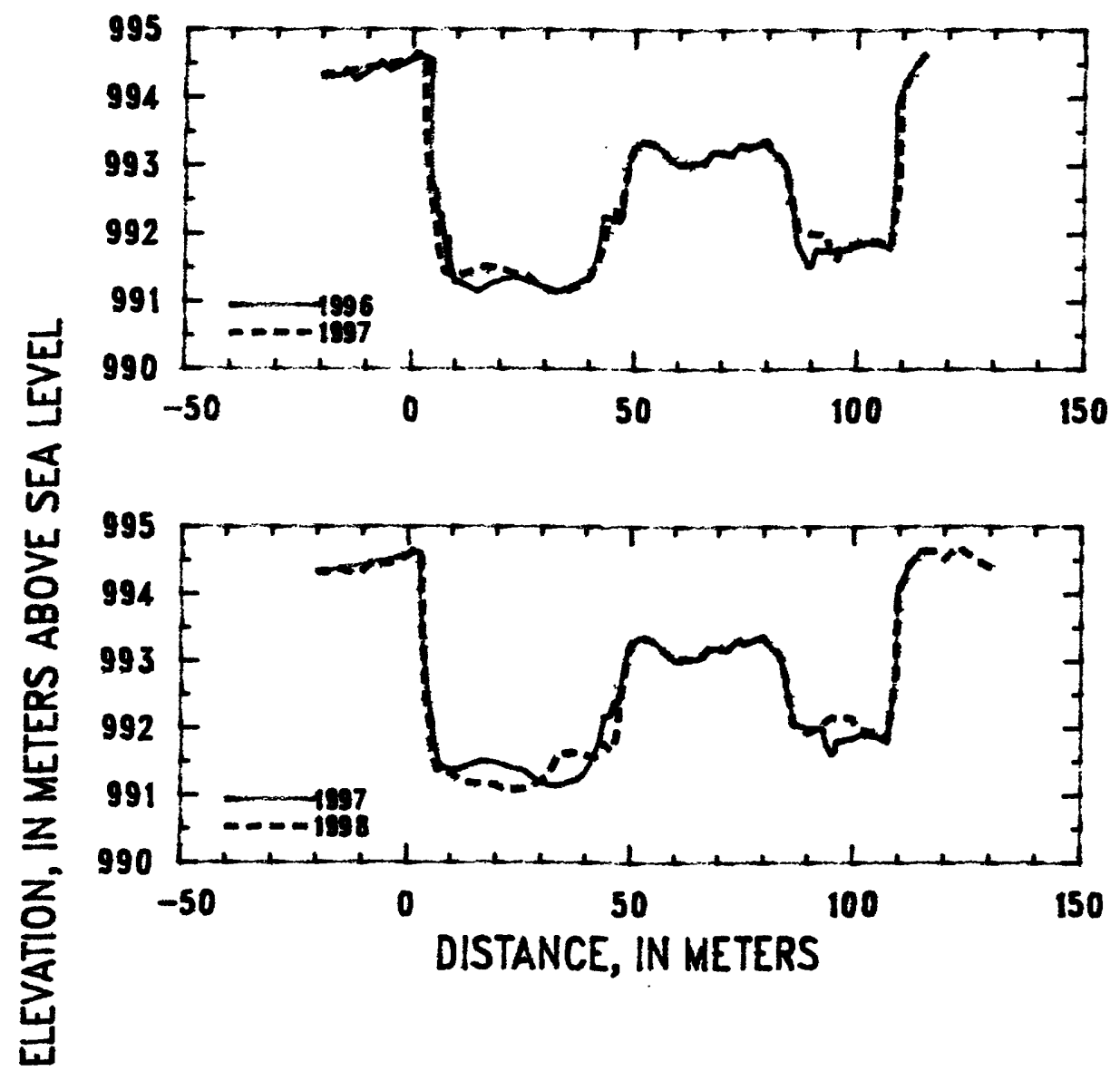

Figure 38. Profiles of cross section PR136 from 1996 to 1998. 
Table 14. Listing of horizontal stations and elevations for cross section PR136

[Sta., station, distance in meters from a reference pin on the left bank; Elev., elevation, in meters above sea level]

\begin{tabular}{|c|c|c|c|c|c|c|c|c|c|}
\hline \multirow{2}{*}{\multicolumn{2}{|c|}{$\begin{array}{c}1989 \\
\text { 18 September } \\
\end{array}$}} & \multicolumn{2}{|c|}{1989} & \multicolumn{2}{|c|}{1989} & \multicolumn{2}{|c|}{1990} & \multicolumn{2}{|c|}{1990} \\
\hline & & $18 \mathrm{Se}$ & ember & $18 \mathrm{Se}$ & ember & $15 \mathrm{Se}$ & ember & $15 \mathrm{Se}$ & ember \\
\hline Sta. & Elev. & Sta. & Elev. & Sta. & Elev. & Sta. & Elev. & Sta. & Elev. \\
\hline-1.5 & 994.52 & 58.0 & 992.64 & 111.0 & 994.21 & -1.5 & 994.52 & 49.6 & 992.71 \\
\hline 0.0 & 994.59 & 60.0 & 992.69 & 112.0 & 994.39 & 0.0 & 994.60 & 50.0 & 992.68 \\
\hline 2.0 & 994.62 & 62.0 & 992.72 & 113.0 & 994.50 & 1.0 & 994.63 & 51.0 & 992.73 \\
\hline 4.0 & 994.60 & 64.0 & 992.61 & 115.0 & 994.64 & 3.0 & 994.60 & 52.0 & 992.76 \\
\hline 6.0 & 994.55 & 66.0 & 992.64 & 116.6 & 994.48 & 5.0 & 994.56 & 53.0 & 992.73 \\
\hline 7.0 & 994.40 & 68.0 & 992.67 & 118.0 & 994.64 & 7.0 & 994.41 & 55.0 & 992.69 \\
\hline 8.0 & 994.14 & 70.0 & 992.67 & 120.0 & 994.53 & 8.0 & 994.16 & 57.0 & 992.67 \\
\hline 8.4 & 994.01 & 72.0 & 992.59 & & & 8.5 & 993.94 & 59.0 & 992.70 \\
\hline 9.0 & 993.76 & 74.0 & 992.66 & & & 9.0 & 993.79 & 61.0 & 992.70 \\
\hline 10.0 & 992.77 & 76.0 & 992.70 & & & 9.4 & 993.30 & 63.0 & 992.67 \\
\hline 11.0 & 992.22 & 78.0 & 992.78 & & & 10.2 & 992.55 & 65.0 & 992.67 \\
\hline 11.5 & 992.03 & 79.5 & 992.70 & & & 11.6 & 991.86 & 67.0 & 992.71 \\
\hline 11.7 & 991.87 & 81.0 & 992.64 & & & 11.7 & 991.51 & 69.0 & 992.73 \\
\hline 11.8 & 991.39 & 81.3 & 992.57 & & & 11.8 & 991.33 & 71.0 & 992.62 \\
\hline 12.3 & 991.24 & 82.5 & 992.56 & & & 12.0 & 991.35 & 73.0 & 992.64 \\
\hline 14.0 & 991.27 & 83.0 & 992.48 & & & 14.0 & 991.42 & 75.0 & 992.73 \\
\hline 16.0 & 991.15 & 83.5 & 992.31 & & & 16.0 & 991.42 & 77.0 & 992.77 \\
\hline 18.0 & 991.20 & 85.0 & 992.24 & & & 18.0 & 991.38 & 79.0 & 992.73 \\
\hline 20.0 & 991.24 & 86.0 & 992.24 & & & 20.3 & 991.36 & 81.0 & 992.68 \\
\hline 22.0 & 991.32 & 86.6 & 992.19 & & & 21.5 & 991.09 & 83.0 & 992.55 \\
\hline 24.0 & 991.35 & 87.2 & 991.71 & & & 23.0 & 991.13 & 84.0 & 992.38 \\
\hline 26.0 & 991.46 & 87.5 & 991.69 & & & 25.0 & 991.25 & 86.0 & 992.33 \\
\hline 28.0 & 991.41 & 87.7 & 991.57 & & & 26.8 & 991.41 & 87.0 & 992.05 \\
\hline 30.0 & 991.37 & 88.5 & 991.61 & & & 27.4 & 991.54 & 87.4 & 991.74 \\
\hline 32.0 & 991.35 & 90.0 & 991.56 & & & 28.0 & 991.58 & 87.7 & 991.55 \\
\hline 34.0 & 991.32 & 91.5 & 991.53 & & & 30.0 & 991.59 & 88.0 & 991.41 \\
\hline 36.0 & 991.32 & 94.0 & 991.59 & & & 32.0 & 991.56 & 90.0 & 991.43 \\
\hline 38.0 & 991.33 & 95.2 & 991.66 & & & 32.4 & 991.53 & 92.0 & 991.49 \\
\hline 40.0 & 991.30 & 96.7 & 991.54 & & & 33.0 & 991.51 & 94.0 & 991.48 \\
\hline 42.0 & 991.33 & 98.0 & 991.43 & & & 34.0 & 991.44 & 96.0 & 991.48 \\
\hline 44.0 & 991.37 & 100.0 & 991.43 & & & 36.0 & 991.37 & 98.0 & 991.47 \\
\hline 45.0 & 991.32 & 102.0 & 991.48 & & & 38.0 & 991.29 & 100.0 & 991.50 \\
\hline 45.5 & 991.36 & 103.3 & 991.46 & & & 40.0 & 991.29 & 101.3 & 991.48 \\
\hline 46.5 & 991.55 & 104.0 & 991.44 & & & 41.0 & 991.33 & 101.7 & 991.37 \\
\hline 47.0 & 991.76 & 104.5 & 991.53 & & & 42.0 & 991.34 & 103.4 & 991.32 \\
\hline 47.2 & 992.00 & 104.7 & 991.73 & & & 44.0 & 991.31 & 104.1 & 991.54 \\
\hline 48.0 & 992.40 & 105.4 & 991.76 & & & 45.2 & 991.33 & 104.2 & 991.61 \\
\hline 49.0 & 992.55 & 106.4 & 992.60 & & & 46.8 & 991.56 & 105.4 & 991.80 \\
\hline 50.0 & 992.60 & 106.6 & 993.28 & & & 47.0 & 991.61 & 106.1 & 992.21 \\
\hline 52.0 & 992.75 & 107.0 & 993.46 & & & 47.1 & 992.02 & 106.5 & 992.78 \\
\hline 54.0 & 992.67 & 108.0 & 993.76 & & & 48.0 & 992.56 & 106.6 & 993.28 \\
\hline 56.0 & 992.60 & 110.0 & 994.18 & & & 48.9 & 992.81 & 107.0 & 993.50 \\
\hline
\end{tabular}


Table 14. (Continued) Listing of horizontal stations and elevations for cross section PR136

[Sta., station, distance in meters from a reference pin on the left bank; Elev., elevation, in meters above sea level]

\begin{tabular}{|c|c|c|c|c|c|c|c|c|c|}
\hline \multirow{2}{*}{\multicolumn{2}{|c|}{$\begin{array}{c}1990 \\
15 \text { September }\end{array}$}} & \multirow{2}{*}{\multicolumn{2}{|c|}{$\begin{array}{l}1991 \\
29 \text { August }\end{array}$}} & \multirow{2}{*}{\multicolumn{2}{|c|}{$\begin{array}{c}1991 \\
29 \text { August }\end{array}$}} & \multirow{2}{*}{\multicolumn{2}{|c|}{$\begin{array}{l}1992 \\
26 \text { August }\end{array}$}} & \multirow{2}{*}{\multicolumn{2}{|c|}{$\begin{array}{l}1992 \\
26 \text { August }\end{array}$}} \\
\hline & & & & & & & & & \\
\hline Sta. & Elev. & Sta. & Elev. & Sta. & Elev. & Sta. & Elev. & Sta. & Elev. \\
\hline 108.0 & 993.77 & -1.5 & 994.53 & 62.0 & 992.68 & -1.5 & 994.53 & 60.0 & 992.72 \\
\hline 110.0 & 994.15 & 0.0 & 994.58 & 64.0 & 992.62 & 0.0 & 994.59 & 62.0 & 992.72 \\
\hline 111.0 & 994.21 & 2.0 & 994.63 & 66.0 & 992.65 & 2.0 & 994.63 & 64.0 & 992.66 \\
\hline 112.0 & 994.39 & 4.0 & 994.60 & 68.0 & 992.71 & 4.0 & 994.60 & 66.0 & 992.67 \\
\hline 113.0 & 994.50 & 6.0 & 994.54 & 70.0 & 992.70 & 6.0 & 994.56 & 68.0 & 992.71 \\
\hline \multirow[t]{37}{*}{115.0} & 994.65 & 7.4 & 994.37 & 72.0 & 992.61 & 7.4 & 994.37 & 70.0 & 992.71 \\
\hline & & 8.5 & 993.94 & 74.0 & 992.70 & 8.4 & 993.99 & 72.0 & 992.62 \\
\hline & & 9.0 & 993.80 & 76.0 & 992.71 & 8.7 & 993.90 & 74.0 & 992.70 \\
\hline & & 9.5 & 993.30 & 78.0 & 992.79 & 9.2 & 993.47 & 76.0 & 992.73 \\
\hline & & 10.1 & 992.55 & 80.0 & 992.74 & 9.5 & 993.56 & 78.0 & 992.81 \\
\hline & & 11.1 & 992.11 & 81.0 & 992.66 & 10.0 & 992.86 & 80.0 & 992.77 \\
\hline & & 11.2 & 991.56 & 82.0 & 992.61 & 10.5 & 992.32 & 82.0 & 992.63 \\
\hline & & 11.5 & 991.41 & 83.3 & 992.50 & 11.0 & 991.69 & 84.0 & 992.51 \\
\hline & & 12.1 & 991.31 & 84.0 & 992.49 & 11.4 & 991.61 & 85.3 & 992.56 \\
\hline & & 13.0 & 991.23 & 85.6 & 992.53 & 12.2 & 991.27 & 86.1 & 992.49 \\
\hline & & 14.0 & 991.23 & 86.8 & 992.07 & 14.0 & 991.29 & 86.6 & 992.35 \\
\hline & & 15.7 & 991.03 & 87.0 & 991.63 & 16.0 & 991.36 & 87.0 & 992.06 \\
\hline & & 17.4 & 991.21 & 88.0 & 991.58 & 18.0 & 991.40 & 87.2 & 991.67 \\
\hline & & 18.7 & 991.16 & 90.0 & 991.58 & 20.0 & 991.41 & 87.5 & 991.52 \\
\hline & & 19.1 & 991.34 & 91.2 & 991.54 & 22.0 & 991.42 & 88.0 & 991.64 \\
\hline & & 21.0 & 991.32 & 93.2 & 991.64 & 24.0 & 991.44 & 90.0 & 991.65 \\
\hline & & 24.0 & 991.34 & 94.2 & 991.46 & 26.0 & 991.46 & 92.0 & 991.72 \\
\hline & & 27.0 & 991.38 & 96.0 & 991.47 & 28.0 & 991.39 & 94.0 & 991.74 \\
\hline & & 30.0 & 991.40 & 98.0 & 991.45 & 30.0 & 991.41 & 96.0 & 991.70 \\
\hline & & 32.0 & 991.36 & 100.0 & 991.49 & 32.0 & 991.43 & 98.0 & 991.71 \\
\hline & & 33.8 & 991.29 & 102.0 & 991.50 & 34.0 & 991.37 & 100.0 & 991.74 \\
\hline & & 36.0 & 991.22 & 103.0 & 991.53 & 36.0 & 991.20 & 102.0 & 991.80 \\
\hline & & 37.8 & 991.17 & 105.0 & 991.62 & 38.0 & 991.16 & 104.0 & 991.96 \\
\hline & & 39.0 & 991.21 & 105.3 & 991.60 & 40.0 & 991.26 & 105.0 & 991.98 \\
\hline & & 41.0 & 991.34 & 105.4 & 991.86 & 42.0 & 991.31 & 106.0 & 992.34 \\
\hline & & 43.6 & 991.44 & 106.5 & 991.61 & 44.0 & 991.35 & 106.3 & 993.42 \\
\hline & & 45.0 & 991.34 & 106.6 & 993.26 & 46.0 & 991.41 & 106.5 & 992.75 \\
\hline & & 47.0 & 991.73 & 108.0 & 993.76 & 47.0 & 991.60 & 106.6 & 993.17 \\
\hline & & 47.2 & 992.26 & 110.0 & 994.17 & 47.2 & 992.17 & 107.0 & 993.39 \\
\hline & & 48.0 & 992.60 & 111.0 & 994.20 & 47.5 & 992.40 & 107.0 & 993.15 \\
\hline & & 48.9 & 992.78 & 112.0 & 994.41 & 48.0 & 992.50 & 107.2 & 993.52 \\
\hline & & 50.0 & 992.70 & 114.0 & 994.60 & 49.0 & 992.79 & 108.0 & 993.74 \\
\hline & & 52.0 & 992.76 & 115.0 & 994.64 & 50.0 & 992.70 & 110.0 & 994.14 \\
\hline & & 54.0 & 992.68 & & & 52.0 & 992.77 & 112.0 & 994.40 \\
\hline & & 56.0 & 992.64 & & & 54.0 & 992.69 & 115.0 & 994.65 \\
\hline & & 58.0 & 992.67 & & & 56.0 & 992.66 & & \\
\hline & & 60.0 & 992.71 & & & 58.0 & 992.67 & & \\
\hline
\end{tabular}


Table 14. (Continued) Listing of horizontal stations and elevations for cross section PR136

[Sta., station, distance in meters from a reference pin on the left bank; Elev., elevation, in meters above sea level]

\begin{tabular}{|c|c|c|c|c|c|c|c|c|c|}
\hline \multirow{2}{*}{\multicolumn{2}{|c|}{$\begin{array}{c}1993 \\
27 \text { August }\end{array}$}} & \multicolumn{2}{|c|}{1993} & \multicolumn{2}{|c|}{1993} & \multicolumn{2}{|c|}{1994} & \multicolumn{2}{|c|}{1994} \\
\hline & & 27 & gust & 27 & gust & $23 \mathrm{Se}$ & ember & $23 \mathrm{Se}$ & ember \\
\hline Sta. & Elev. & Sta. & Elev. & Sta. & Elev. & Sta. & Elev. & Sta. & Elev. \\
\hline-1.5 & 994.53 & 53.5 & 993.08 & 106.6 & 992.86 & -1.5 & 994.55 & 63.0 & 992.81 \\
\hline 0.0 & 994.59 & 54.8 & 993.05 & 106.8 & 992.57 & 0.0 & 994.58 & 65.0 & 992.78 \\
\hline 2.0 & 994.62 & 56.3 & 992.83 & 107.0 & 992.91 & 1.0 & 994.67 & 67.0 & 992.81 \\
\hline 4.0 & 994.59 & 58.0 & 992.78 & 107.3 & 993.55 & 3.0 & 994.61 & 69.0 & 992.84 \\
\hline 6.0 & 994.56 & 60.0 & 992.80 & 108.0 & 993.75 & 5.0 & 994.58 & 71.0 & 992.74 \\
\hline 7.5 & 994.32 & 62.0 & 992.78 & 110.0 & 994.13 & 6.5 & 994.49 & 73.0 & 992.79 \\
\hline 8.5 & 993.99 & 64.0 & 992.71 & 112.0 & 994.41 & 7.3 & 994.38 & 75.0 & 992.87 \\
\hline 9.2 & 993.49 & 66.0 & 992.72 & 113.5 & 994.54 & 8.1 & 994.08 & 76.0 & 992.81 \\
\hline 9.9 & 992.37 & 68.0 & 992.75 & 115.0 & 994.65 & 8.3 & 993.51 & 78.0 & 992.91 \\
\hline 10.1 & 992.14 & 70.0 & 992.76 & & & 8.5 & 992.97 & 80.0 & 992.87 \\
\hline 10.5 & 991.97 & 72.0 & 992.67 & & & 9.3 & 992.53 & 82.0 & 992.85 \\
\hline 11.0 & 991.70 & 74.0 & 992.74 & & & 10.1 & 992.34 & 84.0 & 992.82 \\
\hline 11.5 & 991.40 & 76.0 & 992.77 & & & 10.5 & 991.62 & 85.0 & 992.75 \\
\hline 12.3 & 991.28 & 78.0 & 992.86 & & & 11.2 & 991.47 & 86.0 & 992.61 \\
\hline 14.0 & 991.28 & 80.0 & 992.83 & & & 13.0 & 991.31 & 86.8 & 992.14 \\
\hline 16.0 & 991.25 & 81.0 & 992.82 & & & 15.0 & 991.17 & 87.1 & 992.09 \\
\hline 18.0 & 991.32 & 82.0 & 992.79 & & & 18.0 & 991.26 & 88.0 & 992.15 \\
\hline 20.0 & 991.27 & 83.0 & 992.81 & & & 21.0 & 991.33 & 89.6 & 991.88 \\
\hline 22.0 & 991.27 & 84.0 & 992.82 & & & 24.0 & 991.33 & 91.0 & 991.91 \\
\hline 24.0 & 991.26 & 85.0 & 992.78 & & & 27.0 & 991.28 & 93.0 & 991.93 \\
\hline 26.0 & 991.29 & 85.9 & 992.70 & & & 30.0 & 991.26 & 95.0 & 991.85 \\
\hline 28.0 & 991.34 & 86.1 & 992.53 & & & 33.0 & 991.21 & 97.6 & 991.80 \\
\hline 30.0 & 991.27 & 86.7 & 992.20 & & & 36.0 & 991.15 & 98.0 & 991.84 \\
\hline 32.0 & 991.45 & 88.0 & 991.98 & & & 38.0 & 991.29 & 99.0 & 991.80 \\
\hline 35.0 & 991.33 & 88.5 & 991.93 & & & 40.0 & 991.39 & 100.0 & 991.47 \\
\hline 37.0 & 991.27 & 89.0 & 991.71 & & & 41.2 & 991.48 & 100.8 & 991.49 \\
\hline 39.0 & 991.28 & 89.3 & 991.52 & & & 42.6 & 991.55 & 101.6 & 991.63 \\
\hline 40.0 & 991.25 & 90.0 & 991.46 & & & 43.6 & 991.73 & 103.5 & 991.65 \\
\hline 40.9 & 991.35 & 92.0 & 991.43 & & & 45.3 & 991.78 & 105.0 & 991.79 \\
\hline 42.4 & 991.68 & 93.2 & 991.38 & & & 46.3 & 991.74 & 105.5 & 991.77 \\
\hline 44.0 & 991.69 & 94.5 & 991.75 & & & 46.8 & 991.80 & 106.0 & 992.13 \\
\hline 44.6 & 991.66 & 96.4 & 991.76 & & & 47.1 & 991.96 & 106.5 & 992.78 \\
\hline 45.5 & 991.73 & 98.6 & 991.77 & & & 48.0 & 992.63 & 107.0 & 992.98 \\
\hline 46.4 & 991.58 & 100.0 & 991.62 & & & 49.0 & 993.02 & 107.4 & 993.56 \\
\hline 46.8 & 991.70 & 102.0 & 991.67 & & & 51.0 & 993.13 & 108.0 & 993.74 \\
\hline 47.1 & 991.91 & 103.5 & 991.73 & & & 53.0 & 993.11 & 110.5 & 994.25 \\
\hline 48.0 & 992.62 & 105.0 & 991.60 & & & 54.0 & 993.09 & 112.0 & 994.42 \\
\hline 49.0 & 992.99 & 105.5 & 991.72 & & & 55.0 & 993.07 & 115.0 & 994.64 \\
\hline 50.0 & 993.01 & 105.8 & 991.87 & & & 56.0 & 992.88 & & \\
\hline 50.3 & 993.08 & 105.9 & 992.05 & & & 57.0 & 992.84 & & \\
\hline 51.0 & 993.10 & 106.1 & 992.20 & & & 59.0 & 992.80 & & \\
\hline 52.0 & 993.09 & 106.2 & 992.62 & & & 61.0 & 992.82 & & \\
\hline
\end{tabular}


Table 14. (Continued) Listing of horizontal stations and elevations for cross section PR136

[Sta., station, distance in meters from a reference pin on the left bank; Elev., elevation, in meters above sea level]

\begin{tabular}{|c|c|c|c|c|c|c|c|c|c|}
\hline \multirow{2}{*}{\multicolumn{2}{|c|}{$\begin{array}{c}1995 \\
27 \text { September }\end{array}$}} & \multicolumn{2}{|c|}{1995} & \multicolumn{2}{|c|}{1996} & \multicolumn{2}{|c|}{1996} & \multicolumn{2}{|c|}{1997} \\
\hline & & $27 \mathrm{Se}$ & ember & 18 & tober & 18( & tober & $19 \mathrm{Se}$ & ember \\
\hline Sta. & Elev. & Sta. & Elev. & Sta. & Elev. & Sta. & Elev. & Sta. & Elev. \\
\hline-1.5 & 994.54 & 61.0 & 993.05 & -20.0 & 994.33 & 54.0 & 993.33 & -20.0 & 994.33 \\
\hline 0.0 & 994.58 & 63.0 & 993.02 & -18.0 & 994.32 & 56.0 & 993.26 & -1.4 & 994.55 \\
\hline 2.0 & 994.62 & . 65.0 & 993.01 & -16.0 & 994.33 & 58.0 & 993.11 & 0.0 & 994.59 \\
\hline 4.0 & 994.60 & 67.0 & 993.10 & -14.0 & 994.39 & 60.0 & 993.02 & 1.0 & 994.66 \\
\hline 6.4 & 994.48 & 68.0 & 993.22 & -12.5 & 994.28 & 62.0 & 993.04 & 2.9 & 994.61 \\
\hline 6.5 & 993.33 & 70.0 & 993.20 & -10.0 & 994.39 & 64.0 & 993.03 & 3.2 & 993.52 \\
\hline 6.6 & 993.06 & 72.0 & 993.15 & -7.0 & 994.53 & 66.0 & 993.05 & 4.3 & 992.68 \\
\hline 8.0 & 992.29 & 74.0 & 993.31 & -5.0 & 994.42 & 68.0 & 993.20 & 5.7 & 992.11 \\
\hline 8.2 & 991.99 & 76.0 & 993.26 & -3.0 & 994.47 & 70.0 & 993.21 & 5.8 & 991.86 \\
\hline 8.8 & 991.91 & 78.0 & 993.32 & -1.4 & 994.54 & 72.0 & 993.17 & 6.6 & 991.64 \\
\hline 8.9 & 991.75 & 80.0 & 993.33 & 0.0 & 994.58 & 74.0 & 993.30 & 7.6 & 991.42 \\
\hline 9.1 & 991.70 & 82.0 & 993.17 & 2.0 & 994.62 & 76.0 & 993.26 & 9.0 & 991.38 \\
\hline 10.8 & 991.22 & 83.0 & 993.08 & 4.5 & 994.55 & 78.0 & 993.32 & 11.0 & 991.37 \\
\hline 13.0 & 991.39 & 84.5 & 992.96 & 4.5 & 992.77 & 80.0 & 993.31 & 14.0 & 991.45 \\
\hline 15.0 & 991.44 & 85.2 & 992.84 & 5.0 & 992.67 & 82.0 & 993.17 & 17.0 & 991.51 \\
\hline 17.0 & 991.42 & 86.5 & 992.29 & 5.6 & 992.62 & 83.0 & 993.11 & 20.0 & 991.49 \\
\hline 19.0 & 991.39 & 86.9 & 992.00 & 6.1 & 992.28 & 84.0 & 992.99 & 23.0 & 991.41 \\
\hline 21.0 & 991.33 & 87.0 & 991.67 & 6.7 & 992.28 & 84.6 & 992.92 & 26.0 & 991.38 \\
\hline 23.0 & 991.23 & 87.3 & 991.46 & 7.5 & 991.93 & 85.1 & 992.67 & 29.0 & 991.21 \\
\hline 25.0 & 991.29 & 89.0 & 991.46 & 8.2 & 991.58 & 86.1 & 992.32 & 32.0 & 991.14 \\
\hline 27.0 & 991.26 & 90.5 & 991.38 & 8.5 & 991.77 & 86.7 & 991.85 & 35.0 & 991.16 \\
\hline 29.0 & 991.16 & 91.5 & 991.56 & 8.7 & 991.60 & 88.1 & 991.70 & 38.0 & 991.23 \\
\hline 31.0 & 991.20 & 94.0 & 991.51 & 9.7 & 991.30 & 89.2 & 991.52 & 40.0 & 991.35 \\
\hline 33.0 & 991.15 & 96.0 & 991.52 & 12.0 & 991.25 & 90.0 & 991.52 & 42.2 & 991.65 \\
\hline 35.0 & 991.20 & 98.0 & 991.47 & 15.0 & 991.14 & 91.0 & 991.75 & 43.2 & 991.79 \\
\hline 37.0 & 991.25 & 100.0 & 991.45 & 18.0 & 991.25 & 93.0 & 991.76 & 44.0 & 992.17 \\
\hline 39.0 & 991.40 & 102.0 & 991.57 & 21.0 & 991.33 & 96.0 & 991.75 & 45.6 & 992.21 \\
\hline 42.0 & 991.60 & 104.0 & 991.63 & 24.0 & 991.35 & 99.0 & 991.79 & 46.3 & 992.39 \\
\hline 42.5 & 991.71 & 105.5 & 991.69 & 27.0 & 991.27 & 102.0 & 991.87 & 47.3 & 992.39 \\
\hline 42.9 & 991.78 & 106.0 & 992.04 & 30.0 & 991.20 & 105.0 & 991.84 & 48.8 & 993.04 \\
\hline 45.0 & 991.83 & 106.4 & 991.90 & 33.0 & 991.14 & 107.4 & 991.78 & 50.0 & 993.27 \\
\hline 46.5 & 991.98 & 107.3 & 992.19 & 35.0 & 991.18 & 108.2 & 992.37 & 52.0 & 993.35 \\
\hline 47.3 & 992.14 & 108.7 & 992.96 & 38.0 & 991.29 & 108.8 & 993.01 & 54.0 & 993.33 \\
\hline 49.0 & 993.09 & 108.8 & 993.90 & 40.0 & 991.33 & 108.9 & 993.87 & 56.0 & 993.26 \\
\hline 50.0 & 993.25 & 110.0 & 994.12 & 41.8 & 991.60 & 110.0 & 994.12 & 58.0 & 993.10 \\
\hline 51.0 & 993.35 & 112.0 & 994.41 & 43.2 & 992.25 & 112.0 & 994.42 & 60.0 & 993.00 \\
\hline 53.0 & 993.33 & 114.0 & 994.59 & 45.0 & 992.24 & 115.0 & 994.65 & 62.0 & 993.02 \\
\hline 55.0 & 993.29 & 115.0 & 994.64 & 46.7 & 992.19 & & & 64.0 & 993.02 \\
\hline 57.0 & 993.20 & 117.0 & 994.52 & 47.5 & 992.26 & & & 66.0 & 993.04 \\
\hline 58.0 & 993.09 & 120.0 & 994.52 & 48.8 & 993.02 & & & 68.0 & 993.19 \\
\hline 59.0 & 993.06 & & & 50.0 & 993.26 & & & 70.0 & 993.19 \\
\hline 60.0 & 992.99 & & & 52.0 & 993.35 & & & 72.0 & 993.17 \\
\hline
\end{tabular}


Table 14. (Continued) Listing of horizontal stations and elevations for cross section PR136

[Sta., station, distance in meters from a reference pin on the left bank; Elev., elevation, in meters above sea level]

\begin{tabular}{|c|c|c|c|c|c|}
\hline \multirow{2}{*}{\multicolumn{2}{|c|}{1997}} & \multicolumn{2}{|c|}{1998} & \multicolumn{2}{|c|}{1998} \\
\hline & & \multicolumn{2}{|c|}{28 September } & \multicolumn{2}{|c|}{28 September } \\
\hline Sta. & Elev. & Sta. & Elev. & Sta. & Elev. \\
\hline 74.0 & 993.30 & -20.0 & 994.32 & 62.0 & 993.04 \\
\hline 76.0 & 993.27 & -18.0 & 994.32 & 64.0 & 993.02 \\
\hline 78.0 & 993.32 & -15.0 & 994.37 & 66.0 & 993.06 \\
\hline 80.0 & 993.37 & -13.0 & 994.32 & 68.0 & 993.22 \\
\hline 81.0 & 993.26 & -10.0 & 994.36 & 70.0 & 993.19 \\
\hline 83.0 & 993.11 & -7.0 & 994.51 & 72.0 & 993.18 \\
\hline 84.0 & 993.00 & -6.0 & 994.47 & 74.0 & 993.32 \\
\hline 85.0 & 992.69 & -4.0 & 994.46 & 76.0 & 993.27 \\
\hline 86.8 & 992.07 & -1.5 & 994.52 & 78.0 & 993.33 \\
\hline 89.0 & 992.01 & 0.0 & 994.59 & 80.0 & 993.35 \\
\hline 91.0 & 991.99 & 1.5 & 994.62 & 82.0 & 993.19 \\
\hline 93.2 & 991.99 & 3.0 & 994.58 & 83.0 & 993.14 \\
\hline 94.2 & 991.71 & 3.3 & 993.40 & 84.0 & 993.02 \\
\hline 95.3 & 991.61 & 4.1 & 992.47 & 85.0 & 992.73 \\
\hline 95.8 & 991.64 & 5.1 & 992.01 & 85.5 & 992.48 \\
\hline 96.3 & 991.81 & 5.3 & 991.80 & 86.0 & 992.34 \\
\hline 99.0 & 991.83 & 6.4 & 991.43 & 86.5 & 992.06 \\
\hline 102.0 & 991.91 & 7.3 & 991.33 & 88.0 & 992.00 \\
\hline 105.0 & 991.88 & 10.0 & 991.34 & 90.0 & 991.93 \\
\hline 107.4 & 991.80 & 13.0 & 991.19 & 93.0 & 992.06 \\
\hline 108.0 & 992.05 & 16.0 & 991.17 & 95.0 & 992.16 \\
\hline 109.3 & 992.67 & 19.0 & 991.16 & 97.0 & 992.17 \\
\hline 109.7 & 994.07 & 22.0 & 991.10 & 99.5 & 992.15 \\
\hline 111.0 & 994.23 & 25.0 & 991.09 & 101.0 & 991.99 \\
\hline 112.0 & 994.42 & 28.0 & 991.15 & 104.0 & 991.94 \\
\hline 115.0 & 994.65 & 31.0 & 991.27 & 106.5 & 991.92 \\
\hline & & 34.0 & 991.62 & 107.3 & 991.82 \\
\hline & & 37.0 & 991.65 & 109.3 & 992.78 \\
\hline & & 40.0 & 991.60 & 109.7 & 994.04 \\
\hline & & 42.0 & 991.56 & 111.0 & 994.21 \\
\hline & & 44.0 & 991.78 & 112.0 & 994.42 \\
\hline & & 45.7 & 991.67 & 115.0 & 994.65 \\
\hline & & 46.4 & 991.79 & 118.0 & 994.64 \\
\hline & & 46.8 & 991.89 & 120.0 & 994.53 \\
\hline & & 47.7 & 992.49 & 123.0 & 994.71 \\
\hline & & 49.0 & 993.06 & 125.0 & 994.59 \\
\hline & & 50.0 & 993.27 & 130.0 & 994.39 \\
\hline & & 52.0 & 993.35 & & \\
\hline & & 54.0 & 993.33 & & \\
\hline & & 56.0 & 993.25 & & \\
\hline & & 58.0 & 993.10 & & \\
\hline & & 60.0 & 993.04 & & \\
\hline
\end{tabular}




\section{Description of Cross Section PR141}

Location: Township 7 South/Range 49 East--section 29

U. S. Geological Survey quadrangle (1:24,000): Bloom Creek

Landowners--left bank: U. S. Government

--right bank:U. S. Government

Access: Left bank

Permission from: Gay Ranch

Distance from Moorhead Gaging Station: 26.10 kilometers

Azimuth of Section (degrees magnetic): 021

\section{Reference Monuments}

[Monuments at stations 100.0 and 100.9 were closest to leveling instrument]

\begin{tabular}{|c|c|c|c|c|c|c|}
\hline \multirow[b]{2}{*}{ Description } & \multirow[b]{2}{*}{$\begin{array}{l}\text { Station } \\
(m)\end{array}$} & \multicolumn{2}{|c|}{ GPS-NAD83 (1992) } & \multicolumn{2}{|c|}{ Measurement } & \multirow{2}{*}{$\begin{array}{c}\text { Elevation } \\
\text { (NGVD1929) } \\
\text { (m) }\end{array}$} \\
\hline & & Latitude & Longitude & $\begin{array}{l}\text { Standard } \\
\text { deviation } \\
(m)\end{array}$ & $\begin{array}{l}\text { Horizontal } \\
\text { precision } \\
\text { (m) }\end{array}$ & \\
\hline $\begin{array}{l}\text { 1/2-inch-rebar; } 0.14 \text { meter above } 1998 \text { ground } \\
\text { level }\end{array}$ & -26.5 & $45^{\circ} 11 ' 31.75^{\prime \prime}$ & $105^{\circ} 45^{\prime} 13.37^{\prime \prime}$ & 0.365 & 0.682 & 988.47 \\
\hline $\begin{array}{l}\text { 1/2-inch-rebar; } 0.04 \text { meter above } 1998 \text { ground } \\
\text { level }\end{array}$ & -0.8 & $45^{\circ} 11 ' 32.43^{\prime \prime}$ & $105^{\circ} 45^{\prime} 12.71^{\prime \prime}$ & 0.319 & 0.543 & 988.31 \\
\hline $\begin{array}{l}\text { 1/2-inch-rebar; } 0.09 \text { meter above } 1998 \text { ground } \\
\text { level }\end{array}$ & 100.0 & & & & & 989.06 \\
\hline $\begin{array}{l}\text { 1/2-inch-rebar; } 0.21 \text { meter above } 1998 \text { ground } \\
\text { level }\end{array}$ & 100.9 & & & & & 989.19 \\
\hline Benchmark--brass circular plate & 123.0 & $45^{\circ} 11$ '35.80" & $105^{\circ} 45^{\prime} 09.56^{\prime \prime}$ & 0.336 & 0.486 & 988.93 \\
\hline
\end{tabular}



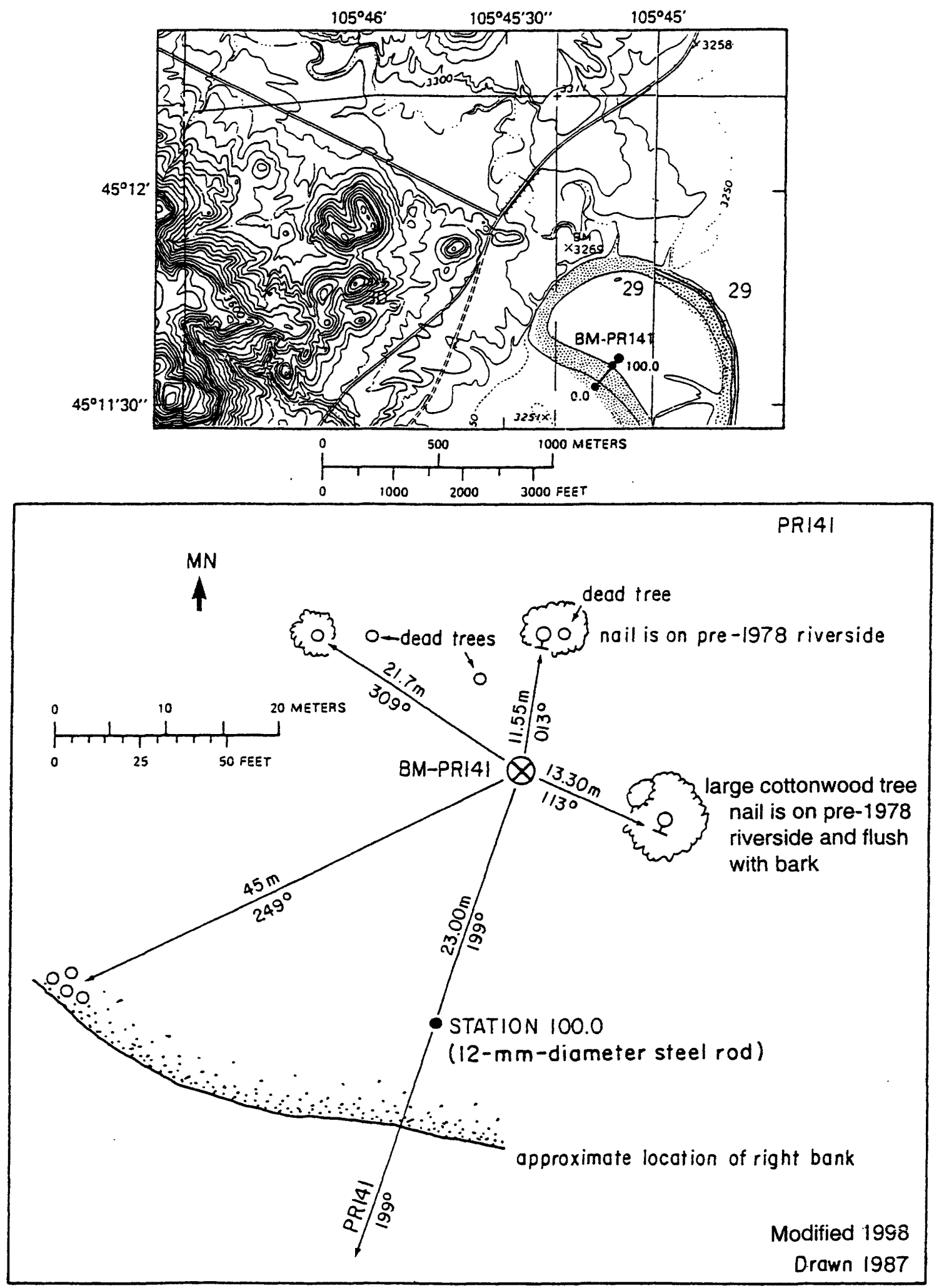

Figure 39. Upper: Location of cross section PR141, bench mark BM-PR141, and the left and right bank reference monuments in the Bloom Creek quadrangle. Lower: Location of the bench mark on the right bank of an abandoned channel. MN is magnetic north. 
No plots were made because this cross section, PR141, was only resurveyed once in 1998 and virtually no change was measured in the profile when compared to the last survey in 1984 . 
Table 15. Listing of horizontal stations and elevations for cross section PR141

[Sta., station, distance in meters from a reference pin on the left bank; Elev., elevation, in meters above sea level]

\begin{tabular}{|c|c|c|c|}
\hline \multirow{2}{*}{\multicolumn{2}{|c|}{$\begin{array}{c}1998 \\
26 \text { September }\end{array}$}} & \multicolumn{2}{|c|}{1998} \\
\hline & & $26 \mathrm{~S}$ & ember \\
\hline Sta. & Elev. & Sta. & Elev. \\
\hline-26.5 & 988.33 & 75.0 & 987.49 \\
\hline-0.8 & 988.26 & 77.0 & 987.43 \\
\hline 0.0 & 988.27 & 79.0 & 987.49 \\
\hline 3.0 & 988.44 & 81.0 & 987.47 \\
\hline 4.5 & 988.55 & 83.0 & 987.47 \\
\hline 6.0 & 988.59 & 85.0 & 987.62 \\
\hline 7.0 & 988.49 & 87.0 & 987.73 \\
\hline 9.0 & 988.52 & 88.3 & 987.77 \\
\hline 10.0 & 988.59 & 89.0 & 988.04 \\
\hline 12.0 & 988.39 & 90.9 & 989.02 \\
\hline 14.0 & 987.93 & 92.0 & 989.08 \\
\hline 15.9 & 987.74 & 94.0 & 989.06 \\
\hline 18.0 & 988.07 & 96.0 & 989.03 \\
\hline 20.0 & 988.11 & 98.0 & 988.98 \\
\hline 22.0 & 988.13 & 100.0 & 988.97 \\
\hline 24.0 & 988.13 & 100.9 & 988.99 \\
\hline 26.0 & 988.25 & & \\
\hline 27.0 & 988.11 & & \\
\hline 29.0 & 988.15 & & \\
\hline 30.0 & 988.13 & & \\
\hline 32.0 & 987.81 & & \\
\hline 34.0 & 987.59 & & \\
\hline 36.0 & 987.50 & & \\
\hline 38.0 & 987.34 & & \\
\hline 40.0 & 987.18 & & \\
\hline 42.0 & 987.13 & & \\
\hline 44.0 & 987.08 & & \\
\hline 46.0 & 987.12 & & \\
\hline 48.0 & 987.10 & & \\
\hline 50.0 & 987.16 & & \\
\hline 52.0 & 987.29 & & \\
\hline 54.0 & 987.24 & & \\
\hline 56.0 & 987.27 & & \\
\hline 58.0 & 987.30 & & \\
\hline 60.0 & 987.35 & & \\
\hline 62.0 & 987.43 & & \\
\hline 64.0 & 987.40 & & \\
\hline 66.0 & 987.38 & & \\
\hline 68.0 & 987.38 & & \\
\hline 70.0 & 987.46 & & \\
\hline 71.0 & 987.46 & & \\
\hline 73.0 & 987.48 & & \\
\hline
\end{tabular}




\section{Description of Cross Section PR141A}

Location: Township 7 South/Range 49 East--section 29

U. S. Geological Survey quadrangle (1:24,000): Huckins School

Landowners--left bank: U. S. Government

--right bank: U. S. Government

Access: Left Bank

Permission from: Gay Ranch

Distance from Moorhead Gaging Station: 26.22 kilometers

Azimuth of Section (degrees magnetic): 181.5

\section{Reference Monuments}

[Monuments at stations $-60.0,-40.6$, and -40.0 were closest to the leveling instrument ]

\begin{tabular}{|c|c|c|c|c|c|c|}
\hline \multirow[b]{2}{*}{ Description } & \multirow[b]{2}{*}{$\begin{array}{c}\text { Station } \\
\text { (m) }\end{array}$} & \multicolumn{2}{|c|}{ GPS-NAD83 (1992) } & \multicolumn{2}{|c|}{ Measurement } & \multirow{2}{*}{$\begin{array}{l}\text { Elevation } \\
\text { (NGVD1929) } \\
\text { (m) }\end{array}$} \\
\hline & & Latitude & Longitude & $\begin{array}{l}\text { Standard } \\
\text { deviation } \\
(\mathrm{m})\end{array}$ & $\begin{array}{l}\text { Horizontal } \\
\text { precision } \\
\text { (m) }\end{array}$ & \\
\hline $\begin{array}{l}\text { 1/2-inch-rebar; } 0.21 \text { meter above above } 1998 \\
\text { ground level }\end{array}$ & -60.0 & $45^{\circ} 11^{\prime} 38.08^{\prime \prime}$ & $105^{\circ} 44^{\prime} 47.90^{\prime \prime}$ & 0.443 & 0.425 & 986.99 \\
\hline $\begin{array}{l}\text { 1/2-inch-rebar; } 0.16 \text { meter above } 1998 \text { ground } \\
\text { level }\end{array}$ & -40.6 & & & & & 987.48 \\
\hline $\begin{array}{l}\text { Benchmark--1/2-inch-rebar; } 0.09 \text { meter } \\
\text { above } 1998 \text { ground level }\end{array}$ & -40.0 & & & & & 987.38 \\
\hline $\begin{array}{l}\text { 1/2-inch-rebar; } 0.14 \text { meter above above } 1998 \\
\text { ground level }\end{array}$ & 110.0 & & & & & 987.05 \\
\hline $\begin{array}{l}\text { 1/2-inch-rebar; } 0.09 \text { meter above above } 1998 \\
\text { ground level }\end{array}$ & 130.0 & & & & & 987.06 \\
\hline $\begin{array}{l}\text { 1/2-inch-rebar; } 0.06 \text { meter above } 1998 \text { ground } \\
\text { level }\end{array}$ & 150.8 & $45^{\circ} 11^{\prime} 31.51^{\prime \prime}$ & $105^{\circ} 44^{\prime} 50.49^{\prime \prime}$ & 0.177 & 0.443 & 987.37 \\
\hline $\begin{array}{l}\text { 1/2-inch-rebar; } 0.17 \text { meter above } 1998 \text { ground } \\
\text { level; } 1.1 \text { meter downstream from side of } \\
\text { cottonwood tree }\end{array}$ & 190.0 & & & & & 988.02 \\
\hline $\begin{array}{l}\text { 1/2-inch-rebar; offsection near cottonwood } \\
\text { tree with nail shown in accompaning fig- } \\
\text { ure }\end{array}$ & -- & & & & & 988.12 \\
\hline
\end{tabular}




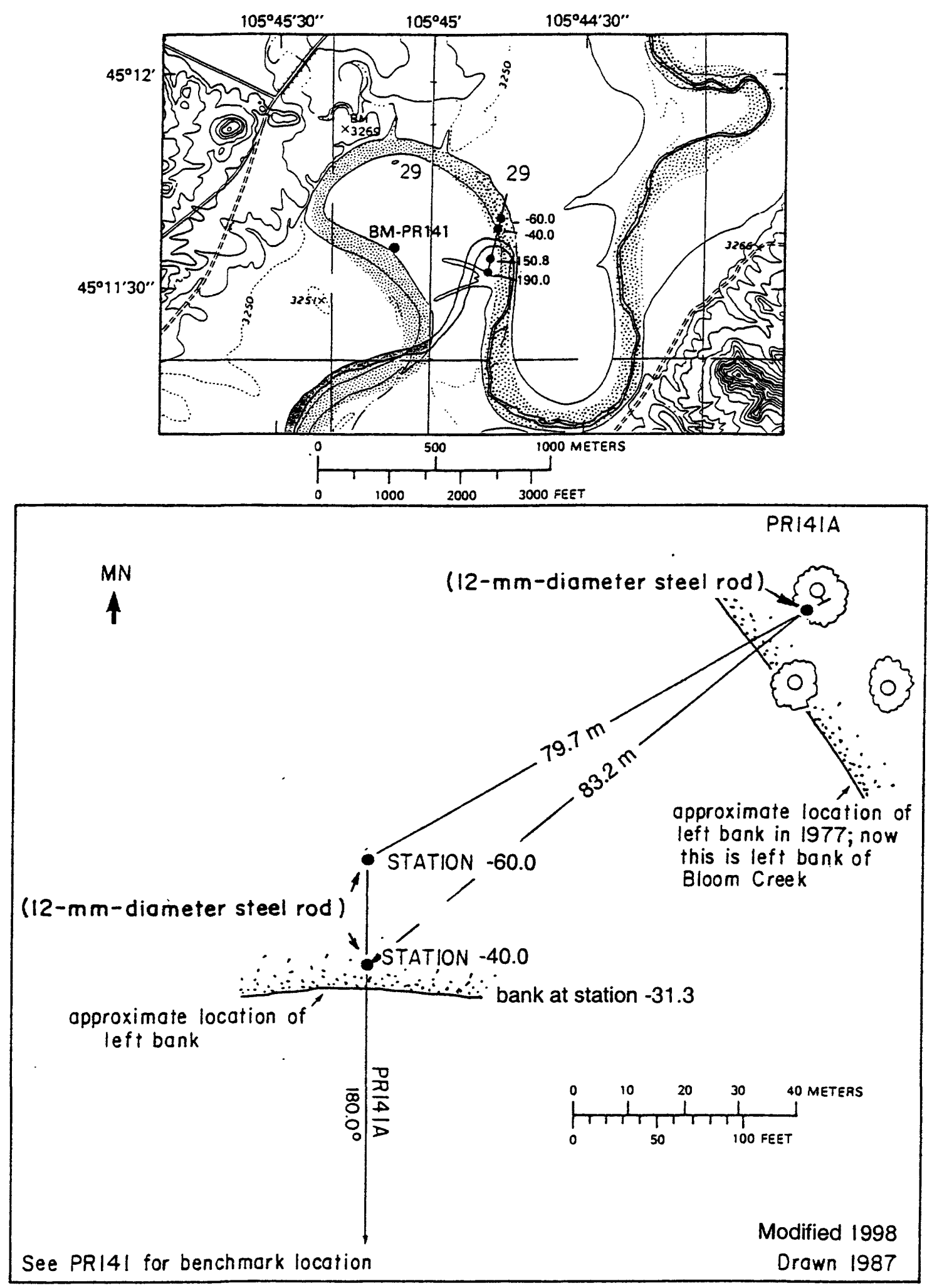

Figure 40. Upper: Location of cross section PR141A, bench mark BM-PR141, and the left and right bank reference monuments of PR-141A in the Huckins School quadrangle. New channel is shown in its approximate location. Lower: Location of the bench marks (12mm-diameter steel rods) at stations -40.0 and -60.0 on the left bank. MN is magnetic north. 

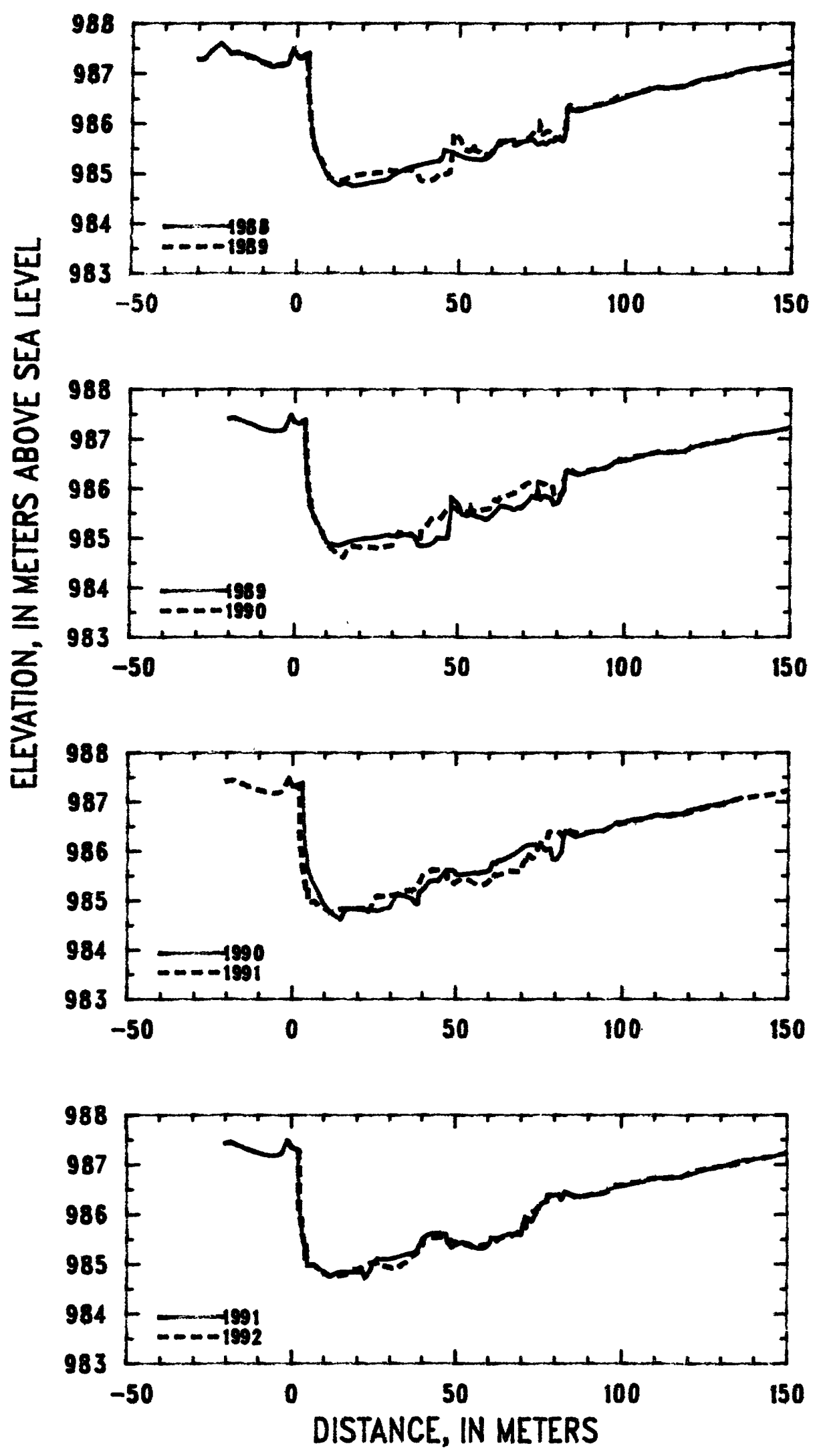

Figure 41. Profiles of cross section PR141A from 1988 to 1992. 

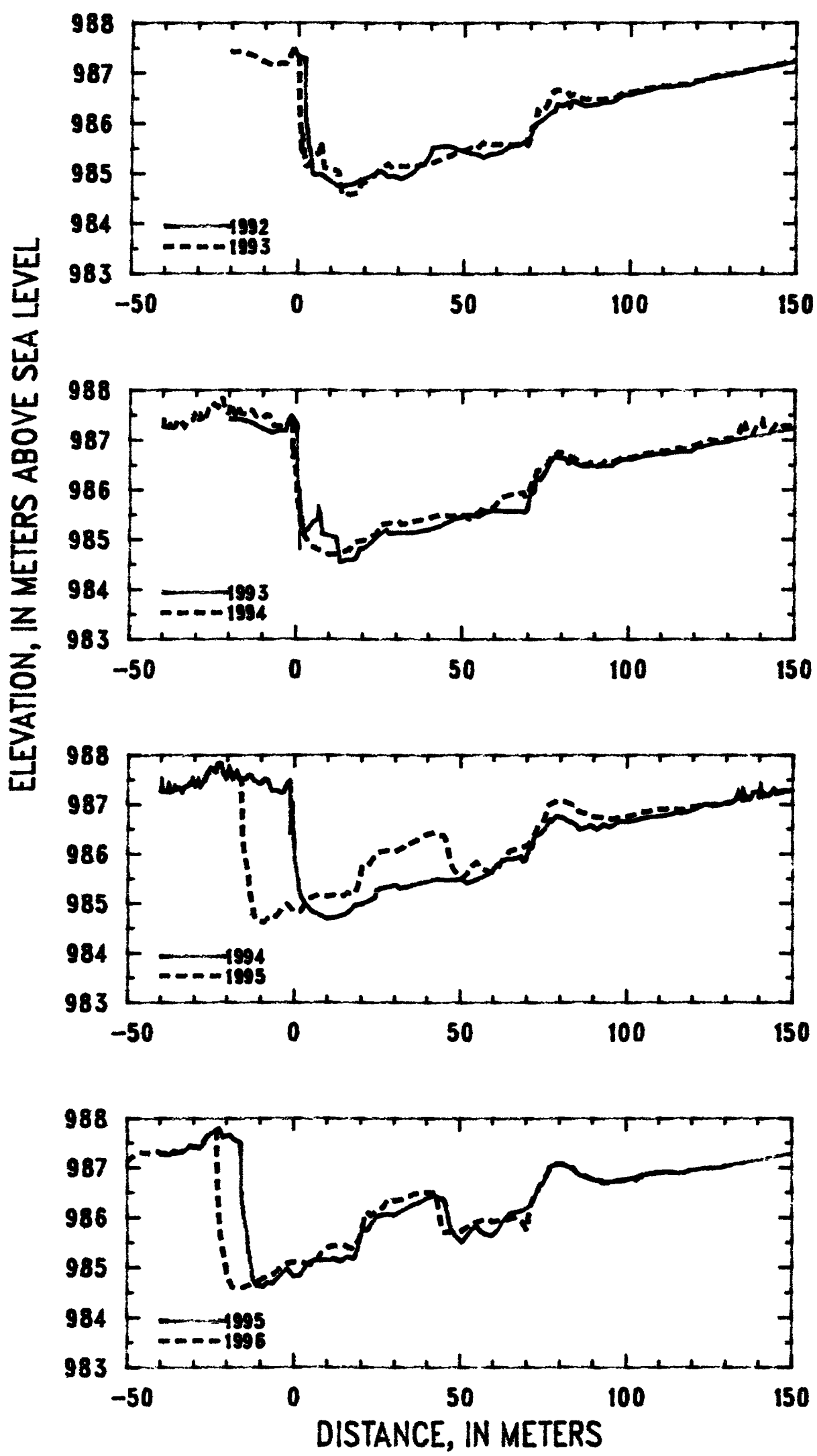

Figure 42. Profiles of cross section PR141A from 1992 to 1996. 


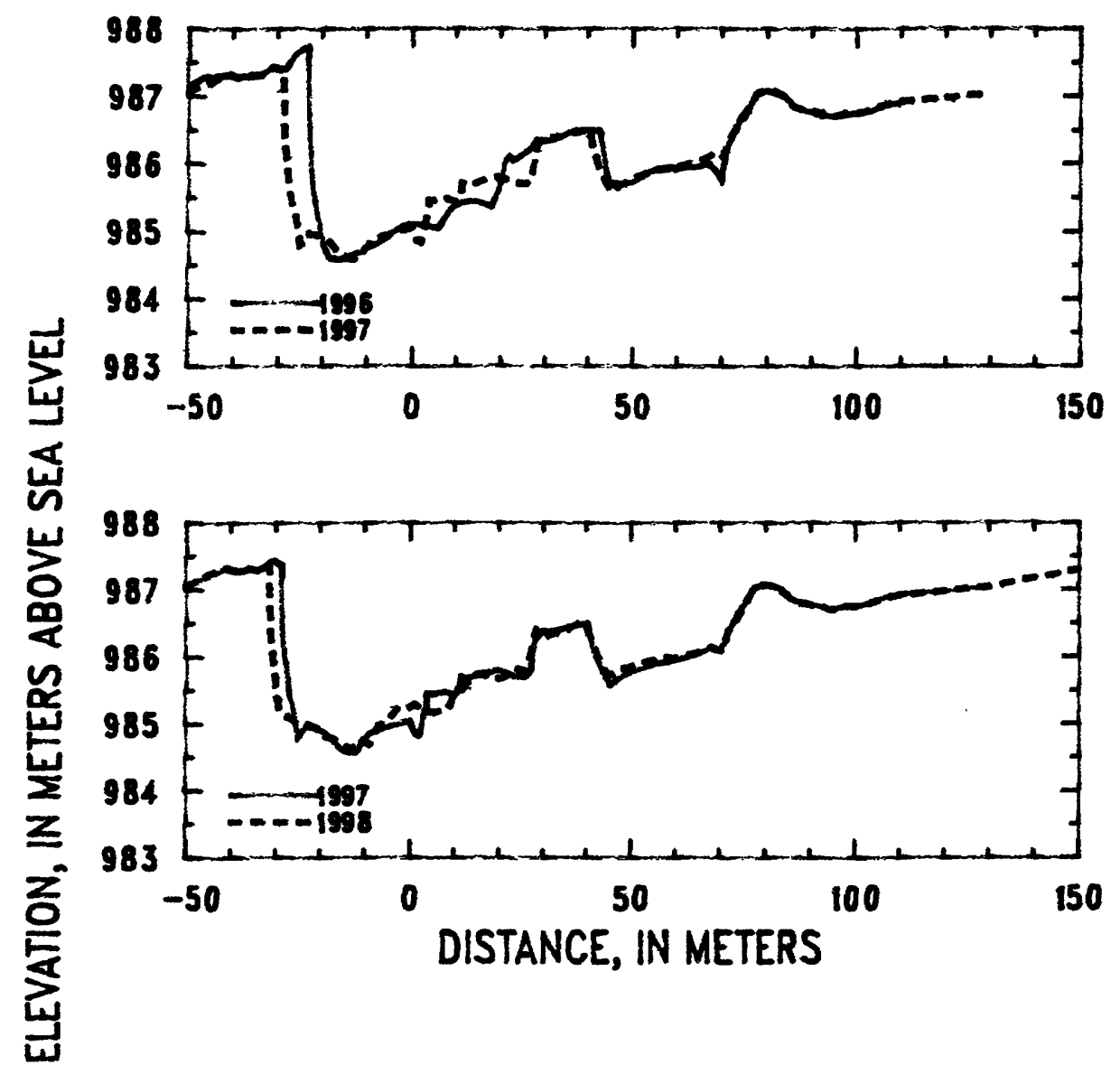

Figure 43. Profiles of cross section PR141A from 1996 to 1998. 
Table 16. Listing of horizontal stations and elevations for cross section PR141A

[Sta., station, distance in meters from a reference pin on the left bank; Elev., elevation, in meters above sea level]

\begin{tabular}{|c|c|c|c|c|c|c|c|c|c|}
\hline \multirow{2}{*}{\multicolumn{2}{|c|}{$\begin{array}{c}1989 \\
18 \text { September }\end{array}$}} & \multicolumn{2}{|c|}{1989} & \multicolumn{2}{|c|}{1989} & \multicolumn{2}{|c|}{1990} & \multicolumn{2}{|c|}{1990} \\
\hline & & $18 \mathrm{Se}$ & ember & $18 \mathrm{Se}$ & ember & $16 \mathrm{Se}$ & ember & $16 \mathrm{Se}$ & tember \\
\hline Sta. & Elev. & Sta. & Elev. & Sta. & Elev. & Sta. & Elev. & Sta. & Elev. \\
\hline-20.0 & 987.41 & 43.0 & 984.93 & 93.0 & 986.39 & -1.2 & 987.47 & 59.0 & 985.59 \\
\hline-18.0 & 987.43 & 44.0 & 985.01 & 96.0 & 986.47 & 0.3 & 987.31 & 60.5 & 985.63 \\
\hline-16.0 & 987.37 & 46.0 & 984.99 & 97.0 & 986.54 & 2.0 & 987.34 & 61.3 & 985.78 \\
\hline-14.0 & 987.33 & 47.0 & 985.01 & 100.0 & 986.56 & 3.3 & 987.38 & 62.3 & 985.75 \\
\hline-12.0 & 987.28 & 47.2 & 985.16 & 103.0 & 986.61 & 4.3 & 986.02 & 62.8 & 985.80 \\
\hline-10.0 & 987.22 & 48.0 & 985.49 & 106.0 & 986.67 & 5.4 & 985.54 & 64.0 & 985.82 \\
\hline-8.0 & 987.18 & 48.1 & 985.81 & 109.0 & 986.72 & 7.0 & 985.34 & 65.0 & 985.86 \\
\hline-6.0 & 987.16 & 49.0 & 985.77 & 112.0 & 986.71 & 8.6 & 985.11 & 67.0 & 985.92 \\
\hline-4.0 & 987.17 & 50.3 & 985.68 & 115.0 & 986.74 & 9.7 & 984.93 & 69.0 & 986.01 \\
\hline-2.8 & 987.20 & 51.3 & 985.48 & 118.0 & 986.75 & 11.0 & 984.83 & 71.0 & 986.12 \\
\hline-1.2 & 987.48 & 53.0 & 985.45 & 121.0 & 986.85 & 13.0 & 984.69 & 73.0 & 986.15 \\
\hline 0.0 & 987.35 & 53.9 & 985.50 & 124.0 & 986.90 & 15.0 & 984.61 & 75.0 & 986.12 \\
\hline 1.0 & 987.31 & 54.1 & 985.62 & 127.0 & 986.93 & 16.6 & 984.82 & 76.3 & 986.09 \\
\hline 2.7 & 987.32 & 54.7 & 985.47 & 130.0 & 986.96 & 18.0 & 984.85 & 76.9 & 986.02 \\
\hline 3.4 & 987.38 & 56.0 & 985.43 & 133.0 & 987.01 & 20.0 & 984.82 & 78.5 & 986.04 \\
\hline 3.5 & 986.82 & 57.0 & 985.43 & 136.0 & 987.08 & 22.0 & 984.82 & 79.0 & 985.84 \\
\hline 3.8 & 986.54 & 58.0 & 985.38 & 139.0 & 987.11 & 24.0 & 984.81 & 80.3 & 985.83 \\
\hline 4.4 & 986.08 & 59.0 & 985.37 & 142.0 & 987.13 & 26.0 & 984.78 & 81.9 & 985.99 \\
\hline 4.9 & 985.73 & 61.0 & 985.49 & 145.0 & 987.16 & 28.0 & 984.83 & 82.1 & 986.20 \\
\hline 5.3 & 985.75 & 63.0 & 985.65 & 148.0 & 987.20 & 29.8 & 984.85 & 82.6 & 986.35 \\
\hline 5.5 & 985.53 & 65.0 & 985.64 & 150.0 & 987.23 & 31.9 & 985.12 & 83.0 & 986.41 \\
\hline 7.0 & 985.34 & 67.0 & 985.58 & 150.8 & 987.24 & 33.4 & 985.11 & 85.0 & 986.33 \\
\hline 8.3 & 985.18 & 68.4 & 985.63 & & & 35.0 & 985.09 & 86.0 & 986.29 \\
\hline 10.0 & 984.91 & 69.0 & 985.61 & & & 37.0 & 985.02 & 88.0 & 986.32 \\
\hline 12.0 & 984.87 & 71.0 & 985.73 & & & 37.7 & 984.94 & 90.0 & 986.37 \\
\hline 14.0 & 984.85 & 72.4 & 985.85 & & & 38.6 & 984.92 & 92.0 & 986.40 \\
\hline 16.0 & 984.92 & 73.8 & 985.82 & & & 39.0 & 985.12 & 94.0 & 986.41 \\
\hline 18.0 & 984.96 & 74.0 & 985.99 & & & 39.1 & 985.17 & 96.0 & 986.48 \\
\hline 20.0 & 984.99 & 74.9 & 985.79 & & & 40.5 & 985.24 & 98.0 & 986.58 \\
\hline 22.0 & 985.00 & 76.0 & 985.85 & & & 42.0 & 985.38 & 100.0 & 986.57 \\
\hline 24.0 & 985.01 & 77.0 & 985.84 & & & 43.8 & 985.41 & 102.0 & 986.62 \\
\hline 26.0 & 985.02 & 78.3 & 985.80 & & & 44.8 & 985.39 & 104.0 & 986.65 \\
\hline 28.0 & 985.07 & 78.8 & 985.69 & & & 46.0 & 985.51 & 106.0 & 986.66 \\
\hline 30.0 & 985.04 & 80.0 & 985.71 & & & 47.4 & 985.62 & 108.0 & 986.69 \\
\hline 32.0 & 985.05 & 80.6 & 985.79 & & & 49.5 & 985.60 & 110.0 & 986.73 \\
\hline 34.0 & 985.08 & 81.8 & 985.93 & & & 50.2 & 985.54 & 112.0 & 986.71 \\
\hline 36.0 & 985.05 & 82.0 & 986.16 & & & 51.0 & 985.52 & 114.0 & 986.75 \\
\hline 37.0 & 985.03 & 82.6 & 986.35 & & & 52.0 & 985.52 & 116.0 & 986.76 \\
\hline 38.0 & 984.86 & 83.0 & 986.37 & & & 54.0 & 985.54 & 118.0 & 986.75 \\
\hline 39.0 & 984.83 & 85.0 & 986.29 & & & 56.0 & 985.58 & 120.0 & 986.84 \\
\hline 41.0 & 984.85 & 87.0 & 986.29 & & & 57.0 & 985.55 & 122.0 & 986.85 \\
\hline 42.0 & 984.87 & 90.0 & 986.35 & & & 58.0 & 985.59 & 124.0 & 986.89 \\
\hline
\end{tabular}


Table 16. (Continued) Listing of horizontal stations and elevations for cross section PR141A

[Sta., station, distance in meters from a reference pin on the left bank; Elev., elevation, in meters above sea level]

\begin{tabular}{|c|c|c|c|c|c|c|c|c|c|}
\hline \multirow{2}{*}{\multicolumn{2}{|c|}{$\begin{array}{c}1990 \\
16 \text { September }\end{array}$}} & \multirow{2}{*}{\multicolumn{2}{|c|}{$\begin{array}{c}1991 \\
31 \text { August }\end{array}$}} & \multirow{2}{*}{\multicolumn{2}{|c|}{$\begin{array}{c}1991 \\
31 \text { August }\end{array}$}} & \multirow{2}{*}{\multicolumn{2}{|c|}{$\begin{array}{c}1991 \\
31 \text { August }\end{array}$}} & \multirow{2}{*}{\multicolumn{2}{|c|}{$\begin{array}{c}1992 \\
27 \text { August }\end{array}$}} \\
\hline & & & & & & & & & \\
\hline Sta. & Elev. & Sta. & Elev. & Sta. & Elev. & Sta. & Elev. & Sta. & Elev. \\
\hline 126.0 & 986.93 & -20.0 & 987.42 & 47.3 & 985.61 & 140.0 & 987.12 & -1.2 & 987.48 \\
\hline 128.0 & 986.95 & -18.0 & 987.44 & 47.5 & 985.45 & 145.0 & 987.14 & 0.0 & 987.36 \\
\hline 130.0 & 986.96 & -16.0 & 987.38 & 48.8 & 985.32 & 150.8 & 987.26 & 1.0 & 987.31 \\
\hline 133.0 & 987.02 & -14.0 & 987.34 & 51.0 & 985.41 & & & 2.4 & 987.29 \\
\hline \multirow[t]{38}{*}{136.0} & 987.08 & -12.0 & 987.29 & 52.0 & 985.43 & & & 2.4 & 986.12 \\
\hline & & -10.0 & 987.23 & 54.0 & 985.41 & & & 3.0 & 985.91 \\
\hline & & -8.0 & 987.19 & 56.0 & 985.34 & & & 3.4 & 985.60 \\
\hline & & -6.0 & 987.17 & 58.0 & 985.31 & & & 3.9 & 985.44 \\
\hline & & -4.0 & 987.18 & 59.3 & 985.33 & & & 4.3 & 985.19 \\
\hline & & -2.7 & 987.22 & 60.4 & 985.42 & & & 4.5 & 985.00 \\
\hline & & -1.2 & 987.48 & 61.0 & 985.54 & & & 5.0 & 984.97 \\
\hline & & 0.0 & 987.35 & 63.0 & 985.51 & & & 7.0 & 984.98 \\
\hline & & 1.0 & 987.31 & 66.0 & 985.61 & & & 9.0 & 984.88 \\
\hline & & 2.3 & 987.30 & 68.0 & 985.60 & & & 11.0 & 984.77 \\
\hline & & 2.3 & 986.34 & 69.8 & 985.59 & & & 13.0 & 984.72 \\
\hline & & 3.3 & 985.71 & 70.3 & 985.78 & & & 15.0 & 984.77 \\
\hline & & 4.2 & 985.30 & 70.9 & 985.82 & & & 17.0 & 984.79 \\
\hline & & 4.5 & 985.20 & 71.4 & 985.76 & & & 19.0 & 984.88 \\
\hline & & 4.8 & 985.29 & 72.0 & 985.93 & & & 21.0 & 984.92 \\
\hline & & 5.0 & 984.95 & 73.1 & 985.86 & & & 23.0 & 985.00 \\
\hline & & 7.0 & 984.98 & 74.0 & 985.98 & & & 25.0 & 985.04 \\
\hline & & 8.0 & 984.91 & 76.0 & 986.22 & & & 27.0 & 984.94 \\
\hline & & 10.0 & 984.85 & 77.6 & 986.26 & & & 29.0 & 984.95 \\
\hline & & 12.0 & 984.76 & 77.9 & 986.39 & & & 31.0 & 984.90 \\
\hline & & 14.0 & 984.82 & 81.0 & 986.39 & & & 33.0 & 984.95 \\
\hline & & 16.0 & 984.83 & 82.0 & 986.30 & & & 35.0 & 985.03 \\
\hline & & 18.0 & 984.83 & 83.0 & 986.45 & & & 36.9 & 985.19 \\
\hline & & 20.0 & 984.84 & 85.0 & 986.38 & & & 37.3 & 985.17 \\
\hline & & 21.7 & 984.85 & 88.0 & 986.35 & & & 38.2 & 985.19 \\
\hline & & 22.4 & 984.71 & 91.0 & 986.39 & & & 39.0 & 985.29 \\
\hline & & 24.2 & 984.91 & 94.0 & 986.40 & & & 40.4 & 985.41 \\
\hline & & 24.4 & 985.00 & 97.0 & 986.52 & & & 40.4 & 985.51 \\
\hline & & 26.0 & 985.10 & 100.0 & 986.56 & & & 42.0 & 985.53 \\
\hline & & 29.0 & 985.09 & 103.0 & 986.61 & & & 44.0 & 985.54 \\
\hline & & 32.0 & 985.13 & 106.0 & 986.64 & & & 46.0 & 985.55 \\
\hline & & 35.0 & 985.20 & 110.0 & 986.73 & & & 48.0 & 985.47 \\
\hline & & 37.0 & 985.21 & 114.0 & 986.72 & & & 50.0 & 985.45 \\
\hline & & 38.4 & 985.26 & 118.0 & 986.76 & & & 52.0 & 985.39 \\
\hline & & 40.0 & 985.49 & 122.0 & 986.83 & & & 54.0 & 985.38 \\
\hline & & 41.0 & 985.55 & 126.0 & 986.90 & & & 56.0 & 985.31 \\
\hline & & 43.0 & 985.62 & 130.0 & 986.96 & & & 58.0 & 985.38 \\
\hline & & 45.0 & 985.62 & 135.0 & 987.06 & & & 60.0 & 985.40 \\
\hline
\end{tabular}


Table 16. (Continued) Listing of horizontal stations and elevations for cross section PR141A

[Sta., station, distance in meters from a reference pin on the left bank; Elev., elevation, in meters above sea level]

\begin{tabular}{|c|c|c|c|c|c|c|c|c|c|}
\hline \multirow{2}{*}{\multicolumn{2}{|c|}{$\begin{array}{c}1992 \\
27 \text { August }\end{array}$}} & \multirow{2}{*}{\multicolumn{2}{|c|}{$\begin{array}{c}1993 \\
28 \text { August }\end{array}$}} & \multirow{2}{*}{\multicolumn{2}{|c|}{$\begin{array}{c}1993 \\
28 \text { August }\end{array}$}} & \multirow{2}{*}{\multicolumn{2}{|c|}{$\begin{array}{c}1993 \\
28 \text { August }\end{array}$}} & \multirow{2}{*}{\multicolumn{2}{|c|}{$\begin{array}{c}1994 \\
23 \text { September }\end{array}$}} \\
\hline & & & & & & & & & \\
\hline Sta. & Elev. & Sta. & Elev. & Sta. & Elev. & Sta. & Elev. & Sta. & Elev. \\
\hline 62.0 & 985.44 & -20.0 & 987.43 & 42.0 & 985.26 & 101.0 & 986.61 & -40.0 & 987.26 \\
\hline 64.0 & 985.54 & -19.0 & 987.42 & 43.0 & 985.26 & 103.0 & 986.65 & -39.7 & 987.43 \\
\hline 65.6 & 985.55 & -17.0 & 987.44 & 45.0 & 985.33 & 105.0 & 986.68 & -39.3 & 987.27 \\
\hline 67.0 & 985.56 & -15.0 & 987.39 & 46.0 & 985.35 & 107.0 & 986.70 & -38.0 & 987.26 \\
\hline 69.0 & 985.64 & -13.0 & 987.34 & 48.0 & 985.43 & 109.0 & 986.73 & -37.6 & 987.39 \\
\hline 70.4 & 985.76 & -11.0 & 987.26 & 50.0 & 985.44 & 111.0 & 986.73 & -37.1 & 987.25 \\
\hline 71.1 & 985.95 & -9.0 & 987.20 & 52.0 & 985.51 & 113.0 & 986.74 & -36.4 & 987.27 \\
\hline 72.0 & 985.99 & -7.0 & 987.15 & 53.7 & 985.47 & 115.0 & 986.77 & -35.8 & 987.38 \\
\hline 74.0 & 986.08 & -5.0 & 987.20 & 55.7 & 985.60 & 117.0 & 986.78 & -35.3 & 987.31 \\
\hline 76.0 & 986.20 & -3.0 & 987.19 & 58.0 & 985.56 & 119.0 & 986.78 & -34.3 & 987.35 \\
\hline 77.4 & 986.24 & -2.0 & 987.38 & 60.0 & 985.58 & 120.0 & 986.84 & -33.5 & 987.26 \\
\hline 78.0 & 986.37 & -1.2 & 987.48 & 62.0 & 985.58 & 122.0 & 986.85 & -33.0 & 987.34 \\
\hline 80.0 & 986.35 & 0.0 & 987.36 & 64.0 & 985.56 & 124.0 & 986.90 & -32.0 & 987.38 \\
\hline 81.3 & 986.40 & 0.4 & 987.30 & 66.0 & 985.57 & 126.0 & 986.94 & -31.0 & 987.42 \\
\hline 82.0 & 986.32 & 0.5 & 986.52 & 68.0 & 985.57 & 128.0 & 986.95 & -30.4 & 987.52 \\
\hline 82.6 & 986.44 & 1.2 & 985.36 & 69.0 & 985.55 & 130.0 & 986.96 & -29.6 & 987.37 \\
\hline 84.0 & 986.42 & 1.3 & 985.79 & 69.6 & 985.60 & 150.8 & 987.25 & -29.0 & 987.40 \\
\hline 86.0 & 986.35 & 2.0 & 985.38 & 70.5 & 985.93 & & & -28.7 & 987.49 \\
\hline 88.0 & 986.35 & 2.5 & 985.13 & 71.4 & 985.94 & & & -28.1 & 987.37 \\
\hline 90.0 & 986.38 & 3.5 & 985.19 & 71.8 & 986.01 & & & -27.7 & 987.43 \\
\hline 92.0 & 986.41 & 5.0 & 985.35 & 72.2 & 986.26 & & & -26.7 & 987.53 \\
\hline 95.0 & 986.43 & 6.6 & 985.40 & 72.8 & 986.22 & & & -26.3 & 987.63 \\
\hline 97.0 & 986.53 & 6.8 & 985.62 & 73.4 & 986.20 & & & -25.6 & 987.67 \\
\hline 98.0 & 986.57 & 7.3 & 985.54 & 74.0 & 986.25 & & & -25.1 & 987.74 \\
\hline 100.0 & 986.57 & 8.0 & 985.11 & 75.0 & 986.36 & & & -24.3 & 987.70 \\
\hline 103.0 & 986.62 & 12.3 & 985.04 & 76.0 & 986.50 & & & -23.4 & 987.65 \\
\hline 106.0 & 986.65 & 13.3 & 984.55 & 77.0 & 986.63 & & & -23.0 & 987.82 \\
\hline 109.0 & 986.72 & 15.0 & 984.57 & 78.0 & 986.66 & & & -22.0 & 987.83 \\
\hline 112.0 & 986.71 & 17.0 & 984.59 & 79.0 & 986.66 & & & -21.3 & 987.64 \\
\hline 115.0 & 986.75 & 18.0 & 984.63 & 80.0 & 986.64 & & & -21.0 & 987.61 \\
\hline 118.0 & 986.75 & 19.0 & 984.81 & 81.0 & 986.62 & & & -20.3 & 987.53 \\
\hline 121.0 & 986.85 & 21.0 & 984.85 & 82.0 & 986.53 & & & -20.0 & 987.60 \\
\hline 124.0 & 986.89 & 23.0 & 984.98 & 83.0 & 986.57 & & & -19.5 & 987.74 \\
\hline 127.0 & 986.93 & 25.0 & 985.10 & 84.0 & 986.54 & & & -19.0 & 987.63 \\
\hline 130.0 & 986.96 & 27.0 & 985.20 & 85.0 & 986.50 & & & -18.3 & 987.51 \\
\hline 135.0 & 987.01 & 28.0 & 985.13 & 87.0 & 986.47 & & & -17.2 & 987.66 \\
\hline 140.0 & 987.11 & 30.0 & 985.13 & 89.0 & 986.47 & & & -17.0 & 987.60 \\
\hline 145.0 & 987.16 & 32.0 & 985.15 & 91.0 & 986.48 & & & -16.6 & 987.54 \\
\hline 150.0 & 987.23 & 34.0 & 985.14 & 93.0 & 986.48 & & & -15.0 & 987.51 \\
\hline \multirow[t]{3}{*}{150.8} & 987.26 & 36.0 & 985.14 & 95.0 & 986.48 & & & -14.4 & 987.52 \\
\hline & & 38.0 & 985.19 & 97.0 & 986.59 & & & -13.8 & 987.59 \\
\hline & & 40.0 & 985.20 & 99.0 & 986.62 & & & -13.0 & 987.59 \\
\hline
\end{tabular}


Table 16. (Continued) Listing of horizontal stations and elevations for cross section PR141A

[Sta., station, distance in meters from a reference pin on the left bank; Elev., elevation, in meters above sea level]

\begin{tabular}{|c|c|c|c|c|c|c|c|c|c|}
\hline \multirow{2}{*}{\multicolumn{2}{|c|}{$\begin{array}{c}1994 \\
23 \text { September }\end{array}$}} & \multicolumn{2}{|c|}{1994} & \multicolumn{2}{|c|}{1994} & \multicolumn{2}{|c|}{1995} & \multicolumn{2}{|c|}{1995} \\
\hline & & $23 \mathrm{Se}$ & tember & $23 \mathrm{~S}$ & ember & $2 \mathrm{C}$ & ober & 20 & ober \\
\hline Sta. & Elev. & Sta. & Elev. & Sta. & Elev. & Sta. & Elev. & Sta. & Elev. \\
\hline-12.0 & 987.47 & 50.0 & 985.51 & 128.0 & 986.98 & -40.0 & 987.27 & 19.0 & 985.36 \\
\hline-11.0 & 987.44 & 52.0 & 985.41 & 130.0 & 987.04 & -39.0 & 987.28 & 20.3 & 985.76 \\
\hline-10.0 & 987.43 & 55.0 & 985.48 & 131.5 & 987.03 & -38.0 & 987.28 & 22.0 & 985.75 \\
\hline-9.0 & 987.50 & 58.0 & 985.57 & 133.2 & 987.11 & -37.0 & 987.26 & 24.5 & 986.01 \\
\hline-8.0 & 987.50 & 59.5 & 985.63 & 133.7 & 987.24 & -36.0 & 987.35 & 26.0 & 986.04 \\
\hline-7.0 & 987.34 & 61.6 & 985.82 & 134.2 & 987.12 & -34.0 & 987.30 & 28.0 & 986.07 \\
\hline-6.6 & 987.28 & 64.0 & 985.90 & 134.7 & 987.14 & -33.0 & 987.31 & 30.0 & 986.04 \\
\hline-5.0 & 987.28 & 66.0 & 985.89 & 135.2 & 987.29 & -31.0 & 987.44 & 32.0 & 986.11 \\
\hline-3.5 & 987.23 & 68.5 & 985.95 & 135.7 & 987.16 & -30.0 & 987.42 & 34.0 & 986.20 \\
\hline-3.0 & 987.25 & 69.0 & 985.85 & 137.4 & 987.11 & -29.0 & 987.42 & 36.0 & 986.25 \\
\hline-2.0 & 987.42 & 69.6 & 985.87 & 138.3 & 987.25 & -28.0 & 987.39 & 38.0 & 986.30 \\
\hline-1.2 & 987.47 & 70.7 & 986.11 & 140.3 & 987.27 & -27.0 & 987.46 & 40.0 & 986.40 \\
\hline-1.0 & 987.35 & 71.6 & 986.14 & 140.5 & 987.37 & -26.0 & 987.63 & 42.0 & 986.43 \\
\hline-1.0 & 986.64 & 72.4 & 986.38 & 141.0 & 987.23 & -24.0 & 987.72 & 44.0 & 986.38 \\
\hline-0.5 & 986.88 & 74.0 & 986.39 & 143.0 & 987.17 & -22.3 & 987.79 & 45.0 & 986.37 \\
\hline-0.1 & 986.35 & 75.5 & 986.50 & 144.4 & 987.31 & -21.0 & 987.62 & 45.5 & 986.30 \\
\hline 0.1 & 986.28 & 77.0 & 986.69 & 145.3 & 987.24 & -20.0 & 987.64 & 47.0 & 985.84 \\
\hline 0.3 & 985.83 & 77.4 & 986.66 & 147.5 & 987.29 & -19.0 & 987.66 & 48.0 & 985.67 \\
\hline 1.3 & 985.45 & 79.0 & 986.77 & 149.0 & 987.27 & -18.0 & 987.57 & 50.5 & 985.51 \\
\hline 1.6 & 985.23 & 81.0 & 986.73 & 150.8 & 987.30 & -15.8 & 987.51 & 52.0 & 985.60 \\
\hline 2.6 & 985.08 & 82.0 & 986.68 & 153.0 & 987.38 & -15.4 & 986.36 & 53.0 & 985.73 \\
\hline 5.0 & 984.84 & 84.0 & 986.62 & 154.5 & 987.35 & -15.0 & 986.05 & 55.0 & 985.83 \\
\hline 8.0 & 984.75 & 85.0 & 986.57 & 155.8 & 987.37 & -13.8 & 985.64 & 56.0 & 985.73 \\
\hline 10.0 & 984.69 & 86.0 & 986.49 & 157.0 & 987.33 & -13.6 & 985.38 & 57.0 & 985.69 \\
\hline 13.0 & 984.72 & 88.0 & 986.52 & 158.3 & 987.37 & -12.0 & 984.74 & 59.0 & 985.63 \\
\hline 16.0 & 984.79 & 89.0 & 986.57 & 158.7 & 987.46 & -11.0 & 984.63 & 60.0 & 985.63 \\
\hline 18.0 & 984.96 & 91.0 & 986.50 & 159.5 & 987.42 & -10.0 & 984.63 & 62.0 & 985.78 \\
\hline 20.0 & 984.98 & 93.0 & 986.59 & 162.0 & 987.44 & -9.0 & 984.61 & 63.0 & 985.91 \\
\hline 22.0 & 985.04 & 95.0 & 986.54 & 162.5 & 987.49 & -8.0 & 984.69 & 64.0 & 985.96 \\
\hline 23.0 & 985.07 & 98.0 & 986.66 & 163.3 & 987.39 & -6.0 & 984.68 & 65.0 & 986.06 \\
\hline 24.3 & 985.13 & 100.5 & 986.64 & 166.0 & 987.39 & -4.0 & 984.85 & 67.0 & 986.10 \\
\hline 24.6 & 985.26 & 103.0 & 986.68 & 169.0 & 987.38 & -2.0 & 984.97 & 69.0 & 986.14 \\
\hline 26.0 & 985.31 & 106.0 & 986.74 & 171.0 & 987.36 & 0.0 & 984.82 & 71.0 & 986.23 \\
\hline 29.0 & 985.35 & 109.0 & 986.77 & 173.0 & 987.30 & 2.0 & 984.83 & 72.0 & 986.34 \\
\hline 30.8 & 985.37 & 112.0 & 986.78 & 176.0 & 987.28 & 4.0 & 985.06 & 73.5 & 986.58 \\
\hline 31.4 & 985.31 & 114.0 & 986.80 & 179.0 & 987.17 & 6.0 & 985.14 & 75.0 & 986.67 \\
\hline 34.0 & 985.33 & 115.4 & 986.85 & 181.0 & 987.15 & 8.0 & 985.15 & 76.7 & 986.99 \\
\hline 37.0 & 985.39 & 116.5 & 986.82 & 182.8 & 987.15 & 10.0 & 985.13 & 78.0 & 987.04 \\
\hline 40.0 & 985.44 & 119.0 & 986.85 & 185.0 & 987.42 & 12.0 & 985.17 & 80.0 & 987.08 \\
\hline 43.0 & 985.50 & 122.0 & 986.94 & 188.5 & 987.58 & 14.0 & 985.12 & 82.0 & 987.05 \\
\hline 46.0 & 985.47 & 124.0 & 987.01 & 190.3 & 987.84 & 16.0 & 985.21 & 84.0 & 986.98 \\
\hline 49.0 & 985.48 & 126.0 & 986.98 & & & 18.0 & 985.18 & 86.0 & 986.84 \\
\hline
\end{tabular}


Table 16. (Continued) Listing of horizontal stations and elevations for cross section PR141A

[Sta., station, distance in meters from a reference pin on the left bank; Elev., elevation, in meters above sea level]

\begin{tabular}{|c|c|c|c|c|c|c|c|c|c|}
\hline \multirow{2}{*}{\multicolumn{2}{|c|}{$\begin{array}{c}1995 \\
2 \text { October }\end{array}$}} & \multirow{2}{*}{\multicolumn{2}{|c|}{$\begin{array}{c}1996 \\
18 \text { October }\end{array}$}} & \multirow{2}{*}{\multicolumn{2}{|c|}{$\begin{array}{c}1996 \\
18 \text { October }\end{array}$}} & \multirow{2}{*}{\multicolumn{2}{|c|}{$\begin{array}{c}1996 \\
18 \text { October }\end{array}$}} & \multicolumn{2}{|c|}{1997} \\
\hline & & & & & & & & $20 \mathrm{Se}$ & ember \\
\hline Sta. & Elev. & Sta. & Elev. & Sta. & Elev. & Sta. & Elev. & Sta. & Elev. \\
\hline 88.0 & 986.81 & -60.0 & 986.77 & 25.0 & 986.13 & 108.0 & 986.88 & -60.0 & 986.79 \\
\hline 90.0 & 986.74 & -57.0 & 986.90 & 27.0 & 986.22 & 110.0 & 986.91 & -40.6 & 987.33 \\
\hline 92.0 & 986.75 & -55.0 & 987.02 & 28.0 & 986.35 & & & -40.0 & 987.30 \\
\hline 94.0 & 986.70 & -52.0 & 987.00 & 30.0 & 986.33 & & & -39.0 & 987.27 \\
\hline 96.0 & 986.71 & -49.0 & 987.19 & 33.0 & 986.37 & & & -37.0 & 987.28 \\
\hline 98.0 & 986.75 & -46.0 & 987.30 & 36.0 & 986.47 & & & -35.5 & 987.31 \\
\hline 100.0 & 986.76 & -43.0 & 987.29 & 39.0 & 986.50 & & & -33.0 & 987.32 \\
\hline 102.0 & 986.79 & -40.6 & 987.32 & 41.0 & 986.50 & & & -31.0 & 987.44 \\
\hline 104.0 & 986.83 & -40.0 & 987.30 & 42.7 & 986.49 & & & -30.0 & 987.43 \\
\hline 106.0 & 986.85 & -37.0 & 987.28 & 44.4 & 985.80 & & & -29.4 & 987.39 \\
\hline 108.0 & 986.88 & -34.0 & 987.31 & 45.2 & 985.70 & & & -28.5 & 987.37 \\
\hline 110.0 & 986.89 & -31.0 & 987.43 & 47.0 & 985.71 & & & -28.4 & 986.36 \\
\hline 112.0 & 986.91 & -28.0 & 987.40 & 50.0 & 985.72 & & & -27.7 & 985.92 \\
\hline 114.0 & 986.90 & -26.0 & 987.63 & 53.0 & 985.85 & & & -26.7 & 985.44 \\
\hline 116.0 & 986.89 & -24.0 & 987.70 & 56.0 & 985.92 & & & -25.8 & 985.20 \\
\hline 118.0 & 986.89 & -23.1 & 987.74 & 58.7 & 985.96 & & & -24.9 & 984.79 \\
\hline 119.0 & 986.94 & -22.7 & 986.43 & 60.0 & 985.91 & & & -23.0 & 984.98 \\
\hline 122.0 & 986.97 & -21.9 & 985.62 & 63.0 & 985.95 & & & -21.0 & 984.95 \\
\hline 124.0 & 986.99 & -20.8 & 985.19 & 65.0 & 985.96 & & & -19.0 & 984.89 \\
\hline 126.0 & 987.00 & -19.6 & 984.79 & 67.0 & 986.00 & & & -16.0 & 984.67 \\
\hline 128.0 & 987.00 & -18.3 & 984.61 & 69.0 & 985.89 & & & -15.0 & 984.61 \\
\hline 130.0 & 987.03 & -16.0 & 984.57 & 70.0 & 985.74 & & & -12.0 & 984.58 \\
\hline 150.8 & 987.30 & -13.0 & 984.65 & 70.5 & 985.93 & & & -9.0 & 984.86 \\
\hline 190.3 & 988.02 & -11.0 & 984.69 & 71.0 & 986.20 & & & -6.0 & 984.94 \\
\hline & & -9.0 & 984.79 & 72.0 & 986.38 & & & -3.0 & 984.99 \\
\hline & & -6.0 & 984.87 & 73.0 & 986.47 & & & 0.0 & 985.05 \\
\hline & & -4.0 & 984.99 & 74.0 & 986.65 & & & 1.0 & 984.86 \\
\hline & & -2.0 & 985.06 & 75.0 & 986.68 & & & 2.0 & 984.82 \\
\hline & & 0.0 & 985.11 & 76.0 & 986.84 & & & 3.4 & 985.20 \\
\hline & & 3.0 & 985.08 & 77.0 & 986.96 & & & 3.7 & 985.27 \\
\hline & & 6.0 & 985.04 & 78.0 & 987.05 & & & 3.8 & 985.45 \\
\hline & & 7.3 & 985.18 & 80.0 & 987.08 & & & 6.0 & 985.44 \\
\hline & & 9.0 & 985.34 & 82.0 & 987.05 & & & 8.0 & 985.47 \\
\hline & & 11.0 & 985.41 & 84.0 & 986.99 & & & 10.4 & 985.43 \\
\hline & & 14.0 & 985.45 & 86.0 & 986.84 & & & 11.2 & 985.56 \\
\hline & & 16.0 & 985.42 & 88.0 & 986.81 & & & 11.3 & 985.71 \\
\hline & & 18.0 & 985.36 & 91.0 & 986.73 & & & 12.5 & 985.67 \\
\hline & & 19.5 & 985.58 & 94.0 & 986.69 & & & 14.0 & 985.70 \\
\hline & & 20.5 & 985.79 & 97.0 & 986.72 & & & 17.0 & 985.77 \\
\hline & & 21.2 & 986.06 & 100.0 & 986.75 & & & 20.0 & 985.80 \\
\hline & & 22.0 & 986.10 & 103.0 & 986.76 & & & 23.0 & 985.71 \\
\hline & & 23.0 & 986.04 & 106.0 & 986.87 & & & 26.0 & 985.70 \\
\hline
\end{tabular}


Table 16. (Continued) Listing of horizontal stations and elevations for cross section PR141A

[Sta., station, distance in meters from a reference pin on the left bank; Elev., elevation, in meters above sea level]

\begin{tabular}{|c|c|c|c|c|c|}
\hline \multirow{2}{*}{\multicolumn{2}{|c|}{$\begin{array}{c}1997 \\
20 \text { September }\end{array}$}} & \multicolumn{2}{|c|}{1998} & \multicolumn{2}{|c|}{1998} \\
\hline & & \multicolumn{2}{|c|}{26 September } & \multicolumn{2}{|c|}{26 September } \\
\hline Sta. & Elev. & Sta. & Elev. & Sta. & Elev. \\
\hline 27.0 & 985.77 & -60.0 & 986.80 & 30.0 & 986.30 \\
\hline 27.3 & 985.82 & -55.0 & 987.00 & 33.0 & 986.35 \\
\hline 28.3 & 986.27 & -50.0 & 987.02 & 36.0 & 986.41 \\
\hline 30.0 & 986.38 & -45.0 & 987.24 & 38.0 & 986.50 \\
\hline 33.0 & 986.38 & -40.6 & 987.32 & 40.0 & 986.47 \\
\hline 36.0 & 986.44 & -40.0 & 987.29 & 42.0 & 986.10 \\
\hline 39.0 & 986.48 & -38.0 & 987.30 & 44.0 & 985.78 \\
\hline 40.4 & 986.51 & -36.0 & 987.32 & 45.4 & 985.72 \\
\hline 41.4 & 986.15 & -33.2 & 987.29 & 48.0 & 985.82 \\
\hline 44.0 & 985.71 & -31.4 & 987.40 & 51.0 & 985.88 \\
\hline 45.5 & 985.57 & -31.2 & 986.70 & 54.0 & 985.93 \\
\hline 47.0 & 985.68 & -30.9 & 986.19 & 57.0 & 985.98 \\
\hline 50.0 & 985.77 & -29.8 & 985.46 & 60.0 & 985.98 \\
\hline 53.0 & 985.85 & -29.0 & 985.18 & 63.0 & 986.03 \\
\hline 56.0 & 985.91 & -27.0 & 985.10 & 66.0 & 986.08 \\
\hline 59.0 & 985.92 & -25.0 & 985.00 & 68.0 & 986.14 \\
\hline 62.0 & 985.99 & -23.0 & 985.00 & 70.0 & 986.09 \\
\hline 65.0 & 986.04 & -21.0 & 984.95 & 71.0 & 986.23 \\
\hline 68.0 & 986.14 & -19.0 & 984.82 & 72.0 & 986.43 \\
\hline 70.0 & 986.08 & -17.0 & 984.77 & 73.0 & 986.50 \\
\hline 72.0 & 986.40 & -15.0 & 984.71 & 75.0 & 986.70 \\
\hline 74.0 & 986.66 & -13.0 & 984.62 & 77.0 & 986.99 \\
\hline 76.0 & 986.83 & -11.0 & 984.77 & 80.0 & 987.08 \\
\hline 77.6 & 987.03 & -9.0 & 984.68 & 83.0 & 987.02 \\
\hline 80.0 & 987.08 & -7.0 & 984.98 & 86.0 & 986.83 \\
\hline 82.0 & 987.07 & -5.0 & 985.03 & 89.0 & 986.79 \\
\hline 84.0 & 986.99 & -3.0 & 985.22 & 92.0 & 986.75 \\
\hline 86.0 & 986.84 & -1.0 & 985.22 & 95.0 & 986.69 \\
\hline 89.0 & 986.79 & 1.0 & 985.29 & 98.0 & 986.75 \\
\hline 92.0 & 986.74 & 3.0 & 985.20 & 101.0 & 986.75 \\
\hline 95.0 & 986.69 & 5.0 & 985.16 & 104.0 & 986.81 \\
\hline 98.0 & 986.75 & 7.0 & 985.19 & 107.0 & 986.87 \\
\hline 101.0 & 986.75 & 9.0 & 985.26 & 110.0 & 986.91 \\
\hline 104.0 & 986.82 & 10.2 & 985.48 & 130.0 & 987.04 \\
\hline 107.0 & 986.89 & 12.0 & 985.51 & 150.0 & 987.30 \\
\hline 110.0 & 986.92 & 14.0 & 985.72 & 190.0 & 987.84 \\
\hline \multirow[t]{6}{*}{130.0} & 987.04 & 16.5 & 985.75 & & \\
\hline & & 18.0 & 985.66 & & \\
\hline & & 21.0 & 985.68 & & \\
\hline & & 24.0 & 985.82 & & \\
\hline & & 26.3 & 985.79 & & \\
\hline & & 28.5 & 986.41 & & \\
\hline
\end{tabular}




\section{Description of Cross Section PR141.7}

Location: Township 7 South/Range 49 East--section 32

U. S. Geological Survey quadrangle (1:24,000): Huckins School

Landowners--left bank: Bowers Ranch

--right bank: U. S. Government

Access: Left bank

Permission from: Gay Ranch

Distance from Moorhead Gaging Station: 26.9 kilometers

Azimuth of Section (degrees magnetic): 240.5

\section{Reference Monuments}

[Monuments at stations $-29.0,-28.95,0.25$, and 1.0 were closest to leveling instrument]

\begin{tabular}{|c|c|c|c|c|c|c|}
\hline \multirow[b]{2}{*}{ Description } & \multirow[b]{2}{*}{$\begin{array}{c}\text { Station } \\
(m)\end{array}$} & \multicolumn{2}{|c|}{ GPS-NAD83 (1992) } & \multicolumn{2}{|c|}{ Measurement } & \multirow{2}{*}{$\begin{array}{l}\text { Elevation } \\
\text { (NGVD1929) } \\
\text { (m) }\end{array}$} \\
\hline & & Latitude & Longitude & $\begin{array}{l}\text { Standard } \\
\text { deviation } \\
(\mathbf{m})\end{array}$ & $\begin{array}{l}\text { Horizontal } \\
\text { precision } \\
\text { (m) }\end{array}$ & \\
\hline $\begin{array}{l}\text { 1/2-inch-rebar; } 0.14 \text { meter above } 1998 \text { ground } \\
\text { level; upstream from a group of buffa- } \\
\text { loberry trees and } 0.05 \text { meter riverward of } \\
\text { wooden stake }\end{array}$ & -29.0 & $45^{\circ} 11^{\prime} 20.20^{\prime \prime}$ & $105^{\circ} 44^{\prime} 44.86^{\prime \prime}$ & 0.527 & 0.759 & 987.34 \\
\hline $\begin{array}{l}\text { Benchmark--1/2-inch-rebar; } 0.18 \text { meter above } \\
1998 \text { ground level }\end{array}$ & 0.25 & & & & & 986.82 \\
\hline $\begin{array}{l}\text { 1/2-inch-rebar; } 0.10 \text { meter above } 1998 \text { ground } \\
\text { level }\end{array}$ & 1.0 & & & & & 986.65 \\
\hline $\begin{array}{l}\text { 1/2-inch-rebar; } 0.16 \text { meter above } 1998 \text { ground } \\
\text { level; } 2 \text { meters upstream from large soli- } \\
\text { tary cottonwood tree near the right bank }\end{array}$ & 149.1 & $45^{\circ} 11^{\prime} 18.74^{\prime \prime}$ & $105^{\circ} 44^{\prime} 52.74^{\prime \prime}$ & 0.590 & 0.534 & 988.62 \\
\hline
\end{tabular}



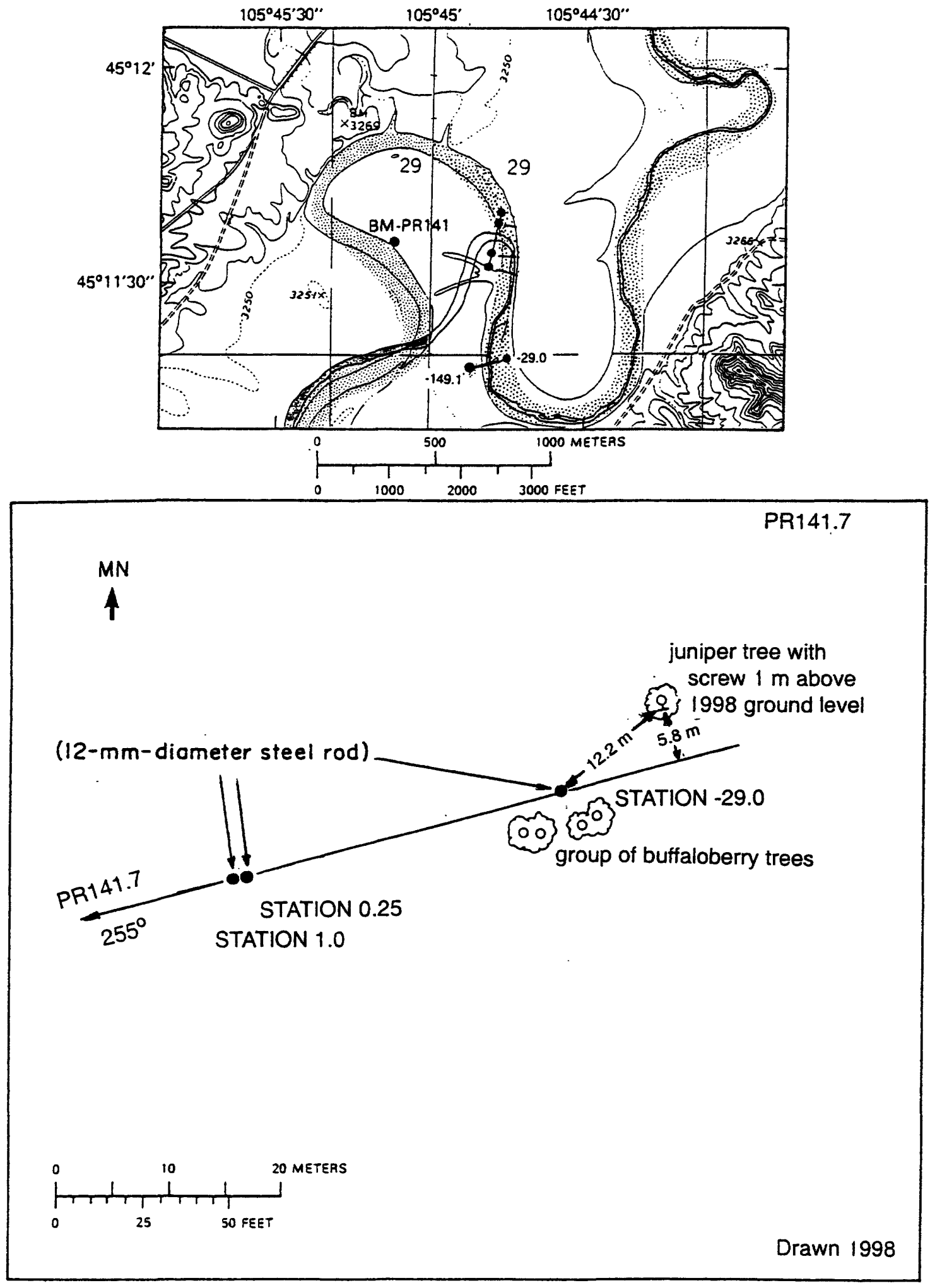

Figure 44. Upper: Location of cross section PR141.7, bench mark BM-PR141, and the left and right bank reference monuments of PR141.7 in the Huckins School quadrangle. Lower: Location of the reference monuments on the left bank. MN is magnetic north. 

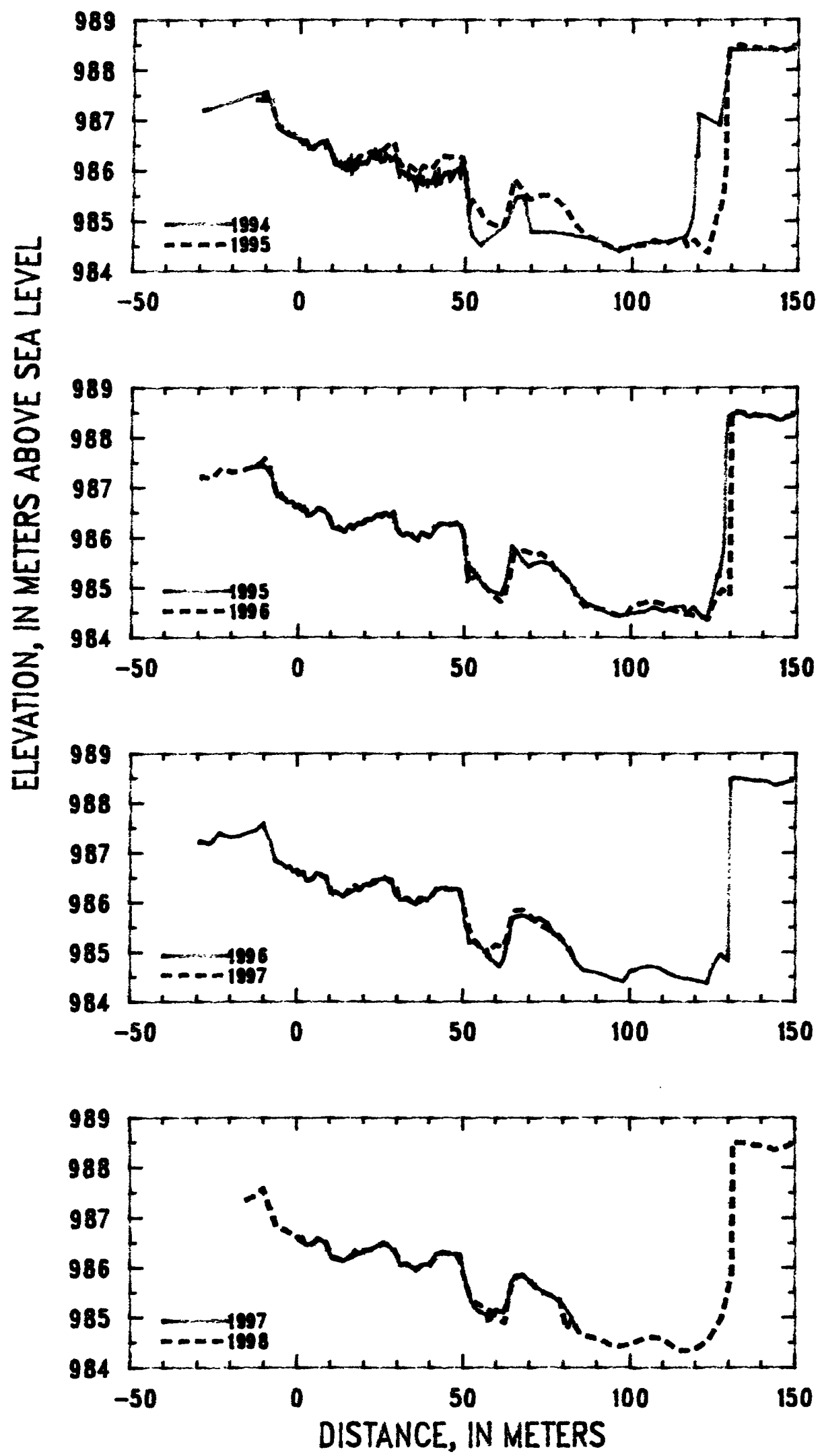

Figure 45. Profiles of cross section PR141.7 from 1994 to 1998. 
Table 17. Listing of horizontal stations and elevations for cross section PR141.7

[Sta., station, distance in meters from a reference pin on the left bank; Elev., elevation, in meters above sea level]

\begin{tabular}{|c|c|c|c|c|c|c|c|c|c|}
\hline \multirow{2}{*}{\multicolumn{2}{|c|}{$\begin{array}{c}1993 \\
\text { see note below }\end{array}$}} & \multirow{2}{*}{\multicolumn{2}{|c|}{$\begin{array}{c}1994 \\
3 \text { September }\end{array}$}} & \multirow{2}{*}{\multicolumn{2}{|c|}{$\begin{array}{c}1994 \\
3 \text { September }\end{array}$}} & \multirow{2}{*}{\multicolumn{2}{|c|}{$\begin{array}{c}1994 \\
3 \text { September }\end{array}$}} & \multirow{2}{*}{\multicolumn{2}{|c|}{$\begin{array}{c}1994 \\
3 \text { September }\end{array}$}} \\
\hline & & & & & & & & & \\
\hline Sta. & Elev. & Sta. & Elev. & Sta. & Elev. & Sta. & Elev. & Sta. & Elev. \\
\hline 0.0 & 985.79 & -29.0 & 987.20 & 17.1 & 986.07 & 32.2 & 985.90 & 42.5 & 985.74 \\
\hline 2.6 & 985.71 & -10.0 & 987.57 & 17.3 & 986.14 & 32.4 & 985.89 & 42.7 & 985.80 \\
\hline 6.8 & 985.66 & -6.0 & 986.81 & 17.5 & 986.25 & 32.6 & 985.94 & 42.9 & 985.85 \\
\hline 12.2 & 985.54 & -3.3 & 986.69 & 18.1 & 986.19 & 32.8 & 985.85 & 43.2 & 985.88 \\
\hline 19.2 & 985.75 & 0.3 & 986.63 & 18.6 & 986.13 & 33.0 & 985.89 & 43.5 & 985.91 \\
\hline 25.0 & 986.01 & 1.0 & 986.54 & 19.5 & 986.16 & 33.2 & 985.92 & 43.7 & 985.97 \\
\hline 30.3 & 986.00 & 1.1 & 986.59 & 20.0 & 986.14 & 33.3 & 985.86 & 43.8 & 985.94 \\
\hline 33.3 & 985.99 & 2.0 & 986.60 & 20.6 & 986.23 & 33.6 & 985.84 & 44.2 & 985.93 \\
\hline 35.4 & 985.86 & 2.9 & 986.45 & 20.8 & 986.28 & 33.8 & 985.89 & 44.5 & 985.96 \\
\hline 37.4 & 985.96 & 3.9 & 986.41 & 21.1 & 986.28 & 33.9 & 985.86 & 44.6 & 985.96 \\
\hline 39.7 & 986.03 & 4.6 & 986.45 & 21.3 & 986.27 & 34.1 & 985.88 & 44.8 & 985.90 \\
\hline 40.7 & 986.28 & 5.1 & 986.50 & 21.4 & 986.34 & 34.4 & 985.84 & 45.0 & 985.93 \\
\hline 42.6 & 986.49 & 5.9 & 986.53 & 21.9 & 986.34 & 34.9 & 985.79 & 45.1 & 985.98 \\
\hline 44.5 & 986.42 & 6.2 & 986.56 & 22.1 & 986.25 & 35.1 & 985.68 & 45.3 & 985.97 \\
\hline 46.0 & 986.31 & 6.8 & 986.56 & 22.5 & 986.25 & 35.3 & 985.74 & 45.5 & 985.80 \\
\hline 48.5 & 986.43 & 7.6 & 986.58 & 22.9 & 986.24 & 35.6 & 985.82 & 45.7 & 985.82 \\
\hline \multicolumn{2}{|c|}{ depths to surface } & 7.9 & 986.49 & 23.3 & 986.31 & 35.7 & 985.78 & 46.3 & 985.92 \\
\hline \multicolumn{2}{|c|}{ measured on } & 8.2 & 986.56 & 23.9 & 986.38 & 36.0 & 985.77 & 46.7 & 985.99 \\
\hline \multirow{4}{*}{\multicolumn{2}{|c|}{3 September 1994}} & 8.4 & 986.50 & 24.2 & 986.17 & 36.2 & 985.88 & 46.9 & 985.98 \\
\hline & & 8.8 & 986.49 & 24.7 & 986.18 & 36.5 & 985.78 & 47.4 & 985.99 \\
\hline & & 9.4 & 986.41 & 25.0 & 986.21 & 36.7 & 985.74 & 47.6 & 986.02 \\
\hline & & 10.0 & 986.31 & 25.4 & 986.20 & 37.2 & 985.72 & 47.6 & 986.05 \\
\hline \multicolumn{2}{|c|}{. } & 10.3 & 986.18 & 25.6 & 986.33 & 37.3 & 985.80 & 47.7 & 986.03 \\
\hline & & 10.9 & 986.11 & 26.3 & 986.27 & 37.5 & 985.75 & 47.8 & 986.06 \\
\hline & & 11.8 & 986.11 & 26.6 & 986.28 & 37.8 & 985.74 & 47.9 & 986.01 \\
\hline & & 12.1 & 986.15 & 26.8 & 986.26 & 38.2 & 985.77 & 48.0 & 986.06 \\
\hline & & 12.2 & 986.09 & 27.2 & 986.20 & 38.5 & 985.79 & 48.1 & 986.03 \\
\hline & & 12.6 & 986.11 & 27.6 & 986.24 & 38.7 & 985.73 & 48.3 & 985.96 \\
\hline & & 12.7 & 986.15 & 28.1 & 986.22 & 39.0 & 985.79 & 48.4 & 986.03 \\
\hline & & 12.8 & 986.05 & 28.3 & 986.27 & 39.1 & 985.79 & 48.5 & 986.02 \\
\hline & & 13.2 & 986.08 & 28.6 & 986.30 & 39.3 & 985.76 & 48.7 & 986.15 \\
\hline & & 13.6 & 986.06 & 28.9 & 986.22 & 39.8 & 985.77 & 49.1 & 986.16 \\
\hline & & 13.9 & 986.02 & 29.1 & 986.20 & 40.0 & 985.76 & 49.6 & 986.02 \\
\hline & & 14.2 & 986.06 & 29.5 & 986.00 & 40.4 & 985.81 & 49.9 & 985.87 \\
\hline & & 14.6 & 986.02 & 29.8 & 985.92 & 40.8 & 985.91 & 50.5 & 985.60 \\
\hline & & 15.0 & 986.10 & 30.0 & 985.99 & 41.0 & 985.75 & 51.5 & 984.84 \\
\hline & & 15.3 & 986.11 & 30.2 & 986.00 & 41.2 & 985.72 & 52.3 & 984.67 \\
\hline & & 15.4 & 986.16 & 30.8 & 985.97 & 41.4 & 985.80 & 54.8 & 984.51 \\
\hline & & 15.7 & 986.00 & 31.0 & 985.96 & 41.7 & 985.83 & 54.8 & 984.53 \\
\hline & & 16.0 & 986.12 & 31.1 & 985.99 & 42.0 & 985.93 & 62.4 & 984.93 \\
\hline & & 16.3 & 986.03 & 31.2 & 985.95 & 42.2 & 985.79 & 65.3 & 985.47 \\
\hline & & 16.7 & 986.05 & 31.8 & 985.91 & 42.4 & 985.74 & 67.7 & 985.50 \\
\hline
\end{tabular}


Table 17. (Continued) Listing of horizontal stations and elevations for cross section PR141.7

[Sta., station, distance in meters from a reference pin on the left bank; Elev., elevation, in meters above sea level]

\begin{tabular}{|c|c|c|c|c|c|c|c|c|c|}
\hline \multirow{2}{*}{\multicolumn{2}{|c|}{$\begin{array}{c}1994 \\
3 \text { September }\end{array}$}} & \multirow{2}{*}{\multicolumn{2}{|c|}{$\begin{array}{c}1995 \\
2 \text { October }\end{array}$}} & \multirow{2}{*}{\multicolumn{2}{|c|}{$\begin{array}{c}1995 \\
2 \text { October }\end{array}$}} & \multirow{2}{*}{\multicolumn{2}{|c|}{$\begin{array}{c}1995 \\
2 \text { October }\end{array}$}} & \multirow{2}{*}{\multicolumn{2}{|c|}{$\begin{array}{c}1996 \\
18 \text { October }\end{array}$}} \\
\hline & & & & & & & & & \\
\hline Sta. & Elev. & Sta. & Elev. & Sta. & Elev. & Sta. & Elev. & Sta. & Elev. \\
\hline 70.0 & 984.78 & -15.0 & 987.37 & 39.5 & 986.01 & 121.0 & 984.45 & -29.0 & 987.20 \\
\hline 75.8 & 984.78 & -13.0 & 987.42 & 41.0 & 986.13 & 123.0 & 984.35 & -29.0 & 987.23 \\
\hline 80.0 & 984.74 & -11.0 & 987.51 & 42.5 & 986.29 & 125.0 & 984.89 & -26.0 & 987.18 \\
\hline 86.4 & 984.66 & -9.0 & 987.38 & 44.0 & 986.28 & 126.2 & 985.25 & -23.0 & 987.40 \\
\hline 90.0 & 984.60 & -8.0 & 987.26 & 46.0 & 986.26 & 126.7 & 985.23 & -20.0 & 987.31 \\
\hline 96.0 & 984.39 & -6.3 & 986.83 & 47.5 & 986.30 & 127.6 & 985.59 & -17.0 & 987.34 \\
\hline 100.1 & 984.52 & -5.0 & 986.89 & 49.0 & 986.25 & 128.3 & 986.07 & -14.0 & 987.41 \\
\hline 104.9 & 984.57 & -3.0 & 986.71 & 50.0 & 986.00 & 128.5 & 987.87 & -12.0 & 987.45 \\
\hline 110.1 & 984.58 & -1.5 & 986.71 & 50.7 & 985.39 & 129.5 & 988.43 & -10.0 & 987.59 \\
\hline 116.3 & 984.67 & 0.0 & 986.61 & 51.0 & 985.31 & 131.0 & 988.49 & -8.0 & 987.27 \\
\hline 118.5 & 985.04 & 1.5 & 986.54 & 51.0 & 985.14 & 133.0 & 988.50 & -6.3 & 986.84 \\
\hline 119.4 & 986.29 & 2.0 & 986.62 & 51.3 & 985.15 & 135.5 & 988.41 & -4.0 & 986.77 \\
\hline 119.9 & 986.29 & 3.0 & 986.44 & 51.4 & 985.38 & 137.0 & 988.45 & -3.0 & 986.70 \\
\hline 120.2 & 987.12 & 4.5 & 986.48 & 52.0 & 985.40 & 138.5 & 988.43 & -2.0 & 986.72 \\
\hline 126.5 & 986.90 & 6.0 & 986.58 & 54.0 & 985.24 & 140.0 & 988.45 & -0.8 & 986.61 \\
\hline 129.4 & 988.41 & 7.0 & 986.57 & 56.0 & 985.03 & 143.0 & 988.42 & 0.3 & 986.65 \\
\hline 138.5 & 988.40 & 9.0 & 986.48 & 58.0 & 984.93 & 145.0 & 988.34 & 1.0 & 986.59 \\
\hline \multirow[t]{25}{*}{149.1} & 988.42 & 10.5 & 986.20 & 60.0 & 984.89 & 147.0 & 988.41 & 2.0 & 986.61 \\
\hline & & 12.0 & 986.19 & 61.5 & 984.86 & 149.1 & 988.47 & 3.0 & 986.45 \\
\hline & & 14.0 & 986.12 & 62.7 & 985.11 & & & 4.5 & 986.47 \\
\hline & & 15.0 & 986.23 & 64.5 & 985.82 & & & 6.0 & 986.59 \\
\hline & & 15.5 & 986.26 & 66.0 & 985.74 & & & 7.0 & 986.58 \\
\hline & & 16.5 & 986.19 & 68.0 & 985.52 & & & 9.0 & 986.49 \\
\hline & & 18.0 & 986.30 & 69.5 & 985.42 & & & 10.5 & 986.19 \\
\hline & & 19.0 & 986.27 & 71.0 & 985.49 & & & 12.0 & 986.22 \\
\hline & & 20.0 & 986.31 & 74.0 & 985.52 & & & 14.0 & 986.13 \\
\hline & & 21.0 & 986.38 & 77.0 & 985.43 & & & 15.0 & 986.21 \\
\hline & & 22.0 & 986.37 & 80.0 & 985.25 & & & 15.5 & 986.26 \\
\hline & & 23.0 & 986.43 & 83.5 & 984.89 & & & 16.5 & 986.22 \\
\hline & & 23.7 & 986.47 & 86.0 & 984.71 & & & 18.0 & 986.29 \\
\hline & & 25.0 & 986.41 & 89.0 & 984.62 & & & 19.0 & 986.25 \\
\hline & & 26.4 & 986.50 & 92.0 & 984.52 & & & 20.0 & 986.36 \\
\hline & & 27.4 & 986.43 & 95.0 & 984.43 & & & 21.0 & 986.38 \\
\hline & & 28.6 & 986.52 & 98.0 & 984.44 & & & 22.0 & 986.36 \\
\hline & & 29.5 & 986.20 & 101.0 & 984.50 & & & 23.0 & 986.46 \\
\hline & & 30.7 & 986.09 & 104.0 & 984.51 & & & 23.7 & 986.45 \\
\hline & & 31.5 & 986.05 & 107.0 & 984.61 & & & 25.0 & 986.47 \\
\hline & & 32.5 & 986.10 & 110.0 & 984.53 & & & 26.4 & 986.50 \\
\hline & & 35.0 & 986.00 & 113.0 & 984.61 & & & 27.4 & 986.47 \\
\hline & & 36.0 & 985.94 & 116.0 & 984.62 & & & 28.6 & 986.46 \\
\hline & & 36.8 & 986.07 & 117.0 & 984.47 & & & 29.5 & 986.20 \\
\hline & & 37.3 & 986.10 & 119.0 & 984.61 & & & 30.7 & 986.08 \\
\hline
\end{tabular}


Table 17. (Continued) Listing of horizontal stations and elevations for cross section PR141.7

[Sta., station, distance in meters from a reference pin on the left bank; Elev., elevation, in meters above sea level]

\begin{tabular}{|c|c|c|c|c|c|c|c|c|c|}
\hline \multirow{2}{*}{\multicolumn{2}{|c|}{$\begin{array}{c}1996 \\
18 \text { October }\end{array}$}} & \multicolumn{2}{|c|}{1996} & \multicolumn{2}{|c|}{1997} & \multicolumn{2}{|c|}{1997} & \multicolumn{2}{|c|}{1998} \\
\hline & & 18 & tober & $20 \mathrm{~S}$ & ember & $20 \mathrm{~S}$ & ember & $26 \mathrm{Se}$ & ember \\
\hline Sta. & Elev. & Sta. & Elev. & Sta. & Elev. & Sta. & Elev. & Sta. & Elev. \\
\hline 31.5 & 986.05 & 95.0 & 984.46 & 0.3 & 986.62 & 60.0 & 985.15 & -15.0 & 987.37 \\
\hline 33.5 & 986.10 & 98.0 & 984.40 & 1.0 & 986.57 & 62.4 & 985.10 & -12.5 & 987.43 \\
\hline 35.0 & 985.99 & 100.0 & 984.61 & 2.0 & 986.61 & 63.3 & 985.28 & -10.0 & 987.59 \\
\hline 36.0 & 985.97 & 103.0 & 984.69 & 3.0 & 986.45 & 64.9 & 985.72 & -7.5 & 987.12 \\
\hline 36.8 & 986.08 & 106.0 & 984.73 & 4.5 & 986.47 & 66.0 & 985.84 & -6.0 & 986.82 \\
\hline 37.3 & 986.10 & 109.0 & 984.70 & 6.5 & 986.59 & 68.0 & 985.86 & -4.0 & 986.78 \\
\hline 39.5 & 986.04 & 112.0 & 984.57 & 8.0 & 986.53 & 70.0 & 985.75 & -2.0 & 986.70 \\
\hline 41.0 & 986.19 & 115.0 & 984.49 & 9.0 & 986.51 & 72.0 & 985.61 & 0.3 & 986.64 \\
\hline 42.5 & 986.26 & 118.0 & 984.46 & 10.5 & 986.21 & 74.0 & 985.52 & 1.0 & 986.55 \\
\hline 44.0 & 986.31 & 121.0 & 984.42 & 12.0 & 986.20 & 76.0 & 985.43 & 4.0 & 986.45 \\
\hline 46.0 & 986.26 & 123.5 & 984.37 & 14.0 & 986.13 & 78.0 & 985.37 & 7.0 & 986.58 \\
\hline 47.5 & 986.29 & 124.5 & 984.61 & 16.0 & 986.22 & 80.0 & 985.24 & 9.0 & 986.49 \\
\hline 49.0 & 986.26 & 126.0 & 984.81 & 16.5 & 986.21 & 82.0 & 985.06 & 11.0 & 986.19 \\
\hline 50.0 & 986.04 & 127.6 & 984.96 & 17.5 & 986.35 & 83.7 & 984.80 & 14.0 & 986.13 \\
\hline 50.7 & 985.57 & 129.9 & 984.83 & 18.5 & 986.28 & & & 17.0 & 986.25 \\
\hline 51.2 & 985.48 & 130.4 & 988.48 & 20.0 & 986.32 & & & 20.0 & 986.33 \\
\hline 52.0 & 985.19 & 132.0 & 988.51 & 22.0 & 986.37 & & & 22.0 & 986.36 \\
\hline 54.0 & 985.23 & 135.0 & 988.47 & 24.0 & 986.43 & & & 24.0 & 986.43 \\
\hline 56.0 & 985.06 & 138.0 & 988.44 & 26.0 & 986.52 & & & 26.0 & 986.48 \\
\hline 58.0 & 984.90 & 141.0 & 988.45 & 28.0 & 986.43 & & & 27.5 & 986.46 \\
\hline 60.0 & 984.79 & 144.0 & 988.36 & 30.0 & 986.28 & & & 29.0 & 986.41 \\
\hline 61.2 & 984.71 & 146.0 & 988.40 & 31.0 & 986.05 & & & 31.3 & 986.07 \\
\hline 62.4 & 984.82 & 149.1 & 988.46 & 33.0 & 986.09 & & & 33.0 & 986.08 \\
\hline 62.8 & 985.00 & & & 35.0 & 986.00 & & & 36.0 & 985.95 \\
\hline 63.5 & 985.19 & & & 36.0 & 985.97 & & & 38.0 & 986.07 \\
\hline 63.8 & 985.21 & & & 38.0 & 986.06 & & & 40.0 & 986.04 \\
\hline 65.0 & 985.68 & & & 40.0 & 986.07 & & & 42.0 & 986.22 \\
\hline 66.0 & 985.71 & & & 42.0 & 986.27 & & & 44.0 & 986.31 \\
\hline 68.0 & 985.75 & & & 44.0 & 986.31 & & & 46.0 & 986.28 \\
\hline 70.0 & 985.70 & & & 46.0 & 986.27 & & & 48.0 & 986.28 \\
\hline 71.0 & 985.65 & & & 48.0 & 986.28 & & & 49.2 & 986.10 \\
\hline 73.0 & 985.69 & & & 49.1 & 986.26 & & & 51.0 & 985.68 \\
\hline 74.3 & 985.62 & & & 50.0 & 985.99 & & & 52.3 & 985.40 \\
\hline 75.5 & 985.59 & & & 51.0 & 985.68 & & & 54.0 & 985.28 \\
\hline 77.2 & 985.36 & & & 51.4 & 985.63 & & & 57.0 & 985.19 \\
\hline 78.5 & 985.32 & & & 52.0 & 985.45 & & & 60.0 & 985.04 \\
\hline 81.0 & 985.14 & & & 53.0 & 985.25 & & & 62.0 & 984.92 \\
\hline 82.5 & 985.00 & & & 55.0 & 985.11 & & & 62.7 & 984.89 \\
\hline 83.8 & 984.80 & & & 57.3 & 985.05 & & & 64.0 & 985.45 \\
\hline 86.0 & 984.63 & & & 57.7 & 984.93 & & & 65.8 & 985.81 \\
\hline 89.0 & 984.60 & & & 58.5 & 984.97 & & & 68.0 & 985.85 \\
\hline 92.0 & 984.55 & & & 58.7 & 985.07 & & & 70.0 & 985.74 \\
\hline
\end{tabular}


Table 17. (Continued) Listing of horizontal stations and elevations for cross section PR141.7

[Sta., station, distance in meters from a reference pin on the left bank; Elev., elevation, in meters above sea level]

1998

\begin{tabular}{cc}
\multicolumn{2}{c}{26 September } \\
\hline Sta. & Elev. \\
\hline 73.0 & 985.53 \\
76.0 & 985.45 \\
79.0 & 985.35 \\
81.0 & 984.76 \\
82.5 & 984.97 \\
85.0 & 984.68 \\
88.0 & 984.61 \\
91.0 & 984.57 \\
94.0 & 984.45 \\
97.0 & 984.42 \\
100.0 & 984.47 \\
103.0 & 984.56 \\
106.0 & 984.61 \\
109.0 & 984.60 \\
112.0 & 984.49 \\
115.0 & 984.33 \\
118.0 & 984.35 \\
121.0 & 984.41 \\
124.0 & 984.56 \\
128.0 & 985.01 \\
131.0 & 985.86 \\
131.4 & 988.51 \\
135.0 & 988.48 \\
138.0 & 988.44 \\
141.0 & 988.43 \\
144.0 & 988.35 \\
147.0 & 988.41 \\
149.1 & 988.47
\end{tabular}




\section{Description of Cross Section PR147}

Location: Township 7 South/Range 49 East--section 21

U. S. Geological Survey quadrangle (1:24,000): Huckins School

Landowners--left bank: Gay Ranch

--right bank: Huckins Ranch

Access: Left or right bank

Permission from: Left bank, Gay Ranch; right bank, Huckins Ranch

Distance from Moorhead Gaging Station: 30.24 kilometers

Azimuth of Section (degrees magnetic): 130

\section{Reference Monuments}

[Monuments at stations -68.9 and 0.0 were closest to leveling instrument]

\begin{tabular}{|c|c|c|c|c|c|c|}
\hline \multirow[b]{2}{*}{ Description } & \multirow[b]{2}{*}{$\begin{array}{c}\text { Station } \\
\text { (m) }\end{array}$} & \multicolumn{2}{|c|}{ GPS-NAD83 (1992) } & \multicolumn{2}{|c|}{ Measurement } & \multirow{2}{*}{$\begin{array}{c}\text { Elevation } \\
\text { (NGVD1929) } \\
\text { (m) }\end{array}$} \\
\hline & & Latitude & Longitude & $\begin{array}{c}\text { Standard } \\
\text { deviation } \\
\text { (m) }\end{array}$ & $\begin{array}{l}\text { Horizontal } \\
\text { precision } \\
\text { (m) }\end{array}$ & \\
\hline $\begin{array}{l}\text { 1/2-inch-rebar; } 0.19 \text { meter above } 1998 \text { ground } \\
\text { level under a fenceline }\end{array}$ & -68.9 & & & & & 984.01 \\
\hline $\begin{array}{l}\text { 1/2-inch-rebar; } 0.05 \text { meter above } 1998 \text { ground } \\
\text { level }\end{array}$ & 0.0 & & & & & 983.91 \\
\hline Benchmark--brass circular plate & -2.0 & $45^{\circ} 12^{\prime} 19.56^{\prime \prime}$ & $105^{\circ} 44^{\prime} 06.61^{\prime \prime}$ & 0.557 & 0.460 & 983.92 \\
\hline $\begin{array}{l}\text { 1/2-inch-rebar; } 0.04 \text { meter below } 1998 \text { ground } \\
\text { level }\end{array}$ & 100.0 & & & & & 983.10 \\
\hline $\begin{array}{l}\text { 1/2-inch-rebar; } 0.09 \text { meter above } 1998 \text { ground } \\
\text { level }\end{array}$ & 100.7 & & & & & 983.27 \\
\hline $\begin{array}{l}\text { 1/2-inch-rebar; } 0.15 \text { meter above } 1998 \text { ground } \\
\text { level }\end{array}$ & 110.0 & $45^{\circ} 12^{\prime} 16.63^{\prime \prime}$ & $105^{\circ} 44^{\prime} 03.58^{\prime \prime}$ & 0.153 & 0.348 & 983.56 \\
\hline
\end{tabular}



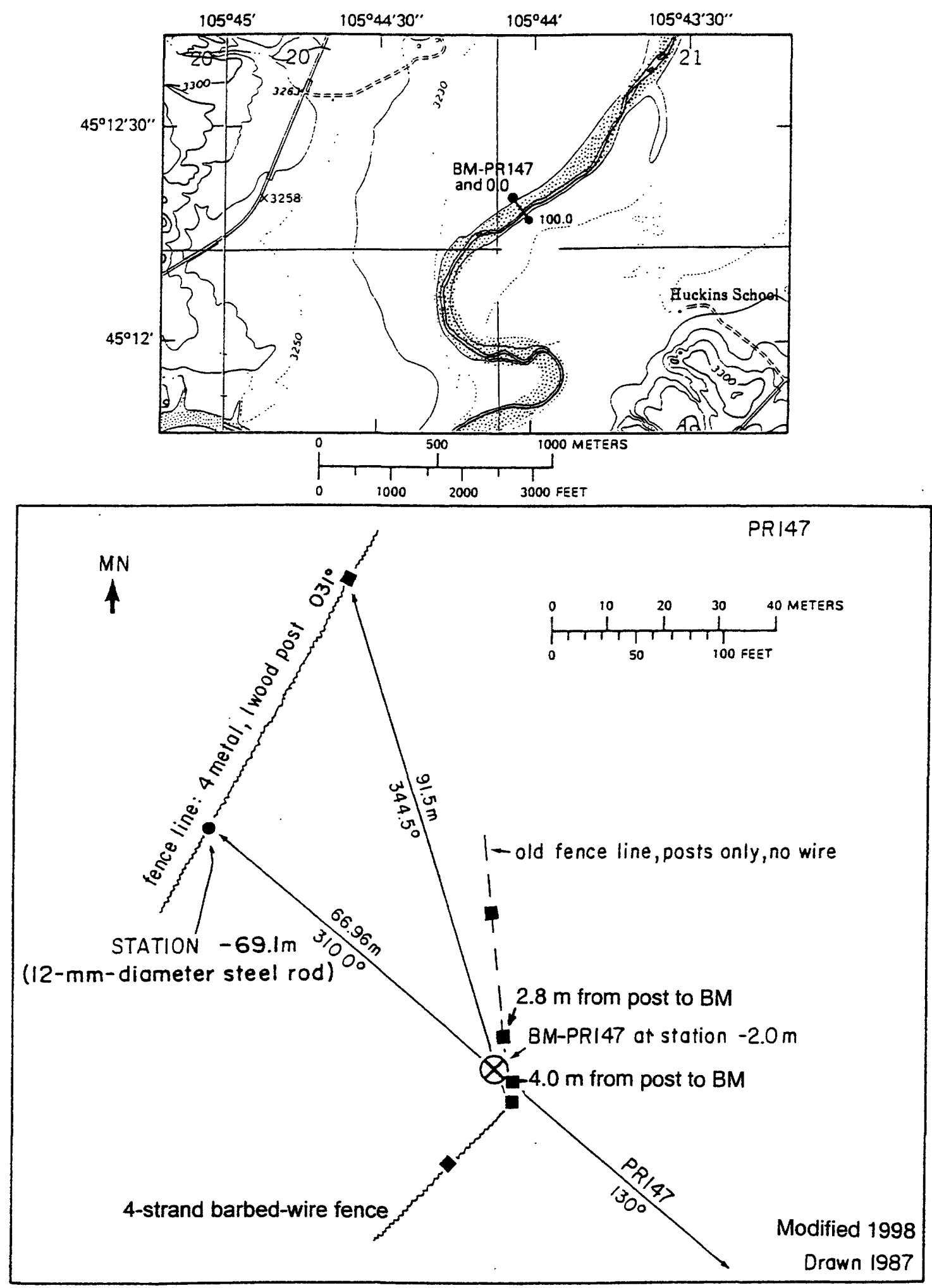

Figure 46. Upper: Location of cross section PR147, bench mark BM-PR147, and the right bank reference monument in the Huckins School quadrangle. Lower: Location of the bench mark on the left bank. $\mathrm{MN}$ is magnetic north. 

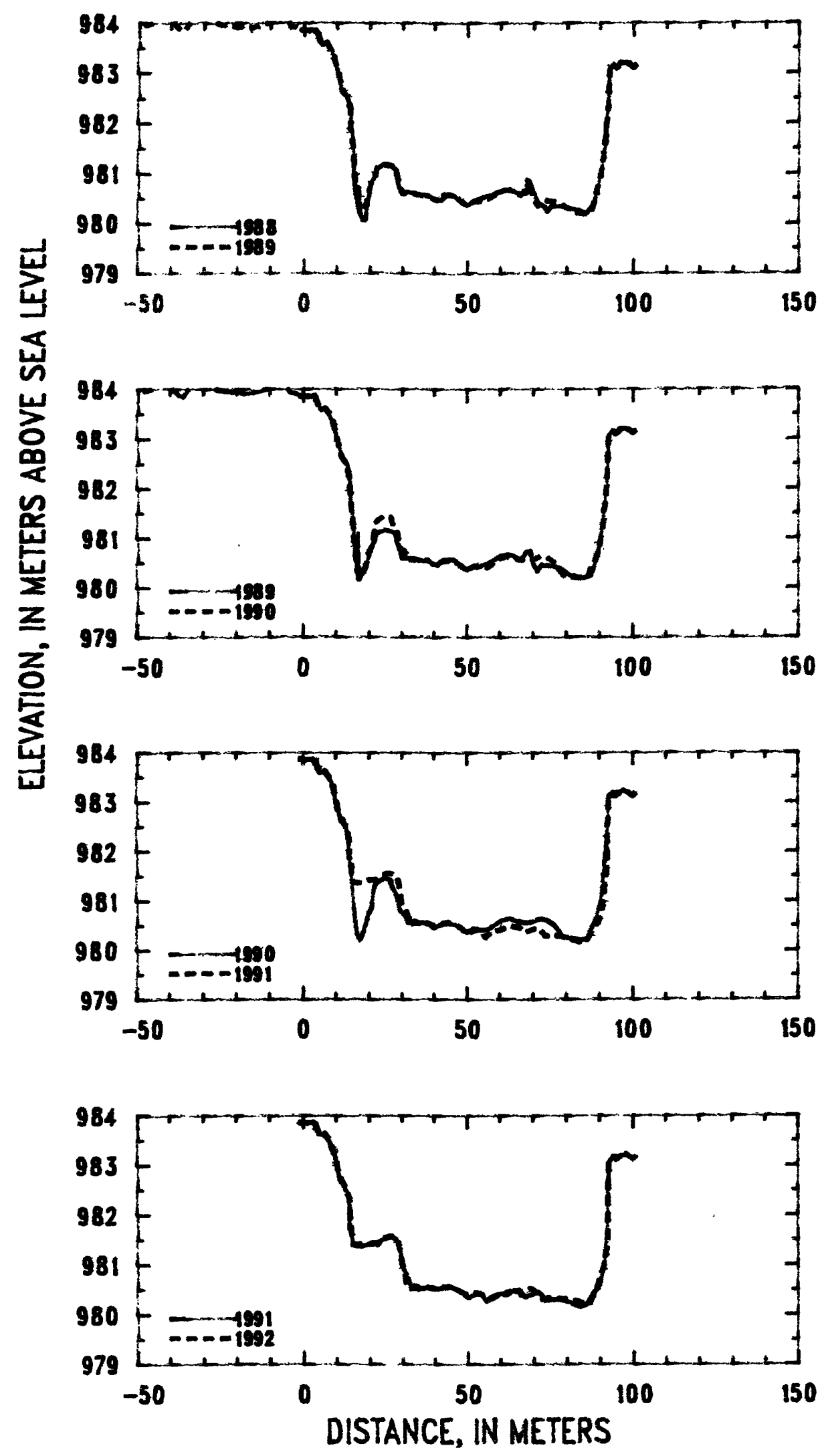

Figure 47. Profiles of cross section PR147 from 1988 to 1992. 

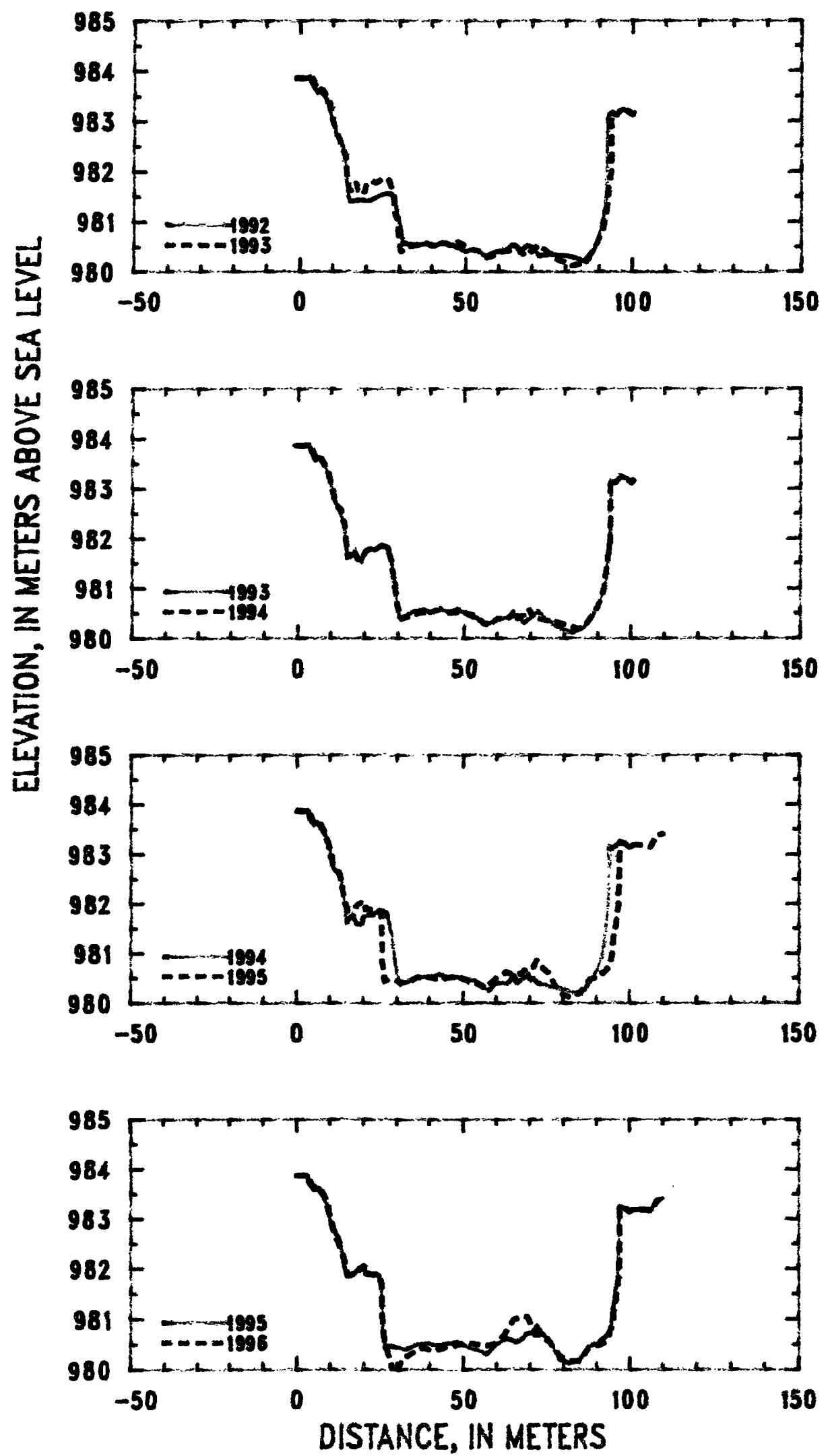

Figure 48. Profiles of cross section PR147 from 1992 to 1996. 


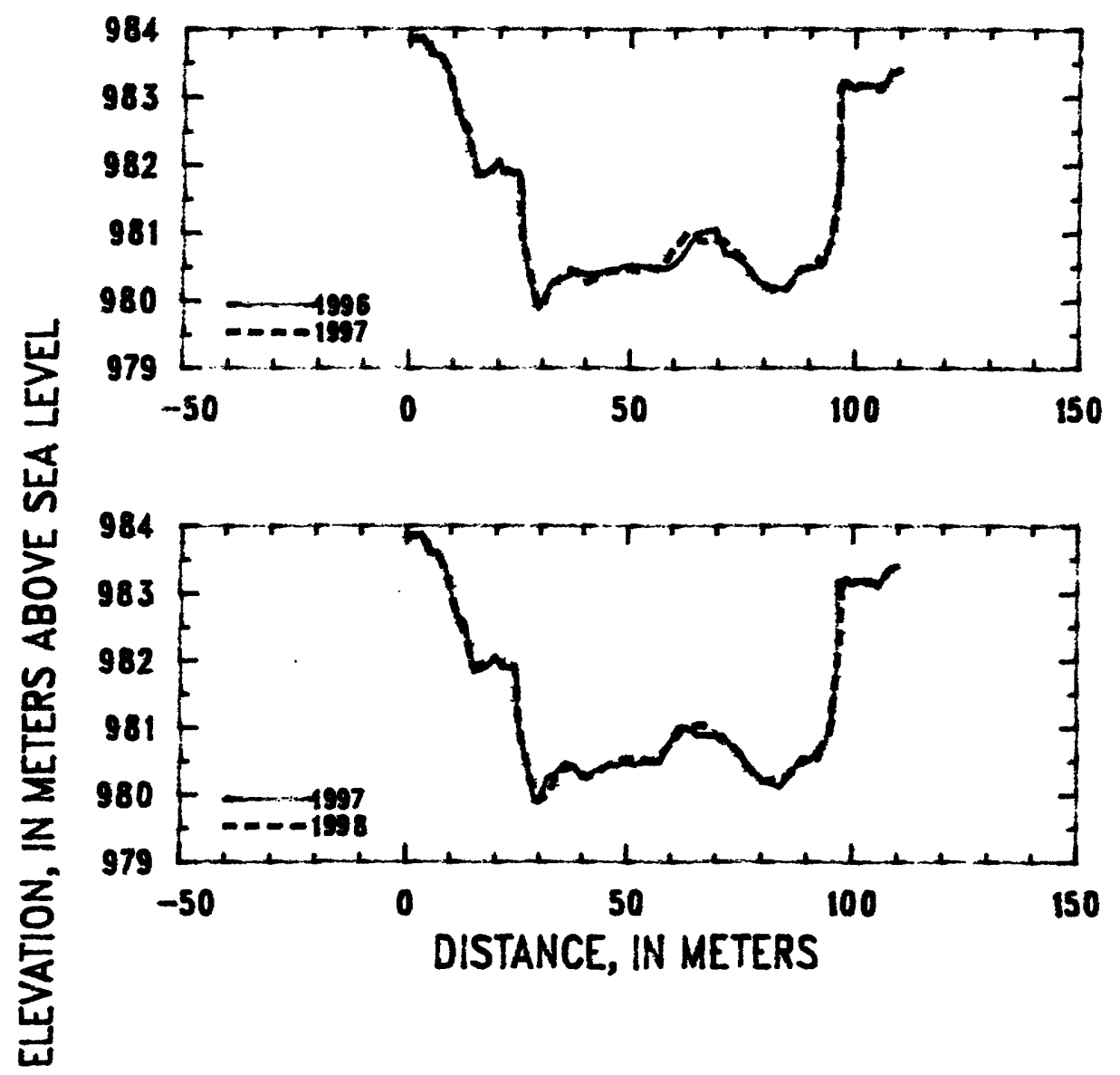

Figure 49. Profiles of cross section PR147 from 1996 to 1998. 
Table 18. Listing of horizontal stations and elevations for cross section PR147

[Sta., station, distance in meters from a reference pin on the left bank; Elev., elevation, in meters above sea level]

\begin{tabular}{|c|c|c|c|c|c|c|c|c|c|}
\hline \multirow{2}{*}{\multicolumn{2}{|c|}{$\begin{array}{c}1989 \\
19 \text { September }\end{array}$}} & \multicolumn{2}{|c|}{1989} & \multicolumn{2}{|c|}{1989} & \multicolumn{2}{|c|}{1990} & \multicolumn{2}{|c|}{1990} \\
\hline & & $19 \mathrm{Se}$ & ember & $19 \mathrm{Se}$ & ember & $23 \mathrm{Se}$ & ember & $23 \mathrm{Se}$ & ember \\
\hline Sta. & Elev. & Sta. & Elev. & Sta. & Elev. & Sta. & Elev. & Sta. & Elev. \\
\hline-69.1 & 983.83 & 15.0 & 981.46 & 69.9 & 980.49 & -0.9 & 983.86 & 58.0 & 980.50 \\
\hline-66.0 & 983.85 & 15.7 & 980.77 & 70.3 & 980.46 & 0.0 & 983.86 & 60.0 & 980.60 \\
\hline-63.0 & 984.17 & 16.4 & 980.37 & 71.3 & 980.34 & 2.0 & 983.85 & 62.0 & 980.65 \\
\hline-61.0 & 984.22 & 16.6 & 980.73 & 72.5 & 980.46 & 3.4 & 983.87 & 64.0 & 980.62 \\
\hline-59.0 & 984.19 & 17.0 & 980.19 & 74.0 & 980.45 & 5.0 & 983.61 & 65.8 & 980.56 \\
\hline-56.0 & 984.25 & 18.0 & 980.29 & 76.0 & 980.44 & 5.4 & 983.58 & 66.0 & 980.58 \\
\hline-53.0 & 984.22 & 19.0 & 980.43 & 78.0 & 980.39 & 6.8 & 983.62 & 68.0 & 980.55 \\
\hline-50.0 & 984.13 & 20.0 & 980.73 & 80.0 & 980.31 & 8.0 & 983.51 & 70.0 & 980.57 \\
\hline-47.6 & 983.96 & 20.2 & 980.78 & 82.0 & 980.20 & 9.0 & 983.34 & 72.0 & 980.63 \\
\hline-45.0 & 984.01 & 21.0 & 980.90 & 84.0 & 980.20 & 10.0 & 983.08 & 74.0 & 980.62 \\
\hline-43.0 & 984.06 & 21.7 & 980.97 & 85.0 & 980.19 & 11.6 & 982.64 & 76.0 & 980.53 \\
\hline-41.0 & 984.15 & 22.0 & 981.10 & 87.5 & 980.25 & 13.0 & 982.53 & 77.0 & 980.47 \\
\hline-39.0 & 983.99 & 24.0 & 981.16 & 88.5 & 980.47 & 13.4 & 982.40 & 79.0 & 980.26 \\
\hline-37.0 & 983.86 & 26.0 & 981.16 & 89.0 & 980.65 & 13.8 & 982.31 & 81.0 & 980.25 \\
\hline-36.0 & 983.85 & 28.0 & 981.10 & 90.2 & 980.91 & 14.4 & 981.78 & 83.0 & 980.21 \\
\hline-34.0 & 984.03 & 28.3 & 980.93 & 91.0 & 981.34 & 15.7 & 980.77 & 85.0 & 980.20 \\
\hline-32.0 & 984.09 & 29.0 & 980.84 & 92.1 & 982.09 & 16.5 & 980.30 & 86.5 & 980.21 \\
\hline-30.0 & 984.07 & 29.2 & 980.76 & 92.6 & 982.68 & 17.3 & 980.21 & 87.6 & 980.45 \\
\hline-28.0 & 984.06 & 30.0 & 980.60 & 92.7 & 983.08 & 18.8 & 980.46 & 88.0 & 980.48 \\
\hline-25.0 & 983.97 & 32.0 & 980.60 & 94.0 & 983.17 & 20.0 & 980.78 & 88.8 & 980.56 \\
\hline-22.0 & 983.94 & 34.0 & 980.57 & 95.0 & 983.10 & 20.8 & 980.84 & 90.3 & 980.96 \\
\hline-19.0 & 983.92 & 36.0 & 980.55 & 96.0 & 983.21 & 21.7 & 981.32 & 91.0 & 981.28 \\
\hline-16.0 & 983.90 & 38.0 & 980.56 & 98.0 & 983.21 & 23.0 & 981.41 & 91.2 & 981.58 \\
\hline-13.0 & 983.96 & 40.0 & 980.48 & 100.0 & 983.12 & 25.0 & 981.46 & 91.5 & 981.63 \\
\hline-10.0 & 984.01 & 41.0 & 980.46 & 100.7 & 983.17 & 26.5 & 981.43 & 91.6 & 981.78 \\
\hline-7.0 & 984.14 & 42.0 & 980.55 & & & 26.9 & 981.30 & 92.0 & 982.04 \\
\hline-6.0 & 984.16 & 44.0 & 980.57 & & & 28.5 & 981.10 & 92.7 & 983.07 \\
\hline-5.0 & 984.06 & 46.0 & 980.57 & & & 29.4 & 980.80 & 93.0 & 983.13 \\
\hline-4.0 & 983.94 & 48.0 & 980.44 & & & 31.3 & 980.69 & 95.0 & 983.11 \\
\hline-2.0 & 983.90 & 50.0 & 980.37 & & & 32.0 & 980.56 & 97.0 & 983.24 \\
\hline-0.9 & 983.86 & 52.0 & 980.44 & & & 34.0 & 980.58 & 99.0 & 983.17 \\
\hline 0.0 & 983.86 & 54.0 & 980.44 & & & 36.0 & 980.55 & 100.0 & 983.12 \\
\hline 2.0 & 983.85 & 56.0 & 980.50 & & & 38.0 & 980.56 & 100.7 & 983.18 \\
\hline 3.6 & 983.87 & 58.0 & 980.56 & & & 40.0 & 980.47 & & \\
\hline 5.1 & 983.59 & 60.0 & 980.66 & & & 42.0 & 980.53 & & \\
\hline 7.0 & 983.61 & 61.0 & 980.66 & & & 44.0 & 980.55 & & \\
\hline 9.0 & 983.34 & 63.0 & 980.68 & & & 46.0 & 980.52 & & \\
\hline 10.0 & 983.08 & 65.0 & 980.61 & & & 48.0 & 980.46 & & \\
\hline 12.0 & 982.60 & 66.0 & 980.57 & & & 50.0 & 980.36 & & \\
\hline 13.2 & 982.52 & 67.2 & 980.63 & & & 52.0 & 980.44 & & \\
\hline 14.0 & 982.31 & 67.7 & 980.74 & & & 54.0 & 980.41 & & \\
\hline 14.2 & 981.88 & 69.0 & 980.75 & & & 56.0 & 980.40 & & \\
\hline
\end{tabular}


Table 18. (Continued) Listing of horizontal stations and elevations for cross section PR147

[Sta., station, distance in meters from a reference pin on the left bank; Elev., elevation, in meters above sea level]

\begin{tabular}{|c|c|c|c|c|c|c|c|c|c|}
\hline \multirow{2}{*}{\multicolumn{2}{|c|}{$\begin{array}{c}1991 \\
31 \text { AUgust }\end{array}$}} & \multirow{2}{*}{\multicolumn{2}{|c|}{$\begin{array}{c}1991 \\
31 \text { August }\end{array}$}} & \multirow{2}{*}{\multicolumn{2}{|c|}{$\begin{array}{c}1992 \\
27 \text { August }\end{array}$}} & \multirow{2}{*}{\multicolumn{2}{|c|}{$\begin{array}{c}1992 \\
27 \text { August }\end{array}$}} & \multirow{2}{*}{\multicolumn{2}{|c|}{$\begin{array}{c}1993 \\
28 \text { August }\end{array}$}} \\
\hline & & & & & & & & & \\
\hline Sta. & Elev. & Sta. & Elev. & Sta. & Elev. & Sta. & Elev. & Sta. & Elev. \\
\hline-0.9 & 983.87 & 64.0 & 980.49 & -0.9 & 983.86 & 64.0 & 980.52 & -0.9 & 983.87 \\
\hline 0.0 & 983.86 & 66.0 & 980.46 & 0.0 & 983.86 & 65.0 & 980.52 & 0.0 & 983.87 \\
\hline 2.0 & 983.86 & 67.4 & 980.35 & 2.0 & 983.86 & 66.0 & 980.46 & 2.0 & 983.86 \\
\hline 3.6 & 983.87 & 69.0 & 980.39 & 3.5 & 983.88 & 66.4 & 980.38 & 3.6 & 983.88 \\
\hline 5.0 & 983.63 & 71.0 & 980.45 & 4.6 & 983.67 & 67.9 & 980.49 & 4.2 & 983.79 \\
\hline 7.0 & 983.61 & 72.0 & 980.43 & 5.7 & 983.57 & 69.0 & 980.52 & 5.0 & 983.62 \\
\hline 8.5 & 983.44 & 73.6 & 980.28 & 6.6 & 983.64 & 70.0 & 980.50 & 5.5 & 983.58 \\
\hline 9.3 & 983.30 & 75.0 & 980.31 & 8.0 & 983.49 & 72.0 & 980.31 & 6.8 & 983.62 \\
\hline 11.0 & 982.75 & 78.0 & 980.29 & 9.6 & 983.19 & 74.0 & 980.33 & 9.0 & 983.37 \\
\hline 13.0 & 982.52 & 80.0 & 980.26 & 11.0 & 982.78 & 76.0 & 980.35 & 11.0 & 982.78 \\
\hline 14.0 & 982.28 & 82.0 & 980.21 & 13.0 & 982.53 & 78.0 & 980.31 & 12.5 & 982.58 \\
\hline 14.2 & 981.74 & 84.0 & 980.15 & 14.0 & 982.30 & 80.0 & 980.31 & 14.0 & 982.28 \\
\hline 14.6 & 981.75 & 86.0 & 980.20 & 14.3 & 981.81 & 82.0 & 980.28 & 14.4 & 981.91 \\
\hline 15.0 & 981.40 & 87.0 & 980.29 & 15.0 & 981.42 & 84.0 & 980.24 & 14.6 & 981.87 \\
\hline 16.0 & 981.37 & 88.4 & 980.46 & 16.0 & 981.40 & 86.0 & 980.20 & 15.0 & 981.62 \\
\hline 18.0 & 981.37 & 89.8 & 980.57 & 18.0 & 981.43 & 86.5 & 980.19 & 16.0 & 981.63 \\
\hline 20.0 & 981.43 & 91.0 & 980.90 & 20.0 & 981.42 & 88.0 & 980.37 & 17.2 & 981.76 \\
\hline 22.0 & 981.43 & 92.3 & 981.77 & 22.0 & 981.43 & 89.0 & 980.50 & 17.8 & 981.60 \\
\hline 24.0 & 981.52 & 92.3 & 982.50 & 24.0 & 981.52 & 91.0 & 980.97 & 18.1 & 981.57 \\
\hline 26.0 & 981.56 & 92.6 & 982.72 & 26.0 & 981.57 & 92.2 & 981.46 & 19.0 & 981.58 \\
\hline 28.0 & 981.52 & 92.7 & 983.07 & 27.5 & 981.57 & 92.4 & 982.48 & 19.3 & 981.55 \\
\hline 29.0 & 981.39 & 94.0 & 983.18 & 28.5 & 981.49 & 92.6 & 983.05 & 20.0 & 981.72 \\
\hline 30.0 & 980.95 & 96.0 & 983.18 & 30.0 & 980.99 & 93.0 & 983.14 & 21.0 & 981.79 \\
\hline 31.0 & 980.85 & 98.0 & 983.21 & 30.5 & 980.87 & 94.0 & 983.17 & 22.0 & 981.81 \\
\hline 32.3 & 980.64 & 100.0 & 983.12 & 31.0 & 980.75 & 95.0 & 983.11 & 23.0 & 981.77 \\
\hline 32.7 & 980.51 & 100.7 & 983.17 & 31.3 & 980.57 & 97.0 & 983.25 & 23.7 & 981.79 \\
\hline 34.0 & 980.59 & & & 33.0 & 980.55 & 100.0 & 983.12 & 25.0 & 981.86 \\
\hline 36.0 & 980.52 & & & 35.0 & 980.51 & 100.7 & 983.17 & 26.0 & 981.85 \\
\hline 38.0 & 980.56 & & & 37.0 & 980.54 & & & 27.0 & 981.82 \\
\hline 40.0 & 980.51 & & & 39.0 & 980.56 & & & 28.2 & 981.61 \\
\hline 42.0 & 980.53 & & & 41.0 & 980.51 & & & 28.9 & 981.11 \\
\hline 44.0 & 980.57 & & & 43.0 & 980.56 & & & 29.4 & 981.09 \\
\hline 46.0 & 980.52 & & & 45.0 & 980.55 & & & 29.7 & 980.80 \\
\hline 48.0 & 980.47 & & & 46.0 & 980.54 & & & 30.5 & 980.41 \\
\hline 50.0 & 980.32 & & & 48.0 & 980.45 & & & 32.0 & 980.42 \\
\hline 52.0 & 980.40 & & & 50.0 & 980.40 & & & 34.0 & 980.51 \\
\hline 54.0 & 980.42 & & & 52.0 & 980.44 & & & 36.0 & 980.54 \\
\hline 55.7 & 980.26 & & & 54.0 & 980.42 & & & 38.0 & 980.58 \\
\hline 57.0 & 980.35 & & & 56.0 & 980.29 & & & 40.0 & 980.51 \\
\hline 59.0 & 980.40 & & & 58.0 & 980.35 & & & 42.0 & 980.56 \\
\hline 60.5 & 980.42 & & & 60.0 & 980.41 & & & 44.0 & 980.56 \\
\hline 62.0 & 980.46 & & & 62.0 & 980.40 & & & 46.0 & 980.51 \\
\hline
\end{tabular}


Table 18. (Continued) Listing of horizontal stations and elevations for cross section PR147

[Sta., station, distance in meters from a reference pin on the left bank; Elev., elevation, in meters above sea level]

\begin{tabular}{|c|c|c|c|c|c|c|c|c|c|}
\hline \multirow{2}{*}{\multicolumn{2}{|c|}{$\begin{array}{c}1993 \\
28 \text { August }\end{array}$}} & \multicolumn{2}{|c|}{1994} & \multicolumn{2}{|c|}{1994} & \multicolumn{2}{|c|}{1995} & \multicolumn{2}{|c|}{1995} \\
\hline & & \multicolumn{2}{|c|}{22 September } & \multicolumn{2}{|c|}{22 September } & \multicolumn{2}{|c|}{2 October } & \multicolumn{2}{|c|}{2 October } \\
\hline Sta. & Elev. & Sta. & Elev. & Sta. & Elev. & Sta. & Elev. & Sta. & Elev. \\
\hline 48.0 & 980.59 & 0.0 & 983.87 & 75.0 & 980.35 & 0.0 & 983.88 & 82.0 & 980.12 \\
\hline 50.0 & 980.50 & 2.0 & 983.86 & 78.0 & 980.32 & 2.0 & 983.86 & 85.0 & 980.20 \\
\hline 52.0 & 980.43 & 3.6 & 983.87 & 81.0 & 980.26 & 3.5 & 983.88 & 88.0 & 980.48 \\
\hline 54.0 & 980.41 & 5.5 & 983.58 & 84.0 & 980.19 & 4.5 & 983.69 & 91.0 & 980.53 \\
\hline 56.0 & 980.27 & 6.6 & 983.63 & 86.5 & 980.27 & 5.5 & 983.58 & 91.5 & 980.60 \\
\hline 58.0 & 980.30 & 8.0 & 983.49 & 87.8 & 980.42 & 7.0 & 983.60 & 93.0 & 980.66 \\
\hline 60.0 & 980.38 & 9.3 & 983.30 & 90.0 & 980.64 & 9.0 & 983.36 & 94.4 & 980.85 \\
\hline 62.0 & 980.40 & 11.4 & 982.69 & 91.7 & 980.97 & 11.0 & 982.78 & 94.8 & 981.24 \\
\hline 64.0 & 980.43 & 13.0 & 982.58 & 93.6 & 981.98 & 13.0 & 982.60 & 95.7 & 981.53 \\
\hline 66.0 & 980.46 & 13.9 & 982.33 & 93.7 & 983.16 & 14.6 & 981.99 & 96.5 & 981.98 \\
\hline 68.0 & 980.30 & 15.0 & 981.62 & 95.0 & 983.12 & 15.5 & 981.85 & 96.9 & 983.25 \\
\hline 70.0 & 980.42 & 17.0 & 981.77 & 97.0 & 983.25 & 17.0 & 981.87 & 98.0 & 983.23 \\
\hline 72.0 & 980.53 & 18.0 & 981.59 & 100.0 & 983.12 & 19.0 & 982.02 & 100.0 & 983.14 \\
\hline 74.0 & 980.43 & 19.5 & 981.58 & 100.7 & 983.18 & 20.5 & 982.05 & 100.7 & 983.18 \\
\hline 76.0 & 980.31 & 20.1 & 981.74 & & & 20.7 & 981.89 & 102.0 & 983.19 \\
\hline 78.0 & 980.21 & 21.0 & 981.78 & & & 22.0 & 981.89 & 104.0 & 983.17 \\
\hline 80.0 & 980.18 & 23.0 & 981.78 & & & 23.0 & 981.86 & 106.0 & 983.14 \\
\hline 82.0 & 980.11 & 25.0 & 981.87 & & & 24.6 & 981.88 & 108.0 & 983.37 \\
\hline 84.0 & 980.14 & 27.0 & 981.82 & & & 25.4 & 981.75 & 110.0 & 983.41 \\
\hline 86.0 & 980.22 & 28.0 & 981.56 & & & 25.7 & 980.81 & & \\
\hline 88.0 & 980.40 & 29.8 & 980.70 & & & 26.5 & 980.43 & & \\
\hline 89.4 & 980.58 & 31.0 & 980.38 & & & 28.0 & 980.47 & & \\
\hline 91.0 & 980.80 & 34.0 & 980.45 & & & 30.0 & 980.44 & & \\
\hline 92.0 & 981.06 & 37.0 & 980.51 & & & 33.0 & 980.40 & & \\
\hline 92.6 & 981.34 & 40.0 & 980.53 & & & 36.0 & 980.51 & & \\
\hline 92.9 & 981.68 & 43.0 & 980.59 & & & 39.0 & 980.54 & & \\
\hline 93.3 & 981.79 & 46.0 & 980.50 & & & 42.0 & 980.49 & & \\
\hline 93.4 & 981.91 & 49.0 & 980.53 & & & 45.0 & 980.50 & & \\
\hline 93.6 & 981.95 & 52.0 & 980.47 & & & 48.0 & 980.55 & & \\
\hline 93.7 & 983.15 & 54.0 & 980.43 & & & 51.0 & 980.45 & & \\
\hline 95.0 & 983.11 & 56.0 & 980.29 & & & 54.0 & 980.40 & & \\
\hline 97.0 & 983.25 & 58.0 & 980.25 & & & 57.0 & 980.30 & & \\
\hline 99.0 & 983.19 & 60.0 & 980.40 & & & 60.0 & 980.50 & & \\
\hline 100.0 & 983.12 & 63.5 & 980.40 & & & 63.0 & 980.63 & & \\
\hline 100.7 & 983.17 & 64.8 & 980.53 & & & 66.0 & 980.54 & & \\
\hline & & 65.6 & 980.54 & & & 68.0 & 980.61 & & \\
\hline & & 66.0 & 980.40 & & & 69.0 & 980.72 & & \\
\hline & & 68.0 & 980.47 & & & 71.0 & 980.73 & & \\
\hline & & 69.5 & 980.59 & & & 72.0 & 980.86 & & \\
\hline & & 70.2 & 980.46 & & & 74.0 & 980.69 & & \\
\hline & & 71.3 & 980.49 & & & 75.5 & 980.61 & & \\
\hline & & 72.5 & 980.39 & & & 79.0 & 980.19 & & \\
\hline
\end{tabular}


Table 18. (Continued) Listing of horizontal stations and elevations for cross section PR147

[Sta., station, distance in meters from a reference pin on the left bank; Elev., elevation, in meters above sea level]

\begin{tabular}{|c|c|c|c|c|c|c|c|c|c|}
\hline \multirow{2}{*}{\multicolumn{2}{|c|}{$\begin{array}{r}1996 \\
25 \text { October } \\
\end{array}$}} & \multirow{2}{*}{\multicolumn{2}{|c|}{$\begin{array}{c}1996 \\
25 \text { October }\end{array}$}} & \multirow{2}{*}{\multicolumn{2}{|c|}{$\begin{array}{c}1997 \\
\text { 20 September }\end{array}$}} & \multirow{2}{*}{\multicolumn{2}{|c|}{$\begin{array}{c}1997 \\
\text { 20 September }\end{array}$}} & \multirow{2}{*}{\multicolumn{2}{|c|}{$\begin{array}{c}1998 \\
28 \text { September }\end{array}$}} \\
\hline & & & & & & & & & \\
\hline Sta. & Elev. & Sta. & Elev. & Sta. & Elev. & Sta. & Elev. & Sta. & Elev. \\
\hline 0.0 & 983.87 & 94.8 & 980.89 & 0.0 & 983.86 & 66.0 & 980.88 & 0.0 & 983.86 \\
\hline 3.0 & 983.87 & 96.5 & 981.73 & 2.0 & 983.85 & 68.0 & 980.88 & 2.0 & 983.86 \\
\hline 5.0 & 983.63 & 96.8 & 981.98 & 3.6 & 983.87 & 70.0 & 980.89 & 3.5 & 983.87 \\
\hline 7.0 & 983.60 & 97.0 & 983.22 & 5.5 & 983.59 & 71.4 & 980.87 & 5.5 & 983.59 \\
\hline 9.0 & 983.36 & 98.0 & 983.23 & 7.0 & 983.60 & 73.0 & 980.72 & 7.0 & 983.57 \\
\hline 11.0 & 982.80 & 100.0 & 983.12 & 8.5 & 983.44 & 75.0 & 980.65 & 9.0 & 983.33 \\
\hline 14.0 & 982.26 & 100.7 & 983.18 & 10.0 & 983.07 & 75.9 & 980.54 & 11.0 & 982.77 \\
\hline 15.3 & 981.86 & 104.0 & 983.17 & 11.5 & 982.66 & 78.0 & 980.33 & 13.0 & 982.45 \\
\hline 17.0 & 981.86 & 106.5 & 983.17 & 13.0 & 982.57 & 81.0 & 980.18 & 15.0 & 981.93 \\
\hline 20.0 & 982.06 & 108.0 & 983.35 & 14.5 & 982.04 & 84.0 & 980.11 & 17.0 & 981.95 \\
\hline 21.0 & 981.90 & 110.0 & 983.41 & 15.5 & 981.85 & 86.0 & 980.26 & 19.0 & 982.05 \\
\hline 23.0 & 981.88 & & & 18.0 & 981.91 & 88.0 & 980.46 & 21.0 & 981.99 \\
\hline 24.5 & 981.89 & & & 20.0 & 982.06 & 89.5 & 980.47 & 23.0 & 981.97 \\
\hline 25.4 & 981.72 & & & 21.5 & 981.92 & 91.2 & 980.54 & 24.0 & 981.94 \\
\hline 25.6 & 981.06 & & & 23.0 & 981.89 & 92.0 & 980.62 & 24.7 & 981.71 \\
\hline 26.0 & 980.69 & & & 24.5 & 981.87 & 92.5 & 980.54 & 25.0 & 981.12 \\
\hline 28.7 & 979.99 & & & 25.0 & 981.67 & 95.0 & 980.92 & 25.9 & 980.80 \\
\hline 29.5 & 979.89 & & & 25.0 & 981.20 & 96.6 & 981.83 & 28.0 & 980.20 \\
\hline 32.0 & 980.23 & & & 25.5 & 980.86 & 96.7 & 983.17 & 30.0 & 979.90 \\
\hline 35.0 & 980.33 & & & 26.3 & 980.69 & 98.5 & 983.19 & 32.0 & 980.00 \\
\hline 38.0 & 980.43 & & & 27.3 & 980.39 & 100.0 & 983.14 & 35.0 & 980.37 \\
\hline 41.0 & 980.37 & & & 29.0 & 979.91 & 100.7 & 983.18 & 38.0 & 980.42 \\
\hline 44.0 & 980.42 & & & 30.0 & 979.89 & 103.0 & 983.18 & 41.0 & 980.25 \\
\hline 47.0 & 980.46 & & & 32.0 & 980.25 & 105.5 & 983.10 & 44.0 & 980.37 \\
\hline 50.0 & 980.52 & & & 34.0 & 980.30 & 108.0 & 983.36 & 47.0 & 980.50 \\
\hline 53.0 & 980.51 & & & 36.0 & 980.46 & 110.0 & 983.40 & 50.0 & 980.54 \\
\hline 56.0 & 980.47 & & & 38.0 & 980.42 & & & 53.0 & 980.54 \\
\hline 58.0 & 980.47 & & & 40.0 & 980.26 & & & 56.0 & 980.51 \\
\hline 60.0 & 980.52 & & & 42.0 & 980.31 & & & 58.0 & 980.62 \\
\hline 62.0 & 980.65 & & & 44.0 & 980.39 & & & 59.7 & 980.75 \\
\hline 65.0 & 980.98 & & & 46.0 & 980.45 & & & 61.7 & 981.00 \\
\hline 67.0 & 981.02 & & & 48.0 & 980.43 & & & 64.0 & 981.02 \\
\hline 69.3 & 981.04 & & & 50.0 & 980.48 & & & 67.0 & 981.04 \\
\hline 71.5 & 980.68 & & & 52.0 & 980.44 & & & 68.0 & 981.02 \\
\hline 73.0 & 980.68 & & & 54.0 & 980.50 & & & 70.0 & 980.88 \\
\hline 75.7 & 980.56 & & & 56.0 & 980.47 & & & 72.0 & 980.78 \\
\hline 79.0 & 980.26 & & & 57.3 & 980.48 & & & 74.4 & 980.68 \\
\hline 82.0 & 980.17 & & & 58.8 & 980.64 & & & 77.0 & 980.40 \\
\hline 85.0 & 980.16 & & & 60.3 & 980.84 & & & 80.0 & 980.20 \\
\hline 88.0 & 980.46 & & & 61.4 & 980.86 & & & 83.0 & 980.23 \\
\hline 91.0 & 980.49 & & & 63.0 & 981.01 & & & 86.0 & 980.28 \\
\hline 93.0 & 980.55 & & & 64.9 & 980.97 & & & 89.0 & 980.55 \\
\hline
\end{tabular}


Table 18. (Continued)Listing of horizontal stations and elevations for cross section PR147

[Sta., station, distance in meters from a reference pin on the left bank; Elev., elevation, in meters above sea level]

1998

28 September

\begin{tabular}{rc}
\hline Sta. & Elev. \\
\hline 91.0 & 980.52 \\
92.8 & 980.69 \\
95.0 & 980.95 \\
97.0 & 982.23 \\
97.5 & 983.24 \\
100.0 & 983.15 \\
100.7 & 983.19 \\
104.0 & 983.17 \\
106.0 & 983.15 \\
107.5 & 983.32 \\
110.0 & 983.41
\end{tabular}




\section{Description of Cross Section PR151}

Location: Township 7 South/Range 49 East--section 15

U. S. Geological Survey quadrangle (1:24,000): Huckins School

Landowners--left bank: Gay Ranch

--right bank: Huckins Ranch

Access: Right bank

Permission from: Floyd and Dora Huckins

Distance from Moorhead Gaging Station: 36.27 kilometers

Azimuth of Section (degrees magnetic): 176.5

\section{Reference Monuments}

[Monuments at stations 100.0, 101.1, and 101.2 were closest to the leveling instrument]

\begin{tabular}{|c|c|c|c|c|c|c|}
\hline \multirow[b]{2}{*}{ Description } & \multirow[b]{2}{*}{$\begin{array}{c}\text { Station } \\
(m)\end{array}$} & \multicolumn{2}{|c|}{ GPS-NAD83 (1992) } & \multicolumn{2}{|c|}{ Measurement } & \multirow{2}{*}{$\begin{array}{c}\text { Elevation } \\
\text { (NGVD1929) } \\
\text { (m) }\end{array}$} \\
\hline & & Latitude & Longitude & $\begin{array}{l}\text { Standard } \\
\text { deviation } \\
\text { (m) }\end{array}$ & $\begin{array}{l}\text { Horizontal } \\
\text { precision } \\
\text { (m) }\end{array}$ & \\
\hline Benchmark--brass circular plate & -1.95 & $45^{\circ} 13^{\prime} 32,83^{\prime \prime}$ & $105^{\circ} 41^{\prime} 47.36^{\prime \prime}$ & 0.251 & 0.531 & 977.46 \\
\hline 1/2-inch-rebar; bent flat on 1998 ground level & -0.9 & & & & & 977.63 \\
\hline $\begin{array}{l}\text { 1/2-inch-rebar; } 0.02 \text { meter above } 1998 \\
\text { ground level }\end{array}$ & 0.0 & & & & & 977.54 \\
\hline $\begin{array}{l}\text { 1/2-inch-rebar; } 0.07 \text { meter above } 1998 \text { ground } \\
\text { level }\end{array}$ & 100.0 & & & & & 977.12 \\
\hline $\begin{array}{l}\text { 1/2-inch-rebar; } 0.02 \text { meter above } 1998 \text { ground } \\
\text { level }\end{array}$ & 101.1 & & & & & 976.98 \\
\hline $\begin{array}{l}\text { 1/2-inch-rebar; } 0.10 \text { meter above } 1998 \text { ground } \\
\text { level }\end{array}$ & 101.2 & $45^{\circ} 13^{\prime} 36.11^{\prime \prime}$ & $105^{\circ} 41^{\prime} 46.45^{\prime \prime}$ & 0.353 & 0.596 & 977.06 \\
\hline
\end{tabular}




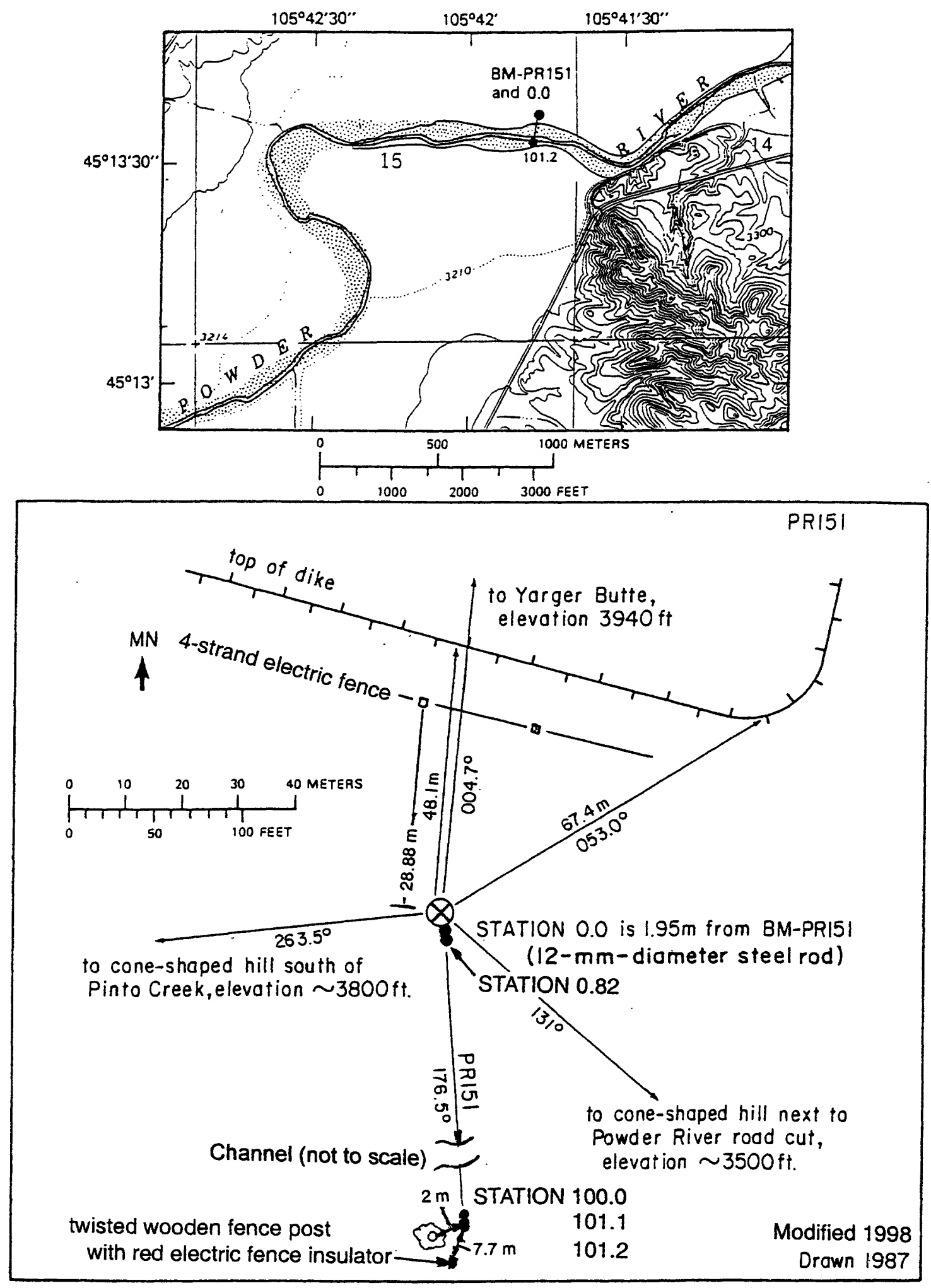

Figure 50. Upper: Location of cross section PR151, bench mark BM-PR151, and the right bank reference monument in the Huckins School quadrangle. Lower: Location of the bench mark on the left bank and reference monuments on the right bank. $\mathrm{MN}$ is magnetic north. 

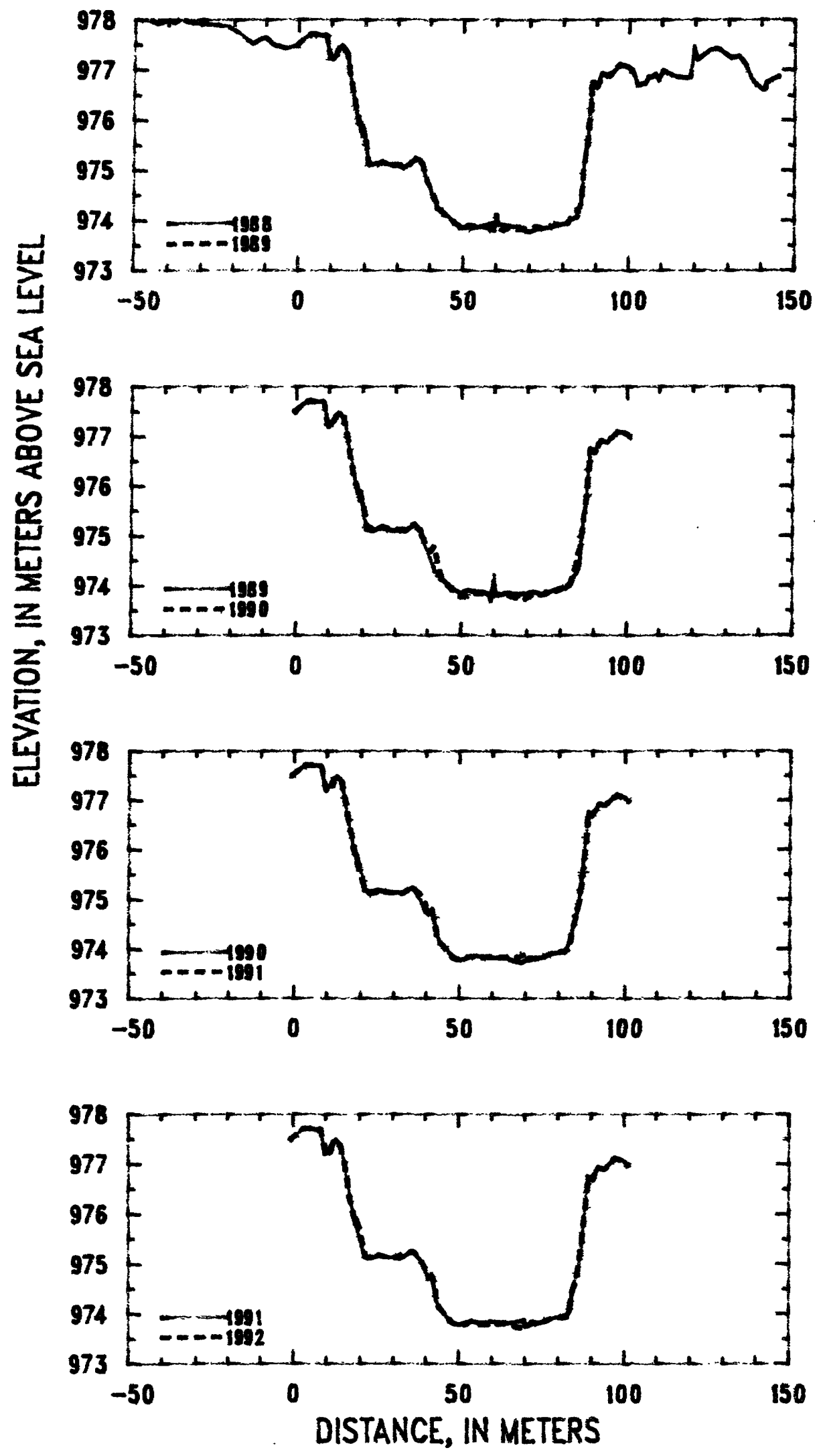

Figure 51. Profiles of cross section PR151 from 1988 to 1992. 

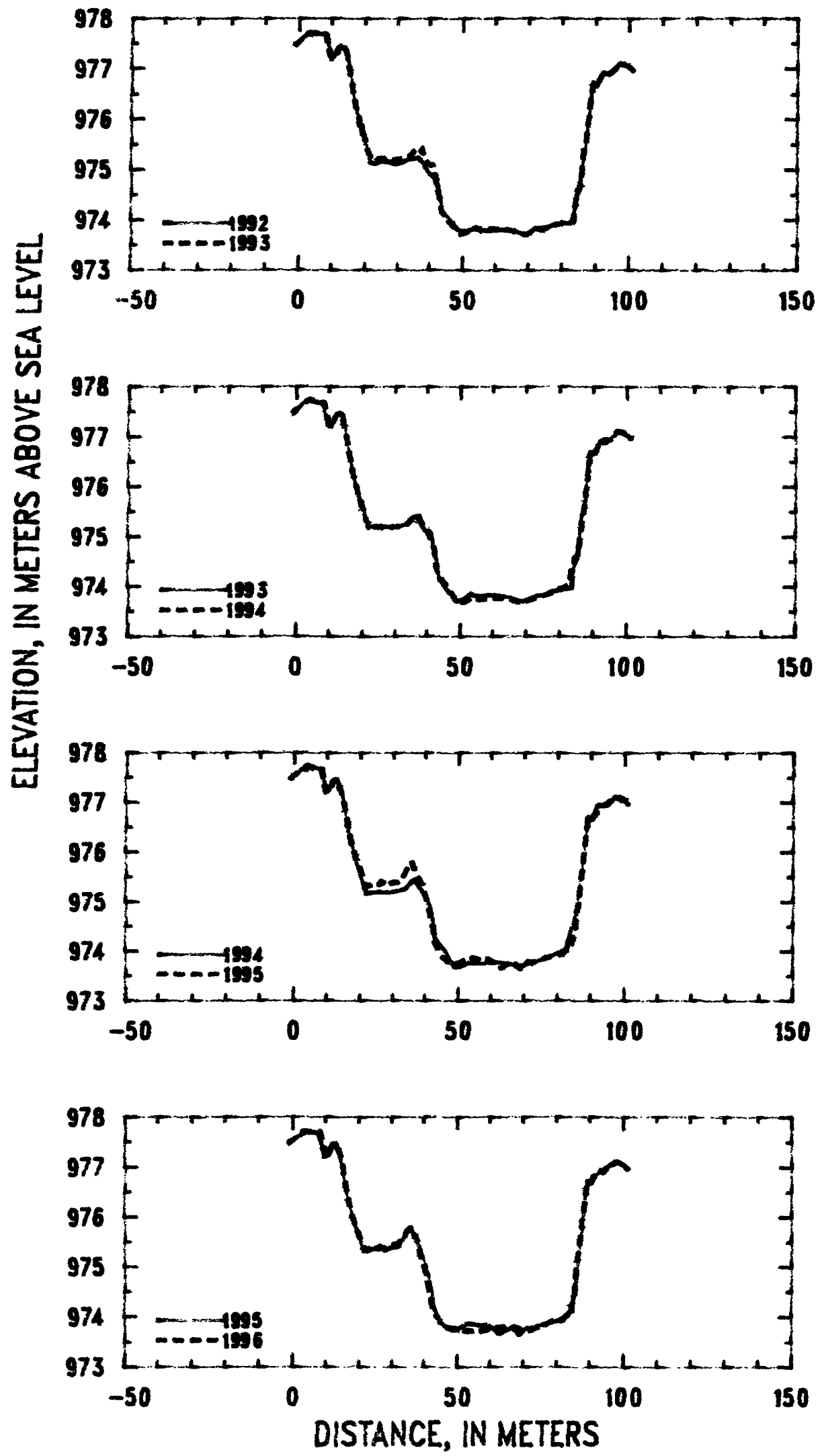

Figure 52. Profiles of cross section PR151 from 1992 to 1996. 


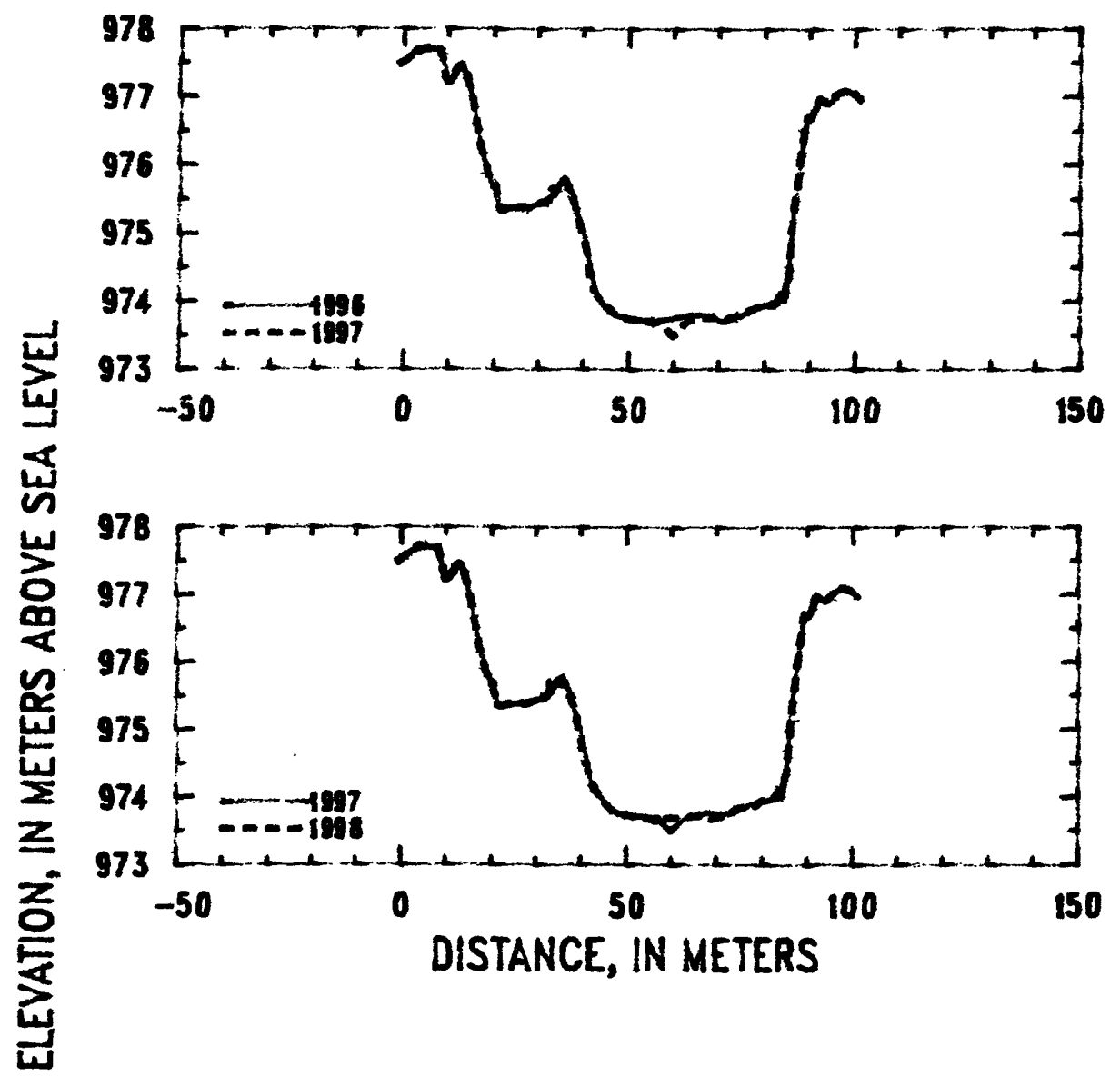

Figure 53. Profiles of cross section PR151 from 1996 to 1998. 
Table 19. Listing of horizontal stations and elevations for cross section PR151

[Sta., station, distance in meters from a reference pin on the left bank; Elev., elevation, in meters above sea level]

\begin{tabular}{|c|c|c|c|c|c|c|c|c|c|}
\hline \multirow{2}{*}{\multicolumn{2}{|c|}{$\begin{array}{c}1989 \\
19 \text { September }\end{array}$}} & \multirow{2}{*}{\multicolumn{2}{|c|}{$\begin{array}{c}1989 \\
19 \text { September }\end{array}$}} & \multirow{2}{*}{\multicolumn{2}{|c|}{$\begin{array}{c}1990 \\
\text { 19 September }\end{array}$}} & \multirow{2}{*}{\multicolumn{2}{|c|}{$\begin{array}{c}1990 \\
19 \text { September } \\
\end{array}$}} & \multirow{2}{*}{\multicolumn{2}{|c|}{$\begin{array}{c}1991 \\
30 \text { August }\end{array}$}} \\
\hline & & & & & & & & & \\
\hline Sta. & Elev. & Sta. & Elev. & Sta. & Elev. & Sta. & Elev. & Sta. & Elev. \\
\hline-0.8 & 977.48 & 62.0 & 973.81 & -0.8 & 977.49 & 65.0 & 973.80 & -0.9 & 977.50 \\
\hline 0.0 & 977.53 & 64.0 & 973.86 & 0.0 & 977.53 & 67.0 & 973.75 & 0.0 & 977.53 \\
\hline 2.0 & 977.65 & 66.0 & 973.84 & 3.0 & 977.70 & 69.0 & 973.73 & 3.0 & 977.71 \\
\hline 4.0 & 977.73 & 68.0 & 973.83 & 6.0 & 977.70 & 71.0 & 973.82 & 5.0 & 977.72 \\
\hline 6.0 & 977.69 & 70.0 & 973.87 & 8.3 & 977.69 & 73.0 & 973.82 & 7.0 & 977.67 \\
\hline 8.4 & 977.70 & 72.0 & 973.88 & 8.6 & 977.62 & 75.0 & 973.82 & 8.4 & 977.66 \\
\hline 9.7 & 977.19 & 74.0 & 973.83 & 9.4 & 977.25 & 77.0 & 973.90 & 9.7 & 977.20 \\
\hline 11.0 & 977.27 & 76.0 & 973.87 & 10.0 & 977.19 & 79.0 & 973.91 & 11.2 & 977.21 \\
\hline 12.0 & 977.43 & 78.0 & 973.92 & 12.0 & 977.43 & 81.0 & 973.96 & 12.0 & 977.42 \\
\hline 13.0 & 977.48 & 80.0 & 973.96 & 13.0 & 977.49 & 82.0 & 973.93 & 13.0 & 977.48 \\
\hline 14.5 & 977.35 & 81.5 & 973.94 & 14.1 & 977.42 & 83.0 & 974.06 & 14.3 & 977.38 \\
\hline 16.0 & 976.74 & 82.5 & 974.07 & 15.0 & 977.13 & 84.0 & 974.28 & 15.0 & 977.14 \\
\hline 18.0 & 976.03 & 83.5 & 974.12 & 17.0 & 976.31 & 85.0 & 974.58 & 18.0 & 976.02 \\
\hline 19.0 & 975.77 & 85.4 & 974.31 & 19.0 & 975.76 & 85.9 & 974.76 & 19.0 & 975.75 \\
\hline 19.2 & 975.85 & 85.7 & 974.43 & 19.2 & 975.84 & 86.9 & 975.38 & 21.3 & 975.17 \\
\hline 20.0 & 975.68 & 86.3 & 975.04 & 20.7 & 975.44 & 87.3 & 975.51 & 23.0 & 975.11 \\
\hline 21.0 & 975.12 & 87.0 & 975.41 & 21.1 & 975.19 & 89.0 & 976.75 & 26.0 & 975.17 \\
\hline 23.0 & 975.09 & 87.3 & 975.49 & 23.0 & 975.14 & 90.1 & 976.68 & 29.0 & 975.13 \\
\hline 25.0 & 975.16 & 88.5 & 976.32 & 25.0 & 975.18 & 92.0 & 976.91 & 32.0 & 975.10 \\
\hline 27.0 & 975.14 & 88.8 & 976.74 & 27.0 & 975.16 & 94.0 & 976.90 & 34.0 & 975.16 \\
\hline 29.0 & 975.10 & 89.8 & 976.74 & 29.0 & 975.13 & 96.0 & 977.03 & 35.0 & 975.23 \\
\hline 31.0 & 975.10 & 90.3 & 976.67 & 31.0 & 975.13 & 97.5 & 977.12 & 36.0 & 975.24 \\
\hline 33.0 & 975.08 & 92.0 & 976.92 & 33.0 & 975.12 & 99.0 & 977.07 & 38.0 & 975.13 \\
\hline 35.0 & 975.19 & 94.0 & 976.88 & 34.0 & 975.18 & 100.0 & 977.05 & 39.7 & 974.90 \\
\hline 36.3 & 975.22 & 96.0 & 977.04 & 36.0 & 975.23 & 101.2 & 976.96 & 40.8 & 974.70 \\
\hline 37.3 & 975.11 & 97.0 & 977.11 & 37.0 & 975.17 & & & 41.9 & 974.76 \\
\hline 37.7 & 975.13 & 98.5 & 977.07 & 38.0 & 975.04 & & & 42.6 & 974.59 \\
\hline 39.0 & 974.79 & 100.0 & 977.05 & 40.0 & 974.73 & & & 43.4 & 974.23 \\
\hline 41.7 & 974.40 & 101.2 & 976.96 & 40.9 & 974.69 & & & 44.4 & 974.12 \\
\hline 42.0 & 974.29 & & & 42.0 & 974.80 & & & 46.4 & 973.96 \\
\hline 43.8 & 974.14 & & & 43.0 & 974.43 & & & 48.0 & 973.82 \\
\hline 44.7 & 974.11 & & & 44.2 & 974.12 & & & 50.0 & 973.77 \\
\hline 46.0 & 974.03 & & & 45.3 & 974.08 & & & 52.0 & 973.82 \\
\hline 48.0 & 973.91 & & & 47.0 & 973.93 & & & 54.0 & 973.85 \\
\hline 50.0 & 973.85 & & & 49.0 & 973.78 & & & 56.0 & 973.81 \\
\hline 52.0 & 973.90 & & & 51.0 & 973.77 & & & 58.0 & 973.83 \\
\hline 54.0 & 973.87 & & & 53.0 & 973.84 & & & 60.0 & 973.85 \\
\hline 56.0 & 973.88 & & & 55.0 & 973.85 & & & 62.0 & 973.83 \\
\hline 58.0 & 973.83 & & & 57.0 & 973.85 & & & 64.0 & 973.83 \\
\hline 59.0 & 973.72 & & & 59.0 & 973.81 & & & 66.0 & 973.82 \\
\hline 60.0 & 974.12 & & & 61.0 & 973.82 & & & 68.0 & 973.86 \\
\hline 60.5 & 973.85 & & & 63.0 & 973.82 & & & 69.6 & 973.89 \\
\hline
\end{tabular}


Table 19. (Continued) Listing of horizontal stations and elevations for cross section PR151

[Sta., station, distance in meters from a reference pin on the left bank; Elev., elevation, in meters above sea level]

\begin{tabular}{|c|c|c|c|c|c|c|c|c|c|}
\hline \multirow{2}{*}{\multicolumn{2}{|c|}{$\begin{array}{c}1991 \\
30 \text { August }\end{array}$}} & \multirow{2}{*}{\multicolumn{2}{|c|}{$\begin{array}{c}1992 \\
31 \text { August }\end{array}$}} & \multirow{2}{*}{\multicolumn{2}{|c|}{$\begin{array}{c}1992 \\
31 \text { August }\end{array}$}} & \multirow{2}{*}{\multicolumn{2}{|c|}{$\begin{array}{c}1993 \\
29 \text { August }\end{array}$}} & \multirow{2}{*}{\multicolumn{2}{|c|}{$\begin{array}{c}1993 \\
29 \text { August }\end{array}$}} \\
\hline & & & & & & & & & \\
\hline Sta. & Elev. & Sta. & Elev. & Sta. & Elev. & Sta. & Elev. & Sta. & Elev. \\
\hline 70.1 & 973.76 & -0.9 & 977.48 & 72.0 & 973.84 & -0.9 & 977.48 & 59.0 & 973.83 \\
\hline 72.0 & 973.81 & 0.0 & 977.53 & 74.0 & 973.80 & 0.0 & 977.53 & 61.0 & 973.83 \\
\hline 74.0 & 973.81 & 3.0 & 977.71 & 76.0 & 973.86 & 2.0 & 977.64 & 63.0 & 973.82 \\
\hline 76.0 & 973.86 & 6.0 & 977.70 & 78.0 & 973.91 & 4.0 & 977.73 & 65.0 & 973.78 \\
\hline 78.0 & 973.92 & 8.5 & 977.69 & 80.0 & 973.94 & 6.0 & 977.70 & 67.0 & 973.73 \\
\hline 80.0 & 973.94 & 9.8 & 977.20 & 82.0 & 973.92 & 7.0 & 977.68 & 69.0 & 973.70 \\
\hline 81.5 & 973.96 & 11.0 & 977.28 & 83.0 & 973.95 & 8.3 & 977.68 & 71.0 & 973.76 \\
\hline 83.4 & 974.02 & 12.0 & 977.43 & 83.6 & 974.13 & 9.5 & 977.27 & 73.0 & 973.83 \\
\hline 84.2 & 974.36 & 14.0 & 977.42 & 84.5 & 974.52 & 10.4 & 977.20 & 75.0 & 973.83 \\
\hline 86.0 & 974.88 & 15.0 & 977.15 & 84.9 & 974.55 & 11.3 & 977.30 & 77.0 & 973.89 \\
\hline 87.3 & 975.52 & 17.0 & 976.32 & 85.5 & 974.74 & 13.0 & 977.48 & 79.0 & 973.92 \\
\hline 88.0 & 975.98 & 19.0 & 975.75 & 87.2 & 975.49 & 14.0 & 977.44 & 81.0 & 973.95 \\
\hline 88.9 & 976.75 & 19.3 & 975.83 & 89.2 & 976.73 & 15.0 & 977.11 & 83.0 & 973.97 \\
\hline 90.0 & 976.68 & 21.6 & 975.14 & 90.2 & 976.67 & 17.0 & 976.30 & 83.6 & 973.98 \\
\hline 92.0 & 976.92 & 23.0 & 975.11 & 92.0 & 976.92 & 19.0 & 975.74 & 83.8 & 974.15 \\
\hline 94.0 & 976.90 & 25.0 & 975.18 & 94.0 & 976.89 & 19.8 & 975.66 & 84.5 & 974.59 \\
\hline 96.0 & 977.05 & 27.0 & 975.15 & 96.0 & 977.04 & 20.9 & 975.38 & 85.6 & 974.64 \\
\hline 98.0 & 977.10 & 29.0 & 975.12 & 97.0 & 977.11 & 22.0 & 975.19 & 86.4 & 975.30 \\
\hline 100.0 & 977.05 & 31.0 & 975.13 & 99.0 & 977.07 & 24.0 & 975.22 & 87.0 & 975.51 \\
\hline \multirow[t]{23}{*}{101.2} & 976.96 & 33.0 & 975.19 & 100.0 & 977.04 & 26.0 & 975.22 & 87.3 & 975.56 \\
\hline & & 35.0 & 975.23 & 101.2 & 976.96 & 28.0 & 975.19 & 88.6 & 976.47 \\
\hline & & 36.6 & 975.23 & & & 30.0 & 975.20 & 89.0 & 976.56 \\
\hline & & 38.0 & 975.13 & & & 32.0 & 975.21 & 89.1 & 976.71 \\
\hline & & 39.6 & 974.92 & & & 34.0 & 975.27 & 90.2 & 976.67 \\
\hline & & 41.0 & 974.88 & & & 35.0 & 975.34 & 92.0 & 976.93 \\
\hline & & 42.0 & 974.74 & & & 36.0 & 975.41 & 93.4 & 976.88 \\
\hline & & 42.5 & 974.68 & & & 37.0 & 975.42 & 95.0 & 976.95 \\
\hline & & 43.7 & 974.20 & & & 37.4 & 975.36 & 97.5 & 977.11 \\
\hline & & 44.1 & 974.13 & & & 37.7 & 975.40 & 100.0 & 977.04 \\
\hline & & 46.0 & 974.00 & & & 38.6 & 975.19 & 101.2 & 976.96 \\
\hline & & 48.0 & 973.82 & & & 40.0 & 975.09 & & \\
\hline & & 50.0 & 973.77 & & & 40.9 & 975.10 & & \\
\hline & & 52.0 & 973.79 & & & 42.2 & 974.68 & & \\
\hline & & 54.0 & 973.85 & & & 43.0 & 974.41 & & \\
\hline & & 56.0 & 973.79 & & & 43.6 & 974.17 & & \\
\hline & & 58.0 & 973.77 & & & 45.0 & 974.02 & & \\
\hline & & 60.0 & 973.81 & & & 47.0 & 973.87 & & \\
\hline & & 62.0 & 973.80 & & & 49.0 & 973.70 & & \\
\hline & & 64.0 & 973.80 & & & 51.0 & 973.74 & & \\
\hline & & 66.0 & 973.78 & & & 53.0 & 973.85 & & \\
\hline & & 68.0 & 973.73 & & & 55.0 & 973.80 & & \\
\hline & & 70.0 & 973.73 & & & 57.0 & 973.82 & & \\
\hline
\end{tabular}


Table 19. (Continued) Listing of horizontal stations and elevations for cross section PR151

[Sta., station, distance in meters from a reference pin on the left bank; Elev., elevation, in meters above sea level]

\begin{tabular}{|c|c|c|c|c|c|c|c|c|c|}
\hline \multirow{2}{*}{\multicolumn{2}{|c|}{$\begin{array}{c}1994 \\
\text { 20 September }\end{array}$}} & \multicolumn{2}{|c|}{1994} & \multicolumn{2}{|c|}{1995} & \multicolumn{2}{|c|}{1995} & \multicolumn{2}{|c|}{1996} \\
\hline & & $20 \mathrm{Se}$ & ember & $27 \mathrm{Se}$ & ember & $27 \mathrm{Se}$ & ember & 19 & tober \\
\hline Sta. & Elev. & Sta. & Elev. & Sta. & Elev. & Sta. & Elev. & Sta. & Elev. \\
\hline-0.8 & 977.48 & 85.5 & 974.63 & -0.8 & 977.49 & 71.0 & 973.78 & -0.9 & 977.49 \\
\hline 0.0 & 977.52 & 87.3 & 975.57 & 0.0 & 977.52 & 73.0 & 973.78 & 0.0 & 977.52 \\
\hline 2.0 & 977.65 & 89.1 & 976.71 & 2.0 & 977.63 & 75.0 & 973.83 & 3.0 & 977.71 \\
\hline 4.0 & 977.74 & 90.3 & 976.66 & 5.0 & 977.71 & 77.0 & 973.87 & 6.0 & 977.70 \\
\hline 6.0 & 977.70 & 92.0 & 976.95 & 8.3 & 977.68 & 79.0 & 973.90 & 8.4 & 977.70 \\
\hline 8.5 & 977.68 & 95.0 & 976.94 & 9.7 & 977.21 & 81.0 & 973.93 & 10.0 & 977.20 \\
\hline 10.0 & 977.20 & 97.0 & 977.12 & 11.0 & 977.25 & 83.0 & 974.02 & 11.0 & 977.26 \\
\hline 11.0 & 977.25 & 99.0 & 977.09 & 12.0 & 977.44 & 83.5 & 974.02 & 12.0 & 977.43 \\
\hline 12.0 & 977.43 & 100.0 & 977.05 & 13.2 & 977.48 & 84.5 & 974.18 & 13.0 & 977.48 \\
\hline 13.0 & 977.47 & 101.1 & 976.97 & 15.0 & 977.04 & 85.5 & 974.50 & 14.6 & 977.24 \\
\hline 14.0 & 977.34 & 101.2 & 976.97 & 17.0 & 976.30 & 87.9 & 975.91 & 16.5 & 976.49 \\
\hline 15.0 & 977.07 & & & 19.0 & 975.79 & 89.2 & 976.73 & 18.0 & 976.02 \\
\hline 17.0 & 976.28 & & & 20.0 & 975.67 & 90.4 & 976.68 & 20.0 & 975.66 \\
\hline 19.0 & 975.76 & & & 21.5 & 975.31 & 93.0 & 976.93 & 21.7 & 975.33 \\
\hline 22.0 & 975.17 & & & 23.0 & 975.33 & 96.0 & 977.05 & 24.0 & 975.39 \\
\hline 25.0 & 975.21 & & & 25.0 & 975.38 & 97.5 & 977.11 & 26.0 & 975.39 \\
\hline 28.0 & 975.19 & & & 26.0 & 975.35 & 100.0 & 977.05 & 28.0 & 975.38 \\
\hline 31.0 & 975.21 & & & 26.5 & 975.42 & 101.1 & 976.97 & 30.0 & 975.43 \\
\hline 34.0 & 975.26 & & & 28.0 & 975.32 & 101.2 & 976.97 & 32.0 & 975.47 \\
\hline 36.0 & 975.40 & & & 30.0 & 975.39 & & & 34.0 & 975.60 \\
\hline 36.8 & 975.45 & & & 32.0 & 975.40 & & & 35.0 & 975.74 \\
\hline 38.0 & 975.33 & & & 34.0 & 975.59 & & & 36.0 & 975.79 \\
\hline 40.0 & 975.12 & & & 35.0 & 975.75 & & & 37.0 & 975.65 \\
\hline 42.0 & 974.82 & & & 36.0 & 975.80 & & & 38.0 & 975.55 \\
\hline 43.0 & 974.27 & & & 37.0 & 975.60 & & & 38.5 & 975.38 \\
\hline 43.5 & 974.21 & & & 39.0 & 975.39 & & & 40.0 & 975.03 \\
\hline 46.0 & 974.01 & & & 41.0 & 974.94 & & & 41.0 & 974.75 \\
\hline 49.0 & 973.68 & & & 42.6 & 974.40 & & & 42.0 & 974.40 \\
\hline 51.0 & 973.69 & & & 43.0 & 974.19 & & & 43.0 & 974.10 \\
\hline 53.0 & 973.77 & & & 45.0 & 973.90 & & & 43.4 & 974.07 \\
\hline 56.0 & 973.75 & & & 47.0 & 973.79 & & & 45.0 & 973.90 \\
\hline 59.0 & 973.77 & & & 49.0 & 973.73 & & & 47.0 & 973.79 \\
\hline 62.0 & 973.78 & & & 51.0 & 973.79 & & & 50.0 & 973.76 \\
\hline 65.0 & 973.76 & & & 53.0 & 973.88 & & & 53.0 & 973.72 \\
\hline 68.0 & 973.68 & & & 55.0 & 973.86 & & & 56.0 & 973.71 \\
\hline 71.0 & 973.74 & & & 57.0 & 973.81 & & & 59.0 & 973.74 \\
\hline 74.0 & 973.80 & & & 59.0 & 973.85 & & & 62.0 & 973.79 \\
\hline 77.0 & 973.86 & & & 61.0 & 973.78 & & & 65.0 & 973.81 \\
\hline 80.0 & 973.96 & & & 63.0 & 973.65 & & & 68.0 & 973.79 \\
\hline 82.0 & 974.01 & & & 65.0 & 973.74 & & & 71.0 & 973.70 \\
\hline 83.0 & 974.07 & & & 67.0 & 973.79 & & & 74.0 & 973.78 \\
\hline 83.4 & 974.33 & & & 69.0 & 973.65 & & & 77.0 & 973.86 \\
\hline
\end{tabular}


Table 19. (Continued) Listing of horizontal stations and elevations for cross section PR151

[Sta., station, distance in meters from a reference pin on the left bank; Elev., elevation, in meters above sea level]

\begin{tabular}{|c|c|c|c|c|c|c|c|c|c|}
\hline \multirow{2}{*}{\multicolumn{2}{|c|}{$\begin{array}{c}1996 \\
19 \text { October }\end{array}$}} & \multicolumn{2}{|c|}{1997} & \multicolumn{2}{|c|}{1997} & \multicolumn{2}{|c|}{1998} & \multicolumn{2}{|c|}{1998} \\
\hline & & \multicolumn{2}{|c|}{23 September } & \multicolumn{2}{|c|}{23 September } & \multicolumn{2}{|c|}{23 September } & \multicolumn{2}{|c|}{23 September } \\
\hline Sta. & Elev. & Sta. & Elev. & Sta. & Elev. & Sta. & Elev. & Sta. & Elev. \\
\hline 80.0 & 973.96 & -0.9 & 977.49 & 69.0 & 973.77 & -0.9 & 977.49 & 73.0 & 973.77 \\
\hline 82.0 & 973.93 & 0.0 & 977.52 & 72.0 & 973.75 & 0.0 & 977.52 & 75.0 & 973.85 \\
\hline 83.2 & 974.06 & 2.0 & 977.64 & 75.0 & 973.82 & 2.0 & 977.64 & 77.0 & 973.84 \\
\hline 83.8 & 974.13 & 5.0 & 977.72 & 78.0 & 973.91 & 4.0 & 977.74 & 79.0 & 973.90 \\
\hline 84.5 & 974.11 & 8.0 & 977.69 & 81.0 & 973.95 & 6.0 & 977.69 & 80.0 & 973.96 \\
\hline 85.6 & 974.51 & 8.5 & 977.64 & 82.8 & 973.99 & 8.3 & 977.69 & 82.0 & 973.96 \\
\hline 86.0 & 974.95 & 9.5 & 977.26 & 83.5 & 974.11 & 10.0 & 977.21 & 83.0 & 974.02 \\
\hline 88.8 & 976.41 & 10.0 & 977.21 & 84.2 & 974.01 & 11.3 & 977.28 & 84.2 & 974.03 \\
\hline 89.3 & 976.73 & 10.5 & 977.22 & 85.6 & 974.42 & 12.4 & 977.45 & 84.7 & 974.19 \\
\hline 90.3 & 976.69 & 12.0 & 977.43 & 86.0 & 975.04 & 13.5 & 977.43 & 85.4 & 974.35 \\
\hline 92.0 & 976.95 & 13.0 & 977.47 & 87.5 & 975.78 & 15.0 & 977.11 & 87.0 & 975.50 \\
\hline 94.0 & 976.90 & 14.0 & 977.35 & 89.1 & 976.70 & 17.0 & 976.31 & 89.2 & 976.72 \\
\hline 96.0 & 977.05 & 15.0 & 977.05 & 90.2 & 976.69 & 19.0 & 975.82 & 90.3 & 976.67 \\
\hline 98.0 & 977.11 & 17.0 & 976.32 & 92.0 & 976.98 & 21.0 & 975.50 & 92.0 & 977.00 \\
\hline 100.0 & 977.05 & 19.0 & 975.82 & 94.0 & 976.91 & 22.0 & 975.35 & 94.0 & 976.90 \\
\hline 101.1 & 976.97 & 20.0 & 975.67 & 96.0 & 977.05 & 24.0 & 975.40 & 97.0 & 977.11 \\
\hline 101.2 & 976.97 & 20.5 & 975.70 & 98.0 & 977.10 & 26.0 & 975.40 & 100.0 & 977.05 \\
\hline & & 21.4 & 975.36 & 100.0 & 977.05 & 28.0 & 975.38 & 101.1 & 976.96 \\
\hline & & 23.0 & 975.37 & 101.1 & 976.97 & 30.0 & 975.45 & 101.2 & 976.95 \\
\hline & & 26.0 & 975.39 & 101.2 & 976.96 & 32.0 & 975.48 & & \\
\hline & & 28.0 & 975.38 & & & 33.7 & 975.58 & & \\
\hline & & 30.0 & 975.44 & & & 34.1 & 975.72 & & \\
\hline & & 32.0 & 975.46 & & & 34.5 & 975.69 & & \\
\hline & & 32.9 & 975.50 & & & 36.0 & 975.69 & & \\
\hline & & 33.2 & 975.67 & & & 37.5 & 975.59 & & \\
\hline & & 33.7 & 975.58 & & & 39.1 & 975.21 & & \\
\hline & & 35.0 & 975.74 & & & 40.7 & 974.63 & & \\
\hline & & 36.0 & 975.79 & & & 42.6 & 974.20 & & \\
\hline & & 37.0 & 975.66 & & & 45.0 & 973.94 & & \\
\hline & & 38.3 & 975.41 & & & 47.0 & 973.77 & & \\
\hline & & 40.0 & 974.94 & & & 49.0 & 973.74 & & \\
\hline & & 41.0 & 974.71 & & & 51.0 & 973.72 & & \\
\hline & & 41.5 & 974.41 & & & 53.0 & 973.68 & & \\
\hline & & 42.7 & 974.11 & & & 55.0 & 973.69 & & \\
\hline & & 45.0 & 973.95 & & & 57.0 & 973.62 & & \\
\hline & & 48.0 & 973.75 & & & 59.0 & 973.69 & & \\
\hline & & 51.0 & 973.73 & & & 61.0 & 973.70 & & \\
\hline & & 54.0 & 973.70 & & & 63.0 & 973.70 & & \\
\hline & & 57.0 & 973.69 & & & 65.0 & 973.72 & & \\
\hline & & 60.0 & 973.49 & & & 67.0 & 973.74 & & \\
\hline & & 63.0 & 973.71 & & & 69.0 & 973.68 & & \\
\hline & & 66.0 & 973.76 & & & 71.0 & 973.72 & & \\
\hline
\end{tabular}




\section{Description of Cross Section PR156A}

Location: Township 7 South/Range 49 East--section 12

U. S. Geological Survey quadrangle (1:24,000): Huckins School

Landowners--left bank: Gay Ranch

--right bank: Twin Butte Ranch

Access: Right bank

Permission from: Butch Samuelson

Distance from Moorhead Gaging Station: 40.24 kilometers

Azimuth of Section (degrees magnetic): 124.5

\section{Reference Monuments}

[Monuments at stations $130.0,131.0$, and 159.2 were closest to leveling instrument]

\begin{tabular}{|c|c|c|c|c|c|c|}
\hline \multirow[b]{2}{*}{ Description } & \multirow[b]{2}{*}{$\begin{array}{c}\text { Station } \\
(\mathrm{m})\end{array}$} & \multicolumn{2}{|c|}{ GPS-NAD83 (1992) } & \multicolumn{2}{|c|}{ Measurement } & \multirow{2}{*}{$\begin{array}{l}\text { Elevation } \\
\text { (NGVD1929) } \\
\text { (m) }\end{array}$} \\
\hline & & Latitude & Longitude & $\begin{array}{l}\text { Standard } \\
\text { deviation } \\
\text { (m) }\end{array}$ & $\begin{array}{l}\text { Horizontal } \\
\text { precision } \\
(\mathbf{m})\end{array}$ & \\
\hline $\begin{array}{l}\text { 1/2-inch-rebar; } 0.27 \text { meter above } 1998 \text { ground } \\
\text { level; on old fence line with no wire but } \\
\text { just post made from tree and riverward of a } \\
\text { dike }\end{array}$ & -141.6 & $45^{\circ} 14^{\prime} 52.42^{\prime \prime}$ & $105^{\circ} 40^{\prime} 05.27^{\prime \prime}$ & 0.416 & 0.660 & 975.93 \\
\hline $\begin{array}{l}\text { 1/2-inch-rebar; bent, } 0.07 \text { meter above } 1998 \\
\text { ground level; } 36.9 \text { meters upstream from } \\
\text { monument at station }-4.2 \text { on PR156 }\end{array}$ & -1.6 & $45^{\circ} 14^{\prime} 48.99^{\prime \prime}$ & $105^{\circ} 40^{\prime} 01.06^{\prime \prime}$ & 0.126 & 0.534 & 971.21 \\
\hline 1/2-inch-rebar; at 1998 ground level & 0.0 & & & & & 971.12 \\
\hline 1/2-inch-rebar; at 1998 ground level & 130.0 & & & & & 971.66 \\
\hline $\begin{array}{l}\text { 1/2-inch-rebar; bent, } 0.18 \text { meter above } 1998 \\
\text { ground level }\end{array}$ & 131.0 & $45^{\circ} 14^{\prime} 45.74^{\prime \prime}$ & $105^{\circ} 39^{\prime} 57.08^{\prime \prime}$ & 0.463 & 0.786 & 971.84 \\
\hline $\begin{array}{l}\text { 1/2-inch-rebar; } 0.11 \text { meter above } 1998 \text { ground } \\
\text { level; under a 3-strand barbed-wire fence }\end{array}$ & 159.2 & & & & & 971.95 \\
\hline Benchmark--brass circular plate; offsection & $\cdots$ & $45^{\circ} 14^{\prime} 44.90^{\prime \prime}$ & $105^{\circ} 39^{\prime} 56.56^{\prime \prime}$ & 1.241 & 0.637 & 972.17 \\
\hline
\end{tabular}




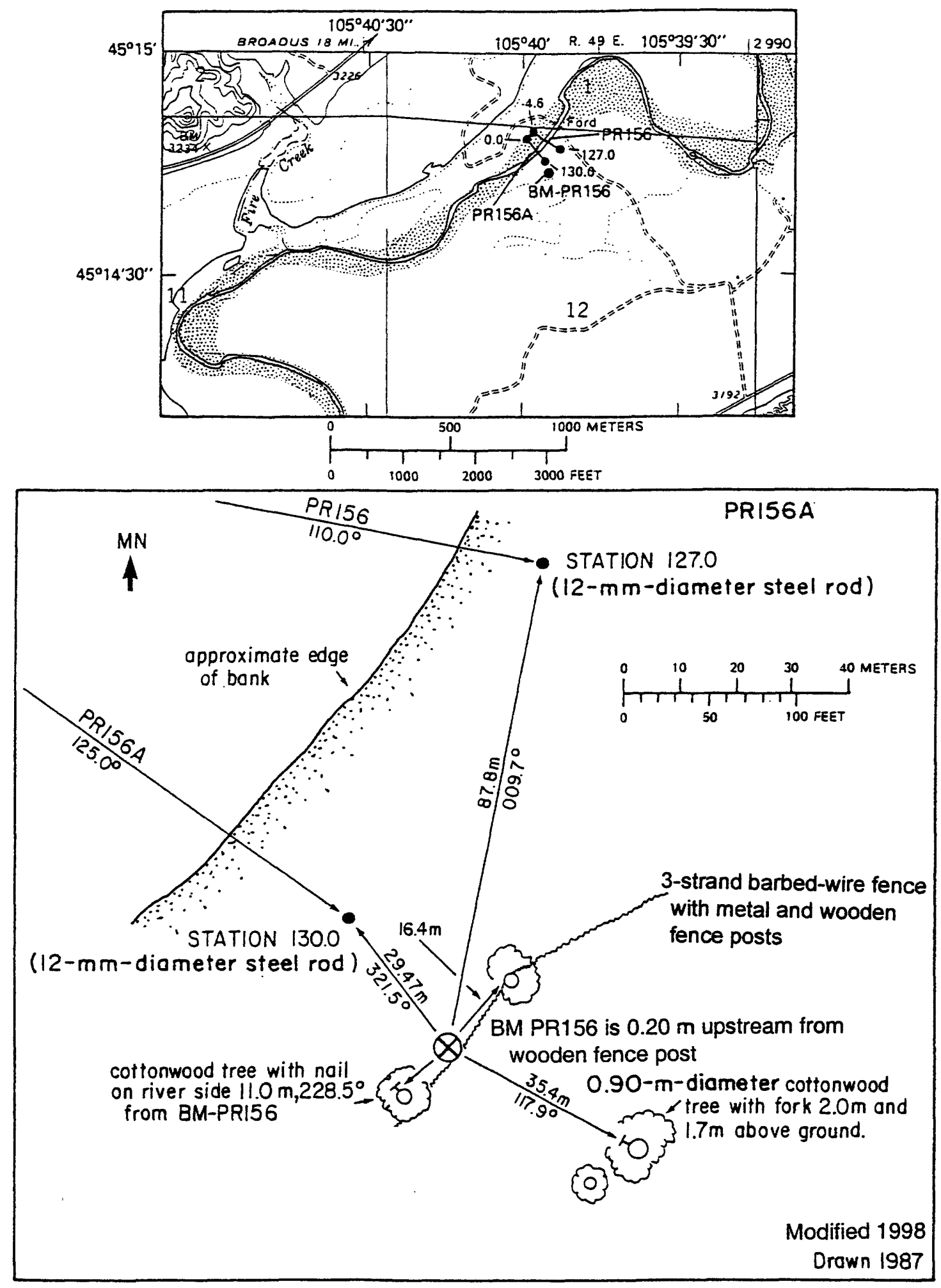

Figure 54. Upper: Location of cross section PR156A, bench mark BM-PR156, and the left and right bank reference monuments in the Huckins School quadrangle. Lower: Location of the bench mark on the left bank. MN is magnetic north. 

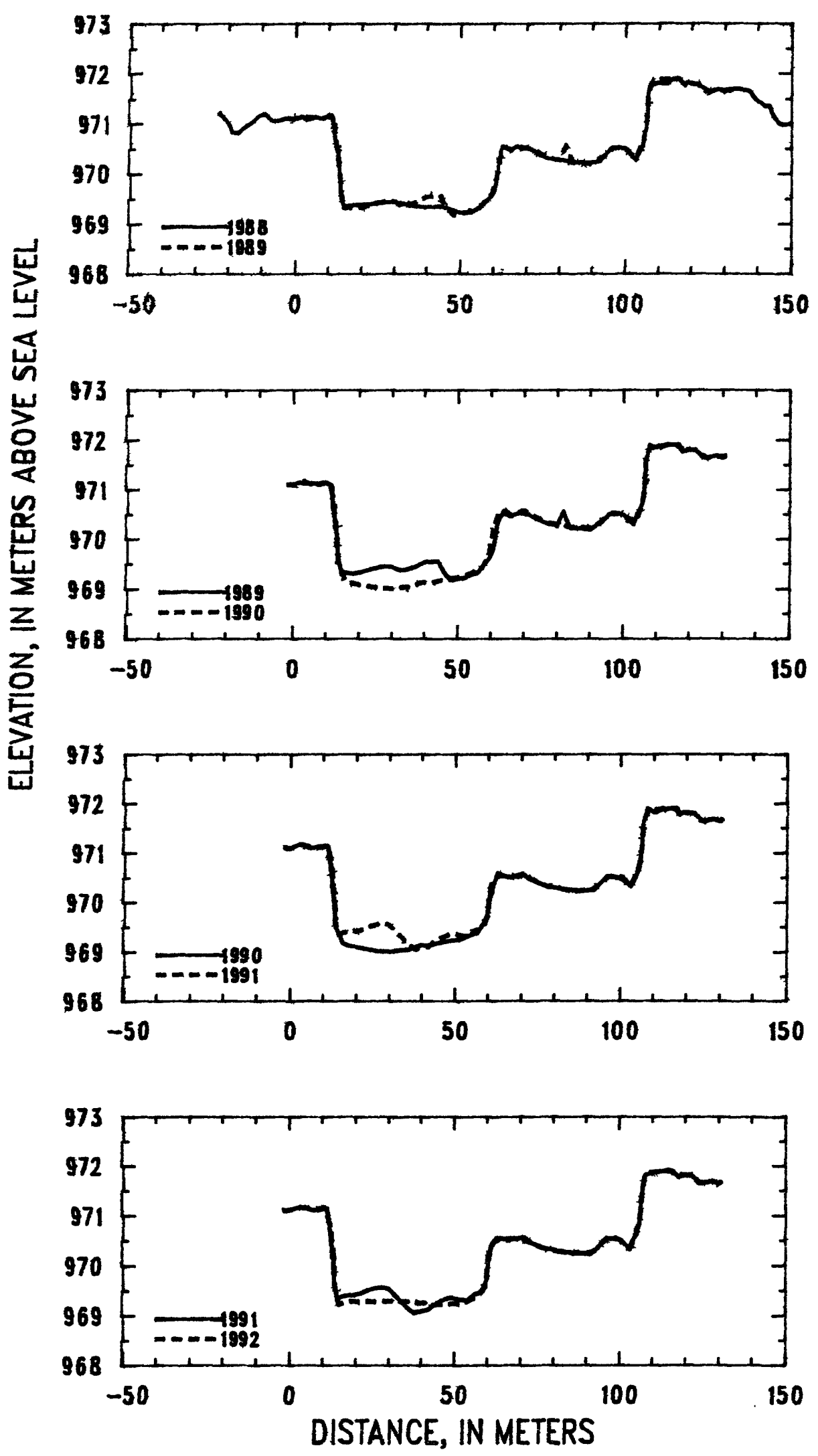

Figure 55. Profiles of cross section PR156A from 1988 to 1992. 

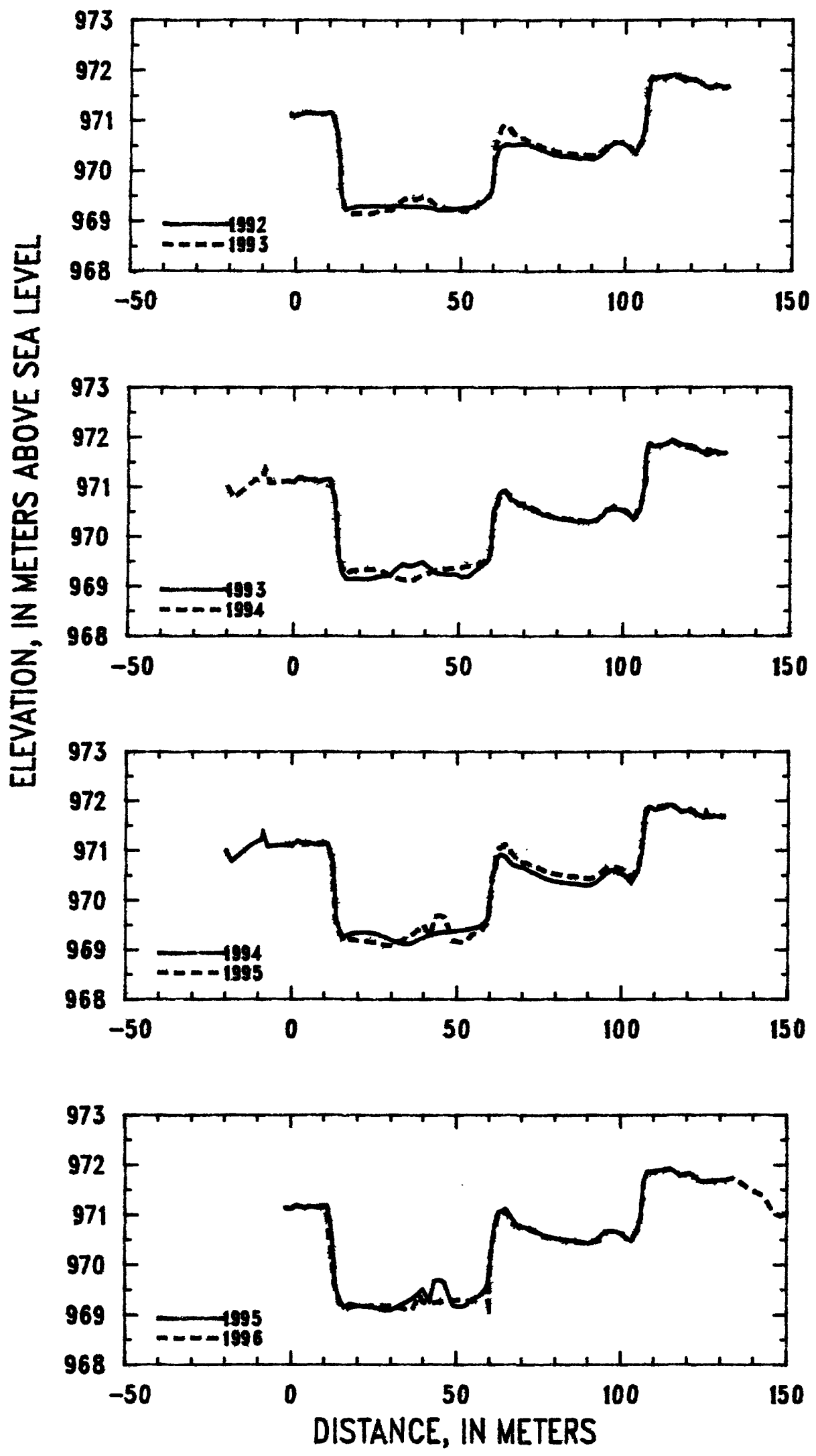

Figure 56. Profiles of cross section PR156A from 1992 to 1996. 


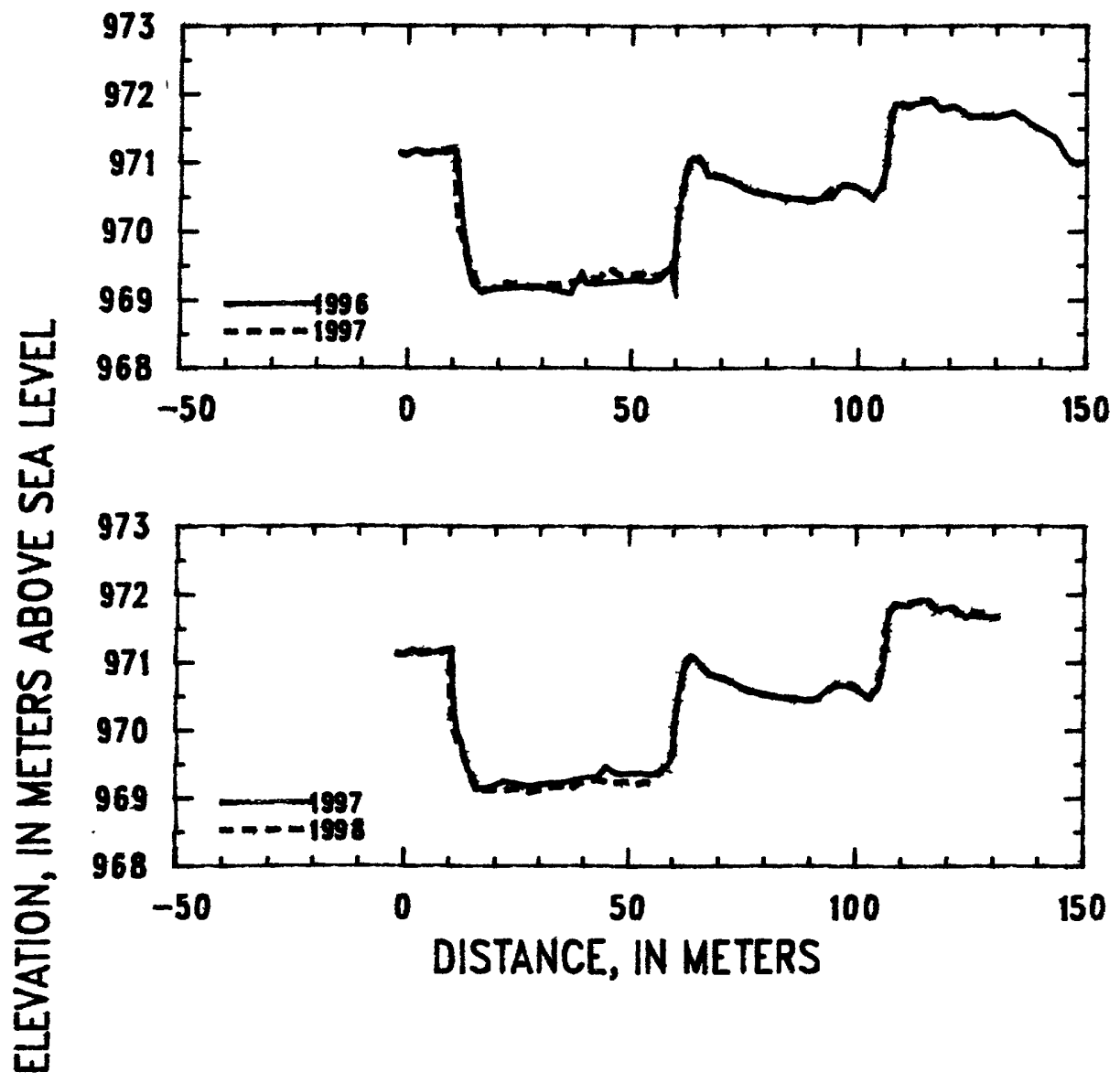

Figure 57. Profiles of cross section PR156A from 1996 to 1998. 
Table 20. Listing of horizontal stations and elevations for cross section PR156A

[Sta., station, distance in meters from a reference pin on the left bank; Elev., elevation, in meters above sea level]

\begin{tabular}{|c|c|c|c|c|c|c|c|c|c|}
\hline \multirow{2}{*}{\multicolumn{2}{|c|}{$\begin{array}{c}1989 \\
20 \text { September }\end{array}$}} & \multicolumn{2}{|c|}{1989} & \multicolumn{2}{|c|}{1990} & \multicolumn{2}{|c|}{1990} & \multicolumn{2}{|c|}{1991} \\
\hline & & $20 \mathrm{Se}$ & ember & $19 \mathrm{Se}$ & ember & $19 \mathrm{Se}$ & ember & 30 & gust \\
\hline Sta. & Elev. & Sta. & Elev. & Sta. & Elev. & Sta. & Elev. & Sta. & Elev. \\
\hline-1.6 & 971.10 & 66.0 & 970.47 & -1.6 & 971.10 & 76.0 & 970.36 & -1.6 & 971.11 \\
\hline 0.0 & 971.10 & 68.0 & 970.52 & 0.0 & 971.09 & 78.0 & 970.32 & 0.0 & 971.10 \\
\hline 2.0 & 971.14 & 70.0 & 970.52 & 1.0 & 971.13 & 80.0 & 970.30 & 3.0 & 971.17 \\
\hline 4.0 & 971.12 & 72.0 & 970.47 & 3.0 & 971.18 & 82.0 & 970.27 & 5.0 & 971.15 \\
\hline 6.0 & 971.14 & 74.0 & 970.41 & 5.0 & 971.12 & 84.0 & 970.26 & 7.0 & 971.10 \\
\hline 8.0 & 971.10 & 76.0 & 970.34 & 7.0 & 971.10 & 86.0 & 970.25 & 9.0 & 971.11 \\
\hline 10.0 & 971.15 & 78.0 & 970.30 & 9.0 & 971.13 & 88.0 & 970.24 & 10.0 & 971.13 \\
\hline 11.6 & 971.09 & 80.0 & 970.27 & 11.0 & 971.15 & 90.0 & 970.23 & 11.4 & 971.14 \\
\hline 12.3 & 970.63 & 82.0 & 970.55 & 11.5 & 971.13 & 92.0 & 970.27 & 12.4 & 970.77 \\
\hline 12.6 & 970.52 & 84.0 & 970.23 & 12.1 & 970.81 & 94.0 & 970.39 & 13.0 & 970.31 \\
\hline 12.9 & 970.28 & 86.0 & 970.22 & 12.4 & 970.72 & 95.0 & 970.47 & 13.4 & 970.10 \\
\hline 13.4 & 970.04 & 88.0 & 970.20 & 13.2 & 970.09 & 97.0 & 970.54 & 13.7 & 969.62 \\
\hline 13.8 & 969.61 & 90.0 & 970.18 & 13.3 & 970.03 & 99.0 & 970.52 & 13.9 & 969.53 \\
\hline 14.9 & 969.30 & 92.0 & 970.28 & 13.7 & 969.60 & 101.0 & 970.44 & 14.5 & 969.34 \\
\hline 16.0 & 969.34 & 94.0 & 970.37 & 14.0 & 969.47 & 103.1 & 970.33 & 16.0 & 969.38 \\
\hline 18.0 & 969.30 & 96.0 & 970.51 & 16.0 & 969.17 & 104.8 & 970.52 & 19.0 & 969.41 \\
\hline 20.0 & 969.32 & 98.0 & 970.52 & 18.0 & 969.11 & 105.8 & 970.81 & 22.0 & 969.46 \\
\hline 22.0 & 969.37 & 100.0 & 970.50 & 21.0 & 969.09 & 106.5 & 971.29 & 23.4 & 969.51 \\
\hline 24.0 & 969.41 & 102.0 & 970.35 & 24.0 & 969.05 & 106.7 & 971.59 & 26.0 & 969.56 \\
\hline 26.0 & 969.44 & 103.0 & 970.30 & 27.0 & 969.01 & 108.0 & 971.90 & 28.0 & 969.57 \\
\hline 28.0 & 969.45 & 104.0 & 970.46 & 30.0 & 969.00 & 110.0 & 971.82 & 30.0 & 969.55 \\
\hline 30.0 & 969.44 & 105.4 & 970.65 & 33.0 & 969.04 & 113.0 & 971.89 & 32.0 & 969.41 \\
\hline 32.0 & 969.38 & 106.0 & 970.94 & 36.0 & 969.05 & 117.0 & 971.91 & 34.0 & 969.28 \\
\hline 34.0 & 969.38 & 106.6 & 971.26 & 39.0 & 969.13 & 118.0 & 971.77 & 36.0 & 969.15 \\
\hline 36.0 & 969.42 & 106.7 & 971.51 & 42.0 & 969.13 & 119.0 & 971.81 & 38.0 & 969.06 \\
\hline 38.0 & 969.47 & 107.0 & 971.67 & 45.0 & 969.18 & 122.0 & 971.79 & 40.0 & 969.09 \\
\hline 40.0 & 969.54 & 108.0 & 971.84 & 48.0 & 969.22 & 125.0 & 971.63 & 42.0 & 969.11 \\
\hline 42.0 & 969.55 & 110.0 & 971.87 & 51.0 & 969.24 & 128.0 & 971.68 & 44.0 & 969.21 \\
\hline 44.0 & 969.56 & 112.0 & 971.88 & 54.0 & 969.32 & 130.0 & 971.64 & 46.0 & 969.29 \\
\hline 46.0 & 969.31 & 114.0 & 971.91 & 57.0 & 969.40 & 130.9 & 971.68 & 48.0 & 969.35 \\
\hline 48.0 & 969.17 & 116.0 & 971.91 & 59.0 & 969.60 & & & 50.0 & 969.35 \\
\hline 50.0 & 969.20 & 118.0 & 971.77 & 59.8 & 969.77 & & & 52.0 & 969.32 \\
\hline 52.0 & 969.23 & 120.0 & 971.80 & 60.6 & 970.17 & & & 54.0 & 969.30 \\
\hline 54.0 & 969.29 & 122.0 & 971.79 & 60.9 & 970.14 & & & 56.0 & 969.42 \\
\hline 56.0 & 969.33 & 124.0 & 971.65 & 62.0 & 970.43 & & & 58.0 & 969.46 \\
\hline 58.0 & 969.48 & 126.0 & 971.65 & 63.0 & 970.59 & & & 58.5 & 969.51 \\
\hline 59.7 & 969.60 & 128.0 & 971.69 & 64.0 & 970.56 & & & 59.7 & 969.65 \\
\hline 60.0 & 969.69 & 130.0 & 971.64 & 66.0 & 970.51 & & & 60.5 & 970.16 \\
\hline 60.8 & 969.80 & 130.9 & 971.67 & 68.0 & 970.53 & & & 61.3 & 970.42 \\
\hline 62.0 & 970.29 & & & 70.0 & 970.58 & & & 62.0 & 970.47 \\
\hline 62.7 & 970.44 & & & 72.0 & 970.49 & & & 63.0 & 970.55 \\
\hline 64.0 & 970.53 & & & 74.0 & 970.44 & & & 65.0 & 970.53 \\
\hline
\end{tabular}


Table 20. (Continued) Listing of horizontal stations and elevations for cross section PR156A

[Sta., station, distance in meters from a reference pin on the left bank; Elev., elevation, in meters above sea level]

\begin{tabular}{|c|c|c|c|c|c|c|c|c|c|}
\hline \multirow{2}{*}{\multicolumn{2}{|c|}{$\begin{array}{c}1991 \\
30 \text { August }\end{array}$}} & \multirow{2}{*}{\multicolumn{2}{|c|}{$\begin{array}{c}1992 \\
31 \text { August }\end{array}$}} & \multirow{2}{*}{\multicolumn{2}{|c|}{$\begin{array}{c}1992 \\
31 \text { August }\end{array}$}} & \multirow{2}{*}{\multicolumn{2}{|c|}{$\begin{array}{c}1993 \\
29 \text { August }\end{array}$}} & \multirow{2}{*}{\multicolumn{2}{|c|}{$\begin{array}{c}1993 \\
29 \text { August }\end{array}$}} \\
\hline & & & & & & & & & \\
\hline Sta. & Elev. & Sta. & Elev. & Sta. & Elev. & Sta. & Elev. & Sta. & Elev. \\
\hline 67.0 & 970.54 & -1.6 & 971.10 & 72.0 & 970.48 & -1.6 & 971.12 & 61.4 & 970.58 \\
\hline 69.0 & 970.52 & 0.0 & 971.10 & 74.0 & 970.43 & 0.0 & 971.10 & 62.4 & 970.86 \\
\hline 71.0 & 970.57 & 3.0 & 971.16 & 76.0 & 970.37 & 2.0 & 971.20 & 63.5 & 970.92 \\
\hline 73.0 & 970.46 & 6.0 & 971.14 & 78.0 & 970.32 & 3.0 & 971.16 & 64.3 & 970.91 \\
\hline 75.0 & 970.40 & 9.0 & 971.14 & 80.0 & 970.29 & 5.0 & 971.15 & 65.7 & 970.75 \\
\hline 77.0 & 970.34 & 10.5 & 971.16 & 82.0 & 970.28 & 7.0 & 971.11 & 67.0 & 970.73 \\
\hline 79.0 & 970.32 & 11.0 & 971.14 & 84.0 & 970.27 & 9.0 & 971.13 & 68.0 & 970.64 \\
\hline 81.0 & 970.29 & 12.2 & 970.83 & 86.0 & 970.24 & 10.0 & 971.15 & 69.0 & 970.66 \\
\hline 83.0 & 970.26 & 12.5 & 970.79 & 88.0 & 970.26 & 11.2 & 971.17 & 70.0 & 970.62 \\
\hline 85.0 & 970.26 & 13.5 & 970.07 & 90.0 & 970.23 & 12.0 & 970.90 & 72.0 & 970.57 \\
\hline 87.0 & 970.23 & 13.7 & 969.62 & 92.0 & 970.27 & 12.4 & 970.82 & 74.0 & 970.49 \\
\hline 89.0 & 970.24 & 15.0 & 969.22 & 94.0 & 970.40 & 13.3 & 970.17 & 76.0 & 970.43 \\
\hline 91.0 & 970.22 & 17.0 & 969.26 & 96.0 & 970.53 & 13.6 & 969.69 & 78.0 & 970.40 \\
\hline 92.0 & 970.26 & 19.0 & 969.29 & 98.0 & 970.56 & 14.7 & 969.27 & 80.0 & 970.37 \\
\hline 94.0 & 970.39 & 21.0 & 969.29 & 100.0 & 970.52 & 16.0 & 969.16 & 82.0 & 970.35 \\
\hline 96.0 & 970.53 & 23.0 & 969.30 & 102.0 & 970.37 & 17.0 & 969.14 & 84.0 & 970.33 \\
\hline 98.0 & 970.54 & 25.0 & 969.29 & 103.0 & 970.32 & 19.0 & 969.16 & 85.0 & 970.33 \\
\hline 100.0 & 970.52 & 27.0 & 969.27 & 104.0 & 970.46 & 21.0 & 969.15 & 86.0 & 970.31 \\
\hline 101.0 & 970.46 & 29.0 & 969.30 & 105.0 & 970.61 & 23.0 & 969.15 & 88.0 & 970.31 \\
\hline 103.0 & 970.32 & 31.0 & 969.30 & 105.8 & 970.82 & 25.0 & 969.19 & 90.0 & 970.30 \\
\hline 104.0 & 970.48 & 33.0 & 969.29 & 107.5 & 971.76 & 27.0 & 969.21 & 92.0 & 970.34 \\
\hline 105.0 & 970.59 & 35.0 & 969.28 & 108.3 & 971.86 & 29.0 & 969.22 & 94.0 & 970.44 \\
\hline 105.9 & 970.84 & 37.0 & 969.29 & 110.0 & 971.87 & 31.0 & 969.35 & 95.0 & 970.55 \\
\hline 107.0 & 971.64 & 39.0 & 969.26 & 112.0 & 971.88 & 33.0 & 969.45 & 96.0 & 970.56 \\
\hline 107.8 & 971.82 & 41.0 & 969.26 & 115.0 & 971.91 & 35.0 & 969.40 & 98.0 & 970.56 \\
\hline 110.0 & 971.86 & 43.0 & 969.20 & 118.0 & 971.78 & 37.0 & 969.44 & 99.0 & 970.55 \\
\hline 112.0 & 971.89 & 45.0 & 969.22 & 119.0 & 971.82 & 39.0 & 969.49 & 100.0 & 970.54 \\
\hline 114.0 & 971.90 & 47.0 & 969.22 & 122.0 & 971.79 & 41.0 & 969.38 & 101.0 & 970.48 \\
\hline 116.0 & 971.90 & 49.0 & 969.24 & 124.0 & 971.67 & 43.0 & 969.28 & 102.3 & 970.40 \\
\hline 118.0 & 971.77 & 51.0 & 969.26 & 126.0 & 971.65 & 45.0 & 969.26 & 103.0 & 970.34 \\
\hline 120.0 & 971.81 & 53.0 & 969.26 & 128.0 & 971.69 & 47.0 & 969.23 & 104.0 & 970.49 \\
\hline 122.0 & 971.80 & 55.0 & 969.32 & 130.0 & 971.64 & 49.0 & 969.25 & 104.9 & 970.54 \\
\hline 124.0 & 971.65 & 57.0 & 969.39 & 130.9 & 971.68 & 51.0 & 969.18 & 105.7 & 970.77 \\
\hline 126.0 & 971.65 & 59.0 & 969.52 & & & 53.0 & 969.19 & 106.1 & 970.96 \\
\hline 128.0 & 971.70 & 59.8 & 969.62 & & & 55.0 & 969.31 & 106.5 & 971.12 \\
\hline 130.0 & 971.64 & 60.2 & 970.10 & & & 57.0 & 969.40 & 106.8 & 971.63 \\
\hline \multirow[t]{6}{*}{130.9} & 971.68 & 60.6 & 970.26 & & & 59.1 & 969.51 & 107.4 & 971.80 \\
\hline & & 62.0 & 970.47 & & & 59.8 & 969.69 & 108.0 & 971.87 \\
\hline & & 64.0 & 970.53 & & & 60.0 & 969.74 & 109.0 & 971.82 \\
\hline & & 66.0 & 970.50 & & & 60.3 & 970.16 & 111.0 & 971.81 \\
\hline & & 68.0 & 970.54 & & & 60.6 & 970.47 & 113.0 & 971.87 \\
\hline & & 70.0 & 970.55 & & & 61.0 & 970.58 & 115.0 & 971.93 \\
\hline
\end{tabular}


Table 20. (Continued) Listing of horizontal stations and elevations for cross section PR156A

[Sta., station, distance in meters from a reference pin on the left bank; Elev., elevation, in meters above sea level]

\begin{tabular}{|c|c|c|c|c|c|c|c|c|c|}
\hline \multirow{2}{*}{\multicolumn{2}{|c|}{$\begin{array}{c}1993 \\
29 \text { August }\end{array}$}} & \multirow{2}{*}{\multicolumn{2}{|c|}{$\begin{array}{c}1994 \\
22 \text { September }\end{array}$}} & \multirow{2}{*}{\multicolumn{2}{|c|}{$\begin{array}{c}1994 \\
22 \text { September }\end{array}$}} & \multirow{2}{*}{\multicolumn{2}{|c|}{$\begin{array}{c}1995 \\
25 \text { September }\end{array}$}} & \multirow{2}{*}{\multicolumn{2}{|c|}{$\begin{array}{c}1995 \\
25 \text { September }\end{array}$}} \\
\hline & & & & & & & & & \\
\hline Sta. & Elev. & Sta. & Elev. & Sta. & Elev. & Sta. & Elev. & Sta. & Elev. \\
\hline 117.0 & 971.87 & -20.0 & 971.00 & 65.0 & 970.87 & -1.6 & 971.13 & 64.0 & 971.07 \\
\hline 119.0 & 971.84 & -18.0 & 970.79 & 66.0 & 970.76 & 0.0 & 971.12 & 65.0 & 971.12 \\
\hline 121.0 & 971.82 & -15.0 & 970.95 & 68.0 & 970.68 & 2.0 & 971.19 & 67.0 & 970.92 \\
\hline 123.0 & 971.73 & -12.0 & 971.12 & 70.0 & 970.67 & 4.0 & 971.14 & 69.0 & 970.76 \\
\hline 125.0 & 971.63 & -9.0 & 971.23 & 72.0 & 970.59 & 6.0 & 971.17 & 71.0 & 970.76 \\
\hline 127.5 & 971.71 & -8.5 & 971.37 & 75.0 & 970.50 & 8.0 & 971.14 & 73.0 & 970.68 \\
\hline 130.0 & 971.67 & -7.0 & 971.08 & 78.0 & 970.40 & 10.0 & 971.14 & 75.0 & 970.64 \\
\hline \multirow[t]{35}{*}{130.9} & 971.68 & -4.0 & 971.11 & 81.0 & 970.37 & 11.0 & 971.17 & 77.0 & 970.57 \\
\hline & & -1.6 & 971.12 & 84.0 & 970.34 & 12.0 & 970.85 & 79.0 & 970.54 \\
\hline & & 0.0 & 971.10 & 87.0 & 970.32 & 12.6 & 970.56 & 81.0 & 970.51 \\
\hline & & 2.0 & 971.20 & 90.0 & 970.29 & 12.8 & 970.22 & 83.0 & 970.49 \\
\hline & & 4.0 & 971.11 & 92.0 & 970.34 & 13.2 & 970.05 & 85.0 & 970.48 \\
\hline & & 6.0 & 971.14 & 94.0 & 970.44 & 13.3 & 969.67 & 87.0 & 970.47 \\
\hline & & 8.0 & 971.11 & 96.5 & 970.60 & 14.0 & 969.42 & 89.0 & 970.45 \\
\hline & & 10.0 & 971.14 & 97.0 & 970.61 & 16.0 & 969.15 & 91.0 & 970.43 \\
\hline & & 11.0 & 971.14 & 99.0 & 970.57 & 18.0 & 969.22 & 93.0 & 970.51 \\
\hline & & 12.0 & 970.89 & 101.0 & 970.51 & 20.0 & 969.18 & 95.0 & 970.63 \\
\hline & & 12.5 & 970.76 & 103.0 & 970.34 & 22.0 & 969.17 & 96.0 & 970.66 \\
\hline & & 13.3 & 970.16 & 104.6 & 970.51 & 24.0 & 969.16 & 98.0 & 970.68 \\
\hline & & 13.7 & 969.57 & 105.5 & 970.75 & 26.0 & 969.14 & 100.0 & 970.63 \\
\hline & & 15.5 & 969.25 & 106.6 & 971.14 & 28.0 & 969.09 & 101.0 & 970.57 \\
\hline & & 18.0 & 969.33 & 106.8 & 971.57 & 30.0 & 969.08 & 102.0 & 970.51 \\
\hline & & 21.0 & 969.35 & 107.4 & 971.82 & 32.0 & 969.15 & 103.5 & 970.48 \\
\hline & & 24.0 & 969.35 & 108.5 & 971.88 & 34.0 & 969.22 & 105.0 & 970.66 \\
\hline & & 27.0 & 969.29 & 110.0 & 971.83 & 36.0 & 969.29 & 106.0 & 970.99 \\
\hline & & 30.0 & 969.19 & 112.5 & 971.86 & 38.0 & 969.38 & 107.0 & 971.67 \\
\hline & & 33.0 & 969.13 & 115.0 & 971.92 & 40.0 & 969.51 & 108.0 & 971.85 \\
\hline & & 36.0 & 969.11 & 118.0 & 971.77 & 42.0 & 969.26 & 110.0 & 971.87 \\
\hline & & 39.0 & 969.22 & 120.0 & 971.80 & 43.6 & 969.67 & 112.0 & 971.89 \\
\hline & & 42.0 & 969.30 & 123.0 & 971.72 & 45.0 & 969.69 & 115.0 & 971.92 \\
\hline & & 45.0 & 969.35 & 125.0 & 971.67 & 46.5 & 969.65 & 118.0 & 971.78 \\
\hline & & 48.0 & 969.37 & 125.5 & 971.78 & 49.0 & 969.19 & 121.0 & 971.83 \\
\hline & & 51.0 & 969.39 & 126.0 & 971.68 & 51.0 & 969.15 & 124.0 & 971.67 \\
\hline & & 54.0 & 969.42 & 128.0 & 971.69 & 53.0 & 969.18 & 127.0 & 971.67 \\
\hline & & 57.0 & 969.46 & 130.0 & 971.66 & 55.0 & 969.32 & 130.9 & 971.69 \\
\hline & & 59.0 & 969.57 & 130.9 & 971.68 & 57.0 & 969.36 & & \\
\hline & & 59.7 & 969.63 & & & 59.4 & 969.53 & & \\
\hline & & 60.1 & 969.98 & & & 59.8 & 969.67 & & \\
\hline & & 61.0 & 970.55 & & & 60.9 & 970.44 & & \\
\hline & & 61.8 & 970.63 & & & 62.0 & 970.80 & & \\
\hline & & 62.4 & 970.87 & & & 62.2 & 970.93 & & \\
\hline & & 63.5 & 970.92 & & & 63.0 & 971.05 & & \\
\hline
\end{tabular}


Table 20. (Continued) Listing of horizontal stations and elevations for cross section PR156A

[Sta., station, distance in meters from a reference pin on the left bank; Elev., elevation, in meters above sea level]

\begin{tabular}{|c|c|c|c|c|c|c|c|c|c|}
\hline \multirow{2}{*}{\multicolumn{2}{|c|}{$\begin{array}{c}1996 \\
21 \text { October }\end{array}$}} & \multicolumn{2}{|c|}{1996} & \multicolumn{2}{|c|}{1996} & \multicolumn{2}{|c|}{1997} & \multicolumn{2}{|c|}{1997} \\
\hline & & 210 & tober & 21 & ober & $23 \mathrm{~S}$ & ember & $23 \mathrm{Se}$ & ember \\
\hline Sta. & Elev. & Sta. & Elev. & Sta. & Elev. & Sta. & Elev. & Sta. & Elev. \\
\hline-1.6 & 971.14 & 74.0 & 970.66 & 165.0 & 972.02 & -1.6 & 971.14 & 79.0 & 970.55 \\
\hline 0.0 & 971.12 & 76.0 & 970.59 & 170.0 & 971.98 & 0.0 & 971.12 & 81.0 & 970.53 \\
\hline 2.0 & 971.19 & 78.0 & 970.54 & 175.0 & 971.96 & 2.0 & 971.20 & 83.0 & 970.49 \\
\hline 4.0 & 971.14 & 80.0 & 970.54 & 180.0 & 972.00 & 4.0 & 971.13 & 85.0 & 970.47 \\
\hline 6.0 & 971.17 & 82.0 & 970.50 & 184.0 & 971.95 & 7.0 & 971.15 & 87.0 & 970.48 \\
\hline 8.0 & 971.15 & 84.0 & 970.46 & 188.0 & 971.70 & 9.0 & 971.20 & 89.0 & 970.43 \\
\hline 10.0 & 971.17 & 86.0 & 970.46 & 189.0 & 971.82 & 10.5 & 971.21 & 91.0 & 970.45 \\
\hline 10.7 & 971.18 & 88.0 & 970.44 & 193.0 & 971.87 & 10.7 & 971.05 & 93.0 & 970.54 \\
\hline 10.9 & 971.14 & 90.0 & 970.44 & 195.0 & 971.74 & 10.8 & 970.51 & $95.0^{\circ}$ & 970.66 \\
\hline 11.1 & 970.94 & 92.0 & 970.47 & 200.0 & 971.75 & 11.7 & 970.00 & 97.0 & 970.67 \\
\hline 12.8 & 969.90 & 94.0 & 970.49 & & & 12.9 & 969.81 & 99.0 & 970.66 \\
\hline 13.2 & 969.80 & 96.0 & 970.66 & & & 13.2 & 969.64 & 101.0 & 970.58 \\
\hline 13.3 & 969.59 & 98.0 & 970.68 & & & 16.0 & 969.13 & 103.0 & 970.48 \\
\hline 14.7 & 969.23 & 100.0 & 970.63 & & & 19.0 & 969.15 & 104.0 & 970.55 \\
\hline 16.5 & 969.11 & 102.0 & 970.52 & & & 22.0 & 969.25 & 105.0 & 970.64 \\
\hline 19.0 & 969.17 & 103.0 & 970.47 & & & 25.0 & 969.21 & 106.1 & 970.98 \\
\hline 22.0 & 969.17 & 104.0 & 970.57 & & & 28.0 & 969.17 & 106.5 & 971.24 \\
\hline 25.0 & 969.19 & 105.0 & 970.65 & & & 31.0 & 969.21 & 107.2 & 971.74 \\
\hline 28.0 & 969.18 & 106.0 & 970.94 & & & 34.0 & 969.22 & 108.5 & 971.87 \\
\hline 31.0 & 969.18 & 106.6 & 971.33 & & & 37.0 & 969.25 & 111.1 & 971.82 \\
\hline 34.0 & 969.12 & 107.0 & 971.68 & & & 40.0 & 969.31 & 114.0 & 971.93 \\
\hline 36.5 & 969.09 & 108.0 & 971.86 & & & 43.0 & 969.31 & 116.0 & 971.92 \\
\hline 39.0 & 969.39 & 109.0 & 971.85 & & & 45.0 & 969.46 & 118.0 & 971.77 \\
\hline 40.0 & 969.25 & 111.0 & 971.83 & & & 47.0 & 969.37 & 121.0 & 971.83 \\
\hline 42.0 & 969.23 & 113.0 & 971.89 & & & 49.0 & 969.36 & 124.0 & 971.66 \\
\hline 45.0 & 969.25 & 116.0 & 971.91 & & & 52.0 & 969.38 & 125.0 & 971.70 \\
\hline 48.0 & 969.28 & 118.0 & 971.78 & & & 55.0 & 969.35 & 127.0 & 971.68 \\
\hline 51.0 & 969.30 & 121.0 & 971.83 & & & 57.0 & 969.38 & 130.0 & 971.66 \\
\hline 54.0 & 969.27 & 124.0 & 971.67 & & & 59.0 & 969.48 & 130.9 & 971.67 \\
\hline 56.0 & 969.29 & 127.0 & 971.67 & & & 60.1 & 969.63 & & \\
\hline 59.0 & 969.49 & 130.0 & 971.66 & & & 60.6 & 970.15 & & \\
\hline 59.9 & 969.19 & 130.9 & 971.68 & & & 61.0 & 970.42 & & \\
\hline 60.0 & 969.67 & 134.0 & 971.73 & & & 62.0 & 970.77 & & \\
\hline 60.6 & 970.24 & 139.0 & 971.50 & & & 63.0 & 971.04 & & \\
\hline 62.0 & 970.80 & 143.0 & 971.37 & & & 64.0 & 971.11 & & \\
\hline 62.5 & 970.94 & 146.0 & 971.05 & & & 65.0 & 971.08 & & \\
\hline 63.0 & 971.04 & 148.0 & 970.97 & & & 67.0 & 970.88 & & \\
\hline 64.0 & 971.07 & 151.0 & 971.04 & & & 69.0 & 970.80 & & \\
\hline 66.0 & 970.99 & 155.0 & 971.28 & & & 71.0 & 970.78 & & \\
\hline 67.0 & 970.82 & 159.0 & 971.80 & & & 73.0 & 970.70 & & \\
\hline 70.0 & 970.77 & 159.2 & 971.83 & & & 75.0 & 970.63 & & \\
\hline 72.0 & 970.74 & 160.0 & 971.87 & & & 77.0 & 970.58 & & \\
\hline
\end{tabular}


Table 20. (Continued) Listing of horizontal stations and elevations for cross section PR156A

[Sta., station, distance in meters from a reference pin on the left bank; Elev., elevation, in meters above sea level]

\begin{tabular}{|c|c|c|c|}
\hline \multirow{2}{*}{\multicolumn{2}{|c|}{$\begin{array}{c}1998 \\
29 \text { September }\end{array}$}} & \multirow{2}{*}{\multicolumn{2}{|c|}{$\begin{array}{c}1998 \\
29 \text { September }\end{array}$}} \\
\hline & & & \\
\hline Sta. & Elev. & Sta. & Elev. \\
\hline-1.6 & 971.14 & 86.0 & 970.46 \\
\hline 0.0 & 971.12 & 88.0 & 970.44 \\
\hline 2.0 & 971.18 & 90.0 & 970.44 \\
\hline 4.0 & 971.16 & 92.0 & 970.47 \\
\hline 6.0 & 971.18 & 94.0 & 970.60 \\
\hline 8.0 & 971.14 & 96.0 & 970.69 \\
\hline 10.2 & 971.20 & 98.0 & 970.69 \\
\hline 10.3 & 970.68 & 100.0 & 970.65 \\
\hline 10.6 & 970.39 & 102.0 & 970.50 \\
\hline 10.7 & 970.12 & 103.0 & 970.48 \\
\hline 11.8 & 969.85 & 104.0 & 970.58 \\
\hline 13.0 & 969.69 & 105.8 & 970.94 \\
\hline 16.0 & 969.15 & 106.8 & 971.64 \\
\hline 19.0 & 969.12 & 107.3 & 971.76 \\
\hline 22.0 & 969.13 & 109.0 & 971.86 \\
\hline 25.0 & 969.14 & 112.3 & 971.88 \\
\hline 28.0 & 969.09 & 115.0 & 971.93 \\
\hline 31.0 & 969.15 & 117.6 & 971.77 \\
\hline 34.0 & 969.18 & 120.0 & 971.82 \\
\hline 37.0 & 969.13 & 123.0 & 971.71 \\
\hline 40.0 & 969.25 & 125.0 & 971.75 \\
\hline 43.0 & 969.28 & 128.0 & 971.72 \\
\hline 46.0 & 969.22 & 130.0 & 971.66 \\
\hline 49.0 & 969.26 & 130.9 & 971.68 \\
\hline 52.0 & 969.21 & & \\
\hline 55.0 & 969.26 & & \\
\hline 58.0 & 969.44 & & \\
\hline 60.2 & 969.68 & & \\
\hline 60.4 & 970.18 & & \\
\hline 61.5 & 970.53 & & \\
\hline 62.4 & 970.94 & & \\
\hline 64.0 & 971.07 & & \\
\hline 66.0 & 970.98 & & \\
\hline 68.0 & 970.83 & & \\
\hline 70.0 & 970.77 & & \\
\hline 72.0 & 970.74 & & \\
\hline 74.0 & 970.66 & & \\
\hline 76.0 & 970.60 & & \\
\hline 78.0 & 970.54 & & \\
\hline 80.0 & 970.53 & & \\
\hline 82.0 & 970.50 & & \\
\hline 84.0 & 970.48 & & \\
\hline
\end{tabular}




\section{Description of Cross Section PR156}

Location: Township 7 South/Range 49 East--section 12

U. S. Geological Survey quadrangle (1:24,000): Huckins School

Landowners--left bank: Gay Ranch

--right bank: Twin Butte Ranch

Access: Right bank

Permission from: Butch Samuelson

Distance from Moorhead Gaging Station: 40.28 kilometers

Azimuth of Section (degrees magnetic): 109.5

\section{Reference Monuments}

[Monuments at stations $127.0,140.0$, and 187.9 were closest to leveling instrument]

\begin{tabular}{|c|c|c|c|c|c|c|}
\hline \multirow[b]{2}{*}{ Description } & \multirow[b]{2}{*}{$\begin{array}{c}\text { Station } \\
\text { (m) }\end{array}$} & \multicolumn{2}{|c|}{ GPS-NAD83 (1992) } & \multicolumn{2}{|c|}{ Measurement } & \multirow{2}{*}{$\begin{array}{l}\text { Elevation } \\
\text { (NGVD1929) } \\
\text { (m) }\end{array}$} \\
\hline & & Latitude & Longitude & $\begin{array}{l}\text { Standard } \\
\text { deviation } \\
(\mathrm{m})\end{array}$ & $\begin{array}{l}\text { Horizontal } \\
\text { precision } \\
(\mathrm{m})\end{array}$ & \\
\hline $\begin{array}{l}\text { 1/2-inch-rebar; } 0.20 \text { meter above } 1998 \text { ground } \\
\text { level; } 36.9 \text { meters downstream from mon- } \\
\text { ument at station -1.6 on PR156A }\end{array}$ & -4.2 & $45^{\circ} 14^{\prime} 49.86^{\prime \prime}$ & $105^{\circ} 39^{\prime} 59.90^{\prime \prime}$ & 0.397 & 0.566 & 971.31 \\
\hline $\begin{array}{l}\text { 1/2-inch-rebar; } 0.01 \text { meter above } 1998 \text { ground } \\
\text { level }\end{array}$ & -1.6 & & & & & 971.05 \\
\hline $\begin{array}{l}\text { 1/2-inch-rebar; } 0.16 \text { meter above } 1998 \text { ground } \\
\text { level }\end{array}$ & 127.0 & & & & & 971.78 \\
\hline $\begin{array}{l}\text { 1/2-inch-rebar; } 0.19 \text { meter above } 1998 \text { ground } \\
\text { level }\end{array}$ & 140.0 & & & & & 971.74 \\
\hline $\begin{array}{l}\text { 1/2-inch-rebar; } 0.18 \text { meter above } 1998 \text { ground } \\
\text { level; } 0.20 \text { meter riverward of } 3 \text {-strand } \\
\text { barbed-wire fence and } 0.36 \text { meter down- } \\
\text { stream from metal fence post near gate }\end{array}$ & 187.9 & & & & & 971.86 \\
\hline Benchmark--brass circular plate; offsection & -.- & $45^{\circ} 14^{\prime} 44.90^{\prime \prime}$ & $105^{\circ} 39^{\prime} 56.56^{\prime \prime}$ & 1.241 & 0.637 & 972.17 \\
\hline
\end{tabular}



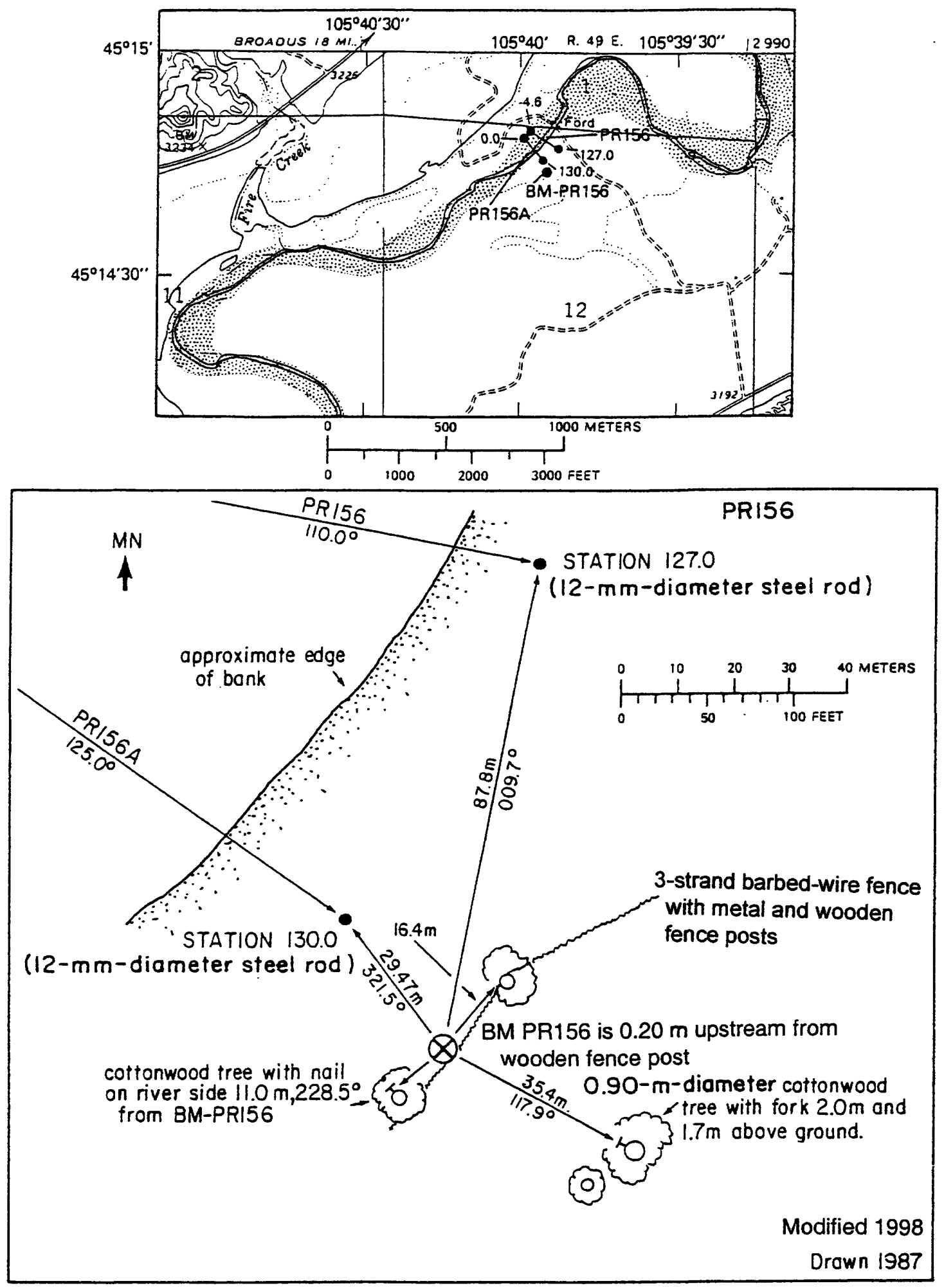

Figure 58. Upper: Location of cross section PR156, bench mark BM-PR156, and the left and right bank reference monuments in the Huckins School quadrangle. Lower: Location of the bench mark on the left bank. $\mathrm{MN}$ is magnetic north. 

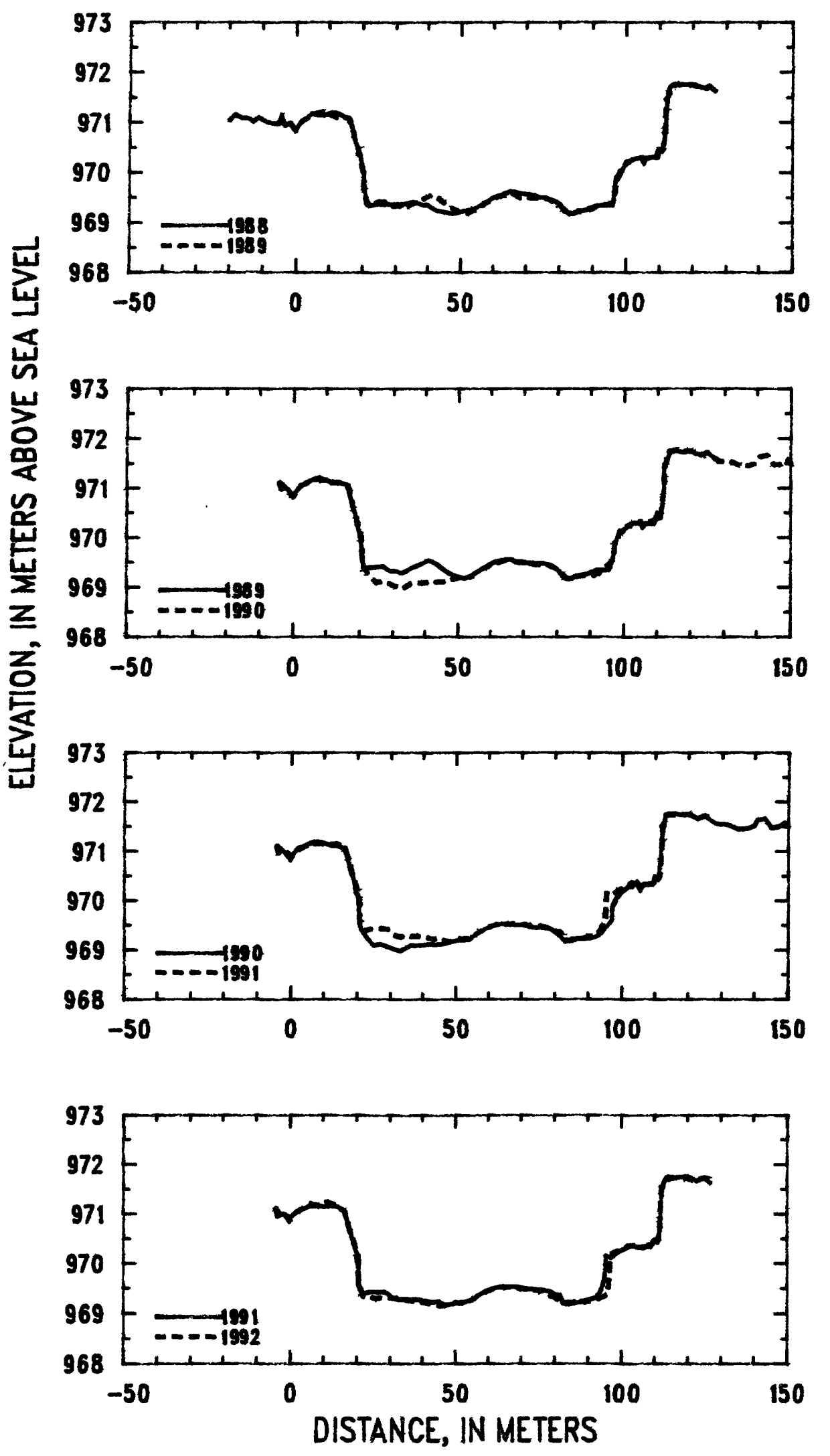

Figure 59. Profiles of cross section PR156 from 1988 to 1992. 

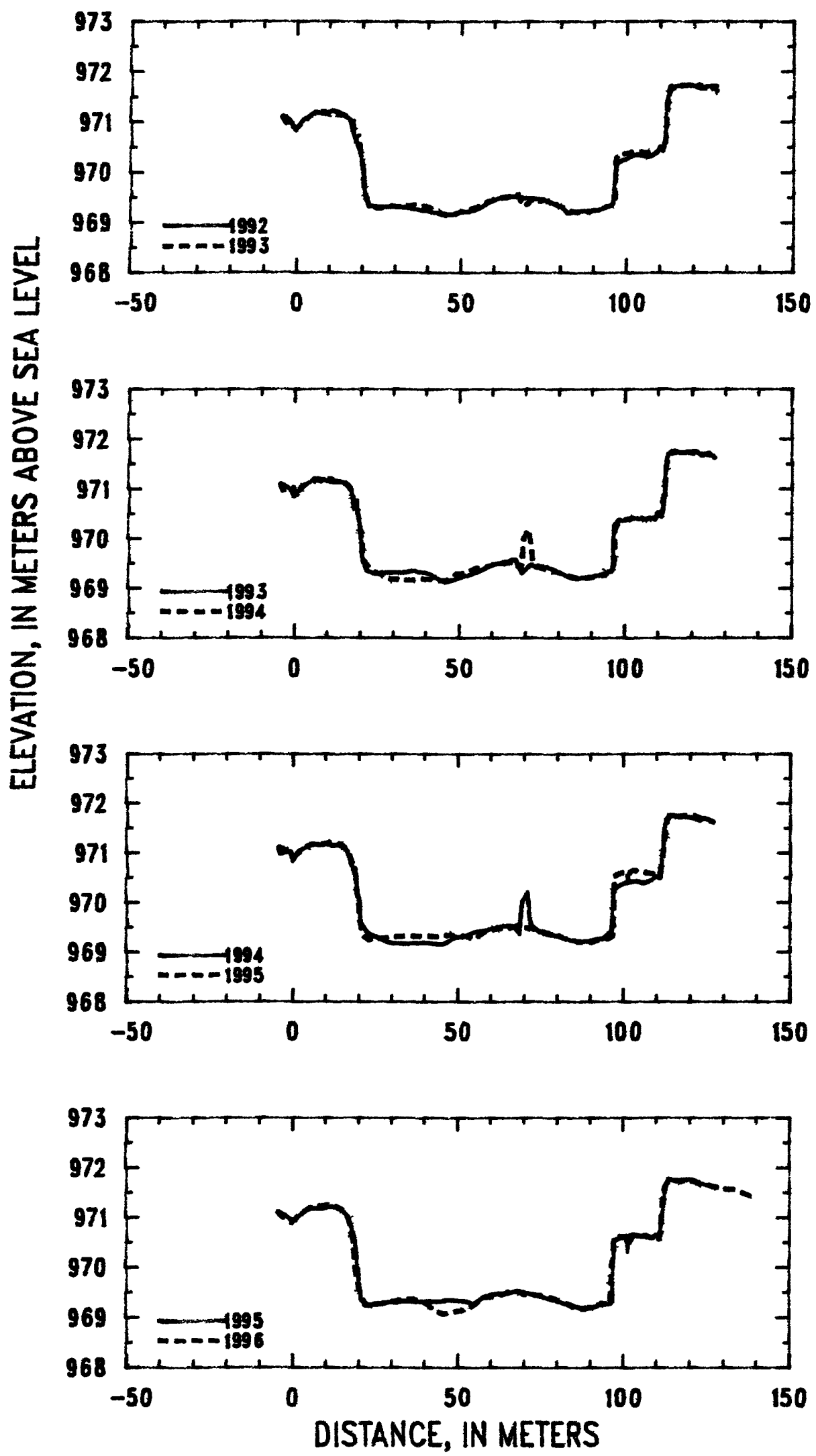

Figure 60. Profiles of cross section PR156 from 1992 to 1996. 


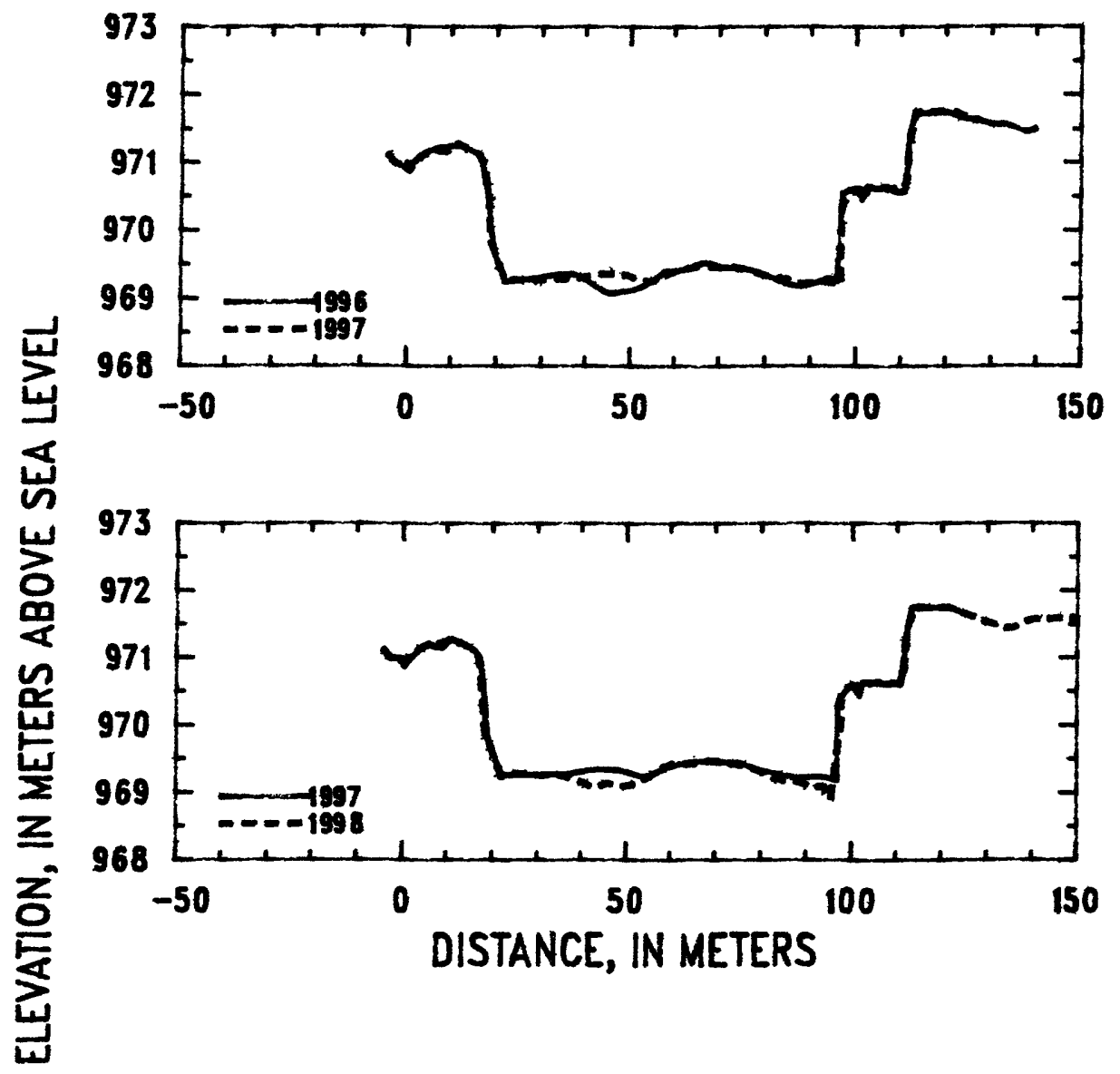

Figure 61. Profiles of cross section PR156 from 1996 to 1998. 
Table 21. Listing of horizontal stations and elevations for cross section PR156

[Sta., station, distance in meters from a reference pin on the left bank; Elev., elevation, in meters above sea level]

\begin{tabular}{|c|c|c|c|c|c|c|c|c|c|}
\hline \multirow{2}{*}{\multicolumn{2}{|c|}{$\begin{array}{c}1989 \\
20 \text { September }\end{array}$}} & \multicolumn{2}{|c|}{1989} & \multicolumn{2}{|c|}{1990} & \multicolumn{2}{|c|}{1990} & \multicolumn{2}{|c|}{1990} \\
\hline & & \multicolumn{2}{|c|}{20 September } & \multicolumn{2}{|c|}{19 September } & \multicolumn{2}{|c|}{19 September } & \multicolumn{2}{|c|}{19 September } \\
\hline Sta. & Elev. & Sta. & Elev. & Sta. & Elev. & Sta. & Elev. & Sta. & Elev. \\
\hline-4.2 & 971.12 & 64.0 & 969.55 & -4.2 & 971.11 & 81.0 & 969.30 & 147.0 & 971.48 \\
\hline-3.5 & 970.97 & 66.0 & 969.57 & -1.6 & 970.98 & 83.0 & 969.17 & 149.0 & 971.57 \\
\hline-2.0 & 971.00 & 68.0 & 969.49 & 0.0 & 970.82 & 85.0 & 969.21 & 149.9 & 971.52 \\
\hline-1.6 & 970.98 & 70.0 & 969.50 & 2.0 & 971.05 & 88.0 & 969.23 & 151.0 & 971.14 \\
\hline 0.0 & 970.83 & 72.0 & 969.49 & 4.0 & 971.10 & 91.0 & 969.24 & 152.1 & 971.09 \\
\hline 2.0 & 971.03 & 74.0 & 969.47 & 6.0 & 971.17 & 93.6 & 969.31 & 153.3 & 971.18 \\
\hline 4.0 & 971.11 & 76.0 & 969.49 & 8.0 & 971.19 & 97.0 & 969.59 & 154.6 & 971.16 \\
\hline 6.0 & 971.17 & 78.0 & 969.41 & 10.0 & 971.17 & 97.1 & 969.89 & 157.0 & 971.46 \\
\hline 8.0 & 971.21 & 80.0 & 969.37 & 12.0 & 971.11 & 98.0 & 969.96 & 159.0 & 971.38 \\
\hline 8.4 & 971.15 & 82.0 & 969.19 & 14.0 & 971.12 & 98.7 & 970.12 & 161.0 & 971.29 \\
\hline 10.0 & 971.17 & 84.0 & 969.16 & 15.0 & 971.07 & 99.0 & 970.08 & 163.0 & 971.23 \\
\hline 12.0 & 971.10 & 86.0 & 969.22 & 16.3 & 971.07 & 100.0 & 970.18 & 165.0 & 971.03 \\
\hline 14.0 & 971.11 & 88.0 & 969.25 & 17.3 & 970.82 & 101.0 & 970.24 & 167.0 & 970.99 \\
\hline 16.0 & 971.07 & 90.0 & 969.29 & 18.1 & 970.55 & 102.0 & 970.29 & 170.0 & 970.95 \\
\hline 17.0 & 970.88 & 92.0 & 969.36 & 18.9 & 970.47 & 103.0 & 970.29 & 172.0 & 970.93 \\
\hline 18.0 & 970.60 & 94.0 & 969.34 & 19.7 & 970.16 & 104.5 & 970.38 & 174.0 & 970.96 \\
\hline 19.0 & 970.43 & 95.4 & 969.35 & 20.2 & 970.13 & 105.6 & 970.21 & 175.0 & 971.00 \\
\hline 19.5 & 970.22 & 96.9 & 969.61 & 20.5 & 969.98 & 106.4 & 970.32 & 176.4 & 971.28 \\
\hline 20.5 & 969.96 & 97.0 & 969.85 & 20.7 & 969.59 & 108.0 & 970.33 & 177.6 & 971.54 \\
\hline 20.7 & 969.59 & 99.0 & 970.12 & 21.2 & 969.40 & 109.6 & 970.36 & 179.0 & 971.62 \\
\hline 21.5 & 969.37 & 101.0 & 970.21 & 23.0 & 969.23 & 110.0 & 970.49 & 182.0 & 971.68 \\
\hline 23.0 & 969.40 & 103.0 & 970.30 & 25.0 & 969.09 & 111.0 & 970.43 & 185.0 & 971.66 \\
\hline 25.0 & 969.41 & 105.0 & 970.28 & 27.0 & 969.12 & 112.0 & 970.95 & 188.0 & 971.68 \\
\hline 27.0 & 969.42 & 107.0 & 970.29 & 30.0 & 969.05 & 112.4 & 971.50 & & \\
\hline 29.0 & 969.33 & 109.0 & 970.28 & 33.0 & 968.98 & 112.7 & 971.51 & & \\
\hline 31.0 & 969.33 & 110.0 & 970.49 & 36.0 & 969.09 & 113.2 & 971.74 & & \\
\hline 33.0 & 969.29 & 110.6 & 970.40 & 39.0 & 969.08 & 114.0 & 971.74 & & \\
\hline 35.0 & 969.36 & 111.0 & 970.40 & 42.0 & 969.12 & 116.0 & 971.78 & & \\
\hline 37.0 & 969.42 & 111.6 & 970.63 & 45.0 & 969.11 & 118.0 & 971.74 & & \\
\hline 39.0 & 969.47 & 112.0 & 970.94 & 48.0 & 969.15 & 120.0 & 971.76 & & \\
\hline 41.0 & 969.54 & 112.2 & 971.48 & 51.0 & 969.20 & 123.0 & 971.66 & & \\
\hline 43.0 & 969.49 & 112.6 & 971.50 & 54.0 & 969.21 & 125.0 & 971.72 & & \\
\hline 44.0 & 969.44 & 114.0 & 971.75 & 57.0 & 969.36 & 127.0 & 971.60 & & \\
\hline 46.0 & 969.33 & 116.0 & 971.77 & 60.0 & 969.45 & 129.0 & 971.54 & & \\
\hline 48.0 & 969.27 & 118.0 & 971.72 & 63.0 & 969.51 & 131.0 & 971.55 & & \\
\hline 50.0 & 969.21 & 120.0 & 971.76 & 65.0 & 969.54 & 133.0 & 971.51 & & \\
\hline 52.0 & 969.17 & 122.0 & 971.68 & 68.0 & 969.50 & 135.0 & 971.44 & & \\
\hline 54.0 & 969.23 & 124.0 & 971.67 & 70.0 & 969.51 & 138.0 & 971.46 & & \\
\hline 56.0 & 969.31 & 125.0 & 971.73 & 73.0 & 969.46 & 140.0 & 971.52 & & \\
\hline 58.0 & 969.39 & 127.0 & 971.62 & 76.0 & 969.44 & 141.0 & 971.64 & & \\
\hline 60.0 & 969.47 & & & 78.0 & 969.44 & 143.0 & 971.66 & & . \\
\hline 62.0 & 969.52 & & & 80.0 & 969.36 & 145.0 & 971.47 & & \\
\hline
\end{tabular}


Table 21. (Continued) Listing of horizontal stations and elevations for cross section PR156

[Sta., station, distance in meters from a reference pin on the left bank; Elev., elevation, in meters above sea level]

\begin{tabular}{|c|c|c|c|c|c|c|c|c|c|}
\hline \multirow{2}{*}{\multicolumn{2}{|c|}{$\begin{array}{c}1991 \\
30 \text { August }\end{array}$}} & \multirow{2}{*}{\multicolumn{2}{|c|}{$\begin{array}{c}1991 \\
30 \text { August }\end{array}$}} & \multirow{2}{*}{\multicolumn{2}{|c|}{$\begin{array}{c}1992 \\
31 \text { August }\end{array}$}} & \multirow{2}{*}{\multicolumn{2}{|c|}{$\begin{array}{c}1992 \\
31 \text { August }\end{array}$}} & \multirow{2}{*}{\multicolumn{2}{|c|}{$\begin{array}{c}1993 \\
29 \text { August }\end{array}$}} \\
\hline & & & & & & & & & \\
\hline Sta. & Elev. & Sta. & Elev. & Sta. & Elev. & Sta. & Elev. & Sta. & Elev. \\
\hline-4.2 & 971.11 & 69.0 & 969.53 & -4.2 & 971.11 & 72.0 & 969.48 & -4.2 & 971.11 \\
\hline-3.5 & 970.95 & 71.0 & 969.50 & -3.0 & 970.97 & 74.0 & 969.46 & -1.6 & 971.02 \\
\hline-1.6 & 970.99 & 73.0 & 969.48 & -1.6 & 971.01 & 76.0 & 969.45 & 0.0 & 970.86 \\
\hline 0.0 & 970.89 & 75.0 & 969.48 & 0.0 & 970.84 & 78.0 & 969.36 & 2.0 & 971.01 \\
\hline 2.0 & 971.02 & 77.0 & 969.46 & 2.0 & 971.04 & 80.0 & 969.33 & 4.0 & 971.11 \\
\hline 4.0 & 971.08 & 79.0 & 969.42 & 4.0 & 971.11 & 82.0 & 969.20 & 6.0 & 971.21 \\
\hline 6.0 & 971.16 & 80.3 & 969.36 & 6.0 & 971.20 & 84.0 & 969.21 & 8.0 & 971.21 \\
\hline 9.0 & 971.15 & 81.6 & 969.36 & 9.0 & 971.16 & 86.0 & 969.23 & 10.0 & 971.17 \\
\hline 12.0 & 971.13 & 82.6 & 969.20 & 11.0 & 971.23 & 88.0 & 969.24 & 12.0 & 971.14 \\
\hline 14.0 & 971.15 & 84.0 & 969.18 & 13.0 & 971.20 & 90.0 & 969.24 & 14.0 & 971.14 \\
\hline 16.0 & 971.08 & 86.0 & 969.20 & 15.0 & 971.10 & 92.0 & 969.26 & 15.0 & 971.12 \\
\hline 16.7 & 970.96 & 88.0 & 969.23 & 16.3 & 971.05 & 94.0 & 969.31 & 16.0 & 971.06 \\
\hline 18.0 & 970.69 & 90.0 & 969.27 & 18.0 & 970.64 & 95.8 & 969.37 & 17.0 & 971.03 \\
\hline 18.5 & 970.54 & 92.0 & 969.30 & 19.5 & 970.38 & 96.0 & 969.48 & 18.0 & 970.64 \\
\hline 20.0 & 970.23 & 93.2 & 969.34 & 20.0 & 970.16 & 96.6 & 970.04 & 18.8 & 970.74 \\
\hline 20.6 & 970.13 & 94.9 & 969.61 & 20.5 & 969.97 & 97.0 & 970.16 & 19.0 & 970.59 \\
\hline 20.7 & 969.57 & 95.4 & 970.15 & 20.7 & 969.57 & 98.0 & 970.23 & 19.6 & 970.37 \\
\hline 20.9 & 969.53 & 97.0 & 970.11 & 21.9 & 969.33 & 100.0 & 970.26 & 20.3 & 969.96 \\
\hline 22.0 & 969.39 & 99.0 & 970.23 & 24.0 & 969.32 & 102.0 & 970.34 & 20.4 & 969.62 \\
\hline 24.0 & 969.43 & 101.0 & 970.27 & 26.0 & 969.31 & 104.0 & 970.35 & 22.0 & 969.36 \\
\hline 26.0 & 969.44 & 103.0 & 970.37 & 28.0 & 969.32 & 106.0 & 970.30 & 24.0 & 969.30 \\
\hline 28.0 & 969.44 & 105.0 & 970.32 & 30.0 & 969.32 & 108.0 & 970.34 & 26.0 & 969.30 \\
\hline 29.0 & 969.41 & 106.0 & 970.32 & 32.0 & 969.30 & 110.0 & 970.46 & 28.0 & 969.32 \\
\hline 31.0 & 969.30 & 108.0 & 970.34 & 34.0 & 969.29 & 111.0 & 970.43 & 30.0 & 969.33 \\
\hline 33.0 & 969.28 & 109.0 & 970.32 & 36.0 & 969.26 & 111.7 & 970.70 & 32.0 & 969.32 \\
\hline 35.0 & 969.29 & 110.0 & 970.48 & 38.0 & 969.24 & 112.0 & 971.44 & 34.0 & 969.33 \\
\hline 37.0 & 969.28 & 111.3 & 970.46 & 40.0 & 969.22 & 113.0 & 971.62 & 36.0 & 969.36 \\
\hline 39.0 & 969.27 & 111.9 & 970.81 & 42.0 & 969.20 & 115.0 & 971.72 & 38.0 & 969.33 \\
\hline 41.0 & 969.25 & 112.3 & 971.48 & 44.0 & 969.17 & 117.0 & 971.72 & 40.0 & 969.32 \\
\hline 43.0 & 969.22 & 113.0 & 971.64 & 46.0 & 969.15 & 120.0 & 971.73 & 42.0 & 969.26 \\
\hline 45.0 & 969.24 & 114.0 & 971.75 & 48.0 & 969.15 & 122.0 & 971.66 & 44.0 & 969.18 \\
\hline 47.0 & 969.16 & 117.0 & 971.73 & 50.0 & 969.22 & 125.0 & 971.73 & 46.0 & 969.12 \\
\hline 49.0 & 969.18 & 120.0 & 971.76 & 52.0 & 969.21 & 127.0 & 971.71 & 48.0 & 969.16 \\
\hline 51.0 & 969.22 & 123.0 & 971.66 & 54.0 & 969.24 & & & 50.0 & 969.22 \\
\hline 53.0 & 969.23 & 125.0 & 971.72 & 56.0 & 969.30 & & & 52.0 & 969.26 \\
\hline 55.0 & 969.26 & 127.0 & 971.62 & 58.0 & 969.39 & & & 54.0 & 969.29 \\
\hline 57.0 & 969.35 & & & 60.0 & 969.45 & & & 56.0 & 969.35 \\
\hline 59.0 & 969.42 & & & 62.0 & 969.50 & & & 58.0 & 969.41 \\
\hline 61.0 & 969.48 & & & 64.0 & 969.53 & & & 60.0 & 969.47 \\
\hline 62.8 & 969.53 & & & 66.0 & 969.52 & & & 62.0 & 969.50 \\
\hline 65.0 & 969.52 & & & 68.0 & 969.51 & & & 64.0 & 969.49 \\
\hline 67.0 & 969.53 & & & 70.0 & 969.48 & & & 66.0 & 969.58 \\
\hline
\end{tabular}


Table 21. (Continued) Listing of horizontal stations and elevations for cross section PR156

[Sta., station, distance in meters from a reference pin on the left bank; Elev., elevation, in meters above sea level]

\begin{tabular}{|c|c|c|c|c|c|c|c|c|c|}
\hline \multirow{2}{*}{\multicolumn{2}{|c|}{$\begin{array}{c}1993 \\
29 \text { August }\end{array}$}} & \multicolumn{2}{|c|}{1994} & \multicolumn{2}{|c|}{1994} & \multicolumn{2}{|c|}{1995} & \multicolumn{2}{|c|}{1995} \\
\hline & & $22 \mathrm{~S}$ & ember & $22 \mathrm{~S}$ & ember & $25 \mathrm{Se}$ & ember & $25 \mathrm{Se}$ & ember \\
\hline Sta. & Elev. & Sta. & Elev. & Sta. & Elev. & Sta. & Elev. & Sta. & Elev. \\
\hline 67.0 & 969.58 & -4.2 & 971.10 & 80.0 & 969.32 & -4.2 & 971.11 & 72.0 & 969.48 \\
\hline 68.8 & 969.32 & -3.5 & 970.98 & 83.0 & 969.24 & -1.6 & 971.04 & 74.0 & 969.42 \\
\hline 71.7 & 969.48 & -1.6 & 971.03 & 86.0 & 969.21 & 0.0 & 970.94 & 76.0 & 969.40 \\
\hline 73.0 & 969.43 & -0.5 & 971.03 & 89.0 & 969.20 & 1.0 & 970.94 & 78.0 & 969.36 \\
\hline 75.0 & 969.45 & -0.1 & 970.93 & 92.0 & 969.27 & 3.0 & 971.07 & 80.0 & 969.34 \\
\hline 77.0 & 969.43 & 0.3 & 970.86 & 94.0 & 969.27 & 5.0 & 971.17 & 82.0 & 969.27 \\
\hline 79.0 & 969.35 & 2.0 & 971.04 & 96.5 & 969.43 & 7.0 & 971.18 & 84.0 & 969.26 \\
\hline 81.0 & 969.30 & 4.0 & 971.11 & 97.0 & 970.23 & 9.0 & 971.17 & 86.0 & 969.21 \\
\hline 83.0 & 969.22 & 6.0 & 971.18 & 98.0 & 970.31 & 11.0 & 971.21 & 88.0 & 969.19 \\
\hline 85.0 & 969.20 & 9.0 & 971.16 & 100.0 & 970.38 & 13.0 & 971.21 & 90.0 & 969.18 \\
\hline 87.0 & 969.21 & 11.0 & 971.20 & 102.0 & 970.41 & 15.0 & 971.14 & 92.0 & 969.23 \\
\hline 89.0 & 969.22 & 12.0 & 971.15 & 104.0 & 970.44 & 16.5 & 971.06 & 94.0 & 969.26 \\
\hline 91.0 & 969.26 & 14.0 & 971.16 & 106.0 & 970.39 & 18.0 & 970.79 & 96.0 & 969.27 \\
\hline 93.0 & 969.29 & 16.4 & 971.06 & 108.0 & 970.42 & 19.0 & 970.54 & 96.8 & 969.32 \\
\hline 95.0 & 969.33 & 18.4 & 970.66 & 110.0 & 970.53 & 19.8 & 969.96 & 97.3 & 970.53 \\
\hline 96.0 & 969.35 & 18.8 & 970.68 & 111.0 & 970.49 & 20.0 & 969.96 & 99.0 & 970.59 \\
\hline 96.4 & 969.40 & 20.4 & 969.97 & 112.0 & 971.07 & 20.3 & 969.66 & 101.0 & 970.63 \\
\hline 96.5 & 969.96 & 20.5 & 969.59 & 112.3 & 971.46 & 21.0 & 969.34 & 101.4 & 970.41 \\
\hline 96.7 & 970.27 & 23.0 & 969.36 & 113.0 & 971.68 & 23.0 & 969.24 & 101.9 & 970.62 \\
\hline 98.0 & 970.38 & 26.0 & 969.29 & 114.5 & 971.77 & 25.0 & 969.26 & 103.0 & 970.66 \\
\hline 100.0 & 970.37 & 29.0 & 969.19 & 117.0 & 971.72 & 27.0 & 969.27 & 105.0 & 970.62 \\
\hline 102.0 & 970.41 & 32.0 & 969.17 & 119.0 & 971.74 & 29.0 & 969.31 & 107.0 & 970.60 \\
\hline 104.0 & 970.41 & 35.0 & 969.16 & 122.0 & 971.67 & 31.0 & 969.31 & 109.0 & 970.58 \\
\hline 106.0 & 970.38 & 38.0 & 969.19 & 124.5 & 971.70 & 33.0 & 969.31 & 110.0 & 970.64 \\
\hline 108.0 & 970.42 & 41.0 & 969.19 & 127.0 & 971.62 & 35.0 & 969.32 & 111.0 & 970.59 \\
\hline 109.0 & 970.38 & 44.0 & 969.16 & & & 37.0 & 969.32 & 112.0 & 971.04 \\
\hline 110.0 & 970.50 & 46.0 & 969.16 & & & 39.0 & 969.32 & 113.0 & 971.63 \\
\hline 111.0 & 970.44 & 49.0 & 969.29 & & & 41.0 & 969.31 & 114.0 & 971.76 \\
\hline 111.5 & 970.59 & 52.0 & 969.32 & & & 43.0 & 969.31 & 117.0 & 971.74 \\
\hline 111.8 & 970.77 & 55.0 & 969.41 & & & 45.0 & 969.32 & 120.0 & 971.78 \\
\hline 112.0 & 970.99 & 58.0 & 969.43 & & & 47.0 & 969.34 & 123.0 & 971.70 \\
\hline 112.3 & 971.46 & 61.0 & 969.49 & & & 49.0 & 969.34 & 127.0 & 971.62 \\
\hline 113.0 & 971.69 & 64.0 & 969.53 & & & 51.0 & 969.33 & & \\
\hline 115.0 & 971.74 & 66.0 & 969.53 & & & 53.0 & 969.31 & & \\
\hline 117.0 & 971.73 & 67.0 & 969.51 & & & 55.0 & 969.26 & & \\
\hline 119.0 & 971.73 & 68.5 & 969.39 & & & 57.0 & 969.36 & & \\
\hline 121.0 & 971.76 & 69.0 & 969.73 & & & 59.0 & 969.42 & & \\
\hline 123.0 & 971.67 & 69.4 & 970.04 & & & 61.0 & 969.46 & & \\
\hline 125.0 & 971.72 & 71.0 & 970.19 & & & 63.0 & 969.47 & & \\
\hline 127.0 & 971.61 & 72.0 & 969.55 & & & 65.0 & 969.47 & & \\
\hline & & 74.0 & 969.42 & & & 68.0 & 969.53 & & \\
\hline & & 77.0 & 969.36 & & & 70.0 & 969.49 & & \\
\hline
\end{tabular}


Table 21. (Continued) Listing of horizontal stations and elevations for cross section PR156

[Sta., station, distance in meters from a reference pin on the left bank; Elev., elevation, in meters above sea level]

\begin{tabular}{|c|c|c|c|c|c|c|c|c|c|}
\hline \multirow{2}{*}{\multicolumn{2}{|c|}{$\begin{array}{c}1996 \\
21 \text { October }\end{array}$}} & \multicolumn{2}{|c|}{1996} & \multicolumn{2}{|c|}{1997} & \multicolumn{2}{|c|}{1997} & \multicolumn{2}{|c|}{1998} \\
\hline & & 21 & tober & $23 \mathrm{~S}$ & ember & $23 \mathrm{Se}$ & ember & $29 \mathrm{Se}$ & ember \\
\hline Sta. & Elev. & Sta. & Elev. & Sta. & Elev. & Sta. & Elev. & Sta. & Elev. \\
\hline-4.2 & 971.10 & 97.1 & 970.54 & -4.2 & 971.12 & 96.9 & 970.32 & -4.2 & 971.12 \\
\hline-3.0 & 971.01 & 99.0 & 970.61 & -3.0 & 970.99 & 98.0 & 970.44 & -3.0 & 971.01 \\
\hline 0.0 & 970.89 & 101.0 & 970.61 & 0.0 & 970.98 & 100.0 & 970.60 & -1.6 & 971.04 \\
\hline 2.0 & 971.04 & 101.5 & 970.46 & 0.5 & 970.88 & 101.7 & 970.45 & 0.0 & 970.93 \\
\hline 5.0 & 971.18 & 103.0 & 970.63 & 3.0 & 971.08 & 102.5 & 970.64 & 2.0 & 971.05 \\
\hline 8.0 & 971.22 & 105.0 & 970.61 & 6.0 & 971.21 & 105.0 & 970.63 & 5.0 & 971.18 \\
\hline 11.0 & 971.26 & 107.0 & 970.64 & 7.0 & 971.16 & 107.5 & 970.62 & 8.0 & 971.21 \\
\hline 14.0 & 971.19 & 109.0 & 970.56 & 9.0 & 971.16 & 110.5 & 970.62 & 11.0 & 971.29 \\
\hline 16.0 & 971.12 & 110.9 & 970.56 & 11.0 & 971.28 & 111.2 & 970.69 & 13.0 & 971.22 \\
\hline 17.0 & 970.96 & 111.4 & 970.70 & 14.0 & 971.19 & 112.4 & 971.44 & 15.0 & 971.16 \\
\hline 17.7 & 970.68 & 112.0 & 971.41 & 16.0 & 971.12 & 113.3 & 971.74 & 17.1 & 971.00 \\
\hline 18.3 & 970.52 & 113.0 & 971.66 & 17.3 & 970.98 & 115.0 & 971.75 & 17.4 & 970.75 \\
\hline 19.0 & 970.00 & 114.0 & 971.73 & 18.5 & 970.40 & 118.0 & 971.75 & 18.0 & 970.34 \\
\hline 20.0 & 969.60 & 117.0 & 971.74 & 18.7 & 969.98 & 121.0 & 971.77 & 18.3 & 969.95 \\
\hline 22.0 & 969.24 & 120.0 & 971.75 & 19.1 & 969.79 & 124.0 & 971.68 & 19.8 & 969.67 \\
\hline 25.0 & 969.26 & 123.0 & 971.67 & 20.0 & 969.62 & 127.0 & 971.62 & 22.0 & 969.24 \\
\hline 28.0 & 969.28 & 127.0 & 971.63 & 22.0 & 969.26 & & & 25.0 & 969.29 \\
\hline 31.0 & 969.32 & 130.0 & 971.57 & 25.0 & 969.26 & & & 28.0 & 969.29 \\
\hline 34.0 & 969.35 & 133.0 & 971.57 & 28.0 & 969.27 & & & 31.0 & 969.25 \\
\hline 37.0 & 969.36 & 138.0 & 971.44 & 31.0 & 969.26 & & & 34.0 & 969.28 \\
\hline 40.0 & 969.30 & 140.0 & 971.50 & 34.0 & 969.28 & & & 37.0 & 969.22 \\
\hline 43.0 & 969.13 & & & 37.0 & 969.27 & & & 40.0 & 969.15 \\
\hline 46.0 & 969.06 & & & 40.0 & 969.31 & & & 43.0 & 969.07 \\
\hline 49.0 & 969.10 & & & 43.0 & 969.35 & & & 46.0 & 969.14 \\
\hline 52.0 & 969.14 & & & 46.0 & 969.35 & & & 49.0 & 969.09 \\
\hline 55.0 & 969.24 & & & 49.0 & 969.34 & & & 52.0 & 969.14 \\
\hline 58.0 & 969.39 & & & 52.0 & 969.26 & & & 55.0 & 969.23 \\
\hline 61.0 & 969.40 & & & 55.0 & 969.24 & & & 58.0 & 969.37 \\
\hline 64.0 & 969.46 & & & 58.0 & 969.34 & & & 61.0 & 969.44 \\
\hline 66.0 & 969.51 & & & 61.0 & 969.41 & & & 64.0 & 969.42 \\
\hline 68.0 & 969.52 & & & 64.0 & 969.44 & & & 67.0 & 969.47 \\
\hline 70.0 & 969.45 & & & 67.0 & 969.47 & & & 70.0 & 969.48 \\
\hline 73.0 & 969.44 & & & 70.0 & 969.45 & & & 73.0 & 969.44 \\
\hline 76.0 & 969.43 & & & 73.0 & 969.45 & & & 76.0 & 969.39 \\
\hline 79.0 & 969.37 & & & 76.0 & 969.42 & & & 79.0 & 969.34 \\
\hline 82.0 & 969.29 & & & 79.0 & 969.35 & & & 82.0 & 969.24 \\
\hline 85.0 & 969.20 & & & 82.0 & 969.30 & & & 85.0 & 969.17 \\
\hline 88.0 & 969.16 & & & 85.0 & 969.27 & & & 88.0 & 969.16 \\
\hline 91.0 & 969.21 & & & 88.0 & 969.20 & & & 91.0 & 969.09 \\
\hline 94.0 & 969.29 & & & 91.0 & 969.23 & & & 94.0 & 969.09 \\
\hline 96.0 & 969.25 & & & 94.0 & 969.24 & & & 95.4 & 968.89 \\
\hline 97.0 & 970.33 & & & 96.7 & 969.18 & & & 97.6 & 970.03 \\
\hline
\end{tabular}


Table 21. (Continued) Listing of horizontal stations and elevations for cross section PR156

[Sta., station, distance in meters from a reference pin on the left bank; Elev., elevation, in meters above sea level]

1998

29 September

\begin{tabular}{rl}
\hline Sta. & Elev. \\
\hline 97.6 & 970.51 \\
99.0 & 970.56 \\
102.0 & 970.60 \\
105.0 & 970.63 \\
108.0 & 970.64 \\
111.0 & 970.61 \\
111.7 & 970.82 \\
112.2 & 971.41 \\
114.0 & 971.76 \\
117.0 & 971.75 \\
119.0 & 971.75 \\
121.5 & 971.75 \\
124.0 & 971.67 \\
127.0 & 971.60 \\
130.0 & 971.52 \\
135.0 & 971.42 \\
140.0 & 971.57 \\
187.9 & 971.69
\end{tabular}




\section{Description of Cross Section PR163}

Location: Township 6 South/Range 50 East--section 32

U. S. Geological Survey quadrangle (1:24,000): Yarger Butte

Landowners--left bank: Gay Ranch

--right bank: Gay Ranch

Access: Left bank

Permission from: Gay Ranch

Distance from Moorhead Gaging Station: 48.30 kilometers

Azimuth of Section (degrees magnetic): 151

\section{Reference Monuments}

[Monument at station -88.0 was closest to leveling instrument]

\begin{tabular}{|c|c|c|c|c|c|c|}
\hline \multirow[b]{2}{*}{ Description } & \multirow[b]{2}{*}{$\begin{array}{c}\text { Station } \\
(m)\end{array}$} & \multicolumn{2}{|c|}{ GPS-NAD83 (1992) } & \multicolumn{2}{|c|}{ Measurement } & \multirow{2}{*}{$\begin{array}{c}\text { Elevation } \\
\text { (NGVD1929) } \\
\text { (m) }\end{array}$} \\
\hline & & Latitude & Longitude & $\begin{array}{c}\text { Standard } \\
\text { deviation } \\
\text { (m) }\end{array}$ & $\begin{array}{l}\text { Horizontal } \\
\text { precision } \\
\text { (m) }\end{array}$ & \\
\hline Benchmark--brass circular plate & -122.0 & $45^{\circ} 16^{\prime} 12.70^{\prime \prime}$ & $105^{\circ} 37^{\prime} 52.08^{\prime \prime}$ & 0.191 & 0.532 & 965.53 \\
\hline $\begin{array}{l}\text { 1/2-inch-rebar; } 0.23 \text { meter above } 1998 \text { ground } \\
\text { level }\end{array}$ & -88.0 & & & & & 965.61 \\
\hline $\begin{array}{l}\text { 1/2-inch-rebar; } 0.04 \text { meter below } 1998 \text { ground } \\
\text { level }\end{array}$ & 30.0 & & & & & 964.21 \\
\hline $\begin{array}{l}\text { 1/2-inch-rebar; } 0.11 \text { meter above } 1998 \text { ground } \\
\text { level }\end{array}$ & 50.0 & $45^{\circ} 16^{\prime} 07.31^{\prime \prime}$ & $105^{\circ} 37^{\prime} 50.04 "$ & 0.426 & 0.632 & 964.09 \\
\hline $\begin{array}{l}\text { 1/2-inch-rebar; } 0.10 \text { meter above } 1998 \text { ground } \\
\text { level }\end{array}$ & 75.0 & & & & & 964.42 \\
\hline $\begin{array}{l}\text { 1/2-inch-rebar; } 0.08 \text { meter above } 1998 \text { ground } \\
\text { level }\end{array}$ & 110.9 & & & & & 964.55 \\
\hline
\end{tabular}




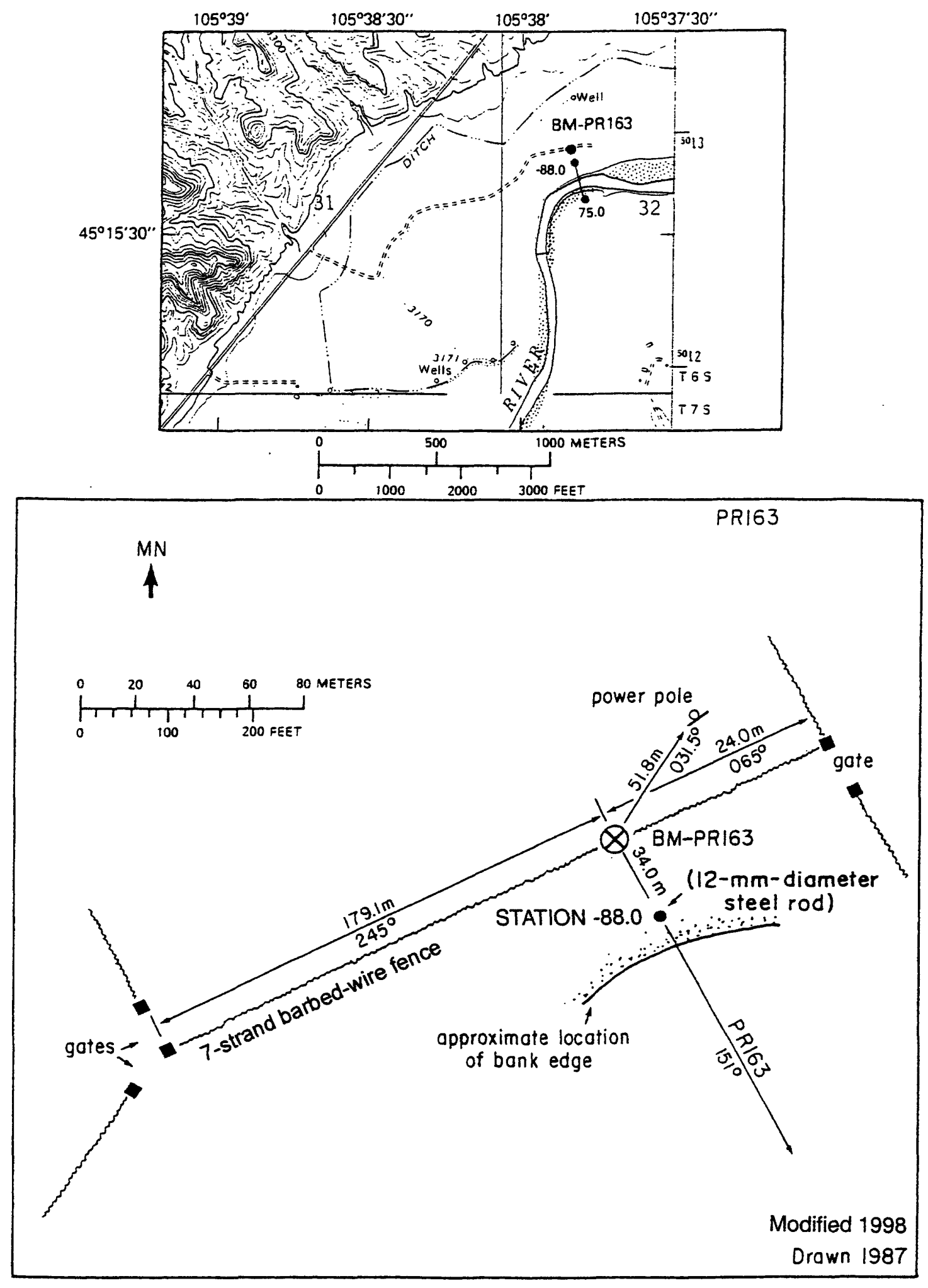

Figure 62. Upper: Location of cross section PR163, bench mark BM-PR163, and the left and right bank reference monuments in the Yarger Butte quadrangle. Lower: Location of the bench mark on the left bank. MN is magnetic north. 

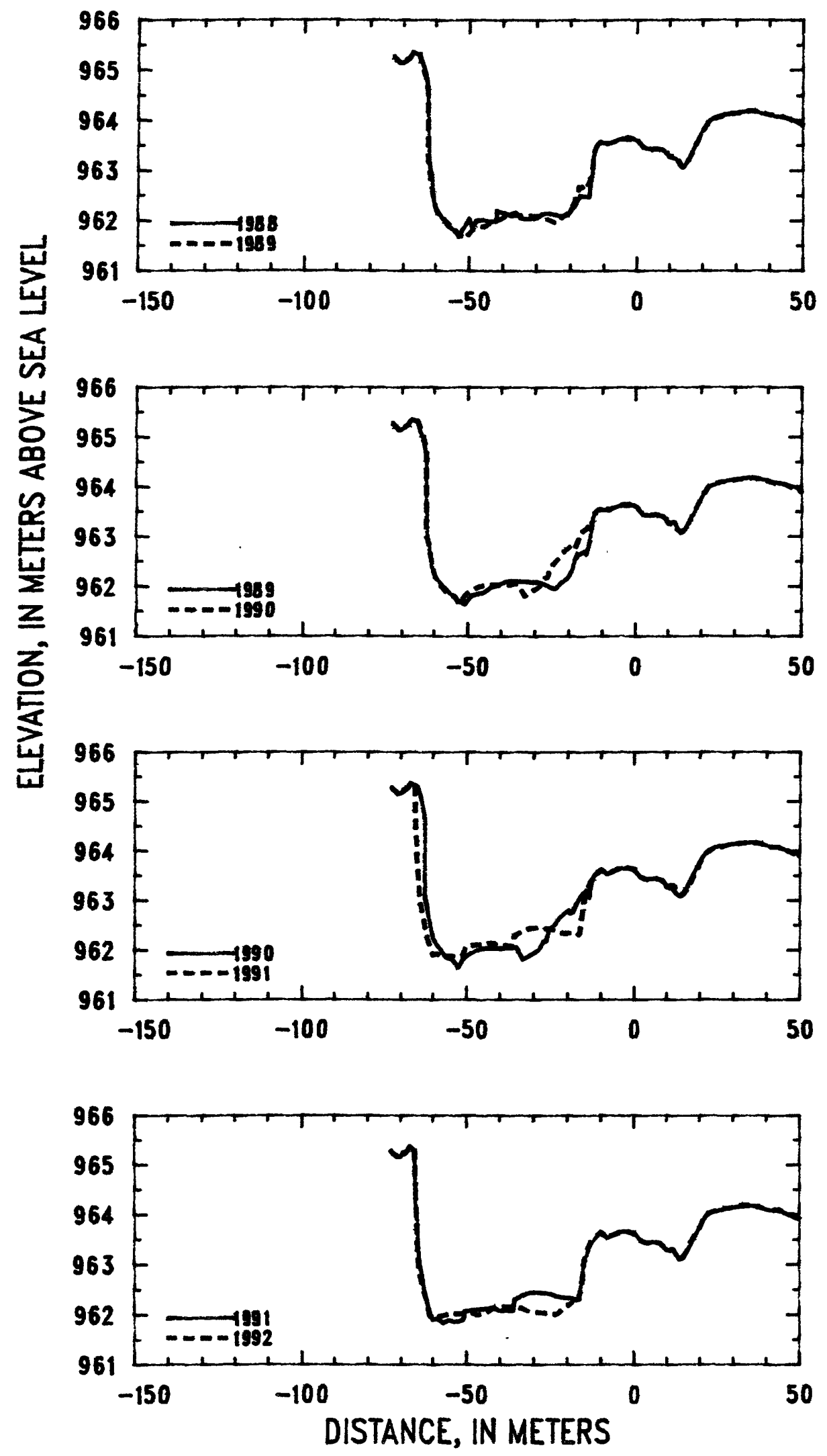

Figure 63. Profiles of cross section PR163 from 1988 to 1992. 


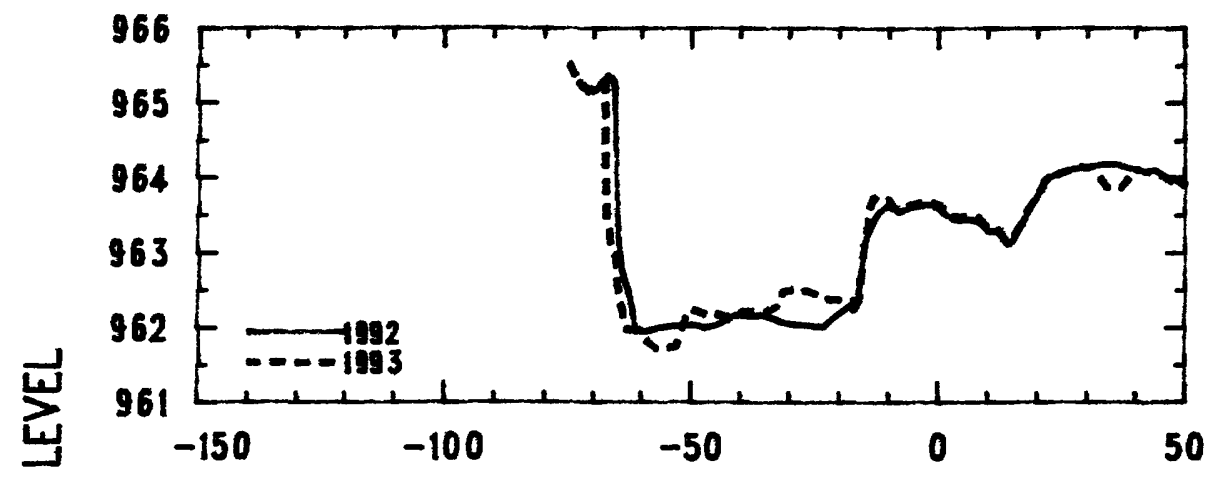

昸

崖

\section{6}

965

964

963

$962[--1993$
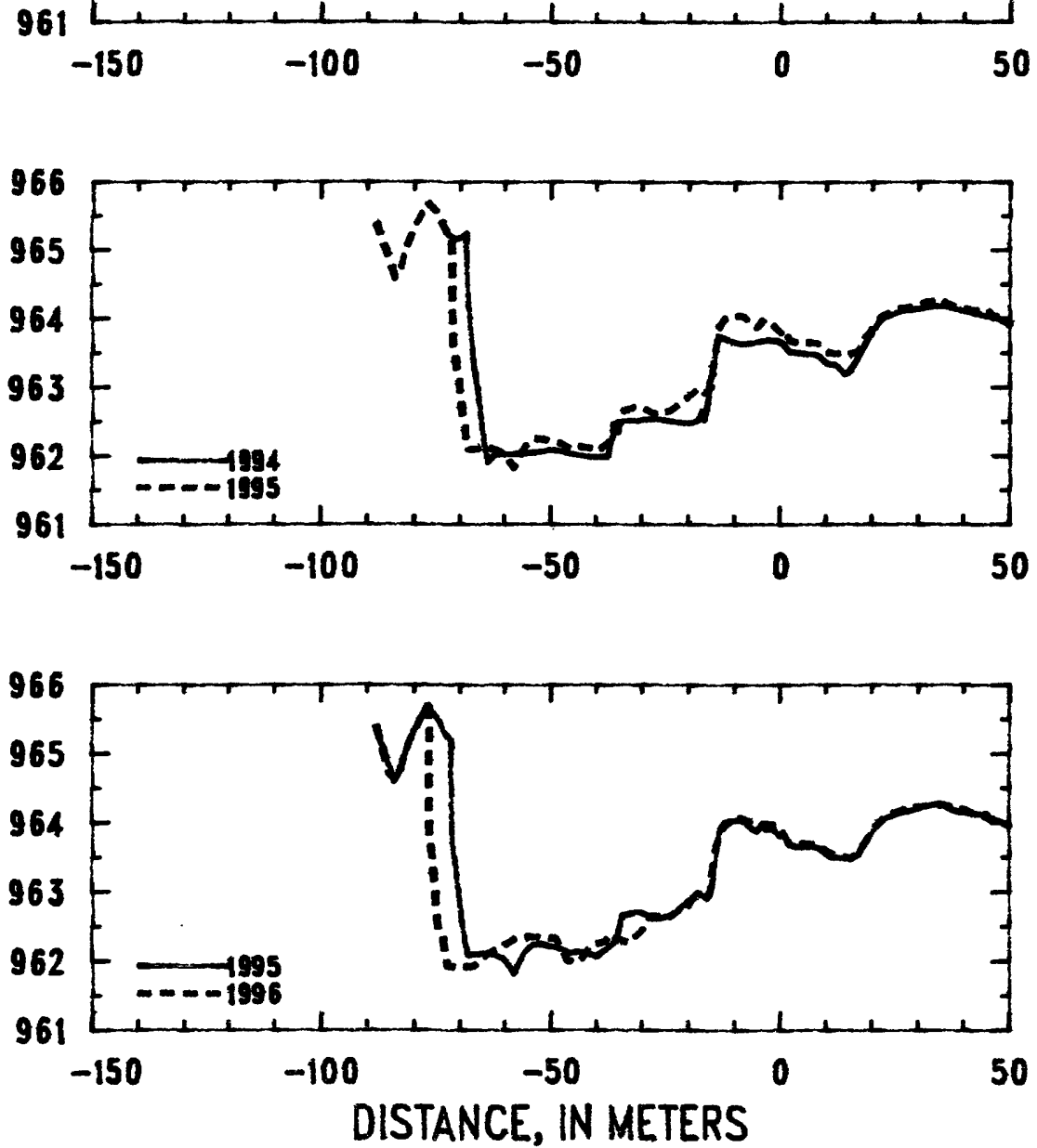

Figure 64. Profiles of cross section PR163 from 1992 to 1996. 


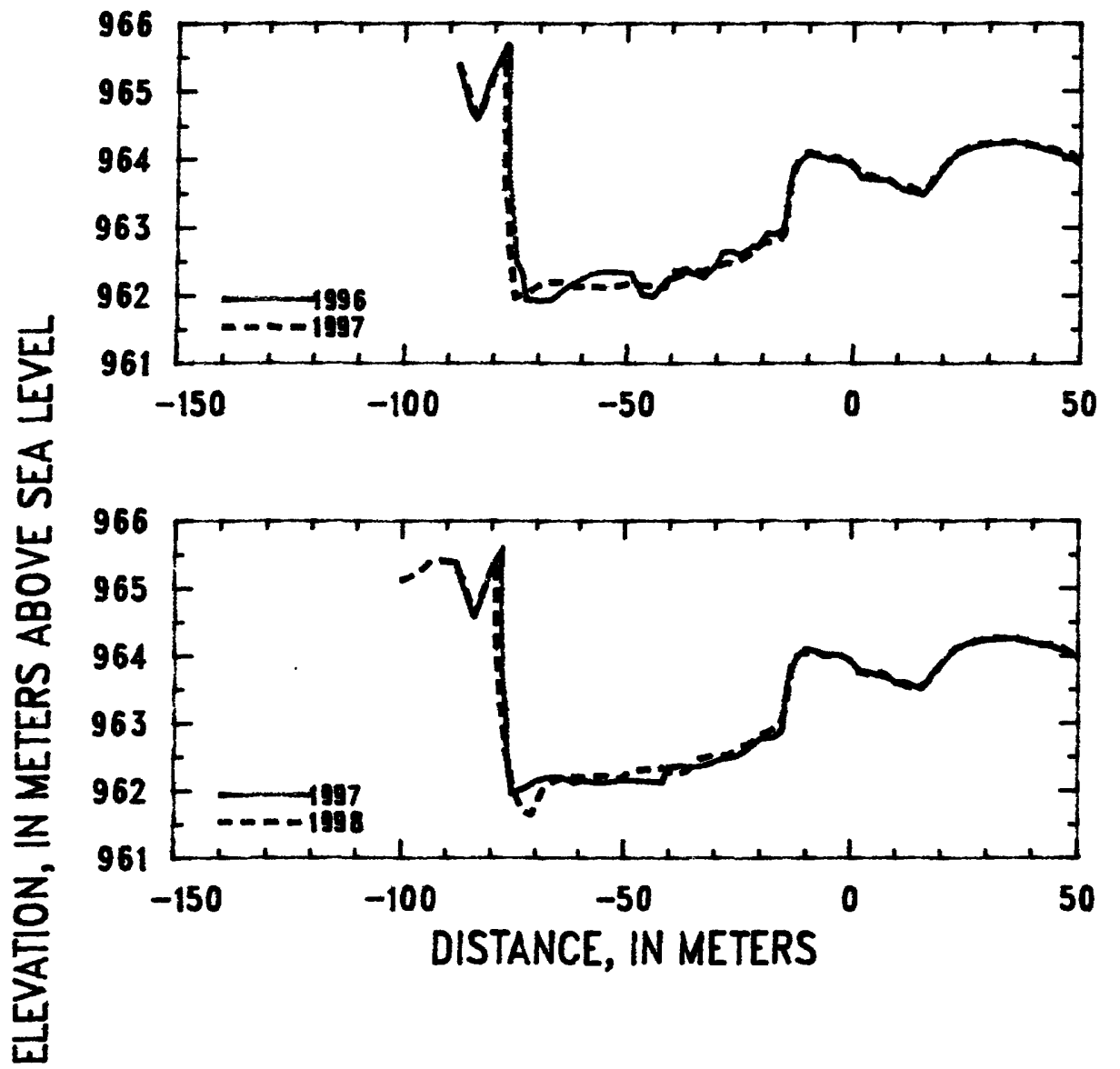

Figure 65. Profiles of cross section PR163 from 1996 to 1998. 
Table 22. Listing of horizontal stations and elevations for cross section PR163

[Sta., station, distance in meters from a reference pin on the left bank; Elev., elevation, in meters above sea level]

\begin{tabular}{|c|c|c|c|c|c|c|c|c|c|}
\hline \multirow{2}{*}{\multicolumn{2}{|c|}{$\begin{array}{c}1989 \\
22 \text { September }\end{array}$}} & \multicolumn{2}{|c|}{1989} & \multicolumn{2}{|c|}{1990} & \multicolumn{2}{|c|}{1990} & \multicolumn{2}{|c|}{1990} \\
\hline & & $22 \mathrm{Se}$ & ember & $21 S$ & ember & $21 \mathrm{Se}$ & ember & $21 \mathrm{~S}$ & ember \\
\hline Sta. & Elev. & Sta. & Elev. & Sta. & Elev. & Sta. & Elev. & Sta. & Elev. \\
\hline-73.0 & 965.28 & -9.0 & 963.53 & -73.0 & 965.29 & -12.8 & 963.39 & 66.8 & 963.66 \\
\hline-71.0 & 965.15 & -7.0 & 963.57 & -71.0 & 965.15 & -12.0 & 963.52 & 67.7 & 963.78 \\
\hline-70.0 & 965.16 & -5.0 & 963.62 & -70.0 & 965.16 & -10.6 & 963.56 & 69.0 & 964.05 \\
\hline-68.0 & 965.26 & -3.0 & 963.61 & -68.0 & 965.26 & -9.7 & 963.63 & 71.0 & 964.19 \\
\hline-67.0 & 965.36 & -1.0 & 963.64 & -67.0 & 965.38 & -8.2 & 963.53 & 73.0 & 964.18 \\
\hline-65.0 & 965.32 & 0.0 & 963.62 & -65.0 & 965.31 & -6.0 & 963.60 & 75.0 & 964.27 \\
\hline-62.4 & 964.69 & 2.0 & 963.45 & -64.0 & 965.15 & -4.0 & 963.64 & 66.8 & 963.66 \\
\hline-62.3 & 963.17 & 4.0 & 963.41 & -62.4 & 964.73 & -2.0 & 963.66 & 67.7 & 963.78 \\
\hline-61.0 & 962.55 & 6.0 & 963.43 & -62.5 & 963.02 & 0.0 & 963.61 & 69.0 & 964.05 \\
\hline-60.0 & 962.26 & 8.0 & 963.41 & -62.0 & 962.93 & 2.0 & 963.46 & 71.0 & 964.19 \\
\hline-59.3 & 962.16 & 9.0 & 963.37 & -60.6 & 962.41 & 4.0 & 963.42 & 73.0 & 964.18 \\
\hline-57.0 & 961.98 & 10.0 & 963.27 & -59.6 & 962.19 & 6.0 & 963.44 & 75.0 & 964.27 \\
\hline-55.0 & 961.85 & 12.0 & 963.28 & -58.0 & 962.03 & 8.0 & 963.42 & & \\
\hline-53.0 & 961.71 & 14.0 & 963.07 & -56.0 & 961.85 & 9.0 & 963.39 & & \\
\hline-51.0 & 961.64 & 15.0 & 963.12 & -54.0 & 961.80 & 10.0 & 963.26 & & \\
\hline-49.0 & 961.81 & 17.0 & 963.36 & -52.5 & 961.65 & 12.0 & 963.24 & & \\
\hline-47.0 & 961.85 & 19.0 & 963.64 & -51.0 & 961.82 & 13.0 & 963.13 & & \\
\hline-45.0 & 961.88 & 21.0 & 963.88 & -49.0 & 961.92 & 14.0 & 963.09 & & \\
\hline-43.0 & 961.99 & 22.0 & 963.99 & -47.0 & 961.99 & 15.0 & 963.13 & & \\
\hline-41.0 & 962.02 & 25.0 & 964.07 & -45.0 & 962.03 & 16.0 & 963.25 & & \\
\hline-39.0 & 962.09 & 28.0 & 964.13 & -43.0 & 962.04 & 18.0 & 963.52 & & \\
\hline-37.0 & 962.11 & 31.0 & 964.15 & -41.0 & 962.03 & 20.4 & 963.81 & & \\
\hline-35.0 & 962.11 & 34.0 & 964.19 & -39.0 & 962.03 & 22.0 & 963.98 & & \\
\hline-33.0 & 962.10 & 37.0 & 964.17 & -37.0 & 962.04 & 24.0 & 964.05 & & \\
\hline-31.0 & 962.09 & 40.0 & 964.11 & -35.0 & 962.05 & 26.0 & 964.10 & & \\
\hline-29.0 & 962.09 & 43.0 & 964.08 & -33.0 & 961.80 & 29.0 & 964.13 & & \\
\hline-27.0 & 962.03 & 45.0 & 964.04 & -31.0 & 961.90 & 32.0 & 964.16 & & \\
\hline-25.0 & 961.97 & 48.0 & 963.99 & -29.0 & 961.98 & 35.0 & 964.18 & & \\
\hline-24.0 & 961.95 & 50.0 & 963.91 & -27.8 & 962.08 & 38.0 & 964.15 & & \\
\hline-22.0 & 962.10 & 52.0 & 963.75 & -27.0 & 962.17 & 41.0 & 964.09 & & \\
\hline-20.3 & 962.16 & 54.0 & 963.76 & -26.3 & 962.18 & 44.0 & 964.07 & & \\
\hline-18.7 & 962.37 & 57.0 & 963.79 & -25.6 & 962.39 & 47.0 & 963.97 & & \\
\hline-18.3 & 962.55 & 60.0 & 963.76 & -24.0 & 962.49 & 49.0 & 963.95 & & \\
\hline-17.7 & 962.58 & 62.0 & 963.71 & -22.0 & 962.68 & 51.0 & 963.81 & & \\
\hline-17.4 & 962.66 & 64.0 & 963.74 & -19.6 & 962.79 & 53.0 & 963.75 & & \\
\hline-17.0 & 962.65 & 66.8 & 963.65 & -19.0 & 962.73 & 55.0 & 963.78 & & \\
\hline-15.5 & 962.71 & 69.0 & 964.03 & -18.3 & 962.76 & 57.0 & 963.79 & & \\
\hline-14.9 & 962.66 & 71.0 & 964.18 & -17.3 & 962.92 & 58.0 & 963.82 & & \\
\hline-13.7 & 962.90 & 73.0 & 964.17 & -16.0 & 963.10 & 59.0 & 963.80 & & \\
\hline-12.7 & 963.41 & 75.0 & 964.27 & -15.0 & 963.15 & 61.0 & 963.74 & & \\
\hline-12.0 & 963.50 & & & -14.4 & 963.20 & 63.0 & 963.66 & & \\
\hline-11.0 & 963.55 & & & -13.5 & 963.08 & 65.0 & 963.63 & & \\
\hline
\end{tabular}


Table 22. (Continued) Listing of horizontal stations and elevations for cross section PR163

[Sta., station, distance in meters from a reference pin on the left bank; Elev., elevation, in meters above sea level]

\begin{tabular}{|c|c|c|c|c|c|c|c|c|c|}
\hline \multirow{2}{*}{\multicolumn{2}{|c|}{$\begin{array}{c}1991 \\
31 \text { August }\end{array}$}} & \multicolumn{2}{|c|}{1991} & \multicolumn{2}{|c|}{1992} & \multicolumn{2}{|c|}{1992} & \multicolumn{2}{|c|}{1992} \\
\hline & & 31 & gust & 27 & gust & 27 & Igust & 27 & gust \\
\hline Sta. & Elev. & Sta. & Elev. & Sta. & Elev. & Sta. & Elev. & Sta. & Elev. \\
\hline-73.0 & 965.29 & -10.0 & 963.62 & -73.0 & 965.29 & -13.3 & 963.40 & 71.0 & 964.18 \\
\hline-71.0 & 965.16 & -8.0 & 963.52 & -72.0 & 965.21 & -12.0 & 963.54 & 73.0 & 964.18 \\
\hline-70.0 & 965.16 & -6.0 & 963.60 & -70.0 & 965.17 & -10.0 & 963.64 & 75.0 & 964.28 \\
\hline-69.0 & 965.25 & -4.0 & 963.66 & -68.0 & 965.26 & -8.0 & 963.54 & & \\
\hline-68.0 & 965.27 & -2.0 & 963.65 & -67.0 & 965.38 & -6.0 & 963.60 & & \\
\hline-67.0 & 965.37 & 0.0 & 963.64 & -66.0 & 965.34 & -4.0 & 963.63 & & \\
\hline-65.5 & 965.28 & 2.0 & 963.47 & -65.5 & 965.26 & -2.0 & 963.66 & & \\
\hline-65.4 & 964.59 & 4.0 & 963.42 & -65.4 & 964.48 & 0.0 & 963.61 & & \\
\hline-64.9 & 963.90 & 6.0 & 963.45 & -65.4 & 964.58 & 2.0 & 963.47 & & \\
\hline-64.4 & 963.35 & 8.0 & 963.42 & -64.6 & 963.22 & 4.0 & 963.43 & & \\
\hline-63.9 & 962.96 & 10.0 & 963.28 & -64.0 & 962.78 & 6.0 & 963.44 & & \\
\hline-63.6 & 962.88 & 12.0 & 963.30 & -63.5 & 962.64 & 8.0 & 963.42 & & \\
\hline-62.0 & 962.28 & 14.0 & 963.10 & -63.0 & 962.56 & 10.0 & 963.28 & & \\
\hline-60.9 & 962.07 & 15.0 & 963.12 & -62.0 & 962.30 & 12.0 & 963.29 & & \\
\hline-60.0 & 961.91 & 17.0 & 963.34 & -61.0 & 961.98 & 14.0 & 963.11 & & \\
\hline-58.6 & 961.92 & 19.0 & 963.60 & -59.2 & 961.94 & 15.0 & 963.15 & & \\
\hline-57.0 & 961.83 & 21.0 & 963.87 & -57.0 & 961.98 & 16.0 & 963.27 & & \\
\hline-55.0 & 961.89 & 22.0 & 963.96 & -55.0 & 962.02 & 18.0 & 963.52 & & \\
\hline-53.0 & 961.85 & 24.0 & 964.04 & -53.0 & 962.02 & 20.0 & 963.74 & & \\
\hline-51.0 & 961.89 & 26.0 & 964.08 & -51.0 & 962.03 & 21.0 & 963.89 & & \\
\hline-50.4 & 962.06 & 29.0 & 964.14 & -49.0 & 962.05 & 22.0 & 963.99 & & \\
\hline-48.0 & 962.09 & 32.0 & 964.15 & -47.0 & 961.99 & 24.0 & 964.05 & & \\
\hline-46.0 & 962.11 & 35.0 & 964.18 & -45.0 & 962.02 & 27.0 & 964.11 & & \\
\hline-44.0 & 962.12 & 38.0 & 964.15 & -43.0 & 962.09 & 30.0 & 964.14 & & \\
\hline-42.0 & 962.14 & 41.0 & 964.08 & -41.0 & 962.17 & 33.0 & 964.18 & & \\
\hline-40.0 & 962.07 & 44.0 & 964.07 & -39.0 & 962.16 & 36.0 & 964.18 & & \\
\hline-38.0 & 962.09 & 47.0 & 963.97 & -37.0 & 962.16 & 39.0 & 964.11 & & \\
\hline-36.0 & 962.08 & 50.0 & 963.90 & -35.0 & 962.17 & 42.0 & 964.08 & & \\
\hline-35.5 & 962.30 & 52.0 & 963.75 & -33.0 & 962.12 & 44.0 & 964.10 & & \\
\hline-33.0 & 962.39 & 54.0 & 963.75 & -31.0 & 962.06 & 46.0 & 964.00 & & \\
\hline-31.0 & 962.44 & 56.0 & 963.78 & -29.0 & 962.05 & 48.0 & 963.99 & & \\
\hline-29.0 & 962.45 & 58.0 & 963.80 & -27.0 & 962.03 & 50.0 & 963.88 & & \\
\hline-27.0 & 962.43 & 60.0 & 963.76 & -25.0 & 962.01 & 52.0 & 963.75 & & \\
\hline-25.0 & 962.42 & 62.0 & 963.71 & -23.0 & 962.01 & 53.0 & 963.74 & & \\
\hline-23.0 & 962.37 & 64.0 & 963.63 & -21.0 & 962.14 & 55.0 & 963.78 & & \\
\hline-21.0 & 962.33 & 66.0 & 963.63 & -19.0 & 962.23 & 57.0 & 963.79 & & \\
\hline-19.0 & 962.34 & 66.5 & 963.59 & -18.0 & 962.29 & 59.0 & 963.79 & & \\
\hline-17.0 & 962.29 & 67.0 & 963.67 & -17.0 & 962.23 & 61.0 & 963.73 & & \\
\hline-16.4 & 962.33 & 69.0 & 964.01 & -16.6 & 962.29 & 63.0 & 963.67 & & \\
\hline-15.0 & 963.01 & 71.0 & 964.15 & -15.7 & 962.64 & 66.0 & 963.64 & & \\
\hline-13.2 & 963.38 & 73.0 & 964.16 & -15.0 & 963.08 & 67.0 & 963.70 & & \\
\hline-11.0 & 963.57 & 75.0 & 964.27 & -14.3 & 963.24 & 69.0 & 964.05 & & \\
\hline
\end{tabular}


Table 22. (Continued) Listing of horizontal stations and elevations for cross section PR163

[Sta., station, distance in meters from a reference pin on the left bank; Elev., elevation, in meters above sea level]

\begin{tabular}{|c|c|c|c|c|c|c|c|c|c|}
\hline \multirow{2}{*}{\multicolumn{2}{|c|}{$\begin{array}{c}1993 \\
30 \text { August }\end{array}$}} & \multirow{2}{*}{\multicolumn{2}{|c|}{$\begin{array}{c}1993 \\
30 \text { August }\end{array}$}} & \multirow{2}{*}{\multicolumn{2}{|c|}{$\begin{array}{c}1993 \\
30 \text { August }\end{array}$}} & \multicolumn{2}{|c|}{1994} & \multicolumn{2}{|c|}{1994} \\
\hline & & & & & & $21 S$ & ember & $21 \mathrm{~S}$ & ember \\
\hline Sta. & Elev. & Sta. & Elev. & Sta. & Elev. & Sta. & Elev. & Sta. & Elev. \\
\hline-75.0 & 965.52 & -13.8 & 963.62 & 64.0 & 963.65 & -73.0 & 965.29 & 8.0 & 963.48 \\
\hline-73.0 & 965.29 & -13.1 & 963.73 & 66.0 & 963.65 & -72.0 & 965.21 & 10.0 & 963.35 \\
\hline-71.0 & 965.16 & -12.0 & 963.69 & 67.0 & 963.69 & -71.0 & 965.16 & 12.0 & 963.33 \\
\hline-69.0 & 965.26 & -10.0 & 963.71 & 67.7 & 963.86 & -70.0 & 965.17 & 14.0 & 963.19 \\
\hline-67.5 & 965.28 & -9.0 & 963.61 & 69.0 & 964.03 & -68.3 & 965.24 & 15.0 & 963.21 \\
\hline-67.5 & 964.08 & -8.0 & 963.59 & 71.0 & 964.16 & -68.0 & 964.36 & 16.0 & 963.32 \\
\hline-67.2 & 963.49 & -6.0 & 963.64 & 73.0 & 964.18 & -66.5 & 963.25 & 17.0 & 963.43 \\
\hline-66.5 & 963.23 & -4.0 & 963.68 & & & -65.9 & 963.01 & 18.0 & 963.58 \\
\hline-66.3 & 963.04 & -2.0 & 963.68 & & & -64.5 & 962.24 & 20.0 & 963.78 \\
\hline-65.4 & 962.97 & 0.0 & 963.65 & & & -63.6 & 961.92 & 22.0 & 964.00 \\
\hline-65.1 & 962.81 & 1.0 & 963.59 & & & -62.0 & 962.03 & 25.0 & 964.07 \\
\hline-65.0 & 962.63 & 2.0 & 963.50 & & & -59.0 & 962.02 & 27.5 & 964.13 \\
\hline-64.7 & 962.42 & 4.0 & 963.47 & & & -56.0 & 962.03 & 30.0 & 964.13 \\
\hline-63.0 & 961.98 & 6.0 & 963.49 & & & -53.0 & 962.06 & 33.0 & 964.18 \\
\hline-61.0 & 961.97 & 8.0 & 963.46 & & & -49.0 & 962.09 & 36.0 & 964.18 \\
\hline-59.0 & 961.82 & 10.0 & 963.32 & & & -46.0 & 962.04 & 39.0 & 964.12 \\
\hline-57.0 & 961.72 & 12.0 & 963.31 & & & -43.0 & 962.00 & 42.0 & 964.07 \\
\hline-55.0 & 961.73 & 13.8 & 963.16 & & & -40.0 & 961.98 & 45.0 & 964.03 \\
\hline-53.0 & 961.74 & 15.0 & 963.19 & & & -37.4 & 962.00 & 48.0 & 963.99 \\
\hline-50.6 & 962.23 & 16.5 & 963.36 & & & -36.5 & 962.24 & 51.0 & 963.83 \\
\hline-49.0 & 962.23 & 18.0 & 963.56 & & & -36.2 & 962.31 & 54.0 & 963.76 \\
\hline-47.0 & 962.17 & 20.0 & 963.76 & & & -36.1 & 962.46 & 57.0 & 963.78 \\
\hline-45.0 & 962.18 & 21.5 & 963.96 & & & -34.0 & 962.52 & 60.0 & 963.78 \\
\hline-43.0 & 962.14 & 23.0 & 964.02 & & & -31.0 & 962.52 & 63.0 & 963.70 \\
\hline-41.0 & 962.17 & 25.0 & 964.06 & & & -28.0 & 962.54 & 65.5 & 963.66 \\
\hline-39.0 & 962.21 & 28.0 & 964.14 & & & -25.0 & 962.53 & 67.0 & 963.70 \\
\hline-37.0 & 962.22 & 30.0 & 964.13 & & & -22.0 & 962.48 & 68.0 & 963.92 \\
\hline-35.0 & 962.21 & 31.0 & 964.14 & & & -19.0 & 962.48 & 70.0 & 964.13 \\
\hline-33.0 & 962.25 & 34.0 & 963.87 & & & -17.6 & 962.53 & 72.5 & 964.17 \\
\hline-31.6 & 962.37 & 37.0 & 963.86 & & & -17.1 & 962.64 & 75.0 & 964.29 \\
\hline-31.5 & 962.48 & 40.0 & 964.10 & & & -16.5 & 962.54 & & \\
\hline-30.0 & 962.50 & 42.0 & 964.08 & & & -15.7 & 962.79 & & \\
\hline-28.0 & 962.50 & 44.0 & 964.07 & & & -15.4 & 963.08 & & \\
\hline-25.7 & 962.48 & 46.0 & 963.99 & & & -14.8 & 963.20 & & \\
\hline-24.0 & 962.40 & 47.0 & 963.96 & & & -13.5 & 963.75 & & \\
\hline-22.0 & 962.38 & 49.0 & 963.95 & & & -12.0 & 963.72 & & \\
\hline-20.0 & 962.37 & 50.0 & 963.89 & & & -9.0 & 963.63 & & \\
\hline-18.0 & 962.37 & 52.0 & 963.76 & & & -6.0 & 963.65 & & \\
\hline-16.2 & 962.35 & 55.0 & 963.79 & & & -3.0 & 963.70 & & \\
\hline-15.7 & 962.54 & 57.0 & 963.77 & & & 0.0 & 963.67 & & \\
\hline-15.3 & 962.95 & 60.0 & 963.77 & & & 2.0 & 963.52 & & . \\
\hline-14.3 & 963.36 & 62.0 & 963.73 & & & 5.0 & 963.49 & & \\
\hline
\end{tabular}


Table 22. (Continued) Listing of horizontal stations and elevations for cross section PR163

[Sta., station, distance in meters from a reference pin on the left bank; Elev., elevation, in meters above sea level]

\begin{tabular}{|c|c|c|c|c|c|c|c|c|c|}
\hline \multirow{2}{*}{\multicolumn{2}{|c|}{$\begin{array}{c}1995 \\
25 \text { September }\end{array}$}} & \multicolumn{2}{|c|}{1995} & \multicolumn{2}{|c|}{1995} & \multicolumn{2}{|c|}{1996} & \multicolumn{2}{|c|}{1996} \\
\hline & & $25 \mathrm{Se}$ & ember & $25 \mathrm{Se}$ & mber & $24 c$ & tober & $24 C$ & ober \\
\hline Sta. & Elev. & Sta. & Elev. & Sta. & Elev. & Sta. & Elev. & Sta. & Elev. \\
\hline-88.0 & 965.39 & -15.1 & 963.03 & 58.0 & 963.85 & -88.0 & 965.39 & 0.0 & 963.89 \\
\hline-86.0 & 965.01 & -13.2 & 963.90 & 60.0 & 963.85 & -85.0 & 964.70 & 2.0 & 963.72 \\
\hline-84.0 & 964.60 & -12.0 & 964.00 & 62.0 & 963.85 & -84.0 & 964.61 & 5.0 & 963.70 \\
\hline-83.0 & 964.68 & -10.0 & 964.04 & 65.0 & 963.68 & -83.0 & 964.69 & 8.0 & 963.68 \\
\hline-81.0 & 965.16 & -8.0 & 964.04 & 66.0 & 963.71 & -81.0 & 965.18 & 11.0 & 963.55 \\
\hline-79.0 & 965.46 & -6.0 & 963.90 & 67.0 & 963.76 & -79.0 & 965.45 & 13.0 & 963.52 \\
\hline-77.0 & 965.69 & -5.0 & 963.88 & 68.0 & 963.95 & -77.0 & 965.69 & 15.5 & 963.48 \\
\hline-75.0 & 965.54 & -4.0 & 963.97 & 70.0 & 964.16 & -76.7 & 965.67 & 17.5 & 963.62 \\
\hline-73.0 & 965.29 & -3.0 & 963.90 & 72.0 & 964.17 & -76.7 & 963.97 & 19.0 & 963.77 \\
\hline-71.7 & 965.20 & -2.0 & 963.92 & & & -74.8 & 962.47 & 21.0 & 963.95 \\
\hline-71.5 & 963.67 & 0.0 & 963.80 & & & -73.6 & 962.35 & 23.0 & 964.09 \\
\hline-70.5 & 963.25 & 0.7 & 963.85 & & & -72.7 & 961.94 & 26.0 & 964.17 \\
\hline-69.8 & 962.83 & 2.0 & 963.68 & & & -70.0 & 961.92 & 28.0 & 964.21 \\
\hline-69.0 & 962.44 & 4.0 & 963.64 & & & -67.0 & 961.93 & 30.0 & 964.22 \\
\hline-68.0 & 962.09 & 6.0 & 963.65 & & & -64.0 & 962.10 & 33.0 & 964.25 \\
\hline-66.0 & 962.10 & 8.0 & 963.64 & & & -61.0 & 962.21 & 36.0 & 964.26 \\
\hline-64.0 & 962.12 & 10.0 & 963.54 & & & -58.0 & 962.32 & 39.0 & 964.21 \\
\hline-62.0 & 962.09 & 11.0 & 963.50 & & & -55.0 & 962.36 & 42.0 & 964.15 \\
\hline-60.0 & 962.02 & 13.0 & 963.49 & & & -52.0 & 962.35 & 46.0 & 964.06 \\
\hline-58.0 & 961.82 & 15.0 & 963.47 & & & -49.0 & 962.33 & 50.0 & 963.95 \\
\hline-56.0 & 962.10 & 17.0 & 963.53 & & & -46.5 & 962.01 & 54.0 & 963.80 \\
\hline-54.0 & 962.24 & 18.0 & 963.67 & & & -44.0 & 961.99 & 57.0 & 963.86 \\
\hline-52.0 & 962.26 & 20.0 & 963.84 & & & -41.0 & 962.24 & 60.0 & 963.87 \\
\hline-50.0 & 962.21 & 22.0 & 964.03 & & & -38.0 & 962.29 & & \\
\hline-48.0 & 962.18 & 24.0 & 964.09 & & & -36.8 & 962.40 & & \\
\hline-46.0 & 962.11 & 26.0 & 964.14 & & & -36.0 & 962.35 & & \\
\hline-44.0 & 962.14 & 28.0 & 964.16 & & & -33.0 & 962.27 & & \\
\hline-42.0 & 962.12 & 30.0 & 964.20 & & & -30.0 & 962.45 & & \\
\hline-40.0 & 962.06 & 32.0 & 964.24 & & & -29.0 & 962.63 & & \\
\hline-38.0 & 962.16 & 34.0 & 964.26 & & & -27.0 & 962.66 & & \\
\hline-35.6 & 962.29 & 35.0 & 964.28 & & & -25.0 & 962.61 & & \\
\hline-35.0 & 962.45 & 37.0 & 964.20 & & & -22.0 & 962.74 & & \\
\hline-34.4 & 962.66 & 39.0 & 964.16 & & & -21.0 & 962.71 & & \\
\hline-32.0 & 962.70 & 41.0 & 964.13 & & & -19.0 & 962.91 & & \\
\hline-30.0 & 962.71 & 43.0 & 964.12 & & & -17.0 & 962.90 & & \\
\hline-28.0 & 962.62 & 45.0 & 964.14 & & & -15.5 & 962.96 & & \\
\hline-26.0 & 962.62 & 46.0 & 964.04 & & & -14.0 & 963.64 & & \\
\hline-24.0 & 962.66 & 48.0 & 964.02 & & & -13.0 & 963.90 & & \\
\hline-22.0 & 962.75 & 50.0 & 963.93 & & & -10.0 & 964.08 & & \\
\hline-20.0 & 962.88 & 52.0 & 963.82 & & & -8.0 & 964.06 & & \\
\hline-18.0 & 962.99 & 54.0 & 963.78 & & & -5.0 & 963.99 & & \\
\hline-16.0 & 962.91 & 56.0 & 963.84 & & & -2.0 & 963.98 & & \\
\hline
\end{tabular}


Table 22. (Continued) Listing of horizontal stations and elevations for cross section PR163

[Sta., station, distance in meters from a reference pin on the left bank; Elev., elevation, in meters above sea level]

\begin{tabular}{|c|c|c|c|c|c|c|c|}
\hline \multirow{2}{*}{\multicolumn{2}{|c|}{$\begin{array}{c}1997 \\
22 \text { September }\end{array}$}} & \multicolumn{2}{|c|}{1997} & \multicolumn{2}{|c|}{1998} & \multicolumn{2}{|c|}{1998} \\
\hline & & $22 \mathrm{~S}$ & ember & $28 \mathrm{Se}$ & ember & $28 \mathrm{~S}$ & ember \\
\hline Sta. & Elev. & Sta. & Elev. & Sta. & Elev. & Sta. & Elev. \\
\hline-88.0 & 965.39 & 2.0 & 963.76 & -100.0 & 965.12 & 2.0 & 963.76 \\
\hline-86.5 & 965.11 & 4.0 & 963.76 & -97.0 & 965.21 & 4.0 & 963.72 \\
\hline-84.0 & 964.61 & 6.0 & 963.70 & -94.0 & 965.39 & 7.0 & 963.75 \\
\hline-83.0 & 964.69 & 8.0 & 963.69 & -91.0 & 965.42 & 10.0 & 963.62 \\
\hline-80.0 & 965.30 & 10.0 & 963.60 & -88.0 & 965.39 & 13.0 & 963.54 \\
\hline-77.7 & 965.57 & 12.0 & 963.61 & -86.0 & 965.03 & 15.0 & 963.52 \\
\hline-77.5 & 963.62 & 14.0 & 963.55 & -83.7 & 964.61 & 17.0 & 963.61 \\
\hline-76.6 & 963.17 & 15.7 & 963.51 & -82.0 & 964.92 & 20.0 & 963.87 \\
\hline-76.5 & 962.72 & 17.0 & 963.60 & -80.0 & 965.29 & 23.0 & 964.10 \\
\hline-76.3 & 962.50 & 18.0 & 963.72 & -79.0 & 965.42 & 26.0 & 964.18 \\
\hline-75.3 & 961.96 & 20.0 & 963.87 & -79.0 & 964.13 & 28.0 & 964.22 \\
\hline-72.0 & 962.06 & 23.0 & 964.09 & -78.0 & 963.28 & 30.0 & 964.24 \\
\hline-70.0 & 962.15 & 26.0 & 964.19 & -76.9 & 962.65 & 33.0 & 964.26 \\
\hline-67.0 & 962.20 & 28.0 & 964.22 & -75.0 & 961.99 & 36.0 & 964.27 \\
\hline-64.0 & 962.21 & 30.0 & 964.23 & -73.0 & 961.70 & 39.0 & 964.21 \\
\hline-61.0 & 962.12 & 33.0 & 964.25 & -71.0 & 961.65 & 42.0 & 964.16 \\
\hline-59.0 & 962.16 & 36.0 & 964.27 & -68.5 & 962.01 & 45.0 & 964.17 \\
\hline-56.0 & 962.12 & 39.0 & 964.22 & -66.0 & 962.14 & 48.0 & 964.07 \\
\hline-53.0 & 962.13 & 42.0 & 964.16 & -63.0 & 962.21 & 50.0 & 963.98 \\
\hline-50.0 & 962.17 & 44.0 & 964.15 & -60.0 & 962.20 & 53.0 & 963.85 \\
\hline-47.0 & 962.15 & 46.0 & 964.08 & -57.0 & 962.22 & 56.0 & 963.87 \\
\hline-44.0 & 962.14 & 48.0 & 964.07 & -54.0 & 962.22 & 59.0 & 963.88 \\
\hline-41.5 & 962.12 & 50.0 & 963.97 & -51.0 & 962.17 & 62.0 & 963.86 \\
\hline-40.0 & 962.35 & & & -48.0 & 962.30 & 65.0 & 963.71 \\
\hline-37.0 & 962.38 & & & -45.0 & 962.32 & 67.0 & 963.79 \\
\hline-34.0 & 962.35 & & & -42.0 & 962.35 & 69.0 & 964.07 \\
\hline-31.0 & 962.41 & & & -39.0 & 962.25 & 72.0 & 964.20 \\
\hline-28.5 & 962.48 & & & -36.0 & 962.28 & 75.0 & 964.32 \\
\hline-26.0 & 962.49 & & & -33.0 & 962.48 & & \\
\hline-23.0 & 962.61 & & & -30.0 & 962.53 & & \\
\hline-20.0 & 962.78 & & & -27.0 & 962.52 & & \\
\hline-17.0 & 962.80 & & & -24.7 & 962.65 & & \\
\hline-15.4 & 962.89 & & & -22.0 & 962.72 & & \\
\hline-14.6 & 963.38 & & & -19.0 & 962.86 & & \\
\hline-13.8 & 963.72 & & & -15.8 & 962.96 & & \\
\hline-12.0 & 963.99 & & & -14.8 & 963.20 & & \\
\hline-10.0 & 964.10 & & & -13.0 & 963.94 & & \\
\hline-8.0 & 964.08 & & & -11.0 & 964.04 & & \\
\hline-6.0 & 964.00 & & & -8.0 & 964.06 & & \\
\hline-4.0 & 964.03 & & & -5.0 & 964.01 & & \\
\hline-2.0 & 964.00 & & & -2.0 & 963.99 & & \\
\hline 0.0 & 963.92 & & & 0.0 & 963.92 & & \\
\hline
\end{tabular}




\section{Description of Cross Section PR164.8}

Location: Township 6 South/Range 50 East--section 28

U. S. Geological Survey quadrangle (1:24,000): Lonesome Peak

Landowners--left bank: U. S. Government

--right bank: U. S. Government

Access: Left bank

Permission from: Gay Ranch

Distance from Moorhead Gaging Station: 49.6 kilometers

Azimuth of Section (degrees magnetic): 185

\section{Reference Monuments}

[Monuments at stations $\mathbf{- 1 . 5}$ and 0.0 were closest to leveling instrument]

\begin{tabular}{|c|c|c|c|c|c|c|}
\hline \multirow[b]{2}{*}{ Description } & \multirow[b]{2}{*}{$\begin{array}{c}\text { Station } \\
(\mathrm{m})\end{array}$} & \multicolumn{2}{|c|}{ GPS-NAD83 (1992) } & \multicolumn{2}{|c|}{ Measurement } & \multirow{2}{*}{$\begin{array}{l}\text { Elevation } \\
\text { (NGVD1929) } \\
\text { (m) }\end{array}$} \\
\hline & & Latitude & Longitude & $\begin{array}{l}\text { Standard } \\
\text { deviation } \\
\text { (m) }\end{array}$ & $\begin{array}{l}\text { Horizontal } \\
\text { precision } \\
\text { (m) }\end{array}$ & \\
\hline $\begin{array}{l}\text { 1/2-inch-rebar; } 0.17 \text { meter above } 1998 \text { ground } \\
\text { level; }\end{array}$ & -1.5 & $45^{\circ} 16^{\prime} 39.98^{\prime \prime}$ & $105^{\circ} 36^{\prime} 35.00^{\prime \prime}$ & 0.413 & 0.475 & 962.75 \\
\hline $\begin{array}{l}\text { 1/2-inch-rebar; } 0.15 \text { meter above } 1998 \text { ground } \\
\text { level; }\end{array}$ & 0.0 & & & & & 962.61 \\
\hline $\begin{array}{l}\text { 1/2-inch-rebar; } 0.10 \text { meter above } 1998 \text { ground } \\
\text { level; } 1.4 \text { meters downstream from a } 10- \\
\text { meter high willow tree (tallest of two wil- } \\
\text { low tress among lower cottonwood trees) }\end{array}$ & 121.0 & & & & & 961.37 \\
\hline $\begin{array}{l}\text { 1/2-inch-rebar; } 0.17 \text { meter above } 1998 \text { ground } \\
\text { level; at riverward edge of } 15-20 \text { meter } \\
\text { high cottonwood trees }\end{array}$ & 150.0 & $45^{\circ} 16^{\prime} 35.38^{\prime \prime}$ & $105^{\circ} 36^{\prime} 37.31^{\prime \prime}$ & 0.664 & 0.957 & 962.29 \\
\hline
\end{tabular}


See Figure 8 for location

of cross section

PR164.8

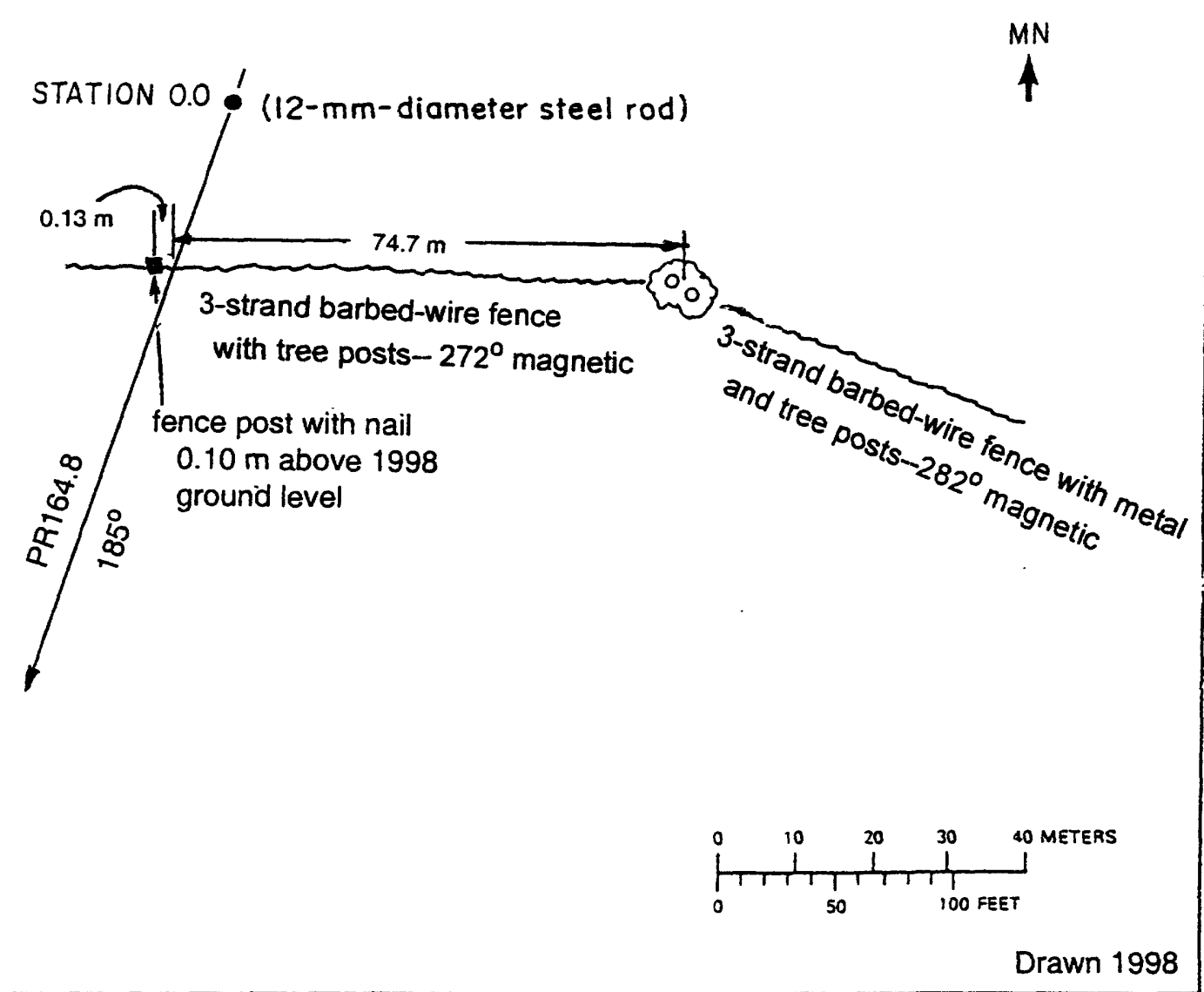

Figure 66. Upper: Location of cross section PR164.8 is shown in figure 8. Lower: Location of the reference monument on the left bank. $\mathrm{MN}$ is magnetic north. 

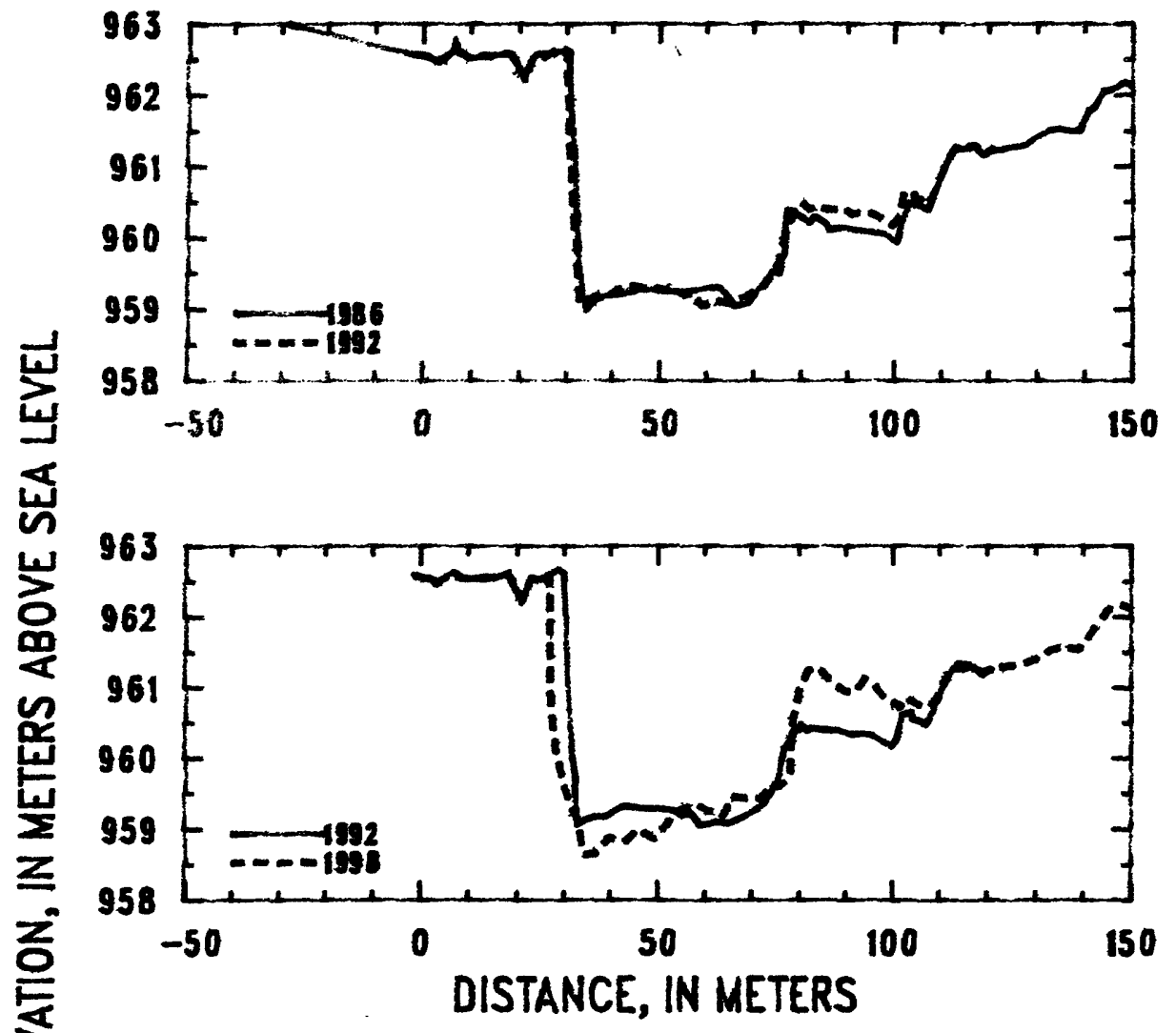

Figure 67. Profiles of cross section PR164.8 from 1986 to 1998. 
Table 23. Listing of horizontal stations and elevations for cross section PR164.8

[Sta., station, distance in meters from a reference pin on the left bank; Elev., elevation, in meters above sea level]

\begin{tabular}{|c|c|c|c|c|c|c|c|c|c|}
\hline \multirow{2}{*}{\multicolumn{2}{|c|}{$\begin{array}{c}1986 \\
25 \text { August }\end{array}$}} & \multirow{2}{*}{\multicolumn{2}{|c|}{$\begin{array}{c}1986 \\
25 \text { August }\end{array}$}} & \multirow{2}{*}{\multicolumn{2}{|c|}{$\begin{array}{c}1992 \\
30 \text { August }\end{array}$}} & \multirow{2}{*}{\multicolumn{2}{|c|}{$\begin{array}{c}1992 \\
30 \text { August }\end{array}$}} & \multirow{2}{*}{\multicolumn{2}{|c|}{$\begin{array}{c}1998 \\
1 \text { October }\end{array}$}} \\
\hline & & & & & & & & & \\
\hline Sta. & Elev. & Sta. & Elev. & Sta. & Elev. & Sta. & Elev. & Sta. & Elev. \\
\hline-63.0 & 963.55 & 74.0 & 959.53 & -1.5 & 962.57 & 67.0 & 959.14 & -1.5 & 962.58 \\
\hline-1.5 & 962.57 & 75.5 & 959.50 & 0.0 & 962.56 & 69.0 & 959.20 & 0.0 & 962.56 \\
\hline 0.0 & 962.56 & 76.8 & 959.83 & 2.0 & 962.54 & 71.0 & 959.27 & 3.0 & 962.46 \\
\hline 2.4 & 962.49 & 76.9 & 960.31 & 3.0 & 962.46 & 72.4 & 959.34 & 6.0 & 962.60 \\
\hline 4.0 & 962.51 & 77.2 & 960.39 & 5.0 & 962.56 & 73.3 & 959.43 & 9.0 & 962.54 \\
\hline 6.2 & 962.62 & 78.0 & 960.37 & 7.0 & 962.63 & 74.0 & 959.50 & 12.0 & 962.53 \\
\hline 6.7 & 962.74 & 80.0 & 960.29 & 9.0 & 962.53 & 75.4 & 959.65 & 15.0 & 962.56 \\
\hline 7.3 & 962.60 & 82.0 & 960.21 & 11.0 & 962.53 & 76.8 & 960.11 & 18.1 & 962.61 \\
\hline 9.0 & 962.53 & 83.0 & 960.30 & 13.0 & 962.57 & 77.4 & 960.20 & 21.0 & 962.24 \\
\hline 12.0 & 962.53 & 85.5 & 960.20 & 15.0 & 962.55 & 78.2 & 960.25 & 23.0 & 962.56 \\
\hline 15.0 & 962.57 & 86.3 & 960.10 & 17.0 & 962.59 & 78.7 & 960.37 & 25.0 & 962.51 \\
\hline 18.0 & 962.59 & 89.0 & 960.14 & 18.1 & 962.59 & 80.0 & 960.41 & 26.9 & 962.56 \\
\hline 19.0 & 962.49 & 92.0 & 960.11 & 19.0 & 962.49 & 80.5 & 960.48 & 27.2 & 960.74 \\
\hline 21.0 & 962.25 & 95.0 & 960.07 & 21.0 & 962.21 & 81.8 & 960.39 & 28.2 & 960.15 \\
\hline 23.0 & 962.55 & 98.0 & 960.05 & 23.0 & 962.55 & 83.0 & 960.44 & 29.8 & 959.69 \\
\hline 24.0 & 962.59 & 100.5 & 959.93 & 25.0 & 962.53 & 85.0 & 960.40 & 30.5 & 959.53 \\
\hline 27.0 & 962.61 & 103.0 & 960.49 & 27.0 & 962.61 & 87.0 & 960.39 & 33.0 & 959.14 \\
\hline 30.0 & 962.62 & 105.0 & 960.46 & 29.0 & 962.67 & 89.0 & 960.40 & 34.5 & 958.64 \\
\hline 30.8 & 962.62 & 107.0 & 960.39 & 30.1 & 962.62 & 91.0 & 960.33 & 37.0 & 958.66 \\
\hline 30.8 & 962.34 & 109.0 & 960.68 & 30.2 & 962.30 & 93.0 & 960.35 & 40.0 & 958.88 \\
\hline 31.2 & 961.83 & 111.0 & 961.05 & 30.6 & 961.98 & 95.0 & 960.33 & 43.0 & 958.81 \\
\hline 32.3 & 961.04 & 113.0 & 961.26 & 30.7 & 961.46 & 97.0 & 960.27 & 46.0 & 958.99 \\
\hline 32.3 & 960.34 & 115.0 & 961.25 & 32.0 & 960.02 & 99.7 & 960.17 & 49.0 & 958.87 \\
\hline 32.6 & 960.30 & 117.0 & 961.30 & 32.5 & 959.85 & 100.8 & 960.27 & 52.0 & 959.08 \\
\hline 32.7 & 959.79 & 119.0 & 961.16 & 32.7 & 959.43 & 102.0 & 960.62 & 55.0 & 959.27 \\
\hline 33.2 & 959.39 & 121.0 & 961.24 & 33.2 & 959.07 & 104.0 & 960.65 & 58.0 & 959.35 \\
\hline 34.5 & 958.99 & 123.0 & 961.24 & 35.0 & 959.14 & 104.4 & 960.55 & 61.0 & 959.25 \\
\hline 36.0 & 959.08 & 125.0 & 961.29 & 37.0 & 959.18 & 106.0 & 960.53 & 64.0 & 959.19 \\
\hline 39.0 & 959.20 & 127.0 & 961.29 & 39.0 & 959.17 & 107.0 & 960.47 & 66.0 & 959.46 \\
\hline 42.0 & 959.20 & 130.0 & 961.41 & 41.0 & 959.26 & 109.0 & 960.71 & 69.0 & 959.43 \\
\hline 45.0 & 959.25 & 133.0 & 961.52 & 43.0 & 959.33 & 111.0 & 961.05 & 72.0 & 959.43 \\
\hline 48.0 & 959.28 & 135.0 & 961.54 & 45.0 & 959.31 & 113.0 & 961.27 & 73.1 & 959.52 \\
\hline 51.0 & 959.24 & 137.0 & 961.51 & 47.0 & 959.29 & 115.0 & 961.27 & 73.7 & 959.63 \\
\hline 54.0 & 959.24 & 139.0 & 961.51 & 49.0 & 959.29 & 117.0 & 961.31 & 76.0 & 959.61 \\
\hline 57.0 & 959.25 & 141.0 & 961.80 & 51.0 & 959.28 & 119.0 & 961.18 & 77.6 & 959.73 \\
\hline 60.0 & 959.30 & 142.0 & 961.82 & 53.0 & 959.27 & 121.0 & 961.26 & 78.2 & 959.82 \\
\hline 63.0 & 959.30 & 144.0 & 962.06 & 55.0 & 959.20 & & & 78.6 & 960.45 \\
\hline 65.0 & 959.13 & 146.0 & 962.08 & 57.0 & 959.22 & & & 80.0 & 960.95 \\
\hline 66.0 & 959.05 & 148.0 & 962.18 & 59.0 & 959.05 & & & 82.0 & 961.25 \\
\hline 69.0 & 959.08 & 150.0 & 962.14 & 61.0 & 959.07 & & & 84.0 & 961.29 \\
\hline 71.0 & 959.26 & & & 63.0 & 959.11 & & & 86.0 & 961.15 \\
\hline 72.7 & 959.39 & & & 65.0 & 959.08 & & & 88.0 & 961.03 \\
\hline
\end{tabular}


Table 23. (Continued) Listing of horizontal stations and elevations for cross section PR164.8

[Sta., station, distance in meters from a reference pin on the left bank; Elev., elevation, in meters above sea level]

1998

\begin{tabular}{cc}
\multicolumn{2}{c}{1 October } \\
\hline Sta. & Elev. \\
\hline 90.0 & 960.94 \\
92.0 & 960.94 \\
94.0 & 961.12 \\
96.0 & 961.04 \\
98.0 & 960.86 \\
100.0 & 960.80 \\
101.5 & 960.71 \\
103.0 & 960.85 \\
106.0 & 960.74 \\
108.0 & 960.75 \\
110.0 & 960.92 \\
112.0 & 961.20 \\
114.0 & 961.34 \\
116.0 & 961.33 \\
119.0 & 961.21 \\
121.0 & 961.27 \\
124.0 & 961.30 \\
127.0 & 961.32 \\
130.0 & 961.40 \\
133.0 & 961.53 \\
136.0 & 961.58 \\
139.0 & 961.54 \\
142.0 & 961.80 \\
145.0 & 962.09 \\
148.0 & 962.16 \\
150.0 & 962.13
\end{tabular}




\section{Description of Cross Section PR165.6}

Location: Township 6 South/Range 50 East--section 28

U. S. Geological Survey quadrangle (1:24,000): Lonesome Peak

Landowners--left bank: Gay Ranch

--right bank: Daily Ranch

Access: Left bank

Permission from: Gay Ranch

Distance from Moorhead Gaging Station: 50.4 kilometers

Azimuth of Section (degrees magnetic): 105

\section{Reference Monuments}

[Monuments at stations -7.3 and -1.0 were closest to leveling instrument]

\begin{tabular}{|c|c|c|c|c|c|c|}
\hline \multirow[b]{2}{*}{ Description } & \multirow[b]{2}{*}{$\begin{array}{c}\text { Station } \\
(m)\end{array}$} & \multicolumn{2}{|c|}{ GPS-NAD83 (1992) } & \multicolumn{2}{|c|}{ Measurement } & \multirow{2}{*}{$\begin{array}{l}\text { Elevation } \\
\text { (NGVD1929) } \\
\text { (m) }\end{array}$} \\
\hline & & Latitude & Longitude & $\begin{array}{l}\text { Standard } \\
\text { deviation } \\
\text { (m) }\end{array}$ & $\begin{array}{l}\text { Horizontal } \\
\text { precision } \\
\quad(\mathbf{m})\end{array}$ & \\
\hline $\begin{array}{l}\text { 1/2-inch-rebar; } 0.10 \text { meter above } 1998 \text { ground } \\
\text { level; under } 3 \text {-strand, barbed-wire fence } \\
\text { with tree posts }\end{array}$ & -7.3 & $45^{\circ} 16^{\prime} 49.90^{\prime \prime}$ & $105^{\circ} 36^{\prime} 13.39^{\prime \prime}$ & 0.334 & 0.513 & 961.60 \\
\hline $\begin{array}{l}\text { 1/2-inch-rebar; } 0.13 \text { meter above } 1998 \text { ground } \\
\text { level; }\end{array}$ & -1.0 & & & & & 961.57 \\
\hline $\begin{array}{l}\text { 1/2-inch-rebar; } 0.10 \text { meter above } 1998 \text { ground } \\
\text { level; }\end{array}$ & 151.0 & $45^{\circ} 16^{\prime} 47.41^{\prime \prime}$ & $105^{\circ} 36^{\prime} 07.06^{\prime \prime}$ & 0.415 & 0.825 & 961.20 \\
\hline
\end{tabular}


See Figure 8 for location

of cross section

PR165.6

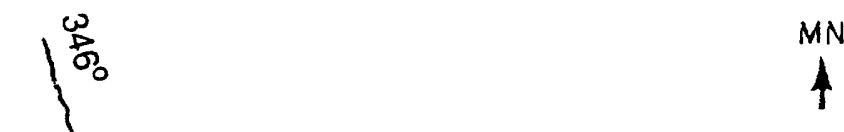

MN

3-strand barbed-wire fence

with tree posts

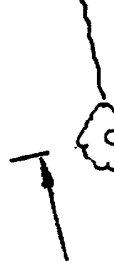

$31.2 \mathrm{~m}$

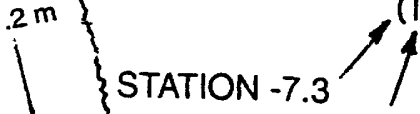

$1-$ STATION -1.0
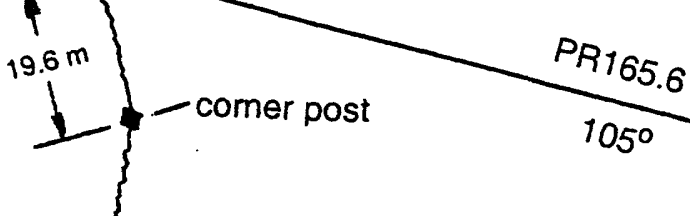

$\stackrel{2}{3}\}$

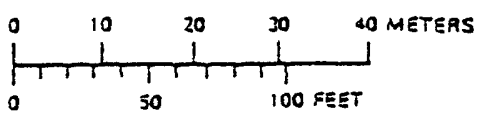

Drawn 1998

Figure 68. Upper: Location of cross section PR165.6 is shown in figure 8. Lower: Location of the reference monument on the left bank. MN is magnetic north. 


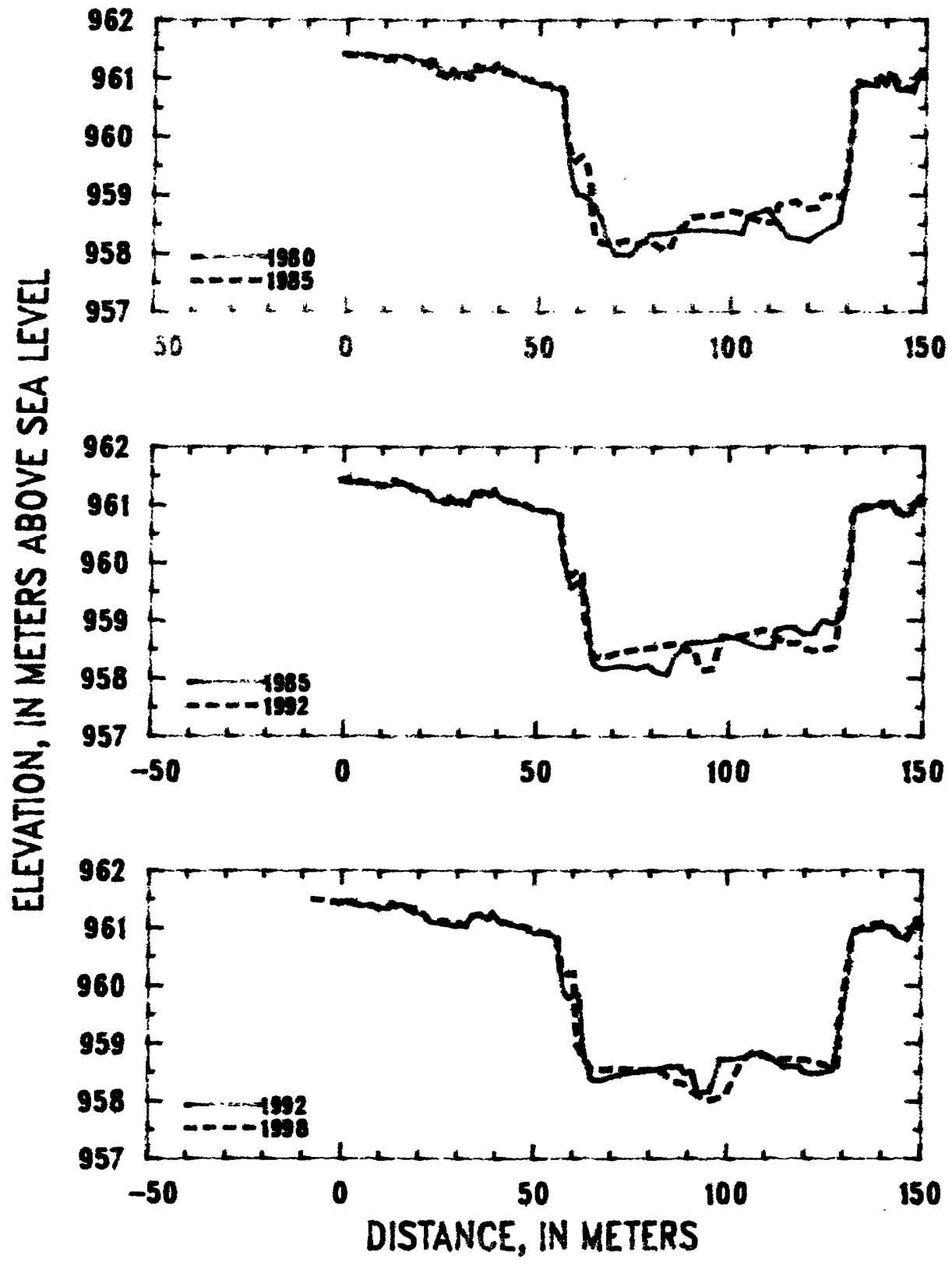

Figure 69. Profiles of cross section PR165.6 from 1980 to 1998. 
Table 24. Listing of horizontal stations and elevations for cross section PR165.6

[Sta., station, distance in meters from a reference pin on the left bank; Elev., elevation, in meters above sea level]

\begin{tabular}{|c|c|c|c|c|c|c|c|c|c|}
\hline \multirow{2}{*}{\multicolumn{2}{|c|}{$\begin{array}{c}1980 \\
20 \text { October }\end{array}$}} & \multirow{2}{*}{\multicolumn{2}{|c|}{$\begin{array}{c}1980 \\
20 \text { October }\end{array}$}} & \multirow{2}{*}{\multicolumn{2}{|c|}{$\begin{array}{c}1985 \\
29 \text { September }\end{array}$}} & \multirow{2}{*}{\multicolumn{2}{|c|}{$\begin{array}{c}1985 \\
29 \text { September }\end{array}$}} & \multirow{2}{*}{\multicolumn{2}{|c|}{$\begin{array}{c}1992 \\
30 \text { August }\end{array}$}} \\
\hline & & & & & & & & & \\
\hline Sta. & Elev. & Sta. & Elev. & Sta. & Elev. & Sta. & Elev. & Sta. & Elev. \\
\hline-1.0 & 961.43 & 128.0 & 958.54 & 0.0 & 961.41 & 86.0 & 958.38 & -1.0 & 961.44 \\
\hline 0.0 & 961.43 & 129.0 & 958.90 & 3.0 & 961.39 & 88.0 & 958.50 & 2.0 & 961.48 \\
\hline 5.0 & 961.40 & 130.0 & 959.23 & 6.0 & 961.39 & 89.0 & 958.61 & 5.0 & 961.38 \\
\hline 10.0 & 961.38 & 131.2 & 960.13 & 9.0 & 961.32 & 91.0 & 958.63 & 8.0 & 961.40 \\
\hline 15.0 & 961.39 & 131.4 & 960.80 & 12.0 & 961.33 & 94.0 & 958.64 & 9.0 & 961.34 \\
\hline 20.0 & 961.28 & 134.0 & 960.91 & 15.0 & 961.38 & 97.0 & 958.66 & 12.0 & 961.34 \\
\hline 22.0 & 961.33 & 137.7 & 960.88 & 18.0 & 961.28 & 100.0 & 958.72 & 13.0 & 961.44 \\
\hline 25.0 & 961.06 & 138.5 & 961.04 & 21.0 & 961.24 & 103.0 & 958.67 & 14.0 & 961.38 \\
\hline 30.0 & 961.09 & 140.0 & 960.89 & 23.0 & 961.10 & 106.0 & 958.58 & 16.0 & 961.41 \\
\hline 35.0 & 961.16 & 141.0 & 961.09 & 25.0 & 961.05 & 109.0 & 958.52 & 18.0 & 961.28 \\
\hline 40.0 & 961.21 & 142.0 & 960.99 & 26.8 & 961.01 & 111.8 & 958.52 & 21.0 & 961.26 \\
\hline 45.0 & 961.02 & 143.0 & 960.81 & 27.4 & 961.14 & 112.2 & 958.82 & 23.0 & 961.10 \\
\hline 51.0 & 960.88 & 146.9 & 960.76 & 29.0 & 961.02 & 114.0 & 958.86 & 25.0 & 961.08 \\
\hline 54.0 & 960.84 & 148.0 & 960.99 & 30.0 & 961.08 & 117.0 & 958.87 & 28.0 & 961.07 \\
\hline 56.6 & 960.77 & 149.0 & 961.14 & 32.4 & 960.99 & 119.5 & 958.74 & 29.0 & 961.03 \\
\hline 56.8 & 960.31 & & & 33.6 & 961.22 & 122.4 & 958.78 & 32.0 & 961.03 \\
\hline 58.0 & 959.50 & & & 35.0 & 961.16 & 123.0 & 958.91 & 34.0 & 961.22 \\
\hline 59.0 & 959.15 & & & 37.0 & 961.22 & 124.5 & 959.00 & 36.0 & 961.24 \\
\hline 60.0 & 959.01 & & & 38.0 & 961.15 & 127.0 & 958.93 & 38.0 & 961.15 \\
\hline 62.0 & 958.98 & & & 39.0 & 961.25 & 129.0 & 959.01 & 39.0 & 961.27 \\
\hline 64.0 & 958.86 & & & 41.0 & 961.11 & 129.7 & 959.13 & 41.0 & 961.11 \\
\hline 66.0 & 958.65 & & & 44.0 & 961.05 & 131.0 & 959.95 & 44.0 & 961.06 \\
\hline 68.1 & 958.17 & & & 47.0 & 960.97 & 131.7 & 960.30 & 47.0 & 961.00 \\
\hline 70.0 & 957.98 & & & 50.0 & 960.90 & 132.0 & 960.80 & 50.0 & 960.90 \\
\hline 73.5 & 957.97 & & & 53.0 & 960.90 & 133.0 & 960.94 & 52.0 & 960.91 \\
\hline 76.0 & 958.14 & & & 56.0 & 960.83 & 134.0 & 960.89 & 54.0 & 960.88 \\
\hline 79.0 & 958.33 & & & 56.4 & 960.77 & 137.0 & 960.96 & 56.3 & 960.82 \\
\hline 83.0 & 958.35 & & & 56.6 & 960.46 & 139.0 & 961.01 & 56.5 & 960.45 \\
\hline 87.0 & 958.39 & & & 57.3 & 959.97 & 140.0 & 960.95 & 56.7 & 960.38 \\
\hline 91.0 & 958.40 & & & 59.0 & 959.56 & 142.0 & 961.03 & 57.1 & 960.11 \\
\hline 95.0 & 958.40 & & & 61.0 & 959.67 & 143.3 & 960.85 & 58.0 & 959.85 \\
\hline 100.0 & 958.37 & & & 62.5 & 959.51 & 146.0 & 960.81 & 59.0 & 959.78 \\
\hline 102.7 & 958.33 & & & 63.5 & 959.24 & 147.0 & 960.85 & 61.0 & 959.89 \\
\hline 104.2 & 958.63 & & & 64.0 & 958.54 & 148.0 & 961.06 & 62.0 & 959.78 \\
\hline 107.5 & 958.72 & & & 65.0 & 958.24 & 149.0 & 961.13 & 62.4 & 959.18 \\
\hline 109.9 & 958.74 & & & 67.0 & 958.16 & 150.0 & 961.03 & 63.0 & 958.90 \\
\hline 112.5 & 958.47 & & & 70.0 & 958.19 & & & 63.6 & 958.69 \\
\hline 115.0 & 958.26 & & & 73.0 & 958.22 & & & 65.0 & 958.37 \\
\hline 117.5 & 958.23 & & & 76.0 & 958.16 & & & 67.0 & 958.35 \\
\hline 120.0 & 958.20 & & & 79.0 & 958.23 & & & 69.0 & 958.40 \\
\hline 123.0 & 958.35 & & & 81.0 & 958.10 & & & 71.0 & 958.44 \\
\hline 126.0 & 958.46 & & & 84.0 & 958.06 & & & 73.0 & 958.47 \\
\hline
\end{tabular}


Table 24. (Continued) Listing of horizontal stations and elevations for cross section PR165.6

[Sta., station, distance in meters from a reference pin on the left bank; Elev., elevation, in meters above sea level]

\begin{tabular}{|c|c|c|c|c|c|c|c|}
\hline \multirow{2}{*}{\multicolumn{2}{|c|}{$\begin{array}{c}1992 \\
30 \text { August }\end{array}$}} & \multirow{2}{*}{\multicolumn{2}{|c|}{$\begin{array}{c}1992 \\
30 \text { August }\end{array}$}} & \multirow{2}{*}{\multicolumn{2}{|c|}{$\begin{array}{c}1998 \\
1 \text { October }\end{array}$}} & \multirow{2}{*}{\multicolumn{2}{|c|}{$\begin{array}{c}1998 \\
1 \text { October }\end{array}$}} \\
\hline & & & & & & & \\
\hline Sta. & Elev. & Sta. & Elev. & Sta. & Elev. & Sta. & Elev. \\
\hline 75.0 & 958.49 & 142.5 & 961.00 & -7.3 & 961.51 & 83.0 & 958.52 \\
\hline 77.0 & 958.51 & 144.0 & 960.84 & -1.0 & 961.45 & 86.0 & 958.35 \\
\hline 79.0 & 958.52 & 146.4 & 960.78 & 0.0 & 961.44 & 89.0 & 958.29 \\
\hline 81.0 & 958.53 & 148.0 & 961.05 & 2.0 & 961.47 & 92.0 & 958.12 \\
\hline 83.0 & 958.56 & 149.0 & 961.16 & 4.0 & 961.40 & 95.0 & 958.00 \\
\hline 85.0 & 958.60 & 150.0 & 961.05 & 7.0 & 961.39 & 98.0 & 958.06 \\
\hline 87.0 & 958.59 & 151.0 & 961.10 & 10.0 & 961.35 & 100.0 & 958.15 \\
\hline 88.0 & 958.60 & & & 12.0 & 961.34 & 102.0 & 958.44 \\
\hline 89.0 & 958.48 & & & 13.0 & 961.43 & 104.0 & 958.72 \\
\hline 91.0 & 958.51 & & & 14.0 & 961.38 & 107.0 & 958.85 \\
\hline 91.7 & 958.36 & & & 17.0 & 961.36 & 110.0 & 958.78 \\
\hline 92.5 & 958.15 & & & 20.0 & 961.32 & 113.0 & 958.70 \\
\hline 94.0 & 958.15 & & & 23.0 & 961.12 & 116.0 & 958.73 \\
\hline 95.7 & 958.17 & & & 26.0 & 961.11 & 119.0 & 958.70 \\
\hline 98.0 & 958.68 & & & 27.0 & 961.12 & 122.0 & 958.70 \\
\hline 98.1 & 958.73 & & & 28.0 & 961.07 & 125.0 & 958.63 \\
\hline 100.0 & 958.72 & & & 30.0 & 961.09 & 128.0 & 958.56 \\
\hline 102.0 & 958.72 & & & 32.5 & 961.03 & 128.5 & 958.58 \\
\hline 105.0 & 958.76 & & & 34.0 & 961.22 & 129.0 & 958.81 \\
\hline 107.0 & 958.81 & & & 36.0 & 961.24 & 129.1 & 959.30 \\
\hline 109.0 & 958.84 & & & 38.0 & 961.17 & 131.0 & 960.20 \\
\hline 111.0 & 958.77 & & & 39.0 & 961.26 & 131.9 & 960.49 \\
\hline 113.3 & 958.66 & & & 41.0 & 961.12 & 132.4 & 960.89 \\
\hline 115.0 & 958.57 & & & 44.0 & 961.07 & 135.0 & 961.01 \\
\hline 115.4 & 958.61 & & & 46.0 & 961.03 & 138.0 & 961.07 \\
\hline 118.0 & 958.60 & & & 48.0 & 961.02 & 141.0 & 961.09 \\
\hline 118.7 & 958.60 & & & 50.0 & 960.93 & 144.0 & 960.87 \\
\hline 120.0 & 958.49 & & & 53.0 & 960.92 & 146.7 & 960.86 \\
\hline 122.0 & 958.47 & & & 55.0 & 960.88 & 148.7 & 961.15 \\
\hline 124.0 & 958.48 & & & 55.9 & 960.80 & 151.0 & 961.12 \\
\hline 126.0 & 958.51 & & & 57.3 & 960.28 & & \\
\hline 127.4 & 958.53 & & & 58.0 & 960.20 & & \\
\hline 128.0 & 958.65 & & & 60.4 & 960.22 & & \\
\hline 128.6 & 959.07 & & & 60.6 & 959.84 & & \\
\hline 129.6 & 959.43 & & & 61.2 & 958.98 & & \\
\hline 131.6 & 960.16 & & & 62.7 & 958.78 & & \\
\hline 132.0 & 960.79 & & & 65.0 & 958.56 & & \\
\hline 133.0 & 960.96 & & & 68.0 & 958.55 & & \\
\hline 135.0 & 960.98 & & & 71.0 & 958.57 & & \\
\hline 137.0 & 960.96 & & & 74.0 & 958.56 & & \\
\hline 139.0 & 961.03 & & & 77.0 & 958.54 & & \\
\hline 141.0 & 961.04 & & & 80.0 & 958.55 & & \\
\hline
\end{tabular}




\section{Description of Cross Section PR166.0}

Location: Township 6 South/Range 50 East--section 28

U. S. Geological Survey quadrangle (1:24,000): Lonesome Peak

Landowners--left bank: Gay Ranch

--right bank: Gay Ranch

Access: Right bank

Permission from: Pat Daily

Distance from Moorhead Gaging Station: 50.8 kilometers

Azimuth of Section (degrees magnetic): 150

\section{Reference Monuments}

[Monuments at stations 0.0 and 20.0 were closest to leveling instrument]

\begin{tabular}{|c|c|c|c|c|c|c|}
\hline \multirow[b]{2}{*}{ Description } & \multirow[b]{2}{*}{$\begin{array}{l}\text { Station } \\
\text { (m) }\end{array}$} & \multicolumn{2}{|c|}{ GPS-NAD83 (1992) } & \multicolumn{2}{|c|}{ Measurement } & \multirow{2}{*}{$\begin{array}{l}\text { Elevation } \\
\text { (NGVD1929) } \\
\text { (m) }\end{array}$} \\
\hline & & Latitude & Longitude & $\begin{array}{l}\text { Standard } \\
\text { deviation } \\
(m)\end{array}$ & $\begin{array}{l}\text { Horizontal } \\
\text { precision } \\
\quad(\mathbf{m})\end{array}$ & \\
\hline $\begin{array}{l}\text { Benchmark--1/2-inch-rebar; } 0.07 \text { meter } \\
\text { above } 1998 \text { ground level; on riverward side } \\
\text { of wooden gate post }\end{array}$ & 0.0 & & & & & 960.96 \\
\hline $\begin{array}{l}\text { 1/2-inch-rebar; } 0.05 \text { meter above } 1998 \text { ground } \\
\text { level }\end{array}$ & 20.0 & $45^{\circ} 17^{\prime} 00.10^{\prime \prime}$ & $105^{\circ} 35^{\prime} 59.67^{\prime \prime}$ & 0.290 & 0.622 & 960.47 \\
\hline $\begin{array}{l}\text { 1/2-inch-rebar; } 0.05 \text { meter below } 1998 \text { ground } \\
\text { level; among } 2-3 \text { meter cottonwoods }\end{array}$ & 170.0 & & & & & 959.58 \\
\hline $\begin{array}{l}\text { 1/2-inch-rebar; } 0.10 \text { meter above } 1998 \text { ground } \\
\text { level; in a row of } 15-20 \text { meter cottonwoods }\end{array}$ & 196.0 & $45^{\circ} 16^{\prime} 54.62^{\prime \prime}$ & $105^{\circ} 35^{\prime} 57.44^{\prime \prime}$ & 0.509 & 0.562 & 960.15 \\
\hline
\end{tabular}


See Figure 8 for location

of cross section

\section{PR166.0}

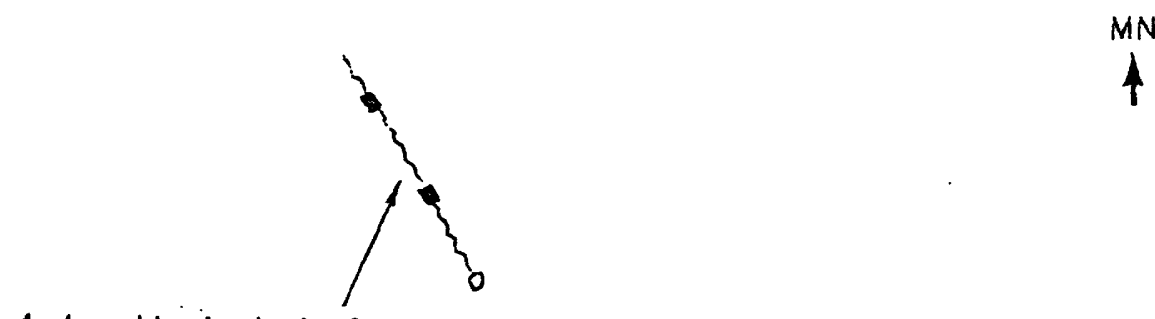

4-strand barbed-wire fence

with tree posts
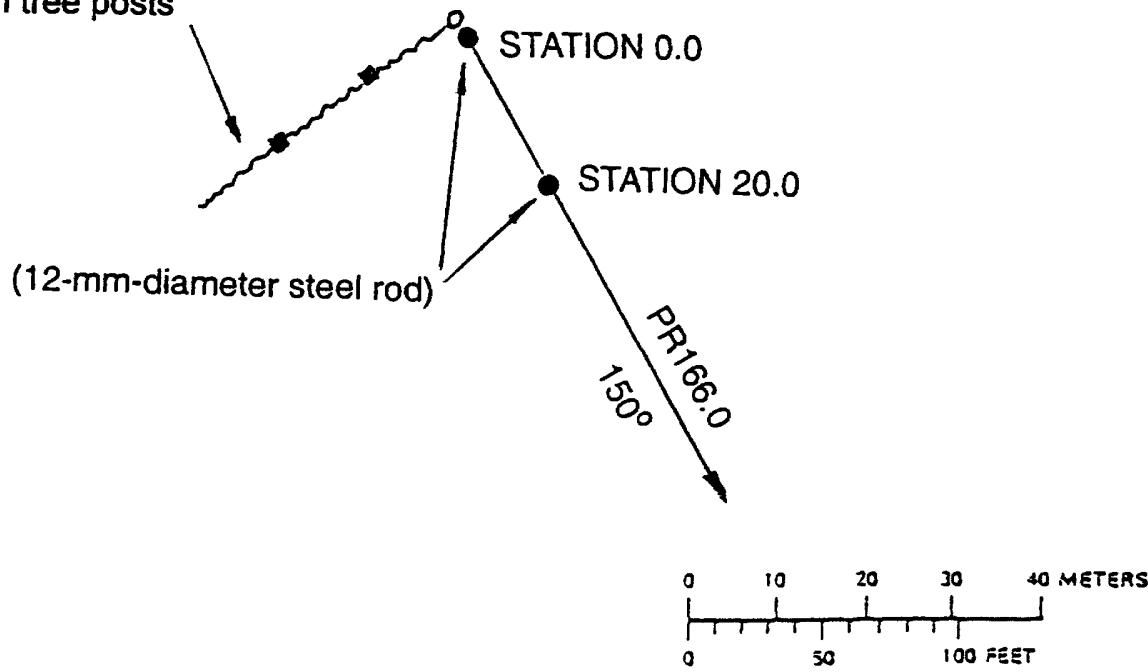

Drawn 1998

Figure 70. Upper: Location of cross section PR166.0 is shown in figure 8. Lower: Location of the reference monument on the left bank. MN is magnetic north. 


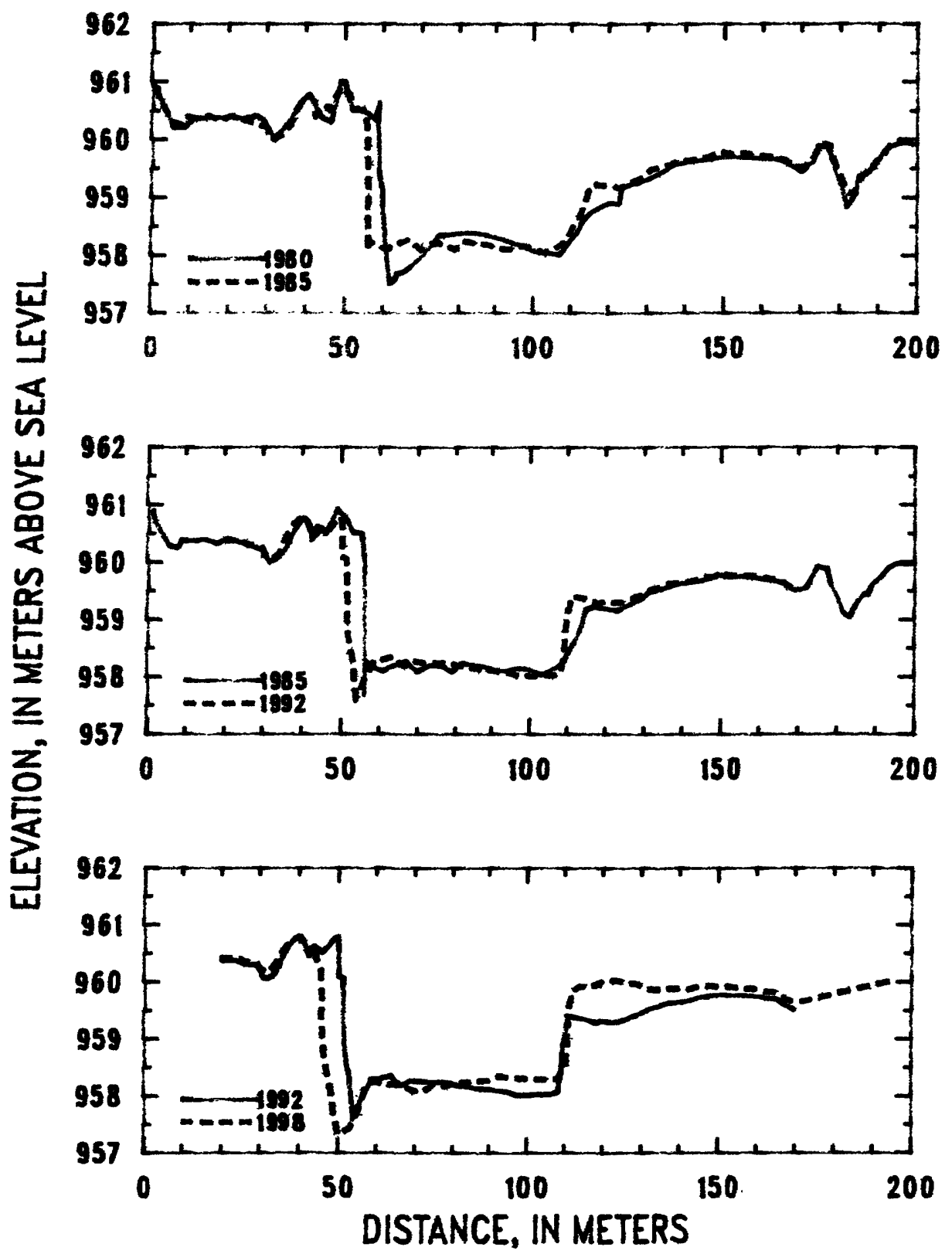

Figure 71. Profiles of cross section PR166.0 from 1980 to 1998. 
Table 25. Listing of horizontal stations and elevations for cross section PR166.0

[Sta., station, distance in meters from a reference pin on the left bank; Elev., elevation, in meters above sea level]

\begin{tabular}{|c|c|c|c|c|c|c|c|c|c|}
\hline \multirow{2}{*}{\multicolumn{2}{|c|}{$\begin{array}{c}1980 \\
19 \text { October }\end{array}$}} & \multirow{2}{*}{\multicolumn{2}{|c|}{$\begin{array}{c}1980 \\
19 \text { October }\end{array}$}} & \multicolumn{2}{|c|}{1985} & \multicolumn{2}{|c|}{1985} & \multicolumn{2}{|c|}{1985} \\
\hline & & & & $29 \mathrm{~S}$ & ember & $29 S$ & ember & $29 \mathrm{~S}$ & ember \\
\hline Sta. & Elev. & Sta. & Elev. & Sta. & Elev. & Sta. & Elev. & Sta. & Elev. \\
\hline 0.0 & 960.96 & 112.0 & 958.40 & 1.0 & 960.89 & 94.0 & 958.10 & 198.0 & 959.97 \\
\hline 2.0 & 960.79 & 113.0 & 958.57 & 3.0 & 960.59 & 97.0 & 958.16 & 200.0 & 959.99 \\
\hline 5.7 & 960.21 & 115.0 & 958.72 & 5.6 & 960.29 & 100.0 & 958.12 & & \\
\hline 8.8 & 960.21 & 119.0 & 958.88 & 8.0 & 960.25 & 103.0 & 958.04 & & \\
\hline 11.0 & 960.36 & 120.0 & 958.91 & 9.0 & 960.38 & 106.0 & 958.12 & & \\
\hline 15.0 & 960.39 & 122.8 & 958.90 & 12.0 & 960.37 & 109.0 & 958.25 & & \\
\hline 20.0 & 960.39 & 123.3 & 959.18 & 15.0 & 960.41 & 110.3 & 958.45 & & \\
\hline 25.0 & 960.36 & 129.0 & 959.28 & 18.0 & 960.32 & 113.0 & 958.71 & & \\
\hline 27.0 & 960.41 & 133.0 & 959.41 & 21.0 & 960.42 & 115.0 & 959.18 & & \\
\hline 29.0 & 960.35 & 136.0 & 959.53 & 24.0 & 960.34 & 117.0 & 959.23 & & \\
\hline 32.0 & 960.01 & 140.0 & 959.62 & 27.0 & 960.28 & 120.0 & 959.21 & & \\
\hline 35.5 & 960.27 & 145.0 & 959.65 & 30.0 & 960.21 & 123.0 & 959.15 & & \\
\hline 39.0 & 960.67 & 150.0 & 959.71 & 31.6 & 960.00 & 126.0 & 959.27 & & 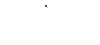 \\
\hline 41.0 & 960.78 & 160.0 & 959.68 & 34.0 & 960.07 & 129.0 & 959.35 & & \\
\hline 44.0 & 960.38 & 165.0 & 959.65 & 36.0 & 960.24 & 131.0 & 959.48 & & \\
\hline 46.6 & 960.30 & 170.0 & 959.44 & 38.0 & 960.57 & 134.0 & 959.52 & & \\
\hline 49.0 & 961.01 & 173.0 & 959.61 & 40.5 & 960.77 & 137.0 & 959.60 & & \\
\hline 50.0 & 960.99 & 174.5 & 959.90 & 42.0 & 960.66 & 140.0 & 959.63 & & \\
\hline 52.0 & 960.51 & 177.0 & 959.88 & 42.5 & 960.40 & 143.0 & 959.67 & & \\
\hline 54.0 & 960.51 & 182.0 & 958.84 & 43.0 & 960.44 & 146.0 & 959.69 & & \\
\hline 56.0 & 960.45 & 183.3 & 958.95 & 45.0 & 960.59 & 149.0 & 959.79 & & \\
\hline 58.0 & 960.34 & 185.0 & 959.36 & 46.0 & 960.50 & 152.0 & 959.74 & & \\
\hline 58.9 & 960.55 & 187.0 & 959.40 & 49.0 & 960.92 & 155.0 & 959.76 & & \\
\hline 59.1 & 959.39 & 190.0 & 959.66 & 50.0 & 960.84 & 158.0 & 959.74 & & \\
\hline 59.8 & 959.13 & 192.2 & 959.85 & 51.0 & 960.79 & 161.0 & 959.70 & & \\
\hline 59.8 & 958.95 & 195.7 & 959.95 & 53.0 & 960.52 & 164.0 & 959.67 & & \\
\hline 60.3 & 958.55 & 200.0 & 959.93 & 55.3 & 960.50 & 166.0 & 959.64 & & \\
\hline 61.0 & 958.06 & & & 56.0 & 960.15 & 169.0 & 959.51 & & \\
\hline 62.0 & 957.51 & & & 56.3 & 958.07 & 172.0 & 959.55 & & \\
\hline 62.9 & 957.54 & & & 56.5 & 958.25 & 175.0 & 959.93 & & \\
\hline 64.0 & 957.68 & & & 58.0 & 958.17 & 177.5 & 959.89 & & \\
\hline 66.0 & 957.72 & & & 61.0 & 958.10 & 178.0 & 959.70 & & \\
\hline 70.0 & 957.96 & & & 64.0 & 958.20 & 180.0 & 959.38 & & \\
\hline 75.0 & 958.35 & & & 67.0 & 958.28 & 182.0 & 959.10 & & \\
\hline 80.0 & 958.38 & & & 70.0 & 958.09 & 183.5 & 959.04 & & \\
\hline 85.0 & 958.40 & & & 73.0 & 958.20 & 185.0 & 959.30 & & \\
\hline 90.0 & 958.31 & & & 76.0 & 958.20 & 187.0 & 959.44 & & \\
\hline 95.0 & 958.21 & & & 79.0 & 958.09 & 188.0 & 959.44 & & \\
\hline 100.0 & 958.08 & & & 82.0 & 958.24 & 189.0 & 959.60 & & \\
\hline 105.0 & 958.03 & & & 85.0 & 958.20 & 191.0 & 959.71 & & \\
\hline 107.0 & 958.01 & & & 88.0 & 958.18 & 193.0 & 959.89 & & \\
\hline 110.0 & 958.25 & & & 91.0 & 958.11 & 195.7 & 959.99 & & \\
\hline
\end{tabular}


Table 25. (Continued) Listing of horizontal stations and elevations for cross section PR166.0

[Sta., station, distance in meters from a reference pin on the left bank; Elev., elevation, in meters above sea level]

\begin{tabular}{|c|c|c|c|c|c|c|c|c|c|}
\hline \multirow{2}{*}{\multicolumn{2}{|c|}{$\begin{array}{c}1992 \\
30 \text { August }\end{array}$}} & \multirow{2}{*}{\multicolumn{2}{|c|}{$\begin{array}{c}1992 \\
30 \text { August }\end{array}$}} & \multirow{2}{*}{\multicolumn{2}{|c|}{$\begin{array}{l}1992 \\
30 \text { August }\end{array}$}} & \multirow{2}{*}{\multicolumn{2}{|c|}{$\begin{array}{c}1998 \\
30 \text { September }\end{array}$}} & \multirow{2}{*}{\multicolumn{2}{|c|}{$\begin{array}{c}1998 \\
30 \text { September }\end{array}$}} \\
\hline & & & & & & & & & \\
\hline Sta. & Elev. & Sta. & Elev. & Sta. & Elev. & Sta. & Elev. & Sta. & Elev. \\
\hline 20.0 & 960.38 & 81.0 & 958.19 & 167.0 & 959.60 & 20.0 & 960.42 & 110.2 & 958.52 \\
\hline 22.0 & 960.38 & 83.0 & 958.17 & 170.0 & 959.51 & 22.0 & 960.42 & 110.7 & 958.82 \\
\hline 24.0 & 960.38 & 85.0 & 958.15 & & & 24.0 & 960.44 & 111.2 & 959.59 \\
\hline 26.0 & 960.31 & 87.0 & 958.12 & & & 26.0 & 960.35 & 112.0 & 959.83 \\
\hline 28.0 & 960.30 & 89.0 & 958.12 & & & 29.0 & 960.35 & 114.0 & 959.93 \\
\hline 29.5 & 960.28 & 91.0 & 958.10 & & & 30.0 & 960.23 & 116.0 & 959.90 \\
\hline 31.0 & 960.07 & 93.0 & 958.08 & & & 32.0 & 960.16 & 118.0 & 959.94 \\
\hline 32.5 & 960.06 & 95.0 & 958.07 & & & 34.0 & 960.31 & 120.0 & 960.02 \\
\hline 34.0 & 960.14 & 97.0 & 958.01 & & & 35.0 & 960.45 & 122.0 & 960.04 \\
\hline 35.0 & 960.26 & 99.0 & 958.02 & & & 37.0 & 960.65 & 124.0 & 960.02 \\
\hline 36.0 & 960.46 & 101.0 & 958.01 & & & 38.5 & 960.75 & 126.0 & 959.98 \\
\hline 38.0 & 960.68 & 103.0 & 958.03 & & & 40.3 & 960.78 & 128.0 & 959.98 \\
\hline 40.0 & 960.80 & 105.0 & 958.03 & & & 42.5 & 960.50 & 130.0 & 959.93 \\
\hline 41.0 & 960.75 & 107.0 & 958.04 & & & 43.5 & 960.64 & 132.0 & 959.87 \\
\hline 42.5 & 960.46 & 108.2 & 958.07 & & & 44.7 & 960.36 & 134.0 & 959.87 \\
\hline 44.0 & 960.62 & 109.2 & 958.38 & & & 45.6 & 960.24 & 136.0 & 959.88 \\
\hline 46.0 & 960.52 & 109.4 & 958.56 & & & 45.8 & 959.38 & 138.0 & 959.89 \\
\hline 48.0 & 960.65 & 109.6 & 958.87 & & & 46.2 & 958.83 & 140.0 & 959.89 \\
\hline 49.0 & 960.75 & 111.0 & 959.40 & & & 47.0 & 958.47 & 142.0 & 959.86 \\
\hline 49.9 & 960.78 & 112.0 & 959.40 & & & 48.0 & 958.15 & 144.0 & 959.91 \\
\hline 50.2 & 960.68 & 114.0 & 959.37 & & & 49.0 & 957.66 & 147.0 & 959.93 \\
\hline 50.2 & 960.09 & 116.0 & 959.34 & & & 50.0 & 957.31 & 150.0 & 959.91 \\
\hline 51.3 & 960.05 & 118.0 & 959.29 & & & 53.0 & 957.43 & 153.0 & 959.91 \\
\hline 51.7 & 958.88 & 120.0 & 959.33 & & & 55.0 & 957.70 & 156.0 & 959.89 \\
\hline 52.3 & 958.53 & 122.0 & 959.30 & & & 57.5 & 958.21 & 159.0 & 959.88 \\
\hline 53.0 & 958.35 & 124.0 & 959.30 & & & 60.0 & 958.25 & 162.0 & 959.84 \\
\hline 53.5 & 957.99 & 126.0 & 959.35 & & & 63.0 & 958.19 & 165.0 & 959.81 \\
\hline 54.0 & 957.62 & 128.0 & 959.40 & & & 66.0 & 958.23 & 167.5 & 959.69 \\
\hline 56.0 & 957.93 & 130.0 & 959.46 & & & 69.0 & 958.11 & 170.0 & 959.64 \\
\hline 58.0 & 958.17 & 132.0 & 959.53 & & & 72.0 & 958.07 & 196.0 & 960.04 \\
\hline 58.8 & 958.29 & 134.0 & 959.57 & & & 75.0 & 958.20 & & \\
\hline 61.0 & 958.30 & 136.0 & 959.62 & & & 78.0 & 958.16 & & \\
\hline 62.0 & 958.33 & 139.0 & 959.64 & & & 81.0 & 958.24 & & \\
\hline 64.0 & 958.35 & 142.0 & 959.66 & & & 84.0 & 958.24 & & \\
\hline 65.5 & 958.21 & 145.0 & 959.74 & & & 87.0 & 958.25 & & \\
\hline 67.0 & 958.19 & 148.0 & 959.75 & & & 90.0 & 958.28 & & \\
\hline 69.0 & 958.27 & 151.0 & 959.78 & & & 93.0 & 958.35 & & . \\
\hline 71.0 & 958.25 & 154.0 & 959.77 & & & 96.0 & 958.32 & & \\
\hline 73.0 & 958.26 & 157.0 & 959.76 & & & 99.0 & 958.31 & & \\
\hline 75.0 & 958.23 & 160.0 & 959.75 & & & 102.0 & 958.28 & & \\
\hline 77.0 & 958.25 & 163.0 & 959.70 & & & 105.0 & 958.30 & & \\
\hline 79.0 & 958.23 & 165.0 & 959.70 & & & 108.0 & 958.29 & & \\
\hline
\end{tabular}




\section{Description of Cross Section PR166.6}

Location: Township 6 South/Range 50 East--section 28

U. S. Geological Survey quadrangle (1:24,000): Lonesome Peak

Landowners--left bank: Gay Ranch

--right bank: Gay Ranch

Access: Right bank

Permission from: Pat Daily

Distance from Moorhead Gaging Station: 51.2 kilometers

Azimuth of Section (degrees magnetic): 078

\section{Reference Monuments}

[Monument at station 151.0 was closest to leveling instrument; latitude and longitude for the monument at station 151.0 was calculated from the measured latitude and longitude of station 147.0]

\begin{tabular}{|c|c|c|c|c|c|c|}
\hline \multirow[b]{2}{*}{ Description } & \multirow[b]{2}{*}{$\begin{array}{l}\text { Station } \\
\text { (m) }\end{array}$} & \multicolumn{2}{|c|}{ GPS-NAD83 (1992) } & \multicolumn{2}{|c|}{ Measurement } & \multirow{2}{*}{$\begin{array}{l}\text { Elevation } \\
\text { (NGVD1929) } \\
\text { (m) }\end{array}$} \\
\hline & & Latitude & Longitude & $\begin{array}{l}\text { Standard } \\
\text { deviation } \\
(\mathbf{m})\end{array}$ & $\begin{array}{l}\text { Horizontal } \\
\text { precision } \\
\text { (m) }\end{array}$ & \\
\hline $\begin{array}{l}\text { 1/2-inch-rebar; } 0.17 \text { meter above } 1998 \text { ground } \\
\text { level; in first row of } 15-20 \text { meters high cot- } \\
\text { tonwoods, } 0.25 \text { meter diameter at breast } \\
\text { height }\end{array}$ & -1.5 & $45^{\circ} 17^{\prime} 07.49^{\prime \prime}$ & $105^{\circ} 35^{\prime} 45.17^{\prime \prime}$ & 1.313 & 0.701 & 960.62 \\
\hline $\begin{array}{l}\text { 1/2-inch-rebar; } 0.17 \text { meter above } 1998 \text { ground } \\
\text { level; in an area with salt cedars about } 2 \\
\text { meters high }\end{array}$ & 21.5 & $45^{\circ} 17^{\prime} 07.45^{\prime \prime}$ & $105^{\circ} 35^{\prime} 44.09^{\prime \prime}$ & 0.359 & 0.489 & 960.30 \\
\hline $\begin{array}{l}\text { Benchmark-1/2-inch-rebar; } 0.29 \text { meter above } \\
1998 \text { ground level; at the base of a large } \\
\text { solitary cottonwood } 11 \text { meters from the } \\
\text { edge of the right bank in 1998; see head- } \\
\text { note }\end{array}$ & 151.0 & $45^{\circ} 17^{\prime} 07.31^{\prime \prime}$ & $105^{\circ} 35^{\prime} 38.21^{\prime \prime}$ & 0.462 & 0.447 & 960.46 \\
\hline
\end{tabular}


See Figure 8 for location of cross section

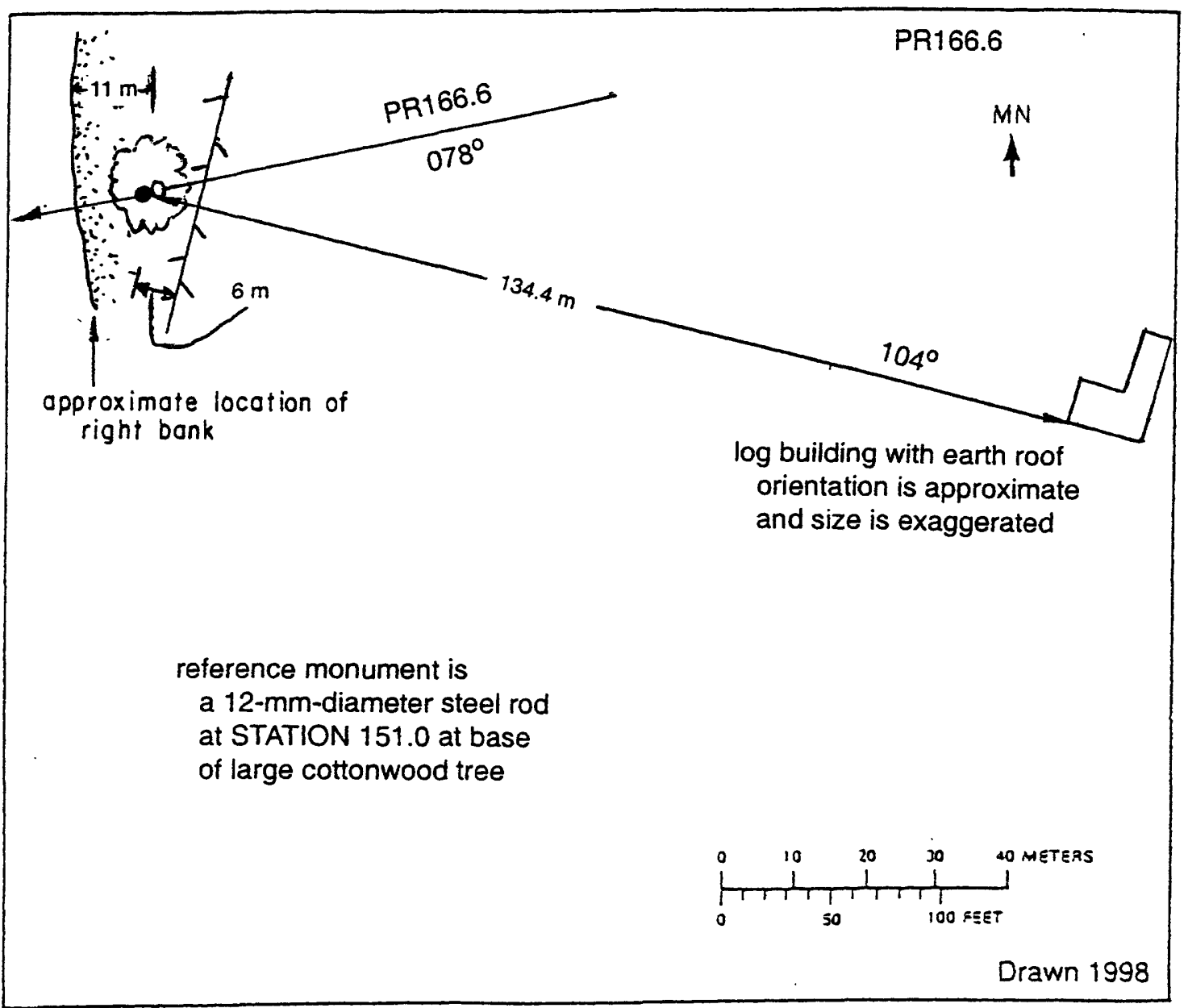

Figure 72. Upper: Location of cross section PR166.6 is shown in figure 8. Lower: Location of the reference monument on the right bank. $\mathrm{MN}$ is magnetic north. 


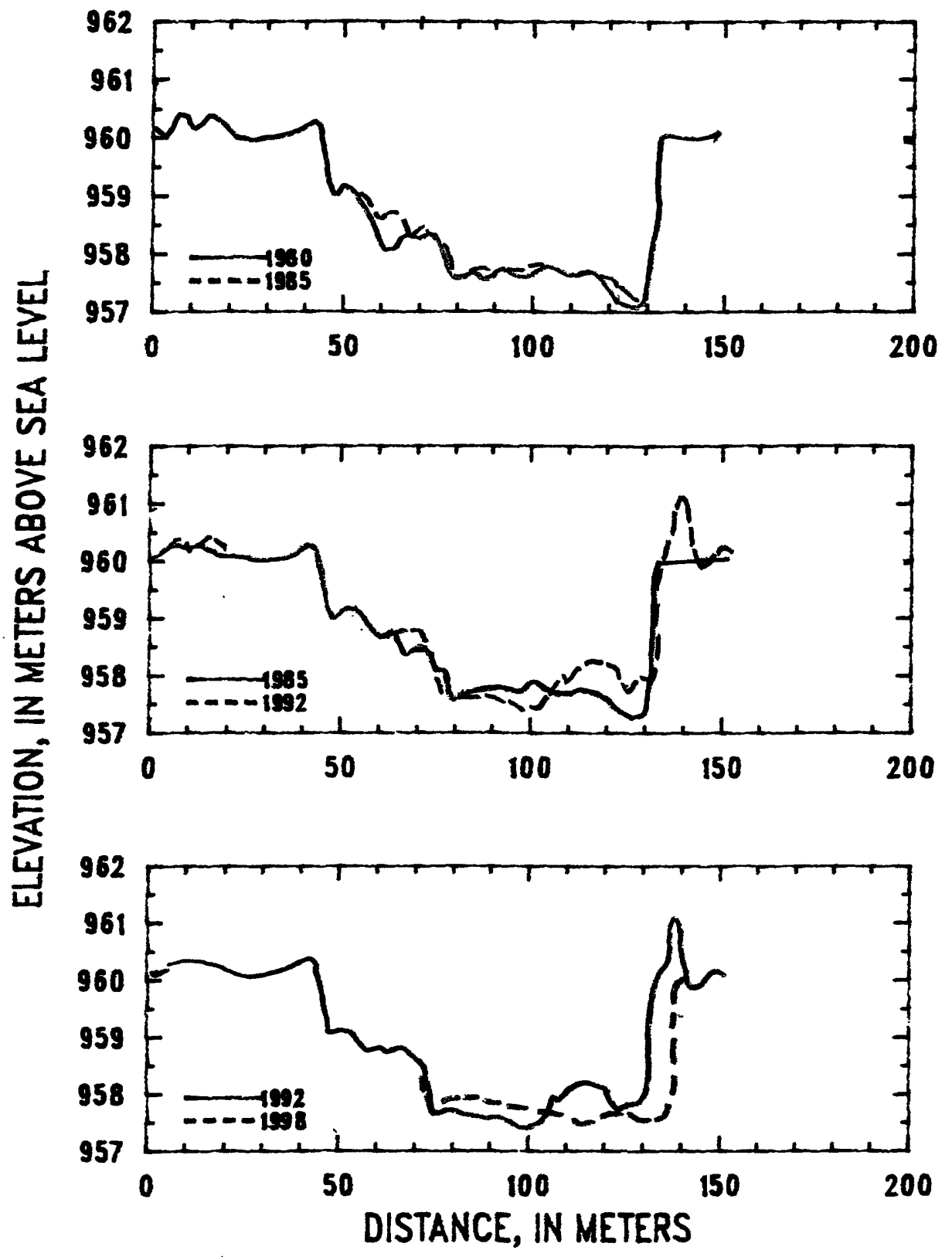

Figure 73. Profiles of cross section PR166.6 from 1980 to 1998. 
Table 26. Listing of horizontal stations and elevations for cross section PR166.6

[Sta., station, distance in meters from a reference pin on the left bank; Elev., elevation, in meters above sea level]

\begin{tabular}{|c|c|c|c|c|c|c|c|c|c|}
\hline \multirow{2}{*}{\multicolumn{2}{|c|}{$\begin{array}{c}1980 \\
19 \text { October }\end{array}$}} & \multirow{2}{*}{\multicolumn{2}{|c|}{$\begin{array}{c}1980 \\
19 \text { October }\end{array}$}} & \multicolumn{2}{|c|}{1985} & \multicolumn{2}{|c|}{1985} & \multicolumn{2}{|c|}{1992} \\
\hline & & & & $29 \mathrm{~S}$ & ember & $29 \mathrm{Se}$ & ember & 30 & gust \\
\hline Sta. & Elev. & Sta. & Elev. & Sta. & Elev. & Sta. & Elev. & Sta. & Elev. \\
\hline-1.5 & 960.41 & 97.0 & 957.66 & 3.0 & 960.13 & 102.0 & 957.79 & 8.0 & 960.34 \\
\hline 0.0 & 960.23 & 100.0 & 957.61 & 5.0 & 960.09 & 105.0 & 957.73 & 10.0 & 960.36 \\
\hline 2.0 & 960.18 & 102.0 & 957.76 & 7.0 & 960.31 & 108.0 & 957.71 & 13.0 & 960.33 \\
\hline 5.4 & 960.11 & 105.0 & 957.76 & 10.0 & 960.28 & 111.0 & 957.72 & 17.0 & 960.31 \\
\hline 6.7 & 960.36 & 110.0 & 957.70 & 13.0 & 960.29 & 114.0 & 957.72 & 20.0 & 960.21 \\
\hline 9.4 & 960.36 & 115.0 & 957.68 & 16.0 & 960.29 & 117.0 & 957.73 & 21.5 & 960.13 \\
\hline 11.0 & 960.20 & 120.0 & 957.54 & 19.0 & 960.19 & 120.0 & 957.69 & 24.0 & 960.07 \\
\hline 12.0 & 960.29 & 124.0 & $95 \underline{7.30}$ & 21.5 & 960.10 & 123.0 & 957.53 & 26.0 & 960.02 \\
\hline 14.0 & 960.33 & 127.0 & 957.17 & 25.0 & 959.99 & 126.0 & 957.31 & 28.0 & 960.09 \\
\hline 17.0 & 960.30 & 129.2 & 957.07 & 28.0 & 960.04 & 129.0 & 957.26 & 31.0 & 960.06 \\
\hline 21.5 & 960.13 & 130.1 & 957.63 & 31.0 & 960.01 & 131.0 & 957.48 & 34.0 & 960.09 \\
\hline 23.0 & 960.12 & 130.9 & 957.57 & 34.0 & 960.06 & 132.2 & 957.91 & 37.0 & 960.11 \\
\hline 26.0 & 959.99 & 131.8 & 958.05 & 37.0 & 960.08 & 132.5 & 958.33 & 40.0 & 960.22 \\
\hline 30.0 & 960.01 & 132.1 & 958.23 & 40.0 & 960.18 & 133.4 & 959.09 & 42.0 & 960.28 \\
\hline 33.0 & 960.03 & 132.4 & 958.38 & 43.0 & 960.30 & 134.0 & 960.03 & 43.5 & 960.34 \\
\hline 36.0 & 960.09 & 132.6 & 959.95 & 44.5 & 960.24 & 135.7 & 960.06 & 44.5 & 960.25 \\
\hline 36.6 & 960.03 & 133.0 & 960.05 & 46.0 & 959.73 & 138.0 & 960.07 & 46.0 & 959.75 \\
\hline 40.0 & 960.21 & 137.0 & 960.07 & 47.3 & 959.19 & 141.0 & 960.04 & 48.0 & 959.14 \\
\hline 42.4 & 960.24 & 141.0 & 960.06 & 48.6 & 958.98 & 144.0 & 960.02 & 49.0 & 959.04 \\
\hline 44.4 & 960.27 & 145.0 & 960.07 & 49.7 & 958.96 & 147.0 & 960.04 & 51.0 & 959.15 \\
\hline 46.0 & 959.69 & 150.0 & 960.10 & 51.0 & 959.10 & 150.0 & 960.09 & 53.0 & 959.14 \\
\hline 47.5 & 959.12 & & & 54.0 & 959.04 & 151.0 & 960.07 & 55.0 & 959.05 \\
\hline 49.0 & 958.97 & & & 57.0 & 958.92 & & & 57.0 & 958.96 \\
\hline 51.0 & 959.11 & & & 58.2 & 958.67 & & & 59.0 & 958.77 \\
\hline 54.0 & 959.02 & & & 60.0 & 958.59 & & & 61.0 & 958.79 \\
\hline 57.0 & 958.90 & & & 63.0 & 958.68 & & & 63.0 & 958.82 \\
\hline 59.7 & 958.35 & & & 65.0 & 958.67 & & & 65.0 & 958.76 \\
\hline 61.2 & 958.27 & & & 67.8 & 958.33 & & & 68.0 & 958.81 \\
\hline 62.0 & 958.06 & & & 69.0 & 958.44 & & & 69.0 & 958.79 \\
\hline 63.8 & 958.02 & & & 71.0 & 958.49 & & & 71.0 & 958.63 \\
\hline 65.0 & 958.22 & & & 73.0 & 958.39 & & & 73.0 & 958.50 \\
\hline 70.0 & 958.23 & & & 75.0 & 958.43 & & & 73.4 & 958.40 \\
\hline 75.0 & 958.25 & & & 76.0 & 958.03 & & & 74.0 & 957.91 \\
\hline 76.0 & 958.06 & & & 77.2 & 957.90 & & & 74.5 & 957.83 \\
\hline 77.0 & 957.92 & & & 79.0 & 957.51 & & & 76.7 & 957.49 \\
\hline 78.0 & 957.75 & & & 81.0 & 957.61 & & & 78.4 & 957.65 \\
\hline 80.0 & 957.60 & & & 84.0 & 957.65 & & & 80.0 & 957.66 \\
\hline 84.0 & 957.62 & & & 87.0 & 957.70 & & & 82.0 & 957.63 \\
\hline 87.0 & 957.60 & & & 90.0 & 957.72 & & & 84.0 & 957.62 \\
\hline 90.0 & 957.59 & & & 93.0 & 957.74 & & & 86.0 & 957.60 \\
\hline 92.0 & 957.69 & & & 96.0 & 957.73 & & & 88.0 & 957.60 \\
\hline 94.0 & 957.61 & & & 99.0 & 957.76 & & & 90.0 & 957.60 \\
\hline
\end{tabular}


Table 26. (Continued) Listing of horizontal stations and elevations for cross section PR166.6

[Sta., station, distance in meters from a reference pin on the left bank; Elev., elevation, in meters above sea level]

\begin{tabular}{|c|c|c|c|c|c|}
\hline \multirow{2}{*}{\multicolumn{2}{|c|}{$\begin{array}{c}1992 \\
1 \text { September }\end{array}$}} & \multicolumn{2}{|c|}{1998} & \multicolumn{2}{|c|}{1998} \\
\hline & & \multicolumn{2}{|c|}{30 September } & \multicolumn{2}{|c|}{30 September } \\
\hline Sta. & Elev. & Sta. & Elev. & Sta. & Elev. \\
\hline 92.0 & 957.55 & -1.5 & 960.45 & 73.3 & 957.95 \\
\hline 94.0 & 957.52 & 0.0 & 960.31 & 75.0 & 957.60 \\
\hline 96.0 & 957.49 & 2.0 & 960.20 & 77.0 & 957.58 \\
\hline 98.0 & 957.44 & 5.0 & 960.15 & 78.0 & 957.67 \\
\hline 100.0 & 957.42 & 6.0 & 960.28 & 78.6 & 957.90 \\
\hline 102.0 & 957.46 & 8.0 & 960.36 & 81.0 & 957.89 \\
\hline 104.0 & 957.57 & 10.0 & 960.32 & 84.0 & 957.88 \\
\hline 105.7 & 957.67 & 12.0 & 960.34 & 87.0 & 957.89 \\
\hline 106.1 & 957.83 & 14.0 & 960.32 & 90.0 & 957.83 \\
\hline 106.8 & 957.87 & 16.0 & 960.33 & 93.0 & 957.84 \\
\hline 107.5 & 957.79 & 18.0 & 960.26 & 96.0 & 957.78 \\
\hline 110.0 & 957.99 & 20.0 & 960.24 & 99.0 & 957.76 \\
\hline 113.0 & 958.08 & 21.5 & 960.15 & 102.0 & 957.67 \\
\hline 116.0 & 958.11 & 24.0 & 960.10 & 105.0 & 957.63 \\
\hline 119.0 & 958.09 & 26.0 & 960.03 & 108.0 & 957.60 \\
\hline 121.5 & 958.06 & 28.0 & 960.11 & 111.0 & 957.50 \\
\hline 121.9 & 957.93 & 30.0 & 960.08 & 114.0 & 957.48 \\
\hline 122.3 & 957.85 & 32.0 & 960.08 & 117.0 & 957.50 \\
\hline 124.0 & 957.62 & 34.0 & 960.09 & 120.0 & 957.57 \\
\hline 126.0 & 957.69 & 36.0 & 960.13 & 123.0 & 957.60 \\
\hline 128.0 & 957.74 & 38.0 & 960.22 & 126.0 & 957.56 \\
\hline 130.0 & 957.76 & 40.0 & 960.23 & 129.0 & 957.57 \\
\hline 132.4 & 957.72 & 42.0 & 960.29 & 132.0 & 957.46 \\
\hline 133.0 & 957.84 & 43.0 & 960.33 & 135.0 & 957.44 \\
\hline 133.2 & 958.21 & 44.0 & 960.28 & 138.0 & 957.45 \\
\hline 133.7 & 959.19 & 45.0 & 960.09 & 139.2 & 957.97 \\
\hline 133.8 & 959.97 & 47.0 & 959.46 & 140.0 & 960.56 \\
\hline 134.3 & 960.11 & 48.0 & 959.31 & 141.0 & 960.07 \\
\hline 135.6 & 960.26 & 50.0 & 959.21 & 142.7 & 959.78 \\
\hline 136.8 & 960.33 & 52.0 & 959.24 & 145.0 & 959.92 \\
\hline 138.0 & 960.93 & 54.0 & 959.17 & 147.1 & 960.06 \\
\hline 138.5 & 961.09 & 56.0 & 959.16 & 150.0 & 960.17 \\
\hline 139.3 & 960.89 & 58.0 & 959.07 & 151.0 & 960.18 \\
\hline 139.8 & 960.99 & 60.0 & 959.03 & & \\
\hline 140.0 & 960.91 & 62.0 & 959.09 & & \\
\hline 141.2 & 960.08 & 64.0 & 959.17 & & \\
\hline 142.8 & 959.79 & 66.0 & 959.15 & & \\
\hline 145.0 & 959.90 & 68.0 & 959.21 & & \\
\hline 148.0 & 960.08 & 69.0 & 959.18 & & \\
\hline 150.0 & 960.16 & 70.0 & 958.96 & & \\
\hline 151.0 & 960.13 & 72.2 & 958.86 & & \\
\hline & & 72.7 & 958.02 & & \\
\hline
\end{tabular}




\section{Description of Cross Section PR167}

Location: Township 6 South/Range 50 East--section 28

U. S. Geological Survey quadrangle (1:24,000): Lonesome Peak

Landowners--left bank: Gay Ranch

--right bank: Gay Ranch

Access: Right bank

Permission from: Pat Daily

Distance from Moorhead Gaging Station: 51.77 kilometers

Azimuth of Section (degrees magnetic): 006

\section{Reference Monuments}

[Monuments at stations 150.0 and 150.6 were closest to leveling instrument]

\begin{tabular}{|c|c|c|c|c|c|c|}
\hline \multirow[b]{2}{*}{ Description } & \multirow[b]{2}{*}{$\begin{array}{l}\text { Station } \\
\text { (m) }\end{array}$} & \multicolumn{2}{|c|}{ GPS-NAD83 (1992) } & \multicolumn{2}{|c|}{ Measurement } & \multirow{2}{*}{$\begin{array}{l}\text { Elevation } \\
\text { (NGVD1929) } \\
\text { (m) }\end{array}$} \\
\hline & & Latitude & Longitude & $\begin{array}{c}\text { Standard } \\
\text { deviation } \\
\text { (m) }\end{array}$ & $\begin{array}{l}\text { Horizontal } \\
\text { precision } \\
\text { (m) }\end{array}$ & \\
\hline $\begin{array}{l}\text { 1/2-inch-rebar; } 0.05 \text { meter above } 1998 \text { ground } \\
\text { level }\end{array}$ & -1.6 & & & & & 959.63 \\
\hline $\begin{array}{l}\text { 1/2-inch-rebar; } 0.19 \text { meter above } 1998 \text { ground } \\
\text { level }\end{array}$ & 10.0 & $45^{\circ} 17^{\prime} 17.69^{\prime \prime}$ & $105^{\circ} 35^{\prime} 51.56^{\prime \prime}$ & 0.319 & 0.542 & 959.84 \\
\hline $\begin{array}{l}\text { 1/2-inch-rebar; } 0.02 \text { meter above } 1998 \text { ground } \\
\text { level }\end{array}$ & 150.0 & & & & & 959.49 \\
\hline $\begin{array}{l}\text { 1/2-inch-rebar; bent, } 0.16 \text { meter above } 1998 \\
\text { ground level }\end{array}$ & 150.6 & $45^{\circ} 17^{\prime} 21.99^{\prime \prime}$ & $105^{\circ} 35^{\prime} 49.34^{\prime \prime}$ & 0.506 & 0.480 & 959.62 \\
\hline Benchmark--brass circular plate; offsection & -- & $45^{\circ} 17^{\prime} 21.96^{\prime \prime}$ & $105^{\circ} 35^{\prime} 49.97^{\prime \prime}$ & 0.527 & 0.490 & 959.79 \\
\hline
\end{tabular}




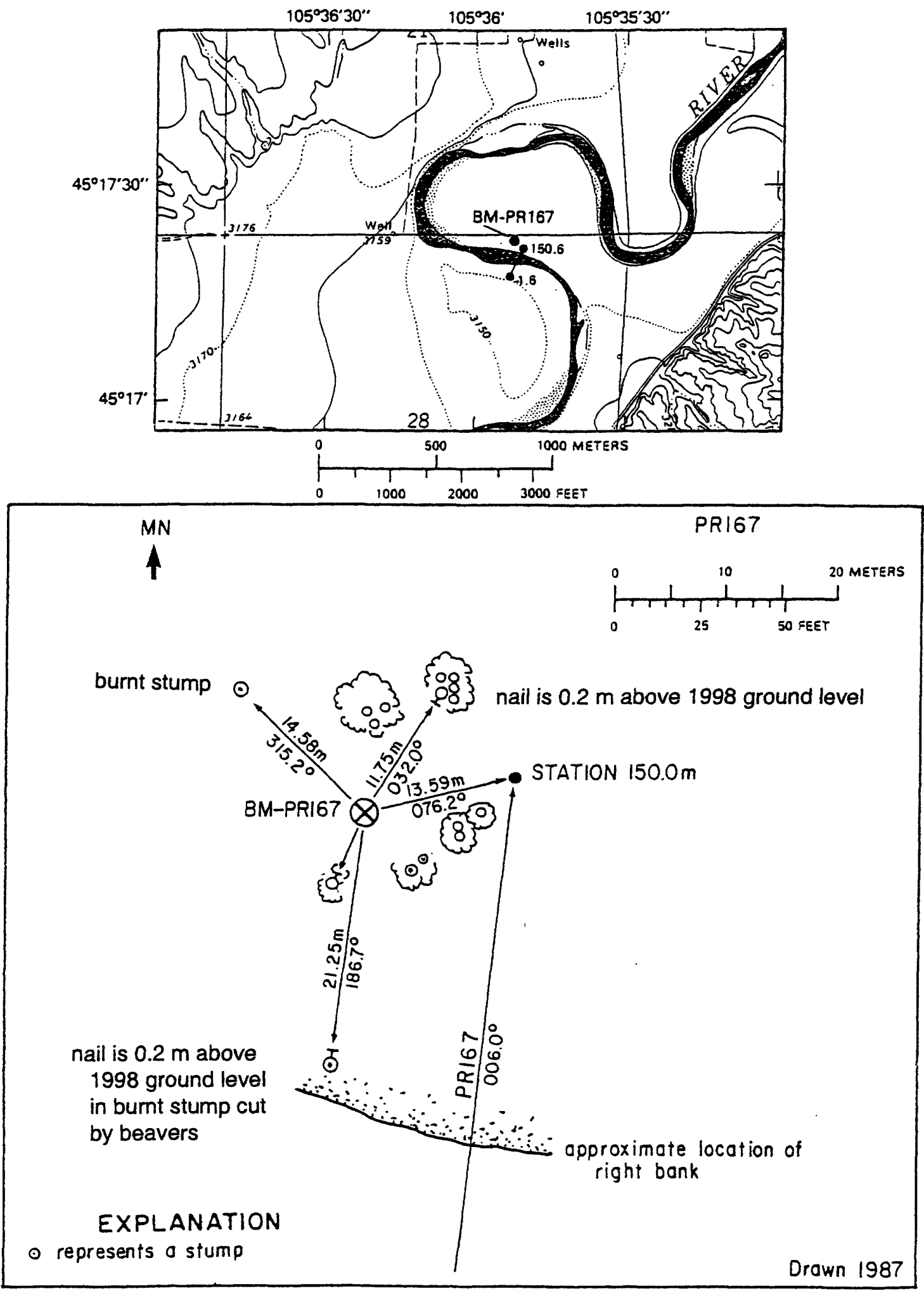

Figure 74. Upper: Location of cross section PR167, bench mark BM-PR167, and the left and right bank reference monuments in the Lonesome Peak quadrangle. Lower: Location of the bench mark on the left bank. MN is magnetic north. 

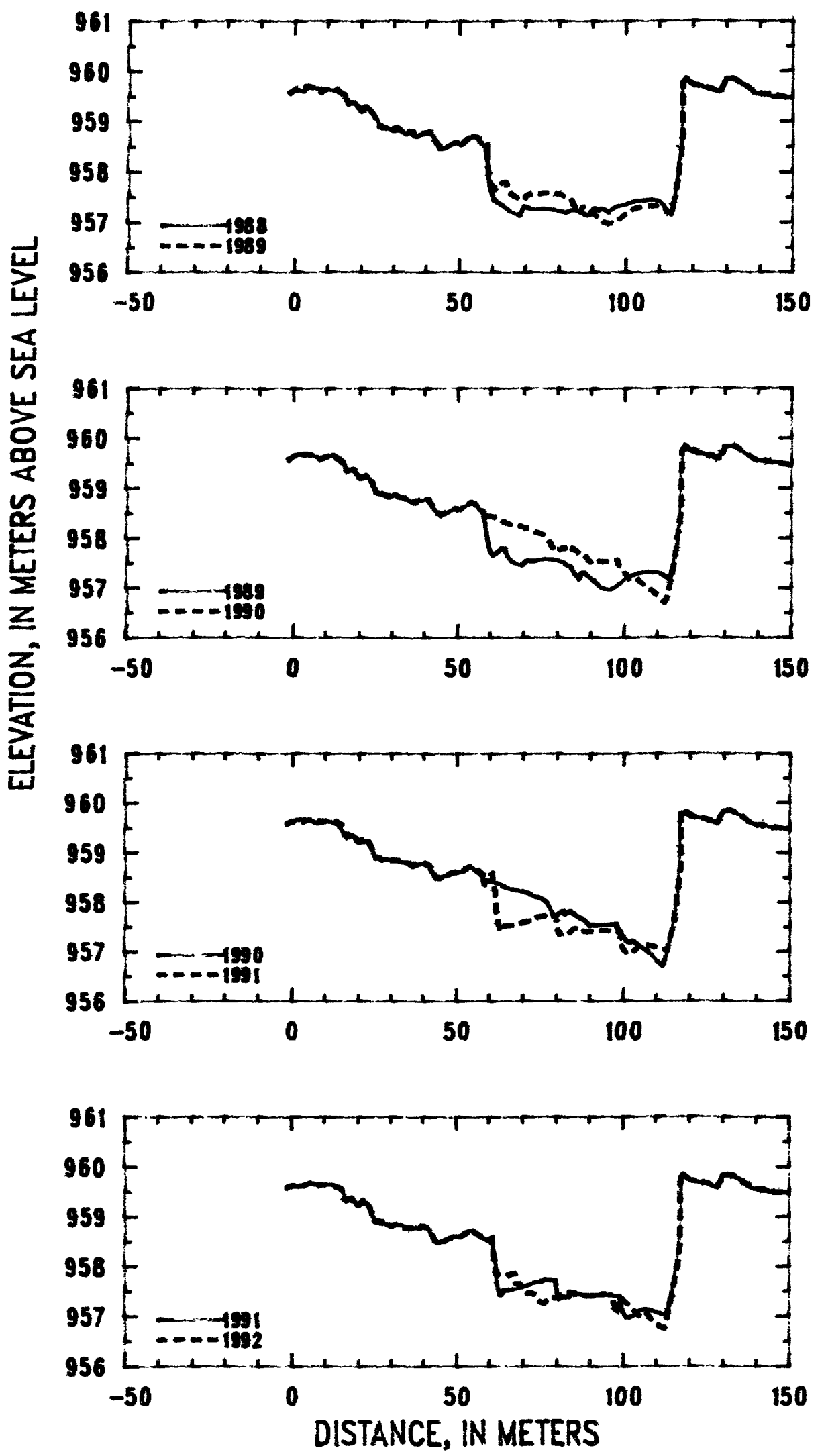

Figure 75. Profiles of cross section PR 167 from 1988 to 1992. 

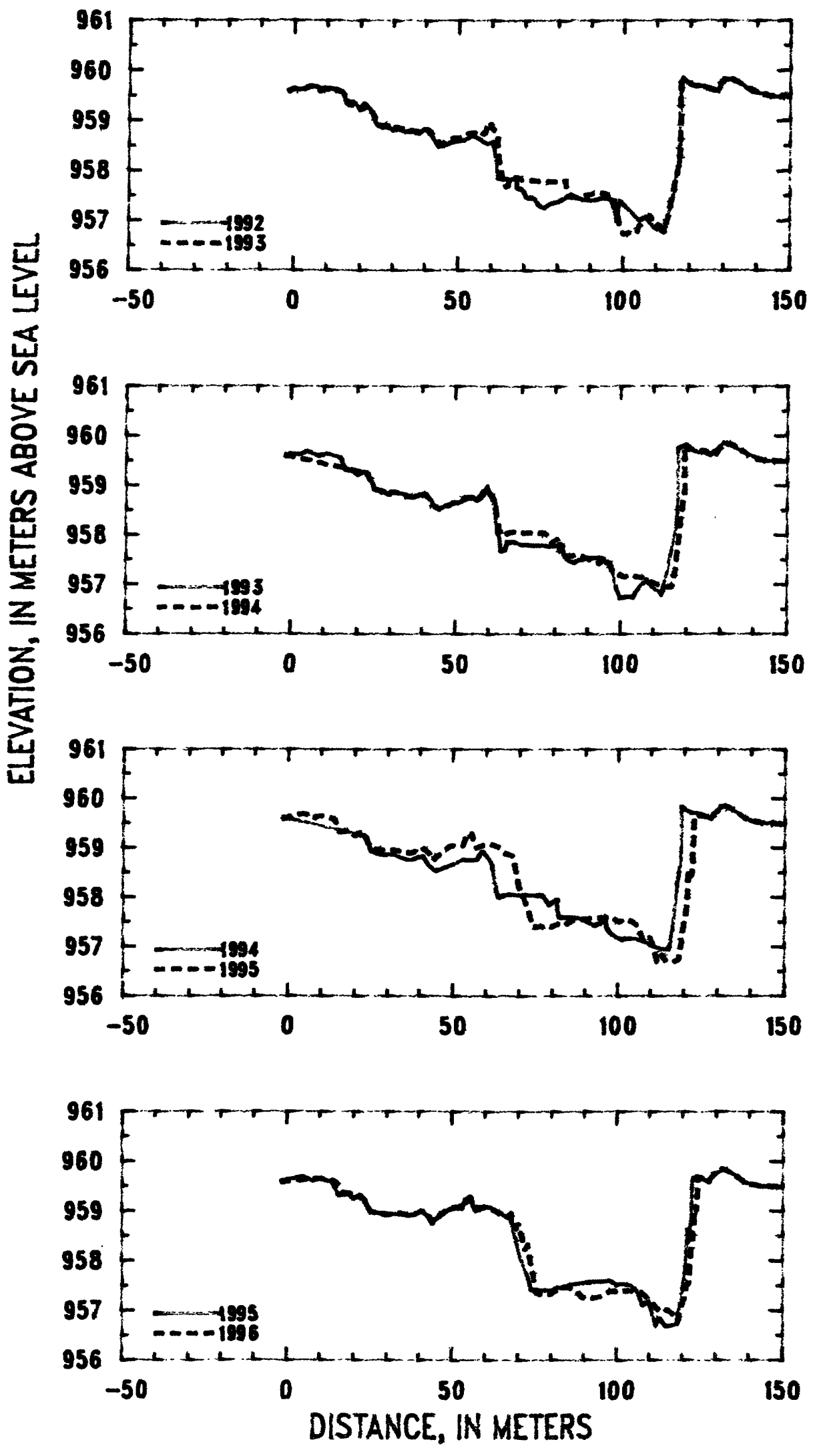

Figure 76. Profiles of cross section PR167 from 1992 to 1996. 


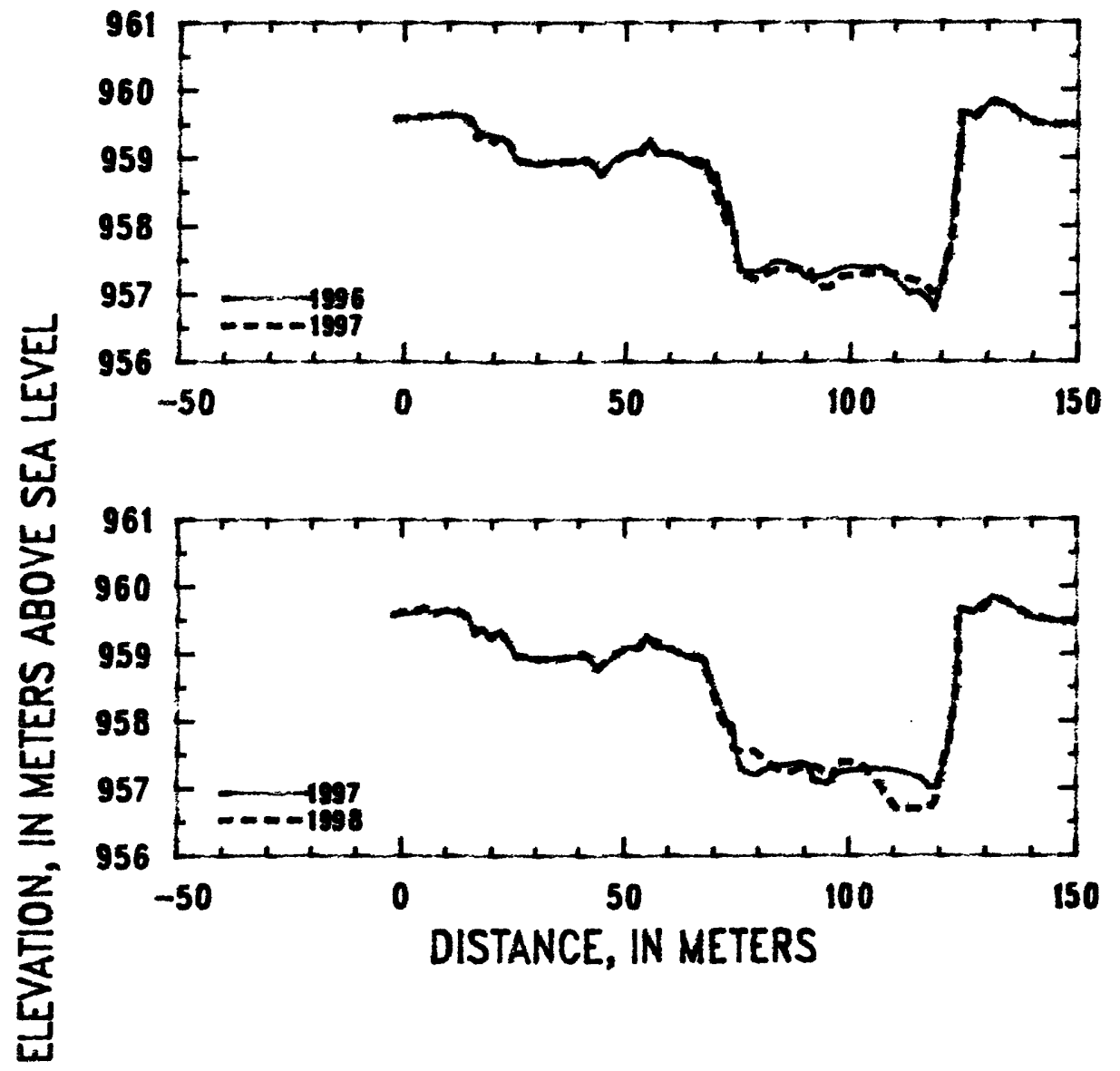

Figure 77. Profiles of cross section PR167 from 1996 to 1998. 
Table 27. Listing of horizontal stations and elevations for cross section PR167

[Sta., station, distance in meters from a reference pin on the left bank; Elev., elevation, in meters above sea level]

\begin{tabular}{|c|c|c|c|c|c|c|c|c|c|}
\hline \multirow{2}{*}{\multicolumn{2}{|c|}{$\begin{array}{c}1989 \\
20 \text { September }\end{array}$}} & \multirow{2}{*}{\multicolumn{2}{|c|}{$\begin{array}{c}1989 \\
20 \text { September }\end{array}$}} & \multirow{2}{*}{\multicolumn{2}{|c|}{$\begin{array}{c}1989 \\
20 \text { September }\end{array}$}} & \multirow{2}{*}{\multicolumn{2}{|c|}{$\begin{array}{c}1990 \\
\text { 20 September }\end{array}$}} & \multirow{2}{*}{\multicolumn{2}{|c|}{$\begin{array}{c}1990 \\
\text { 20 September }\end{array}$}} \\
\hline & & & & & & & & & \\
\hline Sta. & Elev. & Sta. & Elev. & Sta. & Elev. & Sta. & Elev. & Sta. & Elev. \\
\hline-1.6 & 959.57 & 69.6 & 957.45 & 138.0 & 959.58 & -1.6 & 959.58 & 80.0 & 957.72 \\
\hline 0.0 & 959.65 & 70.1 & 957.53 & 140.0 & 959.55 & 0.0 & 959.64 & 81.0 & 957.79 \\
\hline 2.0 & 959.69 & 71.0 & 957.56 & 142.5 & 959.56 & 2.0 & 959.68 & 82.0 & 957.82 \\
\hline 4.0 & 959.71 & 73.0 & 957.53 & 145.0 & 959.52 & 5.0 & 959.68 & 82.4 & 957.76 \\
\hline 6.0 & 959.68 & 75.0 & 957.58 & 147.5 & 959.49 & 8.0 & 959.59 & 84.0 & 957.82 \\
\hline 8.0 & 959.59 & 77.0 & 957.59 & 150.0 & 959.48 & 11.0 & 959.65 & 85.0 & 957.78 \\
\hline 10.0 & 959.65 & 79.0 & 957.57 & 150.6 & 959.44 & 14.0 & 959.57 & 87.0 & 957.68 \\
\hline 12.0 & 959.67 & 81.0 & 957.54 & 138.0 & 959.58 & 16.0 & 959.35 & 87.2 & 957.69 \\
\hline 14.0 & 959.57 & 82.0 & 957.48 & 140.0 & 959.55 & 18.0 & 959.37 & 88.5 & 957.64 \\
\hline 15.0 & 959.55 & 83.7 & 957.45 & 142.5 & 959.56 & 20.0 & 959.21 & 90.0 & 957.51 \\
\hline 16.0 & 959.35 & 85.0 & 957.29 & 145.0 & 959.52 & 23.0 & 959.24 & 91.0 & 957.54 \\
\hline 18.0 & 959.39 & 86.4 & 957.18 & 147.5 & 959.49 & 25.0 & 958.92 & 93.0 & 957.53 \\
\hline 20.0 & 959.21 & 87.0 & 957.31 & 150.0 & 959.48 & 28.0 & 958.87 & 95.0 & 957.54 \\
\hline 22.0 & 959.28 & 88.0 & 957.32 & 150.6 & 959.44 & 31.0 & 958.85 & 97.0 & 957.56 \\
\hline 24.0 & 959.12 & 90.0 & 957.20 & & & 33.0 & 958.82 & 97.8 & 957.57 \\
\hline 25.0 & 958.92 & 92.0 & 957.05 & & & 35.0 & 958.80 & 98.2 & 957.47 \\
\hline 27.0 & 958.89 & 94.0 & 956.98 & & & 37.0 & 958.73 & 98.8 & 957.41 \\
\hline 29.0 & 958.82 & 96.0 & 956.97 & & & 39.0 & 958.78 & 101.0 & 957.20 \\
\hline 31.0 & 958.88 & 98.0 & 957.05 & & & 41.0 & 958.81 & 102.0 & 957.18 \\
\hline 33.0 & 958.82 & 100.0 & 957.16 & & & 43.0 & 958.60 & 103.0 & 957.21 \\
\hline 35.0 & 958.78 & 102.0 & 957.25 & & & 44.0 & 958.50 & 105.0 & 957.09 \\
\hline 37.0 & 958.70 & 104.0 & 957.28 & & & 46.0 & 958.51 & 107.0 & 957.03 \\
\hline 39.0 & 958.77 & 106.0 & 957.32 & & & 48.0 & 958.58 & 109.0 & 956.89 \\
\hline 41.0 & 958.80 & 108.0 & 957.32 & & & 50.0 & 958.60 & 111.0 & 956.75 \\
\hline 43.0 & 958.58 & 110.0 & 957.31 & & & 52.0 & 958.62 & 112.0 & 956.70 \\
\hline 45.0 & 958.45 & 112.0 & 957.23 & & & 54.0 & 958.73 & 113.0 & 956.88 \\
\hline 47.0 & 958.53 & 113.6 & 957.14 & & & 56.0 & 958.64 & 114.7 & 957.41 \\
\hline 49.0 & 958.60 & 114.6 & 957.44 & & & 58.0 & 958.51 & 117.0 & 958.52 \\
\hline 51.0 & 958.57 & 115.8 & 957.84 & & & 58.3 & 958.37 & 117.1 & 959.74 \\
\hline 53.0 & 958.68 & 115.9 & 957.97 & & & 58.7 & 958.35 & 119.0 & 959.82 \\
\hline 55.0 & 958.71 & 116.9 & 958.50 & & & 59.0 & 958.44 & 122.0 & 959.70 \\
\hline 57.0 & 958.55 & 117.1 & 959.77 & & & 60.0 & 958.44 & 125.0 & 959.66 \\
\hline 58.0 & 958.51 & 118.0 & 959.86 & & & 62.0 & 958.41 & 128.0 & 959.57 \\
\hline 59.0 & 957.96 & 120.0 & 959.73 & & & 64.0 & 958.32 & 130.0 & 959.82 \\
\hline 59.6 & 957.78 & 122.0 & 959.71 & & & 66.0 & 958.27 & 131.0 & 959.85 \\
\hline 60.8 & 957.65 & 124.0 & 959.67 & & & 68.0 & 958.26 & 133.0 & 959.84 \\
\hline 62.0 & 957.70 & 126.0 & 959.63 & & & 70.0 & 958.22 & 136.0 & 959.70 \\
\hline 63.2 & 957.80 & 128.0 & 959.58 & & & 72.0 & 958.17 & 139.0 & 959.57 \\
\hline 64.4 & 957.79 & 130.0 & 959.84 & & & 74.0 & 958.10 & 142.0 & 959.55 \\
\hline 64.8 & 957.67 & 132.0 & 959.85 & & & 76.0 & 958.05 & 145.0 & 959.50 \\
\hline 66.0 & 957.55 & 134.0 & 959.79 & & & 77.0 & 957.98 & 147.0 & 959.50 \\
\hline 68.0 & 957.47 & 136.0 & 959.70 & & & 79.0 & 957.74 & 150.0 & 959.46 \\
\hline & & & & & & & & 150.6 & 959.44 \\
\hline
\end{tabular}


Table 27. (Continued) Listing of horizontal stations and elevations for cross section PR167

[Sta., station, distance in meters from a reference pin on the left bank; Elev., elevation, in meters above sea level]

\begin{tabular}{|c|c|c|c|c|c|c|c|c|c|}
\hline \multirow{2}{*}{\multicolumn{2}{|c|}{$\begin{array}{c}1991 \\
\text { 4 September }\end{array}$}} & \multicolumn{2}{|c|}{1991} & \multicolumn{2}{|c|}{1991} & \multicolumn{2}{|c|}{1992} & \multicolumn{2}{|c|}{1992} \\
\hline & & $4 \mathrm{Se}$ & ember & $4 \mathrm{Se}$ & mber & $1 \mathrm{Se}$ & ember & $1 \mathrm{Se}$ & ember \\
\hline Sta. & Elev. & Sta. & Elev. & Sta. & Elev. & Sta. & Elev. & Sta. & Elev. \\
\hline-1.6 & 959.58 & 72.0 & 957.63 & 140.0 & 959.55 & -1.6 & 959.59 & 71.3 & 957.45 \\
\hline 0.0 & 959.63 & 74.0 & 957.69 & 142.0 & 959.56 & 0.0 & 959.63 & 71.7 & 957.47 \\
\hline 3.0 & 959.61 & 75.7 & 957.69 & 144.0 & 959.49 & 3.0 & 959.62 & 73.0 & 957.44 \\
\hline 6.0 & 959.68 & 76.4 & 957.74 & 146.0 & 959.51 & 6.0 & 959.69 & 74.2 & 957.31 \\
\hline 9.0 & 959.63 & 78.0 & 957.72 & 148.0 & 959.49 & 9.0 & 959.63 & 76.0 & 957.25 \\
\hline 12.0 & 959.66 & 79.8 & 957.71 & 150.0 & 959.47 & 11.0 & 959.65 & 78.0 & 957.35 \\
\hline 15.0 & 959.57 & 79.9 & 957.53 & 150.6 & 959.44 & 13.0 & 959.61 & 80.0 & 957.37 \\
\hline 16.4 & 959.30 & 80.8 & 957.33 & 140.0 & 959.55 & 15.0 & 959.56 & 82.0 & 957.45 \\
\hline 18.0 & 959.38 & 82.0 & 957.39 & 142.0 & 959.56 & 16.0 & 959.35 & 83.0 & 957.49 \\
\hline 20.0 & 959.23 & 83.8 & 957.38 & 144.0 & 959.49 & 18.0 & 959.38 & 85.0 & 957.46 \\
\hline 22.0 & 959.28 & 85.3 & 957.48 & 146.0 & 959.51 & 20.0 & 959.23 & 87.0 & 957.43 \\
\hline 23.0 & 959.25 & 87.0 & 957.45 & 148.0 & 959.49 & 21.3 & 959.34 & 89.0 & 957.40 \\
\hline 25.0 & 958.91 & 89.0 & 957.40 & 150.0 & 959.47 & 23.0 & 959.25 & 91.0 & 957.40 \\
\hline 27.0 & 958.87 & 91.0 & 957.41 & 150.6 & 959.44 & 25.0 & 958.92 & 93.0 & 957.45 \\
\hline 29.0 & 958.84 & 93.0 & 957.43 & & & 27.0 & 958.88 & 95.0 & 957.45 \\
\hline 31.0 & 958.86 & 95.0 & 957.43 & & & 28.0 & 958.92 & 96.5 & 957.42 \\
\hline 33.0 & 958.83 & 97.0 & 957.40 & & & 30.0 & 958.81 & 98.0 & 957.02 \\
\hline 36.0 & 958.78 & 99.0 & 957.39 & & & 32.0 & 958.85 & 99.0 & 957.38 \\
\hline 39.0 & 958.78 & 99.2 & 957.24 & & & 34.0 & 958.77 & 101.0 & 957.25 \\
\hline 41.0 & 958.79 & 99.8 & 957.06 & & & 36.0 & 958.79 & 103.0 & 957.11 \\
\hline 42.0 & 958.75 & 101.0 & 956.96 & & & 38.0 & 958.74 & 105.0 & 956.99 \\
\hline 44.0 & 958.48 & 103.0 & 956.99 & & & 40.0 & 958.83 & 107.0 & 957.07 \\
\hline 45.0 & 958.49 & 105.0 & 957.12 & & & 41.0 & 958.79 & 109.0 & 956.88 \\
\hline 47.0 & 958.55 & 107.0 & 957.13 & & & 43.0 & 958.61 & 111.0 & 956.80 \\
\hline 49.0 & 958.62 & 109.0 & 957.10 & & & 44.0 & 958.48 & 112.5 & 956.75 \\
\hline 51.0 & 958.59 & 111.0 & 957.05 & & & 46.0 & 958.50 & 114.9 & 957.47 \\
\hline 53.0 & 958.70 & 113.3 & 957.01 & & & 48.0 & 958.57 & 116.4 & 957.99 \\
\hline 55.0 & 958.73 & 113.8 & 957.18 & & & 50.0 & 958.59 & 116.9 & 958.20 \\
\hline 56.0 & 958.67 & 115.3 & 957.60 & & & 52.0 & 958.61 & 117.1 & 958.38 \\
\hline 58.0 & 958.56 & 117.3 & 958.74 & & & 54.0 & 958.72 & 117.3 & 959.77 \\
\hline 59.7 & 958.51 & 117.4 & 959.78 & & & 56.0 & 958.67 & 118.0 & 959.86 \\
\hline 61.0 & 958.59 & 118.0 & 959.86 & & & 58.0 & 958.56 & 120.0 & 959.73 \\
\hline 61.1 & 958.36 & 120.0 & 959.73 & & & 60.0 & 958.54 & 123.0 & 959.70 \\
\hline 61.8 & 958.19 & 122.0 & 959.70 & & & 60.7 & 958.57 & 124.8 & 959.68 \\
\hline 62.1 & 957.81 & 124.0 & 959.68 & & & 62.1 & 957.84 & 127.0 & 959.63 \\
\hline 62.5 & 957.60 & 126.0 & 959.64 & & & 64.0 & 957.83 & 128.4 & 959.59 \\
\hline 63.2 & 957.42 & 128.0 & 959.57 & & & 66.0 & 957.83 & 130.0 & 959.83 \\
\hline 63.8 & 957.43 & 130.0 & 959.84 & & & 67.7 & 957.86 & 133.0 & 959.84 \\
\hline 64.3 & 957.51 & 132.0 & 959.86 & & & 67.8 & 957.65 & 136.0 & 959.71 \\
\hline 66.0 & 957.52 & 134.0 & 959.79 & & & 69.0 & 957.64 & 138.0 & 959.59 \\
\hline 68.0 & 957.56 & 136.0 & 959.71 & & & 70.0 & 957.56 & 141.0 & 959.55 \\
\hline 70.0 & 957.59 & 138.0 & 959.59 & & & 70.4 & 957.47 & 144.0 & 959.49 \\
\hline
\end{tabular}


Table 27. (Continued) Listing of horizontal stations and elevations for cross section PR167

[Sta., station, distance in meters from a reference pin on the left bank; Elev., elevation, in meters above sea level]

\begin{tabular}{|c|c|c|c|c|c|c|c|c|c|}
\hline \multirow{2}{*}{\multicolumn{2}{|c|}{$\begin{array}{c}1992 \\
1 \text { September }\end{array}$}} & \multirow{2}{*}{\multicolumn{2}{|c|}{$\begin{array}{c}1993 \\
2 \text { September }\end{array}$}} & \multirow{2}{*}{\multicolumn{2}{|c|}{$\begin{array}{c}1993 \\
2 \text { September }\end{array}$}} & \multicolumn{2}{|c|}{1993} & \multicolumn{2}{|c|}{1994} \\
\hline & & & & & & $2 \mathrm{Se}$ & ember & $20 \mathrm{~S}$ & ember \\
\hline Sta. & Elev. & Sta. & Elev. & Sta. & Elev. & Sta. & Elev. & Sta. & Elev. \\
\hline 147.0 & 959.49 & -1.6 & 959.58 & 65.5 & 957.86 & 147.0 & 959.50 & -1.6 & 959.61 \\
\hline 150.0 & 959.48 & 0.0 & 959.64 & 68.0 & 957.87 & 150.0 & 959.48 & 23.0 & 959.25 \\
\hline 150.6 & 959.45 & 3.0 & 959.62 & 70.0 & 957.80 & 150.6 & 959.46 & 25.0 & 958.93 \\
\hline & & 5.0 & 959.70 & 72.0 & 957.81 & & & 27.0 & 958.88 \\
\hline & & 8.0 & 959.59 & 74.0 & 957.79 & & & 29.0 & 958.85 \\
\hline & & 11.0 & 959.63 & 76.0 & 957.78 & & & 32.0 & 958.87 \\
\hline & & 13.0 & 959.61 & 78.0 & 957.77 & & & 35.0 & 958.79 \\
\hline & & 15.0 & 959.55 & 80.0 & 957.77 & & & 37.0 & 958.76 \\
\hline & & 16.5 & 959.31 & 82.6 & 957.80 & & & 39.0 & 958.80 \\
\hline & & 19.0 & 959.30 & 82.7 & 957.61 & & & 41.0 & 958.83 \\
\hline & & 20.0 & 959.22 & 84.0 & 957.50 & & & 43.0 & 958.62 \\
\hline & & 22.0 & 959.28 & 86.0 & 957.45 & & & 45.0 & 958.54 \\
\hline & & 23.0 & 959.26 & 88.0 & 957.49 & & & 47.0 & 958.58 \\
\hline & & 24.3 & 959.08 & 90.0 & 957.53 & & & 50.0 & 958.66 \\
\hline & & 25.0 & 958.93 & 92.0 & 957.55 & & & 53.0 & 958.76 \\
\hline & & 26.0 & 958.90 & 94.0 & 957.54 & & & 56.0 & 958.74 \\
\hline & & 28.0 & 958.89 & 95.0 & 957.52 & & & 57.5 & 958.77 \\
\hline & & 29.5 & 958.81 & 95.6 & 957.43 & & & 58.5 & 958.90 \\
\hline & & 31.0 & 958.87 & 97.0 & 957.44 & & & 59.1 & 958.94 \\
\hline & & 33.0 & 958.85 & 99.0 & 956.85 & & & 60.8 & 958.81 \\
\hline & & 35.0 & 958.82 & 100.0 & 956.72 & & & 61.8 & 958.65 \\
\hline & & 37.0 & 958.76 & 103.0 & 956.75 & & & 63.0 & 958.13 \\
\hline & & 39.0 & 958.80 & 104.0 & 956.74 & & & 64.0 & 957.98 \\
\hline & & 41.0 & 958.82 & 105.0 & 956.89 & & ' & 65.0 & 958.02 \\
\hline & & 42.0 & 958.75 & 108.0 & 957.10 & & & 67.0 & 958.07 \\
\hline & & 44.0 & 958.58 & 110.0 & 956.92 & & & 69.0 & 958.05 \\
\hline & & 45.0 & 958.52 & 112.0 & 956.86 & & & 71.0 & 958.04 \\
\hline & & 47.0 & 958.59 & 112.6 & 956.79 & & & 73.0 & 958.05 \\
\hline & & 49.0 & 958.66 & 115.0 & 957.51 & & & 75.0 & 958.03 \\
\hline & & 51.0 & 958.65 & 116.7 & 958.10 & & & 76.0 & 958.04 \\
\hline & & 53.0 & 958.73 & 117.2 & 958.47 & & & 77.0 & 958.04 \\
\hline & & 55.0 & 958.77 & 117.3 & 959.75 & & & 78.0 & 957.94 \\
\hline & & 56.5 & 958.70 & 119.0 & 959.80 & & & 79.0 & 957.84 \\
\hline & & 58.0 & 958.76 & 121.0 & 959.71 & & & 80.0 & 957.89 \\
\hline & & 59.5 & 958.99 & 124.0 & 959.67 & & & 81.5 & 957.96 \\
\hline & & 60.5 & 958.83 & 127.0 & 959.61 & & & 81.6 & 957.71 \\
\hline & & 62.0 & 958.71 & 129.0 & 959.74 & & & 82.0 & 957.60 \\
\hline & & 62.0 & 958.33 & 131.0 & 959.86 & & & 83.0 & 957.58 \\
\hline & & 62.7 & 958.03 & 134.0 & 959.79 & & & 85.0 & 957.59 \\
\hline & & 63.3 & 957.89 & 137.0 & 959.66 & & & 87.0 & 957.60 \\
\hline & & 63.7 & 957.68 & 140.0 & 959.58 & & & 89.0 & 957.56 \\
\hline & & 65.0 & 957.69 & 144.0 & 959.49 & & & 91.0 & 957.48 \\
\hline
\end{tabular}


Table 27. (Continued) Listing of horizontal stations and elevations for cross section PR167

[Sta., station, distance in meters from a reference pin on the left bank; Elev., elevation, in meters above sea level]

\begin{tabular}{|c|c|c|c|c|c|c|c|c|c|}
\hline \multirow{2}{*}{\multicolumn{2}{|c|}{$\begin{array}{c}1994 \\
20 \text { September }\end{array}$}} & \multirow{2}{*}{\multicolumn{2}{|c|}{$\begin{array}{c}1995 \\
1 \text { October }\end{array}$}} & \multirow{2}{*}{\multicolumn{2}{|c|}{$\begin{array}{l}1995 \\
1 \text { October }\end{array}$}} & \multirow{2}{*}{\multicolumn{2}{|c|}{$\begin{array}{l}1995 \\
1 \text { October }\end{array}$}} & \multirow{2}{*}{\multicolumn{2}{|c|}{$\begin{array}{c}1996 \\
21 \text { October }\end{array}$}} \\
\hline & & & & & & & & & \\
\hline Sta. & Elev. & Sta. & Elev. & Sta. & Elev. & Sta. & Elev. & Sta. & Elev. \\
\hline 93.0 & 957.42 & -1.6 & 959.59 & 76.0 & 957.41 & 146.0 & 959.51 & -1.6 & 959.59 \\
\hline 95.0 & 957.49 & 2.0 & 959.66 & 78.0 & 957.41 & 148.0 & 959.49 & 10.0 & 959.65 \\
\hline 96.2 & 957.54 & 5.0 & 959.69 & 80.0 & 957.40 & 150.0 & 959.48 & 12.5 & 959.65 \\
\hline 96.8 & 957.36 & 8.0 & 959.60 & 82.0 & 957.47 & 150.6 & 959.45 & 15.0 & 959.57 \\
\hline 98.0 & 957.25 & 10.0 & 959.65 & 84.0 & 957.45 & & & 17.0 & 959.35 \\
\hline 101.0 & 957.15 & 12.0 & 959.62 & 86.0 & 957.54 & & & 19.0 & 959.35 \\
\hline 104.0 & 957.17 & 14.0 & 959.57 & 88.0 & 957.54 & & & 21.0 & 959.31 \\
\hline 107.0 & 957.13 & 15.5 & 959.32 & 90.0 & 957.55 & & & 23.0 & 959.26 \\
\hline 110.0 & 957.04 & 18.7 & 959.35 & 92.0 & 957.57 & & & 25.0 & 958.99 \\
\hline 113.0 & 956.95 & 20.0 & 959.24 & 94.0 & 957.58 & & & 27.0 & 958.95 \\
\hline 115.5 & 956.93 & 22.0 & 959.32 & 96.0 & 957.58 & & & 30.0 & 958.91 \\
\hline 116.7 & 957.35 & 23.0 & 959.28 & 98.0 & 957.60 & & & 33.0 & 958.96 \\
\hline 118.0 & 958.27 & 24.0 & 959.12 & 100.0 & 957.50 & & & 36.0 & 958.94 \\
\hline 118.3 & 958.39 & 25.0 & 958.98 & 102.0 & 957.54 & & & 38.0 & 958.93 \\
\hline 119.2 & 958.80 & 26.0 & 958.97 & 104.0 & 957.51 & & & 40.0 & 958.97 \\
\hline 119.2 & 959.83 & 28.0 & 958.95 & 106.0 & 957.42 & & & 41.0 & 959.00 \\
\hline 122.0 & 959.71 & 30.0 & 958.95 & 108.0 & 957.10 & & & 43.0 & 958.91 \\
\hline 125.0 & 959.69 & 33.0 & 958.94 & 110.0 & 957.18 & & & 44.3 & 958.75 \\
\hline 126.0 & 959.65 & 35.0 & 958.93 & 112.0 & 956.71 & & & 47.0 & 958.95 \\
\hline 128.0 & 959.58 & 37.0 & 958.90 & 113.0 & 956.85 & & & 49.0 & 959.05 \\
\hline 130.0 & 959.76 & 39.0 & 958.94 & 115.0 & 956.68 & & & 51.0 & .959 .08 \\
\hline 131.0 & 959.84 & 41.0 & 958.99 & 117.0 & 956.68 & & & 53.0 & 959.10 \\
\hline 133.0 & 959.84 & 43.0 & 958.88 & 118.5 & 956.73 & & & 55.4 & 959.28 \\
\hline 136.0 & 959.72 & 44.0 & 958.74 & 120.5 & 957.62 & & & 57.0 & 959.07 \\
\hline 139.0 & 959.58 & 45.0 & 958.81 & 121.0 & 957.86 & & & 59.0 & 959.07 \\
\hline 141.0 & 959.53 & 47.0 & 958.92 & 121.2 & 958.37 & & & 61.0 & 959.08 \\
\hline 144.0 & 959.48 & 49.0 & 959.03 & 121.5 & 958.55 & & & 63.0 & 959.00 \\
\hline 147.0 & 959.50 & 51.0 & 959.06 & 121.9 & 958.41 & & & 65.0 & 958.93 \\
\hline 150.0 & 959.48 & 53.0 & 959.05 & 122.3 & 958.50 & & & 67.0 & 958.88 \\
\hline 150.6 & 959.45 & 54.0 & 959.23 & 122.7 & 958.90 & & & 68.0 & 958.95 \\
\hline & & 54.5 & 959.17 & 122.8 & 959.51 & & & 69.7 & 958.66 \\
\hline & & 55.6 & 959.28 & 123.2 & 959.67 & & & 70.1 & 958.74 \\
\hline & & 57.0 & 959.00 & 126.0 & 959.65 & & & 72.0 & 958.22 \\
\hline & & 58.0 & 959.01 & 128.0 & 959.57 & & & 72.8 & 958.31 \\
\hline & & 60.0 & 959.09 & 129.0 & 959.69 & & & 73.3 & 958.19 \\
\hline & & 62.0 & 959.07 & 132.4 & 959.85 & & & 75.0 & 957.51 \\
\hline & & 64.0 & 959.00 & 134.0 & 959.80 & & & 75.5 & 957.35 \\
\hline & & 66.0 & 958.88 & 136.0 & 959.71 & & & 77.0 & 957.32 \\
\hline & & 68.0 & 958.84 & 138.0 & 959.60 & & & 80.0 & 957.33 \\
\hline & & 70.0 & 958.20 & 140.0 & 959.56 & & & 83.0 & 957.47 \\
\hline & & 73.3 & 957.62 & 142.0 & 959.52 & & & 85.0 & 957.47 \\
\hline & & 74.0 & 957.41 & 144.0 & 959.49 & & & 88.0 & 957.39 \\
\hline
\end{tabular}


Table 27. (Continued) Listing of horizontal stations and elevations for cross section PR167

[Sta., station, distance in meters from a reference pin on the left bank; Elev., elevation, in meters above sea level]

\begin{tabular}{|c|c|c|c|c|c|c|c|c|c|}
\hline \multirow{2}{*}{\multicolumn{2}{|c|}{$\begin{array}{c}1996 \\
21 \text { October }\end{array}$}} & \multicolumn{2}{|c|}{1997} & \multicolumn{2}{|c|}{1997} & \multicolumn{2}{|c|}{1998} & \multicolumn{2}{|c|}{1998} \\
\hline & & $21 \mathrm{Se}$ & ember & $21 \mathrm{Se}$ & ember & $30 \mathrm{Se}$ & ember & $30 \mathrm{Se}$ & ember \\
\hline Sta. & Elev. & Sta. & Elev. & Sta. & Elev. & Sta. & Elev. & Sta. & Elev. \\
\hline 89.5 & 957.26 & -1.6 & 959.58 & 95.0 & 957.07 & -1.6 & 959.58 & 79.0 & 957.56 \\
\hline 92.0 & 957.23 & 10.0 & 959.64 & 98.0 & 957.25 & 0.0 & 959.62 & 81.0 & 957.44 \\
\hline 95.0 & 957.28 & 12.5 & 959.63 & 101.0 & 957.27 & 3.0 & 959.64 & 84.0 & 957.28 \\
\hline 98.0 & 957.38 & 14.7 & 959.59 & 104.0 & 957.30 & 5.0 & 959.70 & 87.0 & 957.25 \\
\hline 101.0 & 957.40 & 16.4 & 959.29 & 107.0 & 957.28 & 7.5 & 959.60 & 90.0 & 957.34 \\
\hline 104.0 & 957.39 & 18.0 & 959.39 & 110.0 & 957.28 & 10.0 & 959.65 & 93.0 & 957.32 \\
\hline 107.0 & 957.38 & 20.0 & 959.24 & 113.0 & 957.21 & 12.0 & 959.66 & 96.0 & 957.13 \\
\hline 110.0 & 957.22 & 22.0 & 959.34 & 116.0 & 957.16 & 14.0 & 959.59 & 97.5 & 957.40 \\
\hline 113.0 & 957.00 & 23.7 & 959.19 & 118.0 & 957.01 & 15.5 & 959.49 & 100.0 & 957.40 \\
\hline 115.0 & 957.03 & 25.3 & 958.97 & 119.7 & 957.04 & 16.5 & 959.32 & 103.0 & 957.34 \\
\hline 117.0 & 956.94 & 27.0 & 958.95 & 121.3 & 957.51 & 18.0 & 959.39 & 106.0 & 957.12 \\
\hline 118.6 & 956.77 & 30.0 & 958.92 & 122.1 & 957.73 & 20.0 & 959.24 & 108.0 & 956.92 \\
\hline 119.8 & 957.05 & 33.0 & 958.93 & 123.2 & 958.31 & 21.5 & 959.34 & 111.0 & 956.67 \\
\hline 121.3 & 957.51 & 36.0 & 958.95 & 123.3 & 958.64 & 23.0 & 959.26 & 114.0 & 956.71 \\
\hline 121.8 & 957.53 & 39.0 & 958.97 & 124.1 & 959.09 & 25.0 & 958.98 & 117.0 & 956.68 \\
\hline 122.8 & 958.45 & 42.0 & 958.96 & 124.4 & 959.66 & 28.0 & 958.96 & 119.0 & 956.79 \\
\hline 123.2 & 958.53 & 44.2 & 958.76 & 127.4 & 959.62 & 30.0 & 958.93 & 121.1 & 957.57 \\
\hline 123.8 & 959.11 & 45.5 & 958.84 & 129.0 & 959.71 & 33.0 & 958.94 & 122.0 & 957.70 \\
\hline 124.0 & 959.03 & 48.0 & 958.98 & 131.6 & 959.85 & 36.0 & 958.93 & 122.6 & 958.03 \\
\hline 124.3 & 959.10 & 51.0 & 959.10 & 134.0 & 959.79 & 39.0 & 958.97 & 123.9 & 958.59 \\
\hline 124.5 & 959.69 & 53.5 & 959.08 & 136.0 & 959.69 & 41.0 & 959.02 & 123.9 & 959.68 \\
\hline 126.0 & 959.67 & 55.0 & 959.28 & 138.5 & 959.58 & 43.0 & 958.92 & 126.5 & 959.64 \\
\hline 128.0 & 959.62 & 57.0 & 959.11 & 141.0 & 959.52 & 44.0 & 958.79 & 128.5 & 959.61 \\
\hline 130.0 & 959.78 & 59.0 & 959.09 & 144.0 & 959.48 & 46.0 & 958.90 & 131.5 & 959.83 \\
\hline 132.0 & 959.85 & 62.0 & 959.03 & 147.0 & 959.49 & 48.0 & 959.00 & 134.0 & 959.78 \\
\hline 135.0 & 959.77 & 64.0 & 958.96 & 150.0 & 959.48 & 51.0 & 959.09 & 137.0 & 959.66 \\
\hline 138.0 & 959.61 & 66.0 & 958.98 & 150.6 & 959.46 & 53.0 & 959.10 & 140.0 & 959.54 \\
\hline 141.0 & 959.53 & 67.8 & 958.92 & & & 55.0 & 959.28 & 142.0 & 959.53 \\
\hline 144.0 & 959.48 & 69.0 & 958.68 & & & 56.0 & 959.21 & 145.0 & 959.49 \\
\hline 147.0 & 959.49 & 71.0 & 958.30 & & & 58.0 & 959.17 & 150.0 & 959.48 \\
\hline 150.0 & 959.48 & 73.0 & 957.96 & & & 60.0 & 959.09 & 150.6 & 959.46 \\
\hline 150.6 & 959.46 & 74.0 & 957.94 & & & 63.0 & 958.99 & & \\
\hline & & 75.0 & 957.51 & & & 65.0 & 958.96 & & \\
\hline & & 76.0 & 957.30 & & & 67.0 & 958.92 & & \\
\hline & & 78.0 & 957.22 & & & 67.5 & 958.92 & & \\
\hline & & 80.0 & 957.21 & & & 69.2 & 958.60 & & \\
\hline & & 81.0 & 957.27 & & & 71.2 & 958.09 & & \\
\hline & & 83.0 & 957.34 & & & 72.7 & 957.88 & & \\
\hline & & 86.0 & 957.35 & & & 73.4 & 957.97 & & \\
\hline & & 89.0 & 957.39 & & & 74.1 & 957.80 & & \\
\hline & & 91.0 & 957.34 & & & 74.4 & 957.60 & & \\
\hline & & 92.0 & 957.12 & & & 77.0 & 957.52 & & \\
\hline
\end{tabular}




\section{Description of Cross Section PR167.5}

Location: Township 6 South/Range 50 East--section 21

U. S. Geological Survey quadrangle (1:24,000): Lonesome Peak

Landowners--left bank: Gay Ranch

--right bank: Gay Ranch

Access: Left bank

Permission from: Gay Ranch

Distance from Moorhead Gaging Station: 52.3 kilometers

Azimuth of Section (degrees magnetic): 057

\section{Reference Monuments}

[Monument at station 0.0 and 22.0 was closest to leveling instrument; the monuments for this section were not surveyed using the Global Positioning System so that the monuments have been located on an aerial photo and the corresponding latitude and longitude have been measured on a 1:24000 topographic map]

\begin{tabular}{|c|c|c|c|c|c|c|}
\hline \multirow[b]{2}{*}{ Description } & \multirow[b]{2}{*}{$\begin{array}{c}\text { Station } \\
(\mathrm{m})\end{array}$} & \multicolumn{2}{|c|}{ GPS-NAD83 (1992) } & \multicolumn{2}{|c|}{ Measurement } & \multirow{2}{*}{$\begin{array}{l}\text { Elevation } \\
\text { (NGVD1929) } \\
\text { (m) }\end{array}$} \\
\hline & & Latitude & Longitude & $\begin{array}{l}\text { Standard } \\
\text { deviation } \\
(m)\end{array}$ & $\begin{array}{l}\text { Horizontal } \\
\text { precision } \\
\text { (m) }\end{array}$ & \\
\hline $\begin{array}{l}\text { 1/2-inch-rebar; } 0.06 \text { meter above } 1998 \text { ground } \\
\text { level; on a } 6 \text {-meter high terrace; } 0.06 \\
\text { meter riverward of corner fence post }\end{array}$ & 0.0 & & & & & 963.34 \\
\hline $\begin{array}{l}\text { 1/2-inch-rebar; } 0.05 \text { meter above } 1998 \text { ground } \\
\text { level; on a 6-meter high terrace; under a } \\
\text { rock pile }\end{array}$ & 22.0 & & & & & 963.29 \\
\hline $\begin{array}{l}\text { 1/2-inch-rebar; } 0.13 \text { meter above } 1998 \text { ground } \\
\text { level }\end{array}$ & 150.0 & & & & & 959.18 \\
\hline $\begin{array}{l}\text { 1/2-inch-rebar; } 0.10 \text { meter above } 1998 \text { ground } \\
\text { level; on the left bank of a chute channel }\end{array}$ & 220.0 & & & & & 959.32 \\
\hline
\end{tabular}


See Figure 8 for location

of cross section

PR167.5

This cross section was not surveyed in 1998 and no map was made showing the location of the reference monuments.

Figure 78. Upper: Location of cross section PR167.5 is shown in figure 8. Lower: Section was not surveyed in 1998 and a map of the reference monuments was not made. 


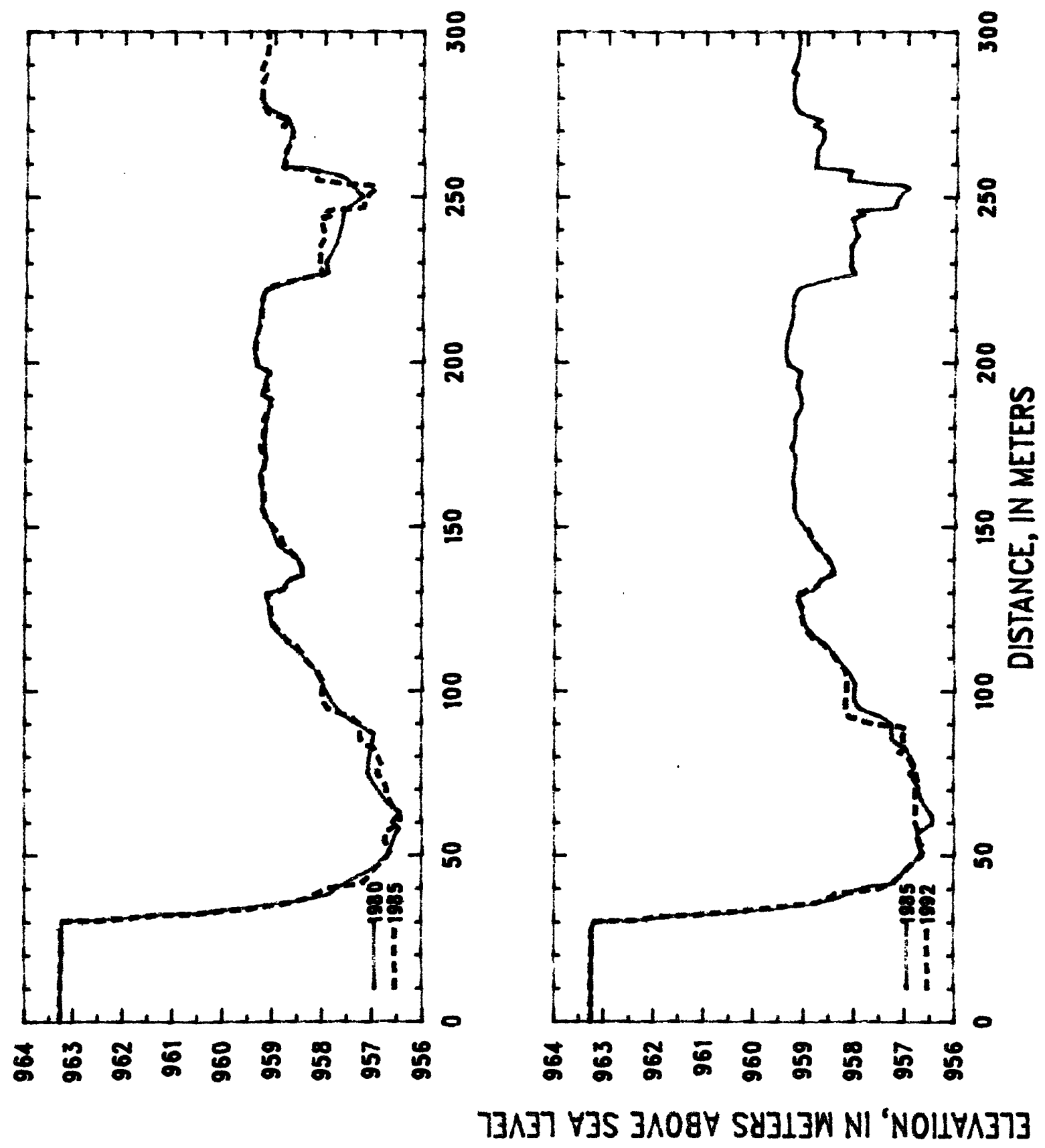

Figure 79. Profiles of cross section PR167.5 from 1980 to 1992. 
Table 28. Listing of horizontal stations and elevations for cross section PR167.5

[Sta., station, distance in meters from a reference pin on the left bank; Elev., elevation, in meters above sea level]

\begin{tabular}{|c|c|c|c|c|c|c|c|c|c|}
\hline \multirow{2}{*}{\multicolumn{2}{|c|}{$\begin{array}{c}1980 \\
20 \text { October }\end{array}$}} & \multirow{2}{*}{\multicolumn{2}{|c|}{$\begin{array}{c}1980 \\
20 \text { October }\end{array}$}} & \multirow{2}{*}{\multicolumn{2}{|c|}{$\begin{array}{c}1985 \\
2 \text { October }\end{array}$}} & \multirow{2}{*}{\multicolumn{2}{|c|}{$\begin{array}{c}1985 \\
2 \text { October }\end{array}$}} & \multirow{2}{*}{\multicolumn{2}{|c|}{$\begin{array}{c}1985 \\
2 \text { October }\end{array}$}} \\
\hline & & & & & & & & & \\
\hline Sta. & Elev. & Sta. & Elev. & Sta. & Elev. & Sta. & Elev. & Sta. & Elev. \\
\hline 0.0 & 963.28 & 131.0 & 958.77 & 0.0 & 963.27 & 90.0 & 957.27 & 199.0 & 959.32 \\
\hline 2.0 & 963.24 & 133.4 & 958.69 & 4.4 & 963.27 & 91.8 & 957.40 & 202.0 & 959.38 \\
\hline 5.0 & 963.26 & 135.0 & 958.38 & 6.9 & 963.27 & 92.8 & 957.59 & 205.0 & 959.37 \\
\hline 10.0 & 963.26 & 140.0 & 958.50 & 10.6 & 963.26 & 94.6 & 957.90 & 208.0 & 959.33 \\
\hline 15.0 & 963.26 & 144.0 & 958.88 & 13.3 & 963.27 & 97.0 & 958.01 & 211.0 & 959.27 \\
\hline 20.0 & 963.22 & 150.0 & 959.04 & 17.5 & 963.24 & 100.0 & 958.01 & 214.0 & 959.22 \\
\hline 22.0 & 963.23 & 155.0 & 959.21 & 21.0 & 963.23 & 102.0 & 957.97 & 217.0 & 959.23 \\
\hline 25.0 & 963.24 & 160.0 & 959.19 & 22.0 & 963.24 & 106.0 & 958.11 & 220.0 & 959.21 \\
\hline 30.0 & 963.21 & 165.0 & 959.26 & 24.4 & 963.24 & 109.0 & 958.31 & 222.0 & 959.14 \\
\hline 30.5 & 963.20 & 170.0 & 959.12 & 28.1 & 963.25 & 111.0 & 958.40 & 223.2 & 959.01 \\
\hline 30.6 & 962.23 & 174.0 & 959.19 & 30.4 & 963.17 & 113.5 & 958.52 & 225.0 & 958.52 \\
\hline 32.3 & 961.26 & 177.0 & 959.16 & 30.4 & 962.45 & 115.0 & 958.69 & 226.6 & 958.01 \\
\hline 32.5 & 960.39 & 180.0 & 959.15 & 32.0 & 961.36 & 117.0 & 958.80 & 229.0 & 958.12 \\
\hline 33.1 & 960.23 & 185.0 & 959.09 & 32.3 & 960.63 & 120.0 & 958.98 & 232.0 & 958.08 \\
\hline 36.2 & 958.43 & 188.7 & 959.04 & 33.1 & 960.09 & 123.0 & 959.04 & 235.0 & 958.11 \\
\hline 38.2 & 957.85 & 190.0 & 959.22 & 34.1 & 959.38 & 126.0 & 959.06 & 238.0 & 957.95 \\
\hline 40.2 & 957.69 & 195.0 & 959.13 & 35.8 & 958.61 & 129.0 & 959.12 & 240.0 & 958.03 \\
\hline 43.4 & 957.30 & 197.0 & 959.08 & 36.7 & 958.34 & 131.0 & 958.76 & 243.3 & 958.09 \\
\hline 46.0 & 956.97 & 199.0 & 959.33 & 38.0 & 958.16 & 133.8 & 958.64 & 244.4 & 957.84 \\
\hline 50.0 & 956.68 & 201.0 & 959.36 & 40.0 & 957.88 & 135.3 & 958.38 & 245.6 & 957.96 \\
\hline 53.0 & 956.60 & 205.0 & 959.39 & 40.9 & 957.53 & 138.0 & 958.43 & 246.0 & 957.80 \\
\hline 57.0 & 956.50 & 210.0 & 959.28 & 41.3 & 957.23 & 141.0 & 958.56 & 246.5 & 957.31 \\
\hline 58.0 & 956.44 & 215.0 & 959.24 & 43.0 & 957.08 & 144.0 & 958.78 & 247.0 & 957.20 \\
\hline 60.0 & 956.60 & 220.0 & 959.19 & 45.0 & 957.00 & 147.0 & 958.86 & 250.0 & 957.14 \\
\hline 63.0 & 956.42 & 222.0 & 959.14 & 48.0 & 956.77 & 150.0 & 959.02 & 252.5 & 956.94 \\
\hline 66.0 & 956.70 & 224.0 & 958.72 & 51.0 & 956.63 & 153.0 & 959.15 & 253.6 & 957.13 \\
\hline 69.0 & 956.84 & 226.0 & 958.30 & 54.0 & 956.74 & 156.0 & 959.21 & 253.8 & 957.56 \\
\hline 72.0 & 956.99 & 227.0 & 957.91 & 57.0 & 956.74 & 159.0 & 959.16 & 254.7 & 957.94 \\
\hline 75.0 & 957.09 & 230.0 & 957.97 & 59.5 & 956.43 & 162.0 & 959.22 & 255.0 & 958.16 \\
\hline 80.0 & 957.04 & 235.0 & 957.76 & 62.0 & 956.42 & 165.0 & 959.23 & 256.0 & 958.17 \\
\hline 84.0 & 956.99 & 240.0 & 957.65 & 65.0 & 956.60 & 168.0 & 959.16 & 257.5 & 958.09 \\
\hline 87.0 & 956.96 & 246.0 & 957.58 & 69.0 & 956.72 & 171.0 & 959.16 & 258.0 & 958.22 \\
\hline 90.0 & 957.30 & 250.0 & 957.21 & 71.0 & 956.72 & 174.0 & 959.27 & 258.8 & 958.82 \\
\hline 91.5 & 957.48 & 256.0 & 957.61 & 74.0 & 956.86 & 177.0 & 959.17 & 261.0 & 958.77 \\
\hline 94.2 & 957.70 & 258.5 & 958.24 & 75.0 & 956.91 & 180.0 & 959.17 & 264.0 & 958.77 \\
\hline 100.0 & 957.95 & 259.0 & 958.84 & 77.0 & 956.78 & 183.0 & 959.18 & 267.0 & 958.64 \\
\hline 105.0 & 958.08 & 270.0 & 958.63 & 80.0 & 956.88 & 186.0 & 959.06 & 269.8 & 958.63 \\
\hline 110.0 & 958.35 & 274.0 & 958.76 & 83.0 & 956.99 & 189.0 & 959.05 & 271.6 & 958.85 \\
\hline 115.0 & 958.73 & 277.0 & 959.17 & 83.4 & 957.07 & 192.0 & 959.16 & 273.0 & 958.70 \\
\hline 120.0 & 959.01 & 280.0 & 959.28 & 84.0 & 957.16 & 195.0 & 959.12 & 274.6 & 958.96 \\
\hline 125.0 & 959.07 & & & 85.5 & 957.25 & 197.0 & 959.07 & 275.4 & 959.14 \\
\hline 129.6 & 959.13 & & & 88.0 & 957.26 & 198.0 & 959.18 & 278.0 & 959.25 \\
\hline
\end{tabular}


Table 28. (Continued) Listing of horizontal stations and elevations for cross section PR167.5

[Sta., station, distance in meters from a reference pin on the left bank; Elev., elevation, in meters above sea level]

\begin{tabular}{|c|c|c|c|c|c|}
\hline \multirow{2}{*}{\multicolumn{2}{|c|}{$\begin{array}{c}1985 \\
2 \text { October }\end{array}$}} & \multirow{2}{*}{\multicolumn{2}{|c|}{$\begin{array}{c}1992 \\
27 \text { August }\end{array}$}} & \multirow{2}{*}{\multicolumn{2}{|c|}{$\begin{array}{c}1992 \\
27 \text { August }\end{array}$}} \\
\hline & & & & & \\
\hline Sta. & Elev. & Sta. & Elev. & Sta. & Elev. \\
\hline 281.0 & 959.26 & 0.0 & 963.29 & 80.3 & 956.95 \\
\hline 284.0 & 959.27 & 1.8 & 963.25 & 81.0 & 957.03 \\
\hline 287.0 & 959.16 & 10.2 & 963.27 & 81.5 & 957.10 \\
\hline 288.0 & 959.28 & 13.3 & 963.28 & 82.2 & 957.02 \\
\hline 291.0 & 959.17 & 16.4 & 963.26 & 83.0 & 956.98 \\
\hline 293.0 & 959.14 & 22.0 & 963.23 & 85.2 & 957.02 \\
\hline 296.0 & 959.11 & 25.6 & 963.25 & 86.5 & 957.04 \\
\hline 299.0 & 959.14 & 27.0 & 963.26 & 88.4 & 956.95 \\
\hline 300.0 & 959.17 & 29.0 & 963.23 & 89.3 & 957.12 \\
\hline \multirow[t]{33}{*}{301.0} & 958.96 & 30.0 & 963.18 & 89.7 & 957.42 \\
\hline & & 30.4 & 962.98 & 90.0 & 957.63 \\
\hline & & 30.6 & 962.28 & 91.0 & 957.93 \\
\hline & & 31.2 & 962.08 & 91.7 & 957.98 \\
\hline & & 31.6 & 961.83 & 91.9 & 958.10 \\
\hline & & 31.9 & 961.15 & 93.0 & 958.15 \\
\hline & & 33.1 & 960.28 & 95.0 & 958.18 \\
\hline & & 34.0 & 959.70 & 97.0 & 958.16 \\
\hline & & 35.6 & 958.75 & 99.0 & 958.13 \\
\hline & & 36.2 & 958.56 & 101.0 & 958.13 \\
\hline & & 37.4 & 958.41 & 103.0 & 958.12 \\
\hline & & 38.4 & 958.36 & 105.0 & 958.15 \\
\hline & & 39.6 & 957.57 & 107.0 & 958.21 \\
\hline & & 40.7 & 957.36 & 110.0 & 958.37 \\
\hline & & 42.0 & 957.19 & 112.0 & 958.46 \\
\hline & & 44.0 & 957.04 & 114.0 & 958.58 \\
\hline & & 46.0 & 956.92 & 116.0 & 958.80 \\
\hline & & 48.0 & 956.79 & 118.0 & 958.93 \\
\hline & & 50.0 & 956.63 & 120.0 & 959.00 \\
\hline & & 52.0 & 956.60 & 123.0 & 959.05 \\
\hline & & 54.0 & 956.72 & 126.0 & 959.06 \\
\hline & & 56.0 & 956.74 & 128.0 & 959.13 \\
\hline & & 58.0 & 956.74 & 129.5 & 959.10 \\
\hline & & 60.0 & 956.80 & 132.0 & 958.72 \\
\hline & & 62.0 & 956.77 & 134.0 & 958.62 \\
\hline & & 64.0 & 956.79 & 135.3 & 958.42 \\
\hline & & 66.0 & 956.77 & 136.0 & 958.42 \\
\hline & & 68.0 & 956.79 & 138.0 & 958.46 \\
\hline & & 70.0 & 956.78 & 140.0 & 958.51 \\
\hline & & 72.0 & 956.74 & 143.0 & 958.70 \\
\hline & & 74.0 & 956.75 & 146.0 & 958.85 \\
\hline & & 77.0 & 956.83 & 149.0 & 958.93 \\
\hline & & 79.0 & 956.90 & & \\
\hline
\end{tabular}




\section{Description of Cross Section PR168.5}

Location: Township 6 South/Range 50 East--section 21

U. S. Geological Survey quadrangle (1:24,000): Lonesome Peak

Landowners--left bank: Gay Ranch

--right bank: Gay Ranch

Access: Left bank--high bank; Right bank--point bar

Permission from: Gay Ranch

Distance from Moorhead Gaging Station: 53.3 kilometers

Azimuth of Section (degrees magnetic): 221

\section{Reference Monuments}

[Monuments at stations -1.0 and 0.0 were closest to leveling instrument before 1998 and monuments at station 151.0 was closest to leveling instrument in 1998]

\begin{tabular}{|c|c|c|c|c|c|c|}
\hline \multirow[b]{2}{*}{ Description } & \multirow[b]{2}{*}{$\begin{array}{l}\text { Station } \\
(\mathbf{m})\end{array}$} & \multicolumn{2}{|c|}{ GPS-NAD83 (1992) } & \multicolumn{2}{|c|}{ Measurement } & \multirow{2}{*}{$\begin{array}{l}\text { Elevation } \\
\text { (NGVD1929) } \\
\text { (m) }\end{array}$} \\
\hline & & Latitude & Longitude & $\begin{array}{c}\text { Standard } \\
\text { deviation } \\
\text { (m) }\end{array}$ & $\begin{array}{l}\text { Horizontal } \\
\text { precision } \\
\text { (m) }\end{array}$ & \\
\hline $\begin{array}{l}\text { 1/2-inch-rebar; } 0.23 \text { meter above } 1998 \text { ground } \\
\text { level }\end{array}$ & -16.0 & $45^{\circ} 17^{\prime} 34.14^{\prime \prime}$ & $105^{\circ} 35^{\prime} 34.22^{\prime \prime}$ & 0.257 & 0.772 & 958.61 \\
\hline $\begin{array}{l}\text { 1/2-inch-rebar; } 0.14 \text { meter above } 1998 \text { ground } \\
\text { level }\end{array}$ & -1.0 & & & & & 958.75 \\
\hline $\begin{array}{l}\text { 1/2-inch-rebar; } 0.03 \text { meter above } 1998 \text { ground } \\
\text { level }\end{array}$ & 0.0 & & & & & 958.67 \\
\hline $\begin{array}{l}\text { 1/2-inch-rebar; } 0.12 \text { meter above } 1998 \text { ground } \\
\text { level }\end{array}$ & 151.0 & $45^{\circ} 17^{\prime} 31.06^{\prime \prime}$ & $105^{\circ} 35^{\prime} 40.53^{\prime \prime}$ & 0.819 & 0.460 & 958.40 \\
\hline
\end{tabular}


See Figure 8 for location

of cross section

PR168.5

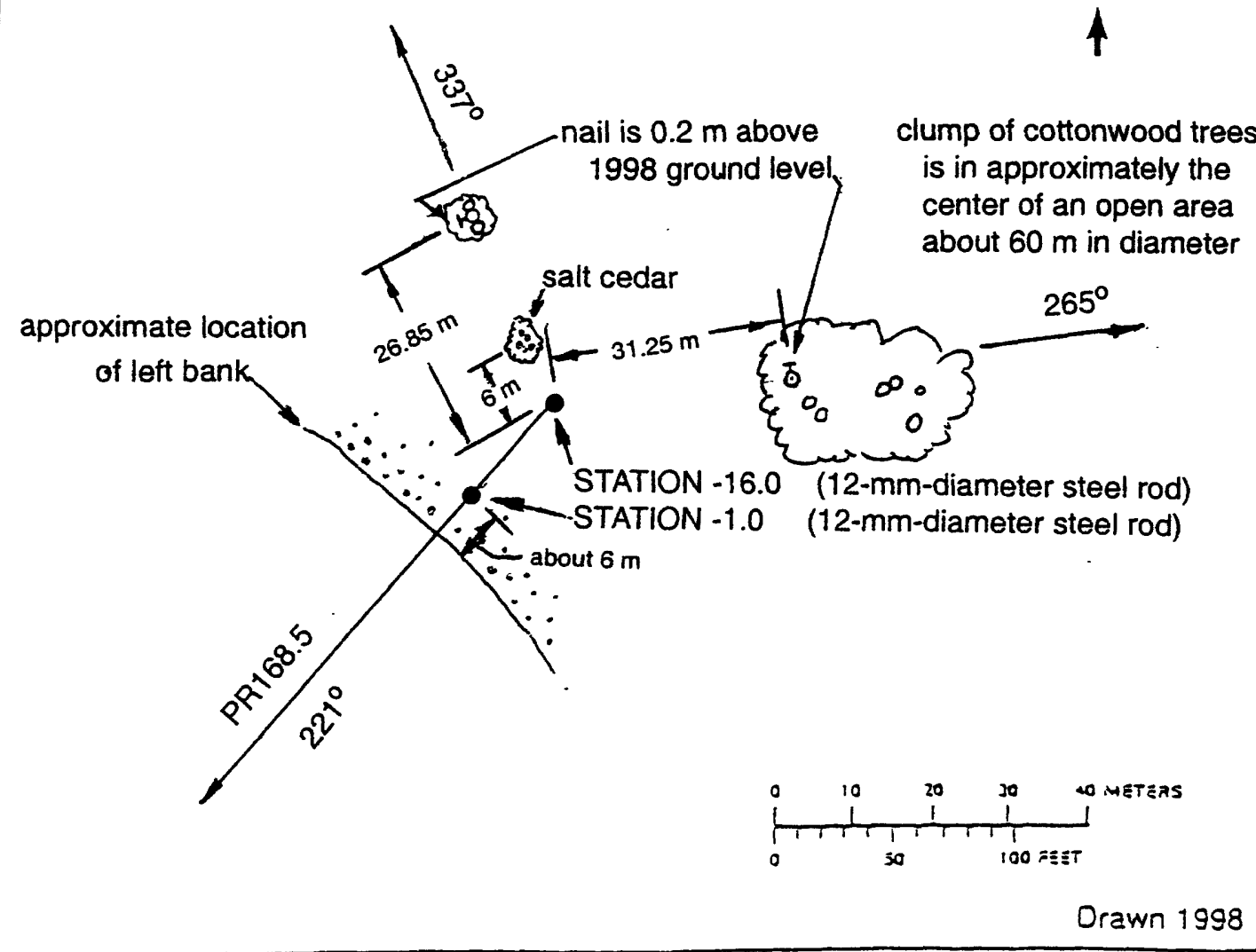

Figure 80. Upper: Location of cross section PR168.5 is shown in figure 8. Lower: Location of the reference monument on the left bank. $\mathrm{MN}$ is magnetic north. 


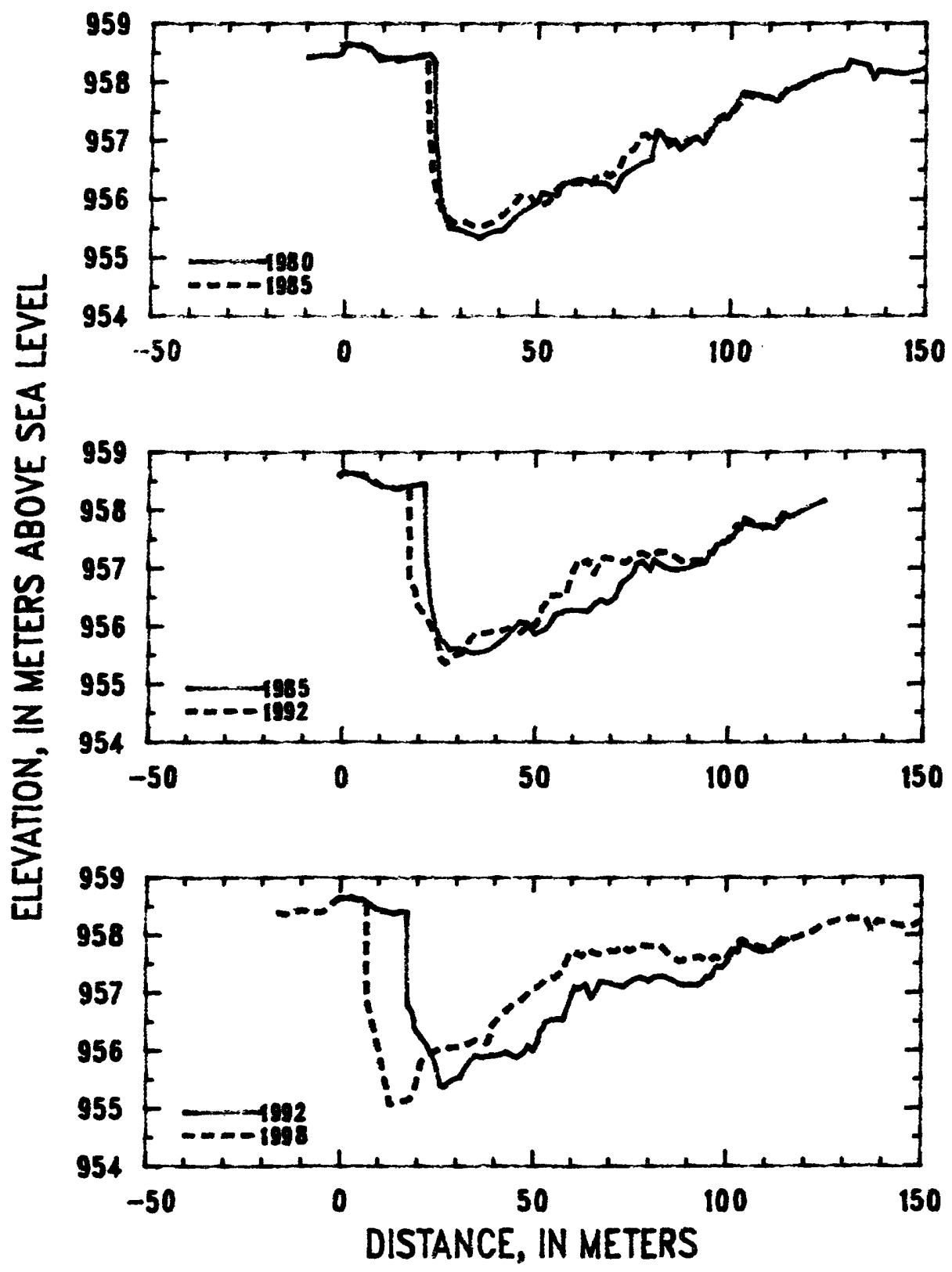

Figure 81. Profiles of cross section PR168.5 from 1980 to 1998. 
Table 29. Listing of horizontal stations and elevations for cross section PR168.5

[Sta., station, distance in meters from a reference pin on the left bank; Elev., elevation, in meters above sea level]

\begin{tabular}{|c|c|c|c|c|c|c|c|c|c|}
\hline \multirow{2}{*}{\multicolumn{2}{|c|}{$\begin{array}{c}1980 \\
20 \text { October }\end{array}$}} & \multicolumn{2}{|c|}{1980} & \multicolumn{2}{|c|}{1985} & \multicolumn{2}{|c|}{1985} & \multicolumn{2}{|c|}{1992} \\
\hline & & $20 c$ & tober & 70 & ober & 70 & ober & $1 \mathrm{Se}$ & mber \\
\hline Sta. & Elev. & Sta. & Elev. & Sta. & Elev. & Sta. & Elev. & Sta. & Elev. \\
\hline-10.0 & 958.43 & 82.0 & 957.14 & -1.0 & 958.61 & 85.0 & 956.98 & -1.0 & 958.60 \\
\hline-5.0 & 958.46 & 84.0 & 956.91 & 0.0 & 958.64 & 88.0 & 956.97 & 0.0 & 958.64 \\
\hline-2.5 & 958.46 & 85.5 & 957.00 & 3.0 & 958.63 & 91.0 & 957.05 & 2.0 & 958.64 \\
\hline-1.0 & 958.49 & 87.0 & 956.86 & 5.0 & 958.60 & 94.0 & 957.09 & 4.0 & 958.61 \\
\hline 0.0 & 958.65 & 91.0 & 957.06 & 8.0 & 958.46 & 97.0 & 957.40 & 6.0 & 958.61 \\
\hline 3.0 & 958.63 & 93.0 & 956.96 & 11.0 & 958.40 & 100.0 & 957.45 & 8.0 & 958.51 \\
\hline 6.0 & 958.60 & 97.0 & 957.39 & 14.0 & 958.37 & 102.0 & 957.62 & 10.0 & 958.42 \\
\hline 7.0 & 958.56 & 99.0 & 957.38 & 17.0 & 958.41 & 104.0 & 957.79 & 12.0 & 958.42 \\
\hline 9.0 & 958.38 & 103.3 & 957.83 & 20.0 & 958.45 & 107.0 & 957.73 & 14.0 & 958.37 \\
\hline 11.0 & 958.40 & 106.0 & 957.81 & 21.4 & 958.46 & 110.0 & 957.74 & 16.0 & 958.42 \\
\hline 13.0 & 958.43 & 110.0 & 957.73 & 21.6 & 957.67 & 112.0 & 957.69 & 17.3 & 958.40 \\
\hline 16.0 & 958.41 & 112.0 & 957.68 & 22.0 & 957.04 & 115.0 & 957.91 & 17.5 & 956.79 \\
\hline 19.0 & 958.43 & 115.0 & 957.88 & 22.5 & 956.81 & 116.0 & 957.87 & 18.0 & 956.73 \\
\hline 22.0 & 958.48 & 120.0 & 958.02 & 22.7 & 956.54 & 119.0 & 957.98 & 19.0 & 956.63 \\
\hline 23.3 & 958.36 & 125.0 & 958.17 & 23.5 & 956.21 & 122.0 & 958.08 & 19.4 & 956.37 \\
\hline 23.5 & 957.11 & 129.6 & 958.21 & 25.0 & 955.83 & 125.0 & 958.16 & 21.0 & 956.23 \\
\hline 24.0 & 956.79 & 130.7 & 958.36 & 28.0 & 955.60 & & & 22.4 & 956.10 \\
\hline 25.0 & 956.05 & 135.5 & 958.28 & 31.0 & 955.62 & & & 23.0 & 956.01 \\
\hline 26.0 & 955.73 & 137.0 & 958.06 & 34.0 & 955.53 & & & 24.6 & 955.80 \\
\hline 27.0 & 955.51 & 138.0 & 958.19 & 37.0 & 955.55 & & & 26.0 & 955.39 \\
\hline 29.0 & 955.50 & 140.0 & 958.19 & 40.0 & 955.67 & & & 27.0 & 955.37 \\
\hline 32.0 & 955.43 & 145.0 & 958.14 & 43.0 & 955.84 & & & 29.0 & 955.50 \\
\hline 35.0 & 955.33 & 150.0 & 958.23 & 46.0 & 956.07 & & & 31.0 & 955.54 \\
\hline 38.0 & 955.44 & 151.0 & 958.26 & 48.0 & 956.05 & & & 33.0 & 955.77 \\
\hline 41.0 & 955.46 & & & 50.0 & 955.85 & & & 35.0 & 955.91 \\
\hline 44.0 & 955.67 & & & 53.0 & 955.95 & & & 37.0 & 955.88 \\
\hline 47.0 & 955.82 & & & 55.3 & 956.21 & & & 39.0 & 955.91 \\
\hline 50.0 & 955.93 & & & 58.0 & 956.28 & & & 41.0 & 955.92 \\
\hline 51.0 & 956.10 & & & 61.0 & 956.29 & & & 43.0 & 955.97 \\
\hline 54.0 & 956.06 & & & 64.0 & 956.26 & & & 45.0 & 955.89 \\
\hline 55.3 & 956.07 & & & 65.3 & 956.38 & & & 46.0 & 955.88 \\
\hline 56.3 & 956.26 & & & 67.5 & 956.46 & . & & 48.0 & 955.99 \\
\hline 61.0 & 956.36 & & & 69.0 & 956.39 & & & 48.7 & 956.08 \\
\hline 65.0 & 956.29 & & & 70.0 & 956.45 & & & 49.9 & 956.00 \\
\hline 68.0 & 956.28 & & & 71.0 & 956.50 & & & 50.6 & 956.09 \\
\hline 70.0 & 956.15 & & & 72.5 & 956.75 & & & 51.0 & 956.14 \\
\hline 71.0 & 956.25 & & & 75.5 & 956.90 & & & 51.7 & 956.33 \\
\hline 72.0 & 956.41 & & & 76.4 & 957.08 & & & 52.5 & 956.37 \\
\hline 74.0 & 956.50 & & & 78.0 & 957.12 & & & 53.0 & 956.48 \\
\hline 77.0 & 956.63 & & & 80.0 & 956.97 & & & 55.0 & 956.54 \\
\hline 79.4 & 956.68 & & & 81.0 & 957.15 & & & 57.0 & 956.55 \\
\hline 81.0 & 957.18 & & & 83.0 & 957.04 & & & 58.0 & 956.54 \\
\hline
\end{tabular}


Table 29. (Continued) Listing of horizontal stations and elevations for cross section PR168.5

[Sta., station, distance in meters from a reference pin on the left bank; Elev., elevation, in meters above sea level]

\begin{tabular}{|c|c|c|c|c|c|}
\hline \multirow{2}{*}{\multicolumn{2}{|c|}{$\begin{array}{c}1992 \\
1 \text { September }\end{array}$}} & \multirow{2}{*}{\multicolumn{2}{|c|}{$\begin{array}{c}1998 \\
2 \text { October }\end{array}$}} & \multirow{2}{*}{\multicolumn{2}{|c|}{$\begin{array}{c}1998 \\
2 \text { October }\end{array}$}} \\
\hline & & & & & \\
\hline Sta. & Elev. & Sta. & Elev. & Sta. & Elev. \\
\hline 58.7 & 956.71 & -16.0 & 958.38 & 75.0 & 957.71 \\
\hline 59.6 & 956.86 & -13.0 & 958.35 & 78.0 & 957.83 \\
\hline 60.0 & 956.98 & -10.0 & 958.43 & 81.0 & 957.81 \\
\hline 60.7 & 957.04 & -7.0 & 958.39 & 83.0 & 957.82 \\
\hline 61.0 & 957.11 & -4.0 & 958.41 & 85.0 & 957.68 \\
\hline 62.0 & 957.07 & -1.0 & 958.61 & 88.0 & 957.53 \\
\hline 63.9 & 957.15 & 0.0 & 958.65 & 91.0 & 957.58 \\
\hline 65.3 & 956.91 & 2.0 & 958.66 & 93.0 & 957.63 \\
\hline 67.0 & 957.08 & 4.0 & 958.62 & 95.4 & 957.52 \\
\hline 67.6 & 957.19 & 6.9 & 958.57 & 97.0 & 957.62 \\
\hline 69.0 & 957.19 & 7.0 & 956.89 & 99.7 & 957.54 \\
\hline 71.0 & 957.15 & 9.4 & 956.20 & 102.0 & 957.79 \\
\hline 73.0 & 957.12 & 12.0 & 955.40 & 104.0 & 957.91 \\
\hline 73.6 & 957.10 & 13.0 & 955.07 & 107.0 & 957.80 \\
\hline 75.0 & 957.18 & 18.0 & 955.15 & 110.0 & 957.77 \\
\hline 76.0 & 957.23 & 19.0 & 955.29 & 113.0 & 957.82 \\
\hline 78.0 & 957.27 & 21.0 & 955.80 & 116.0 & 957.89 \\
\hline 80.0 & 957.20 & 22.5 & 955.93 & 119.0 & 957.98 \\
\hline 82.0 & 957.29 & 24.5 & 955.98 & 121.1 & 958.01 \\
\hline 84.0 & 957.29 & 26.0 & 956.03 & 125.0 & 958.16 \\
\hline 86.0 & 957.22 & 29.0 & 956.05 & 128.0 & 958.24 \\
\hline 88.0 & 957.13 & 32.0 & 956.08 & 131.0 & 958.28 \\
\hline 91.0 & 957.14 & 35.0 & 956.17 & 134.0 & 958.27 \\
\hline 93.0 & 957.13 & 38.0 & 956.12 & 136.0 & 958.28 \\
\hline 95.0 & 957.27 & 38.8 & 956.21 & 137.4 & 958.06 \\
\hline 96.0 & 957.26 & 39.0 & 956.41 & 139.0 & 958.25 \\
\hline 97.5 & 957.45 & 41.0 & 956.55 & 142.0 & 958.19 \\
\hline 99.0 & 957.44 & 44.0 & 956.74 & 145.0 & 958.15 \\
\hline 100.0 & 957.50 & 47.0 & 956.88 & 148.0 & 958.15 \\
\hline 102.0 & 957.74 & 50.0 & 957.05 & 151.0 & 958.27 \\
\hline 103.0 & 957.69 & 53.0 & 957.20 & & \\
\hline 103.5 & 957.85 & 56.0 & 957.28 & & \\
\hline 105.0 & 957.84 & 58.0 & 957.40 & & \\
\hline 107.0 & 957.75 & 59.5 & 957.68 & & \\
\hline 109.0 & 957.70 & 61.0 & 957.70 & & \\
\hline 112.0 & 957.73 & 63.0 & 957.63 & & \\
\hline 114.0 & 957.94 & 64.0 & 957.74 & & \\
\hline 116.0 & 957.90 & 66.0 & 957.70 & & \\
\hline & & 68.0 & 957.65 & & \\
\hline & & 70.0 & 957.72 & & \\
\hline & & 72.0 & 957.73 & & \\
\hline & & 73.0 & 957.82 & & \\
\hline
\end{tabular}




\section{Description of Cross Section PR169.2}

Location: Township 6 South/Range 50 East--section 22 and 27

U. S. Geological Survey quadrangle (1:24,000): Lonesome Peak

Landowners--left bank: EB Ranch

--right bank: Daily Ranch

Access: Left bank

Permission from: EB Ranch

Distance from Moorhead Gaging Station: 54.0 kilometers

Azimuth of Section (degrees magnetic): 114

\section{Reference Monuments}

[Monuments at stations 151.0 and 154.6 were closest to leveling instrument ]

\begin{tabular}{|c|c|c|c|c|c|c|}
\hline \multirow[b]{2}{*}{ Description } & \multirow[b]{2}{*}{$\begin{array}{c}\text { Station } \\
\text { (m) }\end{array}$} & \multicolumn{2}{|c|}{ GPS-NADB3 (1992) } & \multicolumn{2}{|c|}{ Measurement } & \multirow{2}{*}{$\begin{array}{l}\text { Elevation } \\
\text { (NGVD1929) } \\
\text { (m) }\end{array}$} \\
\hline & & Latitude & Longitude & $\begin{array}{l}\text { Standard } \\
\text { deviation } \\
(m)\end{array}$ & $\begin{array}{l}\text { Horizontal } \\
\text { precision } \\
\text { (m) }\end{array}$ & \\
\hline $\begin{array}{l}\text { 1/2-inch-rebar; } 0.17 \text { meter above } 1998 \text { ground } \\
\text { level }\end{array}$ & -1.0 & $45^{\circ} 17^{\prime} 24.67^{\prime \prime}$ & $105^{\circ} 35^{\prime} 23.82^{\prime \prime}$ & 0.466 & 0.495 & 957.69 \\
\hline $\begin{array}{l}\text { 1/2-inch-rebar; } 0.13 \text { meter above } 1998 \text { ground } \\
\text { level; } 0.25 \text { meter downriver from a cotton- } \\
\text { wood tree ( } 0.50 \text { meter diameter at breast } \\
\text { height) with a nail } 1.6 \text { meter above } 1998 \\
\text { ground level }\end{array}$ & 151.0 & & & & & 958.05 \\
\hline $\begin{array}{l}\text { 1/2-inch-rebar; } 0.15 \text { meter above } 1998 \text { ground } \\
\text { level; under } 2 \text {-strand, barbed-wire fence } \\
\text { with tree posts }\end{array}$ & 154.5 & $45^{\circ} 17^{\prime} 21.59^{\prime \prime}$ & $105^{\circ} 35^{\prime} 18.17^{\prime \prime}$ & 0.534 & 0.924 & 958.96 \\
\hline
\end{tabular}


See Figure 8 for location

of cross section

PR169.2

MN

$\uparrow$

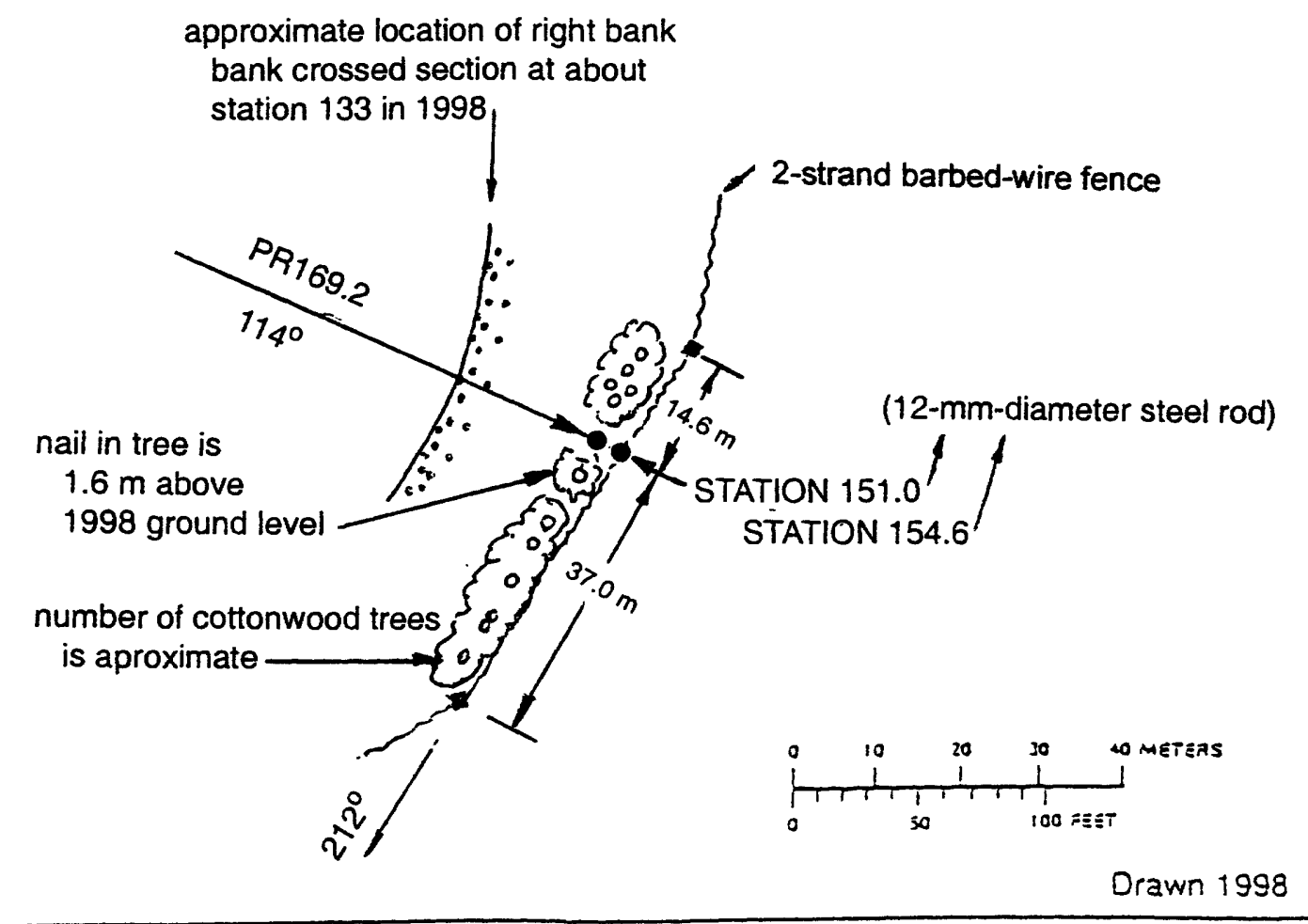

Figure 82. Upper: Location of cross section PR169.2 is shown in figure 8. Lower: Location of the reference monuments on the right bank. $\mathrm{MN}$ is magnetic north. 


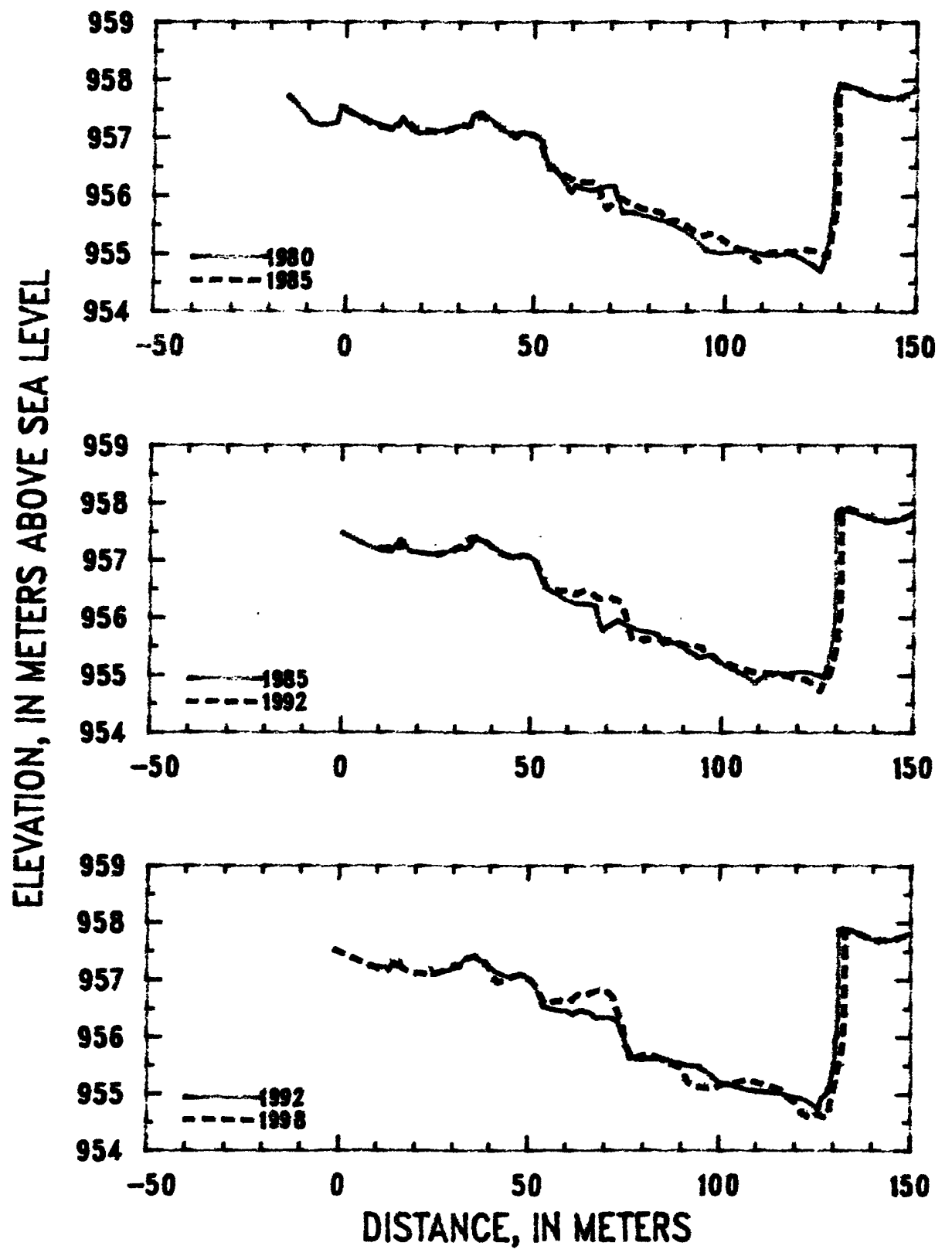

Figure 83. Profiles of cross section PR169.2 from 1980 to 1998. 
Table 30. Listing of horizontal stations and elevations for cross section PR169.2

[Sta., station, distance in meters from a reference pin on the left bank; Elev., elevation, in meters above sea level]

\begin{tabular}{|c|c|c|c|c|c|c|c|c|c|}
\hline \multirow{2}{*}{\multicolumn{2}{|c|}{$\begin{array}{c}1980 \\
21 \text { October }\end{array}$}} & \multirow{2}{*}{\multicolumn{2}{|c|}{$\begin{array}{c}1990 \\
21 \text { October }\end{array}$}} & \multirow{2}{*}{\multicolumn{2}{|c|}{$\begin{array}{c}1985 \\
5 \text { October }\end{array}$}} & \multirow{2}{*}{\multicolumn{2}{|c|}{$\begin{array}{c}1985 \\
5 \text { October }\end{array}$}} & \multirow{2}{*}{\multicolumn{2}{|c|}{$\begin{array}{c}1992 \\
2 \text { September }\end{array}$}} \\
\hline & & & & & & & & & \\
\hline Sta. & Elev. & Sta. & Elev. & Sta. & Elev. & Sta. & Elev. & Sta. & Elev. \\
\hline-15.0 & 957.73 & 110.0 & 954.98 & 0.0 & 957.49 & 106.0 & 955.01 & 10.0 & 957.22 \\
\hline-11.0 & 957.46 & 114.0 & 954.94 & 4.0 & 957.36 & 109.0 & 954.85 & 16.0 & 957.24 \\
\hline-9.0 & 957.29 & 117.0 & 955.00 & 7.0 & 957.26 & 112.0 & 955.01 & 20.0 & 958.12 \\
\hline-6.0 & 957.21 & 120.0 & 954.92 & 10.0 & 957.19 & 115.0 & 955.03 & 25.0 & 957.09 \\
\hline-2.0 & 957.26 & 123.0 & 954.80 & 13.0 & 957.15 & 118.0 & 955.02 & 28.0 & 957.16 \\
\hline-1.0 & 957.55 & 125.0 & 954.68 & 15.4 & 957.36 & 121.0 & 955.07 & 31.0 & 957.21 \\
\hline 0.0 & 957.51 & 126.0 & 954.80 & 18.0 & 957.16 & 124.0 & 955.04 & 34.0 & 957.40 \\
\hline 3.0 & 957.38 & 127.7 & 955.31 & 21.0 & 957.13 & 127.0 & 954.94 & 36.0 & 957.42 \\
\hline 6.0 & 957.30 & 128.6 & 956.05 & 24.0 & 957.10 & 128.7 & 955.48 & 38.0 & 957.32 \\
\hline 9.0 & 957.25 & 128.7 & 957.70 & 27.0 & 957.13 & 129.7 & 955.93 & 41.0 & 957.17 \\
\hline 12.0 & 957.13 & 130.0 & 957.92 & 30.0 & 957.23 & 129.8 & 957.81 & 43.0 & 957.10 \\
\hline 15.0 & 957.35 & 134.0 & 957.87 & 33.0 & 957.21 & 131.0 & 957.90 & 46.0 & 957.04 \\
\hline 17.0 & 957.17 & 138.0 & 957.74 & 36.0 & 957.39 & 134.0 & 957.84 & 48.0 & 957.10 \\
\hline 19.4 & 957.07 & 142.0 & 957.68 & 39.0 & 957.28 & 137.0 & 957.77 & 50.0 & 957.05 \\
\hline 23.0 & 957.10 & 146.0 & 957.69 & 42.0 & 957.14 & 140.0 & 957.70 & 51.5 & 956.95 \\
\hline 27.0 & 957.14 & 150.0 & 957.83 & 45.0 & 957.04 & 143.0 & 957.68 & 54.0 & 956.54 \\
\hline 30.0 & 957.20 & 151.0 & 957.89 & 47.0 & 957.09 & 146.0 & 957.70 & 56.0 & 956.49 \\
\hline 33.0 & 957.23 & 154.6 & 958.82 & 50.0 & 957.05 & 149.0 & 957.79 & 58.0 & 956.46 \\
\hline 34.0 & 957.39 & & & 51.5 & 956.96 & 151.0 & 957.91 & 60.0 & 956.47 \\
\hline 36.0 & 957.44 & & & 53.0 & 956.63 & 154.6 & 958.82 & 62.0 & 956.39 \\
\hline 39.0 & 957.30 & & & 54.0 & 956.51 & & & 64.0 & 956.47 \\
\hline 42.0 & 957.13 & & & 56.0 & 956.44 & & & 66.0 & 956.42 \\
\hline 45.0 & 957.00 & & & 59.0 & 956.32 & & & 67.0 & 956.36 \\
\hline 47.0 & 957.10 & & & 62.0 & 956.23 & & & 68.0 & 956.32 \\
\hline 50.0 & 957.04 & & & 65.0 & 956.24 & & & 70.0 & 956.35 \\
\hline 52.0 & 956.93 & & & 66.9 & 956.20 & & & 72.0 & 956.32 \\
\hline 54.0 & 956.44 & & & 68.1 & 955.86 & & & 73.3 & 956.27 \\
\hline 55.0 & 956.49 & & & 69.0 & 955.77 & & & 74.5 & 956.06 \\
\hline 58.0 & 956.25 & & & 70.0 & 955.83 & & & 76.3 & 955.63 \\
\hline 60.0 & 956.05 & & & 72.6 & 955.96 & & & 78.4 & 955.64 \\
\hline 61.0 & 956.17 & & & 75.0 & 955.86 & & & 79.5 & 955.59 \\
\hline 65.0 & 956.08 & & & 78.0 & 955.77 & & & 80.1 & 955.62 \\
\hline 70.0 & 956.17 & & & 81.0 & 955.75 & & & 82.0 & 955.62 \\
\hline 71.0 & 956.15 & & & 83.3 & 955.70 & & & 84.0 & 955.62 \\
\hline 73.0 & 955.71 & & & 84.3 & 955.55 & & & 86.0 & 955.62 \\
\hline 75.0 & 955.71 & & & 87.0 & 955.58 & & & 88.0 & 955.55 \\
\hline 80.0 & 955.63 & & & 90.0 & 955.48 & & & 89.6 & 955.55 \\
\hline 85.0 & 955.55 & & & 91.0 & 955.42 & & & 91.5 & 955.48 \\
\hline 90.0 & 955.38 & & & 94.0 & 955.30 & & & 93.0 & 955.49 \\
\hline 95.0 & 955.05 & & & 97.0 & 955.35 & & & 94.7 & 955.48 \\
\hline 100.0 & 954.99 & & & 100.0 & 955.20 & & & 95.0 & 955.43 \\
\hline 105.0 & 955.07 & & & 103.0 & 955.09 & & & 97.0 & 955.36 \\
\hline
\end{tabular}


Table 30. (Continued) Listing of horizontal stations and elevations for cross section PR169.2

[Sta., station, distance in meters from a reference pin on the left bank; Elev., elevation, in meters above sea level]

\begin{tabular}{|c|c|c|c|c|c|}
\hline \multirow{2}{*}{\multicolumn{2}{|c|}{$\begin{array}{c}1992 \\
2 \text { September }\end{array}$}} & \multicolumn{2}{|c|}{1998} & \multicolumn{2}{|c|}{1998} \\
\hline & & \multicolumn{2}{|c|}{2 October } & \multicolumn{2}{|c|}{2 October } \\
\hline Sta. & Elev. & Sta. & Elev. & Sta. & Elev. \\
\hline 98.0 & 955.30 & -1.0 & 957.52 & 95.0 & 955.11 \\
\hline 100.0 & 955.19 & 0.0 & 957.50 & 98.0 & 955.08 \\
\hline 102.0 & 955.18 & 2.0 & 957.42 & 101.0 & 955.17 \\
\hline 104.0 & 955.13 & 4.0 & 957.38 & 104.0 & 955.18 \\
\hline 106.0 & 955.10 & 7.0 & 957.27 & 107.0 & 955.24 \\
\hline 108.0 & 955.06 & 10.0 & 957.21 & 110.0 & 955.21 \\
\hline 110.0 & 955.04 & 13.0 & 957.17 & 113.0 & 955.13 \\
\hline 112.0 & 955.02 & 15.3 & 957.36 & 116.0 & 955.08 \\
\hline 114.0 & 955.01 & 17.0 & 957.19 & 118.0 & 954.95 \\
\hline 116.0 & 955.01 & 19.0 & 957.13 & 120.0 & 954.85 \\
\hline 118.0 & 954.96 & 22.0 & 957.11 & 122.0 & 954.64 \\
\hline 120.0 & 954.93 & 25.0 & 957.10 & 124.0 & 954.56 \\
\hline 122.0 & 954.92 & 28.0 & 957.17 & 126.0 & 954.64 \\
\hline 124.0 & 954.84 & 30.0 & 957.25 & 128.0 & 954.57 \\
\hline 126.1 & 954.71 & 32.0 & 957.23 & 129.0 & 954.68 \\
\hline 127.0 & 954.93 & 34.0 & 957.37 & 130.9 & 955.38 \\
\hline 128.5 & 955.00 & 36.0 & 957.42 & 132.5 & 955.90 \\
\hline 129.6 & 955.31 & 39.0 & 957.29 & 132.9 & 956.50 \\
\hline 130.3 & 955.60 & 42.0 & 956.95 & 133.0 & 957.84 \\
\hline 130.5 & 955.94 & 45.0 & 957.03 & 135.0 & 957.84 \\
\hline 130.9 & 955.99 & 48.0 & 957.09 & 137.6 & 957.77 \\
\hline 131.2 & 956.63 & 51.0 & 956.97 & 140.0 & 957.68 \\
\hline 131.3 & 957.86 & 52.0 & 956.88 & 143.0 & 957.69 \\
\hline 132.0 & 957.89 & 54.5 & 956.57 & 146.0 & 957.73 \\
\hline 133.0 & 957.90 & 58.0 & 956.64 & 149.0 & 957.78 \\
\hline 135.0 & 957.86 & 61.0 & 956.62 & 151.0 & 957.91 \\
\hline 138.0 & 957.75 & 63.0 & 956.74 & 154.6 & 958.83 \\
\hline 141.0 & 957.69 & 65.0 & 956.73 & & \\
\hline 144.0 & 957.68 & 68.0 & 956.81 & & \\
\hline 147.0 & 957.74 & 70.0 & 956.82 & & \\
\hline 150.0 & 957.82 & 72.0 & 956.70 & & \\
\hline & & 74.4 & 956.22 & & \\
\hline & & 75.4 & 955.84 & & \\
\hline & & 76.0 & 955.77 & & \\
\hline & & 77.3 & 955.59 & & \\
\hline & & 79.0 & 955.66 & & \\
\hline & & 81.0 & 955.69 & & \\
\hline & & 84.0 & 955.64 & & \\
\hline & & 87.0 & 955.54 & & \\
\hline & & 90.1 & 955.45 & & \\
\hline & & 90.4 & 955.38 & & \\
\hline & & 92.0 & 955.16 & & \\
\hline
\end{tabular}




\section{Description of Cross Section PR169.8}

Location: Township 6 South/Range 50 East--section 22

U. S. Geological Survey quadrangle (1:24,000): Lonesome Peak

Landowners--left bank: E. B. Ranch

--right bank: E. B. Ranch

Access: Left bank

Permission from: E. B. Ranch

Distance from Moorhead Gaging Station: 54.6 kilometers

Azimuth of Section (degrees magnetic): 127

\section{Reference Monuments}

[Monument at station 0.0 was closest to leveling instrument]

\begin{tabular}{|c|c|c|c|c|c|c|}
\hline \multirow[b]{2}{*}{ Description } & \multirow[b]{2}{*}{$\begin{array}{c}\text { Station } \\
\text { (m) }\end{array}$} & \multicolumn{2}{|c|}{ GPS-NAD83 (1992) } & \multicolumn{2}{|c|}{ Measurement } & \multirow{2}{*}{$\begin{array}{l}\text { Elevation } \\
\text { (NGVD1929) } \\
\text { (m) }\end{array}$} \\
\hline & & Latitude & Longitude & $\begin{array}{l}\text { Standard } \\
\text { deviation } \\
(\mathbf{m})\end{array}$ & $\begin{array}{l}\text { Horizontal } \\
\text { precision } \\
\text { (m) }\end{array}$ & \\
\hline $\begin{array}{l}\text { 1/2-inch-rebar; } 0.20 \text { meter above } 1998 \text { ground } \\
\text { level; } 0.05 \text { meter riverward of an orange } \\
\text { metal fence post }\end{array}$ & 0.0 & $45^{\circ} 17^{\prime} 41.85^{\prime \prime}$ & $105^{\circ} 35^{\prime} 16.46^{\prime \prime}$ & 0.268 & 0.779 & 958.45 \\
\hline $\begin{array}{l}\text { 1/2-inch-rebar; } 0.13 \text { meter above } 1998 \text { ground } \\
\text { level; in a clearing landward of first group } \\
\text { of } 15-20 \text { meter high cottonwood trees }\end{array}$ & 151.0 & $45^{\circ} 17^{\prime} 38.07^{\prime \prime}$ & $105^{\circ} 35^{\prime} 12.05^{\prime \prime}$ & 0.390 & 0.438 & 957.42 \\
\hline
\end{tabular}


See Figure 8 for location

of cross section

PR169.8

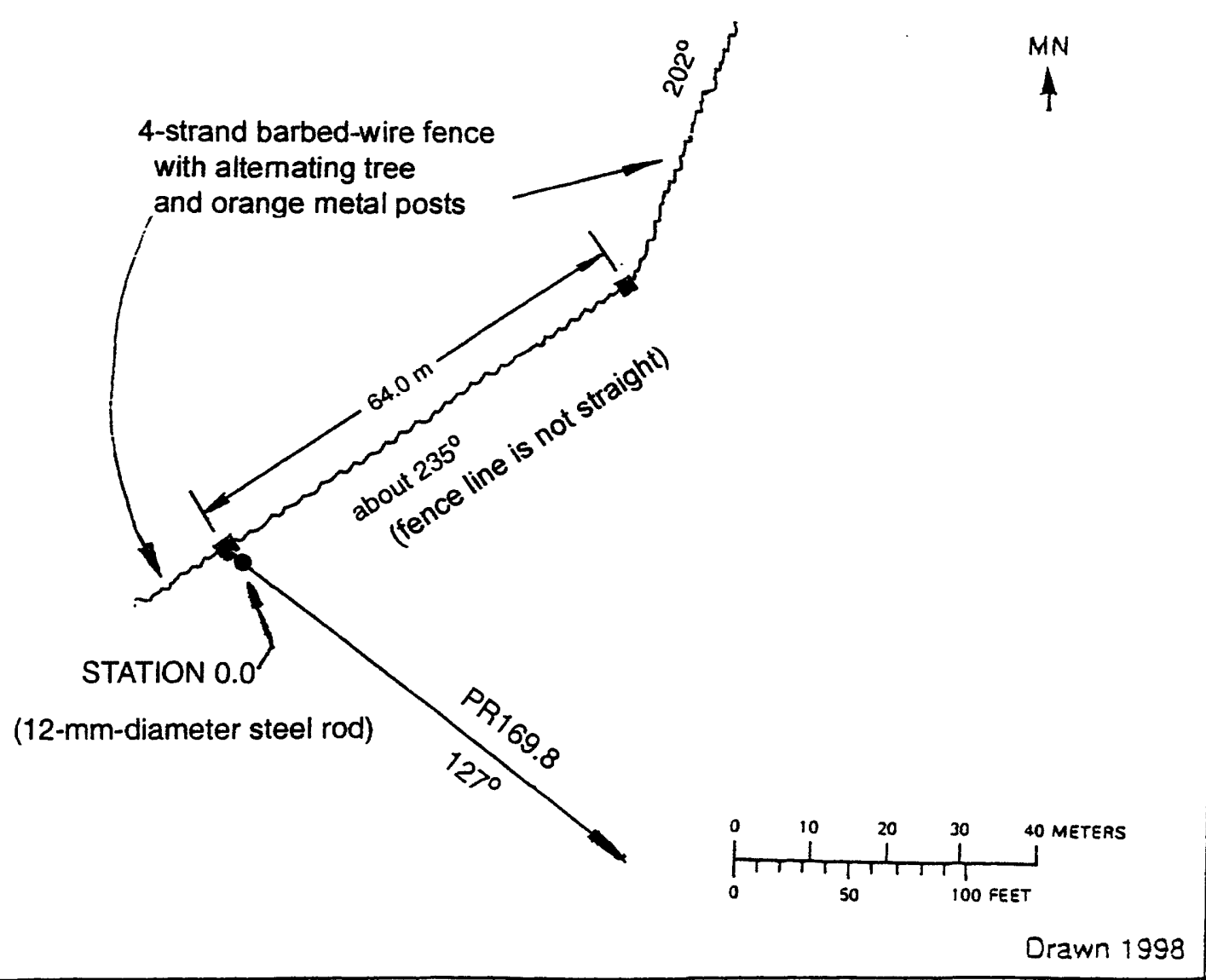

Figure 84. Upper: Location of cross section PR169.8 is shown in figure 8. Lower: Location of the reference monuments on the left bank. $\mathrm{MN}$ is magnetic north. 


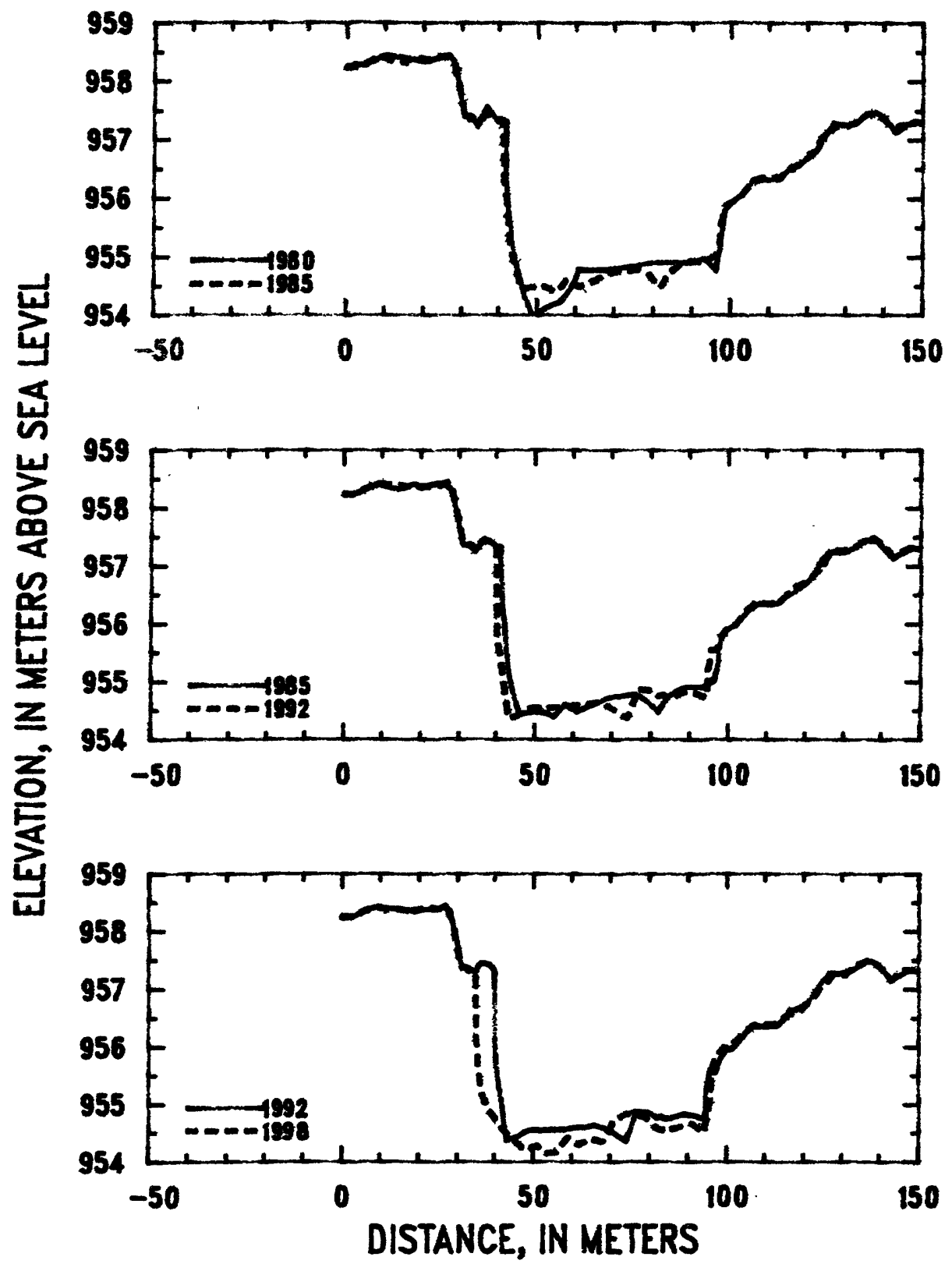

Figure 85. Profiles of cross section PR169.8 from 1980 to 1998. 
Table 31. Listing of horizontal stations and elevations for cross section PR169.8

[Sta., station, distance in meters from a reference pin on the left bank; Elev., elevation, in meters above sea level]

\begin{tabular}{|c|c|c|c|c|c|c|c|c|c|}
\hline \multirow{2}{*}{\multicolumn{2}{|c|}{$\begin{array}{c}1980 \\
21 \text { October }\end{array}$}} & & \multicolumn{2}{|c|}{1985} & \multicolumn{2}{|c|}{1985} & \multicolumn{2}{|c|}{1992} \\
\hline & & $21 \mathrm{C}$ & tober & & ober & & ber & $2 \mathrm{Se}_{1}$ & mber \\
\hline Sta. & Elev. & Sta. & Elev. & Sta. & Elev. & Sta. & Elev. & Sta. & Elev. \\
\hline 0.0 & 958.24 & 120.0 & 956.68 & 0.0 & 958.25 & 97.2 & 955.23 & 0.0 & 958.24 \\
\hline 2.0 & 958.28 & 122.8 & 956.82 & 3.0 & 958.24 & 97.4 & 955.57 & 3.0 & 958.24 \\
\hline 5.0 & 958.28 & 124.0 & 957.06 & 6.0 & 958.34 & 99.0 & 955.88 & 6.0 & 958.35 \\
\hline 10.0 & 958.45 & 126.0 & 957.16 & 9.0 & 958.43 & 102.0 & 955.98 & 9.0 & 958.43 \\
\hline 15.0 & 958.42 & 127.0 & 957.29 & 12.0 & 958.34 & 105.0 & 956.22 & 12.0 & 958.40 \\
\hline 20.0 & 958.34 & 130.0 & 957.25 & 15.0 & 958.33 & 108.0 & 956.35 & 15.0 & 958.39 \\
\hline 25.0 & 958.42 & 133.0 & 957.29 & 18.0 & 958.42 & 111.0 & 956.35 & 17.0 & 958.37 \\
\hline 26.6 & 958.46 & 135.0 & 957.42 & 21.0 & 958.38 & 113.0 & 956.36 & 20.0 & 958.37 \\
\hline 28.2 & 958.32 & 138.0 & 957.48 & 24.0 & 958.43 & 115.0 & 956.47 & 25.0 & 958.39 \\
\hline 30.9 & 957.40 & 140.0 & 957.39 & 26.0 & 958.43 & 118.0 & 956.57 & 26.8 & 958.44 \\
\hline 32.7 & 957.40 & 142.8 & 957.14 & 28.0 & 958.32 & $121.0^{\circ}$ & 956.74 & 28.0 & 958.34 \\
\hline 34.3 & 957.23 & 144.0 & 957.25 & 29.0 & 958.04 & 122.6 & 956.85 & 30.0 & 957.76 \\
\hline 37.0 & 957.56 & 150.0 & 957.32 & 30.0 & 957.73 & 123.7 & 957.04 & 31.2 & 957.38 \\
\hline 40.0 & 957.32 & 151.0 & 957.30 & 31.0 & 957.37 & 125.0 & 957.04 & 33.0 & 957.37 \\
\hline 42.0 & 957.31 & & & 33.0 & 957.35 & 127.0 & 957.25 & 35.0 & 957.28 \\
\hline 42.0 & 956.31 & & & 34.6 & 957.25 & 130.0 & 957.23 & 36.0 & 957.41 \\
\hline 42.6 & 955.82 & & & 36.3 & 957.42 & 133.0 & 957.31 & 37.0 & 957.46 \\
\hline 44.0 & 955.09 & & & 38.0 & 957.44 & 136.0 & 957.45 & 39.0 & 957.42 \\
\hline 45.0 & 954.78 & & & 40.0 & 957.34 & 139.0 & 957.43 & 40.1 & 957.32 \\
\hline 47.1 & 954.26 & & & 41.1 & 957.33 & 142.0 & 957.20 & 40.2 & 955.79 \\
\hline 49.0 & 953.96 & & & 41.6 & 956.22 & 143.0 & 957.13 & 41.5 & 955.21 \\
\hline 53.0 & 954.14 & & & 42.2 & 955.98 & 145.0 & 957.21 & 42.3 & 954.78 \\
\hline 56.0 & 954.21 & & & 43.0 & 955.28 & 148.0 & 957.32 & 43.3 & 954.37 \\
\hline 59.0 & 954.43 & & & 43.8 & 954.99 & 151.0 & 957.27 & 46.0 & 954.43 \\
\hline 61.0 & 954.78 & & & 46.0 & 954.45 & & & 48.0 & 954.53 \\
\hline 65.0 & 954.78 & & & 49.0 & 954.47 & & & 50.0 & 954.56 \\
\hline 68.0 & 954.78 & & & 52.0 & 954.48 & & & 52.0 & 954.54 \\
\hline 75.0 & 954.84 & & & 55.0 & 954.39 & & & 54.0 & 954.55 \\
\hline 80.0 & 954.90 & & & 58.0 & 954.61 & & & 56.0 & 954.56 \\
\hline 85.0 & 954.91 & & & 61.0 & 954.48 & & & 58.0 & 954.55 \\
\hline 90.0 & 954.89 & & & 64.0 & 954.57 & & & 60.0 & 954.59 \\
\hline 93.0 & 954.97 & & & 67.0 & 954.66 & & & 62.0 & 954.60 \\
\hline 96.2 & 954.77 & & & 70.0 & 954.75 & . & & 64.0 & 954.61 \\
\hline 97.2 & 955.10 & & & 73.0 & 954.76 & & & 66.0 & 954.64 \\
\hline 98.4 & 955.78 & & & 76.0 & 954.79 & & & 68.0 & 954.60 \\
\hline 100.0 & 955.91 & & & 79.0 & 954.68 & & & 70.0 & 954.54 \\
\hline 103.0 & 956.05 & & & 82.0 & 954.47 & & & 72.0 & 954.45 \\
\hline 106.0 & 956.32 & & & 84.0 & 954.72 & & & 74.0 & 954.37 \\
\hline 110.0 & 956.31 & & & 87.0 & 954.86 & & & 76.3 & 954.77 \\
\hline 113.0 & 956.33 & & & 90.0 & 954.93 & & & 76.4 & 954.87 \\
\hline 116.0 & 956.55 & & & 93.0 & 954.90 & & & 79.0 & 954.87 \\
\hline 118.0 & 956.57 & & & 96.0 & 955.00 & & & 80.5 & 954.83 \\
\hline
\end{tabular}


Table 31. (Continued) Listing of horizontal stations and elevations for cross section PR169.8

[Sta., station, distance in meters from a reference pin on the left bank; Elev., elevation, in meters above sea level]

\begin{tabular}{|c|c|c|c|c|c|}
\hline \multirow{2}{*}{\multicolumn{2}{|c|}{$\begin{array}{c}1992 \\
2 \text { September }\end{array}$}} & \multirow{2}{*}{\multicolumn{2}{|c|}{$\begin{array}{c}1998 \\
1 \text { October }\end{array}$}} & \multirow{2}{*}{\multicolumn{2}{|c|}{$\begin{array}{c}1998 \\
1 \text { October }\end{array}$}} \\
\hline & & & & & \\
\hline Sta. & Elev. & Sta. & Elev. & Sta. & Elev. \\
\hline 82.0 & 954.75 & 0.0 & 958.25 & 95.6 & 955.17 \\
\hline 83.4 & 954.79 & 3.0 & 958.25 & 96.1 & 955.62 \\
\hline 84.0 & 954.75 & 6.0 & 958.36 & 97.0 & 955.81 \\
\hline 86.0 & 954.75 & 9.0 & 958.42 & 99.0 & 955.98 \\
\hline 88.0 & 954.82 & 12.0 & 958.39 & 101.0 & 956.00 \\
\hline 90.0 & 954.82 & 15.0 & 958.38 & 104.0 & 956.21 \\
\hline 92.0 & 954.79 & 17.0 & 958.36 & 107.0 & 956.38 \\
\hline 94.0 & 954.74 & 18.0 & 958.44 & 110.0 & 956.39 \\
\hline 94.5 & 954.74 & 19.0 & 958.35 & 113.0 & 956.41 \\
\hline 94.6 & 955.21 & 22.0 & 958.42 & 116.0 & 956.57 \\
\hline 95.6 & 955.55 & 25.0 & 958.39 & 119.0 & 956.63 \\
\hline 97.0 & 955.55 & 27.0 & 958.41 & 122.0 & 956.80 \\
\hline 98.0 & 955.77 & 28.0 & 958.34 & 125.0 & 957.06 \\
\hline 100.0 & 955.95 & 29.0 & 958.07 & 128.0 & 957.26 \\
\hline 101.5 & 955.96 & 30.9 & 957.38 & 131.0 & 957.25 \\
\hline 103.0 & 956.07 & 32.0 & 957.38 & 134.0 & 957.42 \\
\hline 105.0 & 956.25 & 34.0 & 957.26 & 137.0 & 957.51 \\
\hline 107.0 & 956.37 & 35.0 & 957.24 & 140.0 & 957.40 \\
\hline 110.0 & 956.35 & 35.2 & 956.13 & 143.0 & 957.15 \\
\hline 113.0 & 956.37 & 36.3 & 955.28 & 146.0 & 957.33 \\
\hline 115.5 & 956.51 & 38.0 & 954.93 & 149.0 & 957.32 \\
\hline 116.4 & 956.62 & 39.4 & 954.82 & 151.0 & 957.30 \\
\hline 118.0 & 956.65 & 42.0 & 954.56 & & \\
\hline 120.0 & 956.71 & 45.0 & 954.37 & & \\
\hline 122.5 & 956.85 & 48.0 & 954.21 & & \\
\hline 124.0 & 957.06 & 51.0 & 954.28 & & \\
\hline 127.0 & 957.28 & 53.0 & 954.15 & & \\
\hline 130.0 & 957.26 & 56.0 & 954.16 & & \\
\hline 133.0 & 957.32 & 59.0 & 954.42 & & \\
\hline 136.0 & 957.46 & 62.0 & 954.29 & & \\
\hline 138.0 & 957.49 & 65.0 & 954.41 & & \\
\hline 140.0 & 957.40 & 68.0 & 954.35 & & \\
\hline 143.0 & 957.15 & 71.0 & 954.72 & & \\
\hline 145.0 & 957.24 & 74.0 & 954.84 & & \\
\hline 148.0 & 957.34 & 76.0 & 954.83 & & \\
\hline 151.0 & 957.29 & 79.0 & 954.77 & & \\
\hline & & 82.0 & 954.57 & & \\
\hline & & 85.0 & 954.54 & & \\
\hline & & 88.0 & 954.62 & & \\
\hline & & 91.0 & 954.66 & & \\
\hline & & 94.0 & 954.51 & & \\
\hline & & 95.0 & 954.83 & & \\
\hline
\end{tabular}




\section{Description of Cross Section PR170.5}

Location: Township 6 South/Range 50 East--section 22

U. S. Geological Survey quadrangle (1:24,000): Lonesome Peak

Landowners--left bank: Daily or Rice Ranch

--right bank: Rice or Daily Ranch

Access: Right bank

Permission from: Daily Ranch

Distance from Moorhead Gaging Station: 55.3 kilometers

Azimuth of Section (degrees magnetic): 101

\section{Reference Monuments}

[Monuments at stations 150.0 and 168.95 were closest to leveling instrument]

\begin{tabular}{|c|c|c|c|c|c|c|}
\hline \multirow[b]{2}{*}{ Description } & \multirow[b]{2}{*}{$\begin{array}{l}\text { Station } \\
\text { (m) }\end{array}$} & \multicolumn{2}{|c|}{ GPS-NAD83 (1992) } & \multicolumn{2}{|c|}{ Measurement } & \multirow{2}{*}{$\begin{array}{c}\text { Elevation } \\
\text { (NGVD1929) } \\
\text { (m) }\end{array}$} \\
\hline & & Latitude & Longitude & $\begin{array}{l}\text { Standard } \\
\text { deviation } \\
\text { (m) }\end{array}$ & $\begin{array}{l}\text { Horizontal } \\
\text { precision } \\
(\mathrm{m})\end{array}$ & \\
\hline $\begin{array}{l}\text { 1/2-inch-rebar; } 0.13 \text { meter above } 1998 \text { ground } \\
\text { level; under 4-strand, barbed-wire and 1- } \\
\text { strand electric-wire fence with mostly } \\
\text { metal and some tree posts }\end{array}$ & -33.7 & $45^{\circ} 17^{\prime} 57.44^{\prime \prime}$ & $105^{\circ} 35^{\prime} 00.38^{\prime \prime}$ & 0.523 & 0.711 & 957.07 \\
\hline $\begin{array}{l}\text { 1/2-inch-rebar; } 0.16 \text { meter above } 1998 \text { ground } \\
\text { level }\end{array}$ & -1.0 & & & & & 956.93 \\
\hline $\begin{array}{l}\text { 1/2-inch-rebar; } 0.14 \text { meter above } 1998 \text { ground } \\
\text { level; } 0.30 \text { meter downstream from a 3- } \\
\text { strand rusty and } 1 \text {-strand galvanized, } \\
\text { barbed-wire fence with tree posts }\end{array}$ & 150.0 & & & & & 957.22 \\
\hline $\begin{array}{l}\text { 1/2-inch-rebar; } 0.12 \text { meter above } 1998 \text { ground } \\
\text { level; under a } 2 \text {-strand, barbed-wire fence } \\
\text { with tree posts }\end{array}$ & 168.95 & $45^{\circ} 17^{\prime} 54.69^{\prime \prime}$ & $105^{\circ} 34^{\prime} 51.91^{\prime \prime}$ & 0.411 & 0.578 & 957.06 \\
\hline
\end{tabular}




$$
\begin{aligned}
& \text { See Figure } 8 \text { for location } \\
& \text { of cross section }
\end{aligned}
$$

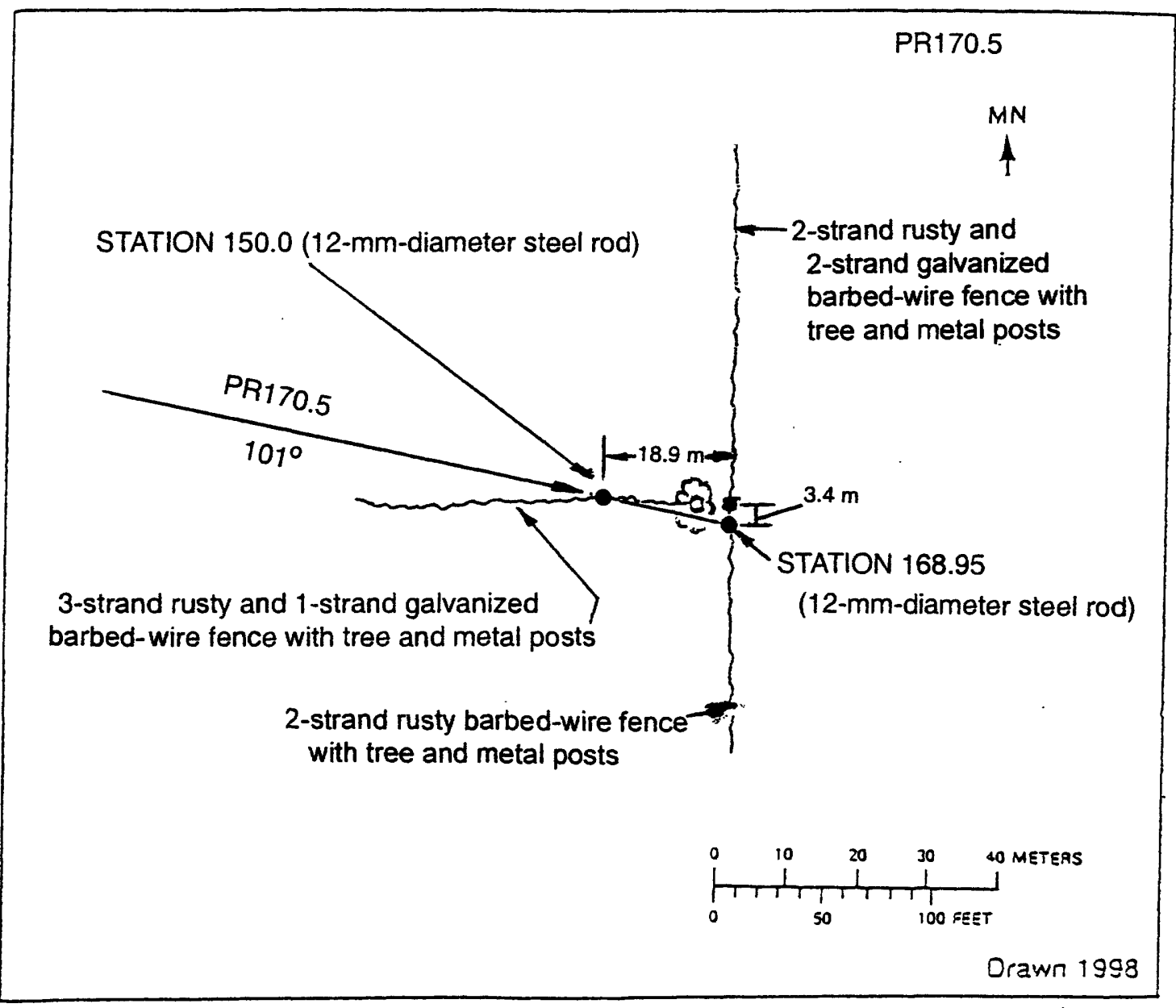

Figure 86. Upper: Location of cross section PR170.5 is shown in figure 8. Lower: Location of the reference monuments on the right bank. $\mathrm{MN}$ is magnetic north. 


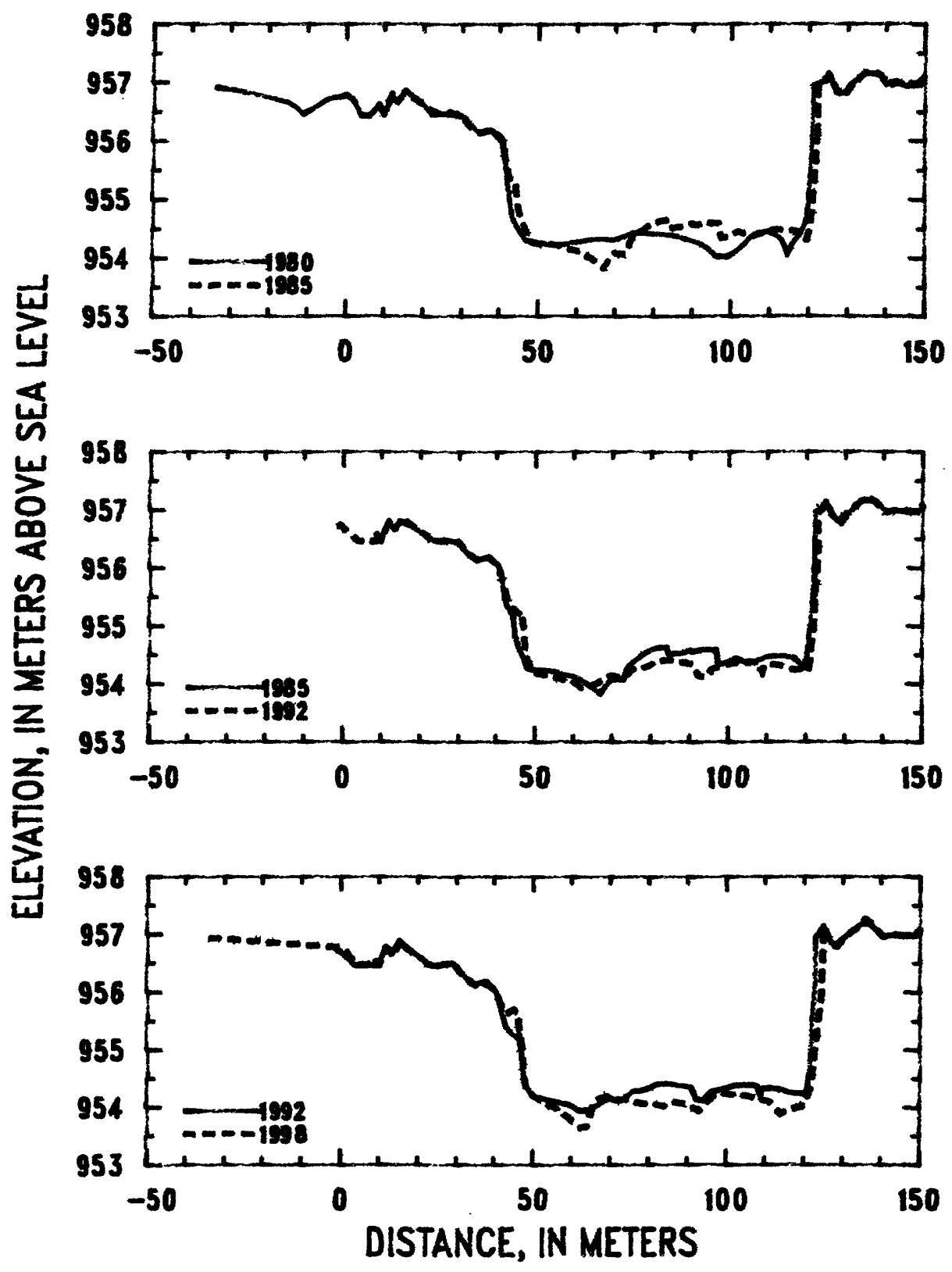

Figure 87. Profiles of cross section PR170.5 from 1980 to 1998. 
Table 32. Listing of horizontal stations and elevations for cross section PR170.5

[Sta., station, distance in meters from a reference pin on the left bank; Elev., elevation, in meters above sea level]

\begin{tabular}{|c|c|c|c|c|c|c|c|c|c|}
\hline \multirow{2}{*}{\multicolumn{2}{|c|}{$\begin{array}{c}1980 \\
21 \text { October }\end{array}$}} & \multirow{2}{*}{\multicolumn{2}{|c|}{$\begin{array}{c}1980 \\
21 \text { October }\end{array}$}} & \multirow{2}{*}{\multicolumn{2}{|c|}{$\begin{array}{c}1985 \\
5 \text { October }\end{array}$}} & \multirow{2}{*}{\multicolumn{2}{|c|}{$\begin{array}{c}1985 \\
5 \text { October }\end{array}$}} & \multirow{2}{*}{\multicolumn{2}{|c|}{$\begin{array}{c}1992 \\
2 \text { September }\end{array}$}} \\
\hline & & & & & & & & & \\
\hline Sta. & Elev. & Sta. & Elev. & Sta. & Elev. & Sta. & Elev. & Sta. & Elev. \\
\hline-33.7 & 956.91 & 113.0 & 954.33 & 8.7 & 956.58 & 99.0 & 954.35 & -1.0 & 956.76 \\
\hline-25.0 & 956.83 & 114.6 & 954.06 & 10.0 & 956.47 & 102.0 & 954.45 & 4.0 & 956.46 \\
\hline-15.0 & 956.67 & 116.0 & 954.25 & 12.0 & 956.80 & 105.0 & 954.35 & 7.0 & 956.46 \\
\hline-11.0 & 956.46 & 118.5 & 954.48 & 13.5 & 956.66 & 108.0 & 954.42 & 10.0 & 956.46 \\
\hline-5.0 & 956.71 & 119.5 & 954.68 & 15.0 & 956.80 & 111.0 & 954.48 & 11.7 & 956.77 \\
\hline-1.0 & 956.76 & 120.6 & 955.53 & 18.0 & 956.71 & 114.0 & 954.48 & 13.5 & 956.66 \\
\hline 0.0 & 956.79 & 121.2 & 956.94 & 21.0 & 956.56 & 117.0 & 954.45 & 15.4 & 956.88 \\
\hline 1.7 & 956.72 & 124.0 & 957.02 & 24.0 & 956.47 & 119.4 & 954.28 & 17.0 & 956.76 \\
\hline 4.0 & 956.43 & 125.4 & 957.12 & 27.0 & 956.49 & 120.5 & 954.54 & 20.0 & 956.64 \\
\hline 6.0 & 956.41 & 127.0 & 956.82 & 30.0 & 956.43 & 121.8 & 955.63 & 23.0 & 956.47 \\
\hline 8.6 & 956.62 & 129.5 & 956.82 & 32.0 & 956.26 & 122.0 & 956.12 & 26.0 & 956.46 \\
\hline 10.0 & 956.45 & 131.0 & 956.98 & 35.0 & 956.13 & 122.1 & 956.01 & 29.0 & 956.51 \\
\hline 12.0 & 956.75 & 133.0 & 957.06 & 38.0 & 956.19 & 122.2 & 956.96 & 32.0 & 956.27 \\
\hline 13.4 & 956.66 & 135.0 & 957.17 & 40.0 & 956.07 & 124.0 & 957.00 & 35.0 & 956.13 \\
\hline 15.5 & 956.86 & 138.5 & 957.14 & 40.8 & 956.02 & 125.0 & 957.13 & 38.0 & 956.19 \\
\hline 17.0 & 956.78 & 140.0 & 956.97 & 42.4 & 955.40 & 127.0 & 956.85 & 40.0 & 956.05 \\
\hline 22.5 & 956.46 & 142.6 & 957.02 & 43.6 & 955.24 & 129.0 & 956.76 & 41.0 & 955.94 \\
\hline 25.0 & 956.47 & 145.0 & 956.93 & 44.2 & 955.25 & 132.0 & 957.01 & 43.0 & 955.39 \\
\hline 30.0 & 956.43 & 148.0 & 956.97 & 45.0 & 954.80 & 135.0 & 957.17 & 45.0 & 955.25 \\
\hline 35.0 & 956.13 & 150.0 & 957.11 & 46.2 & 954.58 & 138.0 & 957.15 & 46.0 & 955.22 \\
\hline 38.0 & 956.18 & 158.0 & 956.82 & 48.0 & 954.27 & 141.0 & 956.95 & 46.4 & 955.26 \\
\hline 40.0 & 956.04 & 165.0 & 957.05 & 50.0 & 954.23 & 143.0 & 956.99 & 47.0 & 955.00 \\
\hline 41.0 & 955.98 & 168.9 & 956.93 & 52.0 & 954.24 & 146.0 & 956.96 & 47.6 & 954.50 \\
\hline 42.5 & 955.12 & & & 55.0 & 954.21 & 149.0 & 956.96 & 47.9 & 954.38 \\
\hline 43.3 & 954.69 & & & 58.0 & 954.15 & & & 50.0 & 954.19 \\
\hline 45.0 & 954.49 & & & 61.0 & 954.11 & & & 52.0 & 954.16 \\
\hline 47.0 & 954.30 & & & 64.0 & 953.99 & & & 54.0 & 954.12 \\
\hline 50.0 & 954.27 & & & 67.0 & 953.82 & & & 56.0 & 954.10 \\
\hline 55.0 & 954.20 & & & 70.0 & 954.09 & & & 58.0 & 954.06 \\
\hline 60.0 & 954.28 & & & 73.0 & 954.08 & & & 60.0 & 954.04 \\
\hline 65.0 & 954.33 & & & 74.0 & 954.29 & & & 62.0 & 953.94 \\
\hline 70.0 & 954.31 & & & 76.0 & 954.41 & & & 64.0 & 953.95 \\
\hline 75.0 & 954.45 & & & 79.0 & 954.56 & & & 66.0 & 954.01 \\
\hline 80.0 & 954.42 & & & 82.0 & 954.64 & & & 68.0 & 954.09 \\
\hline 85.0 & 954.40 & & & 84.2 & 954.64 & & & 70.0 & 954.16 \\
\hline 90.0 & 954.30 & & & 84.7 & 954.51 & & & 72.0 & 954.12 \\
\hline 93.0 & 954.22 & & & 88.0 & 954.53 & & & 74.0 & 954.17 \\
\hline 96.0 & 954.03 & & & 90.0 & 954.59 & & & 76.0 & 954.29 \\
\hline 99.0 & 954.02 & & & 91.0 & 954.56 & & & 78.0 & 954.29 \\
\hline 102.0 & 954.14 & & & 94.0 & 954.60 & & & 80.0 & 954.33 \\
\hline 106.0 & 954.41 & & & 96.9 & 954.59 & & & 82.0 & 954.41 \\
\hline 110.0 & 954.46 & & & 97.4 & 954.34 & & & 85.0 & 954.42 \\
\hline
\end{tabular}


Table 32. (Continued) Listing of horizontal stations and elevations for cross section PR170.5

[Sta., station, distance in meters from a reference pin on the left bank; Elev., elevation, in meters above sea level]

\begin{tabular}{|c|c|c|c|c|c|}
\hline \multirow{2}{*}{\multicolumn{2}{|c|}{$\begin{array}{c}1992 \\
2 \text { September }\end{array}$}} & \multirow{2}{*}{\multicolumn{2}{|c|}{$\begin{array}{c}1998 \\
2 \text { Octiber }\end{array}$}} & \multirow{2}{*}{\multicolumn{2}{|c|}{$\begin{array}{c}1998 \\
2 \text { October }\end{array}$}} \\
\hline & & & & & \\
\hline Sta. & Elev. & Sta. & Elev. & Sta. & Elev. \\
\hline 88.0 & 954.37 & -33.7 & 956.93 & 96.0 & 954.17 \\
\hline 91.0 & 954.34 & -1.0 & 956.77 & 99.0 & 954.25 \\
\hline 92.2 & 954.14 & 1.0 & 956.72 & 102.0 & 954.21 \\
\hline 94.0 & 954.11 & 4.0 & 956.47 & 105.0 & 954.20 \\
\hline 96.0 & 954.28 & 7.0 & 956.47 & 108.0 & 954.13 \\
\hline 98.5 & 954.31 & 8.5 & 956.57 & 111.0 & 954.11 \\
\hline 100.0 & 954.34 & 10.0 & 956.46 & 114.0 & 953.88 \\
\hline 102.0 & 954.38 & 12.0 & 95.6 .78 & 117.0 & 953.97 \\
\hline 104.0 & 954.39 & 14.0 & 956.67 & 120.0 & 954.02 \\
\hline 106.0 & 954.38 & 15.5 & 956.91 & 121.8 & 954.35 \\
\hline 108.0 & 954.37 & 17.0 & 956.76 & 124.5 & 955.97 \\
\hline 108.8 & 954.19 & 20.0 & 956.63 & 124.8 & 956.40 \\
\hline 109.3 & 954.32 & 23.0 & 956.46 & 125.0 & 957.13 \\
\hline 111.0 & 954.34 & 26.0 & 956.47 & 127.0 & 956.85 \\
\hline 113.0 & 954.33 & 29.0 & 956.50 & 128.7 & 956.79 \\
\hline 115.0 & 954.28 & 32.0 & 956.27 & 131.0 & 956.98 \\
\hline 117.0 & 954.24 & 35.0 & 956.12 & 134.0 & 957.11 \\
\hline 119.0 & 954.25 & 37.0 & 956.18 & 136.0 & 957.27 \\
\hline 120.6 & 954.21 & 40.0 & 956.06 & 138.0 & 957.17 \\
\hline 121.0 & 954.37 & 42.0 & 955.76 & 140.5 & 956.96 \\
\hline 121.9 & 954.96 & 43.0 & 955.63 & 143.0 & 957.00 \\
\hline 122.1 & 955.55 & 45.0 & 955.71 & 146.0 & 956.96 \\
\hline 122.9 & 956.11 & 46.0 & 955.59 & 148.0 & 956.97 \\
\hline 123.0 & 956.94 & 47.2 & 954.99 & 150.0 & 957.07 \\
\hline 124.0 & 957.02 & 47.4 & 954.62 & 169.0 & 956.95 \\
\hline 125.0 & 957.13 & 48.1 & 954.32 & & \\
\hline 127.0 & 956.85 & 50.0 & 954.20 & & \\
\hline 128.0 & 956.79 & 53.0 & 954.08 & & \\
\hline 130.0 & 956.92 & 56.0 & 953.99 & & \\
\hline 132.7 & 957.06 & 59.0 & 953.83 & & \\
\hline 135.0 & 957.18 & 62.0 & 953.65 & & \\
\hline 137.0 & 957.18 & 64.0 & 953.65 & & \\
\hline 140.5 & 956.96 & 66.0 & 954.11 & & \\
\hline 144.0 & 957.01 & 69.0 & 954.20 & & \\
\hline 147.0 & 956.97 & 72.0 & 954.13 & & \\
\hline 149.0 & 956.98 & 75.0 & 954.13 & & \\
\hline \multirow[t]{6}{*}{150.0} & 957.10 & 78.0 & 954.07 & & \\
\hline & & 81.0 & 954.06 & & \\
\hline & & 84.0 & 954.02 & & \\
\hline & & 87.0 & 954.09 & & \\
\hline & & 90.0 & 954.05 & & \\
\hline & & 93.0 & 953.93 & & \\
\hline
\end{tabular}




\section{Description of Cross Section PR175}

Location: Township 6 South/Range 50 East--section 15

U. S. Geological Survey quadrangle (1:24,000): Lonesome Peak

Landowners--left bank: Emmons Family

--right bank: Emmons Family

Access: Left bank

Permission from: Emmons Family

Distance from Moorhead Gaging Station: 58.32 kilometers

Azimuth of Section (degrees magnetic): 058

\section{Reference Monuments}

[Russian olive trees block the line of sight between stations -1.7 and 100.0; leveling instrument was setup near station 140.0]

\begin{tabular}{|c|c|c|c|c|c|c|}
\hline \multirow[b]{2}{*}{ Description } & \multirow[b]{2}{*}{$\begin{array}{c}\text { Station } \\
\text { (m) }\end{array}$} & \multicolumn{2}{|c|}{ GPS-NAD83 (1992) } & \multicolumn{2}{|c|}{ Measurement } & \multirow{2}{*}{$\begin{array}{l}\text { Elevation } \\
\text { (NGvD1929) } \\
\text { (m) }\end{array}$} \\
\hline & & Latitude & Longitude & $\begin{array}{l}\text { Standard } \\
\text { deviation } \\
(\mathbf{m})\end{array}$ & $\begin{array}{l}\text { Horizontal } \\
\text { precision } \\
\text { (m) }\end{array}$ & \\
\hline Benchmark--brass circular plate; offsection & -- & $45^{\circ} 18^{\prime} 59.74^{\prime \prime}$ & $105^{\circ} 34^{\prime} 37.31^{\prime \prime}$ & 0.468 & 0.546 & 953.58 \\
\hline $\begin{array}{l}\text { 1/2-inch-rebar; } 0.19 \text { meter above } 1998 \text { ground } \\
\text { level; on a fence line with 4-strands of } \\
\text { barbed-wire and 1-strand of electric wire }\end{array}$ & -39.3 & & & & & 953.49 \\
\hline $\begin{array}{l}\text { 1/2-inch-rebar; } 0.08 \text { meter above } 1998 \text { ground } \\
\text { level }\end{array}$ & -1.7 & & & & & 953.65 \\
\hline $\begin{array}{l}\text { 1/2-inch-rebar; } 0.17 \text { meter above } 1998 \text { ground } \\
\text { level }\end{array}$ & -0.7 & $45^{\circ} 18^{\prime} 59.45^{\prime \prime}$ & $105^{\circ} 34^{\prime} 36.23^{\prime \prime}$ & 0.310 & 0.614 & 953.77 \\
\hline $\begin{array}{l}\text { 1/2-inch-rebar; } 0.05 \text { meter belowe } 1998 \\
\text { ground level }\end{array}$ & 0.0 & & & & & \\
\hline $\begin{array}{l}\text { 1/2-inch-rebar; } 0.11 \text { meter above } 1998 \text { ground } \\
\text { level }\end{array}$ & 99.9 & & & & & 952.95 \\
\hline $\begin{array}{l}\text { 1/2-inch-rebar; } 0.04 \text { meter above } 1998 \text { ground } \\
\text { level }\end{array}$ & 100.0 & & & & & 952.79 \\
\hline $\begin{array}{l}\text { 1/2-inch-rebar; } 0.05 \text { meter below } 1998 \text { ground } \\
\text { level }\end{array}$ & 124.0 & & & & & 952.84 \\
\hline $\begin{array}{l}\text { 1/2-inch-rebar; } 0.12 \text { meter above } 1998 \text { ground } \\
\text { level }\end{array}$ & 125.0 & $45^{\circ} 19^{\prime} 00.71^{\prime \prime}$ & $105^{\circ} 34^{\prime} 30.74^{\prime \prime}$ & 0.225 & 0.549 & 953.05 \\
\hline $\begin{array}{l}\text { 1/2-inch-rebar; } 0.08 \text { meter above } 1998 \text { ground } \\
\text { level; on riverward side of large log; ele- } \\
\text { vation is } \pm 0.02 \text { meter }\end{array}$ & 250.0 & & & & & 952.95 \\
\hline $\begin{array}{l}\text { 1/2-inch-rebar; } 0.03 \text { meter above } 1998 \text { ground } \\
\text { level; elevation is } \pm 0.01 \text { meter }\end{array}$ & 300.0 & & & & & 953.18 \\
\hline $\begin{array}{l}\text { 1/2-inch-rebar; } 0.16 \text { meter above } 1998 \text { ground } \\
\text { level; elevation is } \pm 0.03 \text { meter }\end{array}$ & 320.0 & $45^{\circ} 19^{\prime} 02.68^{\prime \prime}$ & $105^{\circ} 34^{\prime} 22.25^{\prime \prime}$ & 0.485 & 0.565 & 953.56 \\
\hline
\end{tabular}




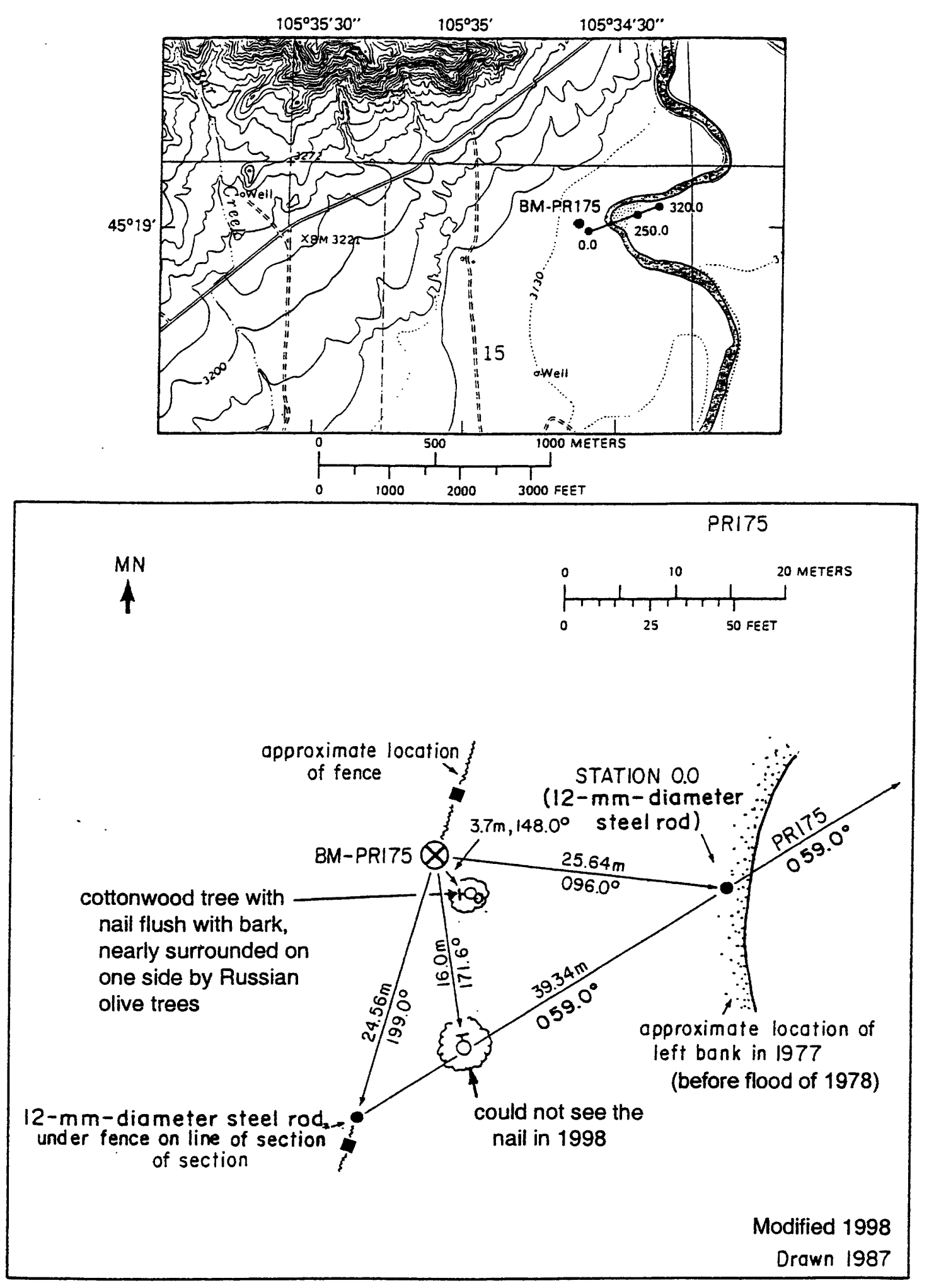

Figure 88. Upper: Location of cross section PR175, bench mark BM-PR175, and the left and right bank reference monuments in the Lonesome Peak quadrangle. Lower: Location of the bench mark on the left bank. MN is magnetic north. 

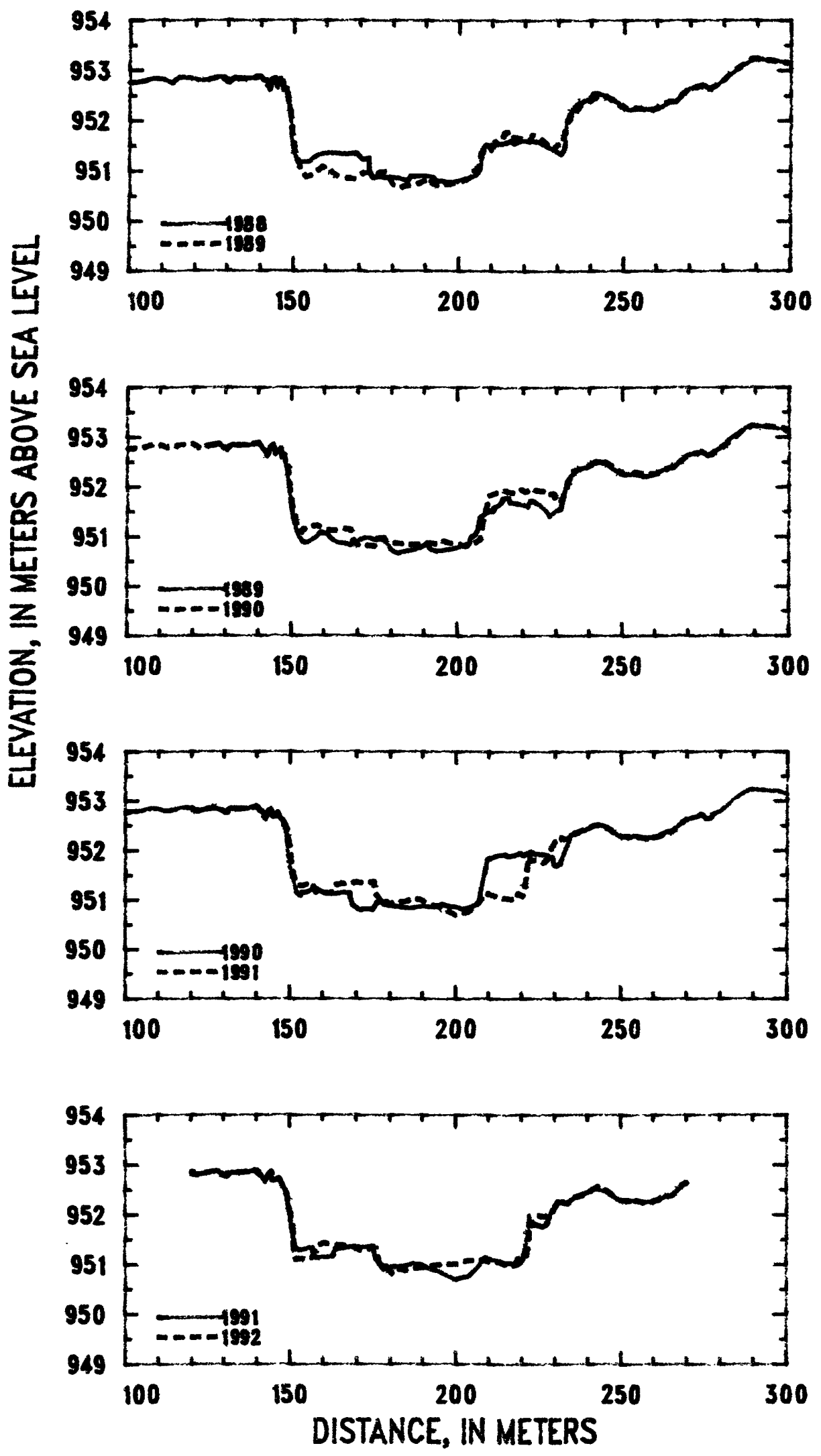

Figure 89. Profiles of cross section PR175 from 1988 to 1992. 

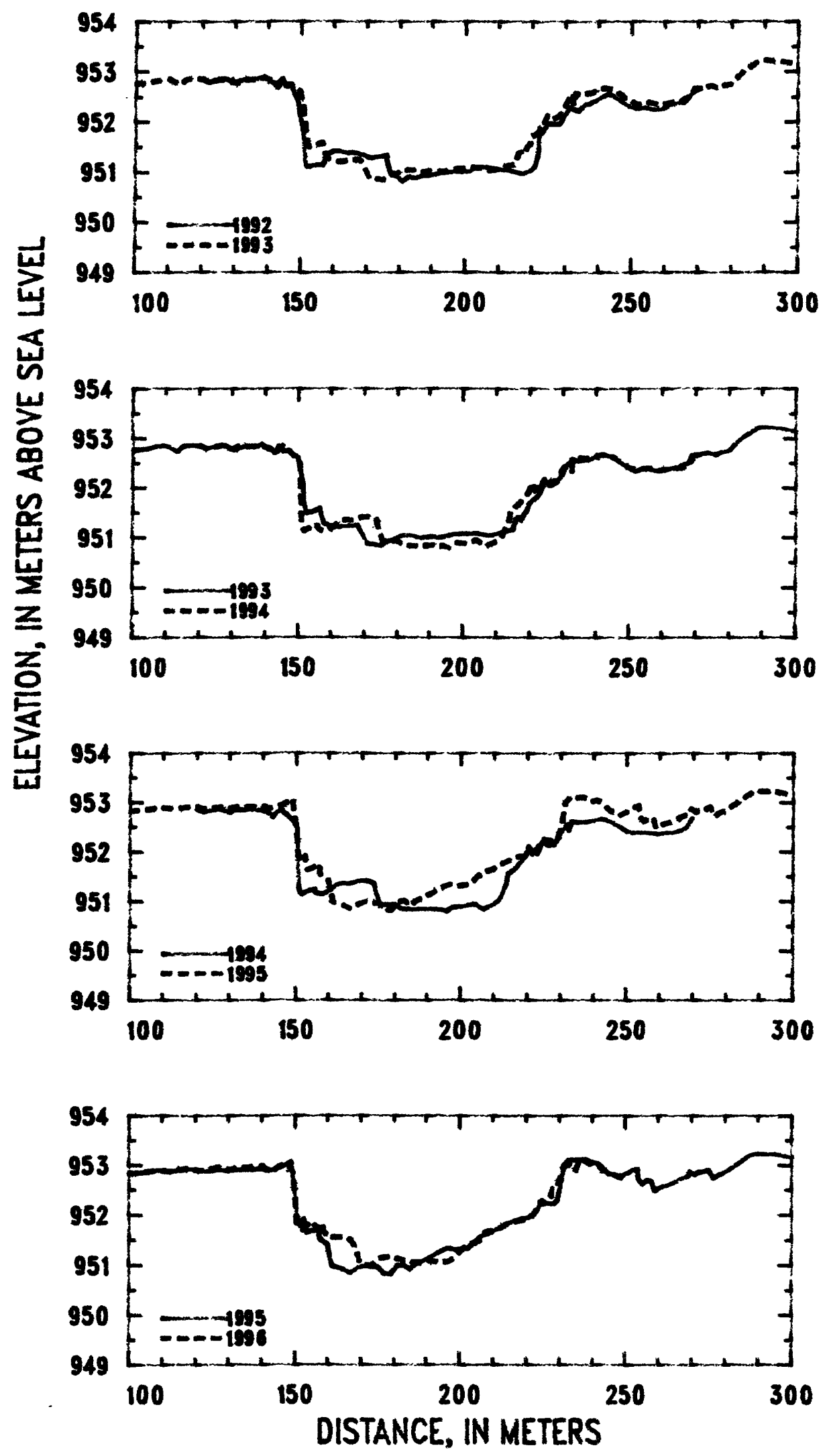

Figure 90. Profiles of cross section PR175 from 1992 to 1996. 


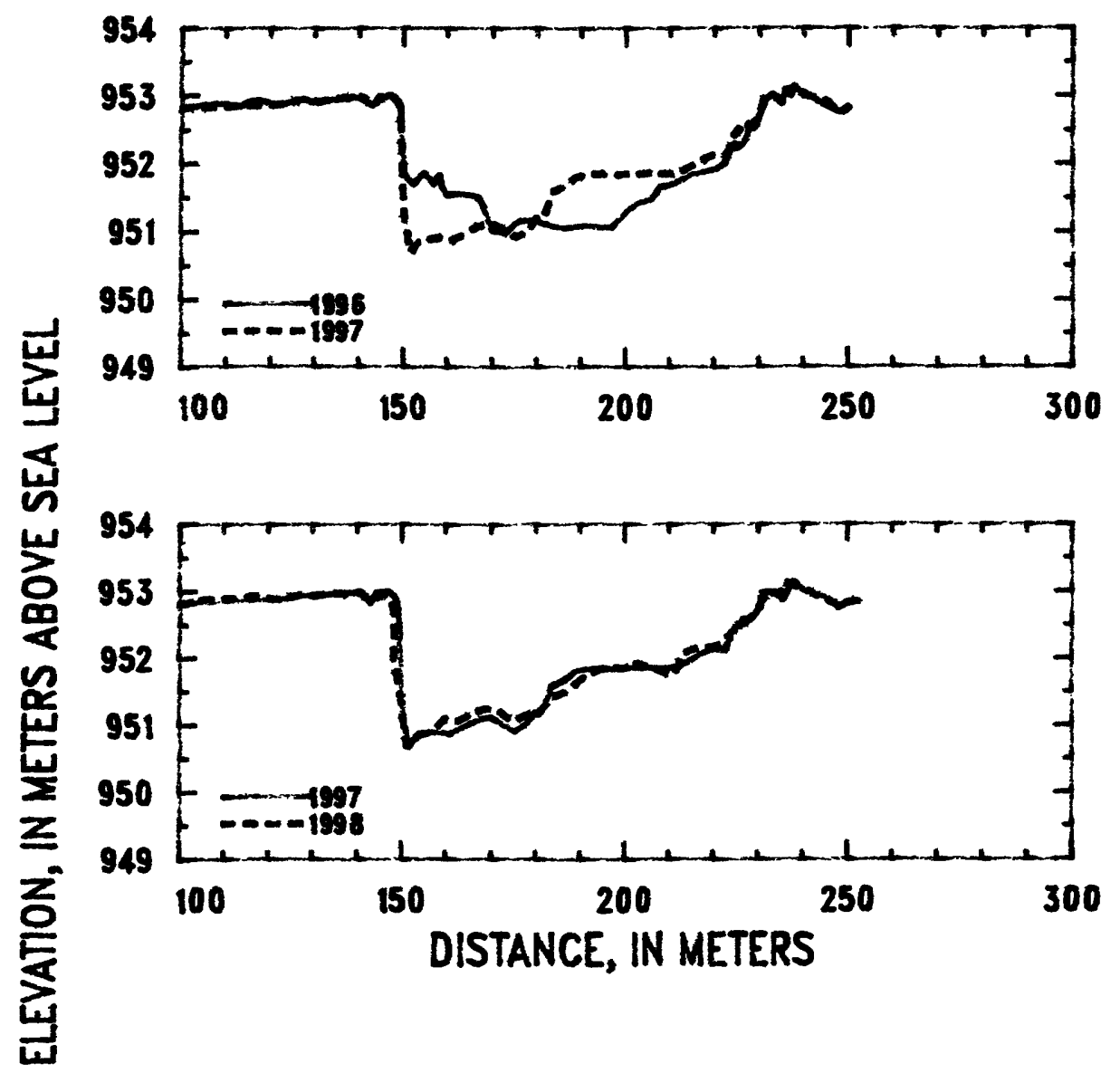

Figure 91. Profiles of cross section PR175 from 1996 to 1998. 
Table 33. Listing of horizontal stations and elevations for cross section PR175

[Sta., station, distance in meters from a reference pin on the left bank; Elev., elevation, in meters above sea level]

\begin{tabular}{|c|c|c|c|c|c|c|c|c|c|}
\hline \multirow{2}{*}{\multicolumn{2}{|c|}{$\begin{array}{c}1989 \\
\text { 22 September } \\
\end{array}$}} & \multicolumn{2}{|c|}{1989} & \multicolumn{2}{|c|}{1989} & \multicolumn{2}{|c|}{1990} & \multicolumn{2}{|c|}{1990} \\
\hline & & $22 \mathrm{Se}$ & ember & $22 \mathrm{Se}$ & mber & $21 \mathrm{Se}$ & ember & $21 \mathrm{Se}$ & ember \\
\hline Sta. & Elev. & Sta. & Elev. & Sta. & Elev. & Sta. & Elev. & Sta. & Elev. \\
\hline 124.0 & 952.82 & 186.0 & 950.72 & 257.0 & 952.20 & 94.8 & 952.95 & 157.0 & 951.22 \\
\hline 126.0 & 952.85 & 188.0 & 950.74 & 260.0 & 952.24 & 95.0 & 952.90 & 158.0 & 951.23 \\
\hline 128.0 & 952.87 & 190.0 & 950.82 & 263.0 & 952.36 & 96.0 & 952.75 & 160.0 & 951.15 \\
\hline 130.0 & 952.76 & 191.0 & 950.86 & 266.0 & 952.39 & 98.0 & $952.75^{\circ}$ & 162.0 & 951.12 \\
\hline 132.0 & 952.85 & 192.0 & 950.75 & 268.0 & 952.54 & 100.0 & 952.76 & 164.0 & 951.12 \\
\hline 134.0 & 952.84 & 194.0 & 950.69 & 270.0 & 952.64 & 102.0 & 952.79 & 166.0 & 951.16 \\
\hline 136.0 & 952.84 & 196.0 & 950.73 & 272.0 & 952.69 & 104.0 & 952.78 & 168.1 & 951.14 \\
\hline 138.0 & 952.86 & 198.0 & 950.75 & 274.0 & 952.71 & 106.0 & 952.83 & 168.2 & 951.05 \\
\hline 140.0 & 952.88 & 200.0 & 950.78 & 276.0 & 952.63 & 108.0 & 952.85 & 169.0 & 950.88 \\
\hline 141.0 & 952.80 & 202.0 & 950.83 & 279.0 & 952.74 & 110.0 & 952.84 & 171.0 & 950.79 \\
\hline 142.8 & 952.63 & 203.8 & 950.86 & 282.0 & 952.94 & 112.0 & 952.79 & 173.0 & 950.82 \\
\hline 143.6 & 952.81 & 204.3 & 950.99 & 285.0 & 953.07 & 114.0 & 952.80 & 175.0 & 950.80 \\
\hline 144.4 & 952.85 & 205.8 & 951.05 & 288.0 & 953.23 & 116.0 & 952.85 & 176.3 & 950.96 \\
\hline 145.3 & 952.66 & 206.4 & 951.15 & 290.0 & 953.23 & 118.0 & 952.87 & 177.7 & 950.98 \\
\hline 146.7 & 952.77 & 206.9 & 951.37 & 295.0 & 953.20 & 120.0 & 952.86 & 178.3 & 950.92 \\
\hline 147.3 & 952.59 & 209.0 & 951.49 & 300.0 & 953.14 & 122.0 & 952.78 & 180.0 & 950.89 \\
\hline 148.0 & 952.63 & 210.9 & 951.51 & & & 124.0 & 952.81 & 182.0 & 950.87 \\
\hline 148.3 & 952.49 & 211.3 & 951.58 & & & 126.0 & 952.85 & 184.0 & 950.85 \\
\hline 149.0 & 952.27 & 213.6 & 951.68 & & & 128.0 & 952.85 & 186.0 & 950.83 \\
\hline 150.0 & 951.63 & 214.0 & 951.77 & & & 130.4 & 952.75 & 188.0 & 950.84 \\
\hline 150.6 & 951.36 & 215.7 & 951.79 & & & 132.0 & 952.85 & 190.0 & 950.87 \\
\hline 152.5 & 950.98 & 216.2 & 951.68 & & & 134.0 & 952.84 & 192.0 & 950.87 \\
\hline 154.0 & 950.87 & 218.0 & 951.66 & & & 136.0 & 952.84 & 194.0 & 950.86 \\
\hline 156.0 & 950.91 & 220.0 & 951.64 & & & 138.0 & 952.86 & 196.0 & 950.92 \\
\hline 157.3 & 950.98 & 221.5 & 951.60 & & & 140.0 & 952.90 & 198.0 & 950.86 \\
\hline 159.0 & 951.08 & 222.4 & 951.73 & & & 141.0 & 952.79 & 200.0 & 950.86 \\
\hline 161.1 & 951.05 & 224.0 & 951.69 & & & 141.7 & 952.79 & 202.0 & 950.81 \\
\hline 162.0 & 950.96 & 226.0 & 951.58 & & & 142.7 & 952.64 & 204.0 & 950.84 \\
\hline 164.0 & 950.88 & 228.0 & 951.40 & & & 143.6 & 952.81 & 205.0 & 950.86 \\
\hline 166.0 & 950.87 & 230.0 & 951.52 & & & 144.5 & 952.83 & 207.0 & 950.94 \\
\hline 168.0 & 950.83 & 231.4 & 951.56 & & & 145.2 & 952.69 & 207.4 & 951.05 \\
\hline 170.0 & 950.90 & 233.0 & 952.01 & & & 146.4 & 952.76 & 208.0 & 951.30 \\
\hline 171.6 & 950.97 & 234.0 & 952.19 & & & 147.3 & 952.63 & 208.7 & 951.49 \\
\hline 173.0 & 950.99 & 236.0 & 952.31 & & & 148.0 & 952.61 & 209.0 & 951.58 \\
\hline 174.1 & 950.98 & 238.0 & 952.38 & & & 148.4 & 952.49 & 209.3 & 951.63 \\
\hline 175.5 & 950.93 & 240.0 & 952.40 & & & 148.8 & 952.48 & 209.8 & 951.82 \\
\hline 176.2 & 950.98 & 243.0 & 952.52 & & & 149.0 & 952.40 & 211.0 & 951.87 \\
\hline 177.4 & 950.98 & 245.0 & 952.47 & & & 149.8 & 951.77 & 213.0 & 951.90 \\
\hline 178.0 & 950.99 & 247.0 & 952.37 & & & 152.1 & 951.13 & 215.0 & 951.93 \\
\hline 180.0 & 950.72 & 250.0 & 952.24 & & & 153.3 & 951.08 & 216.0 & 951.88 \\
\hline 182.0 & 950.65 & 252.0 & 952.26 & & & 153.8 & 951.15 & 218.0 & 951.89 \\
\hline 184.0 & 950.70 & 255.0 & 952.24 & & & 155.0 & 951.14 & 219.6 & 951.95 \\
\hline
\end{tabular}


Table 33. (Continued) Listing of horizontal stations and elevations for cross section PR175

[Sta., station, distance in meters from a reference pin on the left bank; Elev., elevation, in meters above sea level]

\begin{tabular}{|c|c|c|c|c|c|c|c|c|c|}
\hline \multirow{2}{*}{\multicolumn{2}{|c|}{$\begin{array}{c}1990 \\
21 \text { September }\end{array}$}} & \multicolumn{2}{|c|}{1990} & \multicolumn{2}{|c|}{1991} & \multicolumn{2}{|c|}{1991} & \multicolumn{2}{|c|}{1991} \\
\hline & & $21 \mathrm{Se}$ & mber & $1 \mathrm{Se}$ & mber & $1 \mathrm{Se}$ & ember & $1 \mathrm{Se}$ & mber \\
\hline Sta. & Elev. & Sta. & Elev. & Sta. & Elev. & Sta. & Elev. & Sta. & Elev. \\
\hline 220.5 & 951.90 & 289.0 & 953.23 & 120.0 & 952.85 & 180.0 & 950.91 & 257.0 & 952.24 \\
\hline 221.7 & 951.93 & 292.0 & 953.22 & 121.0 & 952.80 & 181.0 & 950.95 & 260.0 & 952.26 \\
\hline 223.0 & 951.98 & 295.0 & 953.20 & 124.0 & 952.82 & 183.0 & 950.95 & 262.0 & 952.28 \\
\hline 224.0 & 951.93 & 298.0 & 953.18 & 126.0 & 952.85 & 185.0 & 950.97 & 264.0 & 952.38 \\
\hline 226.0 & 951.92 & 300.0 & 953.14 & 128.0 & 952.88 & 187.0 & 951.02 & 266.0 & 952.39 \\
\hline 227.7 & 951.90 & 305.0 & 953.21 & 130.0 & 952.78 & 189.0 & 951.02 & 268.0 & 952.53 \\
\hline 228.0 & 951.86 & 310.0 & 953.20 & 131.0 & 952.76 & 191.1 & 950.95 & 270.0 & 952.62 \\
\hline 229.4 & 951.86 & 315.0 & 953.29 & 132.0 & 952.84 & 193.0 & 950.89 & & \\
\hline 229.8 & 951.71 & 320.0 & 953.39 & 134.0 & 952.84 & 195.0 & 950.86 & & \\
\hline 230.3 & 951.68 & & & 136.0 & 952.85 & 197.0 & 950.79 & & \\
\hline 231.7 & 951.72 & & & 138.0 & 952.85 & 200.0 & 950.68 & & \\
\hline 233.0 & 951.96 & & & 140.0 & 952.89 & 202.0 & 950.73 & & \\
\hline 234.0 & 952.15 & & & 142.7 & 952.66 & 204.0 & 950.76 & & \\
\hline 235.0 & 952.32 & & & 144.0 & 952.82 & 205.2 & 950.83 & & \\
\hline 237.0 & 952.36 & & & 145.0 & 952.68 & 207.0 & 950.95 & & \\
\hline 239.0 & 952.40 & & & 147.0 & 952.70 & 209.0 & 951.14 & & \\
\hline 240.0 & 952.43 & & & 148.4 & 952.53 & 211.0 & 951.11 & & \\
\hline 242.0 & 952.51 & & & 149.2 & 952.45 & 214.0 & 951.05 & & \\
\hline 244.0 & 952.52 & & & 149.6 & 952.17 & 217.0 & 951.01 & & \\
\hline 246.0 & 952.46 & & & 150.2 & 952.06 & 218.4 & 951.01 & & \\
\hline 248.0 & 952.35 & & & 150.3 & 951.82 & 219.0 & 951.13 & & \\
\hline 250.0 & 952.26 & & & 151.2 & 951.48 & 220.2 & 951.08 & & \\
\hline 252.0 & 952.24 & & & 152.3 & 951.29 & 220.7 & 951.16 & & \\
\hline 254.0 & 952.30 & & & 154.0 & 951.28 & 222.3 & 951.92 & & \\
\hline 256.0 & 952.26 & & & 156.0 & 951.34 & 222.7 & 951.81 & & \\
\hline 258.0 & 952.25 & & & 157.0 & 951.33 & 224.0 & 951.81 & & \\
\hline 260.0 & 952.26 & & & 157.3 & 951.21 & 226.4 & 951.75 & & \\
\hline 262.0 & 952.30 & & & 158.2 & 951.12 & 227.7 & 951.81 & & \\
\hline 264.0 & 952.38 & & & 160.0 & 951.14 & 229.5 & 952.13 & & \\
\hline 266.0 & 952.40 & & & 162.0 & 951.15 & 231.0 & 952.27 & & \\
\hline 268.0 & 952.53 & & & 163.2 & 951.16 & 233.0 & 952.23 & & \\
\hline 270.0 & 952.61 & & & 164.0 & 951.30 & 234.0 & 952.23 & & \\
\hline 272.0 & 952.67 & & & 166.0 & 951.33 & 236.0 & 952.34 & & \\
\hline 273.4 & 952.68 & & & 168.0 & 951.34 & 238.0 & 952.40 & & \\
\hline 274.5 & 952.74 & & & 170.0 & 951.36 & 240.0 & 952.44 & & \\
\hline 275.4 & 952.67 & & & 172.0 & 951.34 & 242.0 & 952.51 & & \\
\hline 276.0 & 952.63 & & & 174.0 & 951.36 & 244.0 & 952.50 & & \\
\hline 277.3 & 952.65 & & & 175.2 & 951.37 & 246.0 & 952.45 & & \\
\hline 278.0 & 952.72 & & & 175.7 & 951.13 & 248.0 & 952.34 & & \\
\hline 280.0 & 952.78 & & & 177.0 & 951.01 & 250.0 & 952.28 & & \\
\hline 283.0 & 952.95 & & & 178.0 & 950.90 & 252.0 & 952.29 & & \\
\hline 286.0 & 953.11 & & & 179.0 & 950.97 & 254.0 & 952.28 & & \\
\hline
\end{tabular}


Table 33. (Continued) Listing of horizontal stations and elevations for cross section PR175

[Sta., station, distance in meters from a reference pin on the left bank; Elev., elevation, in meters above sea level]

\begin{tabular}{|c|c|c|c|c|c|c|c|c|c|}
\hline \multirow{2}{*}{\multicolumn{2}{|c|}{$\begin{array}{c}1992 \\
28 \text { August }\end{array}$}} & \multicolumn{2}{|c|}{1992} & \multicolumn{2}{|c|}{1993} & \multicolumn{2}{|c|}{1993} & \multicolumn{2}{|c|}{1993} \\
\hline & & \multicolumn{2}{|c|}{28 August } & \multicolumn{2}{|c|}{30 August } & \multicolumn{2}{|c|}{30 August } & \multicolumn{2}{|c|}{30 August } \\
\hline Sta. & Elev. & Sta. & Elev. & Sta. & Elev. & Sta. & Elev. & Sta. & Elev. \\
\hline 120.0 & 952.86 & 187.0 & 950.92 & 100.0 & 952.76 & 162.0 & 951.20 & 228.0 & 952.11 \\
\hline 122.0 & 952.79 & 189.0 & 950.93 & 102.0 & 952.78 & 164.0 & 951.22 & 230.0 & 952.28 \\
\hline 124.0 & 952.83 & 191.0 & 950.94 & 104.0 & 952.78 & 166.0 & 951.24 & 230.5 & 952.39 \\
\hline 127.0 & 952.87 & 193.0 & 950.97 & 106.0 & 952.82 & 168.0 & 951.24 & 232.4 & 952.56 \\
\hline 130.0 & 952.78 & 195.0 & 950.99 & 108.0 & 952.84 & 169.0 & 951.12 & 232.6 & 952.33 \\
\hline 133.0 & 952.85 & 197.0 & 951.01 & 110.0 & 952.86 & 171.0 & 950.87 & 232.7 & 952.33 \\
\hline 136.0 & 952.85 & 199.0 & 951.00 & 112.0 & 952.79 & 173.0 & 950.86 & 233.0 & 952.57 \\
\hline 139.0 & 952.90 & 201.0 & 951.01 & 113.5 & 952.74 & 175.0 & 950.83 & 235.0 & 952.55 \\
\hline 142.0 & 952.75 & 203.0 & 951.07 & 115.0 & 952.84 & 177.0 & 950.94 & 237.0 & 952.58 \\
\hline 143.0 & 952.75 & 205.0 & 951.10 & 117.0 & 952.87 & 179.0 & 950.95 & 238.0 & 952.57 \\
\hline 144.4 & 952.86 & 207.0 & 951.10 & 118.0 & 952.87 & 181.0 & 951.04 & 240.0 & 952.64 \\
\hline 145.4 & 952.70 & 209.0 & 951.06 & 120.0 & 952.85 & 183.0 & 951.04 & 242.0 & 952.68 \\
\hline 147.0 & 952.72 & 211.0 & 951.04 & 122.0 & 952.80 & 185.0 & 951.04 & 244.0 & 952.65 \\
\hline 148.5 & 952.51 & 213.0 & 951.05 & 124.0 & 952.83 & 187.0 & 950.98 & 246.0 & 952.58 \\
\hline 149.3 & 952.41 & 215.0 & 951.00 & 126.0 & 952.84 & 189.0 & 951.01 & 248.0 & 952.48 \\
\hline 149.6 & 952.19 & 217.0 & 950.96 & 128.0 & 952.86 & 191.0 & 951.03 & 250.0 & 952.40 \\
\hline 150.4 & 951.95 & 219.0 & 951.00 & 130.0 & 952.78 & 193.0 & 950.99 & 252.0 & 952.33 \\
\hline 151.0 & 951.73 & 221.0 & 951.11 & 131.0 & 952.77 & 195.0 & 951.02 & 254.0 & 952.39 \\
\hline 151.4 & 951.20 & 221.4 & 951.21 & 133.0 & 952.84 & 197.0 & 951.06 & 256.0 & 952.38 \\
\hline 152.0 & 951.10 & 221.7 & 951.32 & 135.0 & 952.85 & 199.0 & 951.07 & 258.0 & 952.34 \\
\hline 154.0 & 951.12 & 222.0 & 951.39 & 137.0 & 952.84 & 201.0 & 951.09 & 260.0 & 952.35 \\
\hline 156.0 & 951.14 & 222.4 & 951.86 & 139.0 & 952.89 & 203.0 & 951.08 & 262.0 & 952.39 \\
\hline 157.0 & 951.13 & 223.0 & 951.79 & 141.0 & 952.82 & 205.0 & 951.08 & 264.0 & 952.42 \\
\hline 157.4 & 951.20 & 225.0 & 951.98 & 143.0 & 952.68 & 207.0 & 951.03 & 265.0 & 952.40 \\
\hline 157.9 & 951.33 & 227.0 & 951.96 & 144.0 & 952.83 & 209.0 & 951.04 & 266.0 & 952.46 \\
\hline 159.0 & 951.38 & 228.5 & 951.97 & 145.3 & 952.74 & 211.0 & 951.09 & 268.0 & 952.51 \\
\hline 160.3 & 951.43 & 230.0 & 952.14 & 147.0 & 952.74 & 213.0 & 951.13 & 269.0 & 952.66 \\
\hline 162.0 & 951.42 & 231.6 & 952.30 & 148.0 & 952.68 & 215.0 & 951.16 & 270.0 & 952.65 \\
\hline 164.0 & 951.38 & 233.0 & 952.24 & 149.0 & 952.63 & 215.5 & 951.28 & 272.0 & 952.69 \\
\hline 166.0 & 951.39 & 234.0 & 952.21 & 150.0 & 952.60 & 216.0 & 951.38 & 274.0 & 952.72 \\
\hline 168.0 & 951.34 & 236.0 & 952.35 & 151.0 & 952.24 & 216.7 & 951.41 & 276.0 & 952.66 \\
\hline 169.7 & 951.34 & 238.0 & 952.38 & 151.1 & 952.13 & 217.5 & 951.35 & 278.0 & 952.73 \\
\hline 170.5 & 951.29 & 240.0 & 952.44 & 151.4 & 952.09 & 218.5 & 951.49 & 280.0 & 952.75 \\
\hline 172.0 & 951.28 & 243.0 & 952.56 & 151.4 & 951.92 & 219.5 & 951.57 & 281.6 & 952.87 \\
\hline 174.0 & 951.31 & 246.0 & 952.47 & 152.0 & 951.64 & 219.7 & 951.68 & 284.0 & 953.02 \\
\hline 176.2 & 951.34 & 249.0 & 952.30 & 152.5 & 951.49 & 221.0 & 951.76 & 286.0 & 953.10 \\
\hline 176.3 & 951.20 & 252.0 & 952.26 & 154.5 & 951.51 & 222.0 & 951.80 & 288.0 & 953.20 \\
\hline 177.5 & 950.95 & 254.0 & 952.29 & 156.0 & 951.57 & 223.0 & 951.90 & 290.0 & 953.23 \\
\hline 179.0 & 950.92 & 257.0 & 952.23 & 156.7 & 951.59 & 224.5 & 952.13 & 295.0 & 953.21 \\
\hline 181.0 & 950.80 & 260.0 & 952.26 & 156.8 & 951.50 & 225.4 & 952.11 & 300.0 & 953.14 \\
\hline 183.0 & 950.90 & 263.0 & 952.37 & 157.9 & 951.28 & 226.0 & 952.05 & 305.0 & 953.22 \\
\hline 185.0 & 950.87 & 266.0 & 952.39 & 160.0 & 951.23 & 227.0 & 952.08 & 310.0 & 953.21 \\
\hline & & 269.0 & 952.60 & & & & & 315.0 & 953.27 \\
\hline & & 271.0 & 952.67 & & . & & & 320.0 & 953.40 \\
\hline
\end{tabular}


Table 33. (Continued) Listing of horizontal stations and elevations for cross section PR175

[Sta., station, distance in meters from a reference pin on the left bank; Elev., elevation, in meters above sea level]

\begin{tabular}{|c|c|c|c|c|c|c|c|c|c|}
\hline \multirow{2}{*}{\multicolumn{2}{|c|}{$\begin{array}{c}1994 \\
21 \text { September }\end{array}$}} & \multicolumn{2}{|c|}{1994} & \multicolumn{2}{|c|}{1995} & \multicolumn{2}{|c|}{1995} & \multicolumn{2}{|c|}{1995} \\
\hline & & $21 \mathrm{Se}$ & ember & $30 \mathrm{Se}$ & ember & $30 \mathrm{Se}$ & ember & $30 \mathrm{Se}$ & ember \\
\hline Sta. & Elev. & Sta. & Elev. & Sta. & Elev. & Sta. & Elev. & Sta. & Elev. \\
\hline 120.0 & 952.86 & 196.0 & 950.79 & -1.7 & 953.56 & 91.0 & 952.95 & 161.4 & 950.98 \\
\hline 122.0 & 952.80 & 198.0 & 950.89 & -0.7 & 953.59 & 94.0 & 952.98 & 163.0 & 950.94 \\
\hline 124.0 & 952.83 & 201.0 & 950.90 & 0.0 & 953.53 & 96.0 & 952.84 & 165.0 & 950.90 \\
\hline 127.0 & 952.87 & 204.0 & 950.95 & 1.3 & 953.52 & 98.0 & 952.81 & 167.0 & 950.84 \\
\hline 130.0 & 952.79 & 207.0 & 950.83 & 1.4 & 953.33 & 99.9 & 952.83 & 169.0 & 950.93 \\
\hline 132.0 & 952.85 & 210.0 & 950.92 & 3.0 & 952.68 & 100.0 & 952.83 & 171.0 & 950.98 \\
\hline 134.0 & 952.84 & 212.0 & 951.04 & 4.0 & 952.50 & 102.0 & 952.84 & 173.0 & 950.95 \\
\hline 138.0 & 952.86 & 213.3 & 951.25 & 5.0 & 952.50 & 104.0 & 952.85 & 175.0 & 950.97 \\
\hline 141.0 & 952.82 & 213.9 & 951.35 & 6.0 & 952.51 & 106.0 & 952.86 & $177.0^{\circ}$ & 950.83 \\
\hline 143.0 & 952.70 & 214.3 & 951.56 & 7.0 & 952.58 & 108.0 & 952.89 & 179.0 & 950.81 \\
\hline 145.4 & 952.86 & 215.0 & 951.60 & 8.7 & 952.67 & 110.0 & 952.89 & 181.0 & 950.99 \\
\hline 146.4 & 952.77 & 217.0 & 951.69 & 10.5 & 952.54 & 112.0 & 952.88 & 183.0 & 951.01 \\
\hline 147.0 & 952.75 & 218.0 & 951.79 & 12.0 & 952.72 & 114.0 & 952.88 & 185.0 & 950.91 \\
\hline 148.5 & 952.65 & 219.7 & 951.93 & 13.0 & 952.82 & 116.0 & 952.90 & 187.0 & 951.03 \\
\hline 149.9 & 952.59 & 220.3 & 952.11 & 15.0 & 952.82 & 118.0 & 952.92 & 189.0 & 951.08 \\
\hline 150.4 & 952.49 & 221.0 & 952.05 & 18.0 & 952.88 & 120.0 & 952.91 & 191.0 & 951.15 \\
\hline 151.0 & 951.21 & 221.6 & 951.95 & 20.0 & 952.86 & 122.0 & 952.87 & 193.0 & 951.22 \\
\hline 151.8 & 951.13 & 222.4 & 952.03 & 22.0 & 952.87 & 124.0 & 952.87 & 195.0 & 951.29 \\
\hline 152.5 & 951.16 & 223.0 & 951.97 & 24.0 & 952.77 & 126.0 & 952.90 & 197.0 & 951.34 \\
\hline 155.0 & 951.24 & 224.0 & 952.16 & 26.0 & 952.75 & 128.0 & 952.91 & 199.0 & 951.29 \\
\hline 155.8 & 951.24 & 225.0 & 952.24 & 28.0 & 952.80 & 130.0 & 952.87 & 201.0 & 951.33 \\
\hline 155.9 & 951.17 & 226.0 & 952.14 & 31.0 & 952.82 & 132.0 & 952.90 & 202.5 & 951.33 \\
\hline 157.5 & 951.13 & 228.0 & 952.15 & 34.0 & 952.87 & 134.0 & 952.90 & 203.8 & 951.40 \\
\hline 159.0 & 951.14 & 229.2 & 952.28 & 37.0 & 952.87 & 136.0 & 952.91 & 205.0 & 951.51 \\
\hline 161.0 & 951.24 & 229.4 & 952.35 & 39.5 & 952.85 & 138.0 & 952.91 & 207.0 & 951.59 \\
\hline 163.0 & 951.35 & 232.0 & 952.55 & 40.8 & 952.78 & 140.0 & 952.93 & 209.0 & 951.63 \\
\hline 164.5 & 951.36 & 232.6 & 952.45 & 43.0 & 952.96 & 142.0 & 952.88 & 211.0 & 951.70 \\
\hline 166.0 & 951.34 & 233.5 & 952.61 & 46.0 & 952.94 & 144.0 & 952.91 & 213.0 & 951.78 \\
\hline 168.0 & 951.39 & 236.0 & 952.60 & 49.0 & 952.95 & 146.0 & 952.95 & 215.0 & 951.84 \\
\hline 170.0 & 951.41 & 239.0 & 952.62 & 52.0 & 952.95 & 148.0 & 953.03 & 217.0 & 951.90 \\
\hline 172.0 & 951.42 & 242.0 & 952.68 & 55.0 & 952.94 & 148.8 & 953.06 & 219.0 & 951.92 \\
\hline 173.6 & 951.35 & 245.0 & 952.63 & 58.0 & 952.93 & 149.8 & 952.62 & 221.0 & 951.95 \\
\hline 174.4 & 951.15 & 248.0 & 952.50 & 61.0 & 952.93 & 150.8 & 951.87 & 223.0 & 952.05 \\
\hline 174.9 & 951.07 & 250.0 & 952.41 & 64.0 & 952.89 & 151.7 & 951.78 & 224.5 & 952.26 \\
\hline 175.5 & 950.93 & 253.0 & 952.38 & 67.0 & 952.86 & 152.4 & 951.90 & 226.0 & 952.25 \\
\hline 177.0 & 950.92 & 256.0 & 952.40 & 70.0 & 952.87 & 152.8 & 951.91 & 228.0 & 952.22 \\
\hline 180.0 & 950.94 & 259.0 & 952.35 & 73.0 & 952.88 & 153.6 & 951.63 & 229.2 & 952.27 \\
\hline 182.0 & 950.84 & 262.0 & 952.38 & 76.0 & 952.89 & 155.0 & 951.67 & 230.4 & 952.52 \\
\hline 185.0 & 950.84 & 266.0 & 952.42 & 79.0 & 952.89 & 157.3 & 951.74 & 231.5 & 953.01 \\
\hline 188.0 & 950.84 & 268.0 & 952.48 & 82.0 & 952.89 & 157.4 & 951.54 & 233.0 & 953.11 \\
\hline 191.0 & 950.85 & 270.0 & 952.69 & 85.0 & 952.92 & 158.0 & 951.47 & 235.0 & 953.10 \\
\hline 194.0 & 950.83 & & & 88.0 & 952.92 & 160.0 & 951.41 & $237: 0$ & 953.12 \\
\hline
\end{tabular}


Table 33. (Continued) Listing of horizontal stations and elevations for cross section PR175

[Sta., station, distance in meters from a reference pin on the left bank; Elev., elevation, in meters above sea level]

\begin{tabular}{|c|c|c|c|c|c|c|c|c|c|}
\hline \multirow{2}{*}{\multicolumn{2}{|c|}{$\begin{array}{c}1995 \\
30 \text { September }\end{array}$}} & \multicolumn{2}{|c|}{1996} & \multicolumn{2}{|c|}{1996} & \multicolumn{2}{|c|}{1997} & \multicolumn{2}{|c|}{1997} \\
\hline & & 23 & tober & 23 & ober & $17 \mathrm{Se}$ & ember & $17 \mathrm{~S}$ & ember \\
\hline Sta. & Elev. & Sta. & Elev. & Sta. & Elev. & Sta. & Elev. & Sta. & Elev. \\
\hline 239.0 & 953.02 & 99.9 & 952.83 & 200.0 & 951.27 & 99.9 & 952.82 & 211.0 & 951.86 \\
\hline 241.0 & 953.06 & 103.0 & 952.85 & 202.2 & 951.40 & 124.0 & 952.89 & 212.0 & 951.86 \\
\hline 243.0 & 952.96 & 106.0 & 952.88 & 204.0 & 951.44 & 125.0 & 952.93 & 214.0 & 951.94 \\
\hline 245.0 & 952.82 & 109.0 & 952.89 & 206.0 & 951.48 & 128.0 & 952.95 & 217.0 & 952.05 \\
\hline 247.0 & 952.81 & 112.0 & 952.87 & 208.0 & 951.66 & 131.0 & 952.91 & 220.0 & 952.14 \\
\hline 248.0 & 952.75 & 113.5 & 952.85 & 211.0 & 951.70 & 134.0 & 952.97 & 222.0 & 952.13 \\
\hline 250.0 & 952.81 & 115.0 & 952.92 & 214.0 & 951.81 & 136.0 & 952.97 & 222.8 & 952.13 \\
\hline 251.0 & 952.84 & 118.0 & 952.94 & 217.0 & 951.87 & 138.0 & 953.00 & 224.0 & 952.37 \\
\hline 252.0 & 952.90 & 121.0 & 952.88 & 220.0 & 951.91 & 141.0 & 952.98 & 225.3 & 952.51 \\
\hline 253.6 & 952.93 & 124.0 & 952.90 & 222.5 & 952.02 & 143.0 & 952.82 & 226.0 & 952.48 \\
\hline 254.0 & 952.70 & 127.0 & 952.96 & 223.7 & 952.24 & 144.5 & 952.99 & 226.7 & 952.59 \\
\hline 255.2 & 952.62 & 130.0 & 952.90 & 224.7 & 952.29 & 147.0 & 953.01 & 228.0 & 952.57 \\
\hline 256.5 & 952.69 & 133.0 & 952.95 & 225.0 & 952.23 & 148.0 & 952.97 & 229.3 & 952.63 \\
\hline 258.0 & 952.68 & 136.0 & 952.95 & 226.0 & 952.26 & 149.0 & 952.85 & 230.1 & 952.75 \\
\hline 259.0 & 952.47 & 138.5 & 953.00 & 227.5 & 952.38 & 149.7 & 952.50 & 231.0 & 952.98 \\
\hline 261.0 & 952.56 & 140.0 & 952.98 & 227.8 & 952.54 & 150.3 & 951.27 & 233.0 & 952.99 \\
\hline 263.0 & 952.59 & 143.0 & 952.86 & 229.0 & 952.53 & 151.8 & 950.69 & 234.0 & 952.98 \\
\hline 265.0 & 952.66 & 145.0 & 952.96 & 231.0 & 952.86 & 153.5 & 950.84 & 235.5 & 952.87 \\
\hline 267.0 & 952.73 & 147.0 & 953.02 & 231.3 & 952.95 & 155.0 & 950.87 & 238.0 & 953.15 \\
\hline 269.0 & 952.77 & 149.0 & 952.92 & 233.0 & 953.02 & 158.0 & 950.91 & 240.0 & 953.01 \\
\hline 269.5 & 952.87 & 149.4 & 952.84 & 235.0 & 952.89 & 161.0 & 950.87 & 242.0 & 952.97 \\
\hline 271.0 & 952.80 & 150.6 & 951.81 & 236.0 & 953.10 & 164.0 & 950.98 & 245.0 & 952.92 \\
\hline 272.0 & 952.86 & 152.5 & 951.69 & 238.0 & 953.11 & 167.0 & 951.08 & 248.0 & 952.74 \\
\hline 274.0 & 952.87 & 155.0 & 951.87 & 240.0 & 953.04 & 170.0 & 951.12 & 250.0 & 952.83 \\
\hline 275.0 & 952.91 & 157.0 & 951.70 & 242.0 & 952.99 & 173.0 & 951.01 & & \\
\hline 276.5 & 952.72 & 158.4 & 951.83 & 243.0 & 952.91 & 175.5 & 950.91 & & \\
\hline 278.0 & 952.80 & 159.0 & 951.64 & 246.0 & 952.82 & 178.0 & 951.01 & & \\
\hline 280.0 & 952.84 & 160.0 & 951.54 & 248.0 & 952.75 & 180.0 & 951.20 & & \\
\hline 282.0 & 952.95 & 163.0 & 951.56 & 249.0 & 952.75 & 181.0 & 951.17 & & \\
\hline 284.0 & 953.03 & 166.0 & 951.53 & 250.0 & 952.84 & 182.0 & 951.26 & & \\
\hline 286.0 & 953.13 & 167.0 & 951.49 & & & 183.8 & 951.59 & & \\
\hline 288.0 & 953.21 & 167.8 & 951.41 & & & 185.0 & 951.62 & & \\
\hline 290.0 & 953.23 & 170.0 & 951.02 & & & 187.0 & 951.70 & & \\
\hline 292.0 & 953.22 & 173.0 & 950.98 & & & 189.0 & 951.80 & & \\
\hline 295.0 & 953.22 & 176.0 & 951.15 & & & 192.0 & 951.85 & & \\
\hline 300.0 & 953.15 & 179.0 & 951.18 & & & 194.0 & 951.85 & & \\
\hline 305.0 & 953.24 & 182.0 & 951.11 & & & 196.0 & 951.85 & & \\
\hline 310.0 & 953.22 & 185.0 & 951.07 & & & 198.0 & 951.83 & & \\
\hline 315.0 & 953.26 & 188.0 & 951.05 & & & 200.0 & 951.85 & & \\
\hline 320.0 & 953.39 & 191.0 & 951.10 & & & 203.0 & 951.85 & & \\
\hline & & 194.0 & 951.06 & & & 206.0 & 951.87 & & \\
\hline & & 197.0 & 951.07 & & & 209.0 & 951.85 & & \\
\hline
\end{tabular}


Table 33. (Continued) Listing of horizontal stations and elevations for cross section PR175

[Sta., station, distance in meters from a reference pin on the left bank; Elev., elevation, in meters above sea level]

\begin{tabular}{|c|c|c|c|}
\hline \multirow{2}{*}{\multicolumn{2}{|c|}{$\begin{array}{c}1998 \\
27 \text { September }\end{array}$}} & \multirow{2}{*}{\multicolumn{2}{|c|}{$\begin{array}{c}1998 \\
7 \text { September }\end{array}$}} \\
\hline & & & \\
\hline Sta. & Elev. & Sta. & Elev. \\
\hline 100.0 & 952.83 & 209.0 & 951.79 \\
\hline 103.0 & 952.85 & 210.7 & 951.74 \\
\hline 106.0 & 952.88 & 212.3 & 951.87 \\
\hline 109.0 & 952.90 & 213.1 & 952.07 \\
\hline 112.0 & 952.90 & 216.0 & 952.15 \\
\hline 115.0 & 952.91 & 219.0 & 952.17 \\
\hline 118.0 & 952.94 & 222.0 & 952.22 \\
\hline 121.0 & 952.88 & 224.0 & 952.40 \\
\hline 124.0 & 952.89 & 226.0 & 952.47 \\
\hline 125.0 & 952.93 & 228.0 & 952.59 \\
\hline 128.0 & 952.96 & 230.0 & 952.70 \\
\hline 131.0 & 952.94 & 231.5 & 952.93 \\
\hline 134.0 & 952.97 & 233.0 & 952.99 \\
\hline 137.0 & 952.97 & 235.0 & 952.95 \\
\hline 140.0 & 953.00 & 236.0 & 953.14 \\
\hline 143.0 & 952.86 & 238.0 & 953.09 \\
\hline 146.0 & 952.97 & 240.0 & 953.05 \\
\hline 147.7 & 952.89 & 243.0 & 952.94 \\
\hline 148.3 & 952.69 & 246.0 & 952.85 \\
\hline 148.3 & 951.98 & 248.5 & 952.76 \\
\hline 149.3 & 951.63 & 250.0 & 952.84 \\
\hline 151.3 & 950.70 & 300.0 & 953.15 \\
\hline 154.0 & 950.86 & 320.0 & 953.39 \\
\hline 157.0 & 950.90 & & \\
\hline 160.0 & 951.10 & & \\
\hline 163.0 & 951.07 & & \\
\hline 166.0 & 951.19 & & \\
\hline 169.0 & 951.25 & & \\
\hline 172.0 & 951.21 & & \\
\hline 175.0 & 951.06 & & \\
\hline 178.0 & 951.16 & & \\
\hline 181.0 & 951.21 & & \\
\hline 184.0 & 951.43 & & \\
\hline 187.0 & 951.51 & & \\
\hline 189.0 & 951.62 & & \\
\hline 192.0 & 951.77 & & \\
\hline 195.0 & 951.85 & & \\
\hline 198.0 & 951.86 & & \\
\hline 200.0 & 951.86 & & \\
\hline 202.3 & 951.86 & & \\
\hline 203.0 & 951.93 & & \\
\hline 206.0 & 951.87 & & \\
\hline
\end{tabular}




\section{Description of Cross Section PR180}

Location: Township 6 South/Range 50 East--section 12

U. S. Geological Survey quadrangle (1:24,000): Lonesome Peak

Landowners--left bank: EB Ranch

--right bank: U. S. Government

Access: Left bank

Permission from: Dick Wilson

Distance from Moorhead Gaging Station: 63.10 kilometers

Azimuth of Section (degrees magnetic): 103

\section{Reference Monuments}

[Monuments at stations $\mathbf{- 4 0 . 0}$ and $-\mathbf{6 2 . 0}$ were closest to leveling instrument; brass circular plate benchmark was lost to the river after 1996]

\begin{tabular}{|c|c|c|c|c|c|c|}
\hline \multirow[b]{2}{*}{ Description } & \multirow[b]{2}{*}{$\begin{array}{l}\text { Station } \\
\text { (m) }\end{array}$} & \multicolumn{2}{|c|}{ GPS-NAD83 (1992) } & \multicolumn{2}{|c|}{ Measurement } & \multirow{2}{*}{$\begin{array}{l}\text { Elevation } \\
\text { (NGVD1929) } \\
\text { (m) }\end{array}$} \\
\hline & & Latitude & Longitude & $\begin{array}{l}\text { Standard } \\
\text { deviation } \\
(m)\end{array}$ & $\begin{array}{l}\text { Horizontal } \\
\text { precision } \\
\quad(\mathbf{m})\end{array}$ & \\
\hline $\begin{array}{l}\text { 1/2-inch-rebar; } 0.18 \text { meter above } 1998 \text { ground } \\
\text { level and about } 2-3 \text { meters upstream from } \\
\text { a cottonwood tree }\end{array}$ & -62.0 & $45^{\circ} 19^{\prime} 51.46^{\prime \prime}$ & $105^{\circ} 32^{\prime} 38.16^{\prime \prime}$ & 0.615 & 0.711 & 950.15 \\
\hline $\begin{array}{l}\text { 1/2-inch-rebar; bent, } 0.14 \text { meter above } 1998 \\
\text { ground level }\end{array}$ & -40.0 & & & & & 949.99 \\
\hline $\begin{array}{l}\text { 1/2-inch-rebar; } 0.12 \text { meter above } 1998 \text { ground } \\
\text { level }\end{array}$ & 73.0 & $45^{\circ} 19^{\prime} 49.49^{\prime \prime}$ & $105^{\circ} 32^{\prime} 32.61^{\prime \prime}$ & 0.241 & 0.559 & 949.13 \\
\hline $\begin{array}{l}\text { 1/2-inch-rebar; at } 1978 \text { ground level; could } \\
\text { not find pin in } 1998\end{array}$ & 102.0 & & & & & 948.91 \\
\hline $\begin{array}{l}\text { 1/2-inch-rebar; } 0.03 \text { meter above } 1998 \text { ground } \\
\text { level; } 0.40 \text { meter upstream from section }\end{array}$ & 103.4 & & & & & 948.96 \\
\hline $\begin{array}{l}\text { 1/2-inch-rebar; } 0.22 \text { meter above } 1998 \text { ground } \\
\text { level }\end{array}$ & 111.3 & $45^{\circ} 19^{\prime} 48.94^{\prime \prime}$ & 105'32'31.04" & 0.339 & 0.508 & 949.77 \\
\hline
\end{tabular}



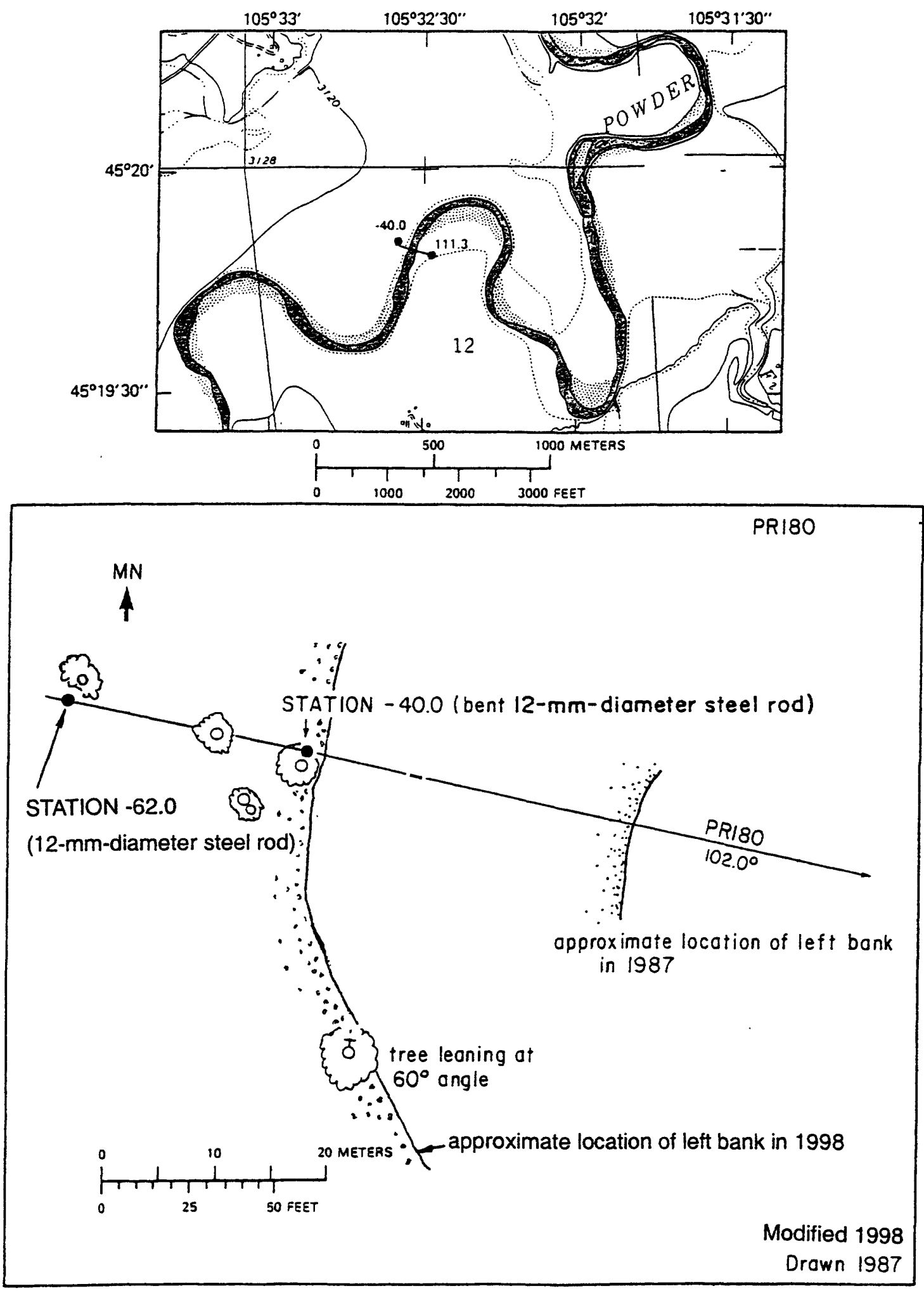

Figure 92. Upper: Location of cross section PR180 and the left and right bank reference monuments in the Lonesome Peak quadrangle. Bench mark PR180 was lost to the river in 1996. Lower: Location of the reference monuments on the left bank. MN is magnetic north. 

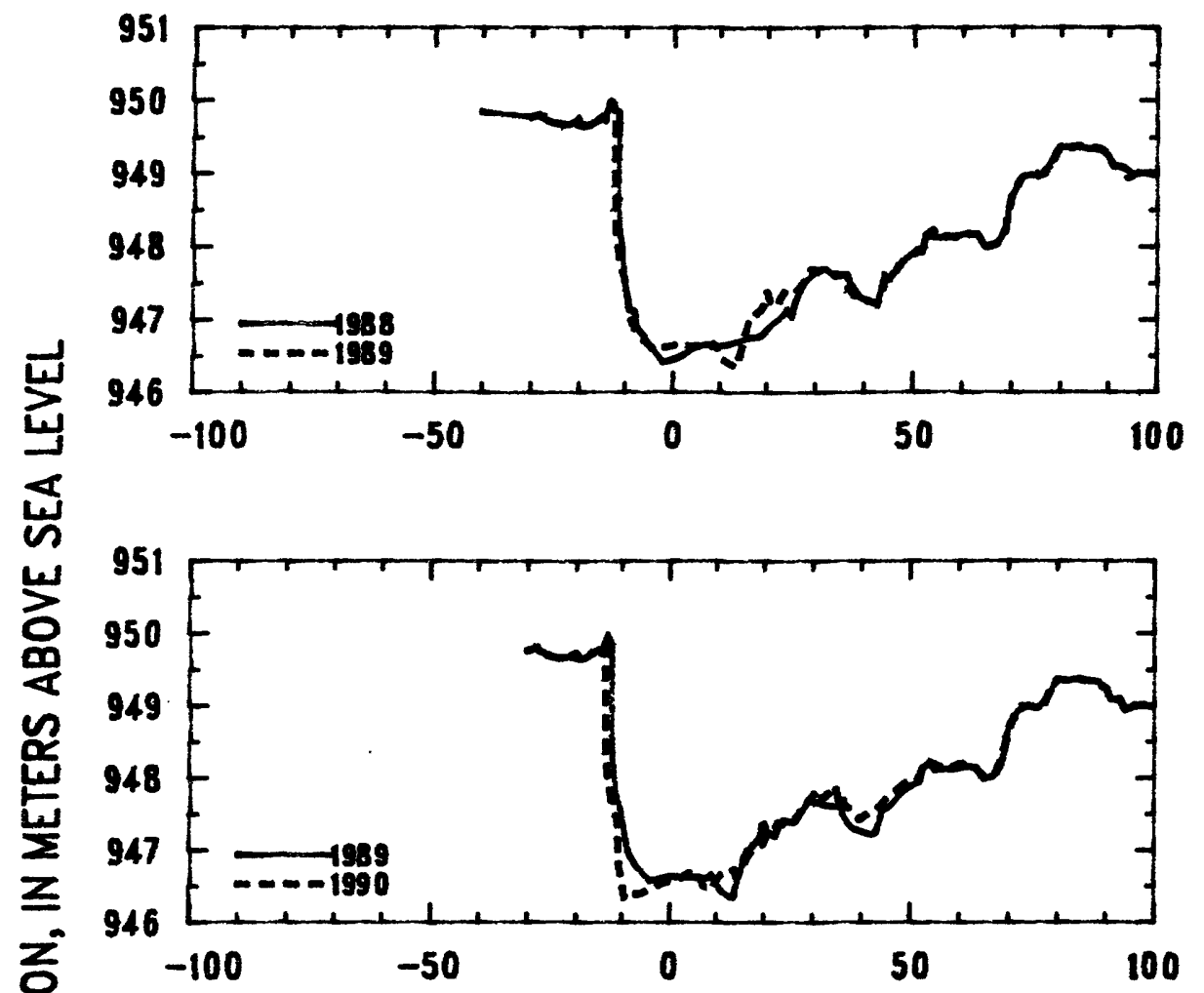

E
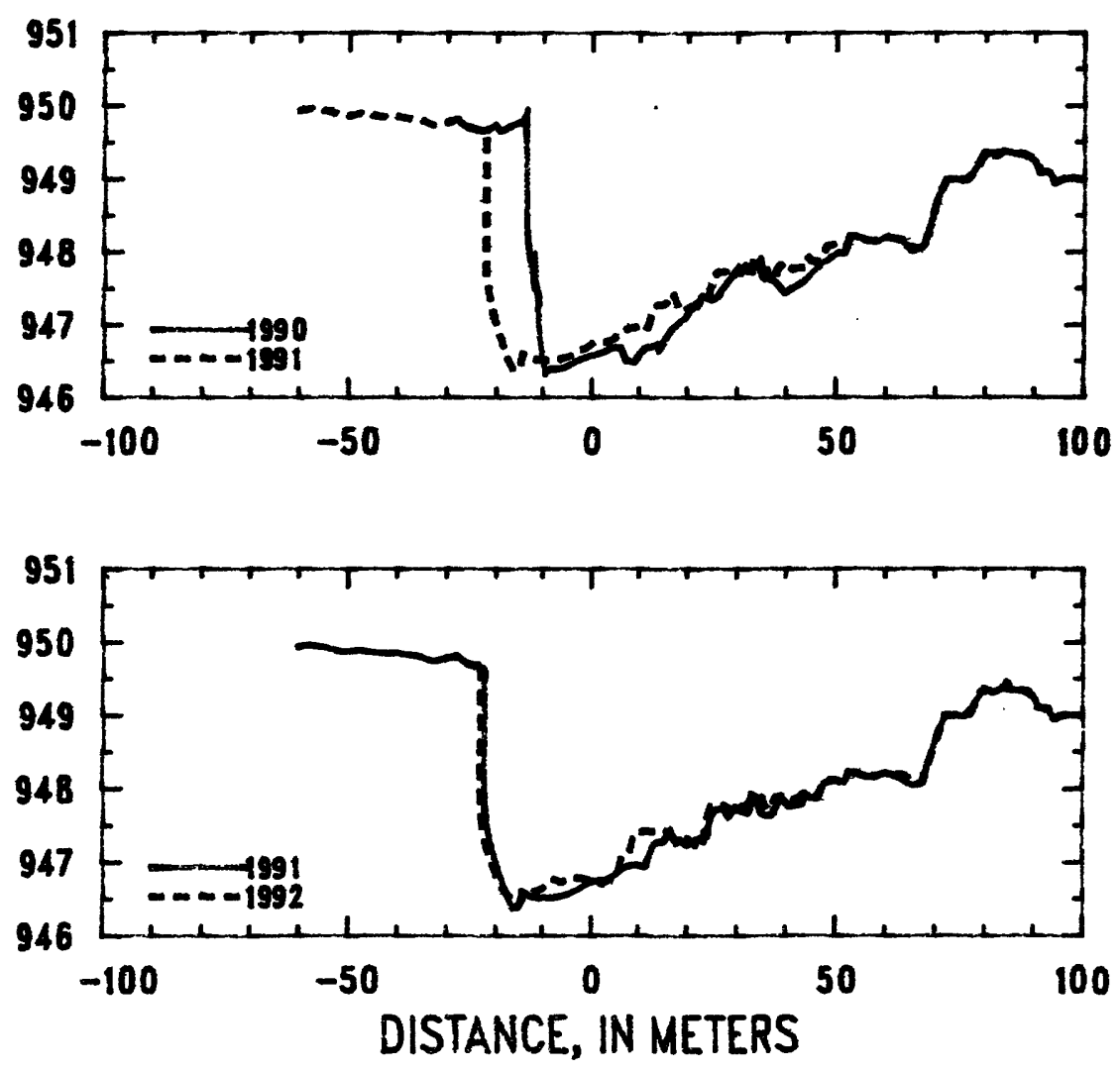

Figure 93. Profiles of cross section PR180 from 1988 to 1992. 

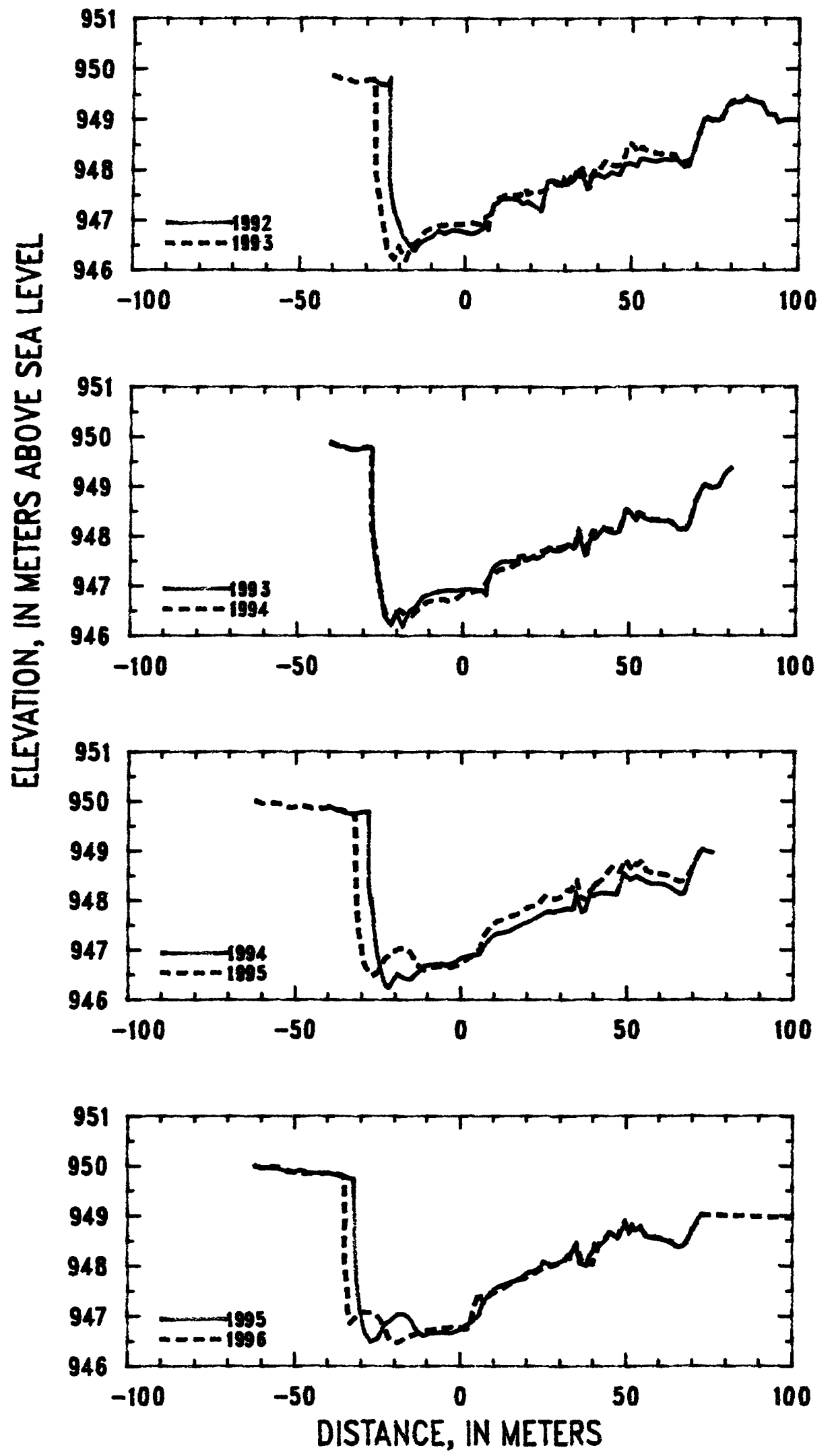

Figure 94. Profiles of cross section PR180 from 1992 to 1996. 


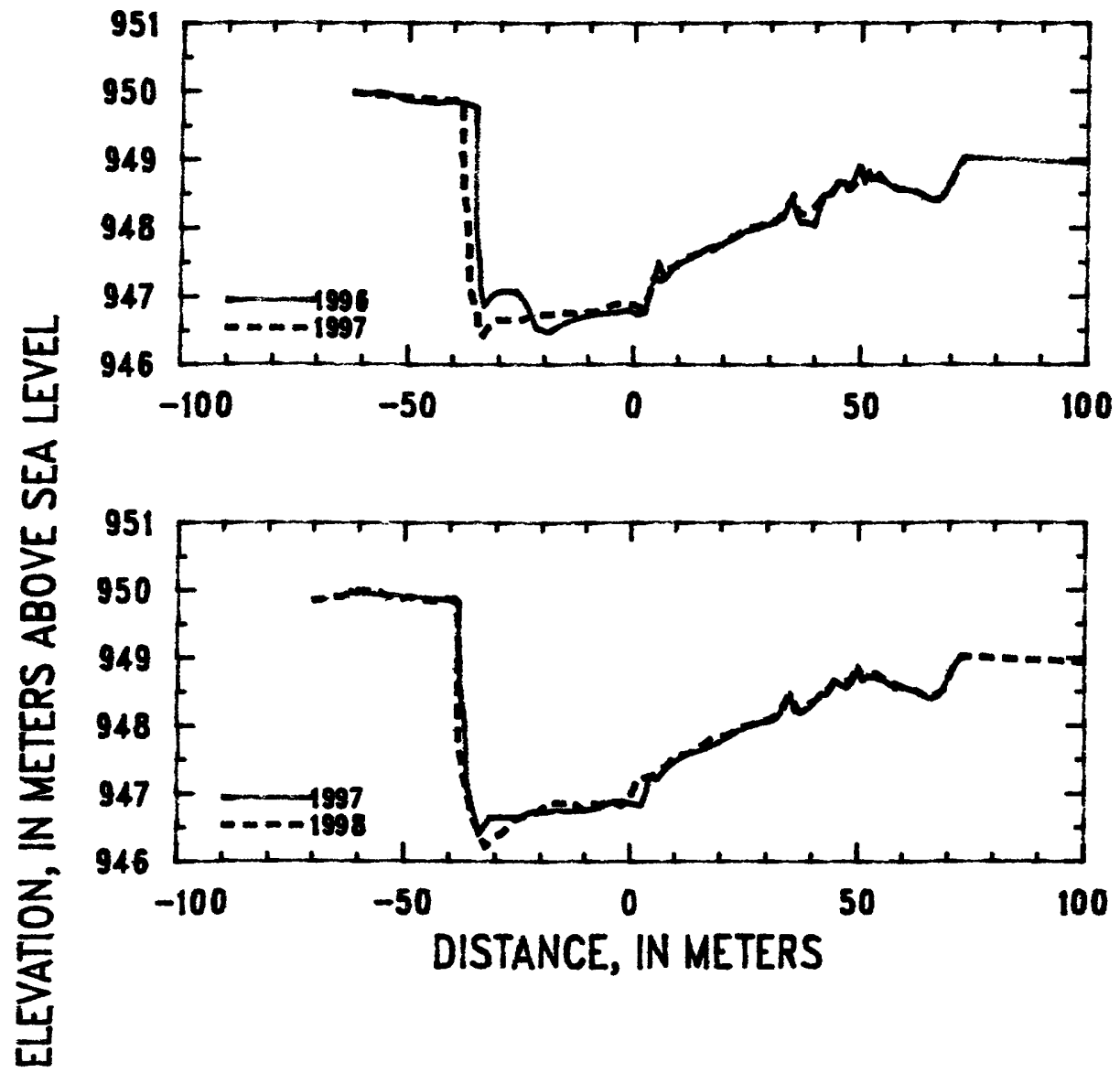

Figure 95. Profiles of cross section PR180 from 1996 to 1998. 
Table 34. Listing of horizontal stations and elevations for cross section PR180

[Sta., station, distance in meters from a reference pin on the left bank; Elev., elevation, in meters above sea level]

\begin{tabular}{|c|c|c|c|c|c|c|c|c|c|}
\hline \multirow{2}{*}{\multicolumn{2}{|c|}{$\begin{array}{c}1989 \\
23 \text { September }\end{array}$}} & \multicolumn{2}{|c|}{1989} & \multicolumn{2}{|c|}{1989} & \multicolumn{2}{|c|}{1990} & \multicolumn{2}{|c|}{1990} \\
\hline & & $23 \mathrm{~S}$ & ember & $23 \mathrm{Se}$ & ember & $22 \mathrm{Se}$ & ember & $22 \mathrm{~S}$ & ember \\
\hline Sta. & Elev. & Sta. & Elev. & Sta. & Elev. & Sta. & Elev. & Sta. & Elev. \\
\hline-30.0 & 949.76 & 24.0 & 947.42 & 94.0 & 948.94 & -30.0 & 949.77 & 26.0 & 947.39 \\
\hline-28.0 & 949.80 & 26.0 & 947.40 & 96.0 & 949.01 & -28.0 & 949.82 & 26.5 & 947.46 \\
\hline-27.0 & 949.76 & 28.0 & 947.55 & 98.0 & 949.00 & -26.0 & 949.71 & 28.0 & 947.59 \\
\hline-25.0 & 949.69 & 29.0 & 947.69 & 100.0 & 948.99 & -24.0 & 949.67 & 29.0 & 947.69 \\
\hline-23.0 & 949.66 & 31.0 & 947.68 & 103.5 & 948.91 & -22.0 & 949.66 & 30.2 & 947.79 \\
\hline-21.0 & 949.69 & 33.0 & 947.62 & 111.3 & 949.55 & -20.0 & 949.74 & 30.6 & 947.75 \\
\hline-19.0 & 949.64 & 35.0 & 947.61 & & & -19.0 & 949.65 & 31.6 & 947.82 \\
\hline-17.0 & 949.69 & 36.0 & 947.61 & & & -17.0 & 949.72 & 32.0 & 947.73 \\
\hline-15.0 & 949.77 & 36.4 & 947.47 & & & -15.0 & 949.78 & 32.5 & 947.73 \\
\hline-14.3 & 949.74 & 38.0 & 947.32 & & & -14.3 & 949.75 & 32.9 & 947.77 \\
\hline-13.0 & 949.98 & 40.0 & 947.27 & & & -13.6 & 949.88 & 34.0 & 947.82 \\
\hline-12.2 & 949.86 & 42.0 & 947.21 & & & -13.4 & 948.36 & 34.7 & 947.90 \\
\hline-12.1 & 948.23 & 42.9 & 947.24 & & & -13.2 & 948.11 & 35.7 & 947.63 \\
\hline-11.0 & 947.71 & 44.3 & 947.55 & & & -12.1 & 947.62 & 36.0 & 947.63 \\
\hline-10.3 & 947.59 & 46.0 & 947.62 & & & -11.9 & 947.78 & 36.3 & 947.71 \\
\hline-9.1 & 947.16 & 48.0 & 947.80 & & & -11.6 & 947.50 & 36.7 & 947.63 \\
\hline-8.5 & 946.96 & 50.0 & 947.88 & & & -11.0 & 947.43 & 38.0 & 947.58 \\
\hline-7.5 & 946.84 & 51.9 & 947.95 & & & -10.8 & 946.86 & 39.6 & 947.43 \\
\hline-6.0 & 946.71 & 52.4 & 948.13 & & & -9.4 & 946.33 & 41.0 & 947.49 \\
\hline-4.0 & 946.58 & 54.0 & 948.22 & & & -8.5 & 946.39 & 43.0 & 947.56 \\
\hline-2.0 & 946.61 & 55.0 & 948.11 & & & -6.0 & 946.40 & 45.0 & 947.71 \\
\hline 0.0 & 946.64 & 57.0 & 948.13 & & & -4.0 & 946.47 & 47.0 & 947.83 \\
\hline 2.0 & 946.63 & 59.0 & 948.11 & & & -2.0 & 946.53 & 49.0 & 947.94 \\
\hline 4.0 & 946.63 & 61.0 & 948.17 & & & 0.0 & 946.58 & 50.5 & 947.99 \\
\hline 6.0 & 946.63 & 63.0 & 948.16 & & & 2.0 & 946.60 & 51.6 & 947.98 \\
\hline 8.0 & 946.61 & 65.0 & 947.99 & & & 4.0 & 946.69 & 52.0 & 948.05 \\
\hline 9.0 & 946.66 & 67.0 & 948.02 & & & 6.0 & 946.69 & 52.6 & 948.20 \\
\hline 11.0 & 946.44 & 68.0 & 948.12 & & & 7.0 & 946.50 & 54.0 & 948.22 \\
\hline 13.0 & 946.36 & 68.7 & 948.20 & & & 9.0 & 946.48 & 56.0 & 948.16 \\
\hline 13.7 & 946.35 & 70.0 & 948.67 & & & 11.0 & 946.66 & 58.0 & 948.14 \\
\hline 15.7 & 946.85 & 72.0 & 948.93 & & & 13.0 & 946.71 & 60.0 & 948.20 \\
\hline 15.8 & 946.90 & 74.0 & 949.01 & & & 14.0 & 946.74 & 62.0 & 948.18 \\
\hline 16.5 & 947.01 & 76.0 & 948.97 & & & 14.3 & 946.66 & 63.0 & 948.18 \\
\hline 17.0 & 947.00 & 78.0 & 949.11 & & & 16.0 & 946.85 & 64.0 & 948.09 \\
\hline 18.0 & 947.10 & 80.0 & 949.37 & & & 16.8 & 946.95 & 65.0 & 948.01 \\
\hline 19.1 & 947.16 & 82.0 & 949.34 & & & 18.0 & 947.01 & 66.0 & 948.03 \\
\hline 19.4 & 947.23 & 84.0 & 949.38 & & & 19.8 & 947.10 & 67.4 & 948.06 \\
\hline 19.6 & 947.36 & 86.0 & 949.34 & & & 20.6 & 947.22 & 69.0 & 948.39 \\
\hline 20.0 & 947.38 & 88.0 & 949.35 & & & 22.0 & 947.25 & 69.3 & 948.44 \\
\hline 20.5 & 947.25 & 90.0 & 949.25 & & & 22.6 & 947.38 & 70.0 & 948.67 \\
\hline 22.3 & 947.20 & 91.0 & 949.10 & & & 23.0 & 947.40 & 71.0 & 948.82 \\
\hline 22.7 & 947.31 & 93.0 & 949.09 & & & 24.6 & 947.34 & 72.1 & 948.99 \\
\hline
\end{tabular}


Table 34. (Continued) Listing of horizontal stations and elevations for cross section PR180

[Sta., station, distance in meters from a reference pin on the left bank; Elev., elevation, in meters above sea level]

\begin{tabular}{|c|c|c|c|c|c|c|c|c|c|}
\hline \multirow{2}{*}{\multicolumn{2}{|c|}{$\begin{array}{c}1990 \\
22 \text { September }\end{array}$}} & \multirow{2}{*}{\multicolumn{2}{|c|}{$\begin{array}{c}1991 \\
1 \text { September }\end{array}$}} & \multirow{2}{*}{\multicolumn{2}{|c|}{$\begin{array}{c}1991 \\
1 \text { September }\end{array}$}} & \multirow{2}{*}{\multicolumn{2}{|c|}{$\begin{array}{c}1991 \\
1 \text { September }\end{array}$}} & \multirow{2}{*}{\multicolumn{2}{|c|}{$\begin{array}{l}1992 \\
28 \text { August }\end{array}$}} \\
\hline & & & & & & & & & \\
\hline Sta. & Elev. & Sta. & Elev. & Sta. & Elev. & Sta. & Elev. & Sta. & Elev. \\
\hline 74.0 & 949.00 & -60.0 & 949.93 & 13.2 & 947.23 & 69.3 & 948.43 & -30.0 & 949.78 \\
\hline 76.0 & 948.97 & -58.0 & 949.96 & 13.9 & 947.28 & 70.4 & 948.74 & -28.0 & 949.81 \\
\hline 77.0 & 949.01 & -56.0 & 949.95 & 15.3 & 947.28 & 72.0 & 948.99 & -26.0 & 949.70 \\
\hline 79.0 & 949.26 & -54.0 & 949.92 & 16.8 & 947.44 & 74.0 & 949.00 & -25.0 & 949.70 \\
\hline 80.0 & 949.37 & -52.0 & 949.88 & 18.0 & 947.26 & 76.0 & 948.98 & -24.0 & 949.68 \\
\hline 82.0 & 949.35 & -50.0 & 949.86 & 18.8 & 947.30 & 78.0 & 949.13 & -23.4 & 949.68 \\
\hline 84.0 & 949.38 & -48.0 & 949.89 & 20.0 & 947.23 & 80.0 & 949.37 & -22.9 & 949.76 \\
\hline 86.0 & 949.35 & -46.0 & 949.87 & 22.0 & 947.31 & 82.0 & 949.31 & -22.9 & 947.82 \\
\hline 88.0 & 949.32 & -44.0 & 949.85 & 24.0 & 947.35 & 84.0 & 949.37 & -22.5 & 947.51 \\
\hline 90.0 & 949.24 & -42.0 & 949.83 & 24.9 & 947.65 & 86.0 & 949.35 & -21.5 & 947.17 \\
\hline 91.0 & 949.09 & -40.0 & 949.86 & 26.0 & 947.73 & 88.0 & 949.34 & -20.5 & 947.00 \\
\hline 93.0 & 949.09 & -38.0 & 949.82 & 27.5 & 947.73 & 90.0 & 949.26 & -19.0 & 946.70 \\
\hline 94.0 & 948.94 & -36.0 & 949.82 & 28.5 & 947.62 & 91.0 & 949.11 & -17.0 & 946.53 \\
\hline 96.0 & 949.00 & -33.0 & 949.74 & 29.6 & 947.73 & 93.0 & 949.09 & -16.0 & 946.42 \\
\hline \multirow[t]{28}{*}{98.0} & 949.01 & -30.0 & 949.77 & 31.0 & 947.70 & 94.0 & 948.95 & -15.0 & 946.39 \\
\hline & & -28.0 & 949.82 & 32.6 & 947.67 & 96.0 & 949.01 & -14.0 & 946.50 \\
\hline & & -26.0 & 949.71 & 32.9 & 947.73 & 98.0 & 949.00 & -12.0 & 946.60 \\
\hline & & -24.0 & 949.67 & 33.0 & 947.85 & 100.0 & 948.99 & -10.0 & 946.64 \\
\hline & & -23.0 & 949.65 & 34.0 & 947.89 & 102.0 & 948.92 & -8.0 & 946.78 \\
\hline & & -22.2 & 949.61 & 35.2 & 947.65 & 104.0 & 948.95 & -6.0 & 946.72 \\
\hline & & -22.4 & 947.78 & 36.0 & 947.63 & 106.0 & 949.07 & -4.0 & 946.79 \\
\hline & & -22.2 & 947.74 & 37.5 & 947.64 & 108.0 & 949.34 & -2.0 & 946.79 \\
\hline & & -21.5 & 947.39 & 38.4 & 947.80 & 111.3 & 949.55 & 0.0 & 946.75 \\
\hline & & -20.0 & 947.01 & 39.5 & 947.85 & & & 2.0 & 946.70 \\
\hline & & -18.2 & 946.65 & 40.8 & 947.75 & & & 4.0 & 946.76 \\
\hline & & -16.4 & 946.37 & 42.0 & 947.77 & & & 5.7 & 946.85 \\
\hline & & -15.4 & 946.40 & 43.3 & 947.78 & & & 6.0 & 946.99 \\
\hline & & -14.8 & 946.44 & 44.1 & 947.93 & & & 6.2 & 947.06 \\
\hline & & -14.3 & 946.60 & 45.0 & 947.87 & & & 8.0 & 947.13 \\
\hline & & -12.0 & 946.52 & 46.5 & 947.87 & & & 8.8 & 947.31 \\
\hline & & -10.0 & 946.52 & 48.0 & 948.08 & & & 10.0 & 947.35 \\
\hline & & -8.0 & 946.51 & 50.0 & 948.12 & & & 10.6 & 947.42 \\
\hline & & -6.0 & 946.53 & 52.0 & 948.08 & & & 12.0 & 947.43 \\
\hline & & -4.0 & 946.58 & 52.7 & 948.22 & & & 13.5 & 947.44 \\
\hline & & -2.2 & 946.65 & 54.0 & 948.21 & & & 15.0 & 947.42 \\
\hline & & 0.0 & 946.75 & 56.0 & 948.16 & & & 16.0 & 947.42 \\
\hline & & 2.0 & 946.75 & 58.0 & 948.15 & & & 17.5 & 947.32 \\
\hline & & 4.0 & 946.78 & 60.0 & 948.21 & & & 19.0 & 947.36 \\
\hline & & 6.0 & 946.89 & 62.0 & 948.19 & & & 21.0 & 947.27 \\
\hline & & 8.0 & 946.96 & 64.0 & 948.09 & & & 22.9 & 947.18 \\
\hline & & 10.0 & 946.97 & 66.0 & 948.04 & & & 23.1 & 947.23 \\
\hline & & 11.5 & 946.93 & 67.4 & 948.07 & & & 24.7 & 947.77 \\
\hline
\end{tabular}


Table 34. (Continued) Listing of horizontal stations and elevations for cross section PR180

[Sta., station, distance in meters from a reference pin on the left bank; Elev., elevation, in meters above sea level]

\begin{tabular}{|c|c|c|c|c|c|c|c|c|c|}
\hline \multirow{2}{*}{\multicolumn{2}{|c|}{$\begin{array}{c}1992 \\
28 \text { August }\end{array}$}} & \multirow{2}{*}{\multicolumn{2}{|c|}{$\begin{array}{c}1992 \\
28 \text { August }\end{array}$}} & \multirow{2}{*}{\multicolumn{2}{|c|}{$\begin{array}{c}1993 \\
31 \text { August }\end{array}$}} & \multirow{2}{*}{\multicolumn{2}{|c|}{$\begin{array}{c}1993 \\
31 \text { August }\end{array}$}} & \multirow{2}{*}{\multicolumn{2}{|c|}{$\begin{array}{c}1993 \\
31 \text { August }\end{array}$}} \\
\hline & & & & & & & & & \\
\hline Sta. & Elev. & Sta. & Elev. & Sta. & Elev. & Sta. & Elev. & Sta. & Elev. \\
\hline 26.0 & 947.80 & 84.6 & 949.45 & -40.0 & 949.87 & 19.0 & 947.52 & 75.0 & 948.96 \\
\hline 28.0 & 947.77 & 85.0 & 949.39 & -38.0 & 949.82 & 21.0 & 947.58 & 77.0 & 949.00 \\
\hline 29.0 & 947.71 & 87.0 & 949.35 & -36.0 & 949.82 & 22.3 & 947.55 & 79.0 & 949.27 \\
\hline 29.6 & 947.83 & 89.0 & 949.33 & -34.0 & 949.74 & 24.0 & 947.60 & 81.0 & 949.38 \\
\hline 30.0 & 947.72 & 91.0 & 949.10 & -32.0 & 949.75 & 26.5 & 947.77 & & \\
\hline 31.0 & 947.73 & 93.0 & 949.09 & -31.0 & 949.75 & 27.0 & 947.72 & & \\
\hline 31.7 & 947.84 & 94.0 & 948.94 & -30.0 & 949.77 & 29.0 & 947.71 & & \\
\hline 32.0 & 947.77 & 96.0 & 949.00 & -29.0 & 949.80 & 31.0 & 947.78 & & \\
\hline 32.7 & 947.82 & 98.0 & 949.00 & -27.4 & 949.77 & 31.6 & 947.84 & & \\
\hline 33.4 & 947.96 & 100.0 & 948.98 & -27.3 & 948.40 & 32.6 & 947.82 & & \\
\hline 34.2 & 947.96 & 102.0 & 948.92 & -27.0 & 947.86 & 33.3 & 947.76 & & \\
\hline 35.0 & 947.91 & 104.0 & 948.95 & -26.4 & 947.54 & 33.9 & 947.80 & & \\
\hline 36.2 & 947.72 & 106.0 & 949.09 & -26.2 & 947.50 & 34.8 & 948.11 & & \\
\hline 37.5 & 947.75 & 108.0 & 949.34 & -25.5 & 947.10 & 36.9 & 947.62 & & \\
\hline 38.0 & 947.88 & 111.3 & 949.56 & -25.2 & 947.07 & 37.5 & 947.65 & & \\
\hline 40.0 & 947.92 & & & -23.5 & 946.38 & 38.0 & 947.85 & & \\
\hline 41.0 & 947.86 & & & -21.5 & 946.22 & 39.0 & 948.00 & & \\
\hline 42.0 & 947.84 & & & -20.0 & 946.47 & 39.8 & 947.97 & & \\
\hline 44.0 & 947.95 & & & -18.0 & 946.20 & 41.0 & 948.06 & & \\
\hline 46.4 & 947.89 & & & -16.0 & 946.50 & 42.3 & 948.18 & & \\
\hline 48.0 & 948.12 & & & -14.0 & 946.57 & 43.5 & 948.10 & & \\
\hline 50.0 & 948.12 & & & -12.0 & 946.78 & 45.0 & 948.07 & & \\
\hline 51.6 & 948.07 & & & -10.0 & 946.83 & 46.0 & 948.07 & & \\
\hline 52.7 & 948.21 & & & -8.0 & 946.87 & 47.0 & 948.10 & & \\
\hline 54.0 & 948.22 & & & -6.0 & 946.89 & 48.0 & 948.32 & & \\
\hline 56.0 & 948.17 & & & -4.0 & 946.92 & 48.6 & 948.51 & & \\
\hline 58.0 & 948.16 & & & -2.0 & 946.89 & 50.0 & 948.50 & & \\
\hline 60.0 & 948.21 & & & 0.0 & 946.92 & 52.0 & 948.32 & & \\
\hline 62.0 & 948.20 & & & 2.0 & 946.93 & 53.0 & 948.45 & & \\
\hline 64.0 & 948.19 & & & 4.0 & 946.91 & 55.0 & 948.34 & & \\
\hline 66.0 & 948.05 & & & 6.0 & 946.90 & 57.0 & 948.33 & & \\
\hline 67.4 & 948.07 & & & 7.0 & 946.82 & 59.0 & 948.31 & & \\
\hline 69.0 & 948.39 & & & 7.5 & 947.07 & 61.0 & 948.30 & & \\
\hline 71.0 & 948.82 & & & 8.2 & 947.19 & 63.0 & 948.29 & & \\
\hline 72.0 & 949.00 & & & 8.7 & 947.32 & 65.0 & 948.14 & & \\
\hline 74.0 & 949.01 & & & 10.0 & 947.42 & 67.0 & 948.14 & & \\
\hline 76.0 & 948.97 & & & 12.0 & 947.49 & 68.0 & 948.20 & & \\
\hline 77.0 & 949.01 & & & 14.0 & 947.49 & 69.0 & 948.40 & & \\
\hline 79.0 & 949.28 & & & 16.0 & 947.50 & 70.0 & 948.65 & & \\
\hline 80.0 & 949.36 & & & 16.9 & 947.50 & 71.0 & 948.82 & & \\
\hline 82.0 & 949.33 & & & 17.1 & 947.60 & 72.0 & 948.95 & & \\
\hline 84.0 & 949.38 & & & 18.5 & 947.61 & 73.0 & 949.02 & & \\
\hline
\end{tabular}


Table 34. (Continued) Listing of horizontal stations and elevations for cross section PR180

[Sta., station, distance in meters from a reference pin on the left bank; Elev., elevation, in meters above sea level]

\begin{tabular}{|c|c|c|c|c|c|c|c|c|c|}
\hline \multirow{2}{*}{\multicolumn{2}{|c|}{$\begin{array}{c}1994 \\
18 \text { September }\end{array}$}} & \multicolumn{2}{|c|}{1994} & \multicolumn{2}{|c|}{1995} & \multicolumn{2}{|c|}{1995} & \multicolumn{2}{|c|}{1996} \\
\hline & & $18 \mathrm{~S}$ & ember & $30 \mathrm{Se}$ & ember & $30 \mathrm{Se}$ & ember & 22 & tober \\
\hline Sta. & Elev. & Sta. & Elev. & Sta. & Elev. & Sta. & Elev. & Sta. & Elev. \\
\hline-40.0 & 949.89 & 36.5 & 947.76 & -62.0 & 950.00 & 17.0 & 947.71 & -62.0 & 949.99 \\
\hline-37.0 & 949.81 & 37.4 & 947.79 & -60.0 & 949.95 & 19.0 & 947.78 & -59.0 & 949.95 \\
\hline-34.0 & 949.74 & 38.0 & 947.93 & -58.0 & 949.96 & 20.5 & 947.87 & -56.5 & 949.99 \\
\hline-32.0 & 949.75 & 39.0 & 948.12 & -55.0 & 949.95 & 22.0 & 947.88 & -54.0 & 949.97 \\
\hline-30.0 & 949.78 & 40.0 & 948.10 & -53.0 & 949.89 & 23.6 & 947.94 & -51.0 & 949.87 \\
\hline-27.8 & 949.79 & 42.0 & 948.15 & -51.0 & 949.86 & 25.0 & 948.08 & -46.5 & 949.84 \\
\hline-27.7 & 948.24 & 44.0 & 948.15 & -48.0 & 949.91 & 27.0 & 948.02 & -44.0 & 949.83 \\
\hline-27.3 & 948.05 & 46.0 & 948.13 & -46.0 & 949.86 & 29.0 & 948.04 & -42.0 & 949.85 \\
\hline-27.2 & 948.02 & 46.8 & 948.12 & -44.0 & 949.84 & 31.0 & 948.11 & -40.0 & 949.86 \\
\hline-26.6 & 947.77 & 49.0 & 948.54 & -42.0 & 949.85 & 32.3 & 948.22 & -37.0 & 949.82 \\
\hline-25.5 & 947.08 & 51.0 & 948.42 & -40.0 & 949.87 & 33.0 & 948.20 & -35.0 & 949.76 \\
\hline-24.5 & 946.71 & 53.0 & 948.48 & -38.0 & 949.82 & 34.6 & 948.41 & -34.9 & 948.02 \\
\hline-22.7 & 946.29 & 55.0 & 948.40 & -36.0 & 949.83 & 35.0 & 948.40 & -34.0 & 947.43 \\
\hline-21.7 & 946.24 & 57.0 & 948.33 & -34.0 & 949.74 & 36.0 & 948.06 & -33.8 & 947.06 \\
\hline-20.0 & 946.42 & 60.0 & 948.33 & -32.1 & 949.74 & 38.0 & 948.00 & -33.0 & 946.86 \\
\hline-19.0 & 946.52 & 62.0 & 948.31 & -31.4 & 947.72 & 39.0 & 948.20 & -31.0 & 947.03 \\
\hline-17.0 & 946.42 & 64.0 & 948.21 & -30.4 & 947.15 & 40.0 & 948.26 & -29.0 & 947.08 \\
\hline-15.0 & 946.41 & 66.0 & 948.13 & -29.0 & 946.67 & 40.6 & 948.41 & -26.0 & 947.07 \\
\hline-14.0 & 946.48 & 67.3 & 948.14 & -27.0 & 946.48 & 41.0 & 948.33 & -24.2 & 946.93 \\
\hline-11.7 & 946.60 & 69.0 & 948.44 & -25.0 & 946.55 & 42.2 & 948.40 & -21.5 & 946.53 \\
\hline-11.0 & 946.67 & 70.5 & 948.76 & -23.0 & 946.82 & 43.5 & 948.48 & -19.0 & 946.47 \\
\hline-8.5 & 946.70 & 72.0 & 948.96 & -21.0 & 946.91 & 45.3 & 948.66 & -16.0 & 946.59 \\
\hline-5.5 & 946.73 & 73.0 & 949.02 & -19.0 & 947.03 & 47.5 & 948.55 & -13.0 & 946.66 \\
\hline-4.4 & 946.69 & 76.0 & 948.96 & -17.0 & 947.04 & 49.5 & 948.82 & -10.0 & 946.71 \\
\hline-2.5 & 946.72 & & & -15.0 & 946.95 & 51.0 & 948.66 & -7.0 & 946.75 \\
\hline-1.8 & 946.75 & & & -13.0 & 946.69 & 51.7 & 948.78 & -4.0 & 946.76 \\
\hline 0.0 & 946.84 & & & -11.0 & 946.60 & 53.0 & 948.71 & -1.0 & 946.80 \\
\hline 3.0 & 946.88 & & & -9.0 & 946.65 & 54.5 & 948.79 & 1.0 & 946.73 \\
\hline 5.6 & 946.92 & & & -7.0 & 946.66 & 56.0 & 948.59 & 2.5 & 946.76 \\
\hline 8.0 & 947.18 & & & -5.0 & 946.68 & 58.0 & 948.55 & 5.5 & 947.48 \\
\hline 11.0 & 947.33 & & & -3.0 & 946.65 & 60.0 & 948.53 & 7.0 & 947.26 \\
\hline 14.0 & 947.36 & & & -1.0 & 946.69 & 62.0 & 948.51 & 9.0 & 947.42 \\
\hline 17.0 & 947.47 & & & 1.0 & 946.78 & 64.0 & 948.43 & 11.0 & 947.50 \\
\hline 20.0 & 947.55 & & & 3.0 & 946.85 & 66.0 & 948.37 & 13.0 & 947.55 \\
\hline 23.0 & 947.66 & & & 5.0 & 946.98 & 67.5 & 948.39 & 15.0 & 947.64 \\
\hline 26.0 & 947.77 & & & 5.8 & 947.02 & 68.5 & 948.48 & 17.0 & 947.72 \\
\hline 28.0 & 947.76 & & & 6.4 & 947.17 & 70.0 & 948.68 & 19.0 & 947.74 \\
\hline 30.5 & 947.81 & & & 7.0 & 947.30 & 71.0 & 948.82 & 21.0 & 947.80 \\
\hline 32.0 & 947.85 & & & 9.0 & 947.45 & 72.0 & 948.96 & 23.0 & 947.88 \\
\hline 33.0 & 947.82 & & & 11.0 & 947.58 & 73.0 & 949.02 & 25.0 & 947.95 \\
\hline 33.8 & 947.83 & & & 13.0 & 947.61 & & & 27.0 & 947.99 \\
\hline 34.6 & 948.13 & & & 15.0 & 947.67 & & & 29.0 & 948.03 \\
\hline
\end{tabular}


Table 34. (Continued) Listing of horizontal stations and elevations for cross section PR180

[Sta., station, distance in meters from a reference pin on the left bank; Elev., elevation, in meters above sea level]

\begin{tabular}{|c|c|c|c|c|c|c|c|c|c|}
\hline \multirow{2}{*}{\multicolumn{2}{|c|}{$\begin{array}{c}1996 \\
22 \text { October }\end{array}$}} & \multirow{2}{*}{\multicolumn{2}{|c|}{$\begin{array}{c}1997 \\
\text { 22 September }\end{array}$}} & \multicolumn{2}{|c|}{1997} & \multicolumn{2}{|c|}{1998} & \multicolumn{2}{|c|}{1998} \\
\hline & & & & $22 \mathrm{~S}$ & ember & $24 \mathrm{Se}$ & ember & $24 S$ & ember \\
\hline Sta. & Elev. & Sta. & Elev. & Sta. & Elev. & Sta. & Elev. & Sta. & Elev. \\
\hline 31.0 & 948.08 & -62.0 & 949.97 & 40.0 & 948.30 & -70.0 & 949.86 & 10.0 & 947.56 \\
\hline 32.3 & 948.18 & -40.0 & 949.86 & 42.0 & 948.47 & -68.0 & 949.87 & 12.5 & 947.58 \\
\hline 33.0 & 948.14 & -39.0 & 949.89 & 43.3 & 948.47 & -66.0 & 949.91 & 15.0 & 947.65 \\
\hline 35.0 & 948.44 & -38.0 & 949.82 & 45.0 & 948.65 & -64.0 & 949.90 & 18.0 & 947.81 \\
\hline 36.3 & 948.14 & -38.0 & 949.05 & 47.5 & 948.55 & -62.0 & 949.98 & 21.0 & 947.85 \\
\hline 37.0 & 948.08 & -37.8 & 948.98 & 49.0 & 948.67 & -59.0 & 950.01 & 24.0 & 947.96 \\
\hline 38.0 & 948.08 & -37.6 & 948.49 & 50.0 & 948.85 & -57.0 & 949.98 & 27.0 & 948.03 \\
\hline 40.0 & 948.03 & -37.0 & 948.28 & 51.0 & 948.69 & -55.0 & 949.95 & 30.0 & 948.07 \\
\hline 42.0 & 948.47 & -36.4 & 947.75 & 53.0 & 948.73 & -53.0 & 949.90 & 33.0 & 948.18 \\
\hline 43.3 & 948.49 & -36.3 & 947.18 & 56.0 & 948.66 & -51.0 & 949.86 & 35.0 & 948.45 \\
\hline 45.0 & 948.66 & -35.8 & 947.04 & 58.0 & 948.55 & -49.0 & 949.91 & 37.5 & 948.21 \\
\hline 46.5 & 948.66 & -33.6 & 946.42 & 61.0 & 948.56 & -47.0 & 949.87 & 40.0 & 948.34 \\
\hline 47.4 & 948.56 & -31.0 & 946.64 & 64.0 & 948.45 & -43.6 & 949.84 & 43.0 & 948.49 \\
\hline 49.5 & 948.88 & -28.0 & 946.65 & 66.0 & 948.38 & -41.0 & 949.88 & 45.0 & 948.68 \\
\hline 50.8 & 948.68 & -25.0 & 946.64 & 68.0 & 948.44 & -40.0 & 949.87 & 48.0 & 948.65 \\
\hline 51.7 & 948.81 & -22.0 & 946.75 & 70.0 & 948.68 & -38.2 & 949.80 & 50.0 & 948.86 \\
\hline 52.4 & 948.70 & -19.0 & 946.73 & 72.0 & 948.94 & -38.1 & 947.71 & 51.0 & 948.67 \\
\hline 54.0 & 948.78 & -16.0 & 946.76 & 73.0 & 949.02 & -37.3 & 947.45 & 54.0 & 948.77 \\
\hline 56.0 & 948.62 & -13.0 & 946.75 & & & -36.8 & 947.24 & 57.0 & 948.63 \\
\hline 58.0 & 948.56 & -10.0 & 946.76 & & & -35.0 & 946.67 & 60.0 & 948.57 \\
\hline 60.0 & 948.55 & -7.0 & 946.79 & & & -33.0 & 946.34 & 63.0 & 948.50 \\
\hline 63.0 & 948.50 & -4.0 & 946.88 & & & -32.0 & 946.21 & 66.0 & 948.39 \\
\hline 65.0 & 948.40 & -1.0 & 946.88 & & & -30.0 & 946.35 & 69.0 & 948.53 \\
\hline 67.0 & 948.40 & 2.3 & 946.81 & & & -28.0 & 946.44 & 71.0 & 948.85 \\
\hline 68.3 & 948.46 & 3.6 & 947.04 & & & -26.0 & 946.60 & 73.0 & 949.02 \\
\hline 70.0 & 948.69 & 4.1 & 947.26 & & & -24.0 & 946.67 & 103.4 & 948.93 \\
\hline 72.0 & 948.96 & 6.0 & 947.22 & & & -22.0 & 946.67 & 111.3 & 949.55 \\
\hline 73.0 & 949.02 & 8.0 & 947.39 & & & -20.0 & 946.77 & & \\
\hline 103.4 & 948.94 & 11.0 & 947.52 & & & -18.0 & 946.88 & & \\
\hline 111.3 & 949.55 & 14.0 & 947.61 & & & -16.0 & 946.86 & & \\
\hline & & 17.0 & 947.67 & & & -14.0 & 946.88 & & \\
\hline & & 20.0 & 947.77 & & & -12.0 & 946.83 & & \\
\hline & & 23.0 & 947.90 & & & -10.0 & 946.75 & & \\
\hline & & 26.0 & 948.00 & & & -8.0 & 946.85 & & \\
\hline & & 29.0 & 948.06 & & & -6.0 & 946.84 & & \\
\hline & & 31.0 & 948.07 & & & -4.0 & 946.91 & & \\
\hline & & 33.0 & 948.17 & & & -2.0 & 946.82 & & \\
\hline & & 34.2 & 948.34 & & & 0.0 & 946.94 & & \\
\hline & & 35.3 & 948.46 & & & 1.5 & 947.22 & & \\
\hline & & 36.3 & 948.23 & & & 2.5 & 947.22 & & \\
\hline & & 37.8 & 948.19 & & & 5.0 & 947.27 & & \\
\hline & & 39.0 & 948.26 & & & 7.5 & 947.38 & & \\
\hline
\end{tabular}




\section{Description of Cross Section PR183}

Location: Township 6 South/Range 50 East--section 1

U. S. Geological Survey quadrangle (1:24,000): Lonesome Peak

Landowners--left bank: EB Ranch

--right bank: EB Ranch

Access: Left bank

Permission from: Dick Wilson

Distance from Moorhead Gaging Station: 67.85 kilometers

Azimuth of Section (degrees magnetic): 059

\section{Reference Monuments}

[Monuments at stations 100.0 and 101.1 were closest to leveling instrument]

\begin{tabular}{|c|c|c|c|c|c|c|}
\hline \multirow[b]{2}{*}{ Description } & \multirow[b]{2}{*}{$\begin{array}{c}\text { Station } \\
(m)\end{array}$} & \multicolumn{2}{|c|}{ GPS-NAD83 (1992) } & \multicolumn{2}{|c|}{ Measurement } & \multirow{2}{*}{$\begin{array}{c}\text { Eievation } \\
\text { (NGVD1929) } \\
\text { (m) }\end{array}$} \\
\hline & & Latitude & Longitude & $\begin{array}{l}\text { Standard } \\
\text { deviation } \\
\text { (m) }\end{array}$ & $\begin{array}{l}\text { Horizontal } \\
\text { precision } \\
(\mathbf{m})\end{array}$ & \\
\hline $\begin{array}{l}\text { 1/2-inch-rebar; } 0.17 \text { meter above } 1998 \text { ground } \\
\text { level }\end{array}$ & -6.9 & $45^{\circ} 20^{\prime} 32.09^{\prime \prime}$ & $105^{\circ} 32^{\prime} 16.66^{\prime \prime}$ & 0.311 & 0.685 & 945.95 \\
\hline $\begin{array}{l}\text { 1/2-inch-rebar; } 0.05 \text { meter above } 1998 \text { ground } \\
\text { level }\end{array}$ & 0.0 & & & & & 945.67 \\
\hline 1/2-inch-rebar; at 1998 ground level & 100.0 & & & & & 945.68 \\
\hline $\begin{array}{l}\text { 1/2-inch-rebar; } 0.10 \text { meter above } 1998 \text { ground } \\
\text { level }\end{array}$ & 101.1 & $45^{\circ} 20^{\prime} 33.12$ & $105^{\circ} 32^{\prime} 11.92$ & 0.688 & 0.602 & 945.86 \\
\hline $\begin{array}{l}\text { Benchmark--brass circular plate; slightly off } \\
\text { section }\end{array}$ & $\sim 127.9$ & $45^{\circ} 20^{\prime} 33.33^{\prime \prime}$ & $105^{\circ} 32 ' 10.76^{\prime \prime}$ & 0.531 & 0.504 & 945.89 \\
\hline
\end{tabular}




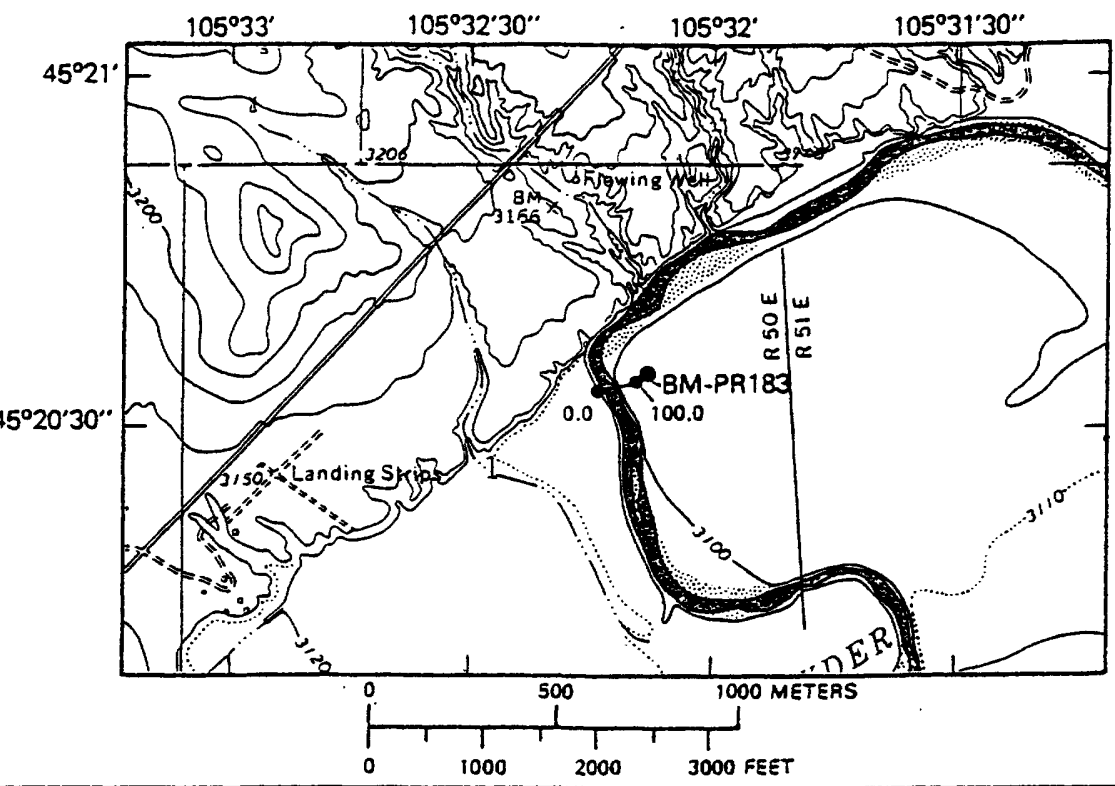

PRI83

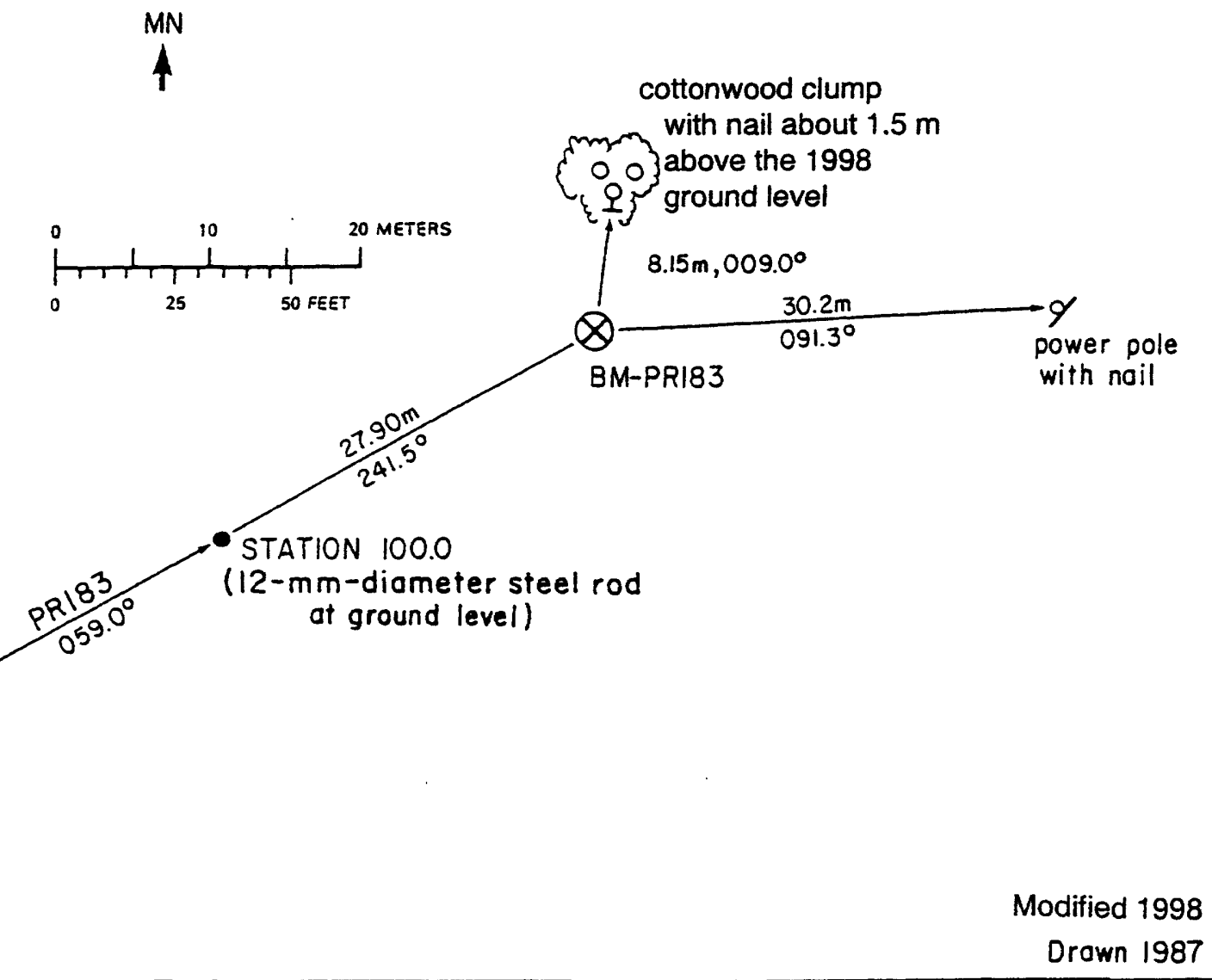

Figure 96. Upper: Location of cross section PR183, bench mark BM-PR183, and the left and right bank reference monuments in the Lonesome Peak quadrangle. Lower: Location of the reference monuments on the left bank. MN is magnetic north. 

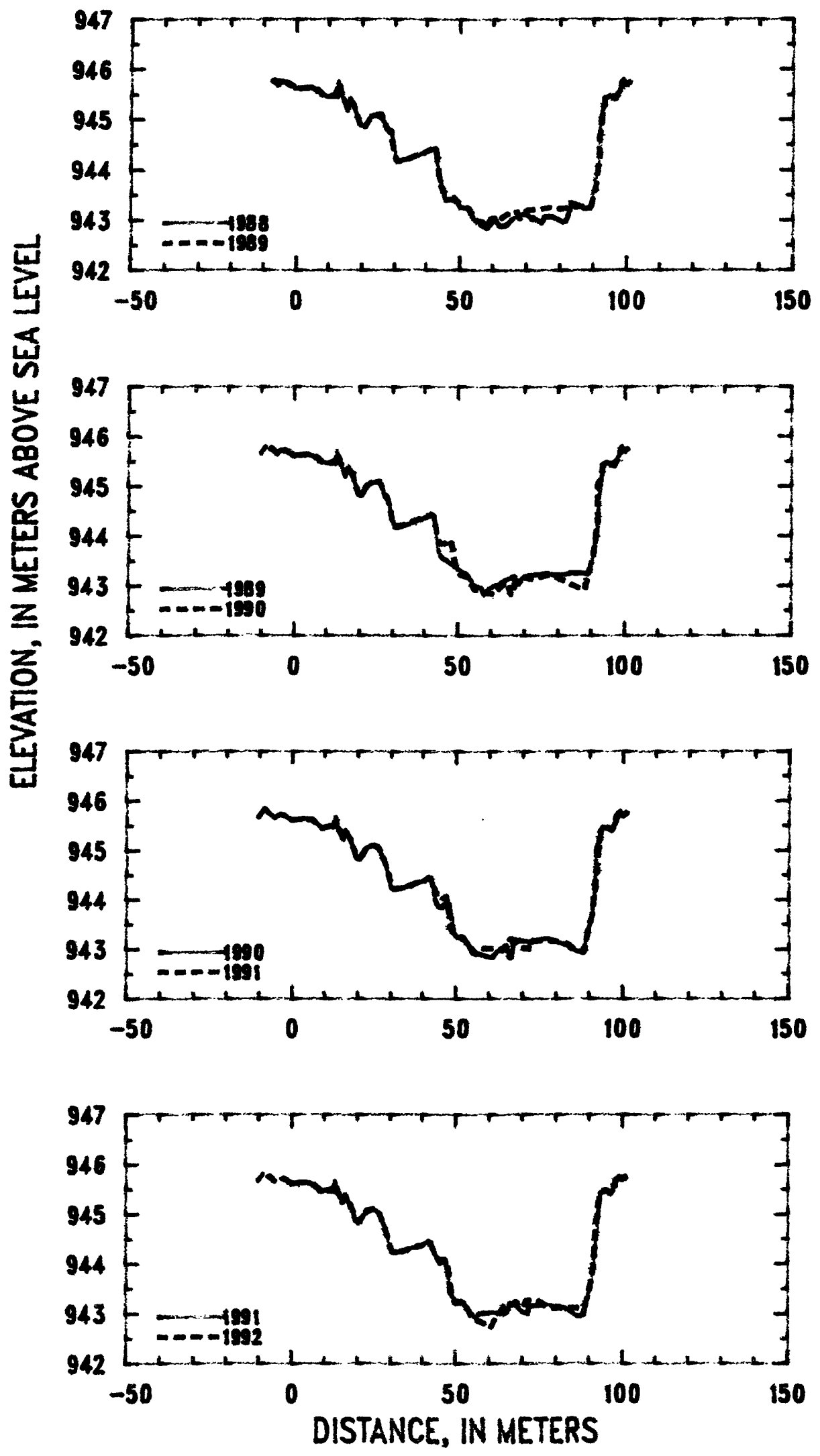

Figure 97. Profiles of cross section PR183 from 1988 to 1992. 

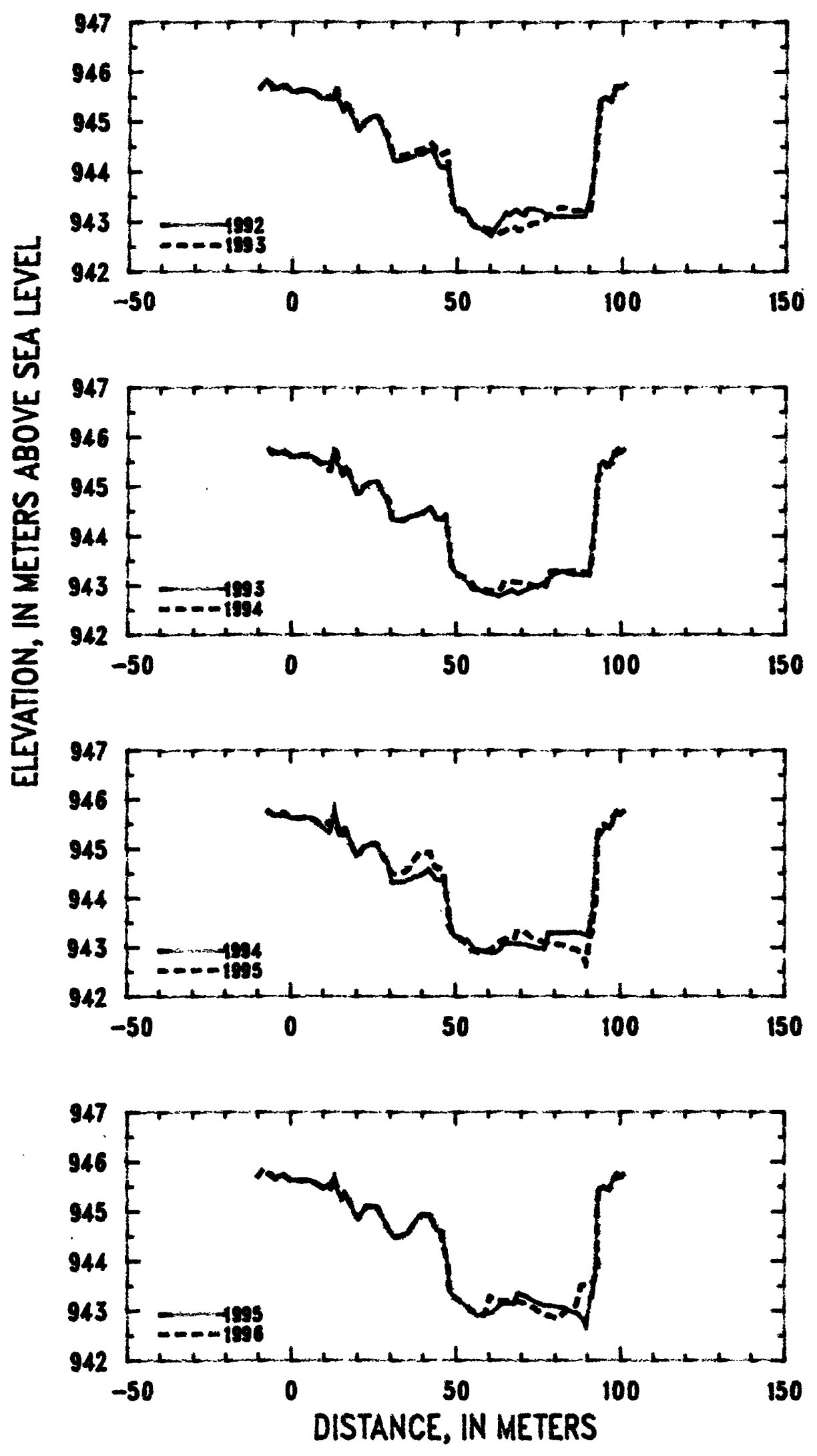

Figure 98. Profiles of cross section PR183 from 1992 to 1996. 


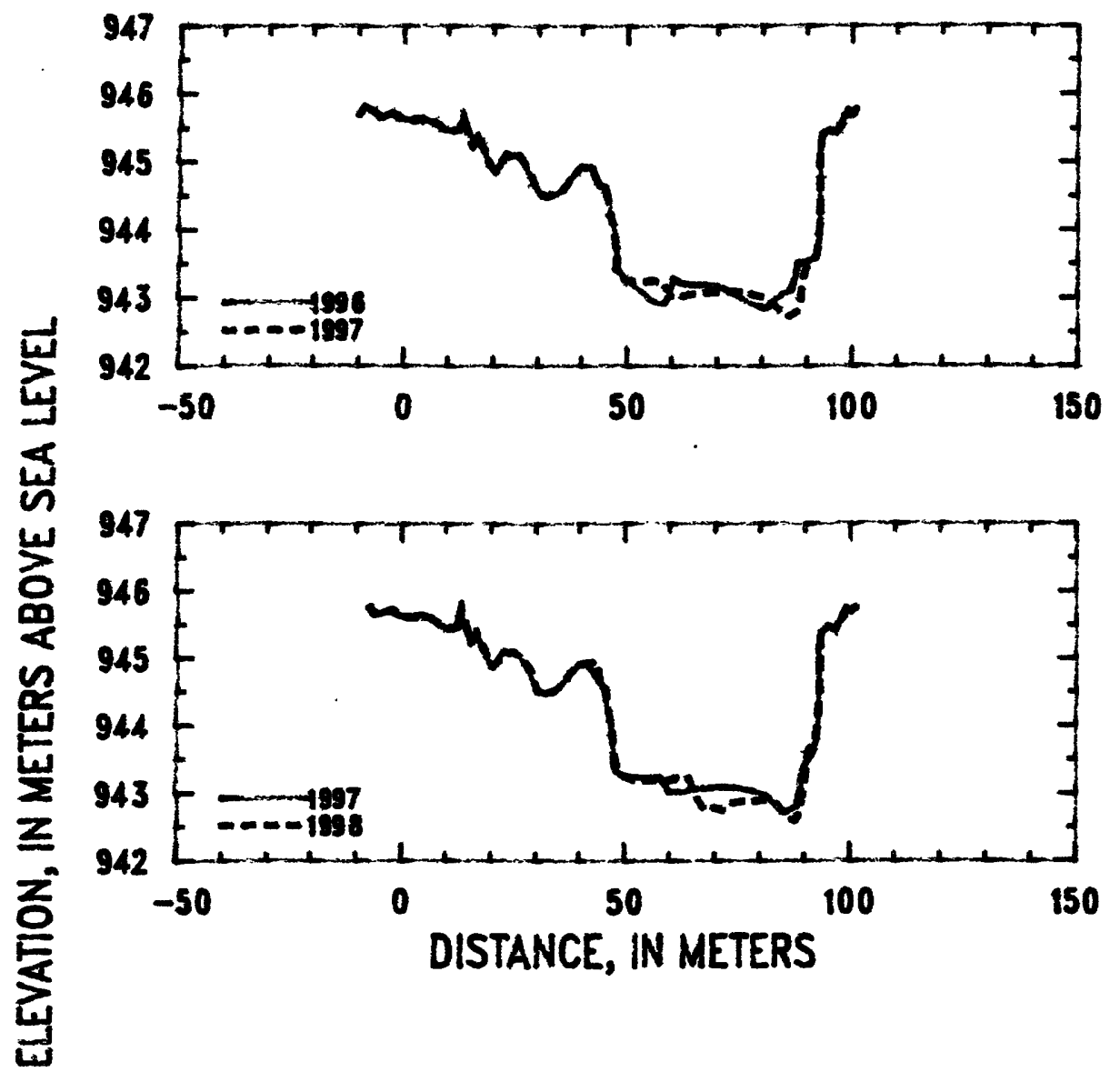

Figure 99. Profiles of cross section PR183 from 1996 to 1998. 
Table 35. Listing of horizontal stations and elevations for cross section PR183

[Sta., station, distance in meters from a reference pin on the left bank; Elev., elevation, in meters above sea level]

\begin{tabular}{|c|c|c|c|c|c|c|c|c|c|}
\hline \multirow{2}{*}{\multicolumn{2}{|c|}{$\begin{array}{c}1989 \\
23 \text { September }\end{array}$}} & \multicolumn{2}{|c|}{1989} & \multicolumn{2}{|c|}{1990} & \multicolumn{2}{|c|}{1990} & \multicolumn{2}{|c|}{1991} \\
\hline & & $23 \mathrm{~S}$ & ember & $22 \mathrm{Se}$ & ember & $22 \mathrm{~S}$ & tember & $2 \mathrm{Se}$ & mber \\
\hline Sta. & Elev. & Sta. & Elev. & Sta. & Elev. & Sta. & Elev. & Sta. & Elev. \\
\hline-7.0 & 945.79 & 57.0 & 942.96 & -10.0 & 945.66 & 47.9 & 943.92 & 0.0 & 945.61 \\
\hline-5.0 & 945.66 & 58.0 & 942.82 & -8.0 & 945.84 & 48.5 & 943.71 & 3.0 & 945.63 \\
\hline-3.0 & 945.75 & 60.0 & 942.98 & -7.0 & 945.77 & 49.5 & 943.35 & 6.0 & 945.63 \\
\hline-1.0 & 945.68 & 62.0 & 943.02 & -5.0 & 945.65 & 50.0 & 943.27 & 9.0 & 945.45 \\
\hline 0.0 & 945.61 & 64.0 & 943.11 & -3.0 & 945.73 & 51.0 & 943.21 & 12.0 & 945.50 \\
\hline 2.0 & 945.62 & 66.0 & 943.17 & -1.0 & 945.67 & 53.0 & 943.17 & 12.7 & 945.45 \\
\hline 4.0 & 945.63 & 67.0 & 943.18 & 0.0 & 945.61 & 55.0 & 942.92 & 13.4 & 945.63 \\
\hline 6.0 & 945.62 & 68.0 & 943.04 & 1.0 & 945.61 & 57.0 & 942.91 & 15.4 & 945.25 \\
\hline 8.0 & 945.50 & 70.0 & 943.19 & 3.0 & 945.64 & 59.0 & 942.85 & 16.5 & 945.38 \\
\hline 10.0 & 945.47 & 72.0 & 943.22 & 5.0 & 945.65 & 61.0 & 942.83 & 18.0 & 945.19 \\
\hline 12.6 & 945.46 & 74.0 & 943.23 & 7.0 & 945.58 & 63.0 & 943.00 & 19.6 & 944.85 \\
\hline 13.3 & 945.68 & 76.0 & 943.25 & 9.0 & 945.45 & 65.0 & 943.09 & 21.0 & 944.87 \\
\hline 14.0 & 945.51 & 77.0 & 943.26 & 11.0 & 945.47 & 66.0 & 942.84 & 23.0 & 945.08 \\
\hline 15.6 & 945.22 & 79.0 & 943.22 & 12.0 & 945.50 & 66.7 & 942.83 & 25.0 & 945.10 \\
\hline 16.5 & 945.39 & 81.0 & 943.24 & 12.8 & 945.47 & 67.2 & 943.11 & 26.3 & 945.06 \\
\hline 17.6 & 945.28 & 83.0 & 943.23 & 13.4 & 945.63 & 69.0 & 943.18 & 28.0 & 944.80 \\
\hline 19.5 & 944.89 & 84.0 & 943.27 & 14.0 & 945.50 & 71.0 & 943.15 & 28.6 & 944.69 \\
\hline 20.6 & 944.82 & 85.0 & 943.26 & 15.5 & 945.24 & 73.0 & 943.13 & 30.5 & 944.23 \\
\hline 21.6 & 944.93 & 87.0 & 943.26 & 16.4 & 945.39 & 75.0 & 943.20 & 32.0 & 944.22 \\
\hline 23.0 & 945.07 & 89.0 & 943.23 & 18.0 & 945.20 & 77.0 & 943.22 & 34.0 & 944.25 \\
\hline 25.0 & 945.10 & 89.6 & 943.32 & 19.6 & 944.84 & 79.0 & 943.19 & 36.0 & 944.28 \\
\hline 26.0 & 945.10 & 91.0 & 943.74 & 20.6 & 944.82 & 81.0 & 943.13 & 38.0 & 944.34 \\
\hline 26.8 & 945.00 & 91.9 & 944.21 & 22.0 & 945.01 & 83.0 & 943.08 & 40.0 & 944.36 \\
\hline 27.7 & 944.79 & 92.0 & 944.64 & 24.0 & 945.10 & 85.0 & 942.99 & 42.0 & 944.46 \\
\hline 28.7 & 944.72 & 93.0 & 945.16 & 26.0 & 945.10 & 87.0 & 942.94 & 42.6 & 944.43 \\
\hline 30.0 & 944.31 & 93.5 & 945.44 & 26.8 & 944.97 & 88.2 & 942.92 & 43.3 & 944.27 \\
\hline 30.6 & 944.18 & 94.6 & 945.49 & 28.5 & 944.75 & 89.3 & 943.29 & 45.6 & 944.00 \\
\hline 31.5 & 944.17 & 96.0 & 945.43 & 30.0 & 944.27 & 90.7 & 943.64 & 47.0 & 944.07 \\
\hline 33.0 & 944.20 & 97.8 & 945.55 & 30.4 & 944.22 & 91.9 & 944.27 & 47.6 & 943.92 \\
\hline 35.0 & 944.24 & 98.6 & 945.75 & 32.0 & 944.21 & 92.0 & 945.05 & 48.3 & 943.42 \\
\hline 37.0 & 944.30 & 100.0 & 945.68 & 34.0 & 944.24 & 92.8 & 945.14 & 50.0 & 943.26 \\
\hline 39.0 & 944.33 & 101.0 & 945.76 & 36.0 & 944.28 & 93.3 & 945.41 & 52.0 & 943.24 \\
\hline 41.0 & 944.40 & & & 38.0 & 944.34 & 94.0 & 945.48 & 53.5 & 943.11 \\
\hline 42.6 & 944.43 & & & 40.0 & 944.36 & 96.0 & 945.43 & 56.0 & 942.91 \\
\hline 43.1 & 944.33 & & & 42.0 & 944.45 & 96.7 & 945.41 & 57.4 & 943.01 \\
\hline 44.4 & 943.67 & & & 42.6 & 944.43 & 97.7 & 945.56 & 60.0 & 943.02 \\
\hline 45.2 & 943.56 & & & 43.0 & 944.33 & 99.0 & 945.78 & 62.0 & 943.03 \\
\hline 47.0 & 943.50 & & & 44.0 & 943.95 & 100.0 & 945.69 & 64.0 & 942.98 \\
\hline 50.0 & 943.33 & & & 44.4 & 943.91 & 101.1 & 945.77 & 65.6 & 943.04 \\
\hline 52.0 & 943.25 & & & 45.0 & 943.85 & & & 66.4 & 943.21 \\
\hline 53.0 & 943.20 & & & 46.0 & 943.85 & & & 68.0 & 943.19 \\
\hline 55.0 & 942.99 & & & 47.0 & 943.93 & & & 70.0 & 943.03 \\
\hline
\end{tabular}


Table 35. (Continued) Listing of horizontal stations and elevations for cross section PR183

[Sta., station, distance in meters from a reference pin on the left bank; Elev., elevation, in meters above sea level]

\begin{tabular}{|c|c|c|c|c|c|c|c|c|c|}
\hline \multirow{2}{*}{\multicolumn{2}{|c|}{$\begin{array}{c}1991 \\
2 \text { September }\end{array}$}} & \multirow{2}{*}{\multicolumn{2}{|c|}{$\begin{array}{c}1992 \\
28 \text { August }\end{array}$}} & \multirow{2}{*}{\multicolumn{2}{|c|}{$\begin{array}{c}1992 \\
28 \text { August }\end{array}$}} & \multirow{2}{*}{\multicolumn{2}{|c|}{$\begin{array}{c}1993 \\
31 \text { August }\end{array}$}} & \multirow{2}{*}{\multicolumn{2}{|c|}{$\begin{array}{c}1993 \\
31 \text { August }\end{array}$}} \\
\hline & & & & & & & & & \\
\hline Sta. & Elev. & Sta. & Elev. & Sta. & Elev. & Sta. & Elev. & Sta. & Elev. \\
\hline 71.7 & 943.00 & -10.0 & 945.67 & 53.4 & 943.11 & -7.0 & 945.78 & 51.0 & 943.24 \\
\hline 72.0 & 943.17 & -8.0 & 945.85 & 55.0 & 942.90 & -6.0 & 945.67 & 53.0 & 943.09 \\
\hline 74.0 & 943.17 & -7.0 & 945.77 & 57.0 & 942.85 & -4.0 & 945.67 & 55.0 & 942.92 \\
\hline 77.0 & 943.18 & -5.0 & 945.66 & 59.0 & 942.78 & -2.0 & 945.73 & 57.0 & 942.91 \\
\hline 80.0 & 943.16 & -3.0 & 945.73 & 60.4 & 942.71 & 0.0 & 945.62 & 59.0 & 942.85 \\
\hline 83.0 & 943.12 & -1.0 & 945.68 & 62.0 & 942.90 & 1.0 & 945.61 & 61.0 & 942.81 \\
\hline 85.0 & 943.02 & 0.0 & 945.61 & 64.0 & 943.08 & 3.0 & 945.64 & 63.0 & 942.77 \\
\hline 87.0 & 942.94 & 1.0 & 945.62 & 65.0 & 943.18 & 5.0 & 945.64 & 65.0 & 942.84 \\
\hline 88.5 & 942.97 & 3.0 & 945.64 & 66.0 & 943.15 & 7.0 & 945.59 & 67.0 & 942.91 \\
\hline 88.9 & 943.13 & 5.0 & 945.64 & 68.0 & 943.24 & 9.0 & 945.46 & 69.0 & 942.83 \\
\hline 91.0 & 943.75 & 7.0 & 945.58 & 70.0 & 943.15 & 11.0 & 945.49 & 71.0 & 942.92 \\
\hline 91.8 & 944.28 & 9.0 & 945.47 & 70.7 & 943.15 & 12.5 & 945.48 & 73.0 & 942.98 \\
\hline 92.8 & 945.09 & 11.0 & 945.47 & 71.0 & 943.25 & 13.6 & 945.68 & 75.0 & 943.01 \\
\hline 93.4 & 945.43 & 12.7 & 945.47 & 73.0 & 943.29 & 14.0 & 945.52 & 77.0 & 943.07 \\
\hline 95.0 & 945.48 & 13.3 & 945.62 & 75.0 & 943.25 & 15.6 & 945.23 & 79.0 & 943.20 \\
\hline 96.6 & 945.41 & 14.0 & 945.47 & 77.0 & 943.16 & 16.7 & 945.39 & 81.0 & 943.29 \\
\hline 97.6 & 945.54 & 15.6 & 945.24 & 79.0 & 943.12 & 18.0 & 945.21 & 83.0 & 943.30 \\
\hline 98.0 & 945.70 & 16.5 & 945.37 & 81.0 & 943.11 & 20.0 & 944.85 & 85.0 & 943.23 \\
\hline 99.0 & 945.76 & 18.0 & 945.18 & 83.0 & 943.12 & 21.0 & 944.87 & 87.0 & 943.23 \\
\hline 100.0 & 945.69 & 20.3 & 944.82 & 85.0 & 943.11 & 22.0 & 945.01 & 89.0 & 943.21 \\
\hline \multirow[t]{22}{*}{101.1} & 945.76 & 22.0 & 945.00 & 87.0 & 943.12 & 24.0 & 945.09 & 90.0 & 943.23 \\
\hline & & 24.0 & 945.10 & 88.8 & 943.11 & 26.0 & 945.11 & 90.6 & 943.26 \\
\hline & & 26.0 & 945.11 & 89.7 & 943.34 & 28.0 & 944.83 & 91.0 & 943.79 \\
\hline & & 28.0 & 944.79 & 90.5 & 943.59 & 29.0 & 944.73 & 92.0 & 944.26 \\
\hline & & 30.0 & 944.35 & 91.0 & 943.78 & 30.0 & 944.41 & 92.5 & 944.73 \\
\hline & & 30.8 & 944.22 & 91.8 & 944.22 & 30.5 & 944.34 & 93.4 & 945.44 \\
\hline & & 32.0 & 944.22 & 92.0 & 944.71 & 31.0 & 944.33 & 94.5 & 945.50 \\
\hline & & 34.0 & 944.25 & 93.0 & 945.22 & 33.0 & 944.33 & 96.5 & 945.41 \\
\hline & & 36.0 & 944.29 & 93.5 & 945.43 & 35.0 & 944.36 & 98.0 & 945.70 \\
\hline & & 38.0 & 944.34 & 95.0 & 945.49 & 37.0 & 944.41 & 100.0 & 945.69 \\
\hline & & 40.0 & 944.37 & 96.6 & 945.41 & 39.0 & 944.45 & 101.1 & 945.76 \\
\hline & & 42.0 & 944.45 & 98.1 & 945.69 & 40.0 & 944.46 & & \\
\hline & & 42.7 & 944.42 & 100.0 & 945.69 & 41.0 & 944.53 & & \\
\hline & & 44.0 & 944.19 & 101.1 & 945.76 & 42.0 & 944.58 & & \\
\hline & & 45.0 & 944.09 & & & 43.0 & 944.51 & & \\
\hline & & 46.0 & 944.08 & & & 44.0 & 944.35 & & \\
\hline & & 47.0 & 944.10 & & & 45.0 & 944.35 & & \\
\hline & & 48.0 & 943.81 & & & 46.3 & 944.37 & & \\
\hline & & 48.6 & 943.48 & & & 46.9 & 944.41 & & \\
\hline & & 49.0 & 943.34 & & & 48.1 & 943.53 & & \\
\hline & & 50.0 & 943.22 & & & 48.4 & 943.39 & & \\
\hline & & 52.0 & 943.23 & & & 49.5 & 943.29 & & \\
\hline
\end{tabular}


Table 35. (Continued) Listing of horizontal stations and elevations for cross section PR183

[Sta., station, distance in meters from a reference pin on the left bank; Elev., elevation, in meters above sea level]

\begin{tabular}{|c|c|c|c|c|c|c|c|c|c|}
\hline \multirow{2}{*}{\multicolumn{2}{|c|}{$\begin{array}{c}1994 \\
18 \text { September }\end{array}$}} & \multirow{2}{*}{\multicolumn{2}{|c|}{$\begin{array}{c}1994 \\
18 \text { September }\end{array}$}} & \multirow{2}{*}{\multicolumn{2}{|c|}{$\begin{array}{c}1995 \\
29 \text { September }\end{array}$}} & \multirow{2}{*}{\multicolumn{2}{|c|}{$\begin{array}{c}1995 \\
29 \text { September }\end{array}$}} & \multirow{2}{*}{\multicolumn{2}{|c|}{$\begin{array}{c}1996 \\
22 \text { October }\end{array}$}} \\
\hline & & & & & & & & & \\
\hline Sta. & Elev. & Sta. & Elev. & Sta. & Elev. & Sta. & Elev. & Sta. & Elev. \\
\hline-7.0 & 945.75 & 75.0 & 942.98 & -7.0 & 945.77 & 48.0 & 943.42 & -10.0 & 945.69 \\
\hline-4.0 & 945.67 & 77.0 & 942.97 & -5.0 & 945.67 & 49.0 & 943.30 & -8.5 & 945.83 \\
\hline-1.0 & 945.68 & 77.9 & 943.16 & -2.0 & 945.74 & 51.0 & 943.20 & -7.0 & 945.79 \\
\hline 0.0 & 945.62 & 78.0 & 943.28 & 0.0 & 945.64 & 53.0 & 943.09 & -4.5 & 945.66 \\
\hline 1.0 & 945.62 & 80.0 & 943.31 & 2.0 & 945.62 & 55.0 & 942.94 & -2.5 & 945.74 \\
\hline 3.0 & 945.64 & 82.0 & 943.29 & 4.0 & 945.63 & 57.0 & 942.88 & 0.0 & 945.63 \\
\hline 6.0 & 945.63 & 84.0 & 943.30 & 6.0 & 945.63 & 59.0 & 942.90 & 3.0 & 945.63 \\
\hline 9.0 & 945.47 & 86.0 & 943.31 & 8.0 & 945.54 & 61.0 & 942.95 & 6.0 & 945.62 \\
\hline 12.0 & 945.32 & 88.0 & 943.29 & 10.0 & 945.46 & 63.0 & 943.08 & 8.0 & 945.55 \\
\hline 12.7 & 945.48 & 90.5 & 943.24 & 12.0 & 945.54 & 65.0 & 943.16 & 10.0 & 945.46 \\
\hline 13.5 & 945.82 & 91.0 & 943.48 & 12.6 & 945.46 & 67.0 & 943.13 & 12.6 & 945.48 \\
\hline 14.5 & 945.46 & 91.8 & 944.11 & 13.5 & 945.70 & 68.0 & 943.16 & 13.3 & 945.70 \\
\hline 16.0 & 945.26 & 93.0 & 945.22 & 14.1 & 945.49 & 69.0 & 943.35 & 14.2 & 945.50 \\
\hline 17.0 & 945.38 & 96.0 & 945.46 & 15.5 & 945.23 & 71.0 & 943.31 & 15.9 & 945.27 \\
\hline 20.0 & 944.86 & 97.3 & 945.47 & 16.5 & 945.39 & 73.0 & 943.21 & 16.7 & 945.38 \\
\hline 22.0 & 945.00 & 99.0 & 945.76 & 18.0 & 945.21 & 75.0 & 943.16 & 19.0 & 945.01 \\
\hline 24.0 & 945.09 & 100.0 & 945.69 & 19.0 & 945.01 & 77.0 & 943.10 & 20.5 & 944.85 \\
\hline 26.0 & 945.11 & 101.1 & 945.76 & 20.0 & 944.87 & 79.0 & 943.09 & 23.0 & 945.10 \\
\hline 28.0 & 944.83 & & & 21.0 & 944.89 & 81.0 & 943.06 & 24.0 & 945.10 \\
\hline 29.5 & 944.72 & & & 22.0 & 945.00 & 83.0 & 943.05 & 26.0 & 945.11 \\
\hline 30.8 & 944.30 & & & 23.0 & 945.09 & 85.0 & 942.98 & 28.0 & 944.85 \\
\hline 32.0 & 944.32 & & & 25.0 & 945.10 & 87.0 & 942.95 & 30.0 & 944.57 \\
\hline 34.0 & 944.33 & & & 26.4 & 945.08 & 89.0 & 942.79 & 31.0 & 944.49 \\
\hline 36.0 & 944.38 & & & 28.0 & 944.85 & 89.7 & 942.66 & 33.0 & 944.49 \\
\hline 38.0 & 944.43 & & & 30.0 & 944.58 & 91.0 & 943.27 & 35.0 & 944.54 \\
\hline 40.0 & 944.47 & & & 31.0 & 944.48 & 91.5 & 943.27 & 37.0 & 944.72 \\
\hline 42.0 & 944.59 & & & 32.0 & 944.49 & 91.7 & 943.43 & 39.0 & 944.87 \\
\hline 44.0 & 944.38 & & & 33.0 & 944.50 & 91.9 & 943.71 & 40.0 & 944.94 \\
\hline 45.7 & 944.35 & & & 34.0 & 944.53 & 92.6 & 943.96 & 41.0 & 944.92 \\
\hline 46.6 & 944.41 & & & 35.0 & 944.55 & 93.0 & 945.13 & 42.3 & 944.93 \\
\hline 48.5 & 943.47 & & & 36.0 & 944.60 & 93.6 & 945.45 & 44.0 & 944.65 \\
\hline 49.6 & 943.23 & & & 37.0 & 944.72 & 95.0 & 945.49 & 45.3 & 944.63 \\
\hline 53.5 & 943.15 & & & 38.0 & 944.82 & 96.6 & 945.42 & 47.6 & 943.85 \\
\hline 56.0 & 942.94 & & & 39.0 & 944.91 & 98.1 & 945.68 & 47.9 & 943.52 \\
\hline 58.0 & 942.93 & & & 40.0 & 944.95 & 99.0 & 945.76 & 48.1 & 943.38 \\
\hline 60.0 & 942.88 & & & 41.5 & 944.92 & 100.0 & 945.69 & 50.0 & 943.25 \\
\hline 62.0 & 942.87 & & & 42.4 & 944.93 & 101.1 & 945.76 & 53.0 & 943.09 \\
\hline 64.0 & 942.95 & & & 43.0 & 944.75 & & & 56.0 & 942.93 \\
\hline 64.6 & 943.07 & & & 44.0 & 944.63 & & & 58.5 & 942.90 \\
\hline 67.0 & 943.08 & & & 45.0 & 944.61 & & & 59.6 & 943.04 \\
\hline 70.0 & 943.07 & & & 46.0 & 944.59 & & & 60.3 & 943.28 \\
\hline 73.0 & 943.02 & & & 47.8 & 943.76 & & & 63.0 & 943.19 \\
\hline
\end{tabular}


Table 35. (Continued) Listing of horizontal stations and elevations for cross section PR183

[Sta., station, distance in meters from a reference pin on the left bank; Elev., elevation, in meters above sea level]

\begin{tabular}{|c|c|c|c|c|c|c|c|c|c|}
\hline \multirow{2}{*}{\multicolumn{2}{|c|}{$\begin{array}{c}1996 \\
22 \text { October }\end{array}$}} & \multicolumn{2}{|c|}{1997} & \multicolumn{2}{|c|}{1997} & \multicolumn{2}{|c|}{1998} & \multicolumn{2}{|c|}{1998} \\
\hline & & \multicolumn{2}{|c|}{22 September } & \multicolumn{2}{|c|}{22 September } & \multicolumn{2}{|c|}{23 September } & \multicolumn{2}{|c|}{23 September } \\
\hline Sta. & Elev. & Sta. & Elev. & Sta. & Elev. & Sta. & Elev. & Sta. & Elev. \\
\hline 66.0 & 943.18 & -7.0 & 945.77 & 72.0 & 943.10 & -7.0 & 945.79 & 64.0 & 943.26 \\
\hline 69.0 & 943.19 & -5.0 & 945.66 & 75.0 & 943.09 & -6.0 & 945.67 & 66.0 & 942.94 \\
\hline 72.0 & 943.12 & -2.5 & 945.73 & 78.0 & 943.05 & -4.0 & 945.69 & 68.0 & 942.74 \\
\hline 75.0 & 942.99 & 0.0 & 945.63 & 81.0 & 943.00 & -2.0 & 945.75 & 70.0 & 942.79 \\
\hline 78.0 & 942.90 & 2.5 & 945.63 & 83.0 & 942.87 & 0.0 & 945.63 & 72.0 & 942.75 \\
\hline 81.0 & 942.83 & 5.0 & 945.65 & 86.0 & 942.72 & 2.0 & 945.61 & 74.0 & 942.86 \\
\hline 84.0 & 943.03 & 7.0 & 945.59 & 88.5 & 942.82 & 4.0 & 945.62 & 76.0 & 942.89 \\
\hline 86.6 & 943.10 & 10.0 & 945.45 & 89.4 & 943.32 & 6.0 & 945.62 & 78.0 & 942.88 \\
\hline 87.7 & 943.37 & 12.5 & 945.49 & 90.0 & 943.44 & 8.0 & 945.54 & 80.0 & 942.91 \\
\hline 88.1 & 943.50 & 13.0 & 945.67 & 91.0 & 943.52 & 10.0 & 945.44 & 82.0 & 942.91 \\
\hline 90.0 & 943.52 & 14.3 & 945.45 & 92.2 & 943.63 & 12.7 & 945.48 & 84.0 & 942.83 \\
\hline 92.0 & 943.58 & 15.5 & 945.23 & 93.0 & 944.09 & 13.4 & 945.77 & 86.0 & 942.65 \\
\hline 92.9 & 943.88 & 16.7 & 945.40 & 93.0 & 945.19 & 14.5 & 945.45 & 88.0 & 942.60 \\
\hline 93.0 & 945.18 & 19.4 & 944.95 & 93.4 & 945.38 & 16.0 & 945.26 & 89.0 & 942.73 \\
\hline 93.5 & 945.43 & 20.5 & 944.87 & 95.0 & 945.48 & 16.7 & 945.40 & 90.4 & 943.40 \\
\hline 95.0 & 945.48 & 23.0 & 945.12 & 96.5 & 945.44 & 19.0 & 945.05 & 91.0 & 943.66 \\
\hline 96.7 & 945.43 & 25.0 & 945.11 & 97.8 & 945.55 & 20.0 & 944.88 & 92.2 & 943.72 \\
\hline 98.1 & 945.68 & 26.5 & 945.05 & 99.0 & 945.75 & 22.0 & 944.98 & 93.1 & 944.21 \\
\hline 100.0 & 945.69 & 28.0 & 944.88 & 100.0 & 945.69 & 23.0 & 945.09 & 93.4 & 945.34 \\
\hline 101.0 & 945.78 & 30.0 & 944.57 & 101.0 & 945.76 & 25.0 & 945.11 & 95.0 & 945.49 \\
\hline & & 32.0 & 944.48 & & & 27.0 & 945.00 & 96.7 & 945.42 \\
\hline & & 34.0 & 944.52 & & & 29.0 & 944.82 & 97.7 & 945.54 \\
\hline & & 36.0 & 944.61 & & & 30.3 & 944.51 & 99.0 & 945.75 \\
\hline & & 38.0 & 944.79 & & & 32.0 & 944.48 & 100.0 & 945.69 \\
\hline & & 40.0 & 944.94 & & & 34.0 & 944.51 & 101.1 & 945.76 \\
\hline & & 42.0 & 944.92 & & & 36.0 & 944.61 & & \\
\hline & & 43.7 & 944.69 & & & 38.0 & 944.80 & & \\
\hline & & 45.0 & 944.62 & & & 40.0 & 944.92 & & \\
\hline & & 45.8 & 944.48 & & & 42.0 & 944.94 & & \\
\hline & & 46.4 & 944.18 & & & 44.0 & 944.95 & & \\
\hline & & 47.1 & 944.06 & & & 44.6 & 944.60 & & \\
\hline & & 47.5 & 943.79 & & & 45.4 & 944.62 & & · \\
\hline & & 47.6 & 943.42 & & & 47.0 & 944.00 & & \\
\hline & & 48.2 & 943.32 & & & 47.8 & 943.55 & & \\
\hline & & 50.0 & 943.25 & & & 48.3 & 943.41 & & \\
\hline & & 52.0 & 943.23 & & & 49.7 & 943.26 & & \\
\hline & & 55.0 & 943.24 & & & 52.0 & 943.19 & & \\
\hline & & 58.0 & 943.24 & & & 54.0 & 943.17 & & \\
\hline & & 60.0 & 943.02 & & & 56.0 & 943.20 & & \\
\hline & & 63.0 & 943.03 & & & 58.0 & 943.17 & & \\
\hline & & 66.0 & 943.07 & & & 60.0 & 943.20 & & \\
\hline & & 69.0 & 943.09 & & & 62.0 & 943.23 & & \\
\hline
\end{tabular}




\section{Description of Cross Section PR191}

Location: Township 5 South/Range 50 East--section 25

U. S. Geological Survey quadrangle (1:24,000): Eldon Mountain

Landowners--left bank: Doug and Lucille Randall

--right bank: Doug and Lucille Randall

Access: Left bank

Permission from: Doug, Lucille, or Craig Randall

Distance from Moorhead Gaging Station: 76.53 kilometers

Azimuth of Section (degrees magnetic): 177

\section{Reference Monuments}

[Monuments at stations -0.4 and 0.0 were closest to leveling instrument]

\begin{tabular}{|c|c|c|c|c|c|c|}
\hline \multirow[b]{2}{*}{ Description } & \multirow[b]{2}{*}{$\begin{array}{c}\text { Station } \\
\text { (m) }\end{array}$} & \multicolumn{2}{|c|}{ GPS-NAD83 (1992) } & \multicolumn{2}{|c|}{ Measurement } & \multirow{2}{*}{$\begin{array}{c}\text { Elevation } \\
\text { (NGVD1929) } \\
\text { (m) }\end{array}$} \\
\hline & & Latitude & Longitude & $\begin{array}{l}\text { Standard } \\
\text { deviation } \\
\text { (m) }\end{array}$ & $\begin{array}{l}\text { Horizontal } \\
\text { precision } \\
\text { (m) }\end{array}$ & \\
\hline $\begin{array}{l}\text { Benchmark--brass circular plate; offsection } \\
\text { under a fenceline }\end{array}$ & -- & $45^{\circ} 22^{\prime} 18.87^{\prime \prime}$ & $105^{\circ} 29^{\prime} 27.95^{\prime \prime}$ & 0.166 & 0.521 & 938.00 \\
\hline $\begin{array}{l}\text { 1/2-inch-rebar; } 0.18 \text { meter above } 1998 \text { ground } \\
\text { level }\end{array}$ & -0.4 & $45^{\circ} 22^{\prime} 18.53^{\prime \prime}$ & $105^{\circ} 29^{\prime} 28.83^{\prime \prime}$ & 0.273 & 0.395 & 937.99 \\
\hline 1/2-inch-rebar; at 1998 ground level & 0.0 & & & & & 937.70 \\
\hline $\begin{array}{l}\text { 1/2-inch-rebar; could not find in } 1998 \text {, about } \\
0.3 \text { meter below } 1998 \text { ground level }\end{array}$ & 130.0 & & & & & 936.09 \\
\hline $\begin{array}{l}\text { 1/2-inch-rebar; could not find in 1998; } 0.27 \\
\text { meter above } 1977 \text { ground level and possi- } \\
\text { ble bent and buried in 1978; may be } \\
\text { slightly offsection when PR191 was re- } \\
\text { established in 1978; elevation was mea- } \\
\text { sured in } 1977\end{array}$ & 150.3 & & & & & 936.63 \\
\hline $\begin{array}{l}\text { 1/2-inch-rebar; } 0.21 \text { meter above } 1998 \text { ground } \\
\text { level }\end{array}$ & 151.6 & & & & & 937.08 \\
\hline $\begin{array}{l}\text { 1/2-inch-rebar; } 0.16 \text { meter above } 1998 \text { ground } \\
\text { level }\end{array}$ & 186.0 & $45^{\circ} 22^{\prime} 12.61^{\prime \prime}$ & $105^{\circ} 27^{\prime} 56.40^{\prime \prime}$ & 0.174 & 0.450 & 936.93 \\
\hline
\end{tabular}




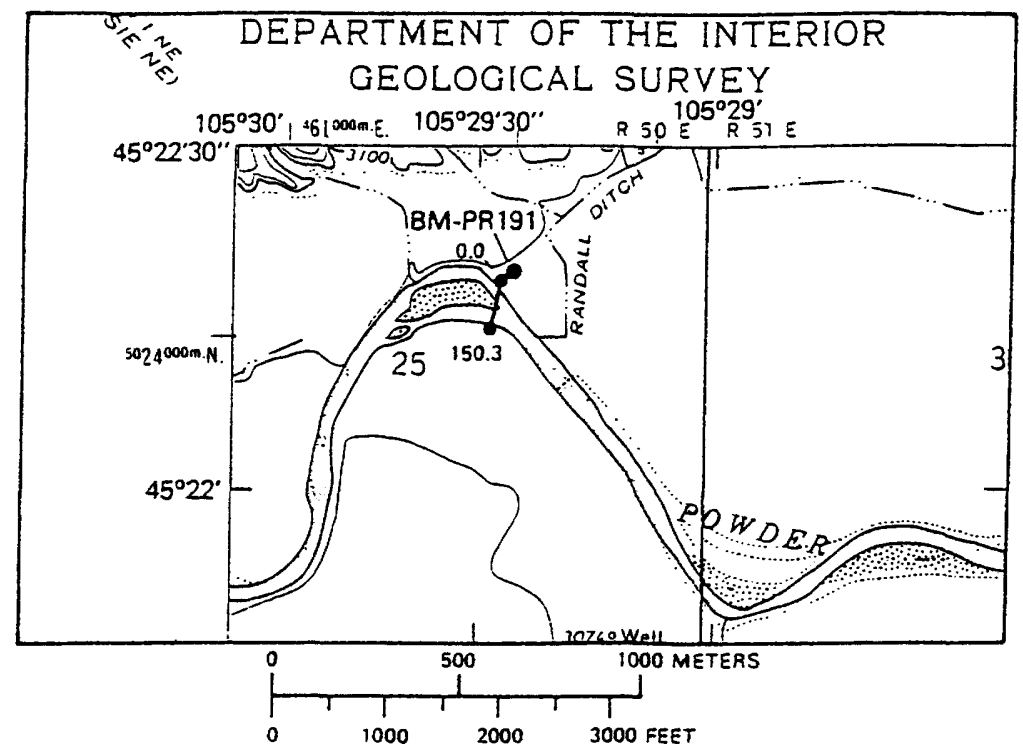

PRI9I

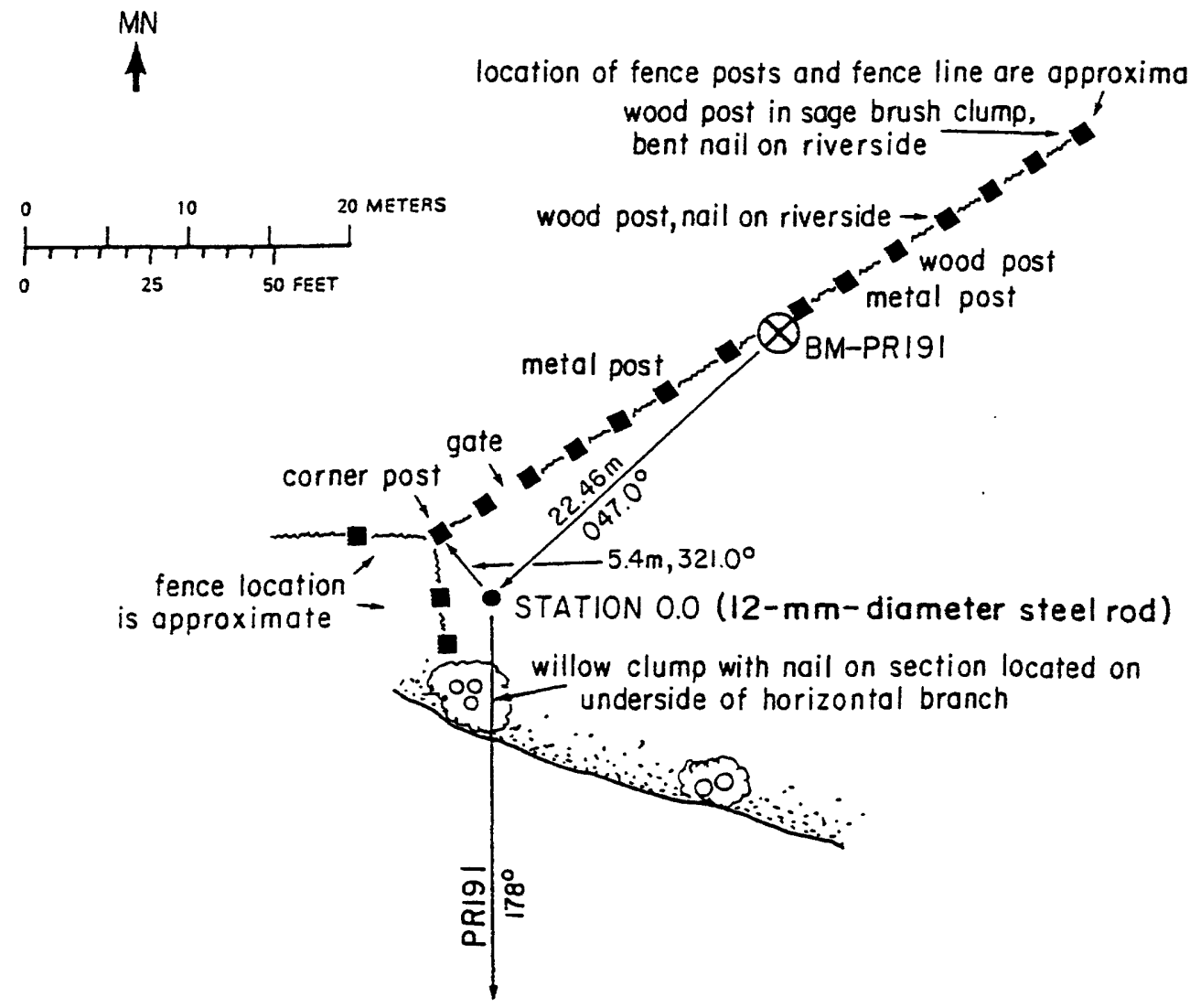

Drawn 1987

Figure 100. Upper: Location of cross section PR191, bench mark BM-PR191, and the left and right bank reference monuments in the Eldon Mountain quadrangle. Lower: Location of the bench mark on the left bank. MN is magnetic north. 

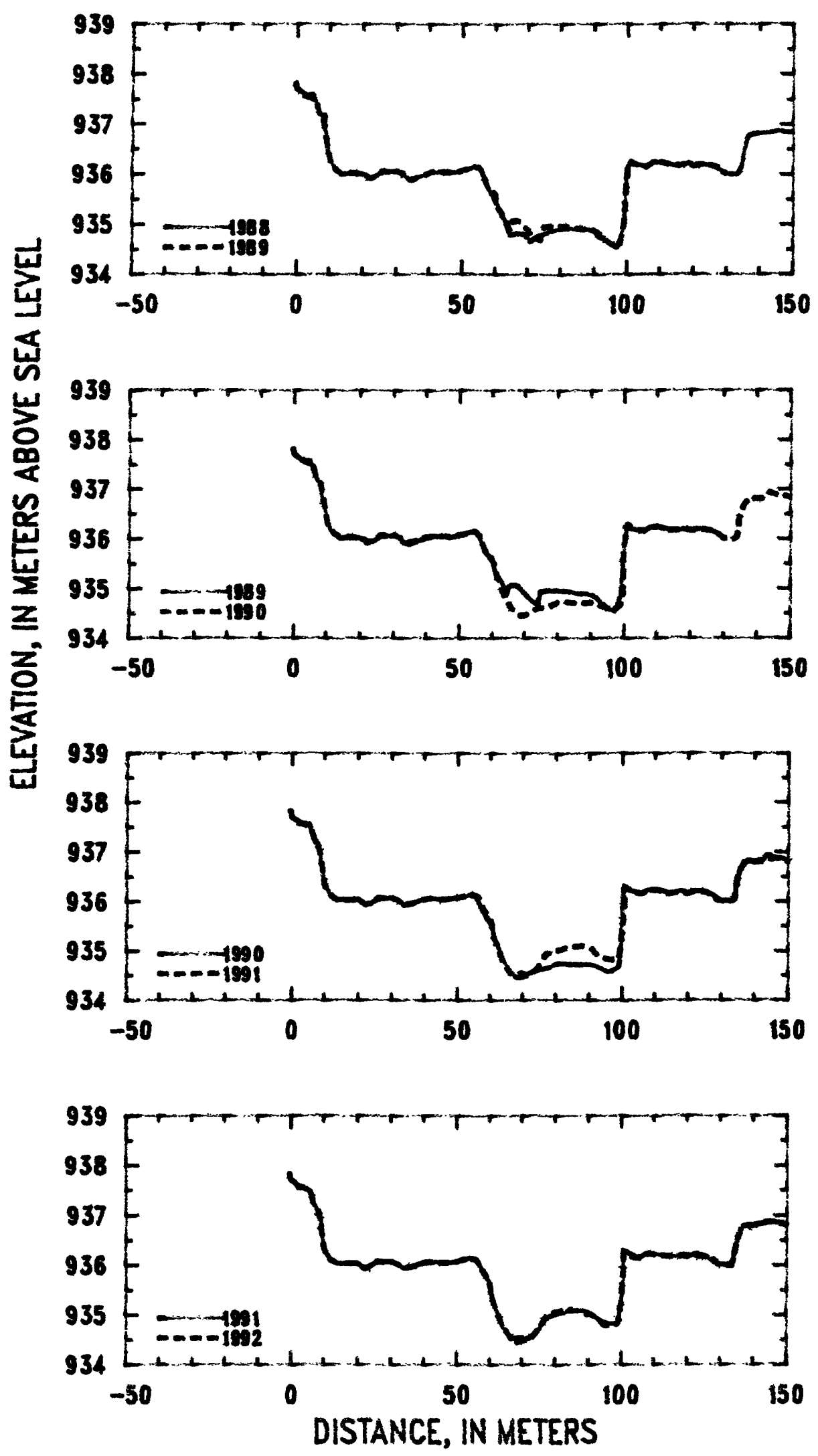

Figure 101. Profiles of cross section PR191 from 1988 to 1992. 

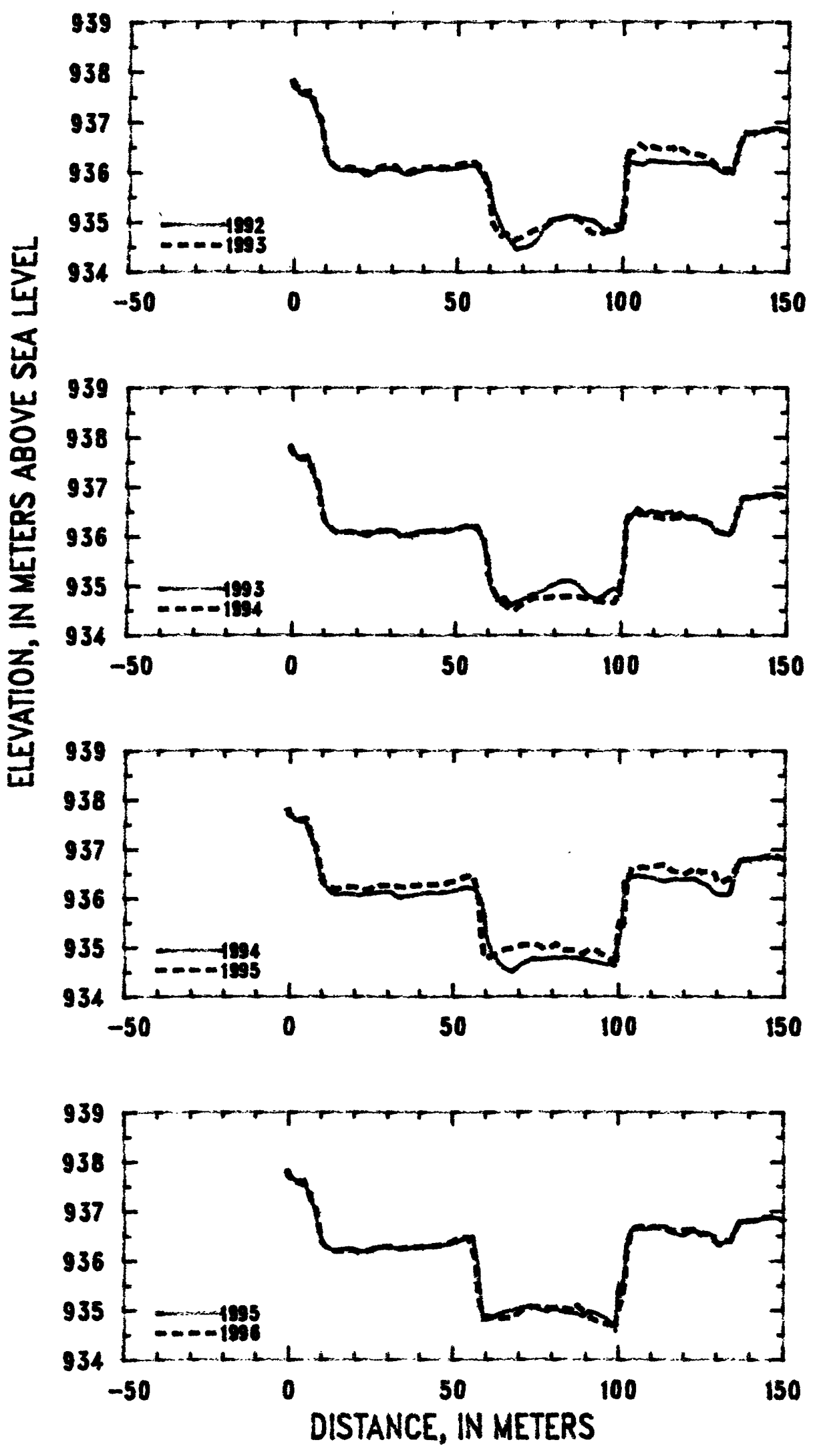

Figure 102. Profiles of cross section PR191 from 1992 to 1996. 


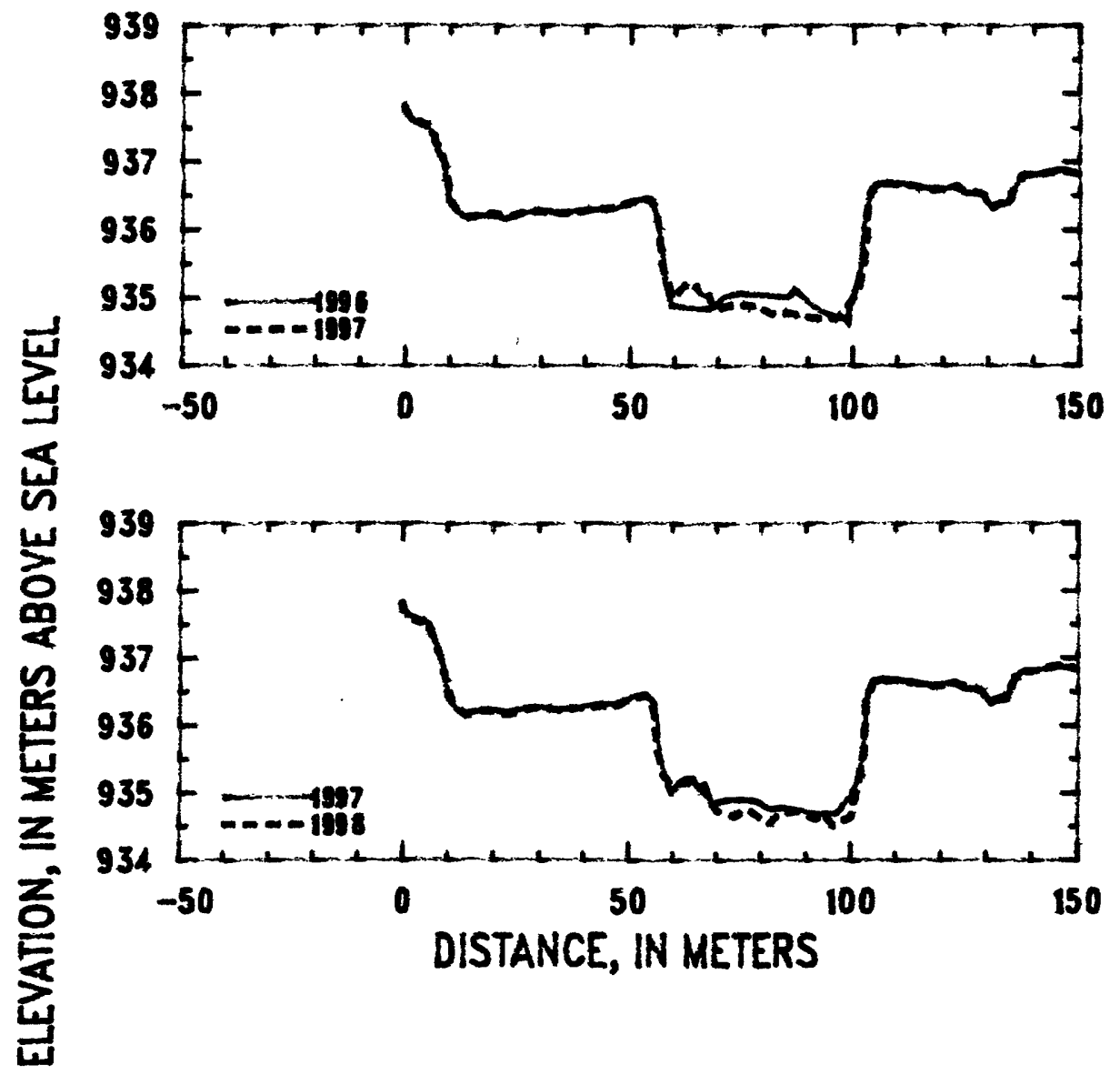

Figure 103. Profiles of cross section PR191 from 1996 to 1998. 
Table 36. Listing of horizontal stations and elevations for cross section PR191

[Sta., station, distance in meters from a reference pin on the left bank; Elev., elevation, in meters above sea level]

\begin{tabular}{|c|c|c|c|c|c|c|c|c|c|}
\hline \multirow{2}{*}{\multicolumn{2}{|c|}{$\begin{array}{c}1989 \\
21 \text { September }\end{array}$}} & \multicolumn{2}{|c|}{1989} & \multicolumn{2}{|c|}{1990} & \multicolumn{2}{|c|}{1990} & \multicolumn{2}{|c|}{1990} \\
\hline & & $21 \mathrm{~S}$ & tember & $23 \mathrm{Se}$ & ember & $23 \mathrm{~S}$ & tember & $23 \mathrm{Se}$ & ember \\
\hline Sta. & Elev. & Sta. & Elev. & Sta. & Elev. & Sta. & Elev. & Sta. & Elev. \\
\hline-0.4 & 937.83 & 67.0 & 935.08 & -0.4 & 937.83 & 70.0 & 934.46 & 138.0 & 936.83 \\
\hline 0.0 & 937.70 & 67.9 & 935.06 & 0.0 & 937.70 & 72.0 & 934.54 & 140.0 & 936.81 \\
\hline 1.0 & 937.67 & 69.0 & 934.96 & 1.0 & 937.66 & 74.0 & 934.61 & 142.0 & 936.82 \\
\hline 2.0 & 937.58 & 70.0 & 934.91 & 3.0 & 937.57 & 76.0 & 934.62 & 144.0 & 936.94 \\
\hline 4.0 & 937.53 & 71.0 & 934.82 & 5.5 & 937.55 & 78.0 & 934.67 & 146.0 & 936.88 \\
\hline 5.0 & 937.50 & 73.0 & 934.71 & 7.0 & 937.19 & 80.0 & 934.74 & 148.0 & 936.87 \\
\hline 6.0 & 937.45 & 74.0 & 934.69 & 8.0 & 937.15 & 82.0 & 934.75 & 150.0 & 936.84 \\
\hline 7.0 & 937.19 & 74.7 & 934.95 & 9.0 & 936.85 & 84.0 & 934.73 & 151.6 & 936.85 \\
\hline 8.0 & 937.13 & 76.0 & 934.96 & 10.2 & 936.31 & 86.0 & 934.72 & & \\
\hline 9.0 & 936.73 & 78.0 & 934.97 & 12.0 & 936.12 & 88.0 & 934.71 & & \\
\hline 10.3 & 936.28 & 80.0 & 934.97 & 14.0 & 936.04 & 90.0 & 934.73 & & \\
\hline 12.0 & 936.09 & 82.0 & 934.96 & 16.0 & 936.04 & 92.0 & 934.69 & & \\
\hline 14.0 & 936.00 & 84.0 & 934.94 & 18.0 & 936.03 & 94.0 & 934.63 & & \\
\hline 16.0 & 936.03 & 86.0 & 934.90 & 20.0 & 936.04 & 96.0 & 934.56 & & \\
\hline 18.0 & 936.02 & 88.0 & 934.90 & 22.0 & 935.94 & 98.0 & 934.63 & & \\
\hline 20.0 & 936.02 & 90.0 & 934.87 & 24.0 & 935.96 & 99.0 & 934.67 & & \\
\hline 22.0 & 935.91 & 91.0 & 934.85 & 26.0 & 936.08 & 99.5 & 934.92 & & \\
\hline 24.0 & 935.95 & 93.0 & 934.71 & 28.0 & 936.06 & 99.7 & 935.51 & & \\
\hline 26.0 & 936.06 & 95.0 & 934.61 & 30.0 & 936.08 & 100.3 & 936.24 & & \\
\hline 28.0 & 936.04 & 97.0 & 934.54 & 32.0 & 936.03 & 101.0 & 936.29 & & \\
\hline 30.0 & 936.07 & 98.0 & 934.62 & 34.0 & 935.93 & 102.0 & 936.23 & & \\
\hline 32.0 & 936.02 & 99.2 & 935.08 & 36.0 & 935.95 & 104.0 & 936.19 & & \\
\hline 33.0 & 935.92 & 99.5 & 935.57 & 38.0 & 936.00 & 106.0 & 936.15 & & \\
\hline 35.0 & 935.88 & 100.6 & 936.26 & 40.0 & 936.05 & 108.0 & 936.24 & & \\
\hline 37.0 & 935.96 & 102.0 & 936.22 & 42.0 & 936.06 & 110.0 & 936.25 & & \\
\hline 39.0 & 936.00 & 104.0 & 936.17 & 44.0 & 936.06 & 112.0 & 936.22 & & \\
\hline 41.0 & 936.05 & 106.0 & 936.15 & 46.0 & 936.05 & 114.0 & 936.17 & & \\
\hline 43.0 & 936.05 & 108.0 & 936.24 & 48.0 & 936.05 & 116.0 & 936.17 & & \\
\hline 45.0 & 936.04 & 110.0 & 936.25 & 50.0 & 936.08 & 118.0 & 936.22 & & \\
\hline 47.0 & 936.04 & 112.0 & 936.22 & 52.0 & 936.10 & 120.0 & 936.16 & & \\
\hline 49.0 & 936.05 & 114.0 & 936.17 & 54.0 & 936.14 & 122.0 & 936.20 & & \\
\hline 51.0 & 936.09 & 116.0 & 936.17 & 54.7 & 936.16 & 124.0 & 936.20 & & \\
\hline 53.0 & 936.13 & 118.0 & 936.20 & 56.0 & 936.07 & 126.0 & 936.17 & & \\
\hline 55.0 & 936.15 & 120.0 & 936.16 & 57.0 & 935.91 & 128.0 & 936.11 & & \\
\hline 56.0 & 936.07 & 122.0 & 936.19 & 59.0 & 935.65 & 130.0 & 936.01 & & \\
\hline 58.0 & 935.72 & 124.0 & 936.19 & 59.2 & 935.67 & 132.0 & 936.01 & & \\
\hline 60.0 & 935.61 & 126.0 & 936.17 & 61.0 & 935.48 & 133.0 & 935.99 & & \\
\hline 61.0 & 935.33 & 128.0 & 936.12 & 61.4 & 935.26 & 133.9 & 936.03 & & \\
\hline 62.0 & 935.25 & 130.0 & 936.00 & 62.5 & 935.13 & 134.2 & 936.06 & & \\
\hline 63.6 & 935.06 & & & 63.3 & 934.92 & 134.5 & 936.30 & & \\
\hline 64.6 & 934.89 & & & 65.6 & 934.65 & 134.8 & 936.44 & & \\
\hline 65.4 & 935.07 & & & 68.0 & 934.46 & 136.0 & 936.67 & & \\
\hline
\end{tabular}


Table 36. (Continued) Listing of horizontal stations and elevations for cross section PR191

[Sta., station, distance in meters from a reference pin on the left bank; Elev., elevation, in meters above sea level]

\begin{tabular}{|c|c|c|c|c|c|c|c|c|c|}
\hline \multirow{2}{*}{\multicolumn{2}{|c|}{$\begin{array}{c}1991 \\
2 \text { September }\end{array}$}} & \multicolumn{2}{|c|}{1991} & \multicolumn{2}{|c|}{1992} & \multicolumn{2}{|c|}{1992} & \multicolumn{2}{|c|}{1993} \\
\hline & & $2 \mathrm{Se}$ & ember & 29 & gust & 29 & Igust & $3 \mathrm{Se}$ & mber \\
\hline Sta. & Elev. & Sta. & Elev. & Sta. & Elev. & Sta. & Elev. & Sta. & Elev. \\
\hline-0.4 & 937.84 & 70.0 & 934.55 & -0.5 & 937.83 & 70.0 & 934.48 & -0.4 & 937.84 \\
\hline 0.0 & 937.70 & 71.0 & 934.50 & 0.0 & 937.70 & 72.0 & 934.51 & 2.0 & 937.58 \\
\hline 1.0 & 937.68 & 73.0 & 934.56 & 1.0 & 937.66 & 74.0 & 934.62 & 5.0 & 937.62 \\
\hline 2.0 & 937.58 & 75.0 & 934.69 & 2.0 & 937.57 & 76.0 & 934.82 & 7.0 & 937.20 \\
\hline 4.0 & 937.54 & 75.8 & 934.77 & 4.0 & 937.55 & 78.0 & 935.02 & 8.5 & 937.06 \\
\hline 6.0 & 937.47 & 77.0 & 934.94 & 6.0 & 937.47 & 80.7 & 935.06 & 10.2 & 936.31 \\
\hline 7.0 & 937.20 & 79.0 & 934.99 & 7.0 & 937.20 & 83.0 & 935.11 & 11.0 & 936.21 \\
\hline 8.4 & 937.06 & 82.0 & 935.04 & 8.0 & 937.12 & 85.0 & 935.11 & 13.0 & 936.10 \\
\hline 9.1 & 936.85 & 85.0 & 935.08 & 8.5 & 937.06 & 87.4 & 935.08 & 15.0 & 936.07 \\
\hline 10.2 & 936.31 & 88.0 & 935.10 & 9.0 & 936.83 & 89.0 & 935.03 & 17.0 & 936.11 \\
\hline 12.4 & 936.09 & 91.0 & 935.03 & 10.3 & 936.29 & 91.0 & 935.02 & 19.0 & 936.09 \\
\hline 14.0 & 936.03 & 92.0 & 934.95 & 12.0 & 936.12 & 93.0 & 934.91 & 21.0 & 936.04 \\
\hline 16.0 & 936.03 & 95.0 & 934.84 & 14.0 & 936.05 & 95.0 & 934.79 & 23.0 & 936.00 \\
\hline 18.0 & 936.03 & 97.0 & 934.81 & 16.0 & 936.04 & 97.0 & 934.79 & 25.0 & 936.08 \\
\hline 20.0 & 936.04 & 99.0 & 934.84 & 18.0 & 936.04 & 99.0 & 934.83 & 27.0 & 936.13 \\
\hline 22.0 & 935.93 & 99.7 & 935.13 & 20.0 & 936.05 & 99.7 & 935.08 & 29.0 & 936.13 \\
\hline 24.0 & 935.98 & 100.6 & 935.93 & 22.0 & 935.93 & 100.4 & 935.71 & 31.0 & 936.12 \\
\hline 26.0 & 936.08 & 100.7 & 936.30 & 24.0 & 935.98 & 100.8 & 936.27 & 33.0 & 936.00 \\
\hline 28.0 & 936.06 & 102.0 & 936.26 & 26.0 & 936.08 & 102.0 & 936.20 & 35.0 & 936.00 \\
\hline 30.0 & 936.08 & 104.0 & 936.19 & 28.0 & 936.07 & 104.0 & 936.16 & 37.0 & 936.05 \\
\hline 32.0 & 936.04 & 106.0 & 936.16 & 30.0 & 936.08 & 106.0 & 936.14 & 39.0 & 936.10 \\
\hline 34.0 & 935.94 & 108.0 & 936.23 & 32.0 & 936.03 & 108.0 & 936.23 & 41.0 & 936.10 \\
\hline 36.0 & 935.96 & 111.0 & 936.23 & 34.0 & 935.95 & 110.0 & 936.21 & 43.0 & 936.11 \\
\hline 38.0 & 936.01 & 114.0 & 936.16 & 36.0 & 935.96 & 113.0 & 936.19 & 45.0 & 936.10 \\
\hline 40.0 & 936.05 & 117.0 & 936.18 & 38.0 & 936.00 & 116.0 & 936.18 & 47.0 & 936.10 \\
\hline 42.0 & 936.07 & 120.0 & 936.15 & 40.0 & 936.06 & 119.0 & 936.20 & 49.0 & 936.13 \\
\hline 44.0 & 936.06 & 123.0 & 936.23 & 42.0 & 936.07 & 122.0 & 936.17 & 51.0 & 936.17 \\
\hline 46.0 & 936.04 & 126.0 & 936.17 & 44.0 & 936.07 & 125.0 & 936.19 & 53.0 & 936.20 \\
\hline 48.0 & 936.05 & 128.5 & 936.04 & 46.0 & 936.05 & 127.0 & 936.16 & 54.0 & 936.20 \\
\hline 50.0 & 936.08 & 130.0 & 936.01 & 48.0 & 936.06 & 128.0 & 936.12 & 55.0 & 936.20 \\
\hline 52.0 & 936.10 & 132.0 & 936.00 & 50.0 & 936.08 & 130.0 & 936.00 & 56.0 & 936.18 \\
\hline 54.0 & 936.14 & 133.0 & 936.00 & 52.0 & 936.11 & 132.0 & 935.99 & 57.0 & 936.11 \\
\hline 56.0 & 936.12 & 134.0 & 936.04 & 54.0 & 936.14 & 133.6 & 935.99 & 58.9 & 935.90 \\
\hline 56.5 & 936.09 & 135.0 & 936.42 & 56.0 & 936.13 & 135.0 & 936.39 & 59.3 & 935.67 \\
\hline 58.0 & 935.84 & 136.0 & 936.66 & 57.0 & 936.01 & 136.0 & 936.63 & 59.7 & 935.63 \\
\hline 60.0 & 935.65 & 137.0 & 936.78 & 59.0 & 935.77 & 137.3 & 936.80 & 60.2 & 935.44 \\
\hline 60.6 & 935.54 & 139.0 & 936.81 & 59.6 & 935.79 & 139.0 & 936.80 & 60.4 & 935.11 \\
\hline 61.0 & 935.31 & 141.0 & 936.81 & 60.9 & 935.31 & 141.0 & 936.80 & 61.0 & 934.96 \\
\hline 62.0 & 935.18 & 144.0 & 936.85 & 62.5 & 935.08 & 144.0 & 936.86 & 63.0 & 934.71 \\
\hline 64.0 & 934.78 & 147.0 & 936.87 & 64.0 & 934.79 & 147.0 & 936.88 & 65.0 & 934.76 \\
\hline 66.7 & 934.52 & 150.0 & 936.80 & 66.0 & 934.61 & 150.0 & 936.79 & 67.0 & 934.61 \\
\hline 68.0 & 934.54 & 151.6 & 936.87 & 68.0 & 934.45 & 151.6 & 936.87 & 70.0 & 934.70 \\
\hline
\end{tabular}


Table 36. (Continued) Listing of horizontal stations and elevations for cross section PR191

[Sta., station, distance in meters from a reference pin on the left bank; Elev., elevation, in meters above sea level]

\begin{tabular}{|c|c|c|c|c|c|c|c|c|c|}
\hline \multirow{2}{*}{\multicolumn{2}{|c|}{$\begin{array}{c}1993 \\
3 \text { September }\end{array}$}} & \multirow{2}{*}{\multicolumn{2}{|c|}{$\begin{array}{c}1993 \\
3 \text { September }\end{array}$}} & \multirow{2}{*}{\multicolumn{2}{|c|}{$\begin{array}{c}1994 \\
19 \text { September }\end{array}$}} & \multirow{2}{*}{\multicolumn{2}{|c|}{$\begin{array}{c}1994 \\
\text { 19 September }\end{array}$}} & \multirow{2}{*}{\multicolumn{2}{|c|}{$\begin{array}{c}1995 \\
30 \text { September }\end{array}$}} \\
\hline & & & & & & & & & \\
\hline Sta. & Elev. & Sta. & Elev. & Sta. & Elev. & Sta. & Elev. & Sta. & Elev. \\
\hline 73.0 & 934.83 & 143.0 & 936.83 & -0.4 & 937.81 & 88.0 & 934.77 & -0.4 & 937.82 \\
\hline 76.0 & 934.87 & 147.0 & 936.87 & 0.0 & 937.70 & 90.0 & 934.75 & 0.0 & 937.70 \\
\hline 79.0 & 935.01 & 150.0 & 936.81 & 2.0 & 937.58 & 92.0 & 934.70 & 1.0 & 937.66 \\
\hline 81.0 & 935.07 & 151.6 & 936.88 & 4.0 & 937.55 & 94.0 & 934.68 & 3.0 & 937.59 \\
\hline 83.0 & 935.10 & & & 5.0 & 937.60 & 96.0 & 934.66 & 5.0 & 937.62 \\
\hline 85.0 & 935.10 & & & 7.0 & 937.18 & 98.0 & 934.66 & 7.0 & 937.20 \\
\hline 87.0 & 935.03 & & & 8.0 & 937.07 & 99.2 & 934.90 & 8.0 & 937.07 \\
\hline 90.0 & 934.78 & & & 10.0 & 936.34 & 100.8 & 935.39 & 10.4 & 936.35 \\
\hline 93.0 & 934.72 & & & 11.0 & 936.25 & 101.5 & 936.30 & 12.0 & 936.25 \\
\hline 95.0 & 934.81 & & & 13.0 & 936.10 & 103.0 & 936.41 & 14.0 & 936.18 \\
\hline 97.0 & 934.92 & & & 15.0 & 936.07 & 105.0 & 936.46 & 16.0 & 936.21 \\
\hline 99.0 & 934.93 & & & 18.0 & 936.09 & 108.0 & 936.44 & 18.0 & 936.23 \\
\hline 99.7 & 934.83 & & & 21.0 & 936.05 & 111.0 & 936.39 & 20.0 & 936.23 \\
\hline 100.6 & 935.20 & & & 25.0 & 936.09 & 114.0 & 936.34 & 22.0 & 936.17 \\
\hline 100.7 & 935.54 & & & 28.0 & 936.12 & 117.0 & 936.39 & 24.0 & 936.18 \\
\hline 101.2 & 935.90 & & & 31.0 & 936.13 & 120.0 & 936.38 & 26.0 & 936.26 \\
\hline 101.3 & 936.22 & & & 33.0 & 936.01 & 123.0 & 936.40 & 28.0 & 936.27 \\
\hline 101.8 & 936.40 & & & 36.0 & 936.03 & 125.0 & 936.31 & 30.0 & 936.27 \\
\hline 103.0 & 936.41 & & & 39.0 & 936.07 & 127.0 & 936.27 & 32.0 & 936.25 \\
\hline 104.0 & 936.46 & & & 42.0 & 936.11 & 129.0 & 936.12 & 34.0 & 936.23 \\
\hline 105.0 & 936.56 & & & 45.0 & 936.11 & 130.0 & 936.08 & 36.0 & 936.24 \\
\hline 106.0 & 936.50 & & & 48.0 & 936.12 & 132.0 & 936.07 & 38.0 & 936.26 \\
\hline 107.0 & 936.43 & & & 51.0 & 936.16 & 133.5 & 936.07 & 40.0 & 936.27 \\
\hline 109.0 & 936.51 & & & 53.0 & 936.21 & 134.2 & 936.15 & 42.0 & 936.27 \\
\hline 111.0 & 936.47 & & & 55.0 & 936.21 & 136.0 & 936.65 & 44.0 & 936.29 \\
\hline 113.0 & 936.47 & & & 56.5 & 936.21 & 137.0 & 936.78 & 46.0 & 936.29 \\
\hline 115.3 & 936.50 & & & 58.6 & 935.95 & 139.0 & 936.80 & 48.0 & 936.32 \\
\hline 116.0 & 936.41 & & & 59.8 & 935.56 & 142.0 & 936.82 & 50.0 & 936.34 \\
\hline 118.0 & 936.47 & & & 59.9 & 935.27 & 145.0 & 936.85 & 52.0 & 936.39 \\
\hline 120.0 & 936.38 & & & 61.7 & 934.89 & 150.0 & 936.80 & 53.3 & 936.43 \\
\hline 122.0 & 936.37 & & & 64.0 & 934.68 & 151.6 & 936.88 & 55.0 & 936.45 \\
\hline 124.0 & 936.33 & & & 66.0 & 934.55 & 186.0 & 936.86 & 56.1 & 936.47 \\
\hline 126.0 & 936.31 & & & 68.0 & 934.50 & & & 57.8 & 935.96 \\
\hline 128.0 & 936.17 & & & 70.0 & 934.62 & & & 58.5 & 935.31 \\
\hline 129.0 & 936.10 & & & 72.0 & 934.71 & & & 59.4 & 934.79 \\
\hline 130.0 & 936.06 & & & 74.0 & 934.77 & & & 61.0 & 934.81 \\
\hline 132.0 & 936.06 & & & 76.0 & 934.76 & & & 63.0 & 934.86 \\
\hline 133.0 & 936.04 & & & 78.0 & 934.76 & & & 65.0 & 934.94 \\
\hline 134.0 & 936.09 & & & 80.0 & 934.79 & & & 67.0 & 934.97 \\
\hline 135.5 & 936.55 & & & 82.0 & 934.77 & & & 69.0 & 935.00 \\
\hline 137.0 & 936.78 & & & 84.0 & 934.79 & & & 71.0 & 935.05 \\
\hline 140.0 & 936.79 & & & 86.0 & 934.78 & & & 73.0 & 935.06 \\
\hline
\end{tabular}


Table 36. (Continued) Listing of horizontal stations and elevations for cross section PR191

[Sta., station, distance in meters from a reference pin on the left bank; Elev., elevation, in meters above sea level]

\begin{tabular}{|c|c|c|c|c|c|c|c|c|c|}
\hline \multirow{2}{*}{\multicolumn{2}{|c|}{$\begin{array}{c}1995 \\
30 \text { September }\end{array}$}} & \multicolumn{2}{|c|}{1995} & \multicolumn{2}{|c|}{1996} & \multicolumn{2}{|c|}{1996} & \multicolumn{2}{|c|}{1997} \\
\hline & & $30 \mathrm{Se}$ & ember & $22 \mathrm{C}$ & tober & 22 & tober & $17 \mathrm{Se}$ & ember \\
\hline Sta. & Elev. & Sta. & Elev. & Sta. & Elev. & Sta. & Elev. & Sta. & Elev. \\
\hline 75.0 & 935.06 & 142.0 & 936.81 & -0.4 & 937.82 & 98.0 & 934.70 & -0.4 & 937.83 \\
\hline 77.0 & 934.98 & 144.0 & 936.86 & 1.0 & 937.65 & 99.0 & 934.61 & 1.0 & 937.65 \\
\hline 79.0 & 934.97 & 146.0 & 936.88 & 2.0 & 937.59 & 99.5 & 934.94 & 3.3 & 937.58 \\
\hline 81.0 & 935.07 & 148.0 & 936.86 & 4.0 & 937.55 & 101.0 & 935.20 & 6.0 & 937.51 \\
\hline 83.0 & 934.95 & 150.0 & 936.81 & 6.0 & 937.45 & 102.0 & 935.59 & 7.0 & 937.26 \\
\hline 85.0 & 934.94 & 151.6 & 936.88 & 7.0 & 937.19 & 102.4 & 936.09 & 8.0 & 937.11 \\
\hline 87.0 & 934.94 & 142.0 & 936.81 & 8.4 & 937.02 & 104.0 & 936.58 & 9.0 & 936.92 \\
\hline 89.0 & 934.86 & 144.0 & 936.86 & 10.0 & 936.40 & 105.5 & 936.67 & 10.0 & 936.43 \\
\hline 91.0 & 934.96 & 146.0 & 936.88 & 11.0 & 936.31 & 107.0 & 936.69 & 12.0 & 936.24 \\
\hline 93.0 & 934.94 & 148.0 & 936.86 & 13.0 & 936.20 & 109.0 & 936.66 & 14.0 & 936.19 \\
\hline 95.0 & 934.86 & 150.0 & 936.81 & 16.0 & 936.20 & 111.0 & 936.68 & 17.0 & 936.22 \\
\hline 97.0 & 934.79 & 151.6 & 936.88 & 19.0 & 936.22 & 114.0 & 936.63 & 20.0 & 936.24 \\
\hline 99.0 & 934.59 & & & 22.0 & 936.16 & 117.0 & 936.60 & 23.0 & 936.19 \\
\hline 99.7 & 935.31 & & & 25.0 & 936.21 & 120.0 & 936.61 & 26.0 & 936.26 \\
\hline 100.0 & 935.47 & & & 28.0 & 936.27 & 123.0 & 936.63 & 29.0 & 936.28 \\
\hline 100.7 & 935.32 & & & 31.0 & 936.28 & 125.0 & 936.54 & 32.0 & 936.27 \\
\hline 101.3 & 935.47 & & & 34.0 & 936.23 & 128.0 & 936.54 & 35.0 & 936.23 \\
\hline 102.3 & 936.25 & & & 37.0 & 936.27 & 129.0 & 936.50 & 38.0 & 936.26 \\
\hline 102.6 & 936.38 & & & 40.0 & 936.28 & 130.0 & 936.38 & 41.0 & 936.30 \\
\hline 104.0 & 936.61 & & & 43.0 & 936.29 & 131.0 & 936.34 & 44.0 & 936.32 \\
\hline 105.0 & 936.64 & & & 46.0 & 936.30 & 133.0 & 936.40 & 47.0 & 936.33 \\
\hline 107.0 & 936.64 & & & 49.0 & 936.36 & 134.5 & 936.39 & 50.0 & 936.41 \\
\hline 109.0 & 936.62 & & & 52.0 & 936.45 & 137.0 & 936.78 & 53.0 & 936.46 \\
\hline 111.0 & 936.66 & & & 54.6 & 936.46 & 138.0 & 936.81 & 54.0 & 936.45 \\
\hline 113.0 & 936.70 & & & 56.0 & 936.24 & 141.0 & 936.81 & 55.7 & 936.35 \\
\hline 115.0 & 936.68 & & & 57.3 & 935.80 & 144.0 & 936.87 & 56.6 & 936.01 \\
\hline 116.0 & 936.57 & & & 57.7 & 935.41 & 147.0 & 936.89 & 57.2 & 935.49 \\
\hline 118.0 & 936.53 & & & 58.2 & 935.18 & 150.0 & 936.81 & 59.6 & 935.00 \\
\hline 120.0 & 936.49 & & & 59.5 & 934.88 & 151.6 & 936.87 & 62.5 & 935.20 \\
\hline 122.0 & 936.57 & & & 62.0 & 934.87 & 186.0 & 936.77 & 64.5 & 935.22 \\
\hline 123.0 & 936.63 & & & 65.0 & 934.84 & & & 65.3 & 935.13 \\
\hline 125.0 & 936.53 & & & 68.0 & 934.84 & & & 66.3 & 935.05 \\
\hline 127.0 & 936.55 & & & 71.0 & 935.00 & & & 67.3 & 935.13 \\
\hline 129.5 & 936.50 & & & 74.0 & 935.08 & & & 69.0 & 934.83 \\
\hline 130.0 & 936.36 & & & 77.0 & 935.07 & & & 70.5 & 934.86 \\
\hline 131.0 & 936.30 & & & 80.0 & 935.04 & & & 73.0 & 934.90 \\
\hline 133.0 & 936.39 & & & 83.0 & 935.03 & & & 76.0 & 934.90 \\
\hline 134.5 & 936.40 & & & 86.0 & 935.01 & & & 79.0 & 934.88 \\
\hline 135.5 & 936.59 & & & 87.0 & 935.13 & & & 82.0 & 934.76 \\
\hline 137.0 & 936.79 & & & 89.0 & 935.01 & & & 85.0 & 934.78 \\
\hline 138.0 & 936.80 & & & 92.0 & 934.82 & & & 88.0 & 934.75 \\
\hline 140.0 & 936.81 & & & 95.0 & 934.75 & & & 91.0 & 934.70 \\
\hline
\end{tabular}


Table 36. (Continued) Listing of horizontal stations and elevations for cross section PR191

[Sta., station, distance in meters from a reference pin on the left bank; Elev., elevation, in meters above sea level]

\begin{tabular}{|c|c|c|c|c|c|}
\hline \multirow{2}{*}{\multicolumn{2}{|c|}{$\begin{array}{c}1997 \\
17 \text { September }\end{array}$}} & \multicolumn{2}{|c|}{1998} & \multicolumn{2}{|c|}{1998} \\
\hline & & \multicolumn{2}{|c|}{24 September } & \multicolumn{2}{|c|}{24 September } \\
\hline Sta. & Elev. & Sta. & Elev. & Sta. & Elev. \\
\hline 94.0 & 934.69 & -0.4 & 937.84 & 92.0 & 934.61 \\
\hline 97.0 & 934.70 & 0.0 & 937.72 & 94.0 & 934.70 \\
\hline 99.5 & 934.93 & 2.0 & 937.58 & 96.0 & 934.51 \\
\hline 101.3 & 935.15 & 4.0 & 937.54 & 98.0 & 934.59 \\
\hline 102.5 & 935.50 & 5.3 & 937.59 & 100.0 & 934.64 \\
\hline 103.0 & 935.85 & 8.0 & 937.08 & 101.8 & 935.27 \\
\hline 103.2 & 936.27 & 11.0 & 936.32 & 102.9 & 935.59 \\
\hline 103.6 & 936.47 & 14.0 & 936.15 & 103.3 & 936.36 \\
\hline 105.0 & 936.65 & 17.0 & 936.22 & 105.0 & 936.67 \\
\hline 107.0 & 936.70 & 19.0 & 936.23 & 108.0 & 936.67 \\
\hline 109.0 & 936.68 & 22.0 & 936.17 & 111.0 & 936.68 \\
\hline 111.0 & 936.67 & 25.0 & 936.20 & 114.0 & 936.64 \\
\hline 114.0 & 936.64 & 28.0 & 936.27 & 117.0 & 936.58 \\
\hline 117.0 & 936.59 & 31.0 & 936.28 & 120.0 & 936.60 \\
\hline 120.0 & 936.59 & 34.0 & 936.22 & 123.0 & 936.63 \\
\hline 123.0 & 936.64 & 37.0 & 936.26 & 126.0 & 936.58 \\
\hline 125.0 & 936.54 & 40.0 & 936.26 & 129.0 & 936.52 \\
\hline 127.0 & 936.54 & 43.0 & 936.29 & 130.0 & 936.41 \\
\hline 129.0 & 936.51 & 45.0 & 936.30 & 131.5 & 936.38 \\
\hline 131.0 & 936.33 & 48.0 & 936.32 & 133.0 & 936.40 \\
\hline 133.0 & 936.39 & 51.0 & 936.41 & 135.0 & 936.48 \\
\hline 134.5 & 936.39 & 54.0 & 936.46 & 136.5 & 936.75 \\
\hline 136.0 & 936.67 & 55.4 & 936.34 & 138.0 & 936.81 \\
\hline 138.0 & 936.81 & 56.1 & 936.03 & 141.0 & 936.81 \\
\hline 140.0 & 936.81 & 56.2 & 935.75 & 144.0 & 936.88 \\
\hline 143.0 & 936.84 & 57.9 & 935.27 & 147.0 & 936.90 \\
\hline 146.0 & 936.89 & 60.0 & 935.10 & 150.0 & 936.84 \\
\hline 149.0 & 936.83 & 62.0 & 935.16 & 151.6 & 936.88 \\
\hline 151.6 & 936.88 & 64.0 & 935.16 & 186.0 & 936.77 \\
\hline & & 66.0 & 935.12 & & \\
\hline & & 68.0 & 934.95 & & \\
\hline & & 70.0 & 934.73 & & \\
\hline & & 72.0 & 934.71 & & \\
\hline & & 74.0 & 934.65 & & \\
\hline & & 76.0 & 934.75 & & \\
\hline & & 78.0 & 934.73 & & \\
\hline & & 80.0 & 934.61 & & \\
\hline & & 82.0 & 934.55 & & \\
\hline & & 84.0 & 934.68 & & \\
\hline & & 86.0 & 934.71 & & \\
\hline & & 88.0 & 934.74 & & \\
\hline & & 90.0 & 934.70 & & \\
\hline
\end{tabular}




\section{Description of Cross Section PR194}

Location: Township 5 South/Range 51 East--section 30

U. S. Geological Survey quadrangle (1:24,000): Eldon Mountain

Landowners--left bank: Doug and Lucille Randall

--right bank: Doug and Lucille Randall

Access: Right bank

Permission from: John Stuver

Distance from Moorhead Gaging Station: 80.08 kilometers

Azimuth of Section (degrees magnetic): 225

\section{Reference Monuments}

[Monuments at stations $96.0,113.3$, and $\mathbf{1 1 3 . 8}$ were closest to leveling instrument]

\begin{tabular}{|c|c|c|c|c|c|c|}
\hline \multirow[b]{2}{*}{ Description } & \multirow[b]{2}{*}{$\begin{array}{c}\text { Station } \\
\text { (m) }\end{array}$} & \multicolumn{2}{|c|}{ GPS-NAD83 (1992) } & \multicolumn{2}{|c|}{ Measurement } & \multirow{2}{*}{$\begin{array}{c}\text { Elevation } \\
\text { (NGVD1929) } \\
\text { (m) }\end{array}$} \\
\hline & & Latitude & Longitude & $\begin{array}{l}\text { Standard } \\
\text { deviation } \\
\quad(m)\end{array}$ & $\begin{array}{l}\text { Horizontal } \\
\text { precision } \\
(\mathbf{m})\end{array}$ & \\
\hline Benchmark--brass circular plate; offsection & -- & $45^{\circ} 22^{\prime} 17.71^{\prime \prime}$ & $105^{\circ} 27^{\prime} 51.05^{\prime \prime}$ & 0.409 & 0.765 & 934.41 \\
\hline $\begin{array}{l}\text { 1/2-inch-rebar; bent, } 0.13 \text { meter above } 1998 \\
\text { ground level }\end{array}$ & -1.2 & $45^{\circ} 22^{\prime} 16.99^{\prime \prime}$ & $105^{\circ} 27^{\prime} 51.85^{\prime \prime}$ & 0.507 & 1.047 & 934.32 \\
\hline 1/2-inch-rebar; flush with 1998 ground level & 0.0 & & & & & 934.19 \\
\hline $\begin{array}{l}\text { 1/2-inch-rebar; bent, } 0.09 \text { meter above } 1998 \\
\text { ground level }\end{array}$ & 96.0 & & & & & 934.21 \\
\hline $\begin{array}{l}\text { 1/2-inch-rebar; } 0.03 \text { meter above } 1998 \text { ground } \\
\text { level }\end{array}$ & 113.3 & & & & & 933.97 \\
\hline $\begin{array}{l}\text { 1/2-inch-rebar; } 0.16 \text { meter above } 1998 \text { ground } \\
\text { level }\end{array}$ & 113.8 & $45^{\circ} 22 ' 15.09^{\prime \prime}$ & $105^{\circ} 27^{\prime} 56.40^{\prime \prime}$ & 0.174 & 0.450 & 934.11 \\
\hline
\end{tabular}




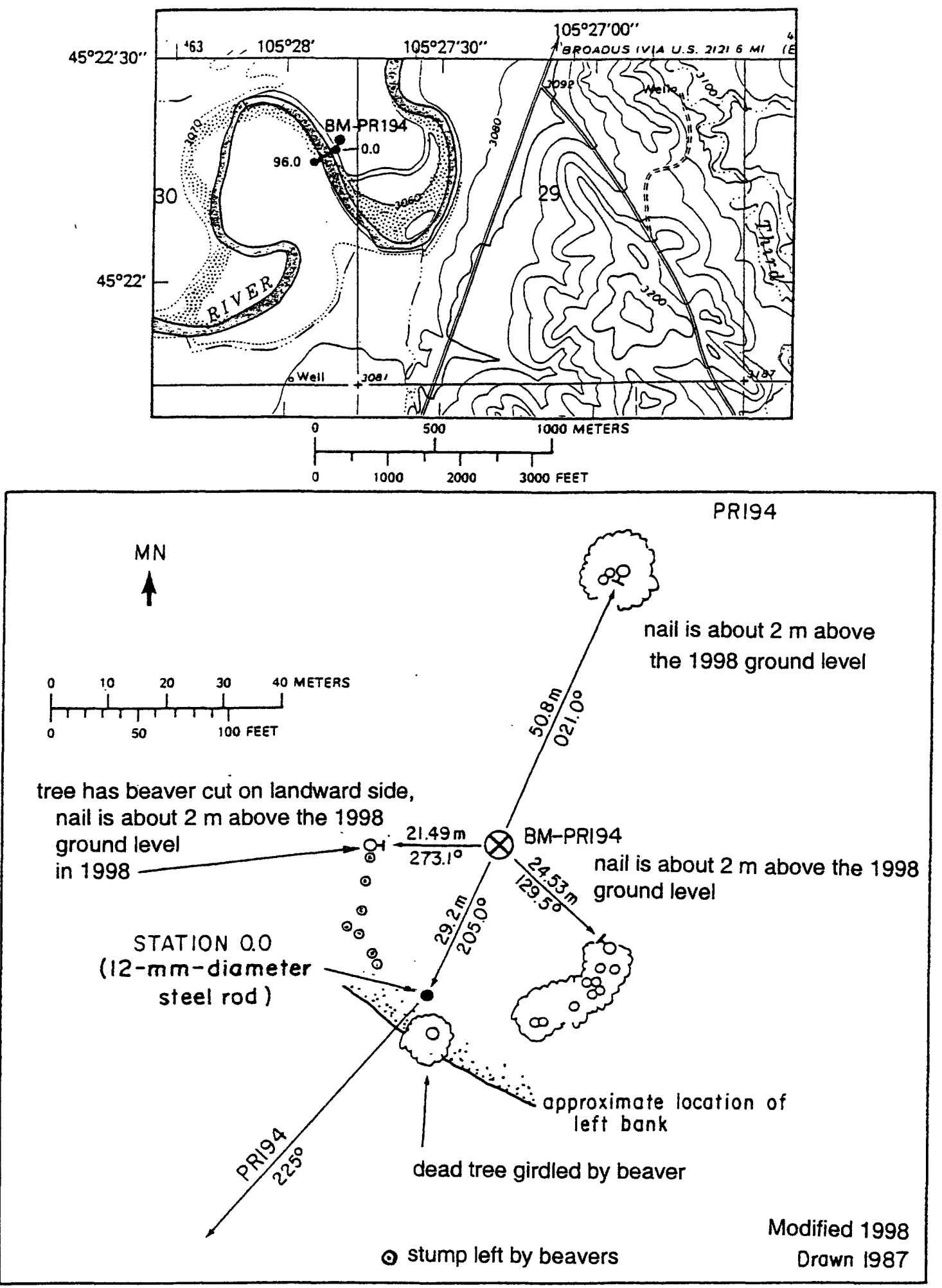

Figure 104. Upper: Location of cross section PR194, bench mark BM-PR194, and the left and right bank reference monuments in the Eldon Mountain quadrangle. Lower: Location of the bench mark on the left bank. MN is magnetic north. 

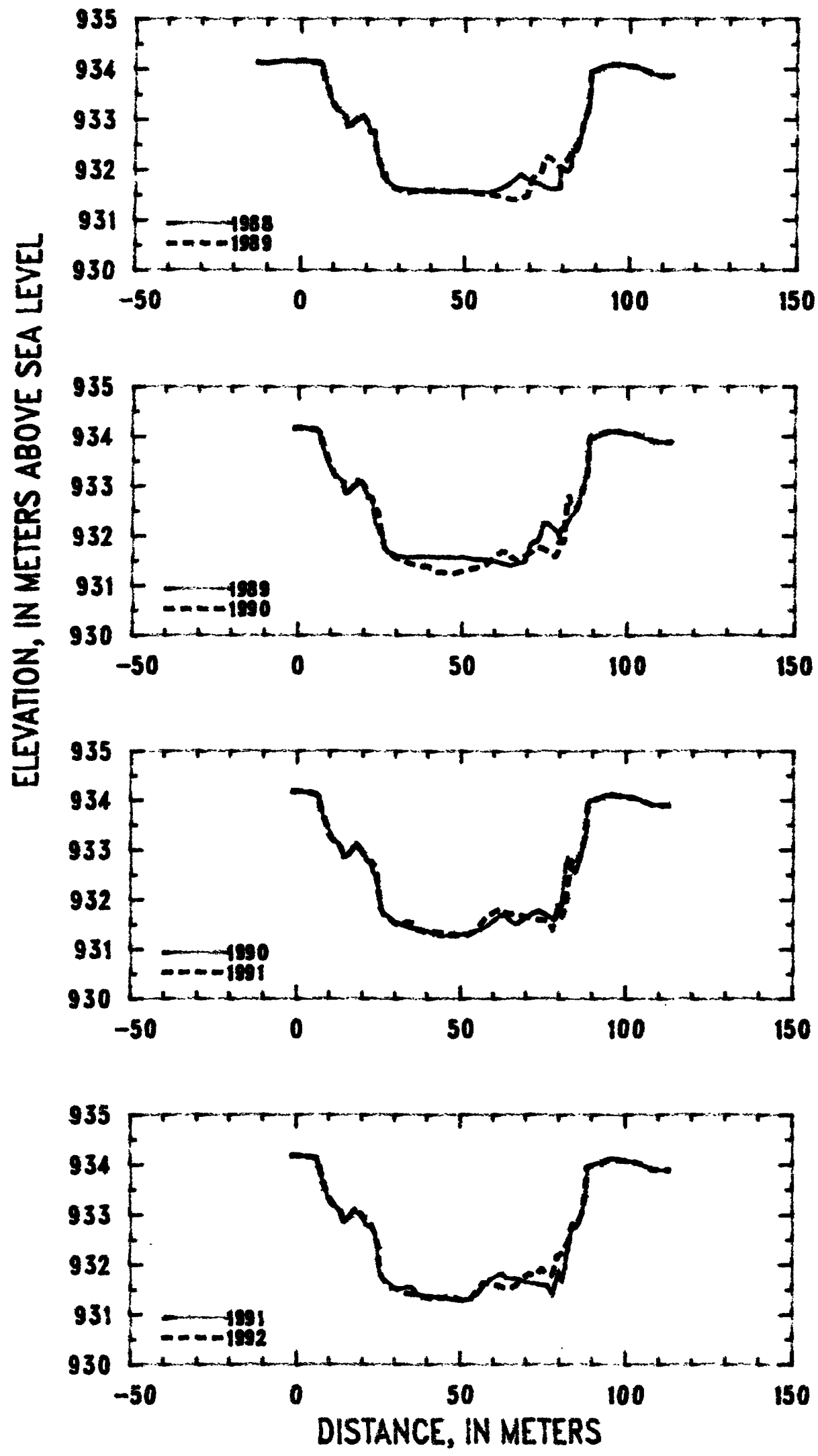

Figure 105. Profiles of cross section PR194 from 1988 to 1992. 

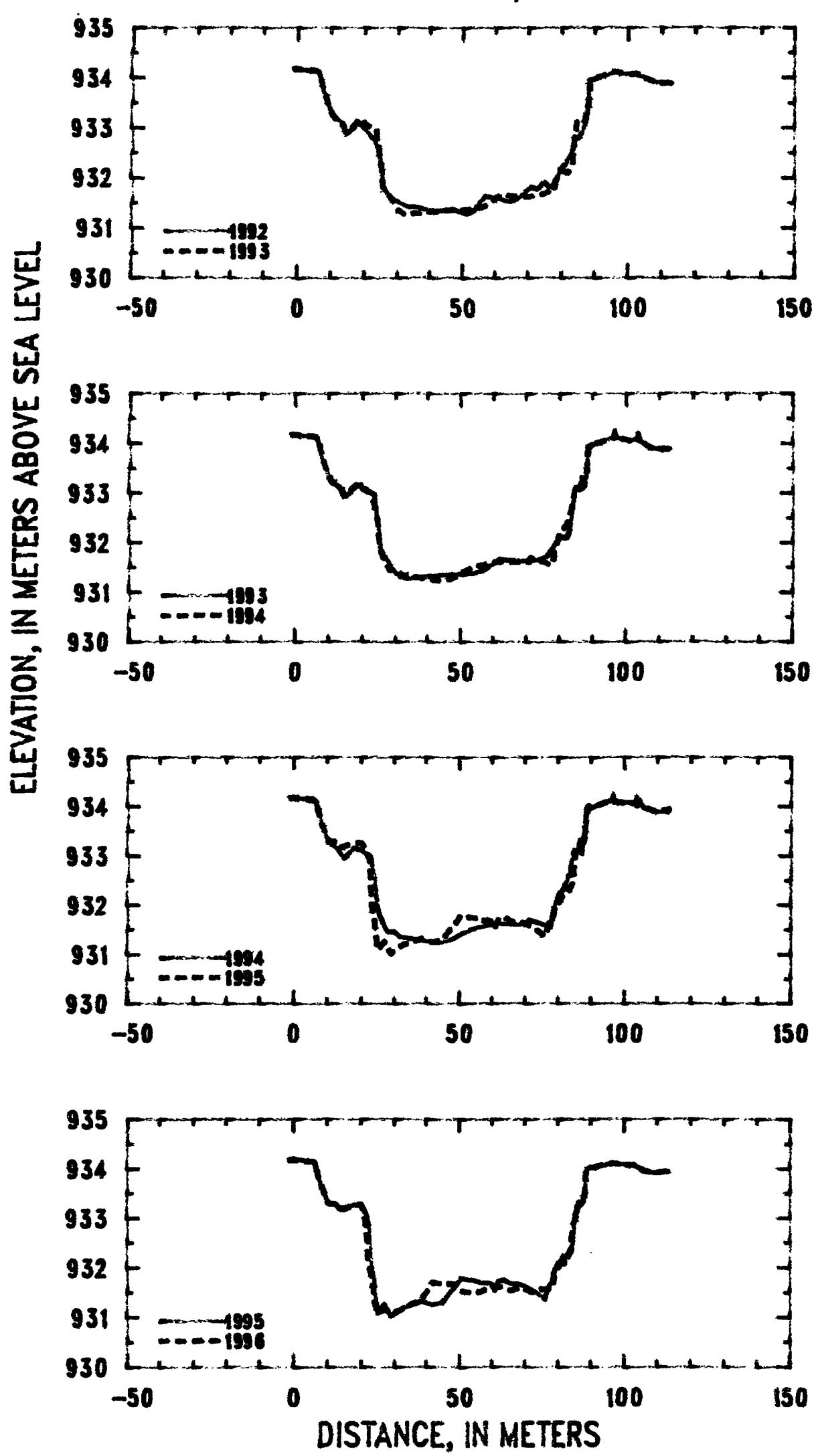

Figure 106. Profiles of cross section PR194 from 1992 to 1996. 


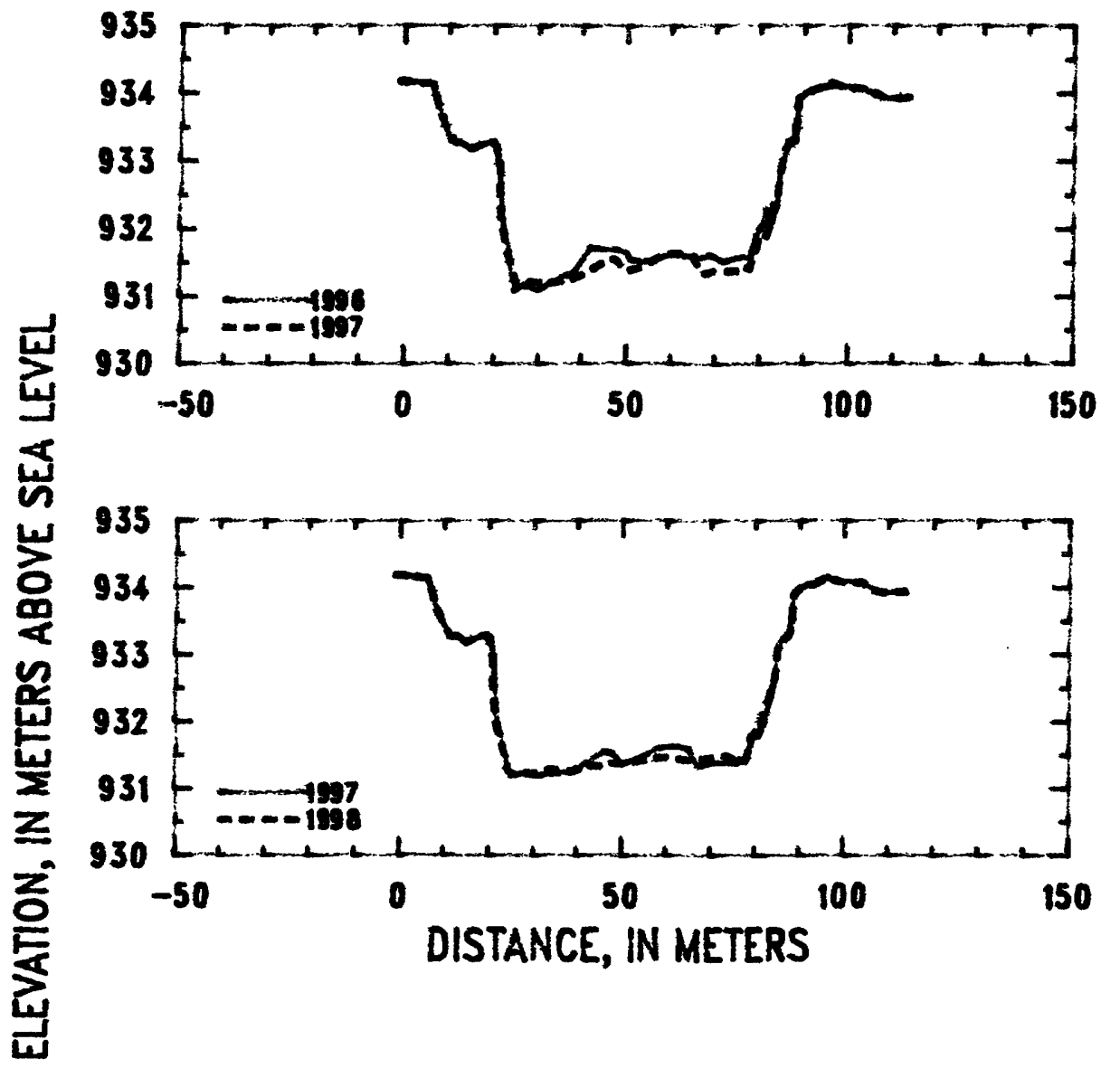

Figure 107. Profiles of cross section PR194 from 1996 to 1998. 
Table 37. Listing of horizontal stations and elevations for cross section PR 194

[Sta., station, distance in meters from a reference pin on the left bank; Elev., elevation, in meters above sea level]

\begin{tabular}{|c|c|c|c|c|c|c|c|c|c|}
\hline \multirow{2}{*}{\multicolumn{2}{|c|}{$\begin{array}{c}1989 \\
\text { 20 September }\end{array}$}} & & \multicolumn{2}{|c|}{1990} & \multicolumn{2}{|c|}{1990} & \multicolumn{2}{|c|}{1991} \\
\hline & & $20 \mathrm{~S}$ & ember & $20 \mathrm{Se}$ & ember & $20 \mathrm{Se}$ & ember & $3 \mathrm{Se}$ & ember \\
\hline Sta. & Elev. & Sta. & Elev. & Sta. & Elev. & Sta. & Elev. & Sta. & Elev. \\
\hline-1.2 & 934.17 & 61.0 & 931.47 & -1.2 & 934.18 & 62.0 & 931.69 & -1.2 & 934.18 \\
\hline 0.0 & 934.18 & 63.0 & 931.44 & 0.0 & 934.18 & 64.3 & 931.69 & 0.0 & 934.18 \\
\hline 2.0 & 934.17 & 65.0 & 931.40 & 3.0 & 934.16 & 65.3 & 931.56 & 2.0 & 934.18 \\
\hline 4.0 & 934.15 & 67.0 & 931.44 & 5.0 & 934.16 & 67.0 & 931.50 & 4.0 & 934.15 \\
\hline 6.0 & 934.13 & 69.3 & 931.48 & 6.8 & 934.09 & 69.0 & 931.59 & 6.7 & 934.09 \\
\hline 6.8 & 934.09 & 70.4 & 931.78 & 7.0 & 934.05 & 71.0 & 931.68 & 7.7 & 933.76 \\
\hline 7.1 & 933.92 & 71.0 & 931.86 & 7.2 & 933.94 & 72.0 & 931.73 & 9.0 & 933.53 \\
\hline 8.0 & 933.72 & 72.0 & 931.89 & 9.0 & 933.55 & 74.0 & 931.78 & 10.4 & 933.25 \\
\hline 10.0 & 933.33 & 73.0 & 931.91 & 11.0 & 933.21 & 75.0 & 931.73 & 11.4 & 933.18 \\
\hline 11.0 & 933.23 & 73.8 & 932.00 & 13.0 & 933.13 & 76.6 & 931.68 & 13.0 & 933.13 \\
\hline 12.0 & 933.17 & 74.6 & 932.26 & 13.7 & 933.08 & 78.5 & 931.59 & 14.6 & 932.86 \\
\hline 13.6 & 933.10 & 76.0 & 932.26 & 14.5 & 932.85 & 79.2 & 931.71 & 16.0 & 932.92 \\
\hline 14.2 & 932.89 & 78.0 & 932.15 & 15.0 & 932.87 & 79.6 & 931.89 & 18.0 & 933.11 \\
\hline 14.5 & 932.86 & 79.0 & 932.10 & 17.0 & 932.99 & 80.9 & 931.90 & 20.0 & 932.95 \\
\hline 15.0 & 932.87 & 79.5 & 931.99 & 18.3 & 933.13 & 81.3 & .932 .30 & 21.3 & 932.78 \\
\hline 17.0 & 933.01 & 80.0 & 932.13 & 20.0 & 933.01 & 82.0 & 932.70 & 23.0 & 932.76 \\
\hline 17.8 & 933.04 & 82.0 & 932.25 & 21.0 & 932.85 & 82.6 & 932.81 & 23.9 & 932.66 \\
\hline 18.1 & 933.13 & 83.0 & 932.40 & 21.5 & 932.78 & 83.0 & 932.68 & 24.4 & 932.48 \\
\hline 20.0 & 933.00 & 84.8 & 932.55 & 22.6 & 932.76 & 84.7 & 932.55 & 25.4 & 931.87 \\
\hline 21.2 & 932.76 & 86.4 & 932.93 & 23.3 & 932.56 & 85.1 & 932.60 & 26.4 & 931.72 \\
\hline 22.6 & 932.76 & 87.9 & 933.26 & 24.2 & 932.48 & 86.1 & 932.75 & 28.0 & 931.66 \\
\hline 23.2 & 932.31 & 88.4 & 933.61 & 24.9 & 932.22 & 87.0 & 932.99 & 30.0 & 931.52 \\
\hline 24.6 & 932.08 & 88.6 & 933.96 & 25.3 & 931.95 & 88.0 & 933.29 & 32.0 & 931.50 \\
\hline 25.4 & 932.06 & 89.0 & 934.04 & 25.9 & 931.88 & 88.7 & 933.97 & 34.0 & 931.56 \\
\hline 26.2 & 931.81 & 90.0 & 933.98 & 26.3 & 931.75 & 91.0 & 934.05 & 35.6 & 931.54 \\
\hline 27.0 & 931.70 & 92.0 & 934.05 & 28.0 & 931.65 & 94.0 & 934.10 & 36.2 & 931.50 \\
\hline 29.0 & 931.63 & 94.0 & 934.09 & 30.0 & 931.55 & 96.0 & 934.11 & 36.8 & 931.41 \\
\hline 31.0 & 931.59 & 96.0 & 934.10 & 32.0 & 931.51 & 99.0 & 934.08 & 39.0 & 931.35 \\
\hline 33.0 & 931.56 & 98.0 & 934.10 & 34.0 & 931.44 & 102.0 & 934.06 & 41.0 & 931.37 \\
\hline 35.0 & 931.57 & 100.0 & 934.06 & 36.0 & 931.43 & 105.0 & 933.98 & 44.0 & 931.34 \\
\hline 37.0 & 931.59 & 102.0 & 934.05 & 38.0 & 931.39 & 108.0 & 933.89 & 46.0 & 931.32 \\
\hline 39.0 & 931.59 & 104.0 & 934.00 & 40.0 & 931.38 & 111.0 & 933.89 & 48.0 & 931.30 \\
\hline 41.0 & 931.59 & 106.0 & 933.95 & 42.0 & 931.29 & 113.3 & 933.88 & 50.0 & 931.28 \\
\hline 43.0 & 931.57 & 108.0 & 933.89 & 44.0 & 931.27 & & & 52.0 & 931.29 \\
\hline 45.0 & 931.57 & 110.0 & 933.87 & 46.0 & 931.27 & & & 54.0 & 931.34 \\
\hline 47.0 & 931.56 & 112.0 & 933.86 & 48.0 & 931.26 & & & 56.0 & 931.49 \\
\hline 49.0 & 931.57 & 113.3 & 933.87 & 50.0 & 931.29 & & & 57.6 & 931.63 \\
\hline 51.0 & 931.57 & & & 52.0 & 931.33 & & & 59.0 & 931.69 \\
\hline 53.0 & 931.52 & & & 54.0 & 931.35 & & & 61.0 & 931.78 \\
\hline 55.0 & 931.51 & & & 56.0 & 931.40 & & & 63.0 & 931.82 \\
\hline 57.0 & 931.52 & & & 58.0 & 931.46 & & & 63.6 & 931.75 \\
\hline 59.0 & 931.49 & & & 60.0 & 931.57 & & & 65.0 & 931.71 \\
\hline
\end{tabular}


Table 37. (Continued) Listing of horizontal stations and elevations for cross section PR194

[Sta., station, distance in meters from a reference pin on the left bank; Elev., elevation, in meters above sea level]

\begin{tabular}{|c|c|c|c|c|c|c|c|c|c|}
\hline \multirow{2}{*}{\multicolumn{2}{|c|}{$\begin{array}{c}1991 \\
3 \text { September }\end{array}$}} & \multicolumn{2}{|c|}{1992} & \multicolumn{2}{|c|}{1992} & \multicolumn{2}{|c|}{1993} & \multicolumn{2}{|c|}{1993} \\
\hline & & \multicolumn{2}{|c|}{1 September } & \multicolumn{2}{|c|}{1 September } & \multicolumn{2}{|c|}{2 September } & \multicolumn{2}{|c|}{2 September } \\
\hline Sta. & Elev. & Sta. & Elev. & Sta. & Elev. & Sta. & Elev. & Sta. & Elev. \\
\hline 67.0 & 931.72 & -1.2 & 934.18 & 71.0 & 931.82 & -1.2 & 934.18 & 72.0 & 931.65 \\
\hline 69.0 & 931.67 & 0.0 & 934.18 & 73.0 & 931.78 & 0.0 & 934.19 & 74.0 & 931.68 \\
\hline 71.0 & 931.63 & 3.0 & 934.16 & 74.4 & 931.90 & 3.0 & 934.16 & 76.0 & 931.72 \\
\hline 73.0 & 931.61 & 5.0 & 934.15 & 75.0 & 931.91 & 5.0 & 934.15 & 77.0 & 931.79 \\
\hline 75.0 & 931.58 & 6.4 & 934.13 & 76.0 & 931.83 & 6.6 & 934.11 & 78.0 & 931.92 \\
\hline 76.5 & 931.59 & 7.0 & 934.00 & 78.0 & 931.80 & 8.0 & 933.73 & 80.0 & 932.14 \\
\hline 78.1 & 931.40 & 7.8 & 933.75 & 79.6 & 932.19 & 10.4 & 933.25 & 82.0 & 932.09 \\
\hline 78.8 & 931.60 & 10.0 & 933.34 & 80.6 & 932.21 & 11.0 & 933.20 & 83.3 & 932.30 \\
\hline 79.9 & 931.85 & 12.0 & 933.17 & 82.5 & 932.43 & 13.0 & 933.15 & 83.8 & 932.73 \\
\hline 80.9 & 931.69 & 13.6 & 933.11 & 83.3 & 932.54 & 14.5 & 932.92 & 84.3 & 932.90 \\
\hline 82.1 & 931.99 & 14.5 & 932.88 & 84.0 & 932.81 & 16.0 & 932.98 & 84.7 & 933.12 \\
\hline 82.3 & 932.19 & 16.0 & 932.94 & 85.5 & 932.79 & 18.0 & 933.14 & 85.5 & 933.12 \\
\hline 82.6 & 932.26 & 18.0 & 933.12 & 87.0 & 933.02 & 19.5 & 933.18 & 86.0 & 933.06 \\
\hline 82.9 & 932.42 & 20.0 & 933.00 & 88.0 & 933.36 & 20.0 & 933.08 & 87.0 & 933.11 \\
\hline 83.8 & 932.76 & 22.0 & 932.83 & 88.3 & 933.58 & 22.0 & 933.00 & 88.0 & 933.36 \\
\hline 84.1 & 932.81 & 23.0 & 932.79 & 88.5 & 933.95 & 23.5 & 932.99 & 88.4 & 933.64 \\
\hline 84.9 & 932.74 & 24.0 & 932.60 & 90.0 & 933.98 & 24.0 & 932.71 & 88.6 & 933.94 \\
\hline 85.6 & 932.78 & 25.0 & 932.35 & 93.0 & 934.03 & 25.0 & 932.29 & 90.0 & 933.99 \\
\hline 86.6 & 932.93 & 25.6 & 931.91 & 96.0 & 934.12 & 25.5 & 931.83 & 93.0 & 934.05 \\
\hline 88.0 & 933.28 & 26.0 & 931.77 & 99.0 & 934.08 & 25.7 & 931.77 & 96.0 & 934.13 \\
\hline 88.4 & 933.63 & 27.0 & 931.67 & 102.0 & 934.06 & 28.0 & 931.60 & 100.0 & 934.07 \\
\hline 88.6 & 933.95 & 29.0 & 931.56 & 105.0 & 934.00 & 30.0 & 931.37 & 103.0 & 934.07 \\
\hline 90.0 & 933.99 & 31.0 & 931.51 & 108.0 & 933.89 & 32.0 & 931.29 & 106.0 & 933.96 \\
\hline 92.0 & 934.05 & 33.0 & 931.42 & 111.0 & 933.89 & 34.0 & 931.27 & 109.0 & 933.88 \\
\hline 94.0 & 934.08 & 35.0 & 931.42 & 113.3 & 933.88 & 36.0 & 931.30 & 111.0 & 933.90 \\
\hline 96.0 & 934.11 & 37.0 & 931.39 & & & 38.0 & 931.30 & 113.3 & 933.88 \\
\hline 99.0 & 934.07 & 39.0 & 931.36 & & & 40.0 & 931.33 & & \\
\hline 102.0 & 934.06 & 41.0 & 931.32 & & & 42.0 & 931.33 & & \\
\hline 105.0 & 933.99 & 43.0 & 931.35 & & & 44.0 & 931.36 & & \\
\hline 108.0 & 933.90 & 45.0 & 931.33 & & & 46.0 & 931.35 & & \\
\hline 111.0 & 933.89 & 47.0 & 931.38 & & & 48.0 & 931.38 & & \\
\hline 113.3 & 933.89 & 49.0 & 931.33 & & & 50.0 & 931.36 & . & \\
\hline & & 51.0 & 931.27 & & & 52.0 & 931.38 & & \\
\hline & & 53.0 & 931.30 & & & 54.0 & 931.39 & & \\
\hline & & 55.0 & 931.48 & & & 56.0 & 931.42 & & \\
\hline & & 57.0 & 931.63 & & & 58.0 & 931.48 & & \\
\hline & & 59.0 & 931.62 & & & 60.0 & 931.58 & & \\
\hline & & 61.0 & 931.59 & & & 62.0 & 931.68 & & \\
\hline & & 63.0 & 931.55 & & & 64.0 & 931.66 & & \\
\hline & & 65.0 & 931.53 & & & 66.0 & 931.64 & & \\
\hline & & 67.0 & 931.58 & & & 68.0 & 931.64 & & \\
\hline & & 69.4 & 931.74 & & & 70.0 & 931.62 & & \\
\hline
\end{tabular}


Table 37. (Continued) Listing of horizontal stations and elevations for cross section PR194

[Sta., station, distance in meters from a reference pin on the left bank; Elev., elevation, in meters above sea level]

\begin{tabular}{|c|c|c|c|c|c|c|c|c|c|}
\hline \multirow{2}{*}{\multicolumn{2}{|c|}{$\begin{array}{c}1994 \\
\text { 20 September }\end{array}$}} & \multicolumn{2}{|c|}{1994} & \multicolumn{2}{|c|}{1995} & \multicolumn{2}{|c|}{1995} & \multicolumn{2}{|c|}{1996} \\
\hline & & $20 \mathrm{~S}$ & ember & 10 & ober & 10 & ober & 16 & tober \\
\hline Sta. & Elev. & Sta. & Elev. & Sta. & Elev. & Sta. & Elev. & Sta. & Elev. \\
\hline-1.2 & 934.18 & 79.0 & 931.96 & -1.2 & 934.18 & 72.0 & 931.56 & -1.2 & 934.18 \\
\hline 0.0 & 934.19 & 80.0 & 932.20 & 0.0 & 934.19 & 74.0 & 931.48 & 0.0 & 934.19 \\
\hline 3.0 & 934.16 & 80.9 & 932.28 & 2.0 & 934.18 & 76.0 & 931.36 & 3.0 & 934.15 \\
\hline 5.0 & 934.16 & 82.0 & 932.34 & 4.0 & 934.16 & 77.5 & 931.65 & 6.0 & 934.15 \\
\hline 6.0 & 934.14 & 83.6 & 932.71 & 6.5 & 934.13 & 78.5 & 931.70 & 6.6 & 934.12 \\
\hline 6.6 & 934.11 & 84.5 & 933.06 & 8.5 & 933.60 & 78.6 & 931.79 & 8.0 & 933.73 \\
\hline 9.0 & 933.52 & 85.2 & 933.14 & 10.0 & 933.37 & 79.0 & 931.93 & 10.5 & 933.30 \\
\hline 10.5 & 933.26 & 86.3 & 933.09 & 10.5 & 933.29 & 79.5 & 931.98 & 13.0 & 933.28 \\
\hline 13.0 & 933.16 & 86.7 & 933.26 & 12.8 & 933.28 & 80.0 & 932.08 & 15.0 & 933.18 \\
\hline 14.9 & 932.94 & 87.3 & 933.10 & 13.9 & 933.17 & 80.5 & 932.06 & 17.0 & 933.24 \\
\hline 17.0 & 933.10 & 87.7 & 933.33 & 16.0 & 933.23 & 81.6 & 932.20 & 20.0 & 933.29 \\
\hline 18.0 & 933.18 & 88.0 & 933.32 & 18.0 & 933.27 & 83.0 & 932.24 & 20.5 & 933.23 \\
\hline 20.0 & 933.12 & 88.4 & 933.62 & 20.0 & 933.27 & 84.4 & 932.44 & 21.7 & 932.81 \\
\hline 22.7 & 933.01 & 88.6 & 933.93 & 21.0 & 933.18 & 85.2 & 933.04 & 22.1 & 932.14 \\
\hline 24.5 & 932.43 & 90.0 & 933.99 & 22.4 & 932.94 & 86.0 & 933.12 & 23.3 & 931.74 \\
\hline 24.9 & 932.02 & 92.0 & 934.03 & 23.3 & 931.93 & 86.2 & 933.26 & 24.6 & 931.08 \\
\hline 25.7 & 931.80 & 95.0 & 934.10 & 23.7 & 931.82 & 87.0 & 933.27 & 27.0 & 931.17 \\
\hline 26.5 & 931.66 & 96.4 & 934.12 & 25.0 & 931.10 & 87.8 & 933.32 & 30.0 & 931.09 \\
\hline 28.0 & 931.45 & 96.6 & 934.20 & 27.0 & 931.26 & 89.0 & 934.01 & 33.0 & 931.19 \\
\hline 30.0 & 931.47 & 97.0 & 934.09 & 29.0 & 931.01 & 90.0 & 934.04 & 36.0 & 931.29 \\
\hline 32.0 & 931.35 & 99.0 & 934.09 & 31.0 & 931.12 & 92.0 & 934.04 & 38.0 & 931.32 \\
\hline 35.0 & 931.33 & 101.0 & 934.06 & 33.0 & 931.21 & 94.0 & 934.09 & 40.0 & 931.49 \\
\hline 38.0 & 931.29 & 103.3 & 934.06 & 35.0 & 931.23 & 97.0 & 934.12 & 42.0 & 931.72 \\
\hline 41.0 & 931.26 & 103.6 & 934.16 & 37.0 & 931.29 & 99.0 & 934.08 & 45.0 & 931.69 \\
\hline 44.0 & 931.24 & 104.0 & 934.09 & 39.0 & 931.33 & 101.0 & 934.07 & 48.0 & 931.68 \\
\hline 47.0 & 931.27 & 104.4 & 934.12 & 41.0 & 931.26 & 103.0 & 934.06 & 50.0 & 931.64 \\
\hline 50.0 & 931.38 & 105.0 & 934.02 & 43.0 & 931.27 & 105.0 & 934.01 & 51.0 & 931.53 \\
\hline 52.0 & 931.46 & 106.5 & 933.92 & 45.0 & 931.30 & 107.0 & 933.95 & 54.0 & 931.50 \\
\hline 55.0 & 931.53 & 109.0 & 933.88 & 47.0 & 931.49 & 109.0 & 933.91 & 57.0 & 931.53 \\
\hline 58.0 & 931.57 & 111.0 & 933.89 & 49.0 & 931.64 & 111.0 & 933.92 & 60.0 & 931.63 \\
\hline 60.0 & 931.61 & 113.3 & 933.88 & 50.6 & 931.80 & 113.3 & 933.94 & 63.0 & 931.61 \\
\hline 62.0 & 931.63 & & & 53.0 & 931.75 & 113.8 & 933.95 & 66.0 & 931.55 \\
\hline 64.0 & 931.62 & & & 55.0 & 931.74 & & & 69.0 & 931.61 \\
\hline 66.0 & 931.61 & & & 57.0 & 931.68 & & & 72.0 & 931.51 \\
\hline 69.0 & 931.61 & & & 59.0 & 931.69 & & & 75.0 & 931.58 \\
\hline 71.0 & 931.63 & & & 60.0 & 931.67 & & & 78.0 & 931.58 \\
\hline 71.3 & 931.70 & & & 61.0 & 931.54 & & & 78.5 & 931.68 \\
\hline 73.0 & 931.67 & & & 62.3 & 931.73 & & & 79.1 & 931.92 \\
\hline 75.0 & 931.63 & & & 64.0 & 931.76 & & & 81.3 & 932.10 \\
\hline 77.0 & 931.56 & & & 66.0 & 931.67 & & & 81.6 & 932.28 \\
\hline 77.7 & 931.56 & & & 68.0 & 931.67 & & & 81.9 & 932.25 \\
\hline 78.0 & 931.63 & & & 70.0 & 931.62 & & & 82.1 & 932.09 \\
\hline
\end{tabular}


Table 37. (Continued) Listing of horizontal stations and elevations for cross section PR194

[Sta., station, distance in meters from a reference pin on the left bank; Elev., elevation, in meters above sea level]

\begin{tabular}{|c|c|c|c|c|c|c|c|c|c|}
\hline \multirow{2}{*}{\multicolumn{2}{|c|}{$\begin{array}{c}1996 \\
16 \text { October }\end{array}$}} & \multicolumn{2}{|c|}{1997} & \multicolumn{2}{|c|}{1997} & \multicolumn{2}{|c|}{1998} & \multicolumn{2}{|c|}{1998} \\
\hline & & \multicolumn{2}{|c|}{24 September } & \multicolumn{2}{|c|}{24 September } & \multicolumn{2}{|c|}{23 September } & \multicolumn{2}{|c|}{23 September } \\
\hline Sta. & Elev. & Sta. & Elev. & Sta. & Elev. & Sta. & Elev. & Sta. & Elev. \\
\hline 82.9 & 932.10 & -1.2 & 934.18 & 85.4 & 933.15 & -1.2 & 934.19 & 74.0 & 931.50 \\
\hline 83.1 & 932.22 & 0.0 & 934.19 & 87.0 & 933.29 & 0.0 & 934.20 & 76.0 & 931.40 \\
\hline 84.0 & 932.33 & 3.0 & 934.15 & 87.7 & 933.30 & 2.5 & 934.18 & 78.0 & 931.45 \\
\hline 84.5 & 932.82 & 6.3 & 934.15 & 88.3 & 933.57 & 4.5 & 934.16 & 79.1 & 931.78 \\
\hline 86.0 & 933.10 & 9.0 & 933.55 & 88.6 & 933.92 & 6.5 & 934.14 & 81.1 & 931.81 \\
\hline 86.5 & 933.32 & 11.5 & 933.28 & 90.0 & 934.00 & 8.0 & 933.74 & 82.0 & 932.13 \\
\hline 87.9 & 933.30 & 13.0 & 933.29 & 92.0 & 934.06 & 10.0 & 933.40 & 84.0 & 932.48 \\
\hline 88.4 & 933.58 & 15.0 & 933.19 & 94.0 & 934.09 & 11.5 & 933.27 & 84.7 & 932.69 \\
\hline 88.6 & 933.92 & 17.0 & 933.24 & 96.0 & 934.16 & 13.0 & 933.30 & 85.4 & 933.19 \\
\hline 90.0 & 934.00 & 18.5 & 933.29 & 99.0 & 934.09 & 15.0 & 933.19 & 87.4 & 933.27 \\
\hline 92.0 & 934.06 & 20.0 & 933.28 & 102.0 & 934.08 & 17.0 & 933.24 & 88.2 & 933.47 \\
\hline 94.0 & 934.09 & 20.5 & 933.23 & 105.0 & 934.04 & 19.0 & 933.29 & 88.6 & 933.89 \\
\hline 95.0 & 934.11 & 21.3 & 932.93 & 108.0 & 933.93 & 20.0 & 933.27 & 90.0 & 933.99 \\
\hline 97.0 & 934.12 & 21.7 & 932.15 & 111.0 & 933.93 & 20.9 & 932.95 & 92.0 & 934.03 \\
\hline 100.0 & 934.08 & 22.8 & 931.71 & 113.3 & 933.94 & 21.0 & 932.38 & 94.0 & 934.08 \\
\hline 103.0 & 934.08 & 25.0 & 931.19 & 113.8 & 933.95 & 21.8 & 931.93 & 96.0 & 934.14 \\
\hline 106.0 & 933.96 & 28.0 & 931.22 & & & 22.4 & 931.81 & 98.0 & 934.11 \\
\hline 109.0 & 933.92 & 31.0 & 931.18 & & & 24.4 & 931.25 & 100.0 & 934.07 \\
\hline 111.0 & 933.93 & 34.0 & 931.21 & & & 26.5 & 931.21 & 102.0 & 934.08 \\
\hline 113.3 & 933.95 & 37.0 & 931.22 & & & 28.5 & .931 .24 & 104.0 & 934.09 \\
\hline 113.8 & 933.95 & 40.0 & 931.30 & & & 30.0 & 931.23 & 106.0 & 933.96 \\
\hline & & 43.0 & 931.41 & & & 32.0 & 931.26 & 108.0 & 933.93 \\
\hline & & 46.0 & 931.55 & & & 34.0 & 931.30 & 110.0 & 933.91 \\
\hline & & 47.6 & 931.54 & & & 36.0 & 931.28 & 112.0 & 933.94 \\
\hline & & 50.0 & 931.36 & & & 38.0 & 931.25 & 113.3 & 933.94 \\
\hline & & 53.0 & 931.42 & & & 40.0 & 931.27 & 113.8 & 933.94 \\
\hline & & 56.0 & 931.52 & & & 42.0 & 931.35 & & \\
\hline & & 59.0 & 931.63 & & & 44.0 & 931.34 & & \\
\hline & & 62.0 & 931.64 & & & 46.0 & 931.33 & & \\
\hline & & 65.0 & 931.61 & & & 48.0 & 931.39 & & \\
\hline & & 67.0 & 931.33 & & & 50.0 & 931.38 & & \\
\hline & & 70.0 & 931.38 & & & 52.0 & 931.39 & & \\
\hline & & 73.0 & 931.38 & & & 54.0 & 931.42 & & \\
\hline & & 76.0 & 931.38 & & & 56.0 & 931.44 & & \\
\hline & & 76.5 & 931.44 & & & 58.0 & 931.47 & & \\
\hline & & 77.8 & 931.41 & & & 60.0 & 931.47 & & \\
\hline & & 79.0 & 931.69 & & & 62.0 & 931.45 & & \\
\hline & & 79.8 & 931.86 & & & 64.0 & 931.43 & & \\
\hline & & 81.0 & 931.83 & & & 66.0 & 931.44 & & \\
\hline & & 82.8 & 932.11 & & & 68.0 & 931.45 & & \\
\hline & & 83.3 & 932.45 & & & 70.0 & 931.46 & & \\
\hline & & 84.3 & 932.53 & & & 72.0 & 931.49 & & \\
\hline
\end{tabular}




\section{Description of Cross Section PR200A}

Location: Township 5 South/Range 51 East--section 8

U. S. Geological Survey quadrangle (1:24,000): Broadus

Landowners--left bank: Doug and Lucille Randall

-right bank: Doug and Lucille Randall

\section{Access: Left bank}

Permission from: Doug and Lucille Randall or Craig Randall

Distance from Moorhead Gaging Station: 86.28 kilometers

Azimuth of Section (degrees magnetic): 140

\section{Reference Monuments}

[Monument at station -6.4 was closest to the first leveling instrument location and monuments at stations 100.0 and 110.0 were closest to the second leveling instrument location]

\begin{tabular}{|c|c|c|c|c|c|c|}
\hline \multirow[b]{2}{*}{ Description } & \multirow[b]{2}{*}{$\begin{array}{c}\text { Station } \\
\text { (m) }\end{array}$} & \multicolumn{2}{|c|}{ GPS-NAD83 (1992) } & \multicolumn{2}{|c|}{ Measurement } & \multirow{2}{*}{$\begin{array}{l}\text { Elevation } \\
\text { (NGVD1929) } \\
\text { (m) }\end{array}$} \\
\hline & & Latitude & Longitude & $\begin{array}{l}\text { Standard } \\
\text { deviation } \\
(\mathbf{m})\end{array}$ & $\begin{array}{l}\text { Horizontal } \\
\text { precision } \\
(\mathbf{m})\end{array}$ & \\
\hline $\begin{array}{l}\text { Benchmark--1/2-inch-rebar; } 0.05 \text { meter } \\
\text { above } 1998 \text { ground level }\end{array}$ & -6.4 & $45^{\circ} 24^{\prime} 32.43^{\prime \prime}$ & $105^{\circ} 27^{\prime} 02.73^{\prime \prime}$ & 0.223 & 0.652 & 927.80 \\
\hline $\begin{array}{l}\text { 1/2-inch-rebar; } 0.17 \text { meter above } 1998 \text { ground } \\
\text { level }\end{array}$ & 100.0 & $45^{\circ} 24^{\prime} 29.33^{\prime \prime}$ & $105^{\circ} 27^{\prime} 00.54^{\prime \prime}$ & 0.275 & 0.496 & 926.41 \\
\hline $\begin{array}{l}\text { 1/2-inch-rebar; bent, } 0.07 \text { meter above } 1998 \\
\text { ground level; } 0.70 \text { meter downstream } \\
\text { from section }\end{array}$ & 110.0 & & & & & 926.32 \\
\hline $\begin{array}{l}\text { 1/2-inch-rebar; } 0.10 \text { meter above } 1998 \text { ground } \\
\text { level }\end{array}$ & 250.0 & & & & & 926.91 \\
\hline $\begin{array}{l}\text { 1/2-inch-rebar; } 0.09 \text { meter above } 1998 \text { ground } \\
\text { level }\end{array}$ & 275.0 & $45^{\circ} 24^{\prime} 24.28^{\prime \prime}$ & $105^{\circ} 26^{\prime} 56.95^{\prime \prime}$ & 0.768 & 0.687 & 926.85 \\
\hline $\begin{array}{l}\text { 1/2-inch-rebar; offsection, } 0.14 \text { meter above } \\
1998 \text { ground level; on riverward side of } \\
\text { only wooden fence post in a 5-strand } \\
\text { barbed-wire fence with metal posts }\end{array}$ & -- & & & & & 927.93 \\
\hline
\end{tabular}




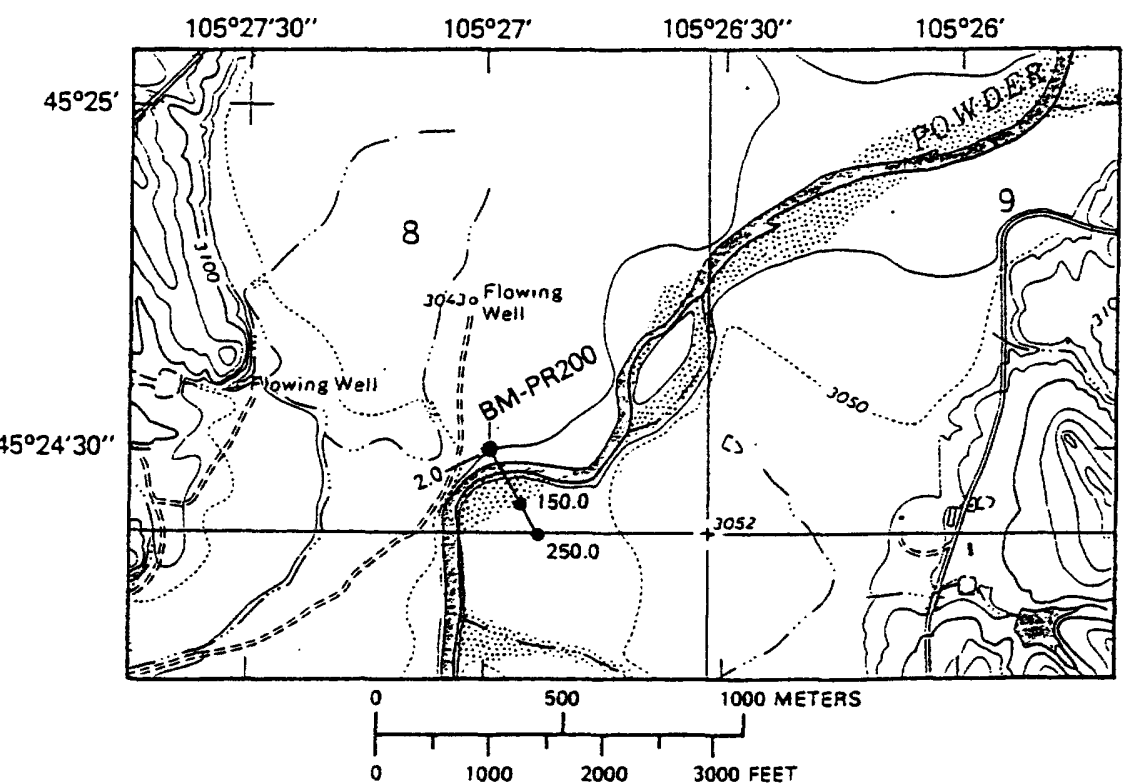

PR200A

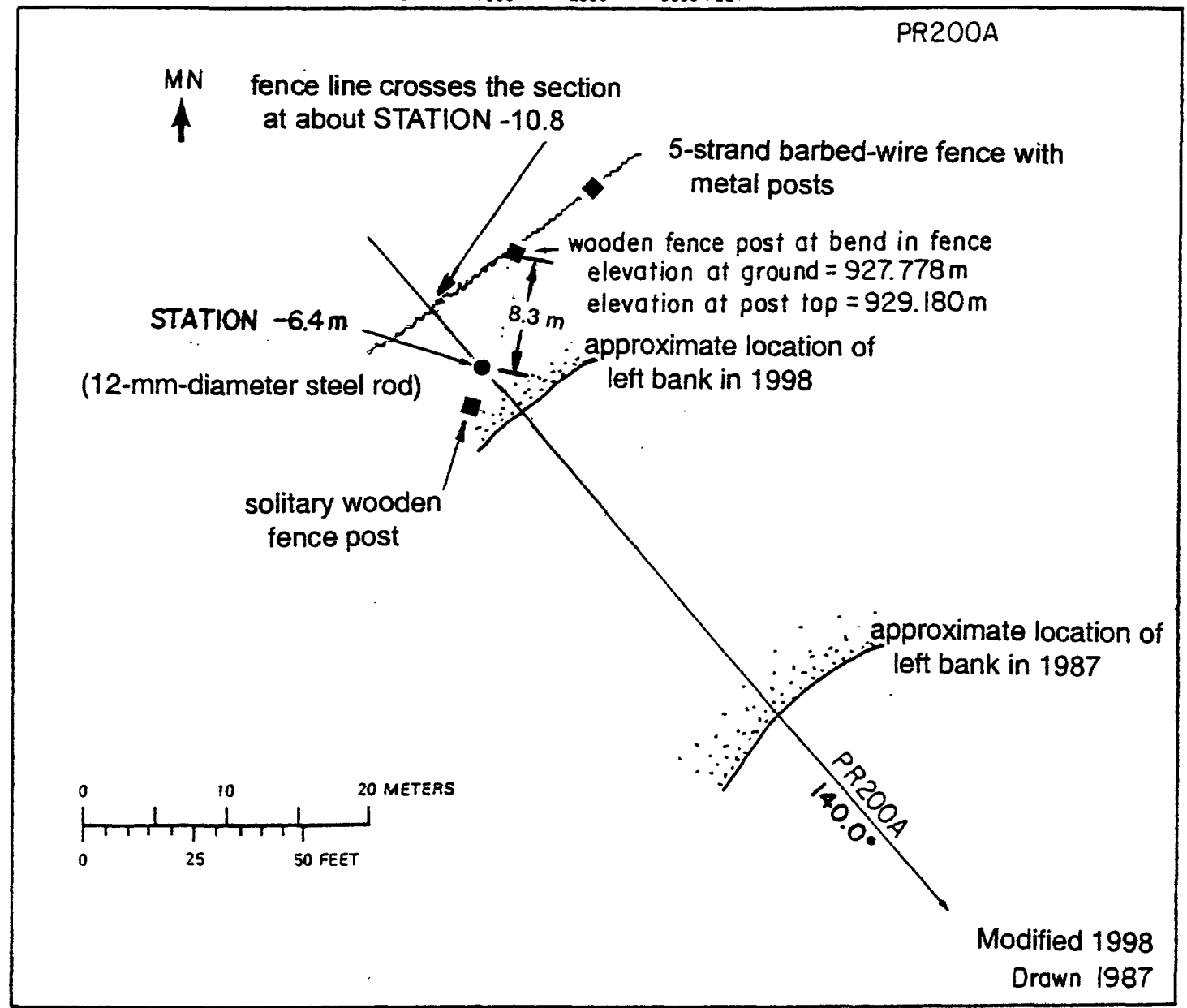

Figure 108. Upper: Location of cross section PR200A and the left and right bank reference monuments in the Broadus quadrangle. Lower: Location of the reference monuments on the left bank. $\mathrm{MN}$ is magnetic north. 

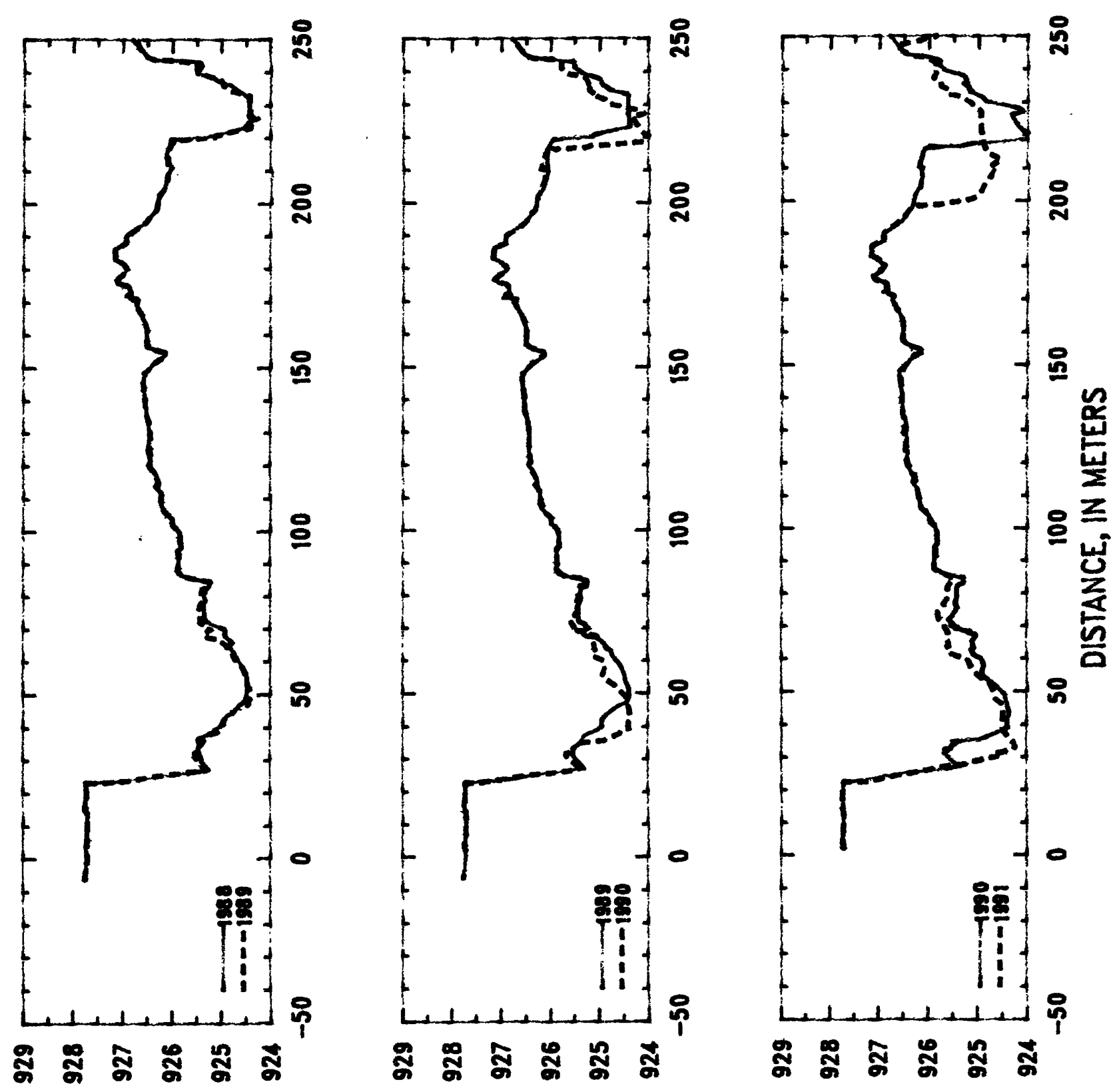

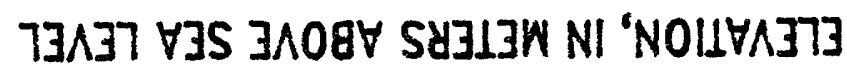



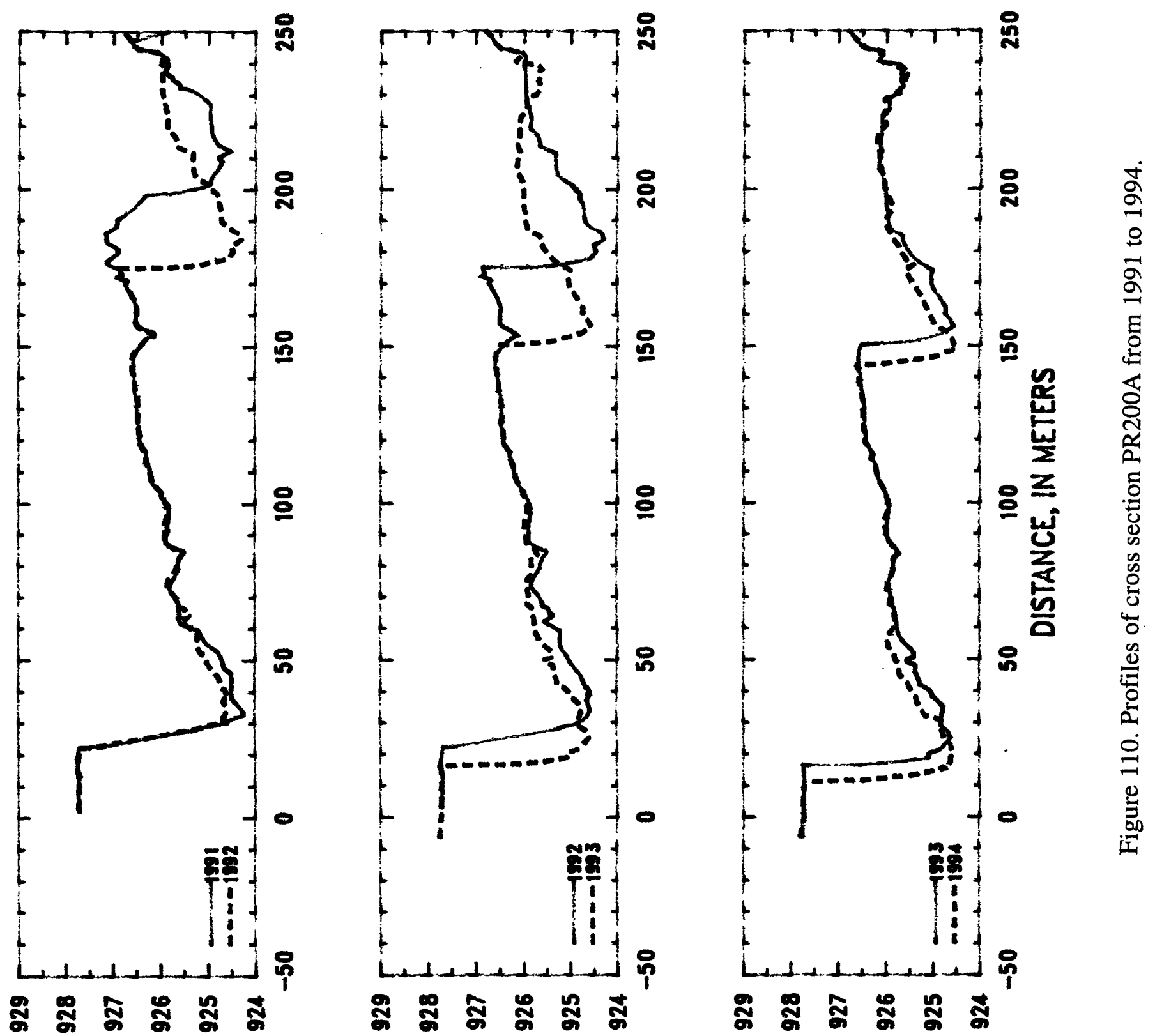

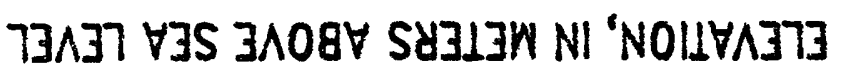



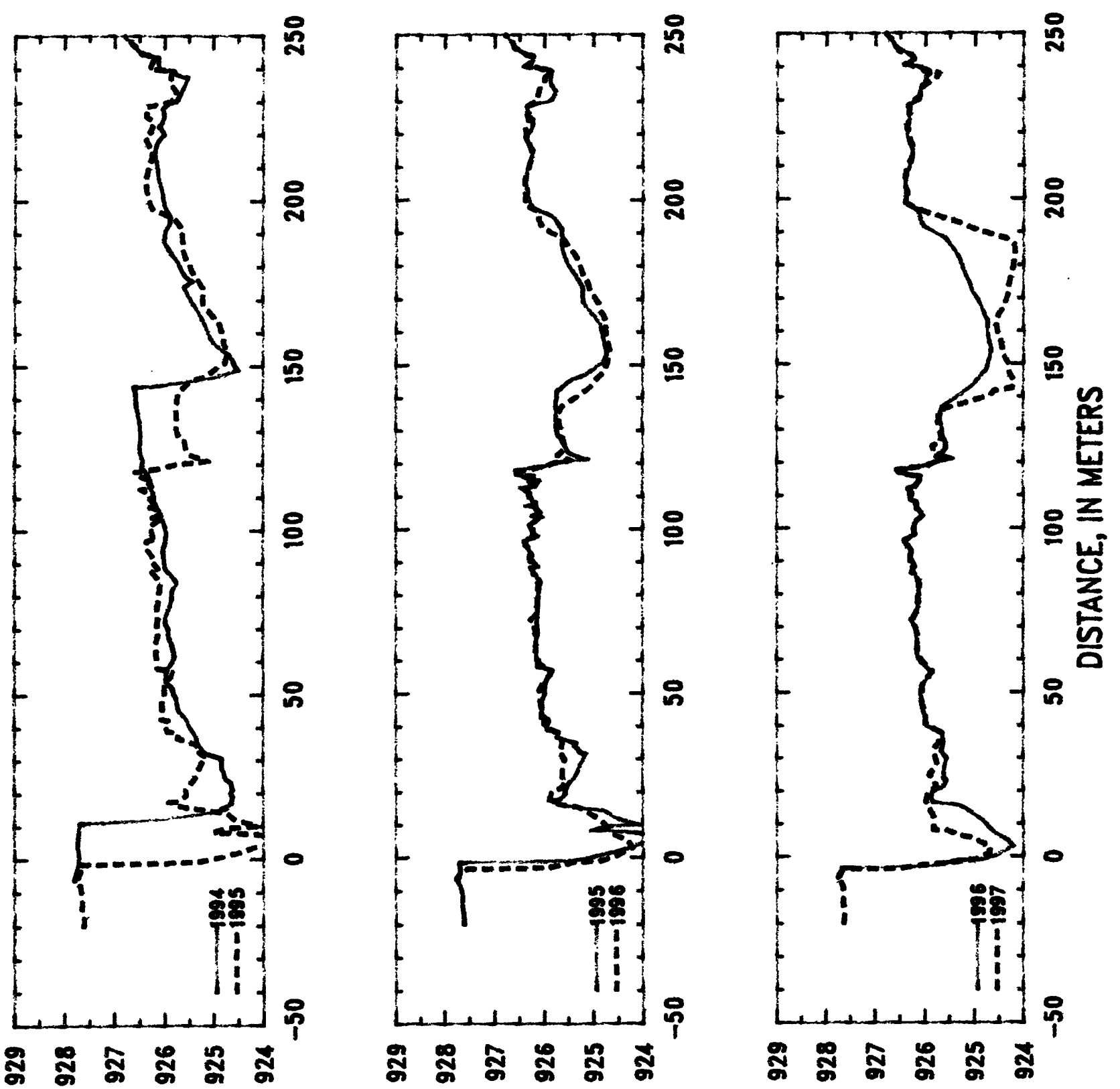

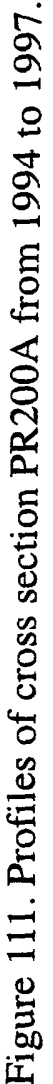

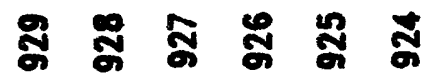

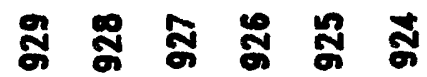

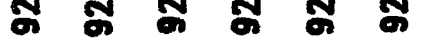

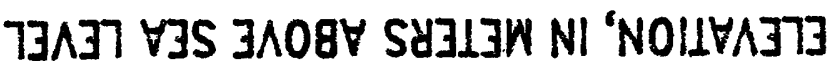




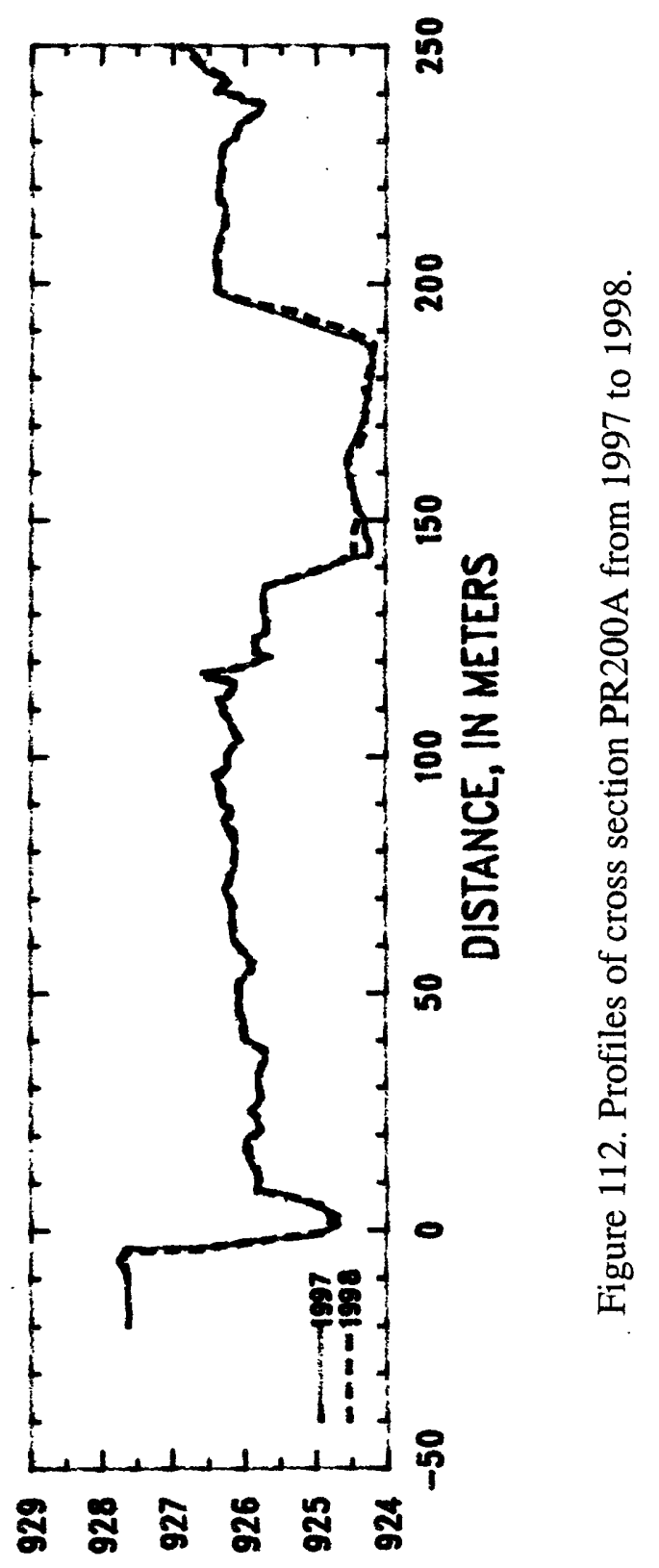


Table 38. Listing of horizontal stations and elevations for cross section PR200A

[Sta., station, distance in meters from a reference pin on the left bank; Elev., elevation, in meters above sea level]

\begin{tabular}{|c|c|c|c|c|c|c|c|c|c|}
\hline \multirow{2}{*}{\multicolumn{2}{|c|}{$\begin{array}{c}1989 \\
21 \text { September }\end{array}$}} & \multicolumn{2}{|c|}{1989} & \multicolumn{2}{|c|}{1989} & \multicolumn{2}{|c|}{1989} & \multicolumn{2}{|c|}{1990} \\
\hline & & $21 \mathrm{Se}$ & ember & $21 \mathrm{Se}$ & ember & $21 \mathrm{Se}$ & ember & $23 \mathrm{Se}$ & ember \\
\hline Sta. & Elev. & Sta. & Elev. & Sta. & Elev. & Sta. & Elev. & Sta. & Elev. \\
\hline-6.4 & 927.76 & 67.4 & 925.27 & 136.0 & 926.53 & 204.0 & 926.16 & 2.0 & 927.70 \\
\hline 0.0 & 927.69 & 68.6 & 925.35 & 138.0 & 926.55 & 206.0 & 926.12 & 3.0 & 927.74 \\
\hline 4.0 & 927.72 & 69.8 & 925.19 & 140.0 & 926.54 & 208.0 & 926.07 & 5.0 & 927.75 \\
\hline 6.0 & 927.70 & 70.2 & 925.26 & 142.0 & 926.58 & 210.0 & 926.07 & 7.0 & 927.70 \\
\hline 8.0 & 927.70 & 71.2 & 925.28 & 144.0 & 926.58 & 212.0 & 926.07 & 10.0 & 927.71 \\
\hline 10.0 & 927.70 & 71.8 & 925.41 & 146.0 & 926.60 & 214.0 & 926.06 & 12.0 & 927.70 \\
\hline 12.0 & 927.69 & 73.0 & 925.43 & 148.0 & 926.56 & 216.0 & 926.08 & 14.0 & 927.70 \\
\hline 14.0 & 927.71 & 75.0 & 925.42 & 150.0 & 926.42 & 218.0 & 926.00 & 17.0 & 927.73 \\
\hline 16.0 & 927.76 & 76.0 & 925.45 & 150.9 & 926.37 & 219.3 & 925.95 & 20.0 & 927.72 \\
\hline 18.0 & 927.72 & 77.0 & 925.42 & 153.3 & 926.13 & 220.2 & 925.13 & 22.0 & 927.70 \\
\hline 20.0 & 927.72 & 79.0 & 925.36 & 154.3 & 926.11 & 220.7 & 925.11 & 22.6 & 927.69 \\
\hline 22.0 & 927.71 & 81.0 & 925.39 & 154.7 & 926.15 & 221.1 & 924.96 & 22.7 & 927.44 \\
\hline 23.0 & 927.72 & 82.3 & 925.36 & 155.6 & 926.37 & 221.7 & 924.82 & 24.0 & 926.88 \\
\hline 23.1 & 927.38 & 82.7 & 925.23 & 156.6 & 926.51 & 223.0 & 924.39 & 25.2 & 926.42 \\
\hline 23.9 & 926.74 & 84.5 & 925.23 & 158.0 & 926.51 & 225.0 & 924.39 & 26.5 & 925.57 \\
\hline 25.0 & 926.44 & 86.0 & 925.71 & 160.0 & 926.50 & 227.0 & 924.46 & 27.2 & 925.40 \\
\hline 27.0 & 925.29 & 86.6 & 925.82 & 162.0 & 926.51 & 228.0 & 924.41 & 29.0 & 925.53 \\
\hline 29.0 & 925.42 & 88.0 & 925.88 & 164.0 & 926.55 & 230.0 & 924.44 & 31.0 & 925.68 \\
\hline 31.0 & 925.52 & 90.0 & 925.85 & 166.0 & 926.60 & 232.0 & 924.44 & 31.7 & 925.68 \\
\hline 33.0 & 925.53 & 92.0 & 925.85 & 168.0 & 926.69 & 233.0 & 924.43 & 31.8 & 925.62 \\
\hline 35.0 & 925.38 & 94.0 & 925.88 & 169.7 & 926.76 & 234.4 & 924.83 & 32.3 & 925.65 \\
\hline 37.0 & 925.31 & 94.8 & 925.80 & 170.8 & 926.69 & 235.1 & 924.86 & 32.6 & 925.53 \\
\hline 38.0 & 925.20 & 96.0 & 925.80 & 171.0 & 926.92 & 236.0 & 924.97 & 33.3 & 925.52 \\
\hline 38.5 & 925.08 & 98.0 & 925.83 & 171.5 & 926.82 & 237.6 & 925.01 & 34.5 & 925.40 \\
\hline 40.0 & 924.93 & 100.0 & 925.83 & 174.0 & 926.88 & 238.3 & 925.13 & 34.8 & 925.46 \\
\hline 42.0 & 924.94 & 102.0 & 925.99 & 174.8 & 926.87 & 238.8 & 925.28 & 35.0 & 925.37 \\
\hline 43.3 & 924.87 & 104.0 & 925.99 & 175.7 & 927.08 & 240.0 & 925.43 & 35.7 & 924.89 \\
\hline 44.6 & 924.74 & 106.0 & 926.15 & 176.7 & 927.16 & 241.0 & 925.52 & 36.1 & 924.84 \\
\hline 46.0 & 924.64 & 108.0 & 926.23 & 178.0 & 927.01 & 242.0 & 925.51 & 38.0 & 924.52 \\
\hline 47.0 & 924.44 & 110.0 & 926.22 & 180.0 & 926.87 & 243.0 & 925.54 & 39.0 & 924.39 \\
\hline 49.0 & 924.39 & 112.0 & 926.22 & 182.0 & 927.01 & 243.8 & 926.13 & 41.0 & 924.43 \\
\hline 50.0 & 924.39 & 114.0 & 926.28 & 183.0 & 927.14 & 244.0 & 926.39 & 43.0 & 924.36 \\
\hline 52.0 & 924.42 & 116.0 & 926.31 & 185.0 & 927.14 & 245.0 & 926.54 & 45.0 & 924.40 \\
\hline 54.0 & 924.48 & 118.0 & 926.34 & 186.0 & 927.15 & 247.5 & 926.67 & 47.0 & 924.45 \\
\hline 56.0 & 924.49 & 120.0 & 926.46 & 188.0 & 926.91 & 249.0 & 926.72 & 49.0 & 924.46 \\
\hline 58.0 & 924.56 & 122.0 & 926.42 & 190.0 & 926.90 & 250.0 & 926.81 & 50.0 & 924.55 \\
\hline 59.0 & 924.59 & 124.0 & 926.43 & 192.0 & 926.71 & 251.0 & 926.82 & 52.0 & 924.67 \\
\hline 61.3 & 924.74 & 126.0 & 926.45 & 194.0 & 926.53 & 253.0 & 926.93 & 53.0 & 924.76 \\
\hline 63.2 & 924.77 & 128.0 & 926.44 & 196.0 & 926.42 & & & 53.8 & 924.82 \\
\hline 63.8 & 924.86 & 130.0 & 926.43 & 198.0 & 926.30 & & & 54.4 & 924.89 \\
\hline 65.0 & 924.88 & 132.0 & 926.45 & 200.0 & 926.29 & & & 55.4 & 924.91 \\
\hline 66.7 & 925.02 & 134.0 & 926.48 & 202.0 & 926.28 & & & 55.6 & 924.93 \\
\hline
\end{tabular}


Table 38. (Continued) Listing of horizontal stations and elevations for cross section PR200A

[Sta., station, distance in meters from a reference pin on the left bank; Elev., elevation, in meters above sea level]

\begin{tabular}{|c|c|c|c|c|c|c|c|c|c|}
\hline & & & & & & & & & 91 \\
\hline $23 \mathrm{Se}$ & ember & $23 \mathrm{Se}$ & ember & $23 \mathrm{Se}$ & ember & $23 \mathrm{Se}$ & ember & $3 \mathrm{Se}$ & ember \\
\hline Sta. & Elev. & Sta. & Elev. & Sta. & Elev. & Sta. & Elev. & Sta. & Elev. \\
\hline 58.0 & 924.86 & 121.0 & 926.41 & 200.0 & 926.29 & 243.6 & 925.97 & 2.0 & 927.70 \\
\hline 60.0 & 924.93 & 124.0 & 926.43 & 202.0 & 926.28 & 244.0 & 926.39 & 3.0 & 927.72 \\
\hline 61.0 & 925.09 & 127.0 & 926.45 & 203.7 & 926.19 & 245.0 & 926.53 & 6.0 & 927.73 \\
\hline 62.8 & 925.14 & 130.0 & 926.43 & 203.9 & 926.16 & 247.0 & 926.64 & 9.0 & 927.72 \\
\hline 63.3 & 925.07 & 133.0 & 926.47 & 205.0 & 926.17 & 250.0 & 926.80 & 12.0 & 927.69 \\
\hline 65.3 & 925.12 & 136.0 & 926.53 & 207.0 & 926.13 & 253.0 & 926.94 & 15.0 & 927.76 \\
\hline 66.7 & 925.03 & 139.0 & 926.55 & 208.0 & 926.13 & 256.0 & 926.98 & 18.0 & 927.73 \\
\hline 67.4 & 925.04 & 142.0 & 926.55 & 209.0 & 926.17 & 262.0 & 926.79 & 21.0 & 927.73 \\
\hline 67.9 & 925.18 & 145.0 & 926.60 & 210.0 & 926.13 & 265.0 & 926.79 & 22.4 & 927.69 \\
\hline 68.3 & 925.17 & 148.0 & 926.58 & 210.4 & 926.22 & 270.0 & 926.75 & 22.4 & 927.22 \\
\hline 69.0 & 925.45 & 150.0 & 926.43 & 212.0 & 926.15 & 275.0 & 926.76 & 24.0 & 926.71 \\
\hline 69.5 & 925.42 & 151.0 & 926.36 & 213.0 & 926.14 & & & 26.0 & 926.00 \\
\hline 70.0 & 925.54 & 152.0 & 926.32 & 215.0 & 926.10 & & & 27.3 & 925.58 \\
\hline 70.4 & 925.49 & 153.3 & 926.14 & 216.0 & 926.06 & & & 27.6 & 925.29 \\
\hline 71.9 & 925.60 & 154.5 & 926.14 & 216.1 & 925.87 & & & 28.6 & 925.07 \\
\hline 72.4 & 925.53 & 156.0 & 926.44 & 216.4 & 925.93 & & & 30.0 & 924.60 \\
\hline 74.0 & 925.47 & 157.4 & 926.53 & 216.6 & 925.31 & & & 30.2 & 924.58 \\
\hline 75.6 & 925.41 & 159.0 & 926.50 & 217.3 & 925.16 & & & 30.7 & 924.66 \\
\hline 77.0 & 925.48 & 161.0 & 926.49 & 217.5 & 924.82 & & & 32.5 & 924.25 \\
\hline 79.0 & 925.41 & 163.0 & 926.51 & 218.8 & 923.90 & & & 33.2 & 924.24 \\
\hline 81.0 & 925.43 & 165.0 & 926.57 & 220.0 & 923.94 & & & 35.0 & 924.30 \\
\hline 82.4 & 925.40 & 167.0 & 926.65 & 221.0 & 924.12 & & & 37.0 & 924.45 \\
\hline 83.0 & 925.29 & 169.6 & 926.75 & 223.0 & 924.23 & & & 39.0 & 924.52 \\
\hline 84.7 & 925.26 & 170.8 & 926.69 & 226.0 & 924.36 & & & 42.0 & 924.50 \\
\hline 86.0 & 925.72 & 172.1 & 926.91 & 227.0 & 924.10 & & & 44.0 & 924.50 \\
\hline 88.0 & 925.90 & 172.5 & 926.82 & 228.0 & 924.19 & & & 46.0 & 924.51 \\
\hline 90.0 & 925.85 & 175.0 & 926.89 & 229.0 & 924.51 & & & 47.4 & 924.65 \\
\hline 92.0 & 925.88 & 176.0 & 927.11 & 230.0 & 924.80 & & & 50.0 & 924.69 \\
\hline 94.0 & 925.89 & 177.0 & 927.12 & 230.3 & 924.88 & & & 52.0 & 924.76 \\
\hline 95.0 & 925.81 & 178.0 & 927.00 & 231.3 & 924.91 & & & 53.0 & 924.81 \\
\hline 97.0 & 925.81 & 180.0 & 926.87 & 233.0 & 925.18 & & & 55.0 & 925.02 \\
\hline 99.0 & 925.82 & 182.0 & 927.02 & 233.3 & 925.14 & & & 56.0 & 925.08 \\
\hline 101.0 & 925.90 & 183.2 & 927.18 & 234.0 & 925.21 & & & 58.0 & 925.16 \\
\hline 103.0 & 925.97 & 185.0 & 927.14 & 234.7 & 925.20 & & & 59.4 & 925.20 \\
\hline 104.0 & 926.00 & 186.0 & 927.16 & 235.6 & 925.31 & & & 61.4 & 925.56 \\
\hline 106.0 & 926.15 & 187.7 & 926.88 & 236.0 & 925.23 & & & 63.4 & 925.63 \\
\hline 109.0 & 926.19 & 189.9 & 926.91 & 237.5 & 925.27 & & & 63.7 & 925.57 \\
\hline 112.0 & 926.23 & 192.0 & 926.68 & 238.3 & 925.35 & & & 66.0 & 925.63 \\
\hline 114.0 & 926.33 & 194.0 & 926.51 & 239.4 & 925.69 & & & 68.0 & 925.61 \\
\hline 114.6 & 926.25 & 196.0 & 926.44 & 241.0 & 925.80 & & & 70.0 & 925.70 \\
\hline 116.0 & 926.29 & 197.4 & 926.34 & 242.0 & 925.81 & & & 72.0 & 925.80 \\
\hline 119.0 & 926.42 & 198.0 & 926.32 & 242.6 & 925.79 & & & 74.0 & 925.82 \\
\hline
\end{tabular}


Table 38. (Continued) Listing of horizontal stations and elevations for cross section PR200A

[Sta., station, distance in meters from a reference pin on the left bank; Elev., elevation, in meters above sea level]

\begin{tabular}{|c|c|c|c|c|c|c|c|c|c|}
\hline \multirow{2}{*}{\multicolumn{2}{|c|}{$\begin{array}{c}1991 \\
3 \text { September }\end{array}$}} & \multirow{2}{*}{\multicolumn{2}{|c|}{$\begin{array}{c}1991 \\
3 \text { September }\end{array}$}} & \multirow{2}{*}{\multicolumn{2}{|c|}{$\begin{array}{c}1991 \\
3 \text { September }\end{array}$}} & \multirow{2}{*}{\multicolumn{2}{|c|}{$\begin{array}{c}1992 \\
29 \text { August }\end{array}$}} & \multirow{2}{*}{\multicolumn{2}{|c|}{$\begin{array}{c}1992 \\
29 \text { August }\end{array}$}} \\
\hline & & & & & & & & & \\
\hline Sta. & Elev. & Sta. & Elev. & Sta. & Elev. & Sta. & Elev. & Sta. & Elev. \\
\hline 76.0 & 925.70 & 170.0 & 926.76 & 233.6 & 925.62 & 3.0 & 927.73 & 75.0 & 925.84 \\
\hline 78.0 & 925.62 & 170.9 & 926.70 & 234.8 & 925.77 & 7.0 & 927.69 & 77.0 & 925.73 \\
\hline 80.0 & 925.60 & 172.0 & 926.92 & 235.5 & 925.78 & 10.0 & 927.71 & 79.0 & 925.65 \\
\hline 82.0 & 925.62 & 172.5 & 926.82 & 237.3 & 925.93 & 13.0 & 927.68 & 81.0 & 925.64 \\
\hline 84.0 & 925.49 & 174.6 & 926.86 & 239.6 & 925.83 & 16.0 & 927.77 & 83.0 & 925.57 \\
\hline 84.7 & 925.49 & 176.0 & 927.11 & 240.6 & 925.89 & 19.0 & 927.72 & 84.0 & 925.51 \\
\hline 86.0 & 925.75 & 176.8 & 927.14 & 241.6 & 925.87 & 22.2 & 927.68 & 84.6 & 925.52 \\
\hline 87.3 & 925.89 & 179.0 & 926.92 & 243.6 & 926.07 & 22.3 & 927.46 & 86.0 & 925.77 \\
\hline 89.0 & 925.90 & 180.0 & 926.86 & 244.0 & 926.35 & 24.0 & 926.78 & 87.5 & 925.90 \\
\hline 90.9 & 925.83 & 181.0 & 926.89 & 245.0 & 926.52 & 26.0 & 926.01 & 89.0 & 925.92 \\
\hline 93.0 & 925.86 & 183.0 & 927.13 & 247.0 & 926.65 & 28.0 & 925.27 & 91.0 & 925.89 \\
\hline 95.0 & 925.81 & 186.0 & 927.16 & 250.0 & 925.82 & 28.6 & 925.15 & 93.0 & 925.90 \\
\hline 97.0 & 925.81 & 188.0 & 926.90 & 253.0 & 926.93 & 29.8 & 924.82 & 95.0 & 925.87 \\
\hline 99.0 & 925.81 & 190.0 & 926.91 & 256.0 & 926.96 & 32.0 & 924.64 & 97.0 & 925.84 \\
\hline 100.2 & 925.85 & 192.6 & 926.65 & 262.0 & 926.79 & 34.0 & 924.59 & 99.0 & 925.86 \\
\hline 102.0 & 925.98 & 195.0 & 926.48 & 265.0 & 926.79 & 36.3 & 924.65 & 101.0 & 925.93 \\
\hline 104.0 & 925.99 & 197.0 & 926.37 & 270.0 & 926.77 & 37.0 & 924.68 & 104.0 & 926.01 \\
\hline 106.0 & 926.15 & 198.3 & 926.28 & 275.0 & 926.75 & 38.7 & 924.58 & 106.0 & 926.15 \\
\hline 109.0 & 926.19 & 198.7 & 925.75 & 277.0 & 926.76 & 39.0 & 924.65 & 108.0 & 926.20 \\
\hline 112.0 & 926.24 & 199.6 & 925.44 & & & 39.9 & 924.60 & 111.0 & 926.22 \\
\hline 114.0 & 926.29 & 200.4 & 925.16 & & & 40.9 & 924.64 & 113.0 & 926.30 \\
\hline 117.0 & 926.30 & 201.4 & 924.97 & & & 41.0 & 924.68 & 116.0 & 926.30 \\
\hline 120.0 & 926.44 & 204.0 & 924.90 & & & 43.0 & 924.75 & 119.0 & 926.44 \\
\hline 123.0 & 926.46 & 206.0 & 924.79 & & & 45.0 & 924.79 & 122.0 & 926.41 \\
\hline 126.0 & 926.47 & 208.0 & 924.73 & & & 47.0 & 924.92 & 125.0 & 926.50 \\
\hline 129.0 & 926.49 & 209.0 & 924.69 & & & 49.0 & 925.03 & 128.0 & 926.45 \\
\hline 132.0 & 926.47 & 210.0 & 924.80 & & & 51.0 & 925.08 & 131.0 & 926.45 \\
\hline 135.0 & 926.53 & 211.3 & 924.66 & & & 53.0 & 925.20 & 134.0 & 926.49 \\
\hline 138.0 & 926.55 & 212.2 & 924.53 & & & 55.0 & 925.24 & 137.0 & 926.53 \\
\hline 141.0 & 926.53 & 212.6 & 924.56 & & & 57.0 & 925.23 & 140.0 & 926.55 \\
\hline 144.0 & 926.57 & 213.0 & 924.65 & & & 59.0 & 925.21 & 143.0 & 926.61 \\
\hline 147.0 & 926.56 & 214.0 & 924.79 & & & 60.0 & 925.35 & 146.0 & 926.59 \\
\hline 150.0 & 926.42 & 216.0 & 924.88 & & & 61.0 & 925.47 & 148.0 & 926.55 \\
\hline 152.0 & 926.33 & 218.0 & 924.93 & & & 62.2 & 925.57 & 150.0 & 926.42 \\
\hline 154.0 & 926.11 & 220.0 & 924.95 & & & 63.5 & 925.41 & 150.9 & 926.37 \\
\hline 154.7 & 926.15 & 223.0 & 924.98 & & & 64.0 & 925.37 & 152.0 & 926.31 \\
\hline 156.0 & 926.45 & 225.0 & 924.98 & & & 64.5 & 925.45 & 153.4 & 926.13 \\
\hline 157.5 & 926.54 & 227.0 & 924.95 & & & 66.5 & 925.50 & 154.6 & 926.24 \\
\hline 160.0 & 926.50 & 228.5 & 925.08 & & & 67.0 & 925.61 & 156.0 & 926.44 \\
\hline 163.0 & 926.51 & 230.0 & 925.18 & & & 69.0 & 925.64 & 157.0 & 926.51 \\
\hline 166.0 & 926.61 & 232.0 & 925.47 & & & 71.0 & 925.75 & 159.0 & 926.51 \\
\hline 168.0 & 926.68 & 232.1 & 925.61 & & & 73.0 & 925.86 & 161.0 & 926.49 \\
\hline
\end{tabular}


Table 38. (Continued) Listing of horizontal stations and elevations for cross section PR200A

[Sta., station, distance in meters from a reference pin on the left bank; Elev., elevation, in meters above sea level]

\begin{tabular}{|c|c|c|c|c|c|c|c|c|c|}
\hline \multirow{2}{*}{\multicolumn{2}{|c|}{$\begin{array}{c}1992 \\
29 \text { August }\end{array}$}} & \multirow{2}{*}{\multicolumn{2}{|c|}{$\begin{array}{c}1992 \\
29 \text { August }\end{array}$}} & \multirow{2}{*}{\multicolumn{2}{|c|}{$\begin{array}{c}1993 \\
3 \text { September }\end{array}$}} & \multirow{2}{*}{\multicolumn{2}{|c|}{$\begin{array}{c}1993 \\
3 \text { September }\end{array}$}} & \multirow{2}{*}{\multicolumn{2}{|c|}{$\begin{array}{c}1993 \\
\text { 3 September }\end{array}$}} \\
\hline & & & & & & & & & \\
\hline Sta. & Elev. & Sta. & Elev. & Sta. & Elev. & Sta. & Elev. & Sta. & Elev. \\
\hline 163.0 & 926.51 & 219.0 & 925.89 & -6.4 & 927.77 & 61.0 & 925.78 & 140.0 & 926.54 \\
\hline 165.0 & 926.56 & 221.0 & 925.86 & 0.0 & 927.72 & 63.0 & 925.80 & 142.0 & 926.58 \\
\hline 167.0 & 926.64 & 223.0 & 925.85 & 2.0 & 927.70 & 65.0 & 925.82 & 144.0 & 926.58 \\
\hline 169.0 & 926.71 & 225.0 & 925.91 & 3.0 & 927.74 & 66.5 & 925.85 & 146.0 & 926.60 \\
\hline 170.0 & 926.75 & 227.0 & 925.93 & 6.0 & 927.71 & 67.5 & 925.94 & 148.0 & 926.57 \\
\hline 170.9 & 926.69 & 229.0 & 925.96 & 9.0 & 927.71 & 69.0 & 925.86 & 150.0 & 926.52 \\
\hline 172.0 & 926.91 & 231.0 & 925.98 & 12.0 & 927.70 & 71.0 & 925.92 & 150.3 & 926.51 \\
\hline 172.7 & 926.81 & 233.0 & 925.96 & 14.0 & 927.71 & 73.0 & 925.95 & 150.4 & 926.44 \\
\hline 174.0 & 926.87 & 235.0 & 925.98 & 16.3 & 927.75 & 75.0 & 925.95 & 150.6 & 926.01 \\
\hline 174.9 & 926.88 & 237.0 & 925.97 & 16.5 & 926.50 & 77.0 & 925.85 & 151.8 & 925.29 \\
\hline 175.0 & 926.05 & 239.0 & 926.02 & 16.7 & 926.19 & 79.0 & 925.83 & 154.0 & 924.78 \\
\hline 175.2 & 925.69 & 241.0 & 925.97 & 17.7 & 925.52 & 81.0 & 925.83 & 156.0 & 924.56 \\
\hline 175.5 & 925.39 & 242.0 & 925.96 & 18.0 & 925.43 & 82.5 & 925.84 & 158.0 & 924.64 \\
\hline 176.0 & 925.12 & 243.0 & 926.02 & 18.7 & 925.08 & 83.5 & 925.71 & 160.0 & 924.77 \\
\hline 176.6 & 925.02 & 243.4 & 926.06 & 20.0 & 925.09 & 85.0 & 925.76 & 162.0 & 924.74 \\
\hline 177.0 & 924.82 & 244.5 & 926.48 & 20.7 & 925.01 & 86.0 & 925.85 & 164.0 & 924.84 \\
\hline 178.0 & 924.56 & 246.0 & 926.61 & 21.0 & 924.89 & 87.0 & 925.93 & 166.0 & 924.91 \\
\hline 180.0 & 924.46 & 248.0 & 926.67 & 23.0 & 924.73 & 89.0 & 925.99 & 168.0 & 925.03 \\
\hline 182.0 & 924.52 & 250.0 & 926.82 & 25.0 & 924.61 & 91.0 & 925.99 & 170.0 & 925.07 \\
\hline 184.0 & 924.29 & 252.0 & 926.89 & 27.0 & 924.68 & 93.0 & 925.99 & 172.0 & 925.07 \\
\hline 186.0 & 924.35 & 254.0 & 926.96 & 29.0 & 924.84 & 95.0 & 925.97 & 174.0 & 925.02 \\
\hline 188.0 & 924.62 & 256.6 & 926.92 & 31.0 & 924.84 & 97.0 & 925.93 & 175.8 & 925.26 \\
\hline 190.0 & 924.72 & 260.0 & 926.93 & 33.0 & 924.78 & 98.0 & 925.93 & 177.0 & 925.36 \\
\hline 192.0 & 924.73 & 262.0 & 926.79 & 35.0 & 924.78 & 100.0 & 925.93 & 179.0 & 925.49 \\
\hline 194.0 & 924.76 & 265.0 & 926.78 & 37.3 & 924.92 & 101.0 & 925.95 & 181.0 & 925.59 \\
\hline 195.0 & 924.75 & 268.0 & 926.76 & 38.0 & 925.01 & 103.0 & 926.03 & 183.5 & 925.67 \\
\hline 197.0 & 924.84 & 271.0 & 926.76 & 39.0 & 925.03 & 105.0 & 926.08 & 185.0 & 925.67 \\
\hline 198.2 & 924.81 & 273.0 & 926.75 & 40.0 & 925.07 & 107.0 & 926.16 & 187.0 & 925.93 \\
\hline 199.0 & 924.84 & 275.0 & 926.75 & 41.0 & 925.15 & 109.0 & 926.20 & 189.0 & 925.98 \\
\hline 199.4 & 924.91 & & & 42.0 & 925.31 & 110.0 & 926.21 & 191.0 & 925.96 \\
\hline 200.0 & 924.90 & & & 44.0 & 925.32 & 112.0 & 926.23 & 194.0 & 926.02 \\
\hline 200.5 & 924.97 & & & 46.0 & 925.40 & 114.0 & 926.30 & 197.0 & 926.02 \\
\hline 201.0 & 924.97 & & & 48.0 & 925.37 & 116.0 & 926.30 & 200.0 & 926.03 \\
\hline 203.0 & 925.18 & & & 48.5 & 925.42 & 119.0 & 926.44 & 203.0 & 926.06 \\
\hline 204.4 & 925.29 & & & 49.0 & 925.57 & 121.0 & 926.42 & 206.0 & 926.17 \\
\hline 206.0 & 925.33 & & & 50.1 & 925.56 & 124.0 & 926.45 & 209.0 & 926.14 \\
\hline 208.0 & 925.34 & & & 50.5 & 925.41 & 127.0 & 926.46 & 212.0 & 926.08 \\
\hline 210.0 & 925.35 & & & 52.0 & 925.40 & 130.0 & 926.45 & 214.0 & 926.15 \\
\hline 211.3 & 925.32 & & & 53.0 & 925.46 & 132.0 & 926.48 & 217.0 & 926.09 \\
\hline 213.5 & 925.68 & & & 55.0 & 925.62 & 134.0 & 926.49 & 220.0 & 926.10 \\
\hline 215.0 & 925.69 & & & 57.0 & 925.71 & 136.0 & 926.52 & 223.0 & 926.07 \\
\hline 217.0 & 925.72 & & & 59.0 & 925.77 & 138.0 & 926.53 & 224.0 & 925.97 \\
\hline
\end{tabular}


Table 38. (Continued) Listing of horizontal stations and elevations for cross section PR200A

[Sta., station, distance in meters from a reference pin on the left bank; Elev., elevation, in meters above sea level]

\begin{tabular}{|c|c|c|c|c|c|c|c|c|c|}
\hline \multirow{2}{*}{\multicolumn{2}{|c|}{$\begin{array}{c}1993 \\
3 \text { September }\end{array}$}} & \multicolumn{2}{|c|}{1994} & \multicolumn{2}{|c|}{1994} & \multicolumn{2}{|c|}{1994} & \multicolumn{2}{|c|}{1994} \\
\hline & & \multicolumn{2}{|c|}{18 September } & \multicolumn{2}{|c|}{18 September } & \multicolumn{2}{|c|}{18 September } & \multicolumn{2}{|c|}{18 September } \\
\hline Sta. & Elev. & Sta. & Elev. & Sta. & Elev. & Sta. & Elev. & Sta. & Elev. \\
\hline 226.0 & 925.93 & -6.4 & 927.80 & 72.0 & 925.99 & 154.0 & 924.73 & 243.6 & 926.13 \\
\hline 228.0 & 925.96 & -1.8 & 927.72 & 74.0 & 925.97 & 157.0 & 925.01 & 244.3 & 926.45 \\
\hline 229.0 & 925.92 & 0.0 & 927.71 & 76.0 & 925.91 & 160.0 & 925.10 & 245.4 & 926.52 \\
\hline 230.0 & 925.71 & 4.0 & 927.74 & 78.0 & 925.85 & 163.0 & 925.19 & 247.0 & 926.62 \\
\hline 232.0 & 925.66 & 6.0 & 927.73 & 80.0 & 925.84 & 166.0 & 925.30 & 250.0 & 926.82 \\
\hline 233.0 & 925.64 & 8.0 & 927.71 & 82.0 & 925.83 & 169.0 & 925.42 & 275.0 & 926.76 \\
\hline 234.3 & 925.71 & 10.9 & 927.69 & 84.0 & 925.73 & 172.0 & 925.56 & 243.6 & 926.13 \\
\hline 235.6 & 925.66 & 11.3 & 927.20 & 86.0 & 925.85 & 174.0 & 925.62 & 244.3 & 926.45 \\
\hline 237.0 & 925.71 & 11.7 & 926.34 & 88.0 & 925.97 & 175.7 & 925.43 & 245.4 & 926.52 \\
\hline 238.0 & 925.62 & 13.5 & 925.30 & 90.0 & 926.01 & 177.3 & 925.60 & 247.0 & 926.62 \\
\hline 239.4 & 925.71 & 15.2 & 924.76 & 92.0 & 926.02 & 180.0 & 925.70 & 250.0 & 926.82 \\
\hline 239.8 & 926.05 & 15.5 & 924.82 & 94.0 & 926.04 & 182.0 & 925.76 & 275.0 & 926.76 \\
\hline 240.2 & 926.19 & 17.0 & 924.63 & 96.0 & 925.97 & 184.0 & 925.87 & 243.6 & 926.13 \\
\hline 242.0 & 926.07 & 19.0 & 924.61 & 98.5 & 925.95 & 185.0 & 925.90 & 244.3 & 926.45 \\
\hline 243.4 & 926.08 & 20.0 & 924.67 & 100.5 & 925.96 & 188.0 & 926.01 & 245.4 & 926.52 \\
\hline 244.4 & 926.48 & 21.2 & 924.60 & 103.0 & 926.04 & 191.0 & 925.99 & 247.0 & 926.62 \\
\hline 246.0 & 926.61 & 23.2 & 924.64 & 105.0 & 926.07 & 193.0 & 925.88 & 250.0 & 926.82 \\
\hline 248.0 & 926.67 & 24.4 & 924.76 & 107.0 & 926.16 & 195.0 & 925.88 & 275.0 & 926.76 \\
\hline 250.0 & 926.81 & 27.0 & 924.81 & 110.0 & 926.21 & 197.0 & 925.96 & 243.6 & 926.13 \\
\hline 253.0 & 926.91 & 28.0 & 924.85 & 112.0 & 926.24 & 200.0 & 926.04 & 244.3 & 926.45 \\
\hline 256.0 & 926.93 & 30.6 & 924.84 & 114.0 & 926.32 & 203.0 & 926.05 & 245.4 & 926.52 \\
\hline 260.0 & 926.92 & 31.4 & 924.96 & 115.0 & 926.26 & 206.0 & 926.11 & & \\
\hline 262.0 & 926.79 & 32.1 & 925.17 & 117.0 & 926.32 & 209.0 & 926.14 & & \\
\hline 270.0 & 926.77 & 34.0 & 925.27 & 119.0 & 926.45 & 212.0 & 926.17 & & \\
\hline 275.0 & 926.76 & 36.0 & 925.34 & 121.0 & 926.43 & 215.0 & 926.21 & & \\
\hline & & 38.0 & 925.38 & 123.0 & 926.48 & 218.0 & 926.14 & & \\
\hline & & 40.0 & 925.48 & 125.0 & 926.51 & 220.0 & 926.00 & & \\
\hline & & 42.5 & 925.55 & 127.0 & 926.47 & 221.5 & 926.04 & & \\
\hline & & 45.0 & 925.72 & 129.0 & 926.47 & 222.5 & 926.12 & & \\
\hline & & 47.0 & 925.74 & 132.0 & 926.48 & 224.0 & 926.03 & & \\
\hline & & 49.0 & 925.76 & 135.0 & 926.53 & 226.0 & 925.99 & & \\
\hline & & 50.0 & 925.82 & 137.0 & 926.56 & 228.0 & 926.05 & & \\
\hline & & 52.0 & 925.84 & 139.0 & 926.58 & 229.0 & 925.96 & & \\
\hline & & 54.0 & 925.97 & 141.0 & 926.59 & 230.4 & 925.74 & & \\
\hline & & 56.0 & 925.98 & 142.0 & 926.58 & 232.0 & 925.69 & & \\
\hline & & 58.0 & 925.92 & 143.7 & 926.63 & 234.0 & 925.62 & & \\
\hline & & 60.0 & 925.81 & 144.0 & 926.15 & 236.0 & 925.56 & & \\
\hline & & 62.0 & 925.78 & 144.3 & 925.98 & 237.5 & 925.52 & & \\
\hline & & 64.0 & 925.83 & 146.4 & 925.01 & 238.4 & 925.77 & & \\
\hline & & 66.0 & 925.86 & 148.8 & 924.49 & 239.4 & 925.81 & & \\
\hline & & 68.0 & 925.92 & 150.7 & 924.59 & 239.8 & 926.10 & & \\
\hline & & 70.0 & 925.91 & 152.5 & 924.64 & 241.0 & 926.13 & & \\
\hline
\end{tabular}


Table 38. (Continued) Listing of horizontal stations and elevations for cross section PR200A

[Sta., station, distance in meters from a reference pin on the left bank; Elev., elevation, in meters above sea level]

\begin{tabular}{|c|c|c|c|c|c|c|c|c|c|}
\hline \multirow{2}{*}{\multicolumn{2}{|c|}{$\begin{array}{c}1995 \\
29 \text { September }\end{array}$}} & \multicolumn{2}{|c|}{1995} & \multicolumn{2}{|c|}{1995} & \multicolumn{2}{|c|}{1995} & \multicolumn{2}{|c|}{1996} \\
\hline & & $29 \mathrm{Se}$ & ember & $29 \mathrm{Se}$ & ember & $29 \mathrm{Se}$ & ember & 23 & ober \\
\hline Sta. & Elev. & Sta. & Elev. & Sta. & Elev. & Sta. & Elev. & Sta. & Elev. \\
\hline-20.0 & 927.62 & 42.6 & 926.06 & 107.0 & 926.31 & 176.5 & 925.34 & -6.4 & 927.76 \\
\hline-17.0 & 927.61 & 44.0 & 926.05 & 107.2 & 926.18 & 179.0 & 925.45 & -6.0 & 927.71 \\
\hline-15.0 & 927.66 & 46.0 & 926.04 & 108.0 & 926.20 & 181.0 & 925.54 & -3.5 & 927.66 \\
\hline-11.0 & 927.63 & 48.0 & 926.03 & 110.0 & 926.29 & 183.0 & 925.59 & -3.1 & 925.96 \\
\hline-8.0 & 927.70 & 50.0 & 925.98 & 111.0 & 926.30 & 185.0 & 925.63 & -1.9 & 925.53 \\
\hline-6.4 & 927.77 & 52.0 & 926.00 & 112.4 & 926.46 & 187.0 & 925.63 & -1.2 & 925.37 \\
\hline-4.0 & 927.67 & 54.0 & 925.95 & 113.0 & 926.22 & 189.0 & 925.66 & -1.2 & 924.89 \\
\hline-1.8 & 927.71 & 55.2 & 925.87 & 114.5 & 926.30 & 191.0 & 925.63 & 0.4 & 924.54 \\
\hline-1.5 & 927.69 & 56.9 & 925.82 & 114.9 & 926.19 & 193.0 & 925.72 & 3.0 & 924.21 \\
\hline-1.2 & 925.91 & 57.7 & 925.99 & 116.0 & 926.39 & 195.0 & 925.84 & 5.0 & 924.32 \\
\hline-0.7 & 925.31 & 58.0 & 926.13 & 116.7 & 926.56 & 197.5 & 926.25 & 7.0 & 924.59 \\
\hline 0.0 & 925.03 & 60.0 & 926.14 & 117.8 & 926.58 & 200.0 & 926.33 & 10.0 & 924.77 \\
\hline 0.9 & 924.77 & 62.0 & 926.16 & 118.0 & 926.45 & 203.0 & 926.36 & 12.0 & 924.93 \\
\hline 2.0 & 924.47 & 64.0 & 926.16 & 119.0 & 926.05 & 206.0 & 926.38 & 14.0 & 925.14 \\
\hline 6.5 & 923.59 & 66.0 & 926.17 & 120.1 & 925.74 & 209.0 & 926.32 & 15.5 & 925.31 \\
\hline 7.5 & 924.15 & 68.0 & 926.11 & 121.2 & 925.12 & 211.0 & 926.30 & 16.5 & 925.83 \\
\hline 8.2 & 924.99 & 70.0 & 926.15 & 123.0 & 925.49 & 213.0 & 926.24 & 18.0 & 925.89 \\
\hline 9.3 & 924.76 & 72.0 & 926.14 & 125.0 & 925.58 & 215.0 & 926.22 & 20.0 & 925.84 \\
\hline 10.0 & 924.11 & 74.0 & 926.12 & 126.5 & 925.60 & 216.5 & 926.32 & 21.3 & 925.63 \\
\hline 12.0 & 924.57 & 76.0 & 926.13 & 127.9 & 925.62 & 218.0 & 926.37 & 23.0 & 925.56 \\
\hline 14.1 & 924.76 & 78.0 & 926.11 & 128.5 & 925.73 & 219.2 & 926.40 & 24.0 & 925.63 \\
\hline 15.0 & 925.19 & 80.0 & 926.10 & 130.0 & 925.76 & 221.0 & 926.26 & 27.0 & 925.63 \\
\hline 16.0 & 925.68 & 82.0 & 926.08 & 132.0 & 925.77 & 223.0 & 926.22 & 30.0 & 925.56 \\
\hline 17.3 & 925.88 & 84.0 & 926.06 & 134.0 & 925.75 & 224.5 & 926.24 & 32.0 & 925.69 \\
\hline 17.6 & 925.68 & 88.0 & 926.27 & 136.0 & 925.77 & 225.5 & 926.36 & 35.0 & 925.62 \\
\hline 19.4 & 925.56 & 88.5 & 926.12 & 138.0 & 925.78 & 227.0 & 926.34 & 37.7 & 925.67 \\
\hline 21.0 & 925.57 & 90.0 & 926.22 & 140.0 & 925.73 & 228.5 & 926.36 & 40.0 & 925.99 \\
\hline 23.0 & 925.46 & 91.0 & 926.28 & 142.0 & 925.73 & 230.0 & 925.86 & 42.0 & 925.99 \\
\hline 25.0 & 925.34 & 92.0 & 926.24 & 143.0 & 925.64 & 231.0 & 925.79 & 45.0 & 926.05 \\
\hline 27.0 & 925.30 & 95.0 & 926.39 & 145.0 & 925.48 & 233.0 & 925.75 & 48.0 & 926.09 \\
\hline 29.0 & 925.23 & 96.8 & 926.34 & 146.0 & 925.36 & 235.0 & 925.88 & 51.0 & 926.09 \\
\hline 31.5 & 925.14 & 97.3 & 926.17 & 146.8 & 925.15 & 237.5 & 925.88 & 54.0 & 925.97 \\
\hline 32.0 & 925.27 & 97.8 & 926.13 & 149.0 & 924.96 & 239.2 & 925.84 & 56.0 & 925.85 \\
\hline 33.0 & 925.31 & 98.0 & 926.22 & 151.0 & 924.77 & 239.4 & 925.95 & 59.0 & 926.09 \\
\hline 34.0 & 925.39 & 100.0 & 926.26 & 154.0 & 924.71 & 239.7 & 925.98 & 62.0 & 926.17 \\
\hline 35.0 & 925.35 & 101.0 & 926.30 & 157.0 & 924.80 & 240.0 & 926.32 & 65.0 & 926.16 \\
\hline 36.7 & 925.74 & 102.0 & 926.20 & 160.0 & 924.83 & 242.0 & 926.22 & 68.0 & 926.15 \\
\hline 38.0 & 925.71 & 103.0 & 926.11 & 163.0 & 924.89 & 243.6 & 926.20 & 70.0 & 926.21 \\
\hline 38.9 & 925.94 & 103.5 & 926.05 & 166.0 & 925.06 & 245.0 & 926.49 & 72.0 & 926.29 \\
\hline 40.0 & 925.96 & 104.0 & 926.22 & 169.0 & 925.21 & 247.0 & 926.62 & 74.0 & 926.16 \\
\hline 41.4 & 925.88 & 105.0 & 926.07 & 172.0 & 925.21 & 250.0 & 926.80 & 77.0 & 926.11 \\
\hline 42.1 & 925.91 & 106.5 & 926.16 & 174.0 & 925.22 & & & 80.0 & 926.13 \\
\hline
\end{tabular}


Table 38. (Continued) Listing of horizontal stations and elevations for cross section PR200A

[Sta., station, distance in meters from a reference pin on the left bank; Elev., elevation, in meters above sea level]

\begin{tabular}{|c|c|c|c|c|c|c|c|c|c|}
\hline \multirow{2}{*}{\multicolumn{2}{|c|}{$\begin{array}{c}1996 \\
23 \text { October }\end{array}$}} & \multirow{2}{*}{\multicolumn{2}{|c|}{$\begin{array}{c}1996 \\
23 \text { October }\end{array}$}} & \multirow{2}{*}{\multicolumn{2}{|c|}{$\begin{array}{c}1997 \\
17 \text { September }\end{array}$}} & \multicolumn{2}{|c|}{1997} & \multicolumn{2}{|c|}{1997} \\
\hline & & & & & & $17 \mathrm{Se}$ & ember & $17 \mathrm{~S}$ & ember \\
\hline Sta. & Elev. & Sta. & Elev. & Sta. & Elev. & Sta. & Elev. & Sta. & Elev. \\
\hline 83.0 & 926.10 & 172.0 & 925.07 & -20.0 & 927.64 & 54.0 & 925.96 & 126.0 & 925.68 \\
\hline 88.0 & 926.28 & 174.6 & 925.11 & -17.0 & 927.61 & 56.5 & 925.86 & 128.0 & 925.67 \\
\hline 89.0 & 926.19 & 177.0 & 925.21 & -14.0 & 927.65 & 58.0 & 925.97 & 130.0 & 925.72 \\
\hline 91.0 & 926.31 & 180.0 & 925.27 & -11.7 & 927.63 & 60.0 & 926.10 & 132.0 & 925.74 \\
\hline 93.0 & 926.27 & 183.0 & 925.36 & -9.0 & 927.64 & 62.0 & 926.16 & 134.0 & 925.74 \\
\hline 96.0 & 926.40 & 186.0 & 925.51 & -6.4 & 927.77 & 64.0 & 926.16 & 136.0 & 925.68 \\
\hline 98.0 & 926.19 & 188.0 & 925.59 & -5.0 & 927.69 & 66.0 & 926.16 & 138.0 & 925.19 \\
\hline 100.0 & 926.23 & 191.5 & 926.04 & -3.8 & 927.66 & 68.0 & 926.16 & 139.5 & 924.86 \\
\hline 102.0 & 926.18 & 194.0 & 926.13 & -3.8 & 926.81 & 70.0 & 926.21 & 140.6 & 924.67 \\
\hline 104.0 & 926.04 & 196.5 & 926.15 & -3.5 & 926.45 & 72.0 & 926.26 & 141.8 & 924.61 \\
\hline 106.0 & 926.16 & 198.5 & 926.42 & -2.7 & 926.11 & 74.0 & 926.18 & 142.5 & 924.23 \\
\hline 108.0 & 926.18 & 200.0 & 926.38 & -2.4 & 926.02 & 76.0 & 926.14 & 144.0 & 924.21 \\
\hline 108.5 & 926.29 & 203.0 & 926.38 & -1.5 & 925.20 & 78.0 & 926.12 & 145.0 & 924.21 \\
\hline 110.0 & 926.30 & 206.0 & 926.39 & -0.6 & 924.91 & 80.0 & 926.13 & 147.0 & 924.31 \\
\hline 112.0 & 926.34 & 209.0 & 926.32 & 1.0 & 924.69 & 82.0 & 926.12 & 150.0 & 924.31 \\
\hline 112.5 & 926.22 & 212.0 & 926.26 & 3.0 & 924.69 & 84.0 & 926.12 & 153.0 & 924.44 \\
\hline 114.0 & 926.11 & 215.0 & 926.25 & 4.5 & 924.91 & 86.0 & 926.28 & 156.0 & 924.47 \\
\hline 115.7. & 926.09 & 218.0 & 926.36 & 5.5 & 925.03 & 89.0 & 926.18 & 159.0 & 924.53 \\
\hline 116.5 & 926.51 & 221.5 & 926.39 & 6.4 & 925.28 & 90.0 & 926.30 & 162.0 & 924.58 \\
\hline 117.6 & 926.50 & 224.0 & 926.31 & 7.9 & 925.57 & 92.0 & 926.28 & 165.0 & 924.51 \\
\hline 119.0 & 925.94 & 227.0 & 926.32 & 8.2 & 925.83 & 93.0 & 926.27 & 168.0 & 924.38 \\
\hline 120.0 & 925.80 & 228.0 & 926.37 & 10.0 & 925.81 & 96.0 & 926.41 & 171.0 & 924.32 \\
\hline 121.0 & 925.47 & 229.0 & 926.27 & 12.0 & 925.81 & 98.0 & 926.20 & 174.0 & 924.30 \\
\hline 122.5 & 925.73 & 230.0 & 926.12 & 15.0 & 925.94 & 100.0 & 926.24 & 177.0 & 924.24 \\
\hline 124.0 & 925.59 & 232.0 & 926.12 & 17.0 & 925.99 & 102.0 & 926.17 & 180.0 & 924.21 \\
\hline 126.0 & 925.58 & 234.0 & 926.08 & 19.5 & 925.94 & 103.5 & 926.03 & 183.0 & 924.18 \\
\hline 128.0 & 925.67 & 235.0 & 926.00 & 21.0 & 925.77 & 105.0 & 926.14 & 186.0 & 924.20 \\
\hline 131.0 & 925.67 & 237.0 & 925.99 & 23.0 & 925.80 & 106.0 & 926.17 & 188.0 & 924.37 \\
\hline 134.0 & 925.72 & 238.0 & 925.91 & 25.0 & 925.92 & 107.9 & 926.20 & 189.5 & 924.73 \\
\hline 137.0 & 925.66 & 239.0 & 925.95 & 27.0 & 925.78 & 109.0 & 926.28 & 191.0 & 925.06 \\
\hline 140.0 & 925.35 & 240.0 & 926.13 & 29.0 & 925.76 & 110.5 & 926.26 & 193.0 & 925.38 \\
\hline 142.0 & 925.15 & 240.5 & 926.39 & 31.0 & 925.81 & 112.0 & 926.36 & 195.0 & 925.84 \\
\hline 144.0 & 924.99 & 241.5 & 926.27 & 33.0 & 925.80 & 112.5 & 926.21 & 197.0 & 926.20 \\
\hline 146.0 & 924.91 & 243.0 & 926.23 & 35.0 & 925.71 & 114.0 & 926.12 & 198.5 & 926.40 \\
\hline 148.0 & 924.76 & 245.0 & 926.50 & 38.0 & 925.69 & 115.6 & 926.11 & 200.0 & 926.37 \\
\hline 151.0 & 924.71 & 246.0 & 926.61 & 40.0 & 925.97 & 116.4 & 926.50 & 202.0 & 926.36 \\
\hline 154.0 & 924.66 & 248.0 & 926.66 & 42.0 & 925.99 & 117.5 & 926.56 & 204.0 & 926.42 \\
\hline 157.0 & 924.73 & 250.0 & 926.80 & 44.0 & 926.00 & 119.0 & 925.96 & 207.0 & 926.40 \\
\hline 160.0 & 924.77 & 275.0 & 926.76 & 46.0 & 926.06 & 121.0 & 925.63 & 210.0 & 926.33 \\
\hline 163.0 & 924.74 & & & 48.0 & 926.08 & 122.0 & 925.84 & 213.0 & 926.26 \\
\hline 166.0 & 924.85 & & & 50.0 & 926.05 & 124.0 & 925.84 & 216.0 & 926.26 \\
\hline 169.0 & 924.91 & & & 52.0 & 926.06 & 125.0 & 925.86 & 219.0 & 926.35 \\
\hline
\end{tabular}


Table 38. (Continued) Listing of horizontal stations and elevations for cross section PR200A

[Sta., station, distance in meters from a reference pin on the left bank; Elev., elevation, in meters above sea level]

\begin{tabular}{|c|c|c|c|c|c|c|c|}
\hline \multirow{2}{*}{\multicolumn{2}{|c|}{$\begin{array}{c}1997 \\
17 \text { September }\end{array}$}} & \multicolumn{2}{|c|}{1998} & \multicolumn{2}{|c|}{1998} & \multicolumn{2}{|c|}{1998} \\
\hline & & \multicolumn{2}{|c|}{27 September } & \multicolumn{2}{|c|}{27 September } & \multicolumn{2}{|c|}{27 September } \\
\hline Sta. & Elev. & Sta. & Elev. & Sta. & Elev. & Sta. & Elev. \\
\hline 222.0 & 926.36 & -9.9 & 927.64 & 88.5 & 926.16 & 187.0 & 924.17 \\
\hline 224.0 & 926.31 & -7.5 & 927.67 & 91.0 & 926.31 & 190.0 & 924.53 \\
\hline 227.0 & 926.32 & -6.4 & 927.75 & 95.0 & 926.36 & 191.8 & 924.93 \\
\hline 229.0 & 926.30 & -6.0 & 927.72 & 98.0 & 926.20 & 194.0 & 925.31 \\
\hline 231.0 & 926.11 & -4.4 & 927.66 & 100.0 & 926.25 & 196.0 & 925.90 \\
\hline 233.0 & 926.10 & -4.0 & 926.58 & 103.0 & 926.09 & 198.2 & 926.36 \\
\hline 234.8 & 925.98 & -3.2 & 926.36 & 104.0 & 926.04 & 200.0 & 926.36 \\
\hline 236.0 & 925.80 & -0.6 & 924.91 & 106.0 & 926.16 & 203.0 & 926.37 \\
\hline 238.0 & 925.73 & 0.2 & 924.82 & 107.5 & 926.16 & 206.0 & 926.38 \\
\hline 238.4 & 925.81 & 2.3 & 924.76 & 109.0 & 926.27 & 209.0 & 926.33 \\
\hline 238.6 & 926.04 & 5.0 & 924.90 & 111.0 & 926.30 & 212.0 & 926.27 \\
\hline 239.8 & 926.10 & 7.0 & 925.35 & 113.0 & 926.21 & 215.0 & 926.27 \\
\hline 240.5 & 926.40 & 8.3 & 925.76 & 114.5 & 926.14 & 218.0 & 926.37 \\
\hline 242.3 & 926.21 & 10.0 & 925.79 & 116.0 & 926.11 & 221.0 & 926.33 \\
\hline 243.5 & 926.29 & 13.0 & 925.83 & 116.5 & 926.49 & 224.0 & 926.29 \\
\hline 244.5 & 926.54 & 16.0 & 925.94 & 117.5 & 926.48 & 227.0 & 926.33 \\
\hline 246.0 & 926.62 & 19.0 & 925.93 & 119.0 & 925.96 & 228.8 & 926.29 \\
\hline 248.0 & 926.67 & 21.0 & 925.76 & 121.1 & 925.67 & 230.0 & 926.14 \\
\hline 250.0 & 926.81 & 23.0 & 925.77 & 122.0 & 925.80 & 232.0 & 926.11 \\
\hline & & 25.0 & 925.90 & 124.0 & 925.85 & 234.0 & 926.03 \\
\hline & & 28.0 & 925.76 & 126.0 & 925.70 & 236.0 & 925.80 \\
\hline & & 30.0 & 925.80 & 128.0 & 925.67 & 238.0 & 925.76 \\
\hline & & 33.0 & 925.79 & 130.0 & 925.71 & 238.6 & 926.05 \\
\hline & & 36.0 & 925.73 & 133.0 & 925.74 & 239.4 & 926.07 \\
\hline & & 38.0 & 925.73 & 136.0 & 925.69 & 240.4 & 926.40 \\
\hline & & 40.0 & 925.98 & 138.0 & 925.20 & 243.0 & 926.23 \\
\hline & & 43.0 & 926.03 & 139.6 & 924.89 & 246.0 & 926.59 \\
\hline & & 46.0 & 926.06 & 142.0 & 924.43 & 250.0 & 926.90 \\
\hline & & 49.0 & 926.09 & 145.0 & 924.46 & & \\
\hline & & 52.0 & 926.07 & 148.0 & 924.44 & & \\
\hline & & 55.0 & 925.88 & 151.0 & 924.36 & & \\
\hline & & 58.0 & 925.96 & 154.0 & 924.45 & & \\
\hline & & 61.0 & 926.14 & 157.0 & 924.50 & & \\
\hline & & 64.0 & 926.15 & 160.0 & 924.53 & & \\
\hline & & 66.0 & 926.15 & 163.0 & 924.56 & & \\
\hline & & 68.0 & 926.11 & 166.0 & 924.36 & & \\
\hline & & 70.0 & 926.20 & 169.0 & 924.30 & & \\
\hline & & 73.0 & 926.22 & 172.0 & 924.30 & & \\
\hline & & 76.0 & 926.15 & 175.0 & 924.23 & & \\
\hline & & 79.0 & 926.11 & 178.0 & 924.33 & & \\
\hline & & 82.0 & 926.11 & 181.0 & 924.24 & & \\
\hline & & 85.0 & 926.24 & 184.0 & 924.21 & & \\
\hline
\end{tabular}




\section{Description of Cross Section PR206}

Location: Township 5 South/Range 51 East--section 2

U. S. Geological Survey quadrangle (1:24,000): Broadus

Landowners--left bank: Ron and Twila Talcott

--right bank: Charles and Shirley Russell

Access: Right bank

Permission from: Charles or Shirley Russell

Distance from Moorhead Gaging Station: 92.19 kilometers

Azimuth of Section (degrees magnetic): 266

\section{Reference Monuments}

[Monuments at stations 101.9 and 139.6 were closest to leveling instrument]

\begin{tabular}{|c|c|c|c|c|c|c|}
\hline \multirow[b]{2}{*}{ Description } & \multirow[b]{2}{*}{$\begin{array}{l}\text { Station } \\
(\mathbf{m})\end{array}$} & \multicolumn{2}{|c|}{ GPS-NAD83 (1992) } & \multicolumn{2}{|c|}{ Measurement } & \multirow{2}{*}{$\begin{array}{l}\text { Elevation } \\
\text { (NGVD1929) } \\
\text { (m) }\end{array}$} \\
\hline & & Latitude & Longitude & $\begin{array}{l}\text { Standard } \\
\text { deviation } \\
\text { (m) }\end{array}$ & $\begin{array}{l}\text { Horizontal } \\
\text { precision } \\
\text { (m) }\end{array}$ & \\
\hline 1/2-inch-rebar; bent flat on 1998 ground level & -50.0 & & & & & 921.06 \\
\hline $\begin{array}{l}\text { 1/2-inch-rebar; } 0.29 \text { meter above } 1998 \text { ground } \\
\text { level }\end{array}$ & -43.25 & $45^{\circ} 26^{\prime} 03.23^{\prime \prime}$ & $105^{\circ} 23^{\prime} 33.50^{\prime \prime}$ & 0.404 & 0.434 & 921.00 \\
\hline $\begin{array}{l}\text { 1/2-inch-rebar; upstream bolt in upstream cir- } \\
\text { cular concrete footing; offsection at station } \\
101.9\end{array}$ & 101.9 & & & & & 921.50 \\
\hline $\begin{array}{l}\text { Benchmark--brass circular plate; off section at } \\
\text { about } 102.0\end{array}$ & $\sim 102.0$ & $45^{\circ} 26^{\prime} 02.44^{\prime \prime}$ & $105^{\circ} 23^{\prime} 26.94^{\prime \prime}$ & 0.409 & 0.775 & 921.32 \\
\hline $\begin{array}{l}\text { 1/2-inch-rebar; } 0.11 \text { meter above } 1998 \text { ground } \\
\text { level; under a } 5 \text {-strand, barbed-wire fence } \\
\text { line }\end{array}$ & 139.6 & $45^{\circ} 26^{\prime} 02.25^{\prime \prime}$ & $105^{\circ} 23^{\prime} 25.23^{\prime \prime}$ & 0.639 & 0.500 & 921.24 \\
\hline
\end{tabular}




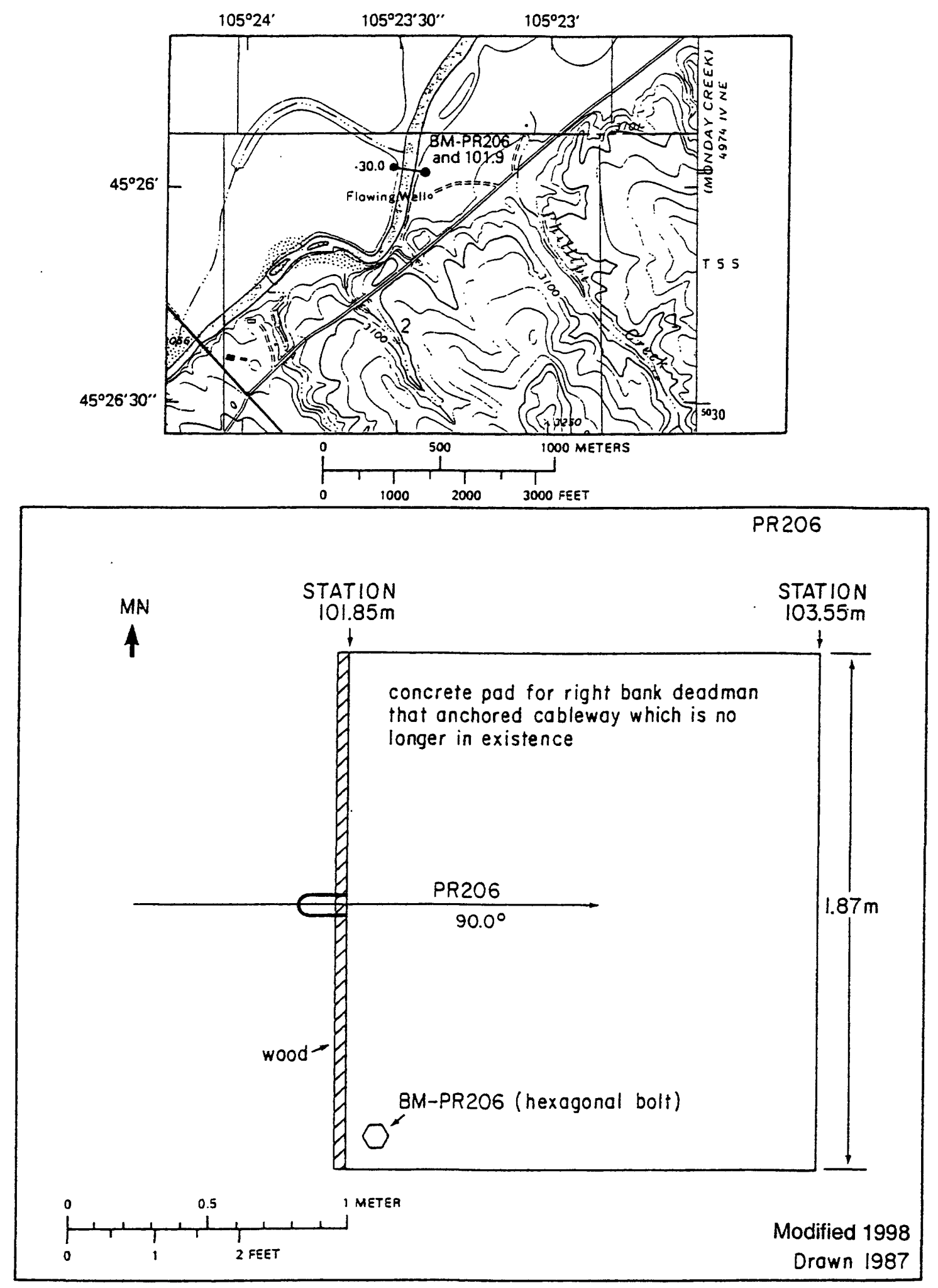

Figure 113. Upper: Location of cross section PR206, bench mark BM-PR206, and the left and right bank reference monuments in the Eldon Mountain quadrangle. Lower: Location of the bench mark on the right bank. MN is magnetic north. 

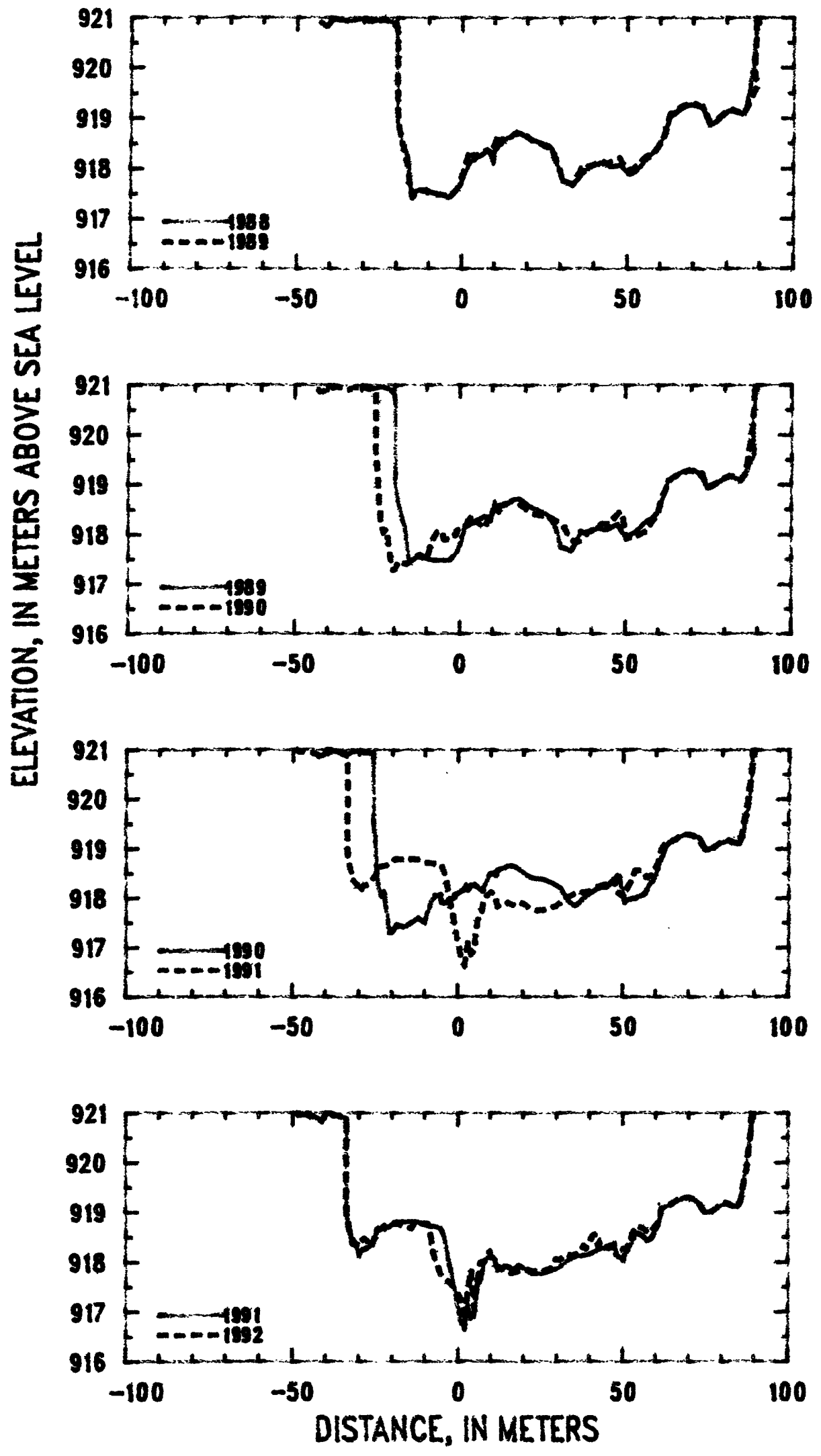

Figure 114. Profiles of cross section PR206 from 1988 to 1992. 

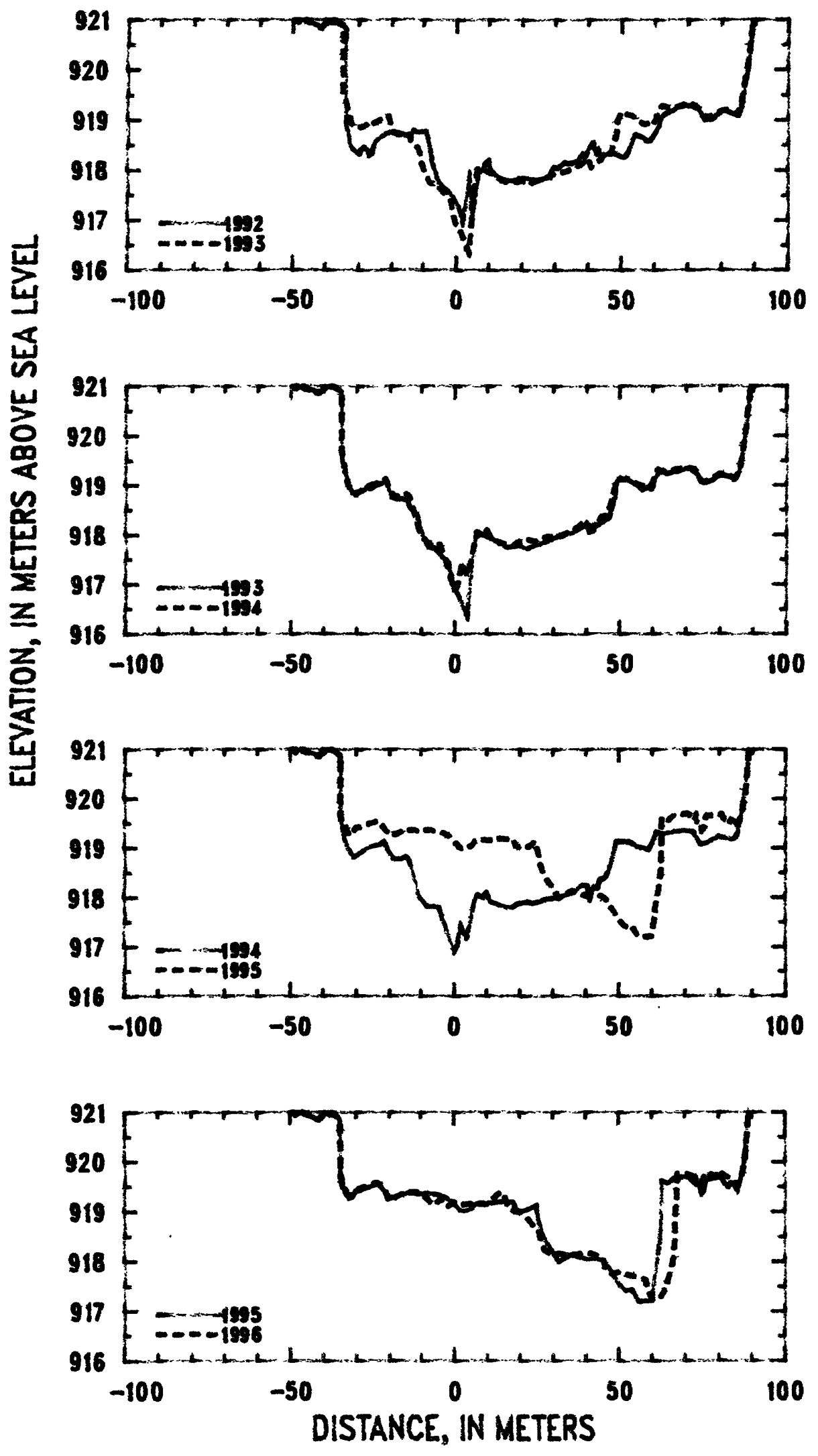

Figure 115. Profiles of cross section PR206 from 1992 to 1996. 


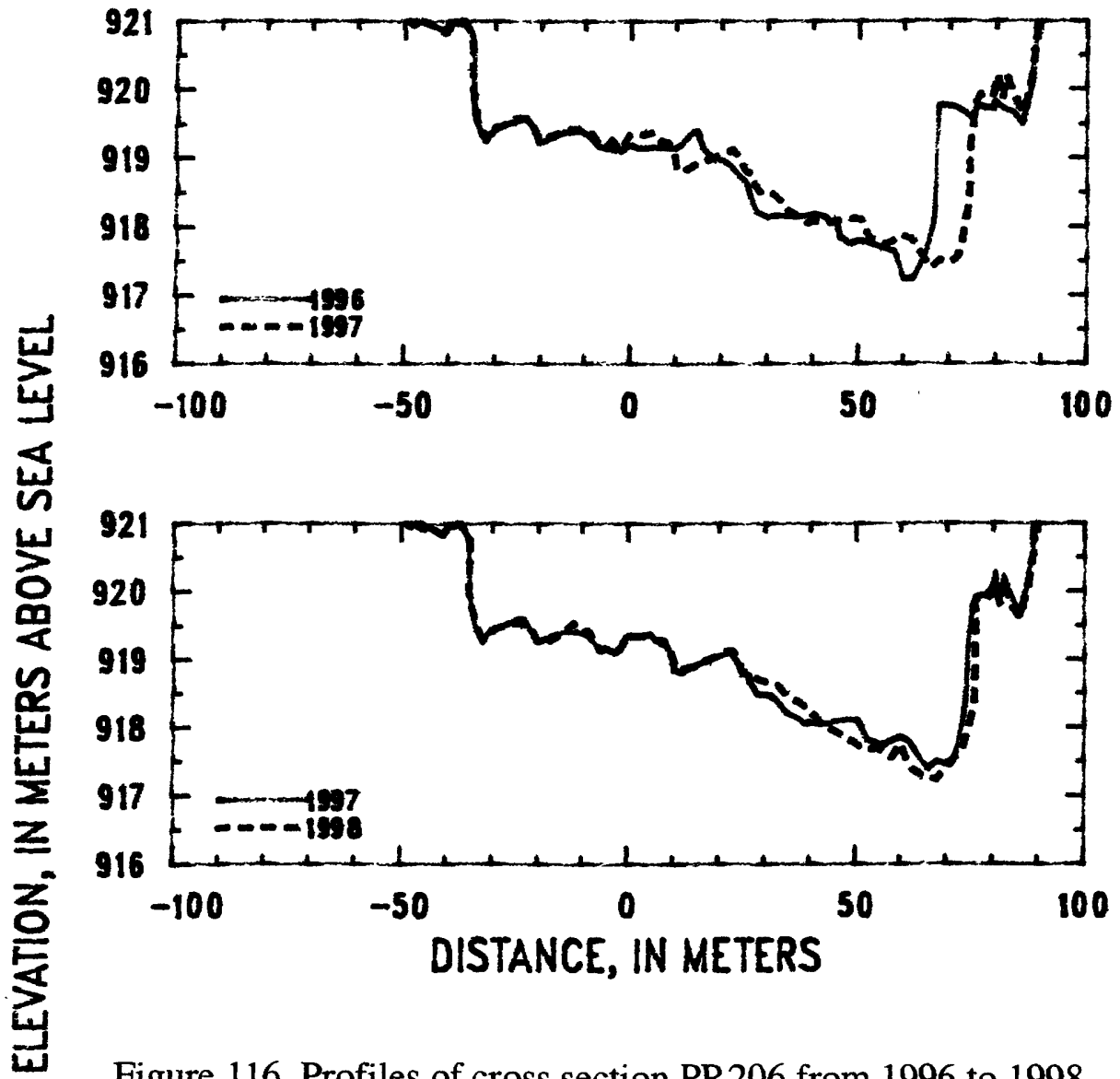

Figure 116. Profiles of cross section PR206 from 1996 to 1998. 
Table 39. Listing of horizontal stations and elevations for cross section PR206

[Sta., station, distance in meters from a reference pin on the left bank; Elev., elevation, in meters above sea level]

\begin{tabular}{|c|c|c|c|c|c|c|c|c|c|}
\hline \multirow{2}{*}{\multicolumn{2}{|c|}{$\begin{array}{c}1989 \\
23 \text { September }\end{array}$}} & \multicolumn{2}{|c|}{1989} & \multicolumn{2}{|c|}{1990} & \multicolumn{2}{|c|}{1990} & \multicolumn{2}{|c|}{1990} \\
\hline & & $23 \mathrm{Se}$ & ember & $24 S$ & ember & $24 \mathrm{~S}$ & ember & $24 \mathrm{Se}$ & ember \\
\hline Sta. & Elev. & Sta. & Elev. & Sta. & Elev. & Sta. & Elev. & Sta. & Elev. \\
\hline-30.0 & 920.98 & 33.7 & 917.67 & -43.0 & 920.91 & 12.3 & 918.57 & 80.0 & 919.13 \\
\hline-28.0 & 920.93 & 34.7 & 917.76 & -42.0 & 920.85 & 14.0 & 918.62 & 82.0 & 919.18 \\
\hline-26.0 & 920.93 & 36.6 & 918.07 & -40.0 & 920.90 & 16.0 & 918.68 & 84.0 & 919.15 \\
\hline-24.0 & 920.98 & 38.0 & 918.04 & -37.0 & 920.98 & 18.0 & 918.63 & 85.0 & 919.08 \\
\hline-21.0 & 920.90 & 40.0 & 918.07 & -34.0 & 920.91 & 20.0 & 918.53 & 86.3 & 919.23 \\
\hline-19.7 & 920.78 & 42.0 & 918.14 & -30.0 & 920.98 & 22.0 & 918.43 & 87.5 & 919.72 \\
\hline-19.6 & 919.10 & 44.0 & 918.09 & -28.0 & 920.93 & 24.0 & 918.39 & 88.1 & 919.99 \\
\hline-19.0 & 918.77 & 46.0 & 918.15 & -25.7 & 920.94 & 26.0 & 918.38 & 88.9 & 920.59 \\
\hline-17.5 & 918.31 & 47.7 & 918.21 & -25.6 & 919.52 & 28.0 & 918.33 & 89.2 & 921.35 \\
\hline-16.5 & 918.13 & 49.0 & 918.07 & -25.0 & 919.24 & 30.0 & 918.26 & 90.0 & 921.39 \\
\hline-16.0 & 917.83 & 50.2 & 917.95 & -24.5 & 918.60 & 31.4 & 918.20 & 92.0 & 921.42 \\
\hline-15.0 & 917.42 & 52.0 & 918.00 & -24.1 & 918.25 & 33.0 & 917.98 & 94.0 & 921.33 \\
\hline-13.0 & 917.55 & 54.0 & 918.11 & -23.2 & 918.03 & 34.1 & 917.89 & 96.0 & 921.30 \\
\hline-11.0 & 917.55 & 56.0 & 918.25 & -22.4 & 918.11 & 35.6 & 917.84 & 98.0 & 921.26 \\
\hline-9.0 & 917.49 & 58.0 & 918.30 & -21.7 & 917.84 & 36.9 & 917.90 & 100.0 & 921.22 \\
\hline-7.0 & 917.48 & 60.0 & 918.47 & -20.6 & 917.29 & 39.0 & 918.06 & & \\
\hline-5.0 & 917.47 & 61.0 & 918.61 & -19.7 & 917.30 & 41.0 & 918.12 & & \\
\hline-3.0 & 917.48 & 63.0 & 919.08 & -18.5 & 917.46 & 43.0 & 918.22 & & \\
\hline-1.0 & 917.62 & 65.0 & 919.16 & -16.0 & 917.41 & 45.0 & 918.21 & & \\
\hline 0.1 & 917.81 & 67.0 & 919.26 & -14.0 & 917.50 & 46.0 & 918.27 & & \\
\hline 1.0 & 918.06 & 69.0 & 919.29 & -13.0 & 917.57 & 46.2 & 918.31 & & \\
\hline 2.0 & 918.27 & 71.0 & 919.29 & -12.0 & 917.60 & 48.2 & 918.46 & & \\
\hline 3.3 & 918.35 & 73.0 & 919.19 & -10.0 & 917.49 & 49.0 & 918.44 & & \\
\hline 3.7 & 918.22 & 74.0 & 919.07 & -8.5 & 917.86 & 50.0 & 918.07 & & \\
\hline 6.0 & 918.31 & 75.0 & 918.93 & -7.0 & 918.05 & 50.5 & 917.91 & & \\
\hline 8.0 & 918.37 & 77.0 & 918.97 & -5.2 & 918.07 & 52.0 & 917.97 & & \\
\hline 9.4 & 918.29 & 79.0 & 919.08 & -4.8 & 917.90 & 54.0 & 917.99 & & \\
\hline 10.8 & 918.63 & 81.0 & 919.19 & -3.2 & 917.90 & 56.5 & 918.07 & & \\
\hline 11.3 & 918.51 & 83.0 & 919.14 & -2.7 & 917.95 & 58.0 & 918.27 & & \\
\hline 12.0 & 918.49 & 85.0 & 919.07 & -1.0 & 918.01 & 59.0 & 918.33 & & \\
\hline 12.4 & 918.59 & 86.0 & 919.20 & -0.9 & 918.08 & 60.2 & 918.49 & & \\
\hline 14.0 & 918.60 & 89.0 & 919.63 & 0.0 & 918.10 & 61.0 & 918.74 & & \\
\hline 16.0 & 918.70 & 88.0 & 919.86 & 1.1 & 918.15 & 62.3 & 918.89 & & \\
\hline 18.0 & 918.73 & 88.8 & 920.50 & 1.9 & 918.17 & 63.0 & 919.05 & & \\
\hline 20.0 & 918.64 & 89.2 & 921.36 & 2.5 & 918.25 & 65.0 & 919.18 & & \\
\hline 22.0 & 918.54 & 91.0 & 921.40 & 3.2 & 918.16 & 67.0 & 919.23 & & \\
\hline 24.0 & 918.49 & 93.0 & 921.37 & 4.0 & 918.26 & 69.0 & 919.30 & & \\
\hline 26.0 & 918.43 & 95.0 & 921.29 & 5.0 & 918.28 & 71.0 & 919.28 & & \\
\hline 28.0 & 918.28 & 97.0 & 921.27 & 7.6 & 918.13 & 73.2 & 919.24 & & \\
\hline 29.6 & 918.06 & 99.0 & 921.23 & 9.9 & 918.48 & 74.9 & 918.98 & & \\
\hline 30.7 & 917.75 & 101.0 & 921.24 & 11.2 & 918.51 & 76.0 & 918.99 & & \\
\hline 32.5 & 917.71 & & & 11.9 & 918.46 & 78.0 & 919.05 & & \\
\hline
\end{tabular}


Table 39. (Continued) Listing of horizontal stations and elevations for cross section PR206

[Sta., station, distance in meters from a reference pin on the left bank; Elev., elevation, in meters above sea level]

\begin{tabular}{|c|c|c|c|c|c|c|c|c|c|}
\hline \multirow{2}{*}{\multicolumn{2}{|c|}{$\begin{array}{c}1991 \\
3 \text { September }\end{array}$}} & \multicolumn{2}{|c|}{1991} & \multicolumn{2}{|c|}{1991} & \multicolumn{2}{|c|}{1992} & \multicolumn{2}{|c|}{1992} \\
\hline & & $3 \mathrm{Se}$ & ember & $3 \mathrm{Se}_{1}$ & mber & 29 & Igust & 29 & gust \\
\hline Sta. & Elev. & Sta. & Elev. & Sta. & Elev. & Sta. & Elev. & Sta. & Elev. \\
\hline-50.0 & 921.06 & 10.3 & 918.13 & 82.0 & 919.17 & -50.0 & 921.05 & 8.3 & 918.00 \\
\hline-48.0 & 920.95 & 11.5 & 918.06 & 85.0 & 919.09 & -48.0 & 920.96 & 8.7 & 918.04 \\
\hline-46.0 & 920.96 & 12.3 & 917.83 & 86.0 & 919.25 & -47.0 & 921.17 & 9.0 & 918.14 \\
\hline-43.0 & 920.91 & 14.2 & 917.91 & 89.0 & 920.60 & -45.0 & 920.94 & 10.0 & 918.21 \\
\hline-41.0 & 920.83 & 15.0 & 917.92 & 89.4 & 921.30 & -43.0 & 920.92 & 10.6 & 918.04 \\
\hline-39.6 & 921.00 & 17.3 & 917.86 & 89.7 & 921.37 & -41.0 & 920.83 & 11.0 & 917.98 \\
\hline-37.0 & 920.98 & 18.6 & 917.93 & 91.0 & 921.41 & -40.0 & 920.97 & 13.0 & 917.87 \\
\hline-35.0 & 920.92 & 20.0 & 917.89 & 94.0 & 921.33 & -38.0 & 920.94 & 15.0 & 917.80 \\
\hline-33.6 & 920.88 & 22.0 & 917.78 & 97.0 & 921.29 & -36.0 & 920.95 & 17.0 & 917.78 \\
\hline-33.6 & 919.00 & 24.0 & 917.75 & 100.0 & 921.22 & -33.7 & 920.83 & 19.0 & 917.83 \\
\hline-33.0 & 918.69 & 26.0 & 917.77 & & & -33.6 & 918.93 & 21.0 & 917.85 \\
\hline-32.3 & 918.54 & 28.0 & 917.81 & & & -33.0 & 918.76 & 23.0 & 917.82 \\
\hline-32.2 & 918.38 & 30.0 & 917.85 & & & -32.4 & 918.49 & 25.0 & 917.80 \\
\hline-30.3 & 918.25 & 32.0 & 917.96 & & & -31.0 & 918.38 & 27.0 & 917.85 \\
\hline-29.8 & 918.12 & 34.0 & 918.04 & & & -29.7 & 918.32 & 28.3 & 917.88 \\
\hline-29.0 & 918.23 & 36.0 & 918.11 & & & -29.4 & 918.41 & 29.5 & 918.03 \\
\hline-27.0 & 918.29 & 38.0 & 918.12 & & & -28.3 & 918.47 & 29.7 & 918.06 \\
\hline-24.8 & 918.48 & 40.0 & 918.14 & & & -26.8 & 918.38 & 31.0 & 918.05 \\
\hline-24.6 & 918.65 & 42.0 & 918.22 & & & -26.6 & 918.29 & 33.0 & 918.15 \\
\hline-23.0 & 918.64 & 44.0 & 918.27 & & & -26.0 & 918.32 & 35.0 & 918.13 \\
\hline-21.0 & 918.69 & 46.0 & 918.27 & & & -25.0 & 918.57 & 37.0 & 918.18 \\
\hline-19.0 & 918.80 & 47.5 & 918.38 & & & -24.0 & 918.65 & 38.4 & 918.33 \\
\hline-17.0 & 918.81 & 48.0 & 918.13 & & & -22.0 & 918.73 & 39.1 & 918.26 \\
\hline-15.0 & 918.81 & 50.2 & 918.02 & & & -20.0 & 918.77 & 41.0 & 918.53 \\
\hline-13.0 & 918.80 & 50.6 & 918.14 & & & -18.0 & 918.76 & 41.6 & 918.55 \\
\hline-11.0 & 918.77 & 52.6 & 918.36 & & & -16.0 & 918.71 & 42.5 & 918.33 \\
\hline-9.0 & 918.72 & 53.7 & 918.57 & & & -14.0 & 918.67 & 43.1 & 918.40 \\
\hline-7.0 & 918.71 & 55.0 & 918.57 & & & -13.0 & 918.79 & 43.3 & 918.31 \\
\hline-5.0 & 918.68 & 56.0 & 918.53 & & & -11.0 & 918.76 & 44.0 & 918.27 \\
\hline-4.0 & 918.51 & 57.5 & 918.41 & & & -9.0 & 918.77 & 46.0 & 918.32 \\
\hline-2.4 & 917.96 & 59.0 & 918.48 & & & -8.0 & 918.48 & 48.0 & 918.33 \\
\hline-1.4 & 917.61 & 60.7 & 918.73 & & & -7.2 & 918.18 & 50.0 & 918.26 \\
\hline 0.0 & 917.13 & 61.7 & 919.01 & & & -6.7 & 918.05 & 51.0 & 918.24 \\
\hline 1.0 & 916.77 & 63.0 & 919.11 & & & -5.0 & 917.72 & 52.2 & 918.37 \\
\hline 2.0 & 916.64 & 65.0 & 919.17 & & & -3.0 & 917.62 & 53.0 & 918.58 \\
\hline 3.0 & 917.11 & 67.0 & 919.24 & & & -1.0 & 917.46 & 54.2 & 918.71 \\
\hline 4.0 & 916.86 & 69.0 & 919.29 & & & 1.0 & 917.23 & 56.0 & 918.68 \\
\hline 5.0 & 916.95 & 71.0 & 919.28 & & & 2.0 & 916.95 & 57.6 & 918.58 \\
\hline 6.0 & 917.56 & 73.0 & 919.21 & & & 4.0 & 917.84 & 58.5 & 918.58 \\
\hline 7.0 & 917.63 & 75.0 & 918.98 & & & 5.0 & 917.04 & 60.0 & 918.71 \\
\hline 8.0 & 917.95 & 77.0 & 919.01 & & & 6.0 & 917.66 & 61.2 & 918.85 \\
\hline 9.0 & 918.06 & 80.0 & 919.15 & & & 7.0 & 917.99 & 61.8 & 919.06 \\
\hline
\end{tabular}


Table 39. (Continued) Listing of horizontal stations and elevations for cross section PR206

[Sta., station, distance in meters from a reference pin on the left bank; Elev., elevation, in meters above sea level]

\begin{tabular}{|c|c|c|c|c|c|c|c|c|c|}
\hline \multirow{2}{*}{\multicolumn{2}{|c|}{$\begin{array}{c}1992 \\
29 \text { August }\end{array}$}} & \multirow{2}{*}{\multicolumn{2}{|c|}{$\begin{array}{c}1993 \\
1 \text { September }\end{array}$}} & \multirow{2}{*}{\multicolumn{2}{|c|}{$\begin{array}{c}1993 \\
1 \text { September }\end{array}$}} & \multicolumn{2}{|c|}{1993} & \multicolumn{2}{|c|}{1994} \\
\hline & & & & & & $1 \mathrm{Se}$ & mber & $19 \mathrm{~S}$ & ember \\
\hline Sta. & Elev. & Sta. & Elev. & Sta. & Elev. & Sta. & Elev. & Sta. & Elev. \\
\hline 63.0 & 919.13 & -50.0 & 921.05 & 10.0 & 917.97 & 72.0 & 919.29 & -50.0 & 921.05 \\
\hline 65.0 & 919.17 & -48.0 & 920.96 & 12.0 & 917.90 & 73.0 & 919.23 & -48.5 & 920.95 \\
\hline 67.0 & 919.27 & -47.0 & 921.05 & 14.0 & 917.81 & 74.0 & 919.14 & -47.0 & 921.03 \\
\hline 69.0 & 919.29 & -45.0 & 920.94 & 16.0 & 917.74 & 75.0 & 919.06 & -45.5 & 920.93 \\
\hline 71.0 & 919.29 & -43.0 & 920.92 & 18.0 & 917.77 & 76.0 & 919.05 & -43.3 & 920.91 \\
\hline 73.0 & 919.22 & -42.0 & 920.85 & 20.0 & 917.79 & 77.0 & 919.07 & -41.0 & 920.87 \\
\hline 75.0 & 918.99 & -41.0 & 920.86 & 22.0 & 917.72 & 78.0 & 919.11 & -39.5 & 920.99 \\
\hline 77.0 & 919.01 & -39.0 & 920.98 & 24.0 & 917.79 & 80.0 & 919.19 & -37.0 & 921.00 \\
\hline 79.0 & 919.11 & -37.0 & 921.00 & 26.0 & 917.82 & 81.0 & 919.21 & -34.8 & 920.89 \\
\hline 81.0 & 919.20 & -35.0 & 920.92 & 28.0 & 917.87 & 82.0 & 919.19 & -34.6 & 920.78 \\
\hline 83.0 & 919.13 & -34.6 & 920.85 & 30.0 & 917.93 & 84.0 & 919.16 & -34.6 & 919.71 \\
\hline 85.0 & 919.08 & -34.6 & 919.54 & 31.5 & 917.97 & 84.5 & 919.11 & -33.5 & 919.22 \\
\hline 86.0 & 919.26 & -34.0 & 919.42 & 33.5 & 917.97 & 85.0 & 919.16 & -32.3 & 918.99 \\
\hline 87.0 & 919.69 & -32.5 & 919.09 & 36.0 & 918.06 & 86.0 & 919.36 & -30.6 & 918.81 \\
\hline 87.9 & 919.94 & -32.3 & 918.97 & 37.7 & 918.10 & 87.0 & 919.75 & -29.0 & 918.88 \\
\hline 89.0 & 920.65 & -31.0 & 918.84 & 39.0 & 918.17 & 88.0 & 920.12 & -27.0 & 918.98 \\
\hline 89.6 & 921.37 & -30.0 & 918.85 & 40.0 & 918.25 & 89.0 & 920.72 & -25.0 & 919.03 \\
\hline 91.0 & 921.41 & -28.0 & 918.89 & 41.0 & 918.05 & 89.3 & 921.38 & -23.0 & 919.07 \\
\hline 94.0 & 921.34 & -26.4 & 918.99 & 43.0 & 918.18 & 91.0 & 921.42 & -21.5 & 919.14 \\
\hline 97.0 & 921.29 & -25.8 & 918.94 & 44.0 & 918.21 & 93.0 & 921.37 & -20.0 & 918.90 \\
\hline 100.0 & 921.22 & -24.0 & 919.00 & 44.5 & 918.32 & 95.0 & 921.30 & -18.5 & 918.78 \\
\hline 101.0 & 921.26 & -23.0 & 919.03 & 45.0 & 918.28 & 97.0 & 921.29 & -16.0 & 918.77 \\
\hline & & -21.0 & 919.10 & 46.0 & 918.35 & 99.0 & 921.24 & -14.5 & 918.83 \\
\hline & & -19.5 & 918.76 & 46.8 & 918.33 & 101.0 & 921.26 & -13.6 & 918.77 \\
\hline & & -18.0 & 918.71 & 48.0 & 918.62 & & & -10.5 & 918.02 \\
\hline & & -16.0 & 918.72 & 48.8 & 918.83 & & & -8.0 & 917.83 \\
\hline & & -14.0 & 918.70 & 48.8 & 919.01 & & & -4.5 & 917.81 \\
\hline & & -14.0 & 918.60 & 50.0 & 919.16 & & & -3.0 & 917.53 \\
\hline & & -13.0 & 918.50 & 51.0 & 919.12 & & & 0.0 & 916.88 \\
\hline & & -12.0 & 918.47 & 53.0 & 919.10 & & & 1.0 & 917.00 \\
\hline & & -11.4 & 918.40 & 55.0 & 919.01 & & & 2.0 & 917.45 \\
\hline & & -10.0 & 918.06 & 57.0 & 918.91 & & & 3.0 & 917.29 \\
\hline & & -8.0 & 917.74 & 58.5 & 918.97 & & & 4.0 & 917.18 \\
\hline & & -6.0 & 917.73 & 59.6 & 918.92 & & & 6.0 & 917.85 \\
\hline & & -4.0 & 917.56 & 60.2 & 919.00 & & & 7.0 & 918.06 \\
\hline & & -2.0 & 917.46 & 60.7 & 919.14 & & & 9.0 & 917.99 \\
\hline & & 0.0 & 916.86 & 62.0 & 919.28 & & & 10.0 & 918.10 \\
\hline & & 1.0 & 916.81 & 63.0 & 919.29 & & & 11.5 & 917.91 \\
\hline & & 4.0 & 916.33 & 64.0 & 919.24 & & & 13.6 & 917.86 \\
\hline & & 5.0 & 917.27 & 66.0 & 919.25 & & & 15.7 & 917.80 \\
\hline & & 6.5 & 918.05 & 68.0 & 919.29 & & & 18.0 & 917.79 \\
\hline & & 8.0 & 918.02 & 70.0 & 919.35 & & & 19.0 & 917.86 \\
\hline
\end{tabular}


Table 39. (Continued) Listing of horizontal stations and elevations for cross section PR206

[Sta., station, distance in meters from a reference pin on the left bank; Elev., elevation, in meters above sea level]

\begin{tabular}{|c|c|c|c|c|c|c|c|c|c|}
\hline \multirow{2}{*}{\multicolumn{2}{|c|}{$\begin{array}{c}1994 \\
19 \text { September }\end{array}$}} & \multirow{2}{*}{\multicolumn{2}{|c|}{$\begin{array}{c}1994 \\
19 \text { September }\end{array}$}} & \multirow{2}{*}{\multicolumn{2}{|c|}{$\begin{array}{c}1995 \\
1 \text { October }\end{array}$}} & \multirow{2}{*}{\multicolumn{2}{|c|}{$\begin{array}{c}1995 \\
1 \text { October }\end{array}$}} & \multirow{2}{*}{\multicolumn{2}{|c|}{$\begin{array}{c}1995 \\
1 \text { October }\end{array}$}} \\
\hline & & & & & & & & & \\
\hline Sta. & Elev. & Sta. & Elev. & Sta. & Elev. & Sta. & Elev. & Sta. & Elev. \\
\hline 22.6 & 917.92 & 98.0 & 921.26 & -50.0 & 921.05 & 21.0 & 919.00 & 84.0 & 919.58 \\
\hline 24.0 & 917.88 & 100.0 & 921.22 & -48.0 & 920.93 & 23.0 & 919.05 & 85.0 & 919.54 \\
\hline 26.0 & 917.93 & 101.0 & 921.27 & -47.0 & 921.04 & 24.8 & 919.15 & 85.6 & 919.41 \\
\hline 28.0 & 917.98 & 139.6 & 921.14 & -45.0 & 920.95 & 26.0 & 918.73 & 87.0 & 919.71 \\
\hline 30.0 & 917.98 & & & -43.3 & 920.91 & 27.0 & 918.51 & 88.0 & 920.10 \\
\hline 33.0 & 918.02 & & & -41.0 & 920.85 & 28.5 & 918.34 & 89.5 & 921.36 \\
\hline 36.0 & 918.09 & & & -39.0 & 920.98 & 29.8 & 918.21 & 90.0 & 921.41 \\
\hline 39.0 & 918.25 & & & -37.0 & 921.00 & 32.0 & 917.98 & 91.0 & 921.41 \\
\hline 40.5 & 918.25 & & & -35.0 & 920.89 & 34.0 & 918.07 & 93.5 & 921.37 \\
\hline 41.5 & 917.97 & & & -34.7 & 920.83 & 36.0 & 918.14 & 96.0 & 921.30 \\
\hline 42.5 & 918.21 & & & -34.6 & 919.66 & 38.0 & 918.10 & 98.0 & 921.26 \\
\hline 43.5 & 918.20 & & & -34.0 & 919.48 & 40.0 & 918.04 & 100.0 & 921.23 \\
\hline 44.3 & 918.37 & & & -33.0 & 919.44 & 42.0 & 918.05 & & \\
\hline 46.0 & 918.39 & & & -32.0 & 919.25 & 44.0 & 918.05 & & \\
\hline 47.0 & 918.57 & & & -31.2 & 919.28 & 46.0 & 917.98 & & \\
\hline 48.0 & 918.78 & & & -30.0 & 919.41 & 48.0 & 917.77 & & \\
\hline 49.2 & 919.14 & & & -28.0 & 919.44 & 50.0 & 917.53 & & \\
\hline 51.0 & 919.13 & & & -26.0 & 919.49 & 52.0 & 917.41 & & \\
\hline 53.0 & 919.14 & & & -24.0 & 919.55 & 54.0 & 917.43 & & \\
\hline 55.0 & 919.04 & & & -23.0 & 919.55 & 56.0 & 917.19 & & \\
\hline 57.0 & 919.00 & & & -20.0 & 919.27 & 60.0 & 917.21 & & \\
\hline 59.0 & 918.96 & & & -18.0 & 919.27 & 62.0 & 918.21 & & \\
\hline 60.0 & 919.07 & & & -16.0 & 919.33 & 62.7 & 918.54 & & \\
\hline 61.3 & 919.33 & & & -14.0 & 919.37 & 63.0 & 919.62 & & \\
\hline 63.0 & 919.29 & & & -12.0 & 919.36 & 64.0 & 919.59 & & \\
\hline 65.0 & 919.30 & & & -10.0 & 919.36 & 65.0 & 919.56 & & \\
\hline 67.0 & 919.33 & & & -8.0 & 919.38 & 67.0 & 919.67 & & \\
\hline 70.0 & 919.36 & & & -6.0 & 919.35 & 69.0 & 919.69 & & \\
\hline 72.3 & 919.33 & & & -4.0 & 919.32 & 71.0 & 919.70 & & \\
\hline 74.6 & 919.07 & & & -2.0 & 919.26 & 72.0 & 919.64 & & \\
\hline 76.0 & 919.08 & & & 0.0 & 919.14 & 73.0 & 919.56 & & \\
\hline 78.0 & 919.15 & & & 2.0 & 919.00 & 73.3 & 919.64 & & \\
\hline 80.0 & 919.24 & & & 4.0 & 919.00 & 73.5 & 919.53 & & \\
\hline 82.0 & 919.20 & & & 5.7 & 919.06 & 74.5 & 919.53 & & \\
\hline 84.0 & 919.17 & & & 6.3 & 919.14 & 75.0 & 919.35 & & \\
\hline 85.0 & 919.19 & & & 8.0 & 919.17 & 75.6 & 919.43 & & \\
\hline 86.0 & 919.38 & & & 10.0 & 919.17 & 76.3 & 919.66 & & \\
\hline 87.2 & 919.81 & & & 12.0 & 919.16 & 78.0 & 919.65 & & \\
\hline 88.8 & 920.75 & & & 14.0 & 919.20 & 80.0 & 919.68 & & \\
\hline 89.2 & 921.34 & & & 16.0 & 919.18 & 81.0 & 919.68 & & \\
\hline 92.0 & 921.40 & & & 18.0 & 919.18 & 82.0 & 919.52 & & \\
\hline 95.5 & 921.31 & & & 19.0 & 919.03 & 83.0 & 919.48 & & \\
\hline
\end{tabular}


Table 39. (Continued) Listing of horizontal stations and elevations for cross section PR206

[Sta., station, distance in meters from a reference pin on the left bank; Elev., elevation, in meters above sea level]

\begin{tabular}{|c|c|c|c|c|c|c|c|c|c|}
\hline \multirow{2}{*}{\multicolumn{2}{|c|}{$\begin{array}{c}1996 \\
16 \text { October }\end{array}$}} & \multicolumn{2}{|c|}{1996} & \multicolumn{2}{|c|}{1996} & \multicolumn{2}{|c|}{1997} & \multicolumn{2}{|c|}{1997} \\
\hline & & 160 & tober & $16 C$ & ober & $24 \mathrm{Se}$ & ember & $24 \mathrm{~S}$ & ember \\
\hline Sta. & Elev. & Sta. & Elev. & Sta. & Elev. & Sta. & Elev. & Sta. & Elev. \\
\hline-50.0 & 921.04 & 18.0 & 919.01 & 87.2 & 919.78 & -50.0 & 921.05 & 26.7 & 918.76 \\
\hline-48.0 & 920.94 & 20.0 & 918.98 & 88.0 & 920.09 & -48.0 & 920.95 & 26.8 & 918.65 \\
\hline-46.5 & 921.01 & 22.0 & 918.90 & 89.0 & 920.83 & -47.0 & 921.04 & 28.5 & 918.49 \\
\hline-43.3 & 920.92 & 24.0 & 918.73 & 89.4 & 921.35 & -45.0 & 920.96 & 31.0 & 918.47 \\
\hline-41.0 & 920.84 & 25.6 & 918.63 & 91.0 & 921.42 & -43.3 & 920.91 & 33.2 & 918.36 \\
\hline-40.0 & 920.98 & 26.0 & 918.48 & 94.0 & 921.35 & -41.0 & 920.82 & 34.5 & 918.22 \\
\hline-38.0 & 920.97 & 27.5 & 918.22 & 97.0 & 921.30 & -39.0 & 920.97 & 37.5 & 918.13 \\
\hline-36.0 & 920.94 & 30.0 & 918.13 & 100.0 & 921.22 & -37.0 & 921.00 & 39.0 & 918.04 \\
\hline-34.7 & 920.73 & 32.0 & 918.16 & & & -35.0 & 920.79 & 40.0 & 918.08 \\
\hline-34.7 & 919.96 & 34.0 & 918.14 & & & -35.0 & 920.08 & 43.0 & 918.04 \\
\hline-34.5 & 919.71 & 36.0 & 918.13 & & & -33.8 & 919.50 & 46.0 & 918.10 \\
\hline-33.9 & 919.50 & 38.0 & 918.15 & & & -32.0 & 919.26 & 49.0 & 918.11 \\
\hline-32.6 & 919.32 & 40.0 & 918.18 & & & -30.0 & 919.44 & 50.5 & 918.10 \\
\hline-31.0 & 919.34 & 42.0 & 918.16 & & & -27.0 & 919.51 & 52.5 & 917.81 \\
\hline-30.0 & 919.41 & 43.1 & 918.13 & & & -25.0 & 919.59 & 54.0 & 917.78 \\
\hline-28.0 & 919.49 & 44.0 & 918.06 & & & -23.0 & 919.59 & 56.0 & 917.73 \\
\hline-26.0 & 919.53 & 45.3 & 918.06 & & & -21.5 & 919.43 & 58.0 & 917.80 \\
\hline-24.0 & 919.58 & 46.0 & 917.83 & & & -20.0 & 919.26 & 60.0 & 917.86 \\
\hline-23.0 & 919.57 & 48.0 & 917.74 & & & -17.0 & 919.34 & 62.0 & 917.80 \\
\hline-21.5 & 919.45 & 50.0 & 917.79 & & & -14.0 & 919.42 & 64.0 & 917.58 \\
\hline-20.4 & 919.23 & 52.0 & 917.76 & & & -11.0 & 919.42 & 66.0 & 917.38 \\
\hline-19.0 & 919.25 & 54.0 & 917.71 & & & -8.5 & 919.35 & 68.0 & 917.50 \\
\hline-17.0 & 919.32 & 56.0 & 917.67 & & & -6.0 & 919.12 & 70.0 & 917.45 \\
\hline-15.0 & 919.38 & 58.0 & 917.64 & & & -4.5 & 919.16 & 72.0 & 917.56 \\
\hline-13.0 & 919.39 & 60.0 & 917.23 & & & -2.8 & 919.10 & 73.7 & 918.15 \\
\hline-11.0 & 919.40 & 62.0 & 917.24 & & & -1.5 & 919.18 & 74.3 & 918.55 \\
\hline-9.0 & 919.32 & 64.0 & 917.44 & & & -0.5 & 919.33 & 74.6 & 919.22 \\
\hline-7.0 & 919.17 & 66.0 & 917.87 & & & 0.5 & 919.36 & 75.6 & 919.77 \\
\hline-5.5 & 919.15 & 66.8 & 918.14 & & & 3.0 & 919.34 & 76.5 & 919.92 \\
\hline-4.0 & 919.27 & 67.0 & 918.50 & & & 5.0 & 919.38 & 78.0 & 919.94 \\
\hline-2.0 & 919.10 & 67.7 & 919.76 & & & 7.0 & 919.24 & 79.6 & 919.91 \\
\hline 0.0 & 919.19 & 69.0 & 919.78 & & & 8.5 & 919.22 & 80.5 & 920.20 \\
\hline 2.0 & 919.13 & 71.0 & 919.75 & & & 9.7 & 919.08 & 81.5 & 919.85 \\
\hline 4.0 & 919.15 & 73.0 & 919.68 & & & 10.3 & 918.84 & 82.5 & 920.19 \\
\hline 6.0 & 919.15 & 75.0 & 919.57 & & & 11.8 & 918.81 & 84.5 & 919.86 \\
\hline 8.0 & 919.15 & 76.0 & 919.77 & & & 14.0 & 918.89 & 86.0 & 919.65 \\
\hline 10.0 & 919.14 & 78.0 & 919.73 & & & 15.5 & 918.94 & 87.0 & 919.86 \\
\hline 11.2 & 919.19 & 79.5 & 919.71 & & & 18.0 & 919.00 & 88.0 & 920.19 \\
\hline 13.0 & 919.33 & 80.0 & 919.82 & & & 21.0 & 919.08 & 89.0 & 920.83 \\
\hline 14.0 & 919.38 & 82.0 & 919.70 & & & 22.6 & 919.12 & 89.5 & 921.36 \\
\hline 15.0 & 919.38 & 84.0 & 919.66 & & & 24.0 & 918.98 & 90.5 & 921.41 \\
\hline 16.0 & 919.13 & 85.8 & 919.51 & & & 25.2 & 918.81 & 92.4 & 921.40 \\
\hline
\end{tabular}


Table 39. (Continued) Listing of horizontal stations and elevations for cross section PR206

[Sta., station, distance in meters from a reference pin on the left bank; Elev., elevation, in meters above sea level]

\begin{tabular}{|c|c|c|c|c|c|}
\hline \multirow{2}{*}{\multicolumn{2}{|c|}{$\begin{array}{c}1997 \\
\text { 24 September }\end{array}$}} & \multicolumn{2}{|c|}{1998} & \multicolumn{2}{|c|}{1998} \\
\hline & & \multicolumn{2}{|c|}{29 September } & \multicolumn{2}{|c|}{29 September } \\
\hline Sta. & Elev. & Sta. & Elev. & Sta. & Elev. \\
\hline 95.0 & 921.32 & -50.0 & 921.05 & 29.0 & 918.69 \\
\hline 98.0 & 921.27 & -47.5 & 921.03 & 31.0 & 918.66 \\
\hline \multirow[t]{40}{*}{100.0} & 921.23 & -45.5 & 920.92 & 33.0 & 918.61 \\
\hline & & -43.3 & 920.91 & 35.0 & 918.45 \\
\hline & & -41.0 & 920.84 & 37.0 & 918.39 \\
\hline & & -39.0 & 920.97 & 38.5 & 918.32 \\
\hline & & -37.0 & 921.00 & 40.3 & 918.21 \\
\hline & & -35.0 & 920.75 & 43.0 & 918.02 \\
\hline & & -35.3 & 920.04 & 46.0 & 917.93 \\
\hline & & -33.4 & 919.49 & 49.0 & 917.79 \\
\hline & & -31.9 & 919.30 & 52.0 & 917.68 \\
\hline & & -29.0 & 919.46 & 55.0 & 917.69 \\
\hline & & -26.0 & 919.54 & 58.0 & 917.55 \\
\hline & & -22.0 & 919.53 & 60.0 & 917.76 \\
\hline & & -20.0 & 919.28 & 62.0 & 917.42 \\
\hline & & -18.0 & 919.26 & 65.0 & 917.28 \\
\hline & & -16.0 & 919.33 & 68.0 & 917.23 \\
\hline & & -14.0 & 919.42 & 70.0 & 917.40 \\
\hline & & -12.0 & 919.55 & 72.0 & 917.57 \\
\hline & & -10.0 & 919.42 & 73.5 & 917.70 \\
\hline & & -8.5 & 919.43 & 75.5 & 918.22 \\
\hline & & -7.0 & 919.23 & 76.0 & 918.36 \\
\hline & & -6.0 & 919.13 & 76.3 & 919.86 \\
\hline & & -4.0 & 919.18 & 79.0 & 919.95 \\
\hline & & -2.0 & 919.14 & 80.5 & 920.12 \\
\hline & & 0.0 & 919.33 & 81.6 & 919.79 \\
\hline & & 2.0 & 919.35 & 82.6 & 920.18 \\
\hline & & 4.0 & 919.36 & 84.0 & 919.81 \\
\hline & & 6.0 & 919.29 & 86.0 & 919.61 \\
\hline & & 8.0 & 919.29 & 87.8 & 920.07 \\
\hline & & 9.0 & 919.22 & 88.8 & 920.57 \\
\hline & & 10.0 & 918.98 & 89.2 & 921.31 \\
\hline & & 11.0 & 918.87 & 91.0 & 921.42 \\
\hline & & 13.0 & 918.88 & 94.6 & 921.31 \\
\hline & & 15.0 & 918.90 & 97.0 & 921.29 \\
\hline & & 17.0 & 918.98 & 100.0 & 921.22 \\
\hline & & 19.0 & 919.04 & 139.6 & 921.12 \\
\hline & & 21.0 & 919.12 & & \\
\hline & & 23.0 & 919.14 & & \\
\hline & & 24.0 & 919.04 & & \\
\hline & & 25.0 & 918.81 & & \\
\hline & & 27.0 & 918.77 & & \\
\hline
\end{tabular}


Appendix. Sand--Silt and Clay analysis of suspended-sediment samples collected at Moorhead and Broadus, Montana, on the Powder River

[Data source is annual publications (U.S. Geological Survey, 1975-97); concentrations of the size fractions of silt and clay and of sand were calculated from the published total concentration and the published value of the percentage of each size fraction; $\mathrm{m}^{3} / \mathrm{s}$, cubic meter per second; $\mathrm{mg} / \mathrm{L}$, milligram per liter]

\begin{tabular}{|c|c|c|c|c|c|c|c|c|}
\hline \multirow[b]{2}{*}{ Date } & \multirow{2}{*}{ Station } & \multirow{2}{*}{$\begin{array}{c}\text { Discharge } \\
\left(\mathrm{m}^{3 / s}\right)\end{array}$} & \multirow[b]{2}{*}{ Stage } & \multicolumn{3}{|c|}{ Concentration (mg/L) } & \multicolumn{2}{|c|}{ Percent of total } \\
\hline & & & & Silt and Clay & Sand & Total & $\begin{array}{c}\text { Silt and } \\
\text { Clay }\end{array}$ & Sand \\
\hline \multicolumn{9}{|c|}{ Ice break-up floods } \\
\hline $3-07-91$ & Moorhead & 13.8 & Rising & 660 & 440 & 1,100 & 60 & 40 \\
\hline $3-19-97$ & Moorhead & 43.8 & Rising & 2,397 & 153 & 2,550 & 94 & 6 \\
\hline $3-19-97$ & Moorhead & 44.0 & Rising & 2,202 & 358 & 2,560 & 86 & 14 \\
\hline $3-19-97$ & Moorhead & 45.3 & Rising & 2,079 & 231 & 2,310 & 90 & 10 \\
\hline $3-19-97$ & Moorhead & 45.7 & Rising & 2,411 & 329 & 2,740 & 88 & 12 \\
\hline $3-19-97$ & Moorhead & 45.9 & Rising & 2,990 & 370 & 3,360 & 89 & 11 \\
\hline $3-19-97$ & Moorhead & 62.0 & Rising & 2,282 & 198 & 2,480 & 92 & 8 \\
\hline $3-19-97$ & Moorhead & 63.7 & Rising & 2,385 & 265 & 2,650 & 90 & 10 \\
\hline $3-19-97$ & Moorhead & 65.4 & Rising & 3,296 & 984 & 4,280 & 77 & 23 \\
\hline $3-19-97$ & Moorhead & 67.1 & Rising & 3,058 & 862 & 3,920 & 78 & 22 \\
\hline $3-19-97$ & Moorhead & 68.8 & Rising & 2,698 & 592 & 3,290 & 82 & 18 \\
\hline $3-19-97$ & Moorhead & 74.5 & Rising & 3,848 & 962 & 4,810 & 80 & 20 \\
\hline $3-19-97$ & Moorhead & 90.1 & Rising & 3,012 & 1,058 & 4,070 & 74 & 26 \\
\hline $3-19-97$ & Moorhead & 110 & Rising & 3,168 & 1,172 & 4,340 & 73 & 27 \\
\hline $3-01-83$ & Broadus & 32.3 & Maximum & 4,910 & 100 & 5,010 & 98 & 2 \\
\hline $3-10-88$ & Broadus & 28.3 & Maximum & 4,704 & 2,016 & 6,720 & 70 & 30 \\
\hline $3-08-90$ & Broadus & 26.8 & Maximum & 6,552 & 1,848 & 8,400 & 78 & 22 \\
\hline $3-24-84$ & Moorhead & 25.5 & Maximum & 6,248 & 772 & 7,020 & 89 & 11 \\
\hline $3-19-97$ & Moorhead & 129 & Maximum & 3,155 & 1,485 & 4,640 & 68 & 32 \\
\hline $3-19-76$ & Broadus & 40.8 & Falling & 5,181 & 4,239 & 9,420 & 55 & 45 \\
\hline $3-14-78$ & Broadus & 96.9 & Falling & 4,130 & 2,870 & 7,000 & 59 & 41 \\
\hline $3-23-78$ & Broadus & 83.0 & Falling & 5,387 & 2,653 & 8,040 & 67 & 33 \\
\hline $3-26-79$ & Broadus & 17.7 & Falling & 2,502 & 248 & 2,750 & 91 & 9 \\
\hline $3-21-84$ & Broadus & 28.6 & Falling & 9,975 & 525 & 10,500 & 95 & 5 \\
\hline $3-10-87$ & Broadus & 64.3 & Falling & 21,840 & 4,160 & 26,000 & 84 & 16 \\
\hline $3-18-75$ & Moorhead & 31.2 & Falling & 4,448 & 2,612 & 7,060 & 63 & 37 \\
\hline $3-15-78$ & Moorhead & 46.2 & Falling & 3,827 & 333 & 4,160 & 92 & 8 \\
\hline $3-23-78$ & Moorhead & 51.5 & Falling & 2,866 & 2,984 & 5,850 & 49 & 51 \\
\hline $3-01-83$ & Moorhead & 24.0 & Falling & 7,251 & 989 & 8,240 & 88 & 12 \\
\hline $3-24-87$ & Moorhead & 14.2 & Falling & 2,214 & 1,246 & 3,460 & 64 & 36 \\
\hline $3-10-88$ & Moorhead & 21.5 & Falling & 2,000 & 740 & 2,740 & 73 & 27 \\
\hline $3-09-90$ & Moorhead & 17.6 & Falling & 2,744 & 56 & 2,800 & 98 & 2 \\
\hline $3-25-93$ & Moorhead & 27.6 & Falling & 8,070 & 1,100 & 9,170 & 88 & 12 \\
\hline $3-10-94$ & Moorhead & 16.1 & Falling & 1,373 & 387 & 1,760 & 78 & 22 \\
\hline $3-19-97$ & Moorhead & 45.8 & Falling & 2,664 & 296 & 2,960 & 90 & 10 \\
\hline $3-19-97$ & Moorhead & 45.7 & Falling & 2,355 & 205 & 2,560 & 92 & 8 \\
\hline $3-19-97$ & Moorhead & 45.3 & Falling & 2,166 & 114 & 2,280 & 95 & 5 \\
\hline
\end{tabular}




\begin{tabular}{|c|c|c|c|c|c|c|c|c|}
\hline \multirow{2}{*}{ Date } & \multirow{2}{*}{ Station } & \multirow{2}{*}{$\begin{array}{c}\text { Discharge } \\
\left(\mathrm{m}^{3} / \mathrm{s}\right)\end{array}$} & \multirow[b]{2}{*}{ Stage } & \multicolumn{3}{|c|}{ Concentration (mg/L) } & \multicolumn{2}{|c|}{ Percent of total } \\
\hline & & & & Silt and Clay & Sand & Total & $\begin{array}{l}\text { Silt and } \\
\text { Clay }\end{array}$ & Sand \\
\hline $3-19-97$ & Moorhead & 45.0 & Falling & 2,242 & 118 & 2,360 & 95 & 5 \\
\hline $3-19-97$ & Moorhead & 90.0 & Falling & 5,180 & 9,620 & 14,800 & 35 & 65 \\
\hline $3-19-97$ & Moorhead & 86.9 & Falling & 4,828 & 9,372 & 14,200 & 34 & 66 \\
\hline $3-19-97$ & Moorhead & 84.5 & Falling & 4,836 & 7,564 & 12,400 & 39 & 61 \\
\hline $3-19-97$ & Moorhead & 82.0 & Falling & 4,914 & 6,786 & 11,700 & 42 & 58 \\
\hline $3-19-97$ & Moorhead & 79.6 & Falling & 4,788 & 7,812 & 12,600 & 38 & 62 \\
\hline $3-19-97$ & Moorhead & 76.3 & Falling & 5,130 & 6,270 & 11,400 & 45 & 55 \\
\hline $3-19-97$ & Moorhead & 76.3 & Falling & 5,920 & 8,880 & 14,800 & 40 & 60 \\
\hline $3-19-97$ & Moorhead & 69.7 & Falling & 6,204 & 7,896 & 14,100 & 44 & 56 \\
\hline $3-19-97$ & Moorhead & 63.1 & Falling & 7,680 & 11,520 & 19,200 & 40 & 60 \\
\hline $3-19-97$ & Moorhead & 60.0 & Falling & 6,960 & 10,440 & 17,400 & 40 & 60 \\
\hline $3-19-97$ & Moorhead & 44.4 & Falling & 6,776 & 8,624 & 15,400 & 44 & 56 \\
\hline $3-20-97$ & Moorhead & 43.3 & Falling & 4,133 & 787 & 4,920 & 84 & 16 \\
\hline $3-21-97$ & Moorhead & 28.3 & Falling & 3,223 & 857 & 4,080 & 79 & 21 \\
\hline \multicolumn{9}{|c|}{ Snowmelt floods } \\
\hline $5-17-77$ & Broadus & 62.0 & Rising & 23,751 & 3,549 & 27,300 & 87 & 13 \\
\hline $5-18-78$ & Broadus & 178 & Rising & 15,826 & 3,474 & 19,300 & 82 & 18 \\
\hline $6-07-83$ & Broadus & 40.8 & Rising & 4,070 & 170 & 4,240 & 96 & 4 \\
\hline $6-04-86$ & Broadus & 28.3 & Rising & 6,502 & 1,058 & 7,560 & 86 & 14 \\
\hline $5-19-88$ & Broadus & 50.7 & Rising & 5,962 & 2,318 & 8,280 & 72 & 28 \\
\hline $6-24-92$ & Broadus & 31.4 & Rising & 6,295 & 1,025 & 7,320 & 86 & 14 \\
\hline $5-25-76$ & Moorhead & 52.7 & Rising & 4,595 & 2,065 & 6,660 & 69 & 31 \\
\hline $5-19-81$ & Moorhead & 8.9 & Rising & 1,110 & 350 & 1,460 & 76 & 24 \\
\hline $5-20-81$ & Moorhead & 33.1 & Rising & 18,232 & 2,968 & 21,200 & 86 & 14 \\
\hline $6-07-83$ & Moorhead & 48.7 & Rising & 5,561 & 1,059 & 6,620 & 84 & 16 \\
\hline $5-02-84$ & Moorhead & 25.0 & Rising & 3,628 & 1,342 & 4,970 & 73 & 27 \\
\hline $6-09-86$ & Moorhead & 123 & Rising & 20,020 & 1,980 & 22,000 & 91 & 9 \\
\hline $6-14-96$ & Moorhead & 51.8 & Rising & 698 & 1,242 & 1,940 & 36 & 64 \\
\hline $5-26-76$ & Broadus & 74.5 & Maximum & 6,873 & 1,827 & 8,700 & 79 & 21 \\
\hline $6-27-82$ & Broadus & 96.9 & Maximum & 20,726 & 3,374 & 24,100 & 86 & 14 \\
\hline $5-30-91$ & Broadus & 64.3 & Maximum & 4,257 & 2,193 & 6,450 & 66 & 34 \\
\hline $6-18-92$ & Broadus & 73.1 & Maximum & 18,360 & 3,240 & 21,600 & 85 & 15 \\
\hline $5-21-75$ & Moorhead & 58.1 & Maximum & 7,986 & 4,114 & 12,100 & 66 & 34 \\
\hline $5-17-77$ & Moorhead & 134 & Maximum & 29,340 & 3,260 & 32,600 & 90 & 10 \\
\hline $5-25-81$ & Moorhead & 56.9 & Maximum & 9,216 & 3,584 & 12,800 & 72 & 28 \\
\hline $5-22-85$ & Moorhead & 15.4 & Maximum & 11,564 & 236 & 11,800 & 98 & 2 \\
\hline $6-05-86$ & Moorhead & 58.1 & Maximum & 17,019 & 1,281 & 18,300 & 93 & 7 \\
\hline
\end{tabular}


Appendix. Sand--Silt and Clay analysis of suspended-sediment samples collected at Moorhead and Broadus, Montana, on the Powder River

\begin{tabular}{|c|c|c|c|c|c|c|c|c|}
\hline \multirow[b]{2}{*}{ Date } & \multirow[b]{2}{*}{ Station } & \multirow{2}{*}{$\begin{array}{c}\text { Discharge } \\
\left(\mathrm{m}^{3} / \mathrm{s}\right)\end{array}$} & \multirow[b]{2}{*}{ Stage } & \multicolumn{3}{|c|}{ Concentration $(\mathrm{mg} / \mathrm{L})$} & \multicolumn{2}{|c|}{ Percent of total } \\
\hline & & & & Silt and Clay & Sand & Total & $\begin{array}{l}\text { Silt and } \\
\text { Clay }\end{array}$ & Sand \\
\hline $5-19-88$ & Moorhead & 49.3 & Maximum & 3,584 & 2,596 & 6,180 & 58 & 42 \\
\hline $5-29-91$ & Moorhead & 66.8 & Maximum & 3,928 & 2,512 & 6,440 & 61 & 39 \\
\hline $6-17-92$ & Moorhead & 88.4 & Maximum & 18,792 & 2,808 & 21,600 & 87 & 13 \\
\hline $5-08-93$ & Moorhead & 142 & Maximum & 45,936 & 6,264 & 52,200 & 88 & 12 \\
\hline $5-11-95$ & Moorhead & 185 & Maximum & 34,196 & 7,004 & 41,200 & 83 & 17 \\
\hline $5-22-78$ & Broadus & 252 & Falling & 20,340 & 2,260 & 22,600 & 90 & 10 \\
\hline 6-05-78 & Broadus & 98.8 & Falling & 3,427 & 1,613 & 5,040 & 68 & 32 \\
\hline $6-04-81$ & Broadus & 33.1 & Falling & 4,410 & 1,470 & 5,880 & 75 & 25 \\
\hline $5-01-87$ & Broadus & 40.2 & Falling & 10,750 & 1,750 & 12,500 & 86 & 14 \\
\hline $6-11-87$ & Broadus & 71.9 & Falling & 29,754 & 4,446 & 34,200 & 87 & 13 \\
\hline $6-05-90$ & Broadus & 28.1 & Falling & 2,471 & 1,009 & 3,480 & 71 & 29 \\
\hline $5-23-91$ & Broadus & 96.9 & Falling & 5,773 & 2,717 & 8,490 & 68 & 32 \\
\hline 6-02-92 & Broadus & 0.68 & Falling & 58 & 8 & 66 & 88 & 12 \\
\hline $6-21-95$ & Broadus & 110 & Falling & 2,668 & 2,272 & 4,940 & 54 & 46 \\
\hline $5-13-81$ & Moorhead & 4.4 & Falling & 1,703 & 17 & 1,720 & 99 & 1 \\
\hline $6-02-81$ & Moorhead & 40.8 & Falling & 4,913 & 1,638 & 6,550 & 75 & 25 \\
\hline $6-27-84$ & Moorhead & 44.5 & Falling & 985 & 365 & 1,350 & 73 & 27 \\
\hline $6-25-87$ & Moorhead & 12.0 & Falling & 1,486 & 444 & 1,930 & 77 & 23 \\
\hline $6-05-90$ & Moorhead & 25.2 & Falling & 2,230 & 1,200 & 3,430 & 65 & 35 \\
\hline $5-22-91$ & Moorhead & 103 & Falling & 5,369 & 2,301 & 7,670 & 70 & 30 \\
\hline $6-21-95$ & Moorhead & 101 & Falling & 1,778 & 1,642 & 3,420 & 52 & 48 \\
\hline 6-06-79 & Moorhead & 12.3 & Minimum & 1,112 & 228 & 1,340 & 83 & 17 \\
\hline $5-14-87$ & Moorhead & 9.1 & Minimum & 1,096 & 164 & 1,260 & 87 & 13 \\
\hline $6-03-92$ & Moorhead & 1.2 & Minimum & 20 & 30 & 50 & 41 & 59 \\
\hline $6-03-93$ & Moorhead & 43.9 & Minimum & 2,499 & 1,531 & 4,030 & 62 & 38 \\
\hline \multicolumn{9}{|c|}{ Flash floods } \\
\hline $6-19-75$ & Moorhead & 274 & Peak & 43,736 & 5,964 & 49,700 & 88 & 12 \\
\hline $9-25-86$ & Moorhead & 25.7 & Peak & 6,899 & 941 & 7,840 & 88 & 12 \\
\hline $7-29-92$ & Moorhead & 23.3 & Peak & 6,252 & 618 & 6,870 & 91 & 9 \\
\hline 7-09-94 & Moorhead & 73.9 & Peak & 40,222 & 3,978 & 44,200 & 91 & 9 \\
\hline $7-31-79$ & Broadus & 22.6 & Falling & 22,310 & 690 & 23,000 & 97 & 3 \\
\hline $8-24-82$ & Broadus & 20.8 & Falling & 24,552 & 248 & 24,800 & 99 & 1 \\
\hline $8-29-82$ & Broadus & 6.6 & Falling & 18,500 & 0 & 18,500 & 100 & 0 \\
\hline $9-23-82$ & Broadus & 22.7 & Falling & 12,816 & 1,584 & 14,400 & 89 & 11 \\
\hline 8-23-90 & Broadus & 25.1 & Falling & 12,512 & 1,088 & 13,600 & 92 & 8 \\
\hline $8-24-76$ & Moorhead & 1.2 & Falling & 232 & 2 & 234 & 99 & 1 \\
\hline $7-31-79$ & Moorhead & 13.8 & Falling & 24,056 & 744 & 24,800 & 97 & 3 \\
\hline
\end{tabular}


Appendix. Sand--Silt and Clay analysis of suspended-sediment samples collected at Moorhead and Broadus, Montana, on the Powder River

\begin{tabular}{|c|c|c|c|c|c|c|c|c|}
\hline \multirow[b]{2}{*}{ Date } & \multirow[b]{2}{*}{ Station } & \multirow{2}{*}{$\begin{array}{c}\text { Discharge } \\
\left(\mathrm{m}^{3} / \mathrm{s}\right)\end{array}$} & \multirow[b]{2}{*}{ Stage } & \multicolumn{3}{|c|}{ Concentration $(\mathrm{mg} / \mathrm{L})$} & \multicolumn{2}{|c|}{ Percent of total } \\
\hline & & & & Silt and Clay & Sand & Total & $\begin{array}{l}\text { Silt and } \\
\text { Clay }\end{array}$ & Sand \\
\hline 8-04-81 & Moorhead & 5.1 & Falling & 5,550 & 0 & 5,550 & 100 & 0 \\
\hline $7-21-87$ & Moorhead & 10.3 & Falling & 2,190 & 190 & 2,380 & 92 & 8 \\
\hline $9-26-89$ & Moorhead & 6.1 & Falling & 25,641 & 259 & 25,900 & 99 & 1 \\
\hline $8-22-90$ & Moorhead & 26.9 & Falling & 10,904 & 696 & 11,600 & 94 & 6 \\
\hline 7-10-94 & Moorhead & 36.5 & Falling & 39,330 & 2,070 & 41,400 & 95 & 5 \\
\hline $7-28-94$ & Moorhead & 1.4 & Falling & 122 & 8 & 130 & 94 & 6 \\
\hline
\end{tabular}



